\title{
5. CENOZOIC PALEOCLIMATIC AND PALEOCEANOGRAPHIC CHANGES IN THE NORTHERN HEMISPHERE REVEALED BY VARIABILITY OF COARSE-FRACTION COMPOSITION IN SEDIMENTS FROM THE VØRING PLATEAU-ODP LEG 104 DRILL SITES ${ }^{1}$
}

\author{
Rüdiger Henrich, ${ }^{2,5}$ Thomas Wolf, ${ }^{2}$ Gerhard Bohrmann, ${ }^{3}$ and Jörn Thiede ${ }^{4}$
}

\begin{abstract}
Coarse-fraction studies of sediments recovered during ODP Leg 104 are used to reconstruct paleoclimatic and paleoceanographic environments on a time scale of 0.1 to $0.5 \mathrm{~m} . \mathrm{y}$. for the past $20 \mathrm{Ma}$. These investigations suggest that relatively warm climates and isolated deep water conditions prevailed prior to $13.6 \mathrm{Ma}$ and between 5.6-4.8 Ma. The first major deep water outflow from the Norwegian-Greenland Sea into the North Atlantic took place at about 13.6 Ma. Progressive cooling linked to increased deep water renewal in the Norwegian-Greenland Sea appears to have occurred between 13.6-5.6 Ma and 4.8-3.1 Ma. A major onset of ice-rafted debris is recorded at 2.56 Ma. Terrigenous coarsefraction components show important fluctuations with two major peaks during the past $0.8 \mathrm{Ma}$.
\end{abstract}

\section{INTRODUCTION}

During the summer of 1985 , ODP Leg 104 sediments were drilled at three sites in the Vøring Plateau region of the Norwegian Sea. Site 643 is located on the lower slope of the outer Vøring Plateau, and Site 642 lies at the top of this submarine feature. Site 644 is located in the inner Vøring Basin and represents the landward end of this northwest to southeast transect (Fig. 1).

One of the main goals in drilling this transect was to understand paleoclimatic and paleoceanographic variations in the Norwegian Sea during the Cenozoic. Because of its high-latitude location adjacent to the cold Arctic Ocean, the modern Norwegian-Greenland Sea is one of the globally most important regimes for modern deep water renewal (Meincke 1983). Over the past two decades, numerous investigations on the evolution of the Norwegian-Greenland Sea and its Cenozoic sediment record have aided in reconstruction of paleoclimatic changes of the Northern Hemisphere. Its origin and structural evolution is well known from magnetic anomaly patterns (Talwani and Eldholm, 1977), permitting reconstructions of its size and shape during Cenozoic times (Thiede 1979, Eldholm and Thiede, 1980).

Interpretations of the paleoenvironmental regimes in the Norwegian-Greenland Sea have been obtained mostly from deepsea drilling and conventional sediment cores (e.g., Talwani, Udintsev, et al., 1976). The oldest sediments collected during DSDP Leg 38 are of early Eocene age (Schrader et al., 1976); they document a pelagic and relatively warm environment at that time. Marine connections were established later with the North Atlantic and the Arctic, but the timing of these paleoceanographic events is still controversial (Bott et al., 1983;

\footnotetext{
${ }^{1}$ Eldholm, O., Thiede, J., Taylor, E., et al., 1989. Proc. ODP, Sci. Results, 104: College Station, TX (Ocean Drilling Program).

2 Geologisch-Päläontologisches Institut und Museum der Christian-AlbrechtsUniversität zu Kiel; Olshausenstr. 40, D-2300 Kiel, Federal Republic of Germany.

${ }^{3}$ Alfred-Wegner-Institut für Polar und Meereforschung, Postfach 120161, D2850 Bremerhaven, Federal Republic of Germany.

${ }^{4}$ GEOMAR, Forschungszentrum für Marine Geowissenschaften an der Universität Kiel, Wischhofstr. 1-3, D-2300 Kiel, Federal Republic of Germany.

5 Present address: GEOMAR, Forschungszentrum für Marine Geowissenschaften an der Universität Kiel, Wischhofstr. 1-3, D-2300 Kiel, Federal Republic of Germany.
}

Thiede and Eldholm, 1983; Berggren and Schnitker, 1983; Kitchell and Clark, 1982).

The onset of glacially influenced deposition in the North Atlantic (Schaeffer and Spiegler, 1986) and the Norwegian Sea was marked by a considerable change in the regional pattern of sediment flux. Isopachs of sediment with ice-rafted components (Warnke and Hansen, 1977) suggest that deposition of this material was controlled by surface water flowing parallel to the Norwegian and East Greenland continental margins. Detailed studies on composition and distribution of surface sediments (Eisma and v.d.Gaast, 1983; Kellogg, 1975b) and stratigraphic studies on Quaternary deposits (Kellogg, 1976; Bjørklund and Goll, 1979) have shown that the relatively warm Norwegian Current, presently the dominant feature of the eastern Norwegian Sea, appears to have reached the Norwegian Sea only during the peak intervals of a few interglacials.

Global models describing changes in the paleoenvironment and paleoclimatology of the Northern Hemisphere have been published by numerous researchers (viz. CLIMAP Project members, 1981; Holtedahl and Bjerkli, 1982; Johnson and Heezen, 1967; Kellogg, 1975a, 1977, 1980; Kellogg et al., 1978; Ruddiman et al., 1986a; Ruddiman et al., 1986b; Shackleton et al., 1984; Thiede, 1980; Thiede et al., 1986; and Vogt, 1986). However, the exact nature of events leading to Northern Hemisphere cooling during the Miocene and Pliocene and the variety of processes and feedback mechanisms that influence the cyclic growth and decay of the large Northern Hemisphere ice sheets during Pliocene and Quaternary remain the subject of discussion today (Ruddiman et al., 1986a).

The primary shortcoming of most previous investigations was the restricted amounts of useful samples recovered by earlier coring techniques. The recently developed hydraulic piston coring technique permits almost continuous coring, which has contributed substantially to improvements in the resolution of paleoenvironmental histories of the world's ocean basins.

The principal objectives of this study are to document compositional variations of major coarse-fraction assemblages in Leg 104 Cenozoic sediments and to use this information as documentation of major changes in surface and bottom-water circulation patterns. Our interpretations have been derived from a voluminous data base, permitting a temporal resolution of events in 0.1 to $0.5 \mathrm{~m} . \mathrm{y}$. in duration, based on changes of coarse-fraction composition. In view of this new data, evolution of cold climates and the onset and variability of large-scale Northern 'Iemisphere glaciations are discussed. Of interest to others us- 


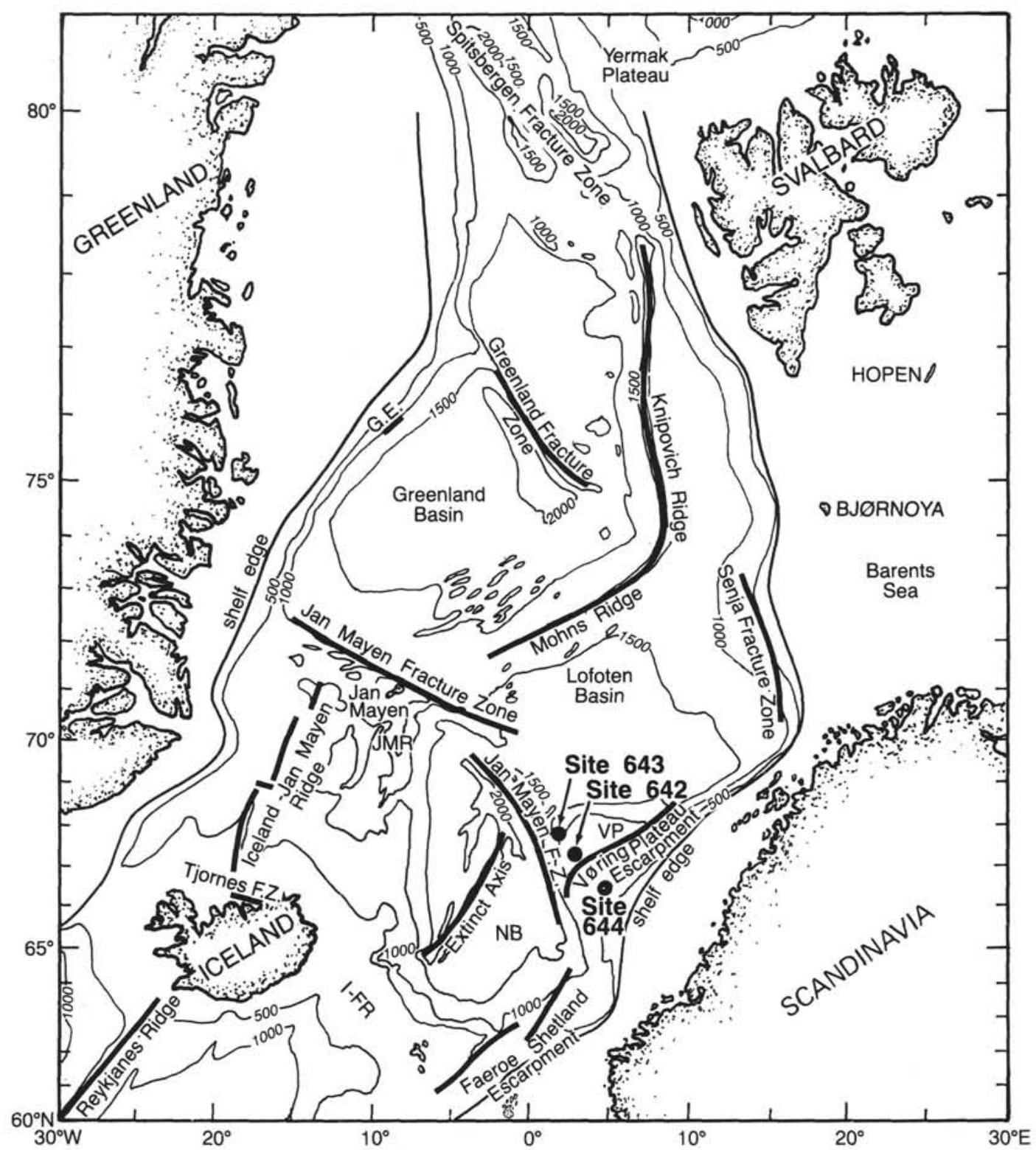

Figure 1. Locations of Leg 104 drill sites. Map based on Talwani and Eldholm (1977).

ing samples from these drill sites, detailed coarse-component analysis permits more precise lithostratigraphic correlation between the Leg 104 drill sites and aids in recognition of hiatuses.

\section{METHODS}

To obtain a high-resolution record of the character and composition of coarse-fraction components, two samples (sample interval approximately $70 \mathrm{~cm}$ ) per section were analyzed from Holes $642 \mathrm{~B} / \mathrm{D}$, while five to seven samples (sample interval approximately $25 \mathrm{~cm}$ ) per section were taken at Hole 643A and six to seven samples (sample interval approximately $20 \mathrm{~cm}$ ) per section were collected from Hole 644A. Data are given in the Appendix. Samples from Holes 643A and 644A were processed partially in Bergen (Group E. Jansen-stable isotopes). The given sample intervals provide a stratigraphic resolution of 3 to $20 \mathrm{k}$.y. for the last $1 \mathrm{Ma}$. Age determinations in the interval from $1 \mathrm{Ma}$ to $2.6 \mathrm{Ma}$ mainly rely on calculations of linear sedimentation rates between paleomagnetic (Bleil, this volume) and a few biostratigraphic datum levels (Donnally, this volume). The stratigraphic framework in sections older than 2.6 Ma is based on combined biostratigraphic and paleomagnetic evidence (Goll, this volume).
After treatment with ammonia-buffered hydrogen peroxide, each sample was split by wet sieving into a fine fraction $<63 \mu \mathrm{m}$ and a coarse fraction $>63 \mu \mathrm{m}$. After drying, both fractions were weighed and the coarse fraction was dry-sieved with a sonic sieving system into 63-125 $\mu \mathrm{m}, 125-250 \mu \mathrm{m}, 250-500 \mu \mathrm{m}, 500-1000 \mu \mathrm{m}$, and $>1000 \mu \mathrm{m}$ subfractions. Subsequently, weight losses were calculated as the starting weight minus the sum of the fraction weights.

To compare coarse-fraction data of ODP Leg 104 sites with other ODP/DSDP data sets, the investigation was limited to the $125-500 \mu \mathrm{m}$ subfractions. These were considered to be representative of the total coarse-fraction composition (Sarnthein, 1971). After subdivision by microsplitting to representative subsamples of countable grain amounts, $500-700$ grains were analyzed microscopically, counted and distinguished as follows:

Biogenic Components-Planktonic foraminifers, calcareous benthic and arenaceous foraminifers, siliceous skeletons such as radiolarians, diatoms, and sponge spicules. Other biogenic components such as ostracods, pelecypods, and echinoid fragments; phosphatic debris were also counted but not discussed in this study.

Terrigenous Components-Quartz, feldspar, rock fragments, mica, and heavy minerals. 
Authigenic Components-Glauconite, pyrite, crystals, and pyritized burrows, including higher amounts of other components such as clay mineral aggregates (gypsum crystals and diagenetic aggregates were excluded). Volcanic Components-Volcanic glass shards and tuffaceous components.

Grain abundance percentages were converted to weight percentages for each component (by referencing grain amount percentages to the total coarse-fraction weight) and plotted vs. core depth. Generally, the variability of coarse-fraction amounts are lower in deeper intervals. In Figures 6,7 , and 8 the scales of biogenic components are outlined as grain amount percentages of the $>63-\mu \mathrm{m}$ fraction. Selected samples were investigated with a S150 Cambridge Scanning Electron Microscope at $10 \mathrm{kV}$ to document typical coarse-particle assemblages and to characterize tuffaceous components and diagenetic aggregates texturally and mineralogically.

\section{RESULTS}

\section{Coarse-fraction Amounts and Grain-size Distribution}

Amounts of coarse-fraction particles of the three sites are outlined in Figures 3 to 11. Data are given in Tables 1.1 through 1.4 in the Appendix. In general, amounts ranging between 5$20 \%$, and in some cases up to $40 \%$ of bulk sediment, are recorded in lithologic Unit I (compare Eldholm, Thiede, Taylor, et al., 1987). These high coarse-fraction contents are mostly caused by a high input of terrigenous sand-sized material derived from ice-rafting and to a lesser degree by planktonic foraminifers. In contrast, at Sites 642 and 643 below lithologic Unit I the coarsefraction amounts are significantly lower and values are typically on the order of 0.5 to $1.5 \%$.

Exceptions are found in the numerous deep levels characterized by volcanic glass shards and some intervals of glauconite formation well defined in the Site 642 cores as single peaks in the $>63-\mu \mathrm{m}$ record (Figs. 4 and 7). Samples with high percentages of coarse-fraction particles are also characterized by their grain-size distribution (Figs. 2A-C). On the basis of microscopic examination, all samples in Hole 643A deeper than 51.26 mbsf, and all samples in Hole 642B and 642D deeper than 66.70 mbsf that contain increased amounts of the fraction $>1000 \mu \mathrm{m}$ are dominated by volcanic ash material (Figs. 2A-C).

\section{Particle Assemblages in Coarse-fraction Sediments}

A limited number of common particle assemblages has been identified. Changes in assemblages are used to characterize the most significant shifts in coarse-particle deposition of upper Cenozoic to Quaternary sediments at the Vøring Plateau. Interpretations based only on changes in coarse-fraction composition have to be used with caution since fine-fraction and bulksediment properties eventually must be considered as well. In addition, splitting the bulk sediment at $63 \mu \mathrm{m}$ may bias the distributions of natural particle assemblages. This factor is of special importance when biogenic pelagic components are analyzed, because mean test diameters of various planktonic siliceous organisms do not correspond to the coarse-fraction grain-size limits. Keeping in mind these limitations, the following major particle assemblages were recognized in Leg 104 sediments (Plates 1 and 2).

\section{Type I: Biogenic Calcareous Particle Assemblage}

The biogenic calcareous particle assemblage consists predominantly of variable amounts of planktonic and benthic calcareous foraminifers (Plate 1-1). Admixtures of other particles occur in minor amounts. Most of the minor components are terrigenous (quartz, feldspar, rock fragments, and mica) and biogenic siliceous particles (sponge spicules, radiolarians, and diatoms). Trace admixtures consist of ostracods, molluscs, and echinoderm shells and fragments, and of phosphatic debris (fish bones, etc.). Bulk sediment properties of this assemblage normally display intermediate to high carbonate contents $(<10$ to $50 \%)$ and low to intermediate organic carbon contents $(0.1$ to $0.5 \%$ ). High carbonate contents in the entire interval reflect large amounts of fine-fraction carbonate (e.g., calcareous nannoplankton). Type I particle assemblages characterize normal interglacial sediments in the glacial/interglacial cycles and dominate the foraminifer-nannofossil oozes and muds that were deposited during Miocene and Pliocene times.

\section{Type II: Biogenic Siliceous Particle Assemblage}

Biogenic siliceous particle assemblages (Plate 1-5) consist of variable proportions of radiolarians, sponge spicules and a low number of diatoms with occasional admixtures of minor amounts of biogenic calcareous particles (planktonic and benthic foraminifers). Variable amounts of vitric tephra fragments are admixed in most samples (Plates 1-6 and 2-1). Coarse terrigenous components are almost completely absent or occur as minor traces, thereby suggesting that these sediments are pelagic and hemipelagic deposits. In this type, terrigenous supply is derived almost exclusively by variable admixtures of mica. Low bulk carbonate contents or carbonate-free deposits and intermediate to high total organic carbon contents $(0.8$ to $5.0 \%)$ are normally observed in deposits characterized by Type II assemblages.

Based on compositional differences, two major subtypes of the biogenic siliceous particle assemblages can be distinguished in the Leg 104 sediments. IIa is sponge spicule-dominated subtype and $\mathrm{IIb}$ is a radiolarian-dominated subtype. These subtypes are considered to reflect major environmental changes. However, because the two subtypes have different mean grain sizes, their contents have to be interpreted with caution.

\section{Type III: Terrigenous Particle Assemblage}

Terrigenous particle assemblages consist of variable amounts of quartz, feldspar, rock fragments, mica, and heavy minerals (Plates 1-3 and 1-4). Generally poor sorting (Fig. 2) and the occasional occurrence of centimeter-sized dropstones suggest icerafting as the prevalent transport mechanism for this particle assemblage. Minor amounts of biogenic calcareous (planktonic and benthic foraminifers) and biogenic silica particles are found in the terrigenous particle assemblages (Plate 1-2). In this assemblage type, bulk carbonate contents are low $(0$ to $5 \%)$ and organic carbon contents are higher $(0.4$ to $1.3 \%)$ than observed in carbonate-rich intervals of type I $(0.1$ to $0.3 \%)$. The organic carbon apparently is derived mainly from terrigenous sources or diagenetically altered organic carbon-rich sediments (see Kvenvolden et al., this volume; Henrich, this volume). The terrigenous particle assemblage is most frequently associated with maximum concentrations of bulk coarse-fraction material. Terrigenous particle assemblages are restricted almost totally to the core sections having glacial/interglacial cycles.

\section{Type IV: Ash-dominated Particle Associations}

Based on their diagenetic alteration characteristics, ash fragment-dominated particle associations can be subdivided in two subassociations. The first includes subsamples dominated by vitric unaltered glass shards showing different degrees of vesicularity and stretching admixed with particles from assemblage types I and II (Plates 1-6, 2-1, and 2-2.). The second subassociation includes partly to strongly altered tephra components forming various diagenetic aggregates (Plates 2-3, 2-4, 2-5, and 2-6), composed of authigenic clay minerals such as smectites and glauconites with impregnations of pyrite (compare Desprairies et al., this volume; Froget et al., this volume). This subassociation was found in the vicinity of distinctive vitric ash layers and 

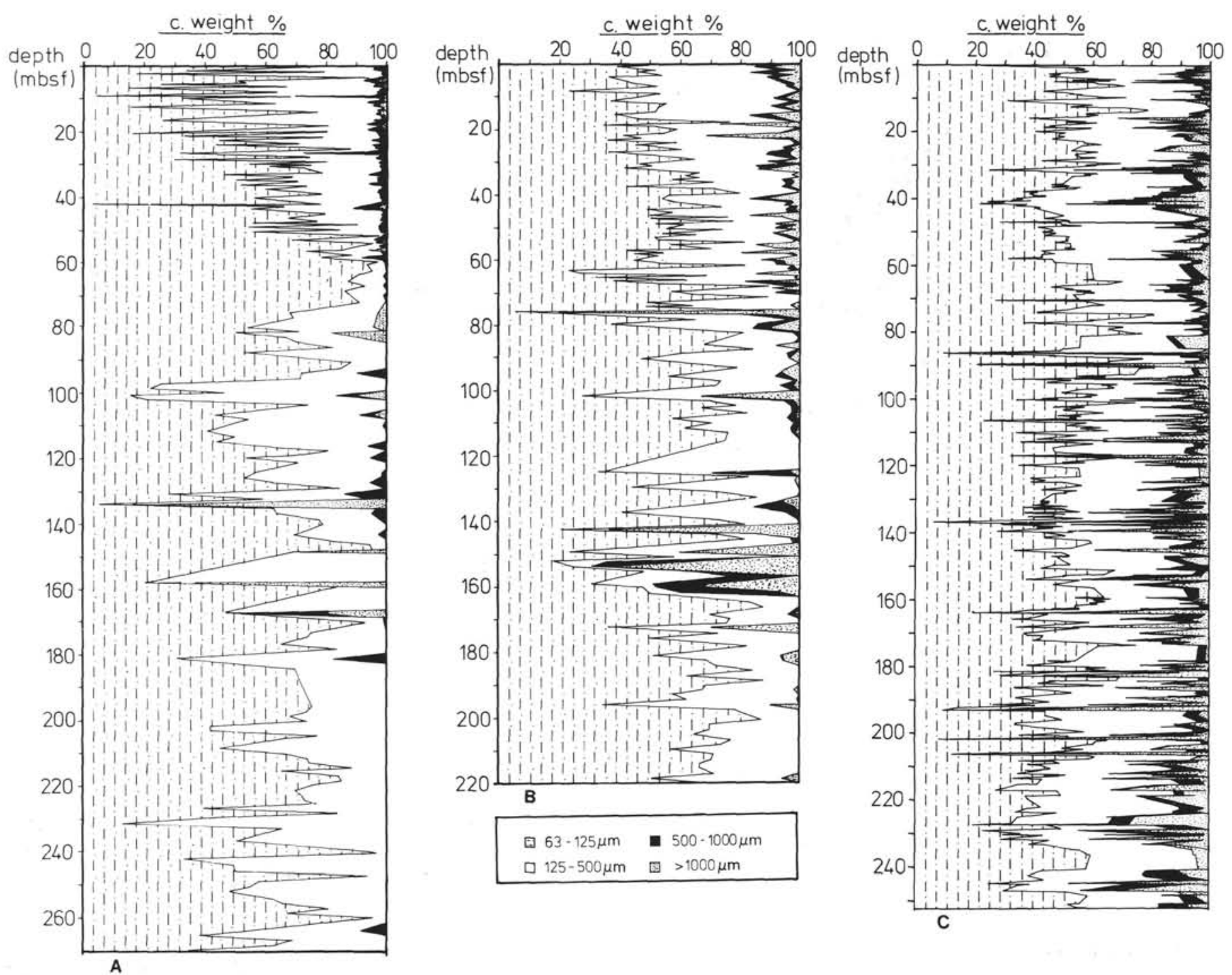

Figure 2. Grain-size distribution in (A) Hole 642B, (B) Hole 643A, and (C) Hole 644A.

ash fragments and seems also to be disseminated throughout the sedimentary column at various levels (Eldholm, Thiede, Taylor, et al., 1987). Strongly altered tephra particles form the majority of coarse particles in the Eocene and Oligocene sediments in Leg 104 cores.

\section{Patterns of Typical Coarse-fraction Particle Assemblages}

Coarse-fraction compositional variations occur in specific sedimentary sections of the Leg 104 drill sites reflecting changes in the depositional environment. The following sections are distinguished and can be correlated, with only minor differences, between the drill sites.

\section{Section A: Hole 642B (Core 104-642B-1H to 104-642B-8H), Hole $643 \mathrm{~A}$ (Cores 104-643A-1H to 104-643A-6H) and Hole $644 \mathrm{~A}$ (Cores 104-644A-1H to 104-644A-30H).}

The coarse-fraction composition of Section A is characterized by high-amplitude variations in coarse-fraction assemblages of type I and III (Figs. 3 to 11). The Section as defined by grains $>63 \mu \mathrm{m}$ corresponds well to lithologic Unit I (Eldholm, Thiede, Taylor et al., 1987). The base of Section A has an age of 2.56 Ma (Fig. 12, for details compare discussion).
Biogenic calcareous assemblage type 1 is rarely found in sediments below $32 \mathrm{msbf}$ in Hole 643A (Fig. 3) and 40 mbsf in Hole 642B (Fig. 4) corresponding to lower productivity for carbonate-producing plankton in surface water combined with carbonate dissolution (Henrich, this volume). This pronounced change in sediment composition corresponds to an age of 1.0 to $1.2 \mathrm{Ma}$ at both sites (Fig. 12). This decrease in calcareous planktonic foraminifers is also documented in Site 644 (Figs. 5, 8) below $110 \mathrm{mbsf}$, corresponding to the same age. Sediments in this lower parts of Section A contain nearly exclusively the terrigeneous assemblage type III. They are characterized by alternate occurrences of intervals with low and high concentrations of coarse-fraction and ice-rafted debris. The immature coarse particle assemblages are composed of variable amounts of quartz, feldspar, mica, and rock fragments (Figs. 3, 4, 5, 9, 10, 11).

Section $\mathrm{A}$ in Hole $644 \mathrm{~A}$ is characterized by alternations of types I and III coarse-fraction assemblages throughout the entire section. The upper 110 mbsf (Fig. 5) has high-amplitude alternations of type I and III similar to those observed in Holes 642B and 643A (Figs. 3, 4). Below, there is a change in coarsefraction composition and high-frequency, low-amplitude oscillations of bulk coarse fraction amount occur. This level corresponds to an age of about $1.0 \mathrm{Ma}$. While in the upper $110 \mathrm{mbsf}$, planktonic foraminifers strongly dominate assemblage type I, 


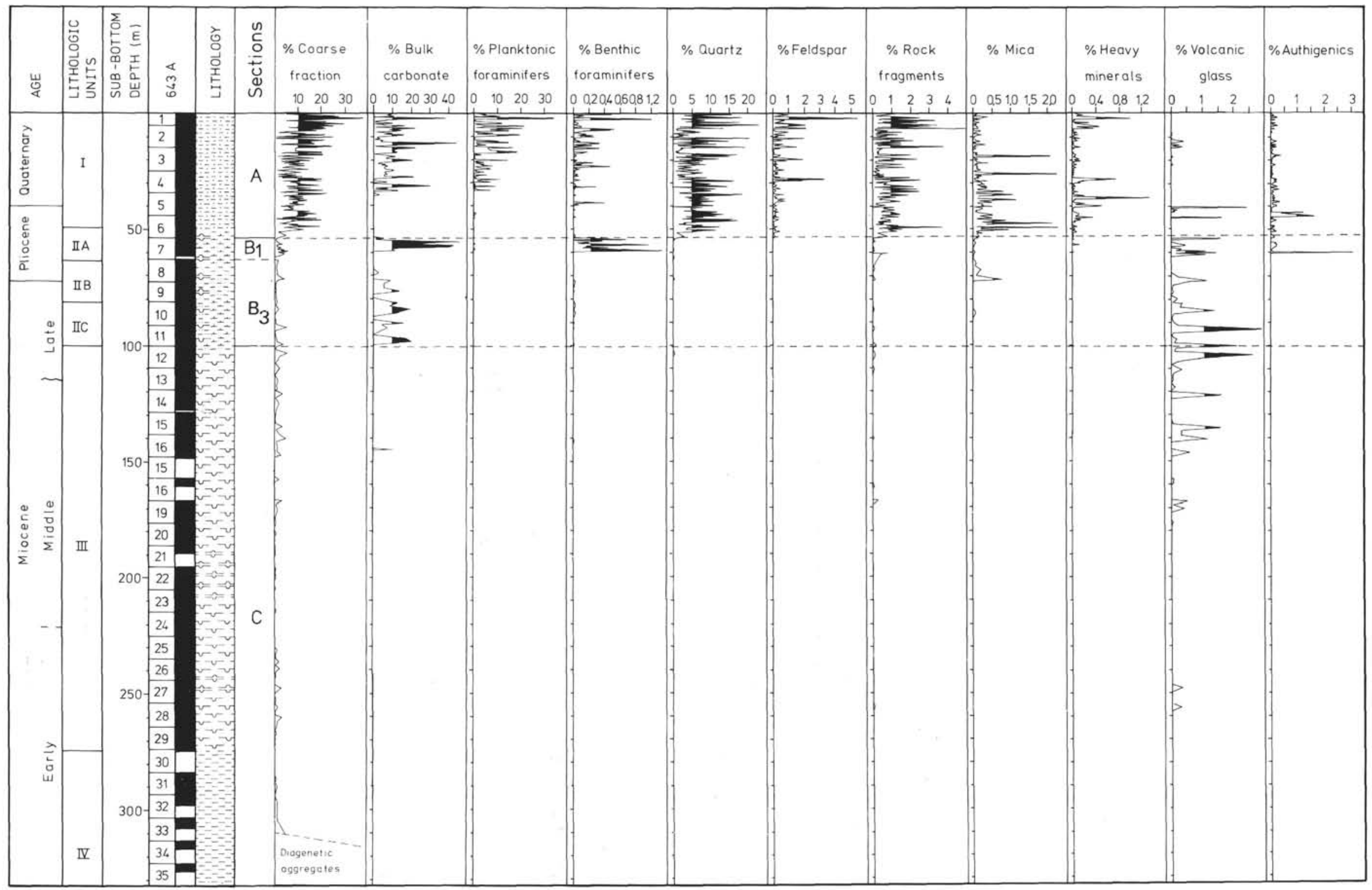

Figure 3. Coarse-fraction components in Hole 643A. (Bulk carbonate data; compare Henrich, this volume. Note: component percentages are with reference to total component weight). 


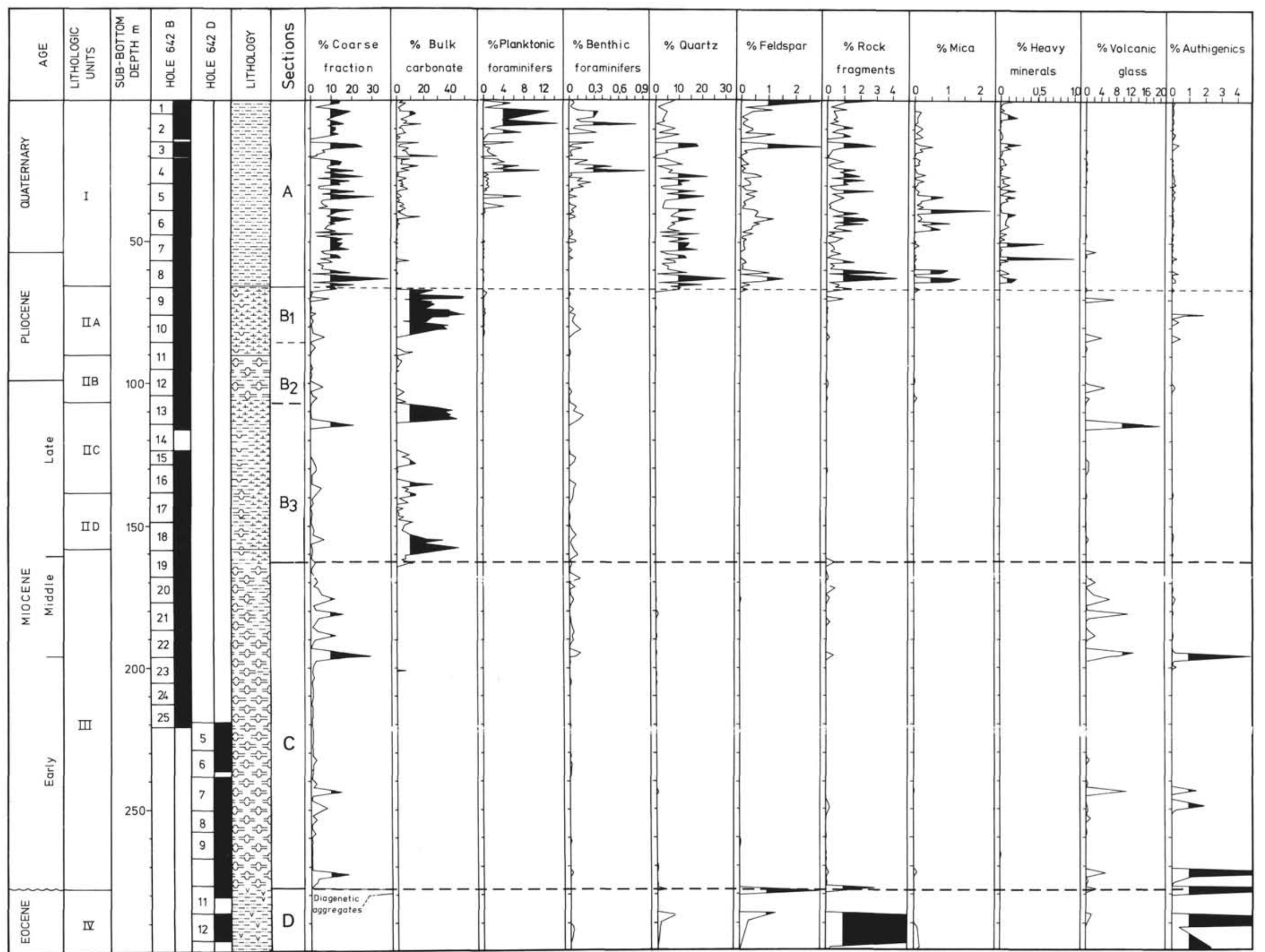




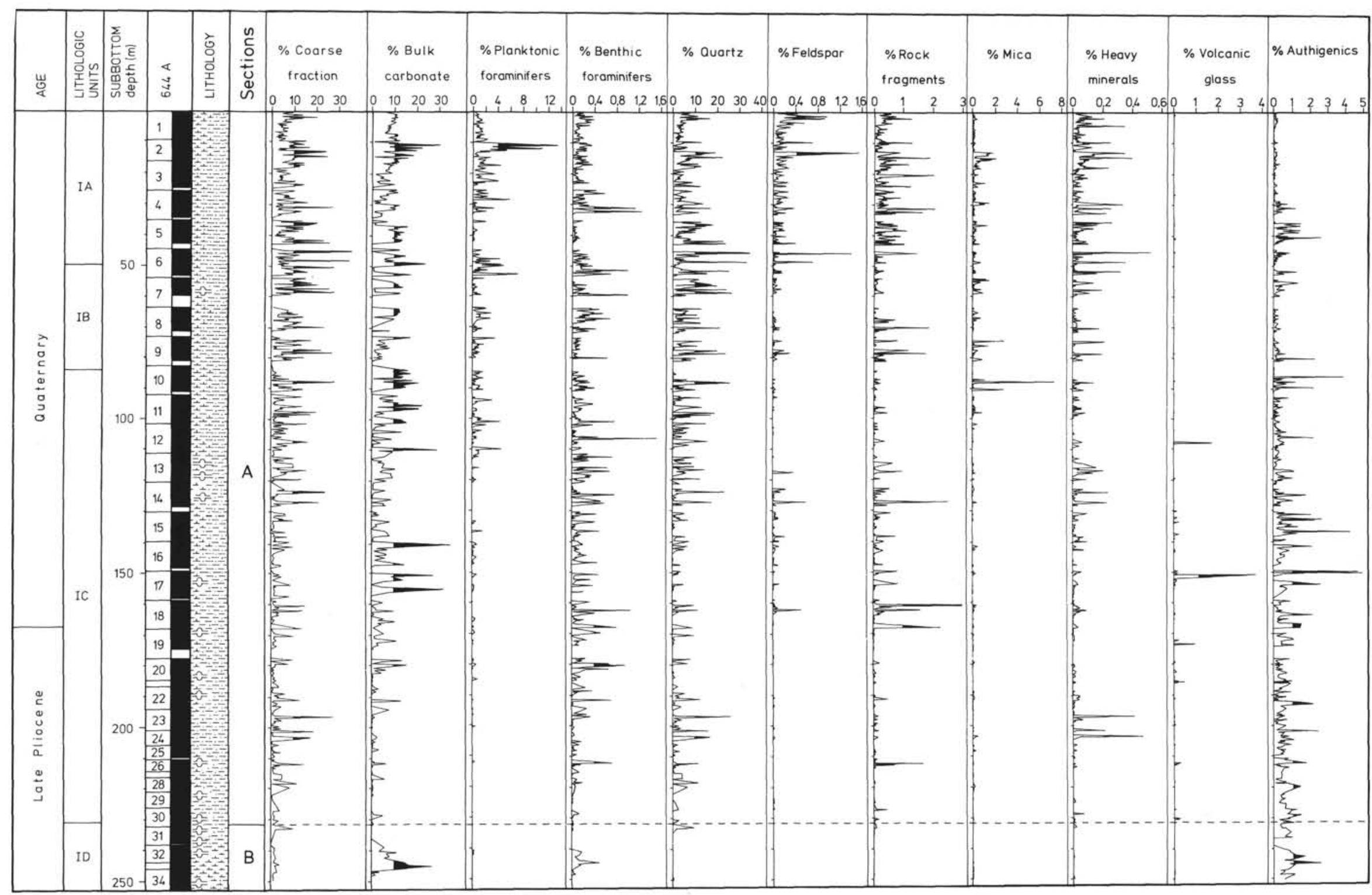

Figure 5. Coarse-fraction components in Hole 644A (Bulk carbonate data; compare Henrich, this volume. Note: component percentages are with reference to total component weight). 




Figure 6. Biogenic components, Hole 643A (biogenic opal data from Bohrmann, 1988; palynomorph counts from Manum et al., this volume. Note: all percentages refer to grain amounts. 


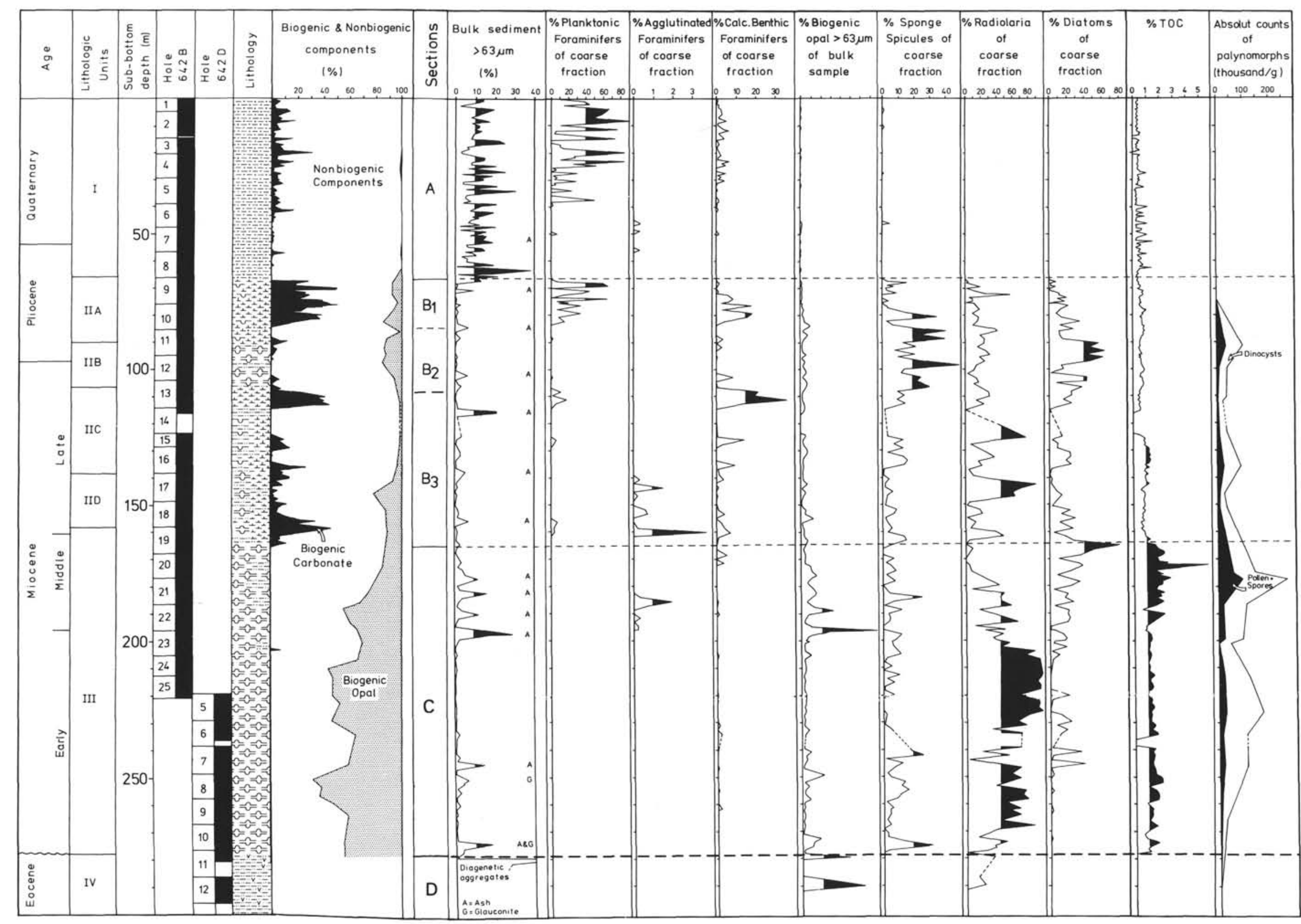




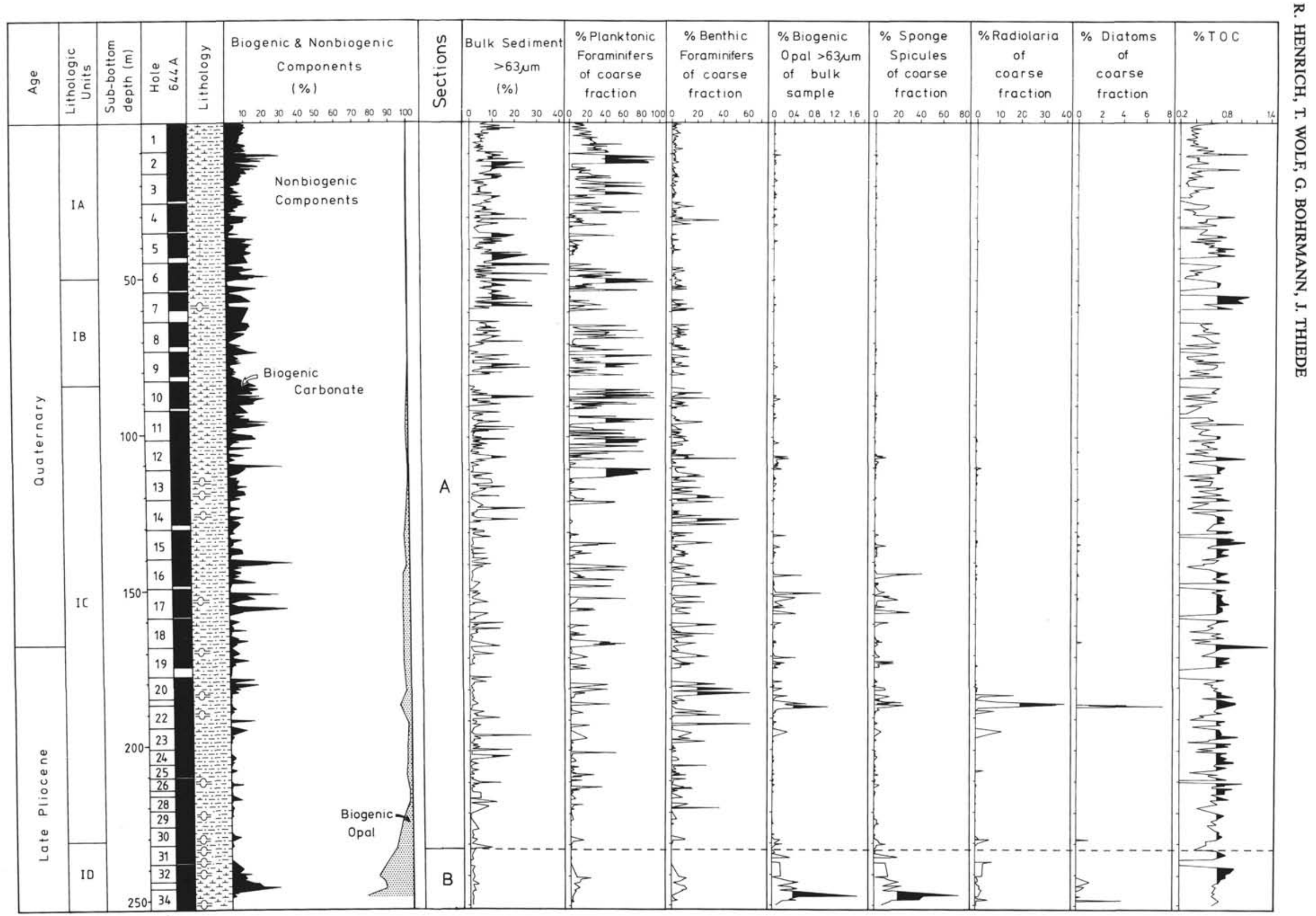

Figure 8. Biogenic components, Hole 644A (biogenic opal data from Bohrmann, 1988; palynomorph counts from Manum et al., this volume. Note: all percentages refer to grain amounts. 


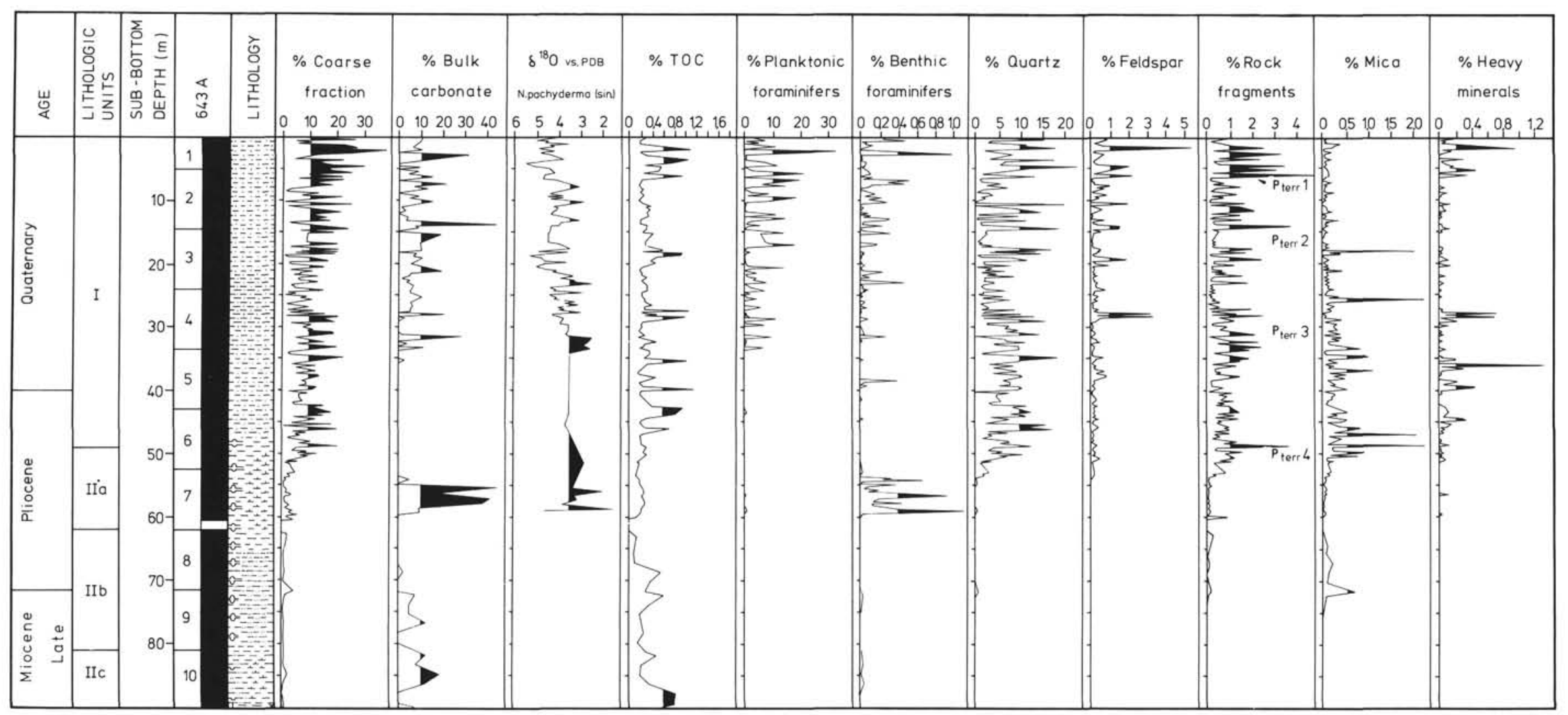

Figure 9. Coarse-fraction components, Hole 643A (0-90 mbsf). (Bulk carbonate data; compare Henrich, this volume. $\delta^{18} \mathrm{O}$ data from Jansen et al., this volume. Note: component percentages are with reference to total weight). 







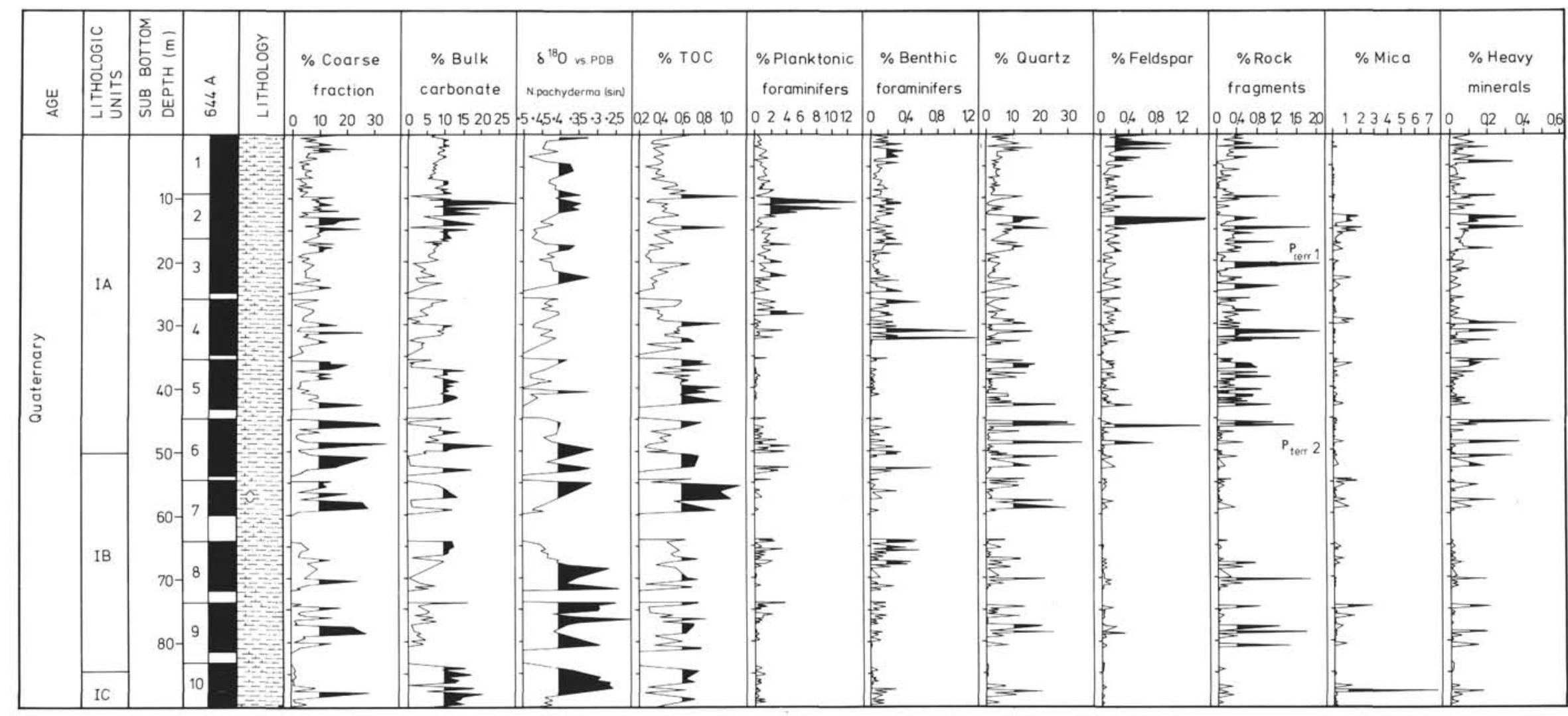




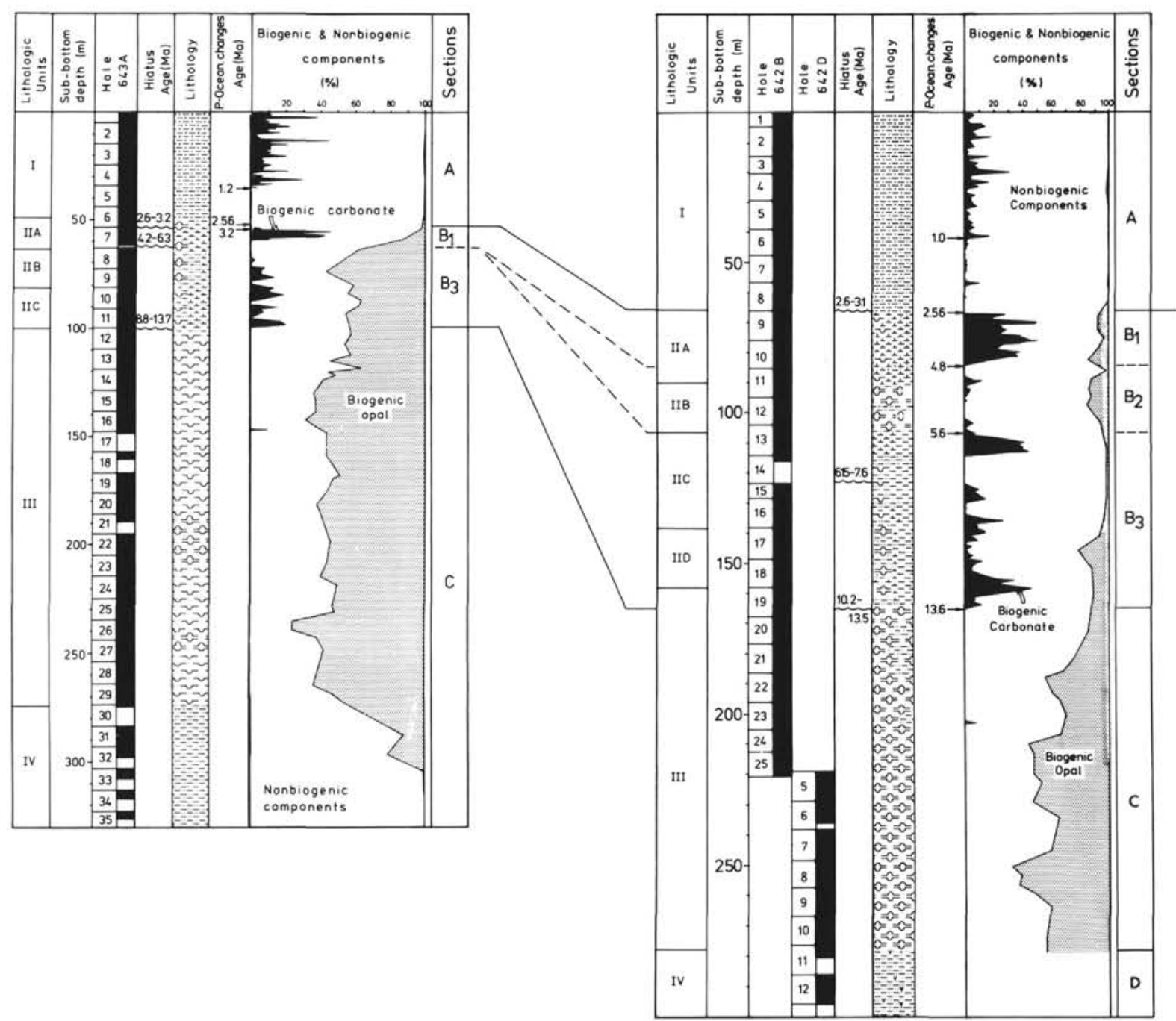

Figure 12. Correlation chart of Leg 104 drill Sites 642, 643, and 644. Major changes in lithofacies, position of hiatuses, and paleoceanographic events are outlined. The following periods have been recognized: 1. Deposition of biogenic siliceous sediments prior to $13.6 \mathrm{Ma}$, correlated with a high eustatic sea level, a warm climate, and weak surface- and deep-water exchange between the Norwegian Sea and the North Atlantic. 2. Sedimentation of mixed biogenic siliceous and calcareous deposits from 13.6 to $5.6 \mathrm{Ma}$, linked to a low global sea level, an increase in Northern Hemisphere cooling with development of steep E-W temperature gradients in the Norwegian-Greenland Sea, and strengthening of surface-water exchange and deep- water outflow to the North Atlantic. A major increase in carbonate deposition is reflected above a hiatus at $10.2 \mathrm{Ma}$. 3. Biogenic siliceous sediments deposited during a phase of reduced surface- and deep-water exchange from 5.6 to 4.8 Ma. 4. Major cooling at 4.8 to 3.1 Ma with deposition of pelagic carbonate over the Vøring Plateau, a strong increase in meridionality in the Northern Hemisphere, and enhanced surface- and deep-water circulation in the Norwegian-Greenland Sea and the North Atlantic. 5. Onset of ice-rafted debris deposition at 2.56 Ma. 6. Increase in strength of episodic intrusions of the Norwegian Current, major increase of pelagic carbonate deposition, and decrease in carbonate dissolution since 1.0 to $1.2 \mathrm{Ma}$. 
below this level they decrease in abundance in favor of high abundances of benthic foraminifers (Fig. 8). At about the same level (115 mbsf), low amounts of biogenic opal occur that consist predominantly of sponge spicules admixed with increased abundances of radiolarians and diatoms at deeper sub-bottom levels (Fig. 8). The interval between 186 and 200 mbsf, corresponding to an age of 2.2 to $2 \mathrm{Ma}$, reflects much higher abundances of radiolarians and diatoms.

\section{Section B: Hole 642B (Cores 104-642B-9H to 104-642B-19H) and Hole 643A (Cores 104-642B-7H to 104-643A-11H) and Hole 644A (Cores 104-644A-31 to $104-644 A-34$ )}

In Section B, particle assemblages of type I and II are found in variable quantities while the terrigenous type III disappears. Section B was divided into three subsections.

\section{Subsection B1: Hole 642B (Cores 104-642B-9H and $104-642 B-10 H)$ and Hole $643 \mathrm{~A}$ (Core 104-643A-7H)}

Subsection B1 is characterized primarily by coarse-fraction assemblages of type I with only minor admixtures of siliceous components (Figs. 6 and 7). Calcareous biogenic particles in this level of Hole 642B are associated with fluctuating bulk carbonate concentrations between $25-60 \%$ (Fig. 4). This carbonate-rich interval is distinctly shorter in Hole 643A (Fig. 3) because of hiatuses at the top and base of this subsection in Core 104-643A-7H. Subsection B1 in Hole 642B corresponds to an age of 3.1 to $4.8 \mathrm{Ma}$ (Goll, this volume; Fig. 12).

\section{Subsection B2: Hole 642B (Cores 104-642B-11H to 104-642B-13H)}

Coarse-fraction assemblages of Subsection B2 are characterized by the alternation between type IIa and IIb (Figs. 6, 7). In Hole 642B an interval dominated by large numbers of diatoms occurs in a section dominated by type IIa assemblages (Fig. 7). Subsection B2 is missing in Hole 643A due to a hiatus of 4.2 to $6.3 \mathrm{Ma}$ (Goll, this volume; Fig. 12). Subsection B2 corresponds to an age of 4.8 to $5.6 \mathrm{Ma}$ in Hole 642B.

\section{Subsection B3: Hole 642B (Cores 104-642B-13H to $104-642 A-19 H)$ and Hole $643 A$ (Cores $104-643 A-8 H$ to $104-634 A-11 H$ )}

Subsection B3 (Figs. 6,7) contains mixtures of coarse fraction assemblages types I and II. In Hole 642B arenaceous benthic foraminifers are consistently present as a minor component. However, they are not found in the corresponding interval of Hole $643 \mathrm{~A}$ but the coarse fraction is accentuated by higher numbers of radiolarians. The absence of arenaceous benthic foraminifers may be due to lower benthic activity caused by the greater depth of this Site. Subsection B3 in Hole 642B corresponds to an age of 5.6 to $13.6 \mathrm{Ma}$ including a hiatus from 10.2 to $13.6 \mathrm{Ma}$ (Goll, this volume). The major increase of carbonate deposition coincides with the top of this hiatus, e.g., at 10.2 Ma. Subsection B3 in Hole 643A reveals two hiatuses, one at the top of the 4.2- to 6.3-Ma interval and the other at the base of the 8.8- to 13.7-Ma interval.

\section{Section C: Hole 642B (Cores 104-642B-20H to 104-642B-25H), Hole 642D (Cores 104-642D-5X to 104-642D-11X) and Hole 643A (Cores 104-643A-12X to 104-643A-30X).}

Section $C$ is defined by coarse-fraction assemblages consisting nearly exclusively of siliceous skeletons, predominantly radiolarians, without any calcareous particles. In the lower part of Hole $643 \mathrm{~A}$, an interval with high numbers of diatom valves in the coarse fraction is recorded (Cores 104-643A-25X to 104643A-28X). This association is not found in Site 642. Strong diagenetic alteration affected all sediments below Core 104-643A$30 \mathrm{X}$ (Fig. 6). Widely distributed silicification and precipitation of authigenic carbonates result in coarse-fraction samples in this interval being composed of numerous diagenetic aggregates that are useless for paleoenvironmental and paleoceanographic interpretations. According to Henrich (this volume), the paleoenvironment has changed little at this diagenetic level except that opal-A of the siliceous components was totally converted to opal-CT.

\section{Section D: Hole 642D (Cores 104-642D-11X to 104-642D-14X)}

The base of Section C in Hole 642D was defined by a strongly altered tuffaceous and glauconitic layer representing a long hiatus between siliceous oozes of Miocene age and underlying Eocene volcaniclastic mudstones. Glauconitic grains are sporadically found in deeper levels of Section C in Hole 642D.

\section{Cyclic Variations of Type I and III Assemblage} Amounts in Section A of Holes 643A, 642B, and 644A

In the Vøring Plateau cores, lithologic cyclicity is recorded as high-frequency fluctuations of grain-size distributions or of coarse-sediment composition (Figs. 9, 10, 11). External mechanisms modulating the cyclicity appear to be linked to variations in the Earth's orbital parameters (Berger 1981, Berger et al., 1984), and may be responsible for high-frequency fluctuations observed in the type I and III assemblages.

The type I assemblage, which is also characterized by its high bulk carbonate content, shows strong fluctuations in Section A of Holes 643A, 642B, and 644A (compare also Henrich, this volume). Six to eight maxima in carbonate content ( $>12-30 \%$ bulk carbonate content) correlate with minima of assemblage type III.

Variation in abundance of terrigenous components is characterized by well-defined repeated maxima. Both quartz and rockfragment abundances reveal four megapeaks, which in the following section are referred to as terrigenous peaks $\left(\mathrm{P}_{\text {terr }}\right)$. Each of these megapeaks consists of 4 to 6 subcycles that contain sets of correlated minima and maxima abundances in terrigenous components. In Hole 643A the megapeaks occur as follows: $\mathrm{P}_{\text {terr }} 1$ at 0-9 mbsf, $\mathrm{P}_{\text {terr }} 2$ at 10-23 mbsf, $\mathrm{P}_{\text {terr }} 3$ at $28-37 \mathrm{mbsf}$ and $\mathrm{P}_{\text {terr }} 4$ at 41-52 mbsf (Fig. 9). In Hole 642B $\mathrm{P}_{\text {terr }} 1$ is found between 9-18 mbsf, $P_{\text {terr }} 2$ between 23-32 mbsf, $P_{\text {terr }} 3$ between 39-48 mbsf, and $P_{\text {terr }} 4$ between 56-65 mbsf (Fig. 10). Finally, in Hole 644A $\mathrm{P}_{\text {terr }} 1$ occurs between $0-35$ mbsf and $\mathrm{P}_{\text {terr }} 2$ between 40-65 mbsf (Fig. 11). The major appearance of terrigenous input occurs in Hole 643A at 51.26 mbsf, in Hole 642B at 66.70 mbsf, and in Hole 644A at 233.10 mbsf corresponding to an age of about $2.56 \mathrm{Ma}$.

\section{DISCUSSION}

Today all three sites of the Leg 104 paleoenvironment transect are situated under the Norwegian Current. Relatively high carbonate shell production within the modern surface water and well-oxygenated, newly formed bottom waters are reflected by high abundances of planktonic and benthic foraminifers in the coarse fraction of surface sediments (e.g., assemblage type I). Bulk sediment parameters reveal high carbonate and low organic carbon contents (compare Henrich, this volume). However, during late Cenozoic times (Quaternary and Neogene) both surface- and deep-water conditions changed dramatically, and these changes are reflected in the coarse-particle assemblages and bulk sediment properties.

Glacial and deglacial surface-water settings are characterized by dense pack ice and/or rapid iceberg drift as well as episodic 
high meltwater discharge from coastal areas (compare Henrich, this volume). As a result a decrease in oxygen content of deep waters due to reduced deep-water renewal is thought to occur. Indications of these environmental conditions are terrigenous coarse-particle assemblages (e.g., assemblage type III) composed of predominantly ice rafted debris with minor admixtures of planktonic foraminifers and most commonly a low abundance and diversity of benthic foraminifers. Bulk sediment properties reveal low carbonate and higher organic carbon contents.

Paleoceanographic conditions characterized by increased fertility of surface waters and sluggish deep-water renewal are indicated by biogenic siliceous coarse-particle assemblages (e.g., assemblage type II) and high organic carbon contents. Variations in abundances of different siliceous components, e.g., diatoms, radiolarians, and sponge spicules, may provide evidence for siliceous productivity in surface waters or proximity to a benthic siliceous sponge population, respectively.

Major changes in coarse-particle assemblage pattern in the Leg 104 drill sites referred to as Sections C, B, and A document three major significant changes in the depositional environment over the Vøring Plateau.

Section C almost exclusively consists of particle assemblage type IIb reflecting persistent highly fertile surface water conditions over the Vøring Plateau. Almost continuous deposition of sediments with biogenic siliceous coarse-particle assemblages persisted during early to middle Miocene, interrupted only by deposition of a short biogenic carbonate particle assemblage section at about 16.9 Ma at Site 642 and 15.2 Ma at Site 643 (compare Goll, this volume). High organic carbon contents and only rare benthic foraminifers most probably indicate rather sluggish deep-water renewal. Nevertheless, the frequent occurrence of bioturbation features reflecting benthic activity (Eldholm, Thiede, Taylor, et al., 1987) give indications that bottom waters were oxygenated. Comparably high biogenic siliceous sediments were recovered in Leg 38 drill sites from other regions of the Norwegian-Greenland Sea. Consequently, regional extensive productive surface waters that do not compare in extent with modern upwelling systems characterize the early to middle Miocene paleoceanography of the Norwegian Sea. An alternative hypothesis for such extensive highly productive surface waters might be increased supply of nutrients by rivers during a warm and humid climate (Frakes, 1979). Only weak surface-water exchange with the North-Atlantic and relative isolation of deep waters would stabilize the specific fertile surface-water conditions. High river discharge fertilizing coastal regions possibly enhanced by weak upwelling conditions induced at internal water mass boundaries within the Norwegian Sea might have caused rapidly siliceous blooms in surface waters.

During late Miocene to early Pliocene, coarse-particle assemblage patterns (summarized in Section B) reflect marked variations in surface and deep-water circulation as indicated by alternate periods of biogenic calcareous, mixed biogenic calcareous and siliceous, and biogenic siliceous sediments. Section B3 contains mixed carbonate and siliceous coarse-particle assemblages, e.g., types $\mathrm{IIb}$ and $\mathrm{I}$, that indicate a strong shift in the depositional environment over the Vøring Plateau at 13.6 to 5.6 Ma. Sediments of Section B2 that were deposited between 5.6 and 4.8 Ma consist of biogenic siliceous assemblages IIa and IIb. Section B1, 4.8 to $3.1 \mathrm{Ma}$, is a calcareous section composed of coarse-particle assemblage type I.

Our approach in interpreting these changes in section B is based on the assumption that deposition of pelagic carbonate (coarse-particle type I) over the Vøring Plateau indicates the Norwegian Current or a precursor current. The modern Norwegian Current has a cold counterpart on the western side of the Norwegian-Greenland Sea in the East Greenland Current. During cold periods dense (saline and cold) water could form in two ways in the Norwegian-Greenland Sea. The most important factor is the salt source because if the water can be cooled to less than approximately $2^{\circ} \mathrm{C}$, density is nearly independent of temperature. Salt could derive either from (a) the rejection during sea-ice formation most likely to occur in greatest quantities on shallow shelf regions or (b) advection of saline water from other sources. Other investigators (e.g., Hurdle, 1986) believe that dense water was first found when the Greenland-Scotland sill became deep enough to permit northwards advection of saline Atlantic water. Atlantic water inflow would also result in more meridional temperature gradients in the Norwegian-Greenland Sea.

We believe that the presence of the Norwegian Current in the past necessarily implies that similar temperature gradients existed in the Norwegian-Greenland Sea. Its first occurrence thus would be confined to periods of major cooling in the Northern Hemisphere. Cooling might have affected the north polar region in an asymmetric manner, with growth of mountain glaciers in Greenland much earlier than in Scandinavia. During these cold periods the potential to create young, dense, deep waters or dense water on the shelves in the northern NorwegianGreenland Sea was increased, provided that the deep sills along the Greenland-Scotland Ridge had attained sufficient depth.

Once this inhibiting factor was overcome, the NorwegianGreenland Sea had the potential of creating new deep-water, given that surface-water flows enabled the import of warm saline water masses. No dense water overflow from the Norwegian-Greenland Sea into the North Atlantic could be established until the sills of the Iceland-Shetland Channel and the Denmark Strait achieved a sufficient depth (Vogt, 1972; Berggren and Schnitker, 1983). This mode of circulation would serve to accelerate meridional temperature gradients through the development of a strong surface water-atmosphere interaction. Subsequently, the alternate occurrence of cold and warm climatic periods in the Northern Hemisphere might have corresponded with global eustatic sea level changes (Berggren and Schnitker, 1983). Warm climate and high sea level could have caused a rather sluggish and stratified surface-water circulation in the North Atlantic and the Norwegian Sea, reducing the possibility of deep convection and therefore reducing the renewal of deep water. Under these conditions Antarctic bottom water would have dominated in the deep-water areas of the North Atlantic.

Following these assumptions, Section B assemblages reflect variations of surface-water exchange between the North Atlantic and the Norwegian-Greenland Sea. The biogenic calcareous deposition appears to be associated with increased surface-water circulation and a relatively high meridionality. These conditions corresponded to a relatively steep temperature gradient in the Norwegian-Greenland Sea, and to an interval of increased deep-water renewal that is coeval with relatively low global sea level stands. Biogenic siliceous sedimentation dominated during intervals with diminished surface-water exchange between the North Atlantic and the Norwegian Sea, high eustatic sea level, warm climate, and isolation of deep-water masses in the Norwegian Sea (Fig. 12).

Based on particle fraction data and the relationships described above, the onset of effective deep-water outflow from the Norwegian Sea and into the North Atlantic may be dated at about 13.6 Ma (Fig. 12), when a correlation is made with respect to the first major carbonate interval. A drastic increase in carbonate deposition is observed above a hiatus of 13.5 to 10.2 $\mathrm{Ma}$ (Goll, this volume; Fig. 12). A small interval characterized by biogenic calcareous deposits is recorded from deeper levels of Hole 642B at about 201 mbsf. This interval corresponds to an age of about $16.9 \mathrm{Ma}$; another small carbonate peak occurs at about 142 mbsf in Hole 643A, corresponding to an age of about 15.2 $\mathrm{Ma}$ (Goll, this volume). A further indicator of major 
change in deep water of the Norwegian-Greenland Sea caused by the initiation of the Greenland-Scotland Ridge overflow may be documented in the total organic carbon (TOC) record of Site 642 (Fig. 7). A major shift from higher TOC values $(>1 \%)$ to distinctly lower values $(<1 \%)$ is observed at $165 \mathrm{mbsf}$, corresponding to an age of about $13.6 \mathrm{Ma}$. In early and middle Miocene higher carbon percentages indicate low oxygen concentration in the bottom waters in the Norwegian-Greenland Sea. The beginning of the deep-water overflow into the North Atlantic is reflected by a drop in TOC content caused by the increased renewal rate of deep water within the Norwegian-Greenland Sea. Initiation of Greenland-Scotland Ridge overflow at this time correlates well with data showing enhanced bottom-current activity in abyssal realms of the North Atlantic, e.g., the development of major sediment drifts at the end of the middle Miocene (Miller and Tucholke, 1983).

Section A is characterized by comparatively high-frequency fluctuations of sediment composition. These data reveal a signal of climate variations which have previously been interpreted to be controlled mainly by complex interactions between the ocean and the atmosphere due to coupling effects of short- and long-term Earth-orbital parameters (Broecker and van Donk, 1970; Berger, 1981; Pisias and Moore, 1981; Imbrie et al., 1984; Ruddiman and McIntyre, 1981a, 1984). Coarse-fraction component analysis of Section A sediments record a rapid alternation of moderate-amplitude variations in biogenic calcareous particles and of high-amplitude changes in terrigenous particle content due to the effects of Plio-Pleistocene glacial and interglacial conditions.

The distinct increase in coarse-fraction percentages in all Leg 104 cores with Section A assemblages must be related to input of ice-rafted debris (IRD). Ice-rafting starts in Hole 642B at $66.70 \mathrm{mbsf}$, Hole 643A at $51.26 \mathrm{mbsf}$, and Hole 644A at 233.10 mbsf (Figs. 3, 4, 5). At Sites 643 and 642 a hiatus (2.6 to 3.1 or $3.2 \mathrm{Ma}$ ) is recorded just below the glacial-interglacial unit based on combined biostratigraphic and paleomagnetic evidence (Goll, this volume, Bleil et al., this volume). The age value was calculated using linear sedimentation rates (LSR) down from the paleomagnetic fix at 64.65 mbsf (Matuyama-Gauss) in Hole 642B and at 49.46 mbsf (Matuyama-Gauss) in Hole 643A. Sedimentological evidence for a major change in LSR is just above 67 mbsf in Hole 642B and 52 mbsf in Hole 643A. As such, the age for the major onset of IRD deposition is around $2.56 \mathrm{Ma}$ in all three holes.

The age of the major onset of IRD represented North American-Eurasian glaciation is still the subject of discussion. The major IRD input to deep-sea sediments was reported at 2.4-2.5 $\mathrm{Ma}$ (Shackleton et al., 1984: Hole 552A $=2.4 \mathrm{Ma}$; Raymo et a1., 1986: Hole 609B = $2.45 \mathrm{Ma}$; Arthur et al., 1986: Site 646 and $647=2.5 \mathrm{Ma}$ ). It is well established that the first glaciation in Iceland started at about 3.1 to $3.0 \mathrm{Ma}$ (McDougall and Wensink, 1966; Einarsson et al., 1967; Eiriksson, 1980). Leg 104 drill sites are located $10^{\circ}$ to $15^{\circ}$ latitude north of Site $552 \mathrm{~A}$ and Site 609B. One might expect a slightly earlier onset of IRD at the Norwegian-Greenland Sea. This idea is consistent with a fluctuating southward progressing glaciation front in the Northern Hemisphere between 3.1 and $2.5 \mathrm{Ma}$. A first input of IRD starting as early as $8 \mathrm{Ma}$ was reported from Site 645 (Baffin Bay), which was drilled during Leg 105 at almost the same latitude as Leg 104 drill sites (Arthur et al., 1986).

The oceanographic regime is mirrored by type I and type III sediment assemblages distributions. These distributions document an alternating advance and retreat of polar water masses which agree with those described in previous studies (McIntyre et al., 1972; Ruddiman and McIntyre, 1977; Kellogg, 1975b). The maximum amplitude of the type III assemblage indicates mainly the transition times both from glacial to interglacial con- ditions and from interglacial to glacial times (compare also Henrich, this volume.). A predominant accumulation of IRD during these transition zones is well known from the Arctic Ocean (Zahn et al., 1985). High- to intermediate-amplitude changes of type I assemblages provide evidence for intervals of selected intrusion of relatively warm North Atlantic surface water during interglacial times. Today, these intrusions reach the Norwegian Sea as the Norwegian Current (compare also Kellogg, 1975b; Bjørklund and Goll, 1979).

Major shifts in coarse-fraction composition, carbonate and dissolution records, and oxygen/carbon isotope records of planktonic and benthic foraminifers in all Leg 104 drill sites reflect three different climatic periods with variable glacial-interglacial regimes in the Norwegian-Greenland Sea during the past 2.56 Ma.

The interval from 2.56 to 1.0 or 1.2 Ma reveals low carbonate plankton productivity associated with enhanced carbonate dissolution at Sites 642, 643, and 644 (Henrich, this volume), a very light planktonic oxygen isotope signal (Jansen et al., in press; Jansen et al., this volume), and low-amplitude, high-frequency oscillations of coarse-fraction percentages and contents of ice-rafted debris. In Hole 644A, assemblage type I is predominantly composed of calcareous benthic foraminifers. Additionally, significant admixtures of biogenic opal preferably occur in coarse-fraction type I. The interval between 2.2 to $2 \mathrm{Ma}$ is characterized by higher percentages of radiolarians and diatoms. The following short section contains increased abundances of ice rafted debris.

These observations indicate rather isolated, strongly corrosive deep waters over the Vøring Plateau. The frequent occurrence of benthic foraminifers at Site 644 indicates that some mechanism of oxygenation of bottom waters was still operating. Surface-water conditions were characterized by pack ice and ice rafting interrupted only by periodic carbonate productivity at Site 644. A tendency to lowered salinities in surface waters is indicated by the light isotopic ratios of planktonic foraminifers (Jansen et al., this volume). Increased nutrient supply at Site 644 close to coastal regions might have favored the production of biogenic silica and the threefold sedimentation rates at this site might have enhanced opaline silica preservation (Bohrmann, 1988). In conclusion, the Norwegian Sea climatic conditions from $2.56 \mathrm{Ma}$ to 1.0 or $1.2 \mathrm{Ma}$ are characterized by an overall temperate glacial climate interrupted only by periodic intrusions of a weak Norwegian Current that was then much narrower in extension and strictly bound to near coastal areas. Such an isolation of the Norwegian-Greenland Sea might be achieved when Greenland and Scandinavia were covered by smaller but much more persistent ice sheets resulting in a more zonal circulation (Jansen et al., in press).

The interval from 1.2 to $0.6 \mathrm{Ma}$ is characterized by a shift toward heavier oxygen isotope ratios of planktonic foraminifers, a gradual decrease in carbonate dissolution (Henrich, this volume), and a change in the frequency of coarse-fraction oscillations. The interval younger than $0.6 \mathrm{Ma}$ contains high-amplitude, low-frequency changes in carbonate records and coarsefraction composition. Hence, extensive carbonate dissolution over the Vøring Plateau is restricted to late glacial/early deglacial configurations and to minor deglaciations within glacial periods (Henrich, this volume). Several mechanisms can be considered as triggers for climatic changes in this time interval. The most likely mechanism is probably variations in the Earth's orbit (Milankovitch cycles).

The cyclic character of type III assemblages, which are excellently developed in rock fragment and quartz abundances is difficult to interpret. In the Brunhes epoch, $\mathrm{P}_{\text {terr }} 1$ and 2 may reflect two long-term fluctuations perhaps associated with the 400-k.y. long-term eccentricity cycle (Berger 1981, Berger et al., 
1984). In contrast, $P_{\text {terr }} 3$ and 4 represent a time span of approximately $1.8 \mathrm{~m} . y$. , which is not easy to explain. Nevertheless, rock fragment and quartz content data clearly document coupling of terrigenous input to the well-known elements of the Milankovitch cyclicity.

\section{CONCLUSIONS}

Analyses of coarse-fraction composition of Leg 104 cores indicate:

1. A major deep-water exchange occurred between the Norwegian-Greenland Sea and the North Atlantic in the middle to late Miocene interval (at approximately 13.6 Ma).

2. Cooling and increased deep-water production took place in the Norwegian-Greenland Sea during the late Miocene and early Pliocene (between 13.6-5.6 Ma and 4.8-3.1 Ma).

3. Relatively warm periods and isolated deep-water conditions are documented for times prior to $13.6 \mathrm{Ma}$ and at 5.6-4.8 Ma.

4. A major onset of IRD deposition occurred at the Vøring Plateau during the late Pliocene at $2.56 \mathrm{Ma}$.

5. Possibly two long-term fluctuations are revealed by rock fragment and quartz contents within the Brunhes epoch.

\section{ACKNOWLEDGMENTS}

We thank the Captain, crew, and technical staff of Leg 104 for assistance in obtaining samples. W. Reimann and C. Samtleben supervised the SEM-laboratory work. For technical assistance and data processing during sedimentological studies we gratefully acknowledge A. Brumm, A. Dettmer, I. Dold, U. Drechsler, U. Gützmacher, K. Lackschewitz, M. Ruff, J. Rust, A. Schröder, and C. Wieger. For editorial help. Thanks are due O. Runze, M. Weinelt, and K. H. Baumann, and for photographic assistance, S. Perbandt, B. Hecht, and U. Schuldt. We thank E. Jansen and his group in Bergen for cooperating with sample processing. Critical reviews supplied by S. Pfirman, C. Schafer, and H. Schrader essentially improved the manuscript and are gratefully acknowledged.

This work was supported by a grant from the German Science Foundation (Th 200/3-4,5). This is publication No. 39 of the Special Research Project SFB 313 at the University of Kiel.

\section{REFERENCES}

Arthur, M., Srivastava, S., Clement, B., et al., 1986. High-latitude palaeoceanography. Nature, 320:17-18.

Berger, A., 1981. The astronomical theory of paleoclimates. In Berger, A. (Ed.), Climatic Variations and Variability: Facts and Theories: Hingham, Mass. (Reidel Publishing Co.), 502-525.

Berger, A., Imbrie, J., Hays, J., Kukla, G., Saltzman, B. (Eds.), 1984. Milankovitch and Climate: NATO ASI Series C, Mathematical and Physical Sciences, 126, pts. 1 and 2. Dordrecht, Netherlands (Reidel Publishing Co.).

Berggren, W.A., and Schnitker, D., 1983. Cenozoic marine environments in the North Atlantic and Norwegian-Greenland Sea. In Bott, M.H.P., Saxov, S., Talwani, M., and Thiede, J. (Eds.), Structure and Development of the Greenland-Scotland Ridge: New York, London (Plenum Press), 495-548.

Bjørklund, K. R., and Goll, R. M., 1979. Ice age climates of the Norwegian-Greenland Sea, Geo-Journal, 3:273-283.

Blanc, P. L., Rabussier, D., Vergnaud-Grazzini, C., Duplessy, J. C., 1980. North Atlantic deep water formed by the later middle miocene. Nature, 283:553-555.

Bohrmann, G., 1988. Zur Sedimentationsgeschichte von biogenem Opal im nördlichen Nordatlantik und dem Europäischen Nordmeer, (DSDP/ ODP Bohrungen 408, 642, 643, 644, 646 und 647): Ber. Sonderforschungsbereich $313,9: 2215$.

Bott, M. H. P., Saxov, S., Talwani, M., and Thiede, J. (Eds.), 1983. Structure and Development of the Greenland-Scotland Ridge: New York: (Plenum Press).

Broecker, W. S., and van Donk, J., 1970. Insolation changes, ice volumes and ${ }^{18} \mathrm{O}$ record in deep-sea sediments. Rev. Geophys. Space Phys., 8:169-198.
CLIMAP Project Members, 1981. Seasonal reconstructions of the Earth's surface at the last glacial maximum. Geol. Soc. Am. Map and Chart Series, M-36.

Einarsson, T., Hopkins, D. M., and Doell, R. R., 1967. The stratigraphy of Tjörnes, Northern Iceland, and the history of the Bering Land Bridge. In Hopkins, D. M. (Ed.), The Barents Land Bridge. Stanford Univ. Press, 312-325.

Eiriksson, J., 1980. Tjörnes North Iceland: A Bibliographical Review of the Geological Research History. Jökull 30: Jöklaránnsoknafelag Island, 1-19.

Eisma, D., and van der Gaast, S. J., 1983. Terrigenous late Quaternary sediment components north and south of the Scotland-Greenland Ridge and in the Norwegian Sea. In Bott, M.H.P., Saxov, S., Talwani, M., and Thiede, J. (Eds.), Structure and Development of the Greenland-Scotland Ridge. New York, London (Plenum Press), 607636.

Eldholm, O., and Thiede, J., 1980. Cenozoic continental separation between Europe and Greenland. Palaeogeogr. Palaeoclimatol. Palaeoecol., 30:243-259.

Eldholm, O., Thiede, J., Taylor, E., et al., 1987. Proc. ODP, Init. Repts., 104: College Station, TX (Ocean Drilling Program).

Frakes, L. A., 1979. Climates Throughout Geologic Times: New York (Elsevier Publ. Co.).

Holtedahl, H., and Bjerkli, K., 1982. Late Quaternary sediments and stratigraphy on the continental shelf off Møre-Trøndelag, W. Norway. Mar. Geol., 45:179-226.

Hurdle, B. G., 1986. The Nordic Seas: New York (Springer Verlag).

Imbrie, J., Hays, J. D., Martinson, D. G., McIntyre, A., Mix, A. C., Morley, J. J., Pisias, N. G., Prell, W. L., and Shackleton, N. J., 1984. The orbital theory of Pleistocene climate: Support from a revised chronology of the marine $\delta^{18} \mathrm{O}$ record. In Berger, A. L., et al. (Eds.), Milankovitch and Climate, Part I: Dordrecht, Netherlands (D. Reidel Publishing Co.), 269-305.

Jansen, E., Bleil, U., Henrich, R., Kringsstad, L. and Slettemark B., in press. Paleoenvironmental changes in the Norwegian Sea and the North Atlantic during the last 2.8 Ma.: DSDP/ODP Sites 610, 642, 643 and 644 . Paleoceanography.

Johnsen, L., and Heezen, B. C., 1967. Morphology and Evolution of the Norwegian-Greenland Sea. Deep-Sea Res., 14:755-771.

Kellogg, T. B., 1975a. Tertiary Stratigraphy and Tectonics in Svalbard and Continental Drift. AAPG Bull., 49:465-485.

Kellogg, T. B., 1975b. Late Quaternary climatic changes in the Norwegian-Greenland sea. In Bowling, S. A., and Weller, G. (Eds.), Climate of the Arctic: College (Univ. of Alaska), 3-36.

Kellogg, T. B., 1976. Late Quaternary climatic changes: Evidence from deep-sea cores of Norwegian and Greenland Seas. In Cline, R. M., and Hays, J. E. (Eds.), Investigation of late Quaternary paleoceanography and paleoclimatology. Geol. Soc. Am. Mem., 145:77-110.

Kellogg, T. B., 1977. Paleoclimatology and paleoceanography of the Norwegian-Greenland Seas: The last 450,000 years. Mar. Micropaleontol. 2:235-249.

Kellogg, T. B., 1980. Paleoclimatology and Paleoceanography of the Norwegian-Greenland Sea: Glacial-interglacial contrasts. Boreas, 9:115-137.

Kellogg, T. B., Duplessy, J. C., and Shackleton, N. J., 1978. Planktonic foraminiferal and oxygen isotopic stratigraphy and paleoclimatology of the Norwegian Sea deep sea cores. Boreas, 7:61-73.

Kitchell, J. A., and Clark, D. L., 1982. Late Cretaceous-Paleogene paleogeography and paleocirculation: Evidence of North Polar Upwelling. Palaeogeogr., Palaeoclimatol., Palaeoecol., 40:135-165.

McDougall, I., and Wensink, H., 1966. Paleomagnetism and geochronology of the Plio-Pleistocene lavas in Iceland. Earth Planet. Sci. Lett., 1:232-236.

McIntyre, A., Ruddiman, W. F., Jantzen, R., 1972. Southward penetrations of the North Atlantic polar front: Faunal and floral evidence of large-scale surface water mass movement over the last 225,000 years. Deep-Sea Res., 19:61-77.

Meincke, J., 1983. The modern current regime across the GreenlandScotland Ridge. In Bott, M.H.P., Saxov, S., Talwani, M., and Thiede, J. (Eds.), Structure and Development of the Greenland-Scotland Ridge: New York, London (Plenum Press), 637-650.

Miller, K. G., and Tucholke, B. E., 1983. Development of Cenozoic Abyssal Circulation South of the Greenland-Scotland Ridge. In Bott, M.H.P., Saxov, S., Talwani, M. and Thiede, J. (Eds.), Structure and Development of the Greenland-Scotland Ridge: New York, London (Plenum Press), 549-590. 
Pisias, N. G., Moore, T. C., 1981. The evolution of Pleistocene climate: A time series approach. Earth Planet. Sci. Lett., 52:450-458.

Raymo, M. E., Ruddiman, W. F., and Clement, B. M., 1986. PliocenePleistocene paleoceanography of the North Atlantic at Deep Sea Drilling Project Site 609. In Ruddiman, W. F., Kidd, R. B., et al., Init. Rpts. DSDP, 94, Pt. 2: Washington (U.S. Govt. Printing Office), 895-902.

Ruddiman, W. F., and McIntyre, A., 1977. Late Quaternary surface ocean kinematics and climatic change in the high-latitude North Atlantic. J. Geophys. Res., 82:3877-3887.

Ruddiman, W. F., and McIntyre, A., 1981a. The North Atlantic Ocean during the last deglaciation. Palaeogeogr., Palaeoclimatol., Palaeoecol., 35:145-214.

Ruddiman, W. F., and McIntyre, A., 1981b. Oceanic mechanisms for amplification of the 23,000-year ice-volume cycle. Science, 212:617627.

Ruddiman, W. F., and McIntyre, A., 1984. Ice-age thermal response and climatic role of the surface Atlantic Ocean, $40^{\circ} \mathrm{N}$ to $63^{\circ} \mathrm{N}$. Geol. Soc. Am. Bull., 95:381-396.

Ruddiman, W. F., Raymo, M., and McIntyre, A., 1986a. Matuyama 41,000-year cycles: North Atlantic Ocean and Northern Hemisphere ice sheets. Earth Planet. Sci. Lett., 80:117-129.

Ruddiman, W. F., McIntyre, A., and Raymo, M., 1986b. Paleoenvironmental results from North Atlantic Sites 607 and 609. In Ruddiman, W. F., Kidd, R. B., et al., Init. Repts. DSDP, 94, Pt. 2: Washington (U.S. Govt. Printing Office), 855-878.

Sarnthein, M., 1971. Oberflächensedimente im Persischen Golf und dem Golf von Oman. II. Quantitative Komponentenanalyse der Grobfraktion. "Meteor" Forsch.-Erg., Reihe C, 5:1-113.

Schaeffer, R., and Spiegler, D., 1986. Neogene Kälteeinbrüche und Vereisungsphasen im Nordatlantik. Z. dt. geol. Ges., 137/2:537-552.

Schrader, H.-J., Bjørklund, K. R., Manum, S., Martini, E., and van Hinte, J., 1976. Cenozoic biostratigraphy, physical stratigraphy and paleoceanography in the Norwegian-Greenland Sea, DSDP Leg 38 Paleontological Synthesis. Init. Repts. DSDP, 38: Washington (U.S. Govt. Printing Office), 1197-1211.

Shackleton, N. J., Backman, J., et al., 1984. Oxygen isotope calibration of the onset of ice-rafting and history of glaciation in the North Atlantic region. Nature, 307:620-623.
Talwani, M., and Eldholm, O., 1977. Evolution of the NorwegianGreenland Sea. Geol. Soc. Am. Bull., 88:969-999.

Talwani, M., and Udintsev, G. et al., 1976. Init. Repts. DSDP, 38: Washington (U.S. Govt. Printing Office).

Thiede, J., 1979. History of the North Atlantic Ocean; Evolution of an Asymmetric Zonal Paleoenvironment in a Latitudinal Ocean Basin. Maurice Ewing Ser.: Washington (Am. Geophys. Union), 3:275296.

Thiede, J., 1980. Paleo-oceanography, margin stratigraphy and paleophysiography of the Tertiary North Atlantic and Norwegian-Greenland Seas. Philos. Trans. R. Soc. London, A 294:177-185.

Thiede, J., and Eldholm, O., 1983. Speculations about the paleodepth of the Greenland-Scotland Ridge during late Mesozoic and Cenozoic times. In Bott, M.H.P., Saxov, S., Talwani, M., and Thiede, J. (Eds.), Structure and Development of the Greenland-Scotland Ridge: New York, London (Plenum Press), 445-456.

Thiede, J., Diesen, G. W., Knudsen, B.-E., and Snaare, T., 1986. Patterns of Cenozoic sedimentation in the Norwegian Greenland Sea. Mar. Geol., 69:323-352.

Thiede, J., Clark, D., Herman, Y., in press. Late Mesozoic and Cenozoic paleoceanography of the north polar oceans. In Grantz, A., Johnson, L. Sweeney, J. F. (Eds.), Decade of North American Geology: Vol. L, Geol. Soc. Am.

Vogt, P. R., 1972. The Faeroe-Iceland-Greenland aseismic ridge and the western boundary undercurrent. Nature, 239:79-81.

Vogt, P. R., 1986. Sea floor topography, sediments, and paleoenvironments. In Hurdle, B. G. (Ed.), The Nordic Seas: New York (Springer Verlag), 237-410.

Warnke, D. A., and Hansen, M. E., 1977. Sediments of glacial origin in the area of operations DSDP Leg 38 (Norwegian-Greenland Seas): Preliminary results from sites 336-344. Ber. Naturforsch. Ges. Freiburg $i \mathrm{Br}$, , 67:371-392.

Zahn, R., Markussen, B., and Thiede, J., 1985. Stable isotope data and depositional environments in the Late Quaternary Arctic Ocean. Nature, 314:433-435.

Date of initial receipt: 8 Sept. 1987

Date of acceptance: 2 May 1988

Ms 104B-196 


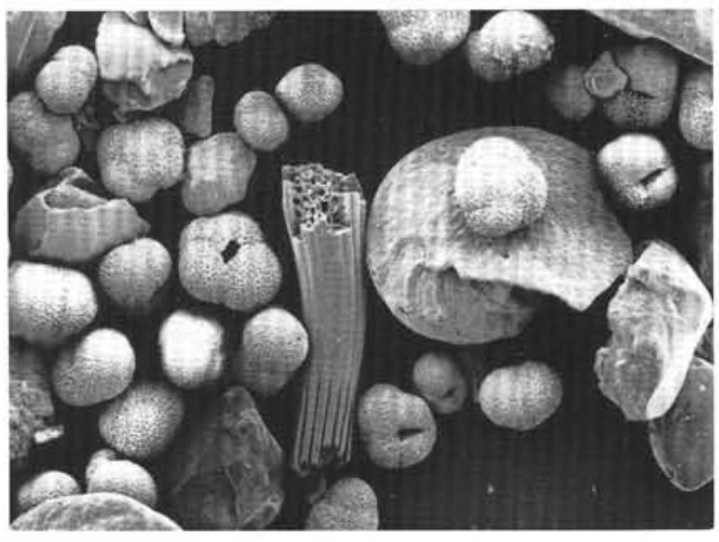

1



3

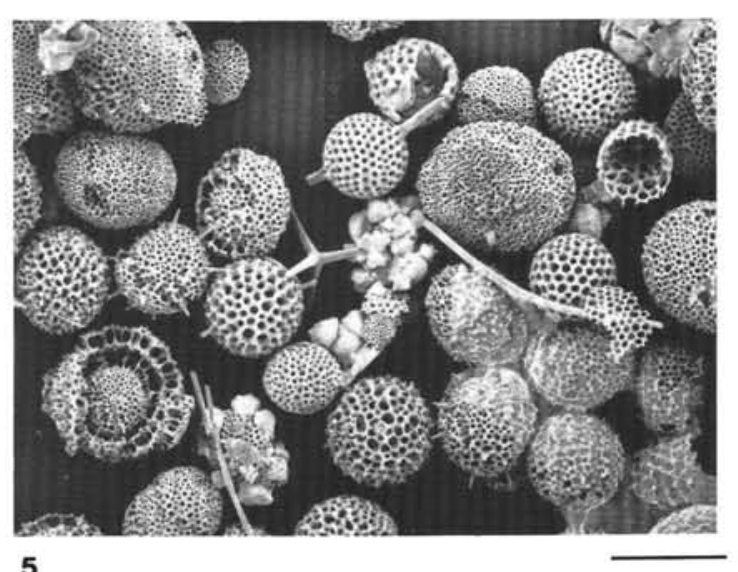

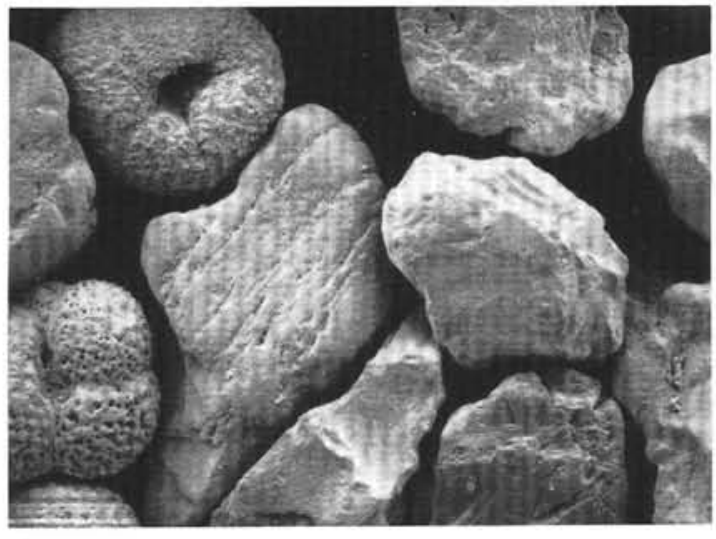

2

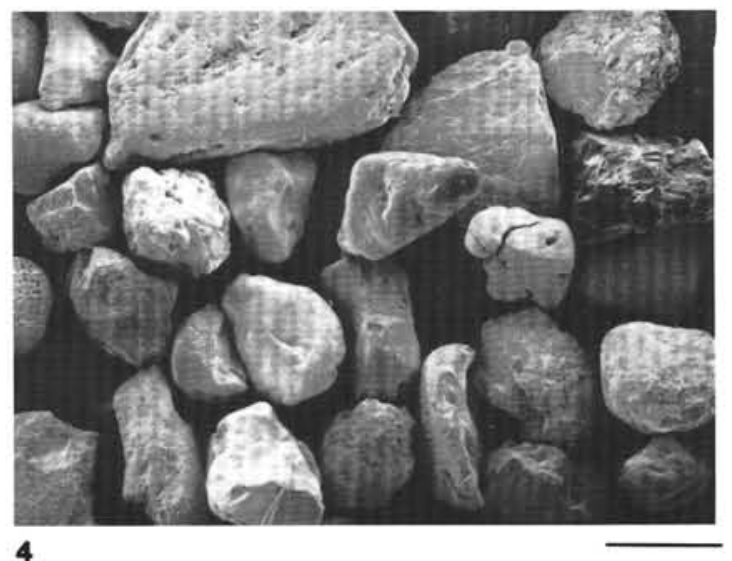

4

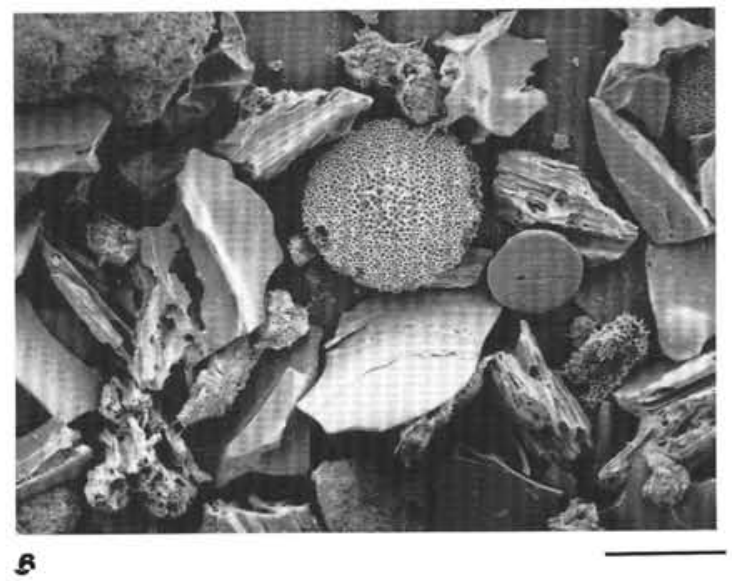

Plate 1. 1. Sample 104-642B-3H-4, $106 \mathrm{~cm}$ (scale bar: $200 \mu \mathrm{m}$ ): Coarse-fraction type I assemblage characterized by planktonic and benthic foraminifers (note some lithogenic particles and an echinoid fragment). 2. Sample 104-644A-lH-5, $82 \mathrm{~cm}$ (scale bar: $100 \mu \mathrm{m}$ ): Mixed particle association of types I and II. 3. Sample 104-642B-9H-1, $28 \mathrm{~cm}$ (scale bar: $400 \mu \mathrm{m}$ ): First onset of type III assemblage in the fraction $>63 \mu \mathrm{m}$. 4. Sample 104642B-3H-2, $106 \mathrm{~cm}$ (scale bar: $200 \mu \mathrm{m}$ ): Coarse-fraction type III assemblage. 5. Sample 104-642D-3X-3, $113 \mathrm{~cm}$ (scale bar: $200 \mu \mathrm{m}$ ): Coarse-fraction assemblage type IIa and IIb characterized by radiolarians and smaller amounts of sponge spicules. 6. Sample 104-642B-20H-6, 103 cm (scale bar: $200 \mu \mathrm{m}$ ): Vitric tephra (type IV assemblage) associated with radiolarians and diatoms. 

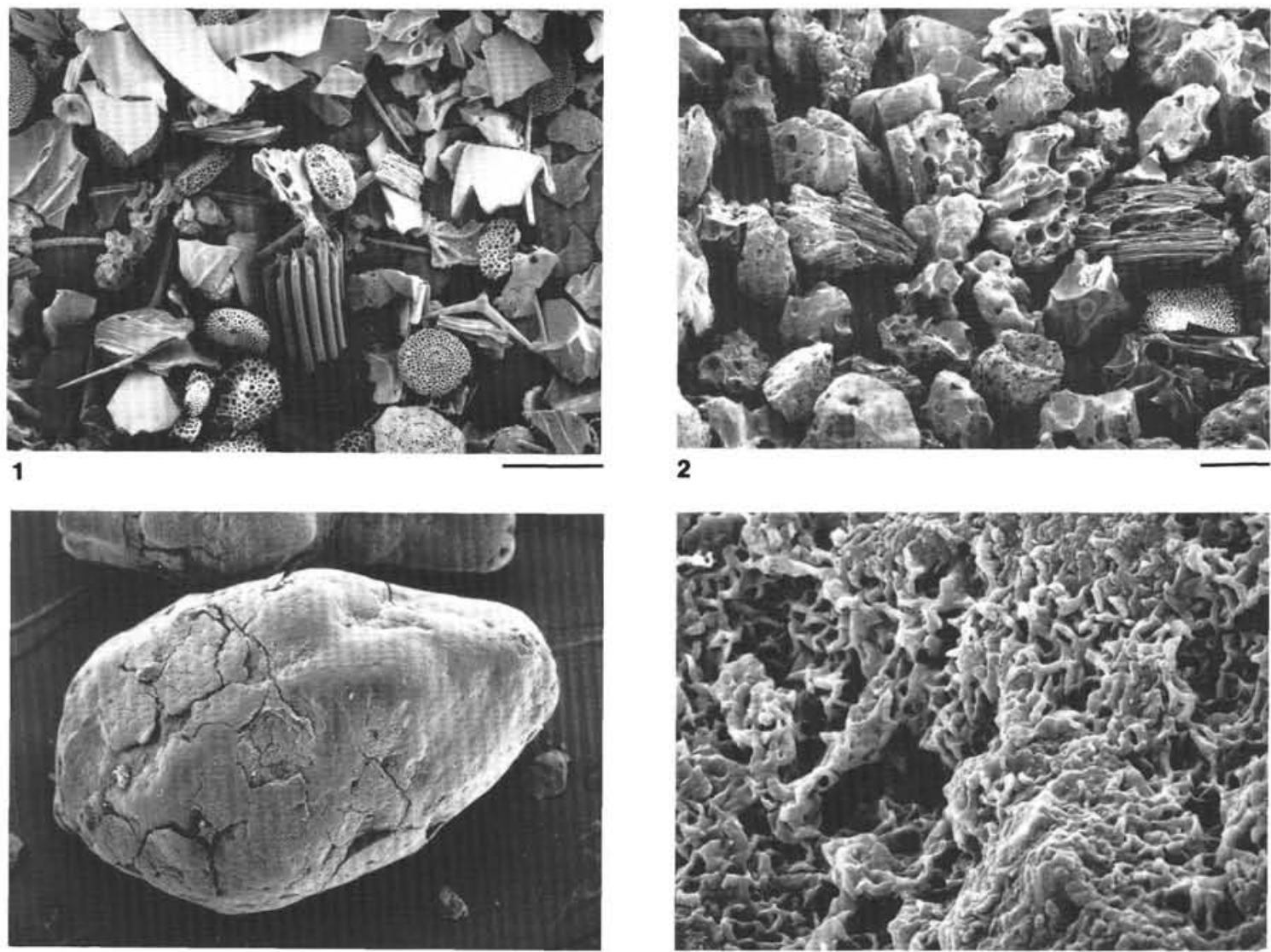

2

3


4

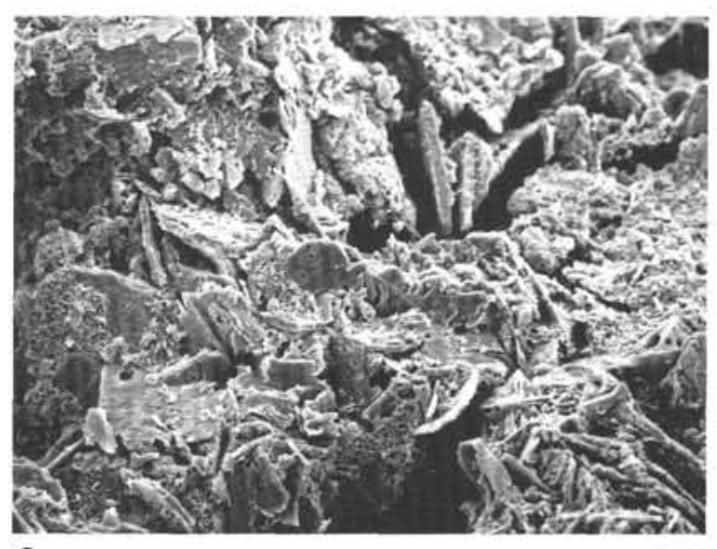

6

Plate 2. 1. Sample 104-642D-7X-4, $103 \mathrm{~cm}$ (scale bar: $200 \mu \mathrm{m}$ ): Volcanic ash layer (type IV assemblage) composed of vitric glass shards showing different degrees of vesicularity and stretching. 2. Sample 104-642B-15H-3, $113 \mathrm{~cm}$ (scale bar: $200 \mu \mathrm{m}$ ). Mixed type of coarse-fraction association (types II and III). 3. Sample 104-642D-13X-3, $105 \mathrm{~cm}$ (scale bar: $100 \mu \mathrm{m}$ ): Strongly altered tephra components showing authigenic clay mineral fabric. 4. Sample 104-642D-13X-3, $105 \mathrm{~cm}$ (scale bar: $4 \mu \mathrm{m}$ ): Detail of 3. 5. Sample 104-642D-13X-4, $40 \mathrm{~cm}$ (scale bar: $200 \mu \mathrm{m}$ ): Different peloids of altered ash fragments. 6. Sample 104-642D-13X-4, $40 \mathrm{~cm}$ (scale bar: $20 \mu \mathrm{m}$ ): Detail of the peloid from 5., showing shard structures with partly authigenic clay minerals. 


\section{APPENDIX}

Table 1.1. Sedimentological data from Hole 642B. Analysis of coarsefraction component.

\begin{tabular}{|c|c|c|c|c|}
\hline $\begin{array}{c}\text { Sample } \\
\text { no. }\end{array}$ & $\begin{array}{l}\text { Depth } \\
\text { (mbsf) }\end{array}$ & $\begin{array}{l}>63 \\
\mu \mathrm{m} \\
(\%)\end{array}$ & $\begin{array}{c}\text { Bulk } \\
\mathrm{CaCO}_{3} \\
(\%)\end{array}$ & $\begin{array}{l}\text { TOC } \\
(\%)\end{array}$ \\
\hline $1 H-1-4 \theta$ & 8.42 & 14.84 & 3.55 & 0.37 \\
\hline $1 H-1-74$ & 0.76 & & 2.68 & 8.41 \\
\hline $1 H-1-96$ & 8.98 & 13.93 & 6.59 & 6. 28 \\
\hline $1 H-2-4 \theta$ & 1.92 & 6.85 & 3.39 & 8.26 \\
\hline $1 H-2-74$ & 2.26 & & 2.82 & 8. 45 \\
\hline $1 H-2-96$ & 2.48 & 2.27 & 1.37 & 0.32 \\
\hline $1 \mathrm{H}-3-48$ & 3.42 & 12.57 & 2.80 & 0.34 \\
\hline $1 \mathrm{H}-3-74$ & 3.76 & & 2.34 & 8.41 \\
\hline $1 \mathrm{H}-3-96$ & 3.98 & 19.45 & 11.56 & 0.28 \\
\hline $1 \mathrm{H}-\mathrm{C}-14$ & 4.66 & & 14.18 & 8.37 \\
\hline $2 \mathrm{H}-1-112$ & 5.94 & 11.38 & 5.81 & 0.24 \\
\hline $2 \mathrm{H}-2-36$ & 6.68 & & 2.88 & 8. 37 \\
\hline $2 \mathrm{H}-2-53$ & 6.85 & 7.65 & 5.44 & 8.24 \\
\hline $2 \mathrm{H}-2-112$ & 7.44 & 11.60 & 7.68 & 8.24 \\
\hline $2 \mathrm{H}-3-36$ & 8.18 & & 4.96 & 8.47 \\
\hline $2 \mathrm{H}-3-53$ & 8.35 & 16.24 & 18.12 & 8.38 \\
\hline $2 \mathrm{H}-3-112$ & 8.94 & 8.36 & 4.14 & 0.31 \\
\hline $2 \mathrm{H}-4-36$ & 9.68 & & 1.54 & 8.41 \\
\hline $2 \mathrm{H}-4-53$ & 9.85 & 12.35 & 4.82 & 8.33 \\
\hline $2 \mathrm{H}-4-112$ & 18.44 & 12.85 & 4.81 & 8.23 \\
\hline $2 \mathrm{H}-5-36$ & 11.18 & & 5.38 & 8.43 \\
\hline $2 \mathrm{H}-5-53$ & 11.35 & 9.85 & 6.81 & 0.27 \\
\hline $2 \mathrm{H}-5-112$ & 11.94 & 13.44 & 0.92 & 0.38 \\
\hline $2 \mathrm{H}-6-36$ & 12.68 & & 8.58 & 0.66 \\
\hline $2 \mathrm{H}-6-53$ & 12.85 & 9.65 & 2.94 & 8.46 \\
\hline $2 \mathrm{H}-\mathrm{C}-1$ & 14.18 & & 1.17 & 0.68 \\
\hline $3 H-1-36$ & 14.68 & & 2.81 & 8.48 \\
\hline $3 H-1-44$ & 14.76 & 8.12 & 16.38 & 0.28 \\
\hline $3 H-1-186$ & 15.38 & 21.41 & 3.29 & 0.58 \\
\hline $3 H-2-36$ & 16.18 & & 1.12 & 8.33 \\
\hline $3 \mathrm{H}-2-44$ & 16.26 & 25.38 & 1.76 & 8.44 \\
\hline $3 H-2-106$ & 16.88 & 12.85 & 8.84 & 0.24 \\
\hline $3 \mathrm{H}-3-36$ & 17.68 & & 3.69 & 0.35 \\
\hline $3 \mathrm{H}-3-44$ & 17.76 & 5.95 & 6.62 & 0.29 \\
\hline $3 H-3-186$ & 18.38 & 7.11 & 7.71 & 0.28 \\
\hline $3 \mathrm{H}-4-36$ & 19.18 & & 8.42 & 0.58 \\
\hline $3 H-4-44$ & 19.26 & 2.93 & 14.18 & 8.26 \\
\hline $3 H-4-186$ & 19.88 & 3.37 & 30.38 & 8.26 \\
\hline $3 \mathrm{H}-\mathrm{C}-9$ & 28.61 & & 9.89 & 0.61 \\
\hline $4 H-1-35$ & 21.87 & & 2.56 & 0.52 \\
\hline $4 H-1-43$ & 21.13 & 18.84 & 3.64 & 8.46 \\
\hline $4 H-1-113$ & 21.78 & 15.87 & 4.18 & 0.36 \\
\hline $4 \mathrm{H}-2-35$ & 22.58 & & 2.78 & 0.44 \\
\hline $4 \mathrm{H}-2-43$ & 22.57 & 14.21 & 3.35 & 8.44 \\
\hline $4 H-2-113$ & 23.14 & 8.25 & 16.87 & 0.44 \\
\hline $4 \mathrm{H}-3-35$ & 23.93 & & 2.58 & 0.39 \\
\hline $4 \mathrm{H}-3-43$ & 24.81 & 8.83 & 3.03 & 8.32 \\
\hline $4 \mathrm{H}-3-113$ & 24.68 & 21.81 & 9.44 & 0.42 \\
\hline $4 H-4-35$ & 25.36 & & 0.28 & 0.41 \\
\hline
\end{tabular}

Table 1.1 (continued).

\begin{tabular}{|c|c|c|c|c|}
\hline $\begin{array}{c}\text { Sample } \\
\text { no. }\end{array}$ & $\begin{array}{l}\text { Depth } \\
\text { (mbsf) }\end{array}$ & $\begin{array}{l}>63 \\
\mu \mathrm{m} \\
(\%)\end{array}$ & $\begin{array}{c}\text { Bulk } \\
\mathrm{CaCO}_{3} \\
(\%)\end{array}$ & $\begin{array}{l}\text { TOC } \\
(\%)\end{array}$ \\
\hline $4 t-4-43$ & 25.44 & 6.68 & 0.87 & 0.38 \\
\hline $414-4-113$ & 26.18 & 16.36 & 1.74 & 0.37 \\
\hline $4 H-5-35$ & 26.88 & & 8. 34 & 8.56 \\
\hline $7 \mathrm{H}-2-131$ & 50.11 & 5.95 & 8.88 & 0.77 \\
\hline $7 \mathrm{H}-3-28$ & 58.49 & 15.57 & 1.25 & 1.82 \\
\hline $7 \mathrm{H}-3-64$ & 51.86 & & 8.84 & 8.51 \\
\hline $7 H-3-131$ & 51.55 & 13.99 & 8.15 & 8.38 \\
\hline $7 \mathrm{H}-4-15$ & 51.88 & 12.64 & 1.12 & 0.31 \\
\hline $7 H-4-64$ & 52.56 & & 0.84 & 0.26 \\
\hline $7 \mathrm{H}-4-185$ & 52.74 & 18.91 & 8.80 & 1.51 \\
\hline $7 \mathrm{H}-5-41$ & 53.56 & 5.58 & 8.88 & 8.31 \\
\hline $7 \mathrm{H}-5-64$ & 54.86 & & 0.03 & 8.68 \\
\hline $7 \mathrm{H}-5-128$ & 54.32 & 6.48 & 8.88 & 0.53 \\
\hline $7 \mathrm{H}-6-32$ & 54.92 & 13.64 & 8.88 & 8.26 \\
\hline $7 \mathrm{H}-6-64$ & 55.26 & & 0.82 & 0.35 \\
\hline $7 H-6-128$ & 55.76 & 14.94 & 0.78 & 0.65 \\
\hline $7 \mathrm{H}-7-12$ & 56.16 & 4.83 & 8.88 & 0.58 \\
\hline $7 \mathrm{H}-7-56$ & 56.59 & 8.93 & 9.54 & 8.47 \\
\hline $7 \mathrm{H}-7-64$ & 57.86 & & 8.03 & 8.66 \\
\hline $7 \mathrm{H}-\mathrm{C}-\quad 4$ & 57.16 & & 0.03 & 8.63 \\
\hline $8 \mathrm{H}-1-55$ & 57.45 & 18.72 & 8.68 & 1.85 \\
\hline $8 H-1-64$ & 57.56 & & 8.35 & 0.89 \\
\hline $8 H-1-88$ & 57.78 & 3.38 & 0.17 & 0.38 \\
\hline $8 \mathrm{H}-2-19$ & 58.56 & 6.32 & 8.88 & 0.57 \\
\hline $8 \mathrm{H}-2-64$ & 59.86 & & 0.83 & 0.78 \\
\hline $8 H-2-137$ & 59.71 & 5.76 & 8.68 & 0.42 \\
\hline $8 \mathrm{H}-3-15$ & 59.98 & 9.85 & 8.68 & 0.49 \\
\hline $8 \mathrm{H}-3-64$ & 68.56 & & 0.03 & 0.66 \\
\hline $8 \mathrm{H}-3-113$ & 68.94 & 19.49 & 1.32 & 0.78 \\
\hline $8 \mathrm{H}-4-19$ & 61.48 & 1.03 & 0.88 & 8.82 \\
\hline $8 H-4-64$ & 62.86 & & 8.82 & 8.56 \\
\hline $8 H-4-112$ & 62.39 & 21.64 & 8.08 & 1.41 \\
\hline $8 \mathrm{H}-5-28$ & 62.95 & 38.85 & 0.80 & 8.53 \\
\hline $8 H-5-64$ & 63.56 & & 8.03 & 0.46 \\
\hline $8 H-5-119$ & 63.91 & 17.81 & 0.16 & 8.37 \\
\hline $8 \mathrm{H}-6-2 \theta$ & 64.41 & 1.58 & 8.89 & 8.34 \\
\hline $8 \mathrm{H}-6-64$ & 64.83 & & 8.85 & 8.48 \\
\hline $8 H-6-94$ & 65.13 & 21.16 & 0.16 & 0.33 \\
\hline $8 H-7-18$ & 65.85 & 7.78 & 8.86 & 8.31 \\
\hline $8 \mathrm{H}-7-44$ & 66.18 & 11.58 & 8.88 & 8.38 \\
\hline $8 H-C-10$ & 66.32 & & 8.80 & 8.38 \\
\hline $9 H-1-28$ & 66.69 & 12.88 & 8.88 & 8.39 \\
\hline $9 H-1-52$ & 66.94 & & 26.82 & 8.35 \\
\hline $9 H-1-137$ & 67.74 & 8.27 & 17.75 & 8.41 \\
\hline $9 H-2-28$ & 68.13 & 1.11 & 17.89 & 8.47 \\
\hline $9 \mathrm{H}-2-52$ & 68.44 & & 9.13 & 0.39 \\
\hline $9 \mathrm{H}-2-137$ & 69.18 & 8.48 & 43.81 & 8.68 \\
\hline $9 \mathrm{H}-3-28$ & 69.58 & 0.32 & 49.15 & 8.56 \\
\hline $9 \mathrm{H}-3-52$ & 69.94 & & 48.21 & 0.53 \\
\hline
\end{tabular}


Table 1.1 (continued).

\begin{tabular}{|c|c|c|c|c|}
\hline $\begin{array}{c}\text { Sample } \\
\text { no. }\end{array}$ & $\begin{array}{l}\text { Depth } \\
\text { (mbsf) }\end{array}$ & $\begin{array}{l}>63 \\
\mu \mathrm{m} \\
(\%)\end{array}$ & $\begin{array}{c}\text { Bulk } \\
\mathrm{CaCO}_{3} \\
(\%)\end{array}$ & $\begin{array}{l}\text { TOC } \\
(\%)\end{array}$ \\
\hline $9 \mathrm{H}-3-96$ & 78.23 & 9.84 & 5.84 & 8.35 \\
\hline $9 H-4-28$ & 71.02 & 0.13 & 25.89 & 0.82 \\
\hline $9 H-4-52$ & 71.44 & & 23.18 & 8.78 \\
\hline $9 H-4-137$ & 72.87 & 0.19 & 27.66 & 0.75 \\
\hline $9 \mathrm{H}-5-28$ & 72.46 & 0.10 & 24.91 & 0.78 \\
\hline $9 H-5-52$ & 72.94 & & 24.16 & 0.61 \\
\hline $9 H-5-137$ & 73.51 & 0.75 & 17.67 & 0.48 \\
\hline $9 H-6-28$ & 73.91 & 8.57 & 38.53 & 0.54 \\
\hline $9 H-6-52$ & 74.44 & & 38.49 & 8.60 \\
\hline $9 H-6-137$ & 74.96 & 0.28 & 42.91 & 0.55 \\
\hline $9 H-7-34$ & 75.41 & 2.89 & 44.98 & 0.64 \\
\hline $9 \mathrm{H}-7-52$ & 75.44 & & 58.16 & 8.58 \\
\hline $9 H-7-56$ & 75.62 & 1.83 & 46.17 & 8.57 \\
\hline $9 \mathrm{H}-\mathrm{C}-8$ & 76.82 & & 35.78 & 8.48 \\
\hline $18 \mathrm{H}-1-52$ & 76.44 & & 38.81 & 0.48 \\
\hline $18 \mathrm{H}-1-58$ & 76.48 & 8.29 & 26.43 & 0.51 \\
\hline $18 \mathrm{H}-2-52$ & 77.86 & & 21.72 & 8.79 \\
\hline $18 \mathrm{H}-2-58$ & 77.92 & 1.42 & 4.74 & 1.85 \\
\hline $18 \mathrm{H}-3-52$ & 79.31 & & 37.92 & 0.69 \\
\hline $18 \mathrm{H}-3-58$ & 79.37 & 8.45 & 33.89 & 8.88 \\
\hline $18 \mathrm{H}-4-52$ & 88.76 & & 36.61 & 8.57 \\
\hline $18 \mathrm{H}-4-58$ & 88.82 & 0.91 & 31.33 & 8.78 \\
\hline $10 \mathrm{H}-5-52$ & 82.28 & & 13.19 & 0.78 \\
\hline $18 \mathrm{H}-5-58$ & 82.26 & 1.34 & 13.38 & 8.78 \\
\hline $18 \mathrm{H}-6-52$ & 83.65 & & 8.83 & 0.72 \\
\hline $10 \mathrm{H}-6-58$ & 83.71 & 6.89 & 8.80 & 8.89 \\
\hline $18 \mathrm{H}-7-52$ & 85.18 & & & 0.81 \\
\hline $18 \mathrm{BH}-7-58$ & 85.16 & 0.63 & 0.80 & 0.83 \\
\hline $10 \mathrm{H}-\mathrm{C}-15$ & 85.63 & & 0.01 & 0.94 \\
\hline $11 \mathrm{H}-1-54$ & 85.94 & 0.28 & 0.80 & 8.78 \\
\hline $11 \mathrm{H}-2-46$ & 87.29 & & 8.11 & 8.82 \\
\hline $11 \mathrm{H}-2-54$ & 87.37 & 2.59 & 0.00 & 8.74 \\
\hline $11 \mathrm{H}-3-46$ & 88.73 & & 5.85 & 0.61 \\
\hline $11 H-3-54$ & 88.81 & 0.63 & 11.59 & 0.56 \\
\hline $11 \mathrm{H}-4-54$ & 98.25 & 0.11 & 8.80 & 0.50 \\
\hline $11 H-5-46$ & 91.68 & & 2.32 & 0.58 \\
\hline $11 \mathrm{H}-5-54$ & 91.68 & 8.19 & 3.68 & 8.45 \\
\hline $11 \mathrm{H}-6-54$ & 93.12 & 0.19 & 1.82 & 0.54 \\
\hline $11 H-7-46$ & 94.48 & & 8.82 & 0.71 \\
\hline $11 \mathrm{H}-7-54$ & 94.56 & 1.85 & 8.80 & 8.45 \\
\hline $11 \mathrm{H}-\mathrm{C}-12$ & 95.84 & & 8.22 & 0.62 \\
\hline $12 \mathrm{H}-1-61$ & 95.53 & 8.18 & 1.48 & 0.42 \\
\hline $12 \mathrm{H}-1-67$ & 95.59 & & 0.89 & 8.56 \\
\hline $12 \mathrm{H}-2-61$ & 97.83 & 0.82 & 8.06 & 0.61 \\
\hline $12 \mathrm{H}-2-67$ & 97.89 & & 8.08 & 0.61 \\
\hline $12 \mathrm{H}-3-61$ & 98.53 & 0.32 & 0.80 & 0.69 \\
\hline $12 \mathrm{H}-4-61$ & 180.03 & 2.24 & 8.88 & 8.64 \\
\hline $12 \mathrm{H}-4-67$ & 180.89 & & 0.80 & 0.93 \\
\hline $12 \mathrm{H}-5-61$ & 181.52 & 6.48 & 0.80 & 8.78 \\
\hline $12 \mathrm{H}-5-67$ & 101.59 & & 8.08 & 0.62 \\
\hline
\end{tabular}

Table 1.1 (continued).

\begin{tabular}{|c|c|c|c|c|}
\hline $\begin{array}{c}\text { Sample } \\
\text { no. }\end{array}$ & $\begin{array}{l}\text { Depth } \\
\text { (mbsf) }\end{array}$ & $\begin{array}{l}>63 \\
\mu \mathrm{m} \\
(\%)\end{array}$ & $\begin{array}{c}\text { Bulk } \\
\mathrm{CaCO}_{3} \\
(\%)\end{array}$ & $\begin{array}{l}\text { TOC } \\
(\%)\end{array}$ \\
\hline $12 \mathrm{H}-6-61$ & 183.82 & 8.36 & 5.64 & 8.57 \\
\hline $12 \mathrm{H}-6-67$ & 103.09 & & 4.38 & 8.71 \\
\hline $12 \mathrm{H}-7-13$ & 184.52 & 1.55 & 8.35 & 8.75 \\
\hline $13 H-1-184$ & 185.26 & 3.37 & 8.88 & 8.82 \\
\hline $13 \mathrm{H}-2-74$ & 186.46 & & 6.78 & 8.77 \\
\hline $13 \mathrm{H}-2-104$ & 186.76 & 1.33 & 8.16 & 8.67 \\
\hline $13 \mathrm{H}-3-104$ & 108.26 & 8.42 & 27.61 & 8.53 \\
\hline $13 \mathrm{H}-4-74$ & 189.46 & & 48.68 & 0.56 \\
\hline $13 \mathrm{H}-4-184$ & 109.76 & 8.28 & 34.48 & 0.38 \\
\hline $13 \mathrm{H}-5-74$ & 110.96 & & 39.66 & 8.52 \\
\hline $13 H-5-184$ & 111.26 & 8.47 & 36.62 & 0.36 \\
\hline $13 \mathrm{H}-6-74$ & 112.46 & & 43.45 & 8.68 \\
\hline $13 H-6-104$ & 112.76 & 0.83 & 28.68 & 8.45 \\
\hline $13 \mathrm{H}-\mathrm{C}-14$ & 113.85 & & 0.80 & 0.49 \\
\hline $14 H-1-95$ & 114.96 & 21.42 & 8.80 & 8.55 \\
\hline $14 \mathrm{H}-\mathrm{C}-6$ & 123.33 & & 0.81 & 0.86 \\
\hline $15 H-1-113$ & 124.90 & 8. 98 & 9.89 & 8.67 \\
\hline $15 \mathrm{H}-2-74$ & 125.76 & & 3.68 & 0.89 \\
\hline $15 H-2-113$ & 126.15 & 8.54 & 7.41 & 0.76 \\
\hline $15 H-3-113$ & 127.65 & 1.52 & 11.94 & 8.89 \\
\hline $15 \mathrm{H}-\mathrm{C}-7$ & 128.84 & & 13.86 & 0.88 \\
\hline $16 \mathrm{H}-1-116$ & 129.28 & 2.65 & 0.82 & 1.18 \\
\hline $16 \mathrm{H}-2-69$ & 138.31 & & 0.82 & 1.17 \\
\hline $16 \mathrm{H}-2-116$ & 138.78 & 2.85 & 0.76 & 1.16 \\
\hline $16 \mathrm{H}-4-69$ & 131.81 & & 8.81 & 1.26 \\
\hline $16 \mathrm{H}-4-116$ & 132.28 & 8.74 & 8.87 & 1.89 \\
\hline $16 \mathrm{H}-5-69$ & 133.31 & & 8.22 & 1.29 \\
\hline $16 \mathrm{H}-5-116$ & 133.78 & 0.65 & 2.45 & 1.29 \\
\hline $16 \mathrm{H}-6-69$ & 134.81 & & 13.41 & 1.19 \\
\hline $16 \mathrm{H}-6-116$ & 135.28 & 8.78 & 26.85 & 0.87 \\
\hline $16 \mathrm{H}-7-69$ & 136.31 & & 3.44 & 1.18 \\
\hline $16 \mathrm{H}-7-116$ & 136.78 & 5.31 & 8.56 & 0.87 \\
\hline $16 \mathrm{H}-\mathrm{C}-8$ & 137.75 & & 6.84 & 0.82 \\
\hline $17 \mathrm{H}-1-71$ & 138.73 & & 13.68 & 1.88 \\
\hline $17 \mathrm{H}-1-114$ & 139.16 & 2.48 & 13.71 & 8.89 \\
\hline $17 \mathrm{H}-2-71$ & 148.23 & & 1.16 & 0.92 \\
\hline $17 \mathrm{H}-2-114$ & 148.66 & 8.73 & 5.88 & 0.94 \\
\hline $17 \mathrm{H}-3-71$ & 141.73 & & 8.22 & 0.98 \\
\hline $17 \mathrm{H}-3-114$ & 142.16 & 1.27 & 8.78 & 1.17 \\
\hline $17 \mathrm{H}-4-71$ & 143.23 & & 8.80 & 0.88 \\
\hline $17 \mathrm{H}-4-114$ & 143.66 & 0.17 & 4.36 & 0.69 \\
\hline $17 \mathrm{H}-5-71$ & 144.73 & & 0.11 & 8.55 \\
\hline $17 \mathrm{H}-5-114$ & 145.16 & 1.89 & 1.62 & 8.81 \\
\hline $17 \mathrm{H}-6-71$ & 146.23 & & 1.44 & 8.88 \\
\hline $17 \mathrm{H}-6-116$ & 146.66 & 8.58 & 4.99 & 0.83 \\
\hline $17 \mathrm{H}-\mathrm{C}-13$ & 147.67 & & 1.19 & 0.83 \\
\hline $18 \mathrm{H}-1-72$ & 148.54 & & 11.58 & 0.75 \\
\hline $18 \mathrm{H}-1-114$ & 148.96 & 8.27 & 7.88 & 0.83 \\
\hline $18 \mathrm{H}-2-72$ & 158.84 & & 4.64 & 8.77 \\
\hline $18 \mathrm{H}-2-114$ & 150.46 & 1.48 & 5.18 & 0.89 \\
\hline
\end{tabular}


Table 1.1 (continued).

\begin{tabular}{|c|c|c|c|c|}
\hline $\begin{array}{c}\text { Sample } \\
\text { no. }\end{array}$ & $\begin{array}{l}\text { Depth } \\
\text { (mbsf) }\end{array}$ & $\begin{array}{l}>63 \\
\mu \mathrm{m} \\
(\%)\end{array}$ & $\begin{array}{c}\text { Bulk } \\
\mathrm{CaCO}_{3} \\
(\%)\end{array}$ & $\begin{array}{l}\text { TOC } \\
(\%)\end{array}$ \\
\hline $18 \mathrm{H}-3-72$ & 151.54 & & 3.82 & 0.71 \\
\hline $18 \mathrm{H}-3-114$ & 151.96 & 2.81 & 5.76 & 8.98 \\
\hline $18 \mathrm{H}-4-72$ & 153.84 & & 9.48 & 8.82 \\
\hline $18 \mathrm{H}-4-114$ & 153.46 & 1.55 & 12.28 & 8.67 \\
\hline $18 \mathrm{H}-5-82$ & 154.64 & & 21.37 & 8.78 \\
\hline $18 \mathrm{H}-5-114$ & 154.96 & 7.82 & 33.17 & 8.63 \\
\hline $18 \mathrm{H}-6-72$ & 156.84 & & 16.48 & 8.88 \\
\hline $18 H-6-114$ & 156.46 & 0.55 & 26.96 & 8.56 \\
\hline $18 \mathrm{H}-\mathrm{C}-\theta$ & 157.64 & & 45.32 & 8.64 \\
\hline $19 \mathrm{H}-1-72$ & 158.44 & & 33.49 & 8.75 \\
\hline $19 H-1-105$ & 158.77 & 1.30 & 27.88 & 8.56 \\
\hline $19 H-2-89$ & 160.11 & & 9.81 & 0.81 \\
\hline $19 H-2-105$ & 168.27 & 8.92 & 5.42 & 8.78 \\
\hline $19 \mathrm{H}-3-72$ & 161.44 & & 6.46 & 8.89 \\
\hline $19 \mathrm{H}-3-185$ & 161.77 & 0.73 & 2.81 & 8.89 \\
\hline $19 \mathrm{H}-4-72$ & 162.94 & & 11.18 & 1.28 \\
\hline $19 \mathrm{H}-4-185$ & 163.27 & 2.74 & 7.25 & 1.83 \\
\hline $19 \mathrm{H}-5-72$ & 164.44 & & 8.88 & 8.98 \\
\hline $19 H-5-105$ & 164.77 & 1.82 & 8.88 & 1.55 \\
\hline $19 \mathrm{H}-6-72$ & 165.94 & & 0.00 & 1.79 \\
\hline $19 H-6-185$ & 166.27 & 8.51 & 8.80 & 1.83 \\
\hline $19 H-C-1$ & 167.31 & & 8.08 & 2.26 \\
\hline $28 \mathrm{H}-1-71$ & 168.13 & & 8.88 & 2.29 \\
\hline $28 \mathrm{H}-1-103$ & 168.44 & 2.37 & 8.88 & 2.19 \\
\hline $28 \mathrm{H}-2-71$ & 169.63 & & 8.88 & 1.69 \\
\hline $28 \mathrm{H}-2-183$ & 169.92 & 3.59 & 0.80 & 1.77 \\
\hline $28 \mathrm{H}-3-71$ & 171.13 & & 8.80 & 1.86 \\
\hline $28 \mathrm{H}-3-103$ & 171.41 & 1.69 & 8.80 & 2.51 \\
\hline $28 \mathrm{H}-4-71$ & 172.88 & & 8.88 & 2.83 \\
\hline $28 \mathrm{H}-4-183$ & 172.48 & 3.91 & 8.88 & 5.59 \\
\hline $28 \mathrm{H}-5-71$ & 174.13 & & 8.80 & 1.68 \\
\hline $28 \mathrm{H}-5-103$ & 174.38 & 5.53 & 8.88 & 1.54 \\
\hline $28 \mathrm{H}-6-71$ & 175.63 & & 8.80 & 2.17 \\
\hline $28 \mathrm{H}-6-183$ & 175.87 & 11.69 & 0.80 & 1.68 \\
\hline $28 \mathrm{H}-\mathrm{C}-9$ & 176.97 & & 8.88 & 2.75 \\
\hline $21 \mathrm{H}-1-74$ & 177.86 & & 8.88 & 2.29 \\
\hline $21 \mathrm{H}-1-104$ & 178.16 & 1.56 & 8.88 & 2.12 \\
\hline $21 \mathrm{H}-2-74$ & 179.36 & & 8.80 & 2.12 \\
\hline $21 \mathrm{H}-2-184$ & 179.66 & 2.72 & 8.80 & 1.83 \\
\hline $21 \mathrm{H}-3-74$ & 180.86 & & 8.80 & 1.78 \\
\hline $21 \mathrm{H}-3-184$ & 181.16 & 15.87 & 8.88 & 1.53 \\
\hline $21 \mathrm{H}-4-74$ & 182.36 & & 8.88 & 2.21 \\
\hline $21 \mathrm{H}-4-184$ & 182.66 & 3.98 & 8.80 & 1.92 \\
\hline $21 \mathrm{H}-5-74$ & 183.86 & & 8.88 & 2.84 \\
\hline $21 \mathrm{H}-5-185$ & 184.16 & 2.16 & 8.08 & 1.56 \\
\hline $21 \mathrm{H}-6-74$ & 185.36 & & 8.08 & 2.14 \\
\hline $21 \mathrm{H}-6-184$ & 185.66 & 1.82 & 8.80 & 2.13 \\
\hline $22 \mathrm{H}-1-55$ & 187.26 & 3.58 & 8.08 & 1.54 \\
\hline $22 \mathrm{H}-2-55$ & 188.74 & 12.84 & 8.88 & 8.59 \\
\hline $22 \mathrm{H}-3-55$ & 190.22 & 2.34 & 8.80 & 2.26 \\
\hline
\end{tabular}

Table 1.1 (continued).

\begin{tabular}{|c|c|c|c|c|}
\hline $\begin{array}{c}\text { Sample } \\
\text { no. }\end{array}$ & $\begin{array}{l}\text { Depth } \\
\text { (mbsf) }\end{array}$ & $\begin{array}{l}>63 \\
\mu \mathrm{m} \\
(\%)\end{array}$ & $\begin{array}{c}\text { Bulk } \\
\mathrm{CaCO}_{3} \\
(\%)\end{array}$ & $\begin{array}{l}\text { TOC } \\
(\%)\end{array}$ \\
\hline $22 \mathrm{H}-4-55$ & 191.78 & 8.87 & 0.88 & 1.83 \\
\hline $22 \mathrm{H}-5-55$ & 193.17 & 8.58 & 8.80 & 1.41 \\
\hline $22 \mathrm{H}-6-55$ & 194.65 & 15.87 & 0.88 & 0.90 \\
\hline $22 \mathrm{H}-7-45$ & 196.03 & 29.22 & 8.88 & 0.93 \\
\hline $23 \mathrm{H}-1-6$ & 196.87 & 18.83 & 8.88 & 0.80 \\
\hline $23 \mathrm{H}-2-12$ & 197.62 & 2.58 & 8.88 & 1.22 \\
\hline $23 \mathrm{H}-3-12$ & 199.11 & 1.57 & 0.88 & 1.30 \\
\hline $23 H-4-12$ & 288.68 & 8.64 & 0.88 & 1.89 \\
\hline $23 \mathrm{H}-\mathrm{S}-12$ & 282.89 & 8.53 & 6.08 & 1.12 \\
\hline $23 H-6-12$ & 203.58 & 1.36 & 8.88 & 1.02 \\
\hline $23 \mathrm{H}-7-12$ & 285.87 & 8.34 & 8.88 & 1.28 \\
\hline $24 \mathrm{H}-1-183$ & 286.64 & 8.54 & 8.88 & 1.88 \\
\hline $24 \mathrm{H}-2-183$ & 288.14 & 0.92 & 8.80 & 8. 97 \\
\hline $24 \mathrm{H}-3-183$ & 289.64 & 8.88 & 8.88 & 1.18 \\
\hline $24 \mathrm{H}-4-183$ & 211.14 & 8.68 & 8.80 & 1.85 \\
\hline $24 \mathrm{H}-5-183$ & 212.64 & 8.15 & 8.80 & 1.41 \\
\hline $25 \mathrm{H}-1-96$ & 214.67 & 8.56 & 0.80 & 1.88 \\
\hline $25 \mathrm{H}-2-96$ & 215.56 & 0.47 & 8.08 & 1.15 \\
\hline $25 \mathrm{H}-3-96$ & 217.05 & 8.65 & 0.00 & 1.33 \\
\hline $25 \mathrm{H}-4-96$ & 218.53 & 8. 39 & 8.80 & 1.88 \\
\hline $25 \mathrm{H}-5-96$ & 228.02 & 8.38 & 0.80 & 1.02 \\
\hline
\end{tabular}


Table 1.1 (continued).

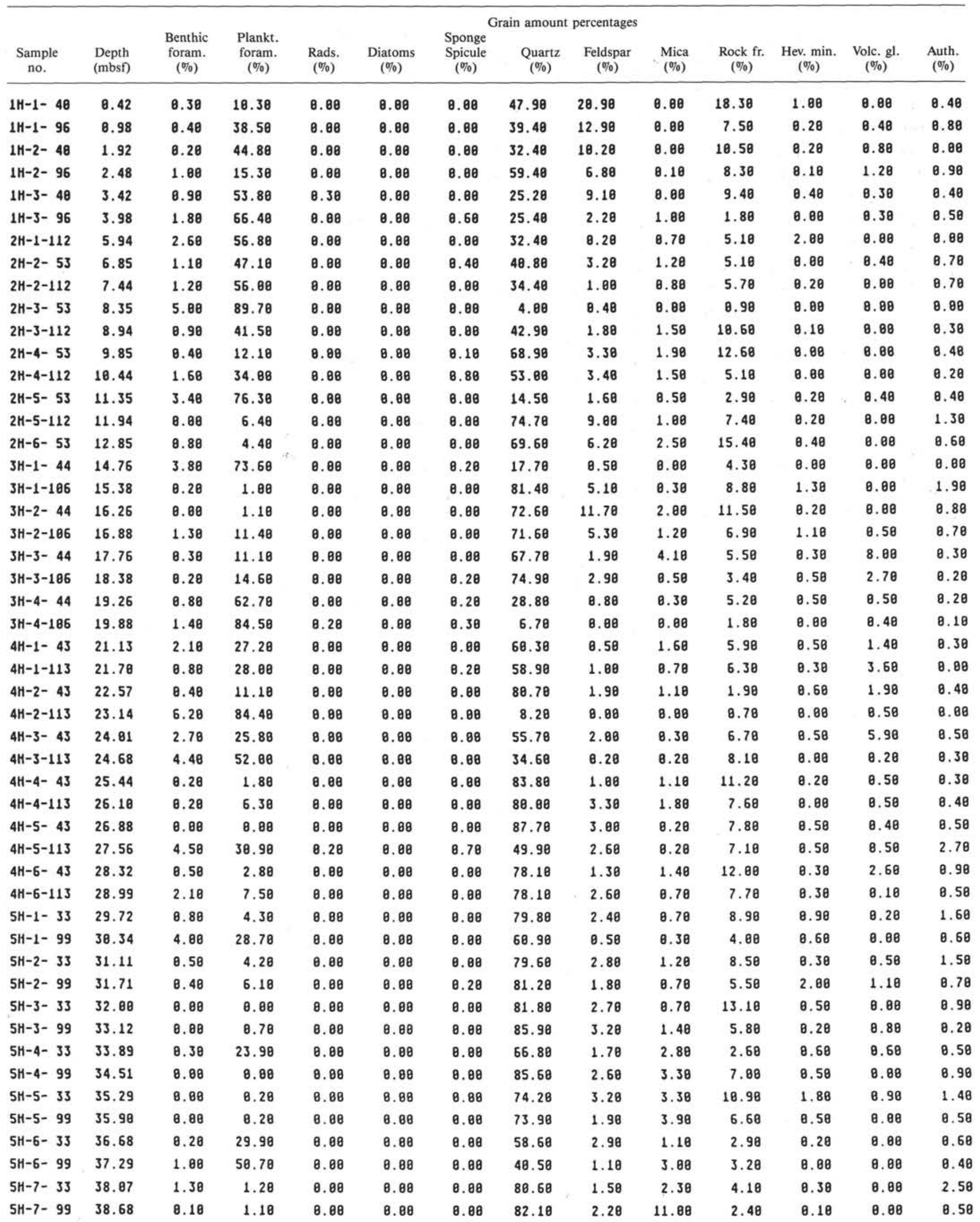


Table 1.1 (continued).

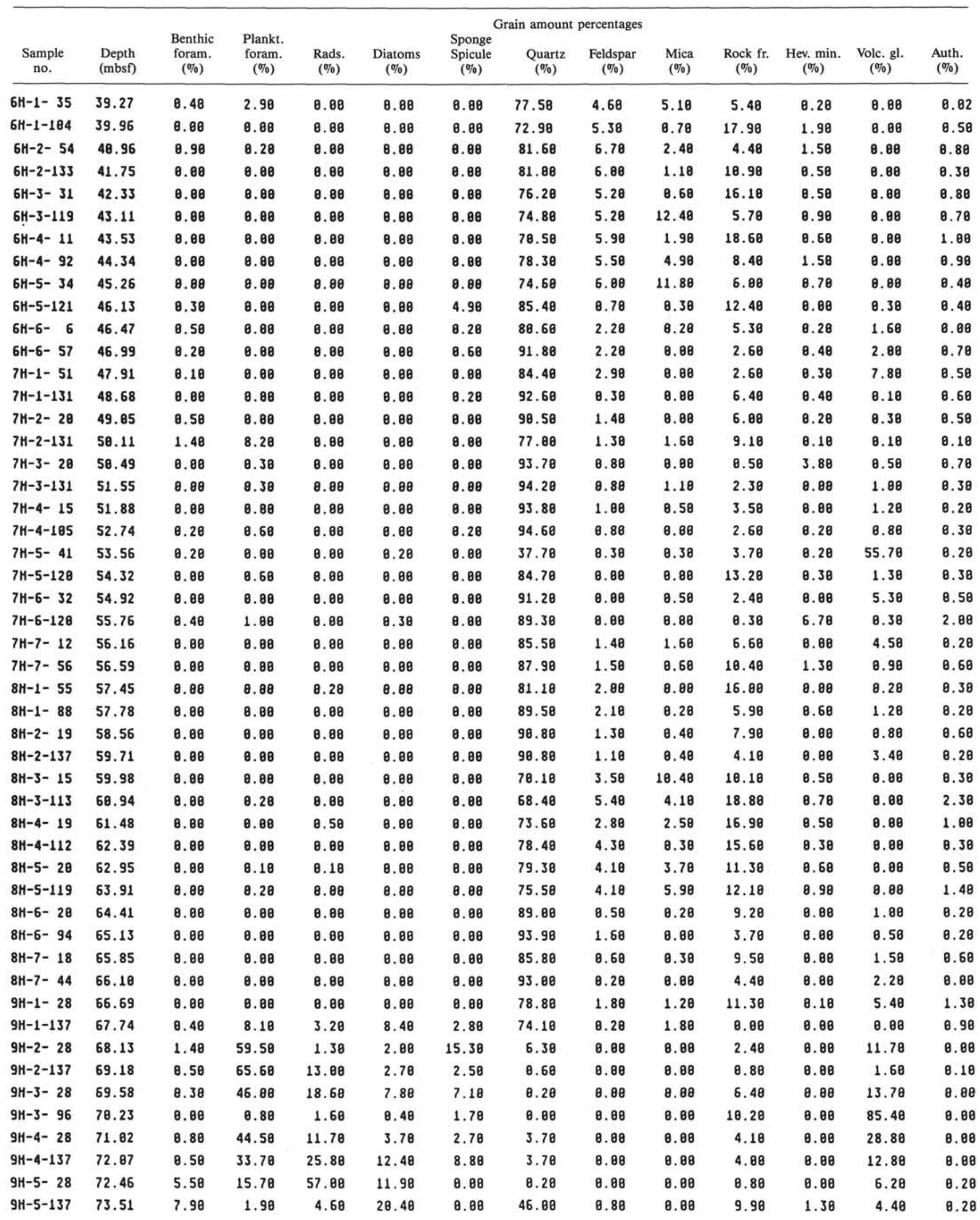


Table 1.1 (continued).

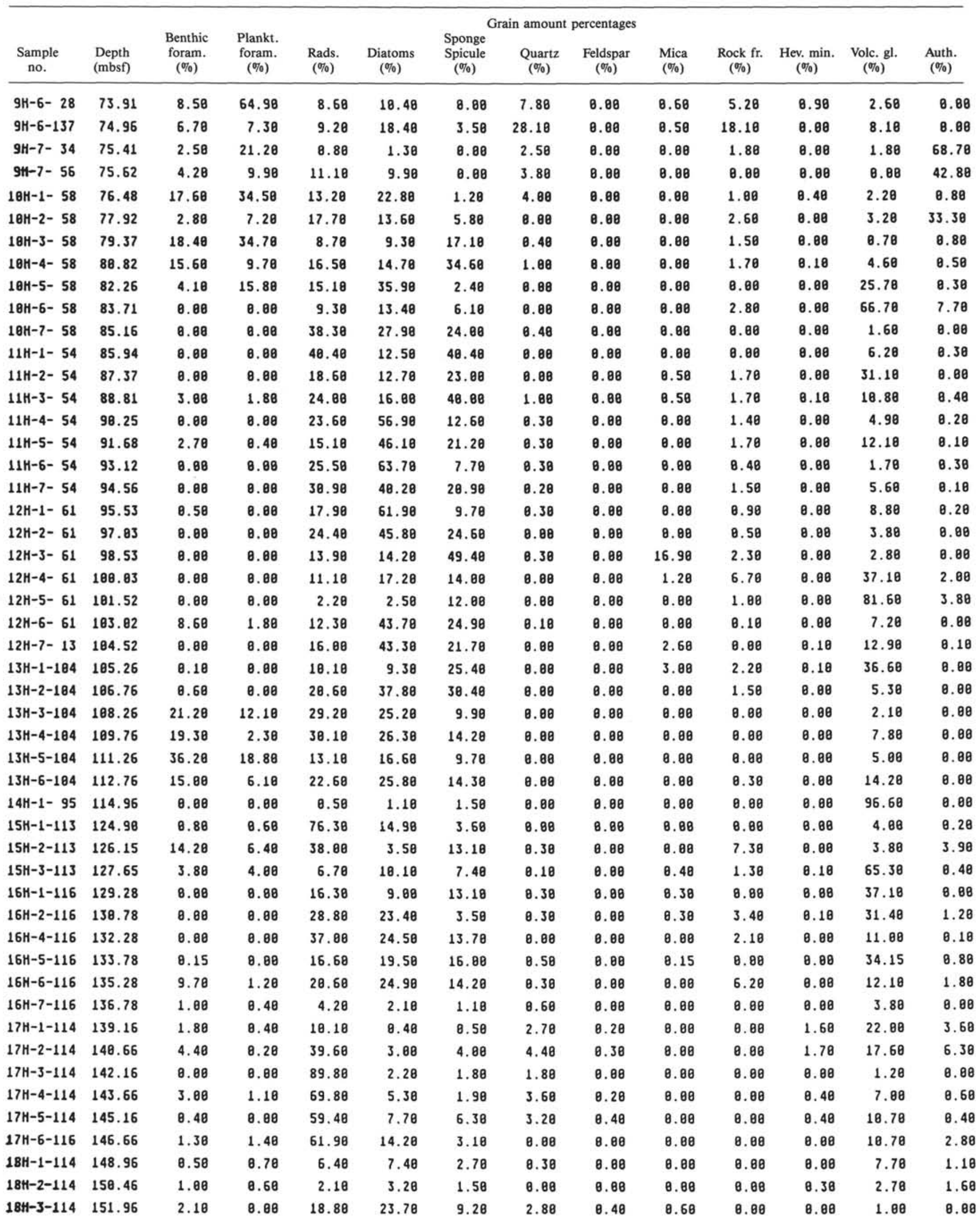


Table 1.1 (continued).

\begin{tabular}{|c|c|c|c|c|c|c|c|c|c|c|c|c|c|}
\hline \multirow[b]{2}{*}{$\begin{array}{c}\text { Sample } \\
\text { no. }\end{array}$} & \multirow[b]{2}{*}{$\begin{array}{l}\text { Depth } \\
\text { (mbsf) }\end{array}$} & \multirow[b]{2}{*}{$\begin{array}{l}\text { Benthic } \\
\text { foram. } \\
(\%)\end{array}$} & \multirow[b]{2}{*}{$\begin{array}{l}\text { Plankt. } \\
\text { foram. } \\
(\%)\end{array}$} & \multirow[b]{2}{*}{$\begin{array}{c}\text { Rads. } \\
(\%)\end{array}$} & \multirow[b]{2}{*}{$\begin{array}{c}\text { Diatoms } \\
(\%)\end{array}$} & \multicolumn{3}{|c|}{$\begin{array}{l}\text { Grain amount percentages } \\
\text { Sange }\end{array}$} & \multirow[b]{2}{*}{$\begin{array}{l}\text { Mica } \\
(\%)\end{array}$} & \multirow[b]{2}{*}{$\begin{array}{c}\text { Rock fr. } \\
(\%)\end{array}$} & \multirow[b]{2}{*}{$\begin{array}{l}\text { Hev. min. } \\
(\%)\end{array}$} & \multirow[b]{2}{*}{$\begin{array}{l}\text { Volc. gl. } \\
(\%)\end{array}$} & \multirow[b]{2}{*}{$\begin{array}{l}\text { Auth. } \\
(\%)\end{array}$} \\
\hline & & & & & & $\begin{array}{c}\text { Sponge } \\
\text { Spicule } \\
(\%)\end{array}$ & $\begin{array}{l}\text { Quartz } \\
(\%)\end{array}$ & $\begin{array}{c}\text { Feldspar } \\
(\%)\end{array}$ & & & & & \\
\hline $18 H-4-114$ & 153.46 & 5.48 & 8.78 & 21.98 & 13.68 & 7.48 & 2.98 & 8.18 & 8.48 & 8.88 & 1.78 & 8.30 & 8.58 \\
\hline $18 \mathrm{H}-5-114$ & 154.96 & 0.30 & 8.88 & 2.80 & 38.88 & 1.98 & 8.48 & 8.88 & 8.80 & 8.08 & 8.10 & 13.38 & 1.10 \\
\hline $18 H-6-114$ & 156.46 & 2.98 & 8.88 & 13.30 & 11.78 & 2.98 & 8.98 & 8.88 & 8.80 & 8.88 & 8.38 & 21.88 & 1.58 \\
\hline $19 H-1-105$ & 158.77 & 5.88 & 3.98 & 6.78 & 27.38 & 5.88 & 0.98 & 8.88 & 0.80 & 8.80 & 0.80 & 7.80 & 8.88 \\
\hline $19 H-2-185$ & 168.27 & 11.88 & 4.68 & 39.88 & 5.38 & 3.18 & 2.68 & 8.88 & 8.80 & 8.30 & 8.88 & 18.78 & 9.78 \\
\hline $19 H-3-185$ & 151.77 & 1.48 & 8.80 & 48.78 & 19.98 & 13.78 & 1.58 & 8.88 & 8.80 & 8.30 & 8.88 & 5.68 & 2.80 \\
\hline $19 H-4-185$ & 163.27 & 1.80 & 8.28 & 5.88 & 27.98 & 14.98 & 8.88 & 8.88 & 0.80 & 19.78 & 8.00 & 14.78 & 0.28 \\
\hline $19 H-5-105$ & 164.77 & 8.30 & 8.80 & B. 98 & 88.18 & 6.48 & 8.80 & 8.88 & 0.80 & 8.50 & 8.88 & 18.68 & 0.30 \\
\hline $19 H-6-105$ & 166.27 & 8.30 & 0.88 & 8.78 & 58.38 & 0.80 & 0.88 & 8.80 & 8.80 & 1.48 & 8.80 & 11.48 & 0.80 \\
\hline $28 \mathrm{H}-1-103$ & 168.44 & 5.78 & 8.88 & 3.48 & 36.80 & 8.88 & 8.88 & 8.80 & 8.68 & 6.88 & 0.08 & 23.78 & 8.40 \\
\hline $28 \mathrm{H}-2-183$ & 169.92 & 8.88 & 0.80 & 0.68 & 18.48 & 6.88 & 8.88 & 8.88 & 0.80 & 1.80 & 0.88 & 75.78 & 8.00 \\
\hline $28 \mathrm{H}-3-103$ & 171.41 & 4.88 & 8.80 & 1.80 & 33.38 & 1.80 & 8.88 & 0.80 & 0.88 & 18.88 & 8.80 & 21.28 & 8.58 \\
\hline $2 \theta \mathrm{H}-4-103$ & 172.48 & 8.88 & 8.88 & 4.58 & 37.78 & 4.80 & 8.88 & 8.80 & 0.88 & 13.68 & 8.98 & 14.78 & 1.60 \\
\hline $28 \mathrm{H}-5-183$ & 174.38 & 8.28 & 0.28 & 7.28 & 23.88 & 8.00 & 8.80 & 8.88 & 8.88 & 8.88 & 8.88 & 41.78 & 8.80 \\
\hline $28 \mathrm{H}-6-183$ & 175.87 & 8.48 & 0.88 & 15.98 & 8.60 & 2.28 & 2.48 & 8.38 & 8.80 & 1.80 & 8.88 & 56.68 & 1.78 \\
\hline $21 \mathrm{H}-1-104$ & 178.16 & 8.80 & 8.88 & 31.48 & 34.18 & 7.68 & 8.88 & 8.88 & 8.08 & 8.80 & 8.88 & 4.68 & 8.88 \\
\hline $21 \mathrm{H}-2-104$ & 179.66 & 8.88 & 0.80 & 42.68 & 25.80 & 8.88 & 4.48 & 8.80 & 0.80 & 8.88 & 8.80 & 16.78 & 8.38 \\
\hline $21 \mathrm{H}-3-184$ & 181.16 & $\theta .88$ & 8.88 & 9.30 & 5.30 & 2.28 & 7.28 & 8.80 & 8.88 & 0.88 & 0.80 & 78.78 & 8.38 \\
\hline $21 \mathrm{H}-4-184$ & 182.66 & 8.38 & 8.88 & 48.78 & 21.58 & 4.10 & 2.18 & 8.88 & 8.80 & 0.80 & 8.88 & 21.28 & 8.88 \\
\hline $21 \mathrm{H}-5-185$ & 184.16 & 0.68 & 8.88 & 44.88 & 17.80 & 24.88 & 1.38 & 8.88 & 8.88 & 9.68 & 8.80 & 0.88 & 0.18 \\
\hline $21 \mathrm{H}-6-184$ & 185.66 & 2.88 & 0.88 & 44.98 & 27.10 & 7.18 & 1.78 & 0.88 & 0.80 & 1.98 & 8.08 & 6.80 & 1.98 \\
\hline $22 \mathrm{H}-1-55$ & 187.26 & 1.38 & 0.88 & 57.68 & 5.48 & 8.68 & 2.88 & 8.88 & 8.88 & 8.38 & 8.88 & 28.88 & 8.78 \\
\hline $22 \mathrm{H}-2-55$ & 188.74 & 0.28 & 8.88 & 17.98 & 22.88 & 11.98 & 1.88 & 8.88 & 0.88 & 0.88 & 8.88 & 22.78 & 0.30 \\
\hline $22 \mathrm{H}-3-55$ & 190.22 & 1.58 & 8.88 & 35.68 & 28.78 & 3.48 & 2.88 & 8.88 & 8.88 & 8.88 & 0.80 & 24.58 & 1.18 \\
\hline $22 \mathrm{H}-4-55$ & 191.78 & 0.30 & 8.88 & 51.88 & 23.48 & 4.38 & 8.78 & 8.88 & 8.88 & 1.38 & 8.08 & 13.48 & 0.10 \\
\hline $22 \mathrm{H}-5-55$ & 193.17 & 0.80 & $\theta .88$ & 66.48 & 28.38 & 3.78 & 8.88 & 0.88 & 8.88 & 0.80 & 8.80 & 4.58 & 8.38 \\
\hline $22 \mathrm{H}-6-55$ & 194.65 & 8.88 & 8.88 & 12.38 & 1.58 & 8.80 & 2.88 & 8.88 & 0.80 & 8.88 & 8.80 & 81.28 & 1.80 \\
\hline $22 \mathrm{H}-7-45$ & 196.83 & 0.28 & 0.80 & 58.58 & 1.48 & 8.88 & 8.58 & 8.88 & 8.80 & 1.78 & 8.88 & 29.88 & 15.58 \\
\hline $23 \mathrm{H}-1-6$ & 196.07 & 0.80 & 0.80 & 24.78 & 5.68 & 0.60 & 8.68 & 8.88 & 0.80 & 1.98 & 8.88 & 35.28 & 27.58 \\
\hline $23 \mathrm{H}-2-12$ & 197.62 & 8.30 & 8.08 & 48.10 & 16.88 & 11.78 & 8.58 & 8.80 & 0.80 & 8.88 & 0.80 & 17.78 & 1.58 \\
\hline $23 \mathrm{H}-3-12$ & 199.11 & 8.80 & 8.88 & 34.78 & 28.88 & 9.10 & 8.78 & 8.88 & 0.80 & 0.80 & 8.88 & 16.28 & 2.90 \\
\hline $23 \mathrm{H}-4-12$ & 280.68 & 0.50 & 8.80 & 43.88 & 15.78 & 5.68 & 8.88 & 8.88 & 8.88 & 8.88 & 8.80 & 16.90 & 1.58 \\
\hline $23 \mathrm{H}-5-12$ & 282.89 & 0.80 & 8.80 & 22.78 & 13.88 & 6.38 & 0.48 & 8.88 & 0.80 & 8.88 & 8.80 & 5.98 & 8.88 \\
\hline $23 \mathrm{H}-6-12$ & 283.58 & 8.48 & 0.88 & 64.78 & 16.78 & 9.28 & 8.88 & 8.88 & 8.88 & 8.28 & 8.88 & 1.38 & 8.80 \\
\hline $23 \mathrm{H}-7-12$ & 285.87 & 8.28 & 0.80 & 77.38 & 18.88 & 11.48 & 8.88 & 8.88 & 8.88 & 8.80 & 8.88 & 8.28 & 8.88 \\
\hline $24 \mathrm{H}-1-183$ & 286.64 & 8.88 & 0.80 & 98.28 & 6.88 & 2.28 & 8.80 & 8.88 & 8.08 & 8.80 & 8.08 & 8.88 & 0.10 \\
\hline $24 \mathrm{H}-2-103$ & 288.14 & 0.88 & 8.88 & 95.88 & 1.88 & 8.98 & 8.88 & 8.88 & 8.88 & 0.88 & 8.80 & 8.28 & 8.28 \\
\hline $24 \mathrm{H}-3-183$ & 289.64 & 8.08 & 8.88 & 80.98 & 9.88 & 5.48 & 8.80 & 8.80 & 8.88 & 8.88 & 8.08 & 8.38 & 8.38 \\
\hline $24 \mathrm{H}-4-183$ & 211.14 & 8.80 & 8.80 & 86.68 & 5.38 & 8.88 & 8.80 & 0.80 & 8.80 & 8.88 & 8.08 & 2.28 & 8.98 \\
\hline $24 \mathrm{H}-5-183$ & 212.64 & 8.88 & 0.88 & 83.48 & 5.88 & 18.38 & 8.80 & 8.88 & 8.08 & 8.88 & 8.80 & 8.68 & 8.88 \\
\hline $25 \mathrm{H}-1-96$ & 214.87 & 8.88 & 0.88 & 65.78 & 23.10 & 2.68 & 0.88 & 8.88 & 0.80 & 0.48 & 8.88 & 4.18 & 2.18 \\
\hline $25 \mathrm{H}-2-96$ & 215.56 & 8.88 & 0.88 & 67.28 & 18.88 & 7.18 & 8.88 & 8.80 & 8.88 & 8.10 & 8.88 & 1.88 & 8.18 \\
\hline $25 \mathrm{H}-3-96$ & 217.85 & 8.80 & 0.80 & 73.18 & $18.4 \theta$ & 1.80 & 8.88 & 8.88 & 8.08 & $\theta .8 \theta$ & 8.88 & 8.38 & 0.00 \\
\hline $25 \mathrm{H}-4-96$ & 218.53 & 8.88 & 0.28 & 64.88 & 17.78 & 1.38 & 0.88 & 8.80 & $\theta . \theta 0$ & 8.28 & 0.80 & 2.18 & 2.88 \\
\hline $25 \mathrm{H}-5-96$ & 228.82 & 8.88 & 8.28 & 62.18 & 19.18 & 7.38 & 8.38 & 8.80 & 8.80 & 8.58 & 8.58 & 5.50 & 1.50 \\
\hline
\end{tabular}


Table 1.1 (continued).

\begin{tabular}{|c|c|c|c|c|c|c|}
\hline \multirow[b]{2}{*}{$\begin{array}{l}\text { Sample } \\
\text { no. }\end{array}$} & \multirow[b]{2}{*}{$\begin{array}{l}\text { Depth } \\
\text { (mbsf) }\end{array}$} & \multicolumn{5}{|c|}{ Subfraction of coarse fraction $(\mu \mathrm{m})$} \\
\hline & & $\begin{array}{l}>63 \\
(\%)\end{array}$ & $\begin{array}{c}63-125 \\
(\%)\end{array}$ & $\begin{array}{c}125-500 \\
(\%)\end{array}$ & $\begin{array}{c}500-1000 \\
(\%)\end{array}$ & $\begin{array}{c}>1000 \\
(\%)\end{array}$ \\
\hline $1 H-1-4 \theta$ & 0.42 & 14.84 & 48.91 & 37.11 & 7.82 & 6.96 \\
\hline $1 H-1-96$ & 8.98 & 13.93 & 38.95 & 45.75 & 3.99 & 11.31 \\
\hline $1 H-2-48$ & 1.92 & 6.85 & 51.81 & 41.67 & 4.29 & 2.23 \\
\hline $1 \mathrm{H}-2-96$ & 2.48 & 2.27 & 45.86 & 37.48 & 6.84 & 10.62 \\
\hline $1 \mathrm{H}-3-48$ & 3.42 & 12.57 & 54.15 & 48.54 & 2.55 & 2.77 \\
\hline $1 H-3-96$ & 3.98 & 19.45 & 36.21 & 52.87 & 2.82 & 8.90 \\
\hline $2 \mathrm{H}-1-112$ & 5.94 & 11.38 & 44.91 & 49.99 & 2.84 & 2.26 \\
\hline $2 \mathrm{H}-2-53$ & 6.85 & 7.65 & 52.39 & 42.84 & 2.83 & 2.74 \\
\hline $2 \mathrm{H}-2-112$ & 7.44 & 11.68 & 48.58 & 57.14 & 1.23 & 1.05 \\
\hline $2 \mathrm{H}-3-53$ & 8.35 & 16.24 & 23.45 & 76.33 & 8.22 & 0.08 \\
\hline $2 \mathrm{H}-3-112$ & 8.94 & 8.36 & 53.72 & 43.13 & 1.81 & 1.34 \\
\hline $2 \mathrm{H}-4-53$ & 9.85 & 12.35 & 48.58 & 44.86 & 2.28 & 4.45 \\
\hline $2 \mathrm{H}-4-112$ & 10.44 & 12.85 & 46.22 & 44.83 & 3.46 & 5.49 \\
\hline $2 \mathrm{H}-5-53$ & 11.35 & 9.85 & 36.93 & 68.58 & 1.78 & 0.78 \\
\hline $2 H-5-112$ & 11.94 & 13.44 & 55.46 & 38.36 & 4.76 & 1.43 \\
\hline $2 \mathrm{H}-6-53$ & 12.85 & 9.65 & 53.28 & 36.78 & 3.45 & 6.65 \\
\hline $3 H-1-44$ & 14.76 & 8.12 & 51.94 & 47.43 & 0.63 & 8.88 \\
\hline $3 H-1-186$ & 15.38 & 21.41 & 38.86 & 44.58 & 5.81 & 11.63 \\
\hline $3 \mathrm{H}-2-44$ & 16.26 & 25.38 & 43.03 & 44.45 & 5.48 & 7.84 \\
\hline $3 \mathrm{H}-2-106$ & 16.88 & 12.65 & 46.41 & 42.83 & 5.24 & 5.51 \\
\hline $3 \mathrm{H}-3-44$ & 17.76 & 5.95 & 76.22 & 22.36 & 1.26 & 0.16 \\
\hline $3 H-3-186$ & 18.38 & 7.11 & 34.23 & 38.98 & 3.66 & 23.12 \\
\hline $3 \mathrm{H}-4-44$ & 19.26 & 2.93 & 55.11 & 43.31 & 1.58 & 8.80 \\
\hline $3 H-4-186$ & 19.88 & 3.37 & 58.97 & 39.73 & 1.38 & 0.08 \\
\hline $4 H-1-43$ & 21.13 & 18.84 & 54.53 & 37.42 & 1.57 & 6.48 \\
\hline $4 H-1-113$ & 21.78 & 15.87 & 41.11 & 27.88 & 1.58 & 29.68 \\
\hline $4 H-2-43$ & 22.57 & 14.21 & 47.13 & 44.35 & 5.63 & 2.89 \\
\hline $4 \mathrm{H}-2-113$ & 23.14 & 8.25 & 35.79 & 68.51 & 1.18 & 2.51 \\
\hline $4 \mathrm{H}-3-43$ & 24.81 & 8.83 & 58.83 & 38.92 & 1.73 & 0.52 \\
\hline $4 H-3-113$ & 24.68 & 21.81 & 45.27 & 52.39 & 1.68 & 8.65 \\
\hline $4 H-4-43$ & 25.44 & 6.68 & 54.49 & 39.17 & 2.14 & 4.21 \\
\hline $4 H-4-113$ & 26.10 & 16.36 & 68.67 & 34.14 & 2.63 & 2.55 \\
\hline $4 H-5-43$ & 26.88 & 25.48 & 36.46 & 52.98 & 6.36 & 4.28 \\
\hline $4 H-5-113$ & 27.56 & 3.56 & 46.63 & 41.79 & 7.53 & 4.85 \\
\hline $4 H-6-43$ & 28.32 & 18.98 & 54.15 & 48.31 & 2.47 & 3.87 \\
\hline $4 H-6-113$ & 28.99 & 12.76 & 64.78 & 33.85 & 1.15 & 1.82 \\
\hline $5 H-1-33$ & 29.72 & 14.14 & 54.37 & 34.83 & 3.86 & 6.94 \\
\hline $5 H-1-99$ & 38.34 & 4.14 & 46.33 & 49.43 & 4.24 & 8.80 \\
\hline $5 H-2-33$ & 31.11 & 3.81 & 50.96 & 33.88 & 12.80 & 3.16 \\
\hline $5 H-2-99$ & 31.71 & 18.82 & 41.79 & 45.83 & 2.65 & 18.53 \\
\hline $5 \mathrm{H}-3-33$ & 32.68 & 21.21 & 45.55 & 48.28 & 3.71 & 18.46 \\
\hline $5 H-3-99$ & 33.12 & 5.54 & 66.88 & 32.44 & 1.18 & 0. 38 \\
\hline $5 H-4-33$ & 33.89 & 38.74 & 61.49 & 35.45 & 8.94 & 2.11 \\
\hline $5 H-4-99$ & 34.51 & 15.85 & 66.53 & 32.84 & 8.78 & 8.66 \\
\hline $5 H-5-33$ & 35.29 & 5.86 & 57.68 & 35.89 & 2.99 & 3.43 \\
\hline $5 H-5-99$ & 35.98 & 5.17 & 71.03 & 24.12 & 2.55 & 2.29 \\
\hline $5 H-6-33$ & 36.68 & 5.89 & 48.37 & 46.82 & 2.31 & 2.58 \\
\hline $5 H-6-99$ & 37.29 & 8.82 & 42.39 & 51.47 & 2.26 & 3.89 \\
\hline $5 \mathrm{H}-7-33$ & 38.87 & 3.81 & 71.68 & 25.65 & 1.22 & 1.44 \\
\hline $5 \mathrm{H}-7-99$ & 38.68 & 28.89 & 74.21 & 25.23 & 8.56 & 8.88 \\
\hline $6 \mathrm{H}-1-35$ & 39.27 & 13.32 & 79.73 & 19.29 & 8.94 & 8.84 \\
\hline $6 H-1-184$ & 39.96 & 18.84 & 56.38 & 34.88 & 3.80 & 5.82 \\
\hline
\end{tabular}


Table 1.1 (continued).

\begin{tabular}{|c|c|c|c|c|c|c|}
\hline \multirow[b]{2}{*}{$\begin{array}{l}\text { Sample } \\
\text { no. }\end{array}$} & \multirow[b]{2}{*}{$\begin{array}{l}\text { Depth } \\
\text { (mbsf) }\end{array}$} & \multicolumn{5}{|c|}{ Subfraction of coarse fraction $(\mu \mathrm{m})$} \\
\hline & & $\begin{array}{l}>63 \\
(\%)\end{array}$ & $\begin{array}{c}63-125 \\
(\%)\end{array}$ & $\begin{array}{c}125-500 \\
(\%)\end{array}$ & $\begin{array}{c}500-1000 \\
(\%)\end{array}$ & $\begin{array}{c}>1000 \\
(\%)\end{array}$ \\
\hline $6 \mathrm{H}-2-54$ & 48.96 & 7.81 & 54.01 & 29.33 & 4.44 & 12.22 \\
\hline $6 \mathrm{H}-2-133$ & 41.75 & 19.73 & 57.27 & 36.98 & 2.52 & 3.31 \\
\hline $6 \mathrm{H}-3-31$ & 42.33 & 15.74 & 64.73 & 31.88 & 1.88 & 2.39 \\
\hline $6 H-3-119$ & 43.11 & 8.66 & 66.45 & 28.86 & 8.74 & 4.75 \\
\hline $6 H-4-11$ & 43.53 & 11.78 & 73.89 & 23.08 & 2.84 & 8.99 \\
\hline $6 \mathrm{H}-4-92$ & 44.34 & 7.95 & 49.42 & 45.82 & 2.53 & 2.22 \\
\hline $6 \mathrm{H}-5-34$ & 45.26 & 6.64 & 57.89 & 37.85 & 3.65 & 8.62 \\
\hline $6 H-5-121$ & 46.13 & 12.83 & 58.34 & 33.81 & 4.65 & 12.88 \\
\hline $6 H-6-6$ & 46.47 & 2.28 & 75.82 & 28.55 & 1.93 & 1.70 \\
\hline $6 \mathrm{H}-6-57$ & 46.99 & 28.17 & 49.29 & 44.72 & 1.76 & 4.23 \\
\hline $7 \mathrm{H}-1-51$ & 47.91 & 2.84 & 77.61 & 21.47 & 0.93 & 8.80 \\
\hline $7 H-1-131$ & 48.68 & 15.43 & 52.24 & 39.58 & 3.82 & 5.17 \\
\hline $7 \mathrm{H}-2-78$ & 49.85 & 14.47 & 61.17 & 34.42 & 2.79 & 1.62 \\
\hline $7 \mathrm{H}-2-131$ & 58.11 & 5.95 & 55.68 & 35.97 & 2.15 & 6.28 \\
\hline $7 \mathrm{H}-3-28$ & 58.49 & 15.57 & 73.93 & 22.96 & 1.67 & 1.44 \\
\hline $7 \mathrm{H}-3-131$ & 51.55 & 13.99 & 54.47 & 36.16 & 1.45 & 7.93 \\
\hline $7 \mathrm{H}-4-15$ & 51.88 & 12.64 & 65.46 & 27.55 & 1.61 & 5.38 \\
\hline $7 H-4-185$ & 52.74 & 18.91 & 51.78 & 43.83 & 2.16 & 2.31 \\
\hline $7 \mathrm{H}-5-41$ & 53.56 & 5.58 & 53.41 & 44.50 & 1.88 & 0.21 \\
\hline $7 \mathrm{H}-5-128$ & 54.32 & 6.48 & 81.48 & 15.97 & $\theta . \theta 0$ & 2.55 \\
\hline $7 \mathrm{H}-6-32$ & 54.92 & 13.64 & 57.28 & 28.13 & 1.26 & 13.41 \\
\hline $7 \mathrm{H}-6-128$ & 55.76 & 14.94 & 62.69 & 26.76 & 4.78 & 5.77 \\
\hline $7 \mathrm{H}-7-12$ & 56.16 & 4.83 & 78.38 & 26.35 & 2.68 & 8.58 \\
\hline $7 \mathrm{H}-7-56$ & 56.59 & 8.93 & 42.18 & 47.85 & 5.34 & 5.51 \\
\hline $8 \mathrm{H}-1-55$ & 57.45 & 18.72 & 54.38 & 26.35 & 2.68 & 16.67 \\
\hline $8 H-1-88$ & 57.78 & 3.38 & 44.63 & 49.96 & 2.44 & 2.96 \\
\hline $8 H-2-19$ & 58.56 & 6.32 & 52.91 & 45.32 & 8.87 & 8.98 \\
\hline $8 \mathrm{H}-2-137$ & 59.71 & 5.76 & 44.73 & 41.86 & 3.49 & 9.92 \\
\hline $8 H-3-15$ & 59.98 & 9.85 & 46.88 & 45.15 & 2.86 & 5.91 \\
\hline $8 H-3-113$ & 68.94 & 19.49 & 57.82 & 33.97 & 3.12 & 5.88 \\
\hline $8 H-4-19$ & 61.48 & 1.83 & 81.48 & 15.81 & 8.80 & 2.78 \\
\hline $8 \mathrm{H}-4-112$ & 62.39 & 21.64 & 47.92 & 47.69 & 2.87 & 1.52 \\
\hline $8 \mathrm{H}-5-28$ & 62.95 & 38.85 & 23.65 & 74.87 & 0.84 & 0.63 \\
\hline $8 H-5-119$ & 63.91 & 17.81 & 27.94 & 57.35 & 5.28 & 9.52 \\
\hline $8 H-6-28$ & 64.41 & 1.58 & 69.14 & 30.58 & 8.28 & 8.80 \\
\hline $8 \mathrm{H}-6-94$ & 65.13 & 21.16 & 32.18 & 65.71 & 0.95 & 1.24 \\
\hline $8 \mathrm{H}-7-18$ & 65.85 & 7.78 & 62.83 & 37.21 & 0.76 & 8.88 \\
\hline $8 H-7-44$ & 66.18 & 11.58 & 38.88 & 43.75 & 3.28 & 14.89 \\
\hline $9 H-1-28$ & 66.69 & 12.88 & 45.56 & 44.95 & 7.29 & 2.28 \\
\hline $9 H-1-137$ & 67.74 & 0.27 & 89.34 & 9.82 & 0.82 & 8.82 \\
\hline $9 \mathrm{H}-2-28$ & 68.13 & 1.11 & 68.58 & 21.71 & 0.77 & 8.94 \\
\hline $9 H-2-137$ & 69.18 & 0.48 & 57.22 & 48.33 & 2.18 & 8.27 \\
\hline $9 H-3-28$ & 69.58 & 0.32 & 67.02 & 24.61 & 5.76 & 2.62 \\
\hline $9 H-3-96$ & 78.23 & 9.84 & 67.37 & 32.29 & 0.19 & 0.15 \\
\hline $9 \mathrm{H}-4-28$ & 71.82 & 0.13 & 88.89 & 11.11 & 8.88 & 8.80 \\
\hline $9 H-4-137$ & 72.87 & 0.19 & 62.58 & 37.58 & 8.88 & 8.88 \\
\hline $9 H-5-28$ & 72.46 & 0.18 & 58.08 & 58.08 & 8.08 & 8.88 \\
\hline $9 H-5-137$ & 73.51 & 0.75 & 65.25 & 31.18 & 3.58 & 8.88 \\
\hline $9 H-6-28$ & 73.91 & 8.57 & 48.57 & 49.71 & 1.71 & 8.80 \\
\hline $9 \mathrm{H}-6-137$ & 74.96 & 0.28 & 85.45 & 14.55 & 0.80 & 8.80 \\
\hline $9 H-7-34$ & 75.41 & 2.89 & 5.88 & 21.19 & 25.88 & 47.86 \\
\hline $9 t-7-56$ & 75.62 & 1.83 & 6.81 & 18.94 & 18.97 & 63.28 \\
\hline
\end{tabular}


Table 1.1 (continued).

\begin{tabular}{|c|c|c|c|c|c|c|}
\hline \multirow[b]{2}{*}{$\begin{array}{l}\text { Sample } \\
\text { no. }\end{array}$} & \multirow[b]{2}{*}{$\begin{array}{l}\text { Depth } \\
\text { (mbsf) }\end{array}$} & \multicolumn{5}{|c|}{ Subfraction of coarse fraction $(\mu \mathrm{m})$} \\
\hline & & $\begin{array}{l}>63 \\
(\%)\end{array}$ & $\begin{array}{c}63-125 \\
(\%)\end{array}$ & $\begin{array}{c}125-500 \\
(\%)\end{array}$ & $\begin{array}{c}500-1000 \\
(\%)\end{array}$ & $\begin{array}{c}>1000 \\
(\%)\end{array}$ \\
\hline $1 \theta \mathrm{H}-1-58$ & 76.48 & 0.29 & 41.67 & 40.91 & 8.33 & 9.89 \\
\hline $18 \mathrm{H}-2-58$ & 77.92 & 1.42 & 65.48 & 31.88 & 2.61 & 8.11 \\
\hline $18 \mathrm{H}-3-58$ & 79.37 & 8.45 & 37.32 & 47.84 & 7.18 & 7.66 \\
\hline $18 \mathrm{H}-4-58$ & 88.82 & 8.91 & 55.56 & 28.89 & 5.48 & 18.16 \\
\hline $10 \mathrm{H}-5-58$ & 82.26 & 1.34 & 81.22 & 16.27 & 2.58 & 8.80 \\
\hline $18 H-6-58$ & 83.71 & 6.89 & 75.55 & 23.98 & 8.48 & 8.88 \\
\hline $18 H-7-58$ & 85.16 & 8.63 & 69.93 & 28.71 & 1.35 & 8.88 \\
\hline $11 H-1-54$ & 85.94 & 0.28 & 68.18 & 23.93 & 1.84 & 6.13 \\
\hline $11 H-2-54$ & 87.37 & 2.59 & 84.28 & 15.29 & 0.42 & 8.88 \\
\hline $11 H-3-54$ & 88.81 & 0.63 & 59.64 & 35.67 & 2.34 & 2.34 \\
\hline $11 \mathrm{H}-4-54$ & 98.25 & 0.11 & 47.27 & 49.89 & 8.80 & 3.64 \\
\hline $11 \mathrm{H}-5-54$ & 91.68 & 0.19 & 62.79 & 37.21 & 8.80 & 8.88 \\
\hline $11 H-6-54$ & 93.12 & 8.19 & 79.83 & 16.94 & 8.88 & 4.83 \\
\hline $11 \mathrm{H}-7-54$ & 94.56 & 1.85 & 65.59 & 27.59 & 8.85 & 5.96 \\
\hline $12 \mathrm{H}-1-61$ & 95.53 & 0.18 & 56.16 & 35.62 & 4.11 & 4.11 \\
\hline $12 \mathrm{H}-2-61$ & 97.03 & 0.82 & 73.72 & 24.78 & 0.59 & 8.99 \\
\hline $12 \mathrm{H}-3-61$ & 98.53 & 8.32 & 72.41 & 28.69 & 4.31 & 2.59 \\
\hline $12 \mathrm{H}-4-61$ & 180.83 & 2.24 & 48.75 & 58.80 & 0.94 & 8.31 \\
\hline $12 \mathrm{H}-5-61$ & 181.52 & 6.48 & 28.15 & 39.48 & 2.91 & 29.55 \\
\hline $12 \mathrm{H}-6-61$ & 103.02 & 8.36 & 69.47 & 24.78 & 3.18 & 2.65 \\
\hline $12 \mathrm{H}-7-13$ & 184.52 & 1.55 & 78.86 & 17.82 & 2.22 & 1.11 \\
\hline $13 H-1-184$ & 185.26 & 3.37 & 67.38 & 29.49 & 2.48 & 0.64 \\
\hline $13 H-2-184$ & 186.76 & 1.33 & 82.18 & 15.82 & 1.59 & 1.21 \\
\hline $13 H-3-184$ & 188.26 & 8.42 & 57.76 & 37.88 & 3.11 & 1.24 \\
\hline $13 H-4-184$ & 109.76 & 0.28 & 78.55 & 27.27 & 2.18 & 8.88 \\
\hline $13 H-5-184$ & 111.26 & 0.47 & 61.75 & 35.14 & 2.29 & 0.83 \\
\hline $13 H-6-184$ & 112.76 & 8.83 & 76.87 & 21.80 & 1.10 & 1.83 \\
\hline $14 \mathrm{H}-1-95$ & 114.96 & 21.42 & 75.44 & 24.52 & 8.88 & 8.84 \\
\hline $15 \mathrm{H}-1-113$ & 124.98 & 8.98 & 33.25 & 37.61 & 25.83 & 3.31 \\
\hline $15 H-2-113$ & 126.15 & 8.54 & 83.08 & 16.85 & 8.66 & 8.22 \\
\hline $15 \mathrm{H}-3-113$ & 127.65 & 1.52 & 62.99 & 35.21 & 1.79 & 8.88 \\
\hline $16 \mathrm{H}-1-116$ & 129.28 & 2.65 & 44.34 & 54.68 & 8.93 & 8.84 \\
\hline $16 \mathrm{H}-2-116$ & 138.78 & 2.85 & 73.88 & 25.93 & 0.28 & 0.88 \\
\hline $16 \mathrm{H}-4-116$ & 132.28 & 0.74 & 85.62 & 13.48 & 0.98 & 8.80 \\
\hline $16 \mathrm{H}-5-116$ & 133.78 & 8.65 & 76.35 & 15.31 & 1.74 & 6.61 \\
\hline $16 \mathrm{H}-6-116$ & 135.28 & 8.78 & 60.21 & 24.44 & 13.11 & 2.24 \\
\hline $16 \mathrm{H}-7-116$ & 136.78 & 5.31 & 48.72 & 54.87 & 4.62 & 8.68 \\
\hline $17 \mathrm{H}-1-114$ & 139.16 & 2.48 & 71.73 & 27.56 & 0.71 & $\theta . \theta \theta$ \\
\hline $17 \mathrm{H}-2-114$ & 148.66 & 8.73 & 81.68 & 18.32 & 8.80 & 8.88 \\
\hline $17 \mathrm{H}-3-114$ & 142.16 & 1.27 & 28.73 & 11.86 & 1.33 & 66.89 \\
\hline $17 \mathrm{H}-4-114$ & 143.66 & 8.17 & 72.39 & 11.94 & 8.75 & 14.93 \\
\hline $17 H-5-114$ & 145.16 & 1.89 & 81.84 & 14.75 & 2.49 & 8.92 \\
\hline $17 H-6-116$ & 146.66 & 0.50 & 62.31 & 35.51 & 1.87 & 8.31 \\
\hline $18 \mathrm{H}-1-114$ & 148.96 & 0.27 & 23.89 & 36.18 & 4.38 & 35.55 \\
\hline $18 \mathrm{H}-2-114$ & 158.46 & 1.48 & 58.69 & 37.98 & 4.81 & 8.88 \\
\hline $18 \mathrm{H}-3-114$ & 151.96 & 2.81 & 18.85 & 17.67 & 22.24 & 41.24 \\
\hline $18 \mathrm{H}-4-114$ & 153.46 & 1.55 & 24.44 & 6.78 & 5.98 & 62.82 \\
\hline $18 \mathrm{H}-5-114$ & 154.96 & 7.02 & 48.37 & 16.81 & 3.57 & 31.26 \\
\hline $18 \mathrm{H}-6-114$ & 156.46 & 0.55 & 43.28 & 34.88 & 21.85 & 0.00 \\
\hline $19 H-1-185$ & 158.77 & 1.30 & 38.96 & 28.11 & 18.76 & 38.17 \\
\hline $19 H-2-185$ & 168.27 & 0.92 & 47.63 & 6.31 & 13.28 & 32.86 \\
\hline $19 H-3-185$ & 161.77 & 0.73 & 49.48 & 10.69 & 32.88 & 7.7 \\
\hline
\end{tabular}


Table 1.1 (continued).

\begin{tabular}{|c|c|c|c|c|c|c|}
\hline \multirow[b]{2}{*}{$\begin{array}{l}\text { Sample } \\
\text { no. }\end{array}$} & \multirow[b]{2}{*}{$\begin{array}{l}\text { Depth } \\
\text { (mbsf) }\end{array}$} & \multicolumn{5}{|c|}{ Subfraction of coarse fraction $(\mu \mathrm{m})$} \\
\hline & & $\begin{array}{l}>63 \\
(\%)\end{array}$ & $\begin{array}{c}63-125 \\
(\%)\end{array}$ & $\begin{array}{c}125-500 \\
(\%)\end{array}$ & $\begin{array}{c}500-1000 \\
(\%)\end{array}$ & $\begin{array}{c}>1000 \\
(\%)\end{array}$ \\
\hline $19 \mathrm{H}-4-105$ & 163.27 & 2.74 & 59.87 & 39.73 & 8.48 & 0.88 \\
\hline $19 H-5-105$ & 164.77 & 1.82 & 83.55 & 16.44 & 8.88 & 8.88 \\
\hline $19 H-6-105$ & 166.27 & 8.51 & 87.91 & 12.89 & 8.88 & 8.88 \\
\hline $28 \mathrm{H}-1-103$ & 168.44 & 2.37 & 69.89 & 26.38 & 2.58 & 1.31 \\
\hline $28 \mathrm{H}-2-183$ & 169.92 & 3.59 & 76.75 & 23.05 & 8.19 & 8.80 \\
\hline $28 \mathrm{H}-3-183$ & 171.41 & 1.69 & 76.85 & 23.95 & 8.88 & 8.80 \\
\hline $20 \mathrm{H}-4-103$ & 172.48 & 3.91 & 36.52 & 34.23 & 1.57 & 27.68 \\
\hline $28 \mathrm{H}-5-103$ & 174.38 & 5.53 & 72.97 & 27.82 & 8.80 & 8.88 \\
\hline $28 \mathrm{H}-6-183$ & 175.87 & 11.69 & 58.35 & 49.54 & 0.11 & 8.88 \\
\hline $21 \mathrm{H}-1-184$ & 178.16 & 1.56 & 82.43 & 17.57 & 8.88 & 0.80 \\
\hline $21 \mathrm{H}-2-184$ & 179.66 & 2.72 & 69.38 & 38.62 & 8.88 & 0.88 \\
\hline $21 \mathrm{H}-3-184$ & 181.16 & 15.87 & 51.63 & 42.87 & 0.74 & 5.57 \\
\hline $21 \mathrm{H}-4-104$ & 182.66 & 3.90 & 68.25 & 26.27 & 0.18 & 5.38 \\
\hline $21 \mathrm{H}-5-105$ & 184.16 & 2.16 & 72.58 & 27.42 & 8.88 & 8.88 \\
\hline $21 \mathrm{H}-6-184$ & 185.66 & 1.82 & 84.56 & 15.44 & 8.80 & 8.88 \\
\hline $22 \mathrm{H}-1-55$ & 187.26 & 3.58 & 62.73 & 37.27 & 8.88 & 8.88 \\
\hline $22 \mathrm{H}-2-55$ & 188.74 & 12.84 & 88.18 & 11.57 & 8.24 & 8.80 \\
\hline $22 \mathrm{H}-3-55$ & 198.22 & 2.34 & 69.83 & 38.97 & 8.80 & 0.88 \\
\hline $22 \mathrm{H}-4-55$ & 191.78 & 8.87 & 67.84 & 38.22 & 1.95 & 8.88 \\
\hline $22 \mathrm{H}-5-55$ & 193.17 & 0.58 & 57.73 & 42.27 & 8.88 & $8 . \theta 8$ \\
\hline $22 \mathrm{H}-6-55$ & 194.65 & 15.87 & 62.98 & 36.31 & 8.79 & 8.88 \\
\hline $22 \mathrm{H}-7-55$ & 196.03 & 29.22 & 34.16 & 65.64 & 8.28 & 8.88 \\
\hline $23 \mathrm{H}-1-6$ & 196.07 & 18.83 & 46.13 & 44.33 & 8.75 & 8.79 \\
\hline $23 \mathrm{H}-2-12$ & 197.62 & 2.58 & 78.61 & 21.39 & 0.80 & 8.80 \\
\hline $23 \mathrm{H}-3-12$ & 199.11 & 1.57 & 82.50 & 17.49 & 8.80 & 8.80 \\
\hline $23 \mathrm{H}-4-12$ & 280.68 & 8.64 & 87.56 & 12.44 & 8.80 & 8.80 \\
\hline $23 \mathrm{H}-5-12$ & 282.89 & 8.53 & 78.31 & 29.69 & 8.88 & 0.88 \\
\hline $23 \mathrm{H}-6-12$ & 283.58 & 1.36 & 78.25 & 29.54 & 8.28 & 0.80 \\
\hline $23 \mathrm{H}-7-12$ & 285.87 & 8.34 & 65.18 & 34.98 & 8.80 & 8.88 \\
\hline $24 \mathrm{H}-1-183$ & 286.64 & 0.54 & 77.29 & 22.71 & 8.88 & 8.80 \\
\hline $24 \mathrm{H}-2-103$ & 288.14 & 8.92 & 74.18 & 25.98 & 8.88 & 0.88 \\
\hline $24 \mathrm{H}-3-103$ & 289.64 & 0.80 & 57.44 & 42.56 & 8.80 & 8.88 \\
\hline $24 \mathrm{H}-4-103$ & 211.14 & 0.68 & 71.57 & 28.43 & 8.88 & 8.88 \\
\hline $24 \mathrm{H}-5-103$ & 212.64 & 0.15 & 78.78 & 29.22 & 8.80 & 0.08 \\
\hline $25 \mathrm{H}-1-96$ & 214.07 & 8.56 & 66.67 & 33.34 & 8.88 & 8.88 \\
\hline $25 \mathrm{H}-2-96$ & 215.56 & 8.47 & 67.82 & 32.98 & 8.80 & 0.88 \\
\hline $25 \mathrm{H}-3-96$ & 217.85 & 8.65 & 71.88 & 28.12 & 8.88 & 8.88 \\
\hline $25 \mathrm{H}-4-96$ & 218.53 & 0.39 & 58.75 & 43.61 & 5.64 & 8.88 \\
\hline $25 \mathrm{H}-5-96$ & 228.82 & $\theta .30$ & 69.18 & 38.98 & 0.88 & 8.88 \\
\hline
\end{tabular}


Table 1.2. Sedimentological data from Hole 642D. Analysis of coarsefraction component.

\begin{tabular}{|c|c|c|c|c|}
\hline $\begin{array}{c}\text { Sample } \\
\text { no. }\end{array}$ & $\begin{array}{l}\text { Depth } \\
\text { (mbsf) }\end{array}$ & $\begin{array}{l}>63 \\
\mu \mathrm{m} \\
(\%)\end{array}$ & $\begin{array}{l}\text { Bulk } \\
\mathrm{CaCO}_{3} \\
(\%)\end{array}$ & $\begin{array}{l}\text { TOC } \\
(\%)\end{array}$ \\
\hline $5 x-2-183$ & 221.41 & 0.77 & 0.80 & 1.24 \\
\hline $5 x-3-103$ & 222.89 & 0.87 & 0.80 & 1.15 \\
\hline $5 x-4-103$ & 224.38 & 0.62 & 0.08 & 1.48 \\
\hline $5 x-5-103$ & 225.85 & 0.82 & 0.80 & 1.16 \\
\hline $5 x-6-103$ & 227.32 & 8.44 & 0.80 & 1.17 \\
\hline $6 x-1-184$ & 229.56 & 0.65 & 0.00 & 1.89 \\
\hline $6 x-2-184$ & 231.06 & 0.48 & 0.80 & 1.41 \\
\hline $6 x-3-104$ & 232.56 & 2.38 & 0.00 & 1.14 \\
\hline $6 x-4-104$ & 234.86 & 0.83 & 0.00 & 1.68 \\
\hline $7 x-1-103$ & 239.23 & 0.41 & 8.80 & 1.45 \\
\hline $7 x-2-103$ & 248.78 & 2.58 & 0.00 & 1.28 \\
\hline $7 x-3-103$ & 242.17 & 1.01 & 0.00 & 1.37 \\
\hline $7 X-4-103$ & 243.65 & 14.63 & 0.00 & 1.27 \\
\hline $7 x-5-183$ & 245.12 & 0.61 & 0.80 & 1.28 \\
\hline $7 X-6-183$ & 246.59 & 0.37 & 0.80 & 1.36 \\
\hline $8 x-1-113$ & 248.95 & 6.97 & 0.80 & 1.45 \\
\hline $8 x-2-113$ & 250.45 & 4.29 & 0.08 & 1.95 \\
\hline $8 x-3-113$ & 251.95 & 8.78 & 0.80 & 1.92 \\
\hline $8 x-4-113$ & 253.45 & 2.72 & 8.80 & 0.89 \\
\hline $8 x-5-113$ & 254.95 & 0.45 & 8.80 & 1.51 \\
\hline $8 x-6-113$ & 256.45 & 8.59 & 8.88 & 1.66 \\
\hline $9 x-1-78$ & 258.28 & 2.04 & 8.80 & 1.64 \\
\hline $9 x-2-45$ & 259.42 & 0.31 & 8.80 & 0.79 \\
\hline $9 x-3-127$ & 261.68 & 8.66 & 0.80 & 1.25 \\
\hline $9 x-4-21$ & 262.12 & 0.35 & 0.80 & 1.18 \\
\hline $9 x-5-64$ & 264.81 & 0.79 & 0.08 & 1.22 \\
\hline $9 x-6-126$ & 266.88 & 0.46 & 0.00 & 0.96 \\
\hline $9 x-7-17$ & 266.48 & 0.33 & 8.88 & 1.11 \\
\hline $10 x-1-77$ & 267.88 & 0.59 & 8.88 & 1.53 \\
\hline $10 x-2-79$ & 269.39 & 0.51 & 0.80 & 0.94 \\
\hline $10 x-3-84$ & 278.93 & 0.52 & 0.80 & 0.59 \\
\hline $18 x-4-84$ & 272.42 & 18.51 & 0.00 & 0.72 \\
\hline $10 x-5-85$ & 273.92 & 3.63 & 0.80 & 1.73 \\
\hline $10 x-6-85$ & 275.41 & 3.18 & 0.80 & 1.03 \\
\hline $10 x-7-17$ & 276.22 & 1.45 & 0.80 & 0.82 \\
\hline $11 x-1-47$ & 277.29 & 0.32 & 8.80 & 1.85 \\
\hline $11 x-1-121$ & 278.83 & 53.26 & 0.80 & 0.85 \\
\hline $11 x-2-114$ & 279.46 & 27.81 & 0.80 & 0.01 \\
\hline $12 x-1-49$ & 287.80 & 28.16 & 8.80 & 0.08 \\
\hline
\end{tabular}


Table 1.2 (continued).

\begin{tabular}{|c|c|c|c|c|c|c|c|c|c|c|c|c|c|}
\hline \multirow[b]{2}{*}{$\begin{array}{l}\text { Sample } \\
\text { no. }\end{array}$} & \multirow[b]{2}{*}{$\begin{array}{l}\text { Depth } \\
\text { (mbsf) }\end{array}$} & \multirow[b]{2}{*}{$\begin{array}{l}\text { Benthic } \\
\text { foram. } \\
(\%)\end{array}$} & \multirow[b]{2}{*}{$\begin{array}{l}\text { Plankt. } \\
\text { foram. } \\
(\%)\end{array}$} & \multirow[b]{2}{*}{$\begin{array}{c}\text { Rads. } \\
(\%)\end{array}$} & \multirow[b]{2}{*}{$\begin{array}{c}\text { Diatoms } \\
(\%)\end{array}$} & \multicolumn{3}{|c|}{ Grain amount percentages } & \multirow[b]{2}{*}{$\begin{array}{c}\text { Mica } \\
(\%)\end{array}$} & \multirow[b]{2}{*}{$\begin{array}{c}\text { Rock fr. } \\
(\%)\end{array}$} & \multirow[b]{2}{*}{$\begin{array}{c}\text { Hev. min. } \\
(\%)\end{array}$} & \multirow[b]{2}{*}{$\begin{array}{l}\text { Volc. gl. } \\
(\%)\end{array}$} & \multirow[b]{2}{*}{$\begin{array}{l}\text { Auth. } \\
(\%)\end{array}$} \\
\hline & & & & & & $\begin{array}{c}\text { Sponge } \\
\text { Spicule } \\
(\%)\end{array}$ & $\begin{array}{c}\text { Quartz } \\
(\%)\end{array}$ & $\begin{array}{c}\text { Feldspar } \\
(\%)\end{array}$ & & & & & \\
\hline $5 x-2-103$ & 221.41 & 8.88 & 0.80 & 92.78 & 3.48 & 0.98 & 1.38 & 8.88 & 8.28 & 8.28 & 8.88 & 0.40 & 0.28 \\
\hline $5 x-3-103$ & 222.89 & 0.80 & 8.80 & 94.48 & 1.98 & 0.08 & 0.48 & 0.00 & 0.80 & 8.80 & 0.80 & 3.10 & 0.20 \\
\hline $5 x-4-103$ & 224.38 & 8.28 & 8.80 & 91.60 & 1.88 & 8.48 & 3.88 & 8.80 & 0.80 & 0.28 & 0.80 & 2.88 & 0.08 \\
\hline $5 x-5-103$ & 225.85 & 0.88 & 0.00 & 98.38 & 0.68 & 0.28 & 0.08 & 0.08 & 0.88 & 0.00 & 0.80 & 0.68 & 0.28 \\
\hline $5 x-6-103$ & 227.32 & 8.40 & 0.00 & 80.60 & 14.30 & 2.10 & 8.48 & 0.80 & 0.00 & 8.58 & 0.00 & 1.10 & 0.78 \\
\hline $6 x-1-184$ & 229.56 & 0.88 & 8.80 & 65.68 & 24.68 & 1.68 & 1.38 & 8.80 & 0.28 & 8.80 & 8.08 & 6.68 & 8.20 \\
\hline $6 x-2-104$ & 231.86 & 1.30 & 0.00 & 77.88 & 8.10 & 0.98 & 2.28 & 8.80 & 0.80 & 8.48 & 0.28 & 1.88 & 8.10 \\
\hline $6 x-3-104$ & 232.56 & 8.80 & 0.88 & 33.88 & 28.18 & 4.60 & 3.28 & 0.08 & 0.80 & 8.88 & 0.08 & 38.38 & 0.80 \\
\hline $6 x-4-184$ & 234.86 & 2.80 & 0.00 & 78.98 & 13.10 & 6.50 & 2.28 & 0.80 & 0.80 & 1.78 & 0.28 & 2.60 & 1.80 \\
\hline $7 x-1-103$ & 239.23 & 8.48 & 8.00 & 69.78 & 3.80 & 16.28 & 6.48 & 8.80 & 8.80 & 8.48 & 8.80 & 2.48 & 1.50 \\
\hline $7 x-2-103$ & 248.78 & 8.28 & 8.80 & 28.78 & 35.28 & 18.28 & 3.78 & 0.60 & 0.08 & 8.48 & 0.28 & 21.58 & 0.80 \\
\hline $7 x-3-183$ & 242.17 & 8.28 & 0.80 & 54.68 & 3.50 & 25.80 & 2.10 & 8.80 & 0.08 & 1.80 & 0.00 & 11.30 & 0.78 \\
\hline $7 x-4-183$ & 243.65 & 8.88 & 0.00 & 3.60 & 1.68 & 1.88 & 5.50 & 8.28 & 8.28 & 8.88 & 8.08 & 76.38 & 10.78 \\
\hline $7 x-5-103$ & 245.12 & 8.08 & 8.80 & 39.10 & $4 \theta .2 \theta$ & 12.88 & 0.28 & 8.80 & 8.80 & 0.08 & 0.80 & 7.78 & 0.80 \\
\hline $7 x-6-103$ & 246.59 & 0.50 & 8.08 & 69.48 & 0.90 & 7.98 & 1.38 & 0.08 & 0.28 & 4.10 & 8.48 & 4.10 & 11.38 \\
\hline $8 x-1-113$ & 248.95 & 0.00 & 8.80 & 56.88 & 4.30 & 3.80 & 0.30 & 0.80 & 8.28 & 2.90 & 0.08 & 3.78 & 28.98 \\
\hline $8 x-2-113$ & 258.45 & 0.00 & 8.80 & 68.28 & 0.38 & 5.40 & 0.58 & 8.00 & 0.50 & 4.98 & 0.80 & 13.58 & 6.60 \\
\hline $8 x-3-113$ & 251.95 & 8.80 & 8.08 & 51.28 & 0.80 & 14.18 & 0.28 & 8.00 & 8.00 & 6.10 & 0.08 & 28.38 & 0.80 \\
\hline $8 x-4-113$ & 253.45 & 8.08 & 8.00 & 35.68 & 0.08 & 11.78 & 0.90 & 0.08 & 0.00 & 1.80 & 0.20 & 50.58 & 0.28 \\
\hline $8 x-5-113$ & 254.95 & 8.68 & 8.88 & 73.28 & 4.80 & 16.80 & 0.60 & 0.08 & 0.00 & 1.28 & 0.80 & 4.48 & 0.80 \\
\hline $8 x-6-113$ & 256.45 & 8.88 & 8.80 & 69.48 & 8.80 & 11.48 & 8.80 & 8.80 & $\theta .00$ & 2.80 & 0.08 & 9.80 & 7.48 \\
\hline $9 x-1-78$ & 258.28 & 8.80 & 8.80 & 76.78 & 8.48 & 8.60 & 0.28 & 8.80 & 8.88 & 0.28 & 0.08 & 13.58 & 0.40 \\
\hline $9 x-2-45$ & 259.42 & 8.88 & 0.80 & 47.38 & 0.00 & 2.28 & 2.80 & 8.80 & 8.80 & 3.98 & 8.80 & 44.18 & 0.48 \\
\hline $9 x-3-127$ & 261.68 & 2.80 & 8.00 & 67.48 & 2.38 & 6.30 & 7.38 & 6.80 & 0.80 & 0.58 & 8.00 & 6.80 & 2.80 \\
\hline $9 x-4-21$ & 262.12 & 0.80 & 8.80 & 52.50 & 2.80 & 4.60 & 2.60 & 18.60 & 8.08 & 0.78 & 0.80 & 26.28 & 0.80 \\
\hline $9 x-5-64$ & 264.81 & 0.28 & 8.80 & 67.88 & 0.28 & 4.80 & 9.10 & 0.40 & 8.80 & 0.28 & 0.70 & 15.38 & 1.30 \\
\hline $9 x-6-126$ & 266.88 & 8.68 & 8.28 & 43.68 & 0.60 & 2.50 & 31.10 & 0.08 & 8.88 & 1.50 & 1.98 & 15.60 & 2.38 \\
\hline $9 x-7-17$ & 266.48 & 0.28 & 8.88 & 44.78 & 8.80 & 3.28 & 32.68 & 8.88 & 0.88 & 1.80 & 0.48 & 9.80 & 9.80 \\
\hline $10 x-1-77$ & 267.88 & 0.08 & 0.80 & 85.68 & 0.80 & 4.98 & 1.58 & 8.80 & 8.80 & 1.18 & 8.08 & 0.90 & 5.98 \\
\hline $18 x-2-79$ & 269.39 & 8.48 & 8.80 & 39.88 & 8.48 & 0.48 & 54.80 & 0.48 & 0.00 & 8.80 & B. 28 & 1.80 & 2.78 \\
\hline $10 x-3-84$ & 270.93 & 0.80 & 8.88 & 25.88 & 0.80 & 3.80 & 63.68 & 8.80 & 0.98 & 2.48 & 0.08 & 1.38 & 3.08 \\
\hline $10 x-4-84$ & 272.42 & 8.28 & 0.80 & 14.78 & 2.18 & 3.88 & 0.80 & 0.80 & 0.60 & 0.80 & 0.00 & 29.90 & 48.88 \\
\hline $10 x-5-85$ & 273.92 & 8.88 & 8.80 & 50.80 & 0.50 & 14.20 & 0.50 & 8.00 & 0.00 & 0.20 & 0.80 & 8.78 & 33.98 \\
\hline $10 x-6-85$ & 275.41 & 0.08 & 8.80 & 34.98 & 0.40 & 31.48 & 1.38 & 8.00 & 0.00 & 0.28 & 0.08 & 18.90 & 12.98 \\
\hline $10 x-7-17$ & 276.22 & 0.38 & 0.80 & 36.10 & 0.28 & 18.48 & 18.98 & 8.00 & 0.80 & 2.58 & 8.28 & 14.58 & 8.98 \\
\hline $11 x-1-47$ & 277.29 & 8.08 & 8.08 & 24.98 & 0.80 & 3.68 & 65.80 & 8.80 & 8.80 & 8.48 & 0.80 & 2.98 & 3.38 \\
\hline $11 x-1-121$ & 278.03 & 8.00 & 0.80 & 0.68 & 8.00 & 0.48 & 8.08 & 0.08 & 0.08 & 5.58 & 0.80 & 5.98 & 79.78 \\
\hline $11 x-2-114$ & 279.46 & 0.80 & 8.08 & 34.58 & 8.08 & 0.80 & 17.58 & 11.80 & 0.80 & 1.30 & 0.00 & 0.48 & 35.38 \\
\hline $12 x-1-49$ & 287.88 & 8.80 & 8.80 & 15.38 & 8.00 & 0.80 & 47.18 & 6.60 & 8.80 & 11.18 & 0.00 & 8.18 & 11.98 \\
\hline
\end{tabular}


Table 1.2 (continued).

\begin{tabular}{|c|c|c|c|c|c|c|c|c|c|c|c|c|}
\hline \multirow[b]{2}{*}{ Sample no. } & \multirow[b]{2}{*}{$\begin{array}{l}\text { Depth } \\
\text { mbsf }\end{array}$} & \multirow[b]{2}{*}{$\begin{array}{c}>63 \mu \mathrm{m} \\
(\%)\end{array}$} & \multicolumn{10}{|c|}{ Coarse fraction component analysis (wt \%) } \\
\hline & & & $\begin{array}{c}\text { Benthic } \\
\text { foram. } \\
(\%)\end{array}$ & $\begin{array}{c}\text { Plankt. } \\
\text { foram. } \\
(\%)\end{array}$ & $\begin{array}{l}\text { Biogenic } \\
\text { opal cf } \\
(\%)\end{array}$ & $\begin{array}{c}\text { Quartz } \\
(\%)\end{array}$ & $\begin{array}{c}\text { Feldspar } \\
(\%)\end{array}$ & $\begin{array}{l}\text { Mica } \\
(\%)\end{array}$ & $\begin{array}{c}\text { Rock fr. } \\
(\%)\end{array}$ & $\begin{array}{l}\text { Hev. min. } \\
\quad(\%)\end{array}$ & $\begin{array}{c}\text { Volc. gl. } \\
(\%)\end{array}$ & $\begin{array}{l}\text { Auth. } \\
(\%)\end{array}$ \\
\hline $5 x-2-103$ & 221.41 & 0.77 & 0.01 & 8.00 & 0.75 & 8.81 & 8.00 & 0.00 & 8.80 & 8.00 & 8.80 & 8.88 \\
\hline $5 x-3-103$ & 222.89 & 8.87 & 0.08 & 0.80 & 8.84 & 8.88 & 8.88 & 8.80 & 8.08 & 8.00 & 0.03 & 8.80 \\
\hline $5 x-4-183$ & 224.38 & 8.62 & 0.80 & 0.08 & 0.58 & 0.82 & 0.80 & 8.80 & 0.80 & 0.80 & 0.02 & 0.80 \\
\hline $5 x-5-103$ & 225.85 & 8.82 & 8.80 & 8.80 & 0.81 & 8.88 & 8.80 & 0.80 & 0.08 & 0.00 & 8.00 & 0.08 \\
\hline $5 x-6-103$ & 227.32 & 8.44 & 8.80 & 0.00 & 0.43 & 8.08 & 8.80 & 0.80 & 8.80 & 0.00 & 0.00 & 8.08 \\
\hline $6 x-1-104$ & 229.56 & 8.65 & 0.80 & 0.00 & 0.68 & 0.01 & 0.80 & 8.00 & 8.80 & 0.80 & 0.84 & 0.08 \\
\hline $6 x-2-104$ & 231.06 & 0.48 & 0.01 & 0.80 & 0.41 & 0.01 & 0.00 & 0.80 & 8.08 & 0.80 & 0.81 & 0.84 \\
\hline $6 x-3-104$ & 232.56 & 2.38 & 0.80 & 8.80 & 1.39 & 8.08 & 8.88 & 0.88 & 8.08 & 8.88 & 8.91 & 8.00 \\
\hline $6 x-4-104$ & 234.86 & 0.83 & 0.82 & 0.08 & 8.75 & 8.82 & 0.00 & 8.80 & 8.01 & 0.80 & 8.82 & 0.01 \\
\hline $7 x-1-103$ & 239.23 & 8.41 & 8.08 & 0.80 & 0. 36 & 8.83 & 0.80 & 0.88 & 8.80 & 0.88 & 0.01 & 8.01 \\
\hline $7 x-2-183$ & 248.78 & 2.58 & 0.80 & 0.80 & 1.85 & 8.89 & 0.08 & 8.88 & 0.01 & 0.08 & 0.54 & 0.80 \\
\hline $7 x-3-183$ & 242.17 & 1.01 & 8.80 & 0.80 & 8.85 & 0.82 & 0.80 & 0.88 & 0.82 & 0.80 & 0.11 & 0.01 \\
\hline $7 x-4-103$ & 243.65 & 14.63 & 8.80 & 8.80 & 1.82 & 0.80 & 0.03 & 0.03 & 0.08 & 8.80 & 11.16 & 1.57 \\
\hline $7 x-5-103$ & 245.12 & 0.61 & 0.80 & 0.80 & 8.56 & 0.00 & 8.80 & 0.08 & 8.80 & 0.00 & 0.85 & 8.00 \\
\hline $7 x-6-103$ & 246.59 & 8.37 & 8.88 & 0.08 & 0.29 & 8.08 & 8.80 & 8.88 & 8.82 & 8.88 & 0.82 & 8.04 \\
\hline $8 x-1-113$ & 248.95 & 6.97 & 0.88 & 0.00 & 4.47 & 0.02 & 8.08 & 0.01 & 8.28 & 0.08 & 0.26 & 2.01 \\
\hline $8 x-2-113$ & 250.45 & 4.29 & 8.80 & 0.80 & 3.17 & 0.02 & 0.80 & 0.02 & 8.21 & 8.08 & 8.58 & 0.28 \\
\hline $8 x-3-113$ & 251.95 & 0.78 & 0.80 & 0.80 & 0.46 & 8.80 & 8.80 & 0.08 & 0.84 & 0.08 & 8.28 & 0.00 \\
\hline $8 x-4-113$ & 253.45 & 2.72 & 8.80 & 0.88 & 1.29 & 0.82 & 8.88 & 8.08 & 0.03 & 0.01 & 1.37 & 0.01 \\
\hline $8 x-5-113$ & 254.95 & 0.45 & 8.80 & 0.08 & 0.42 & 8.80 & 8.80 & 0.00 & 0.81 & 8.80 & 0.82 & 0.08 \\
\hline $8 x-6-113$ & 256.45 & 0.59 & 0.88 & 0.88 & 8.48 & 8.88 & 8.88 & 0.80 & 8.01 & 0.88 & 8.86 & 0.84 \\
\hline $9 x-1-78$ & 258.28 & 2.84 & 0.80 & 0.80 & 1.75 & 8.80 & 0.80 & 0.80 & 0.00 & 0.80 & 0.28 & 0.01 \\
\hline $9 x-2-45$ & 259.42 & 0.31 & 0.80 & 0.08 & 8.15 & 0.81 & 0.08 & 0.88 & 0.01 & 8.80 & 0.14 & 0.08 \\
\hline $9 x-3-127$ & 261.68 & 0.66 & 0.01 & 0.00 & 8.50 & 0.85 & 0.84 & 0.80 & 0.00 & 0.80 & 8.84 & 0.81 \\
\hline $9 x-4-21$ & 262.12 & 0.35 & 0.88 & 0.08 & 0.21 & 0.01 & 8.84 & 0.00 & 0.08 & 8.00 & 0.09 & 0.08 \\
\hline $9 x-5-64$ & 264.81 & 8.79 & 8.80 & 8.80 & 8.58 & 8.87 & 8.88 & 8.80 & 8.88 & 0.81 & 0.12 & 8.01 \\
\hline $9 x-6-126$ & 266.88 & 0.46 & 0.80 & 0.08 & 0.21 & 0.14 & 8.08 & 0.00 & 0.01 & 0.01 & 0.87 & 0.01 \\
\hline $9 x-7-17$ & 266.48 & 0.33 & 0.08 & 0.08 & 0.16 & 0.11 & 0.00 & 0.00 & 0.80 & 0.00 & 0.03 & 0.03 \\
\hline $10 x-1-77$ & 267.88 & 8.59 & 0.80 & 0.00 & 0.53 & 0.01 & 0.08 & 0.08 & 0.01 & 0.00 & 8.01 & 0.03 \\
\hline $10 x-2-79$ & 269.39 & 8.51 & 8.80 & 0.08 & 0.21 & 0.28 & 0.00 & 0.00 & 0.00 & 0.08 & 0.81 & 0.01 \\
\hline $18 x-3-84$ & 278.93 & 8.52 & 8.80 & 8.88 & 0.15 & 0.33 & 0.80 & 0.00 & 0.01 & 0.80 & 8.81 & 0.02 \\
\hline $10 x-4-84$ & 272.42 & 18.51 & 0.04 & 0.08 & 3.66 & 8.15 & 0.00 & 0.11 & 0.15 & 8.88 & 5.53 & 8.88 \\
\hline $10 x-5-85$ & 273.92 & 3.63 & 0.08 & 8.80 & 2.35 & 0.02 & 0.08 & 0.80 & 0.01 & 8.80 & 0.83 & 1.23 \\
\hline $10 x-6-85$ & 275.41 & 3.18 & 0.80 & 0.00 & 2.12 & 0.84 & 0.00 & 0.00 & 0.01 & 0.80 & 0.60 & 0.41 \\
\hline $10 x-7-17$ & 276.22 & 1.45 & 0.80 & 8.80 & 0.79 & 0.27 & 0.08 & 0.08 & 8.84 & 8.80 & 0.21 & 8.13 \\
\hline $11 x-1-47$ & 277.29 & 0.32 & 0.80 & 0.08 & 8.09 & 0.21 & 0.80 & 0.00 & 0.00 & 8.08 & 0.01 & 0.01 \\
\hline $11 x-1-121$ & 278.83 & 53.26 & 8.08 & 0.08 & 0.53 & 4.26 & 0.80 & 0.08 & 2.93 & 0.08 & 3.14 & 42.45 \\
\hline $11 x-2-114$ & 279.46 & 27.81 & 0.80 & 8.80 & 9.59 & 4.87 & 3.86 & 0.80 & 0.36 & 0.00 & 0.11 & 9.82 \\
\hline $12 x-1-49$ & 287.08 & 28.16 & 8.80 & 0.80 & 3.88 & 9.50 & 1.33 & 0.80 & 2.24 & 0.00 & 1.63 & 2.48 \\
\hline
\end{tabular}


Table 1.2 (continued).

\begin{tabular}{|c|c|c|c|c|c|c|}
\hline \multirow[b]{2}{*}{$\begin{array}{c}\text { Sample } \\
\text { no. }\end{array}$} & \multirow[b]{2}{*}{$\begin{array}{l}\text { Depth } \\
\text { (mbsf) }\end{array}$} & \multicolumn{5}{|c|}{ Subfraction of coarse fraction $(\mu \mathrm{m})$} \\
\hline & & $\begin{array}{l}>63 \\
(\%)\end{array}$ & $\begin{array}{c}63-125 \\
(\%)\end{array}$ & $\begin{array}{c}125-500 \\
(\%)\end{array}$ & $\begin{array}{c}500-1000 \\
(\%)\end{array}$ & $\begin{array}{c}>1000 \\
(\%)\end{array}$ \\
\hline $5 x-2-103$ & 221.41 & 0.77 & 76.86 & 23.94 & 0.00 & 8.00 \\
\hline $5 x-3-183$ & 222.89 & 8.87 & 25.50 & 74.58 & 8.80 & 8.80 \\
\hline $5 x-4-183$ & 224.38 & 8.62 & 69.52 & 38.48 & 0.00 & 8.00 \\
\hline $5 x-5-103$ & 225.85 & 8.82 & 45.45 & 54.55 & 8.08 & 8.08 \\
\hline $5 x-6-103$ & 227.32 & 0.44 & 57.99 & 42.81 & 8.80 & 8.80 \\
\hline $6 x-1-104$ & 229.56 & 0.65 & 67.43 & 32.57 & 8.80 & 8.80 \\
\hline $6 x-2-104$ & 231.86 & 0. 48 & 57.87 & 42.13 & 0.08 & 0.00 \\
\hline $6 x-3-104$ & 232.56 & 2.38 & 88.15 & 19.85 & 8.00 & 8.80 \\
\hline $6 x-4-104$ & 234.86 & 8.83 & 69.31 & 30.69 & 8.08 & 8.00 \\
\hline $7 x-1-103$ & 239.23 & 0.41 & 81.21 & 18.79 & 0.08 & 8.00 \\
\hline $7 x-2-103$ & 248.78 & 2.50 & 84.38 & 15.78 & 8.00 & 0.00 \\
\hline $7 x-3-103$ & 242.17 & 1.01 & 79.33 & 28.67 & 8.08 & 8.80 \\
\hline $7 x-4-103$ & 243.65 & 14.63 & 14.44 & 85.56 & 0.00 & 0.00 \\
\hline $7 x-5-103$ & 245.12 & 0.61 & 79.55 & 20.45 & 8.08 & 0.00 \\
\hline $7 x-6-103$ & 246.59 & 0.37 & 68.84 & 31.16 & 0.00 & 0.00 \\
\hline $8 x-1-113$ & 248.95 & 6.97 & 68.56 & 31.44 & 8.80 & 8.80 \\
\hline $8 x-2-113$ & 258.45 & 4.29 & 63.48 & 36.60 & 0.80 & 0.80 \\
\hline $8 x-3-113$ & 251.95 & 0.78 & 83.88 & 16.12 & 0.08 & 0.08 \\
\hline $8 x-4-113$ & 253.45 & 2.72 & 81.62 & 18.38 & 0.80 & 8.88 \\
\hline $8 x-5-113$ & 254.95 & 8.45 & 68.89 & 31.91 & 0.80 & 0.08 \\
\hline $8 x-6-113$ & 256.45 & 8.59 & 78.42 & 29.58 & 8.08 & 8.80 \\
\hline $9 x-1-78$ & 258.28 & 2.84 & 78.99 & 21.81 & 0.08 & 0.00 \\
\hline $9 x-2-45$ & 259.42 & 8.31 & 49.87 & 38.89 & 12.84 & 8.80 \\
\hline $9 x-3-127$ & 261.68 & 8.66 & 82.59 & 17.41 & 0.08 & 8.08 \\
\hline $9 x-4-21$ & 262.12 & 8. 35 & 59.55 & 48.45 & 0.68 & 8.80 \\
\hline $9 x-5-64$ & 264.81 & 8.79 & 75.38 & 21.57 & 3.13 & 0.00 \\
\hline $9 x-6-126$ & 266.88 & 8.46 & 56.36 & 31.36 & 12.29 & 8.80 \\
\hline $9 x-7-17$ & 266.48 & 0.33 & 35.89 & 64.91 & 0.80 & 0.00 \\
\hline $18 x-1-77$ & 267.88 & 8.59 & 21.32 & 10.45 & 6.61 & 61.62 \\
\hline $10 x-2-79$ & 269.39 & 0.51 & 48.14 & 51.86 & 8.00 & 0.88 \\
\hline $10 x-3-84$ & 270.93 & 8.52 & 31.98 & 24.48 & 8.80 & 43.78 \\
\hline $10 x-4-84$ & 272.42 & 18.51 & 65.15 & 34.85 & 8.08 & 8.08 \\
\hline $18 x-5-85$ & 273.92 & 3.63 & 78.88 & 21.28 & 8.08 & 8.08 \\
\hline $18 x-6-85$ & 275.41 & 3.18 & 86.37 & 13.46 & 0.17 & 0.00 \\
\hline $10 x-7-17$ & 276.22 & 1.45 & 55.66 & 38.17 & 6.17 & 0.00 \\
\hline $11 x-1-47$ & 277.29 & 0.32 & 43.68 & 48.80 & 7.60 & 0.80 \\
\hline $11 x-1-121$ & 278.03 & 53.26 & 27.62 & 35.52 & 17.86 & 19.80 \\
\hline $11 x-2-114$ & 279.46 & 27.81 & 64.44 & 35.45 & 8.11 & 0.08 \\
\hline $12 x-1-49$ & 287.80 & 28.16 & 43.98 & 53.59 & 2.22 & 0.21 \\
\hline
\end{tabular}


Table 1.3. Sedimentological data from Hole 643A. Analysis of coarsefraction component.

\begin{tabular}{|c|c|c|c|c|}
\hline $\begin{array}{c}\text { Sample } \\
\text { no. }\end{array}$ & $\begin{array}{l}\text { Depth } \\
\text { (mbsf) }\end{array}$ & $\begin{array}{l}>63 \\
\mu \mathrm{m} \\
(\%)\end{array}$ & $\begin{array}{l}\text { Bulk } \\
\mathrm{CaCO}_{3} \\
(\%)\end{array}$ & $\begin{array}{l}\text { TOC } \\
(\%)\end{array}$ \\
\hline $1 \mathrm{H}-1-28$ & 8.28 & 18.81 & 18.28 & 8.28 \\
\hline $1 H-1-68$ & 0.68 & 4.95 & 8.45 & 0.19 \\
\hline $1 H-1-181$ & 1.81 & 5.64 & 6.79 & 8.28 \\
\hline $1 H-1-141$ & 1.41 & 24.87 & 11.81 & 8.42 \\
\hline $1 \mathrm{H}-2-41$ & 1.98 & 19.33 & 0.80 & 1.11 \\
\hline $1 \mathrm{H}-2-81$ & 2.31 & 38.63 & 37.93 & 0.19 \\
\hline $1 \mathrm{H}-2-121$ & 2.71 & & 28.51 & 8.27 \\
\hline $1 \mathrm{H}-3-48$ & 2.71 & 13.88 & 18.37 & 8.19 \\
\hline $1 \mathrm{H}-3-82$ & 3.62 & 21.61 & 0.08 & 1.86 \\
\hline $1 \mathrm{H}-3-121$ & 4.81 & 13.29 & 8.55 & 8.76 \\
\hline $1 H-3-141$ & 4.41 & 18.33 & 10.15 & 8.29 \\
\hline $1 \mathrm{H}-4-21$ & 4.71 & 29.88 & 0.15 & 8.57 \\
\hline $1 H-4-41$ & 4.91 & 16.78 & 9.25 & 0.56 \\
\hline $2 \mathrm{H}-1-21$ & 5.31 & 17.31 & 4.97 & 8.53 \\
\hline $2 H-1-48$ & 5.78 & 24.82 & 15.97 & 8.25 \\
\hline $2 \mathrm{H}-1-82$ & 6.12 & 18.23 & 0.08 & 8. 95 \\
\hline $2 \mathrm{H}-1-142$ & 6.72 & 21.53 & 22.18 & 8.23 \\
\hline $2 H-2-48$ & 7.28 & 11.13 & 7.29 & 0.28 \\
\hline $2 \mathrm{H}-2-82$ & 7.62 & 17.78 & 13.83 & 8.18 \\
\hline $2 \mathrm{H}-2-128$ & 8.80 & 9.17 & 5.28 & 0.28 \\
\hline $2 \mathrm{H}-2-142$ & 8.22 & 3.18 & 0.08 & 0.25 \\
\hline $2 \mathrm{H}-3-4 \mathrm{~B}$ & 8.78 & 2.93 & 9.46 & 0.28 \\
\hline $2 \mathrm{H}-3-82$ & 9.12 & 6.83 & 5.41 & 0.25 \\
\hline $2 \mathrm{H}-3-128$ & 9.58 & 21.16 & 15.98 & 0.21 \\
\hline $2 \mathrm{H}-4-21$ & 18.81 & 18.48 & 10.98 & 8.18 \\
\hline $2 \mathrm{H}-4-6 \theta$ & 18.48 & 0.28 & 1.74 & 0.24 \\
\hline $2 \mathrm{H}-4-186$ & 18.86 & 8.02 & 2.86 & 8.39 \\
\hline $2 \mathrm{H}-4-12 \theta$ & 11.00 & 6.98 & 3.25 & 8.38 \\
\hline $2 \mathrm{H}-5-21$ & 11.51 & 14.48 & 0.84 & 0.37 \\
\hline $2 \mathrm{H}-5-68$ & 11.98 & 28.95 & 4.68 & 8.32 \\
\hline $2 \mathrm{H}-5-128$ & $12.5 \theta$ & 11.58 & 3.93 & 8.35 \\
\hline $2 \mathrm{H}-6-21$ & 13.81 & 18.87 & 44.34 & 0.19 \\
\hline $2 \mathrm{H}-6-82$ & 13.62 & 2.55 & 8.18 & 0.28 \\
\hline $2 \mathrm{H}-6-111$ & 13.91 & 9.24 & 9.26 & 0.32 \\
\hline $2 \mathrm{H}-6-142$ & 14.22 & 28.32 & 2.12 & 0.32 \\
\hline $2 \mathrm{H}-7-21$ & 14.51 & 23.48 & 8.80 & 0.31 \\
\hline $2 \mathrm{H}-7-4 \theta$ & 14.78 & 6.42 & 5.81 & 0.25 \\
\hline $3 H-1-25$ & 15.85 & 18.89 & 28.28 & 0.23 \\
\hline $3 H-1-42$ & 15.21 & 9.10 & 18.84 & 8.42 \\
\hline $3 H-2-28$ & 16.58 & 8.78 & 10.48 & 0.29 \\
\hline $3 \mathrm{H}-2-82$ & 17.12 & 15.11 & 18.92 & 0.27 \\
\hline $3 \mathrm{H}-2-142$ & 17.72 & 28.18 & 8.88 & 8.59 \\
\hline $3 \mathrm{H}-3-28$ & 18.80 & 6.84 & 5.48 & 0.63 \\
\hline $3 \mathrm{H}-3-25$ & 18.85 & 19.68 & 8.57 & 0.23 \\
\hline $3 \mathrm{H}-3-48$ & 18.28 & 4.48 & 7.86 & 8.96 \\
\hline $3 \mathrm{H}-3-82$ & 18.62 & 0.69 & 11.22 & 0.94 \\
\hline $3 H-3-116$ & 18.96 & 2.88 & 8.28 & 8.49 \\
\hline $3 H-4-28$ & 19.58 & 16.68 & 6.16 & 8.37 \\
\hline $3 H-4-58$ & 19.88 & 2.30 & 12.28 & 0.46 \\
\hline $3 \mathrm{H}-4-122$ & 28.52 & 14.78 & 28.83 & 8.23 \\
\hline $3 H-5-28$ & 21.88 & 2.41 & 5.96 & 8.18 \\
\hline
\end{tabular}

Table 1.3 (continued).

\begin{tabular}{|c|c|c|c|c|}
\hline $\begin{array}{c}\text { Sample } \\
\text { no. }\end{array}$ & $\begin{array}{l}\text { Depth } \\
\text { (mbsf) }\end{array}$ & $\begin{array}{l}>63 \\
\underset{\mathrm{m}}{(\%)}\end{array}$ & $\begin{array}{l}\text { Bulk } \\
\mathrm{CaCO}_{3} \\
(\%)\end{array}$ & $\begin{array}{l}\text { TOC } \\
(\%)\end{array}$ \\
\hline $3 H-5-58$ & 21.38 & 6.44 & 5.46 & 8.23 \\
\hline $3 H-5-18 \theta$ & 21.88 & 4.11 & 2.12 & 0.23 \\
\hline $3 H-5-148$ & 22.28 & 6.52 & 5.61 & 0.32 \\
\hline $3 H-6-28$ & 22.58 & 5.78 & 4.11 & 0.26 \\
\hline $3 H-6-48$ & 22.78 & 7.78 & 7.38 & 0.23 \\
\hline $3 H-6-188$ & 23.38 & 2.98 & 5.51 & 0.33 \\
\hline $3 H-7-48$ & 24.28 & 14.38 & 6.15 & 0.34 \\
\hline $4 H-1-28$ & $24.5 \theta$ & 2.10 & 11.14 & 0.22 \\
\hline $4 H-1-80$ & 25.18 & 4.88 & 9.84 & 0.23 \\
\hline $4 H-1-128$ & 25.58 & 6.58 & 5.99 & 0.28 \\
\hline $4 H-2-28$ & 26.88 & 5.48 & 6.87 & 0.27 \\
\hline $4 H-2-88$ & 26.60 & 2.58 & 6.15 & 0.41 \\
\hline $4 H-2-18 \theta$ & 26.88 & 8.92 & 5.56 & 0.27 \\
\hline $4 H-2-14 \theta$ & 27.38 & 1.67 & 5.79 & 0.27 \\
\hline $4 H-3-28$ & 27.48 & 11.58 & 0.88 & 1.88 \\
\hline $4 H-3-48$ & 27.68 & 2.95 & 21.82 & 0.23 \\
\hline $4 H-3-6 \theta$ & 27.88 & 3.80 & 18.63 & 0.23 \\
\hline $4 H-3-80$ & 28.80 & 15.83 & 8.88 & 0.48 \\
\hline $4 \mathrm{H}-3-188$ & 28.28 & 1.72 & 3.15 & 0.29 \\
\hline $4 H-3-12 \theta$ & 28.48 & 28.84 & 0.80 & 1.01 \\
\hline $4 H-4-48$ & 29.28 & 18.28 & 0.80 & 8.24 \\
\hline $4 H-4-88$ & 29.68 & 18.38 & 6.95 & 0.21 \\
\hline $4 H-4-128$ & 38.80 & 6.78 & 0.08 & 0.23 \\
\hline $4 H-5-18$ & 38.48 & 18.95 & 0.80 & 0.18 \\
\hline $4 H-5-128$ & 38.58 & 9.99 & 29.33 & 0.17 \\
\hline $4 H-5-94$ & 31.24 & 18.48 & 2.14 & 8.29 \\
\hline $4 H-6-48$ & 32.28 & 9.78 & 0.80 & 0.47 \\
\hline $4 H-6-8 \theta$ & 32.68 & 6.28 & 4.73 & 0.27 \\
\hline $4 H-6-128$ & 33.80 & 12.28 & 0.80 & 0.27 \\
\hline $4 H-6-14 \theta$ & 33.28 & 28.38 & 11.88 & 8.19 \\
\hline $4 \mathrm{H}-7-48$ & 33.78 & 18.48 & 8.08 & 0.37 \\
\hline $5 H-1-42$ & 34.22 & 2.08 & 0.08 & 8.37 \\
\hline $5 H-1-102$ & 34.82 & 22.18 & 8.80 & 8.27 \\
\hline $5 H-1-142$ & 35.22 & 12.48 & 3.37 & 1.83 \\
\hline $5 H-2-42$ & 35.72 & 7.50 & 8.80 & 0.34 \\
\hline $5 H-2-82$ & 36.12 & 11.82 & 8.88 & 8.28 \\
\hline $5 \mathrm{H}-2-102$ & 36.32 & 7.80 & 0.08 & 0.26 \\
\hline $5 \mathrm{H}-2-123$ & 36.43 & 5.10 & 8.06 & 8.25 \\
\hline $5 \mathrm{H}-3-17$ & 36.97 & 12.18 & 8.88 & 8.18 \\
\hline $5 H-3-62$ & 37.42 & 5.88 & 0.80 & 0.18 \\
\hline $5 H-3-182$ & 37.82 & 13.63 & 0.08 & 8.48 \\
\hline $5 H-4-42$ & 38.72 & 8.72 & 8.88 & 0.28 \\
\hline $5 H-4-102$ & 39.32 & 6.18 & 8.88 & 8.26 \\
\hline $5 H-4-143$ & 39.62 & 12.58 & 0.80 & 1.08 \\
\hline $5 H-5-17$ & 39.97 & 10.72 & $8.8 \theta$ & 8.23 \\
\hline $5 H-5-82$ & 48.62 & 5.80 & 0.08 & 0.21 \\
\hline $5 H-5-123$ & 41.03 & 6.05 & 0.80 & 0.27 \\
\hline $5 H-6-42$ & 41.72 & 7.64 & 0.80 & 0.33 \\
\hline $5 H-6-82$ & 42.12 & 4.82 & 8.88 & 0.39 \\
\hline $5 H-6-122$ & 42.42 & 14.58 & 0.88 & 0.41 \\
\hline $5 H-6-142$ & 42.62 & 10.21 & 8.80 & 0.99 \\
\hline
\end{tabular}


Table 1.3 (continued).

\begin{tabular}{|c|c|c|c|c|}
\hline $\begin{array}{c}\text { Sample } \\
\text { no. }\end{array}$ & $\begin{array}{l}\text { Depth } \\
\text { (mbsf) }\end{array}$ & $\begin{array}{l}>63 \\
\mu \mathrm{m} \\
(\%)\end{array}$ & $\begin{array}{l}\text { Bulk } \\
\mathrm{CaCO}_{3} \\
(\%)\end{array}$ & $\begin{array}{l}\text { TOC } \\
(\%)\end{array}$ \\
\hline $6 \mathrm{H}-1-2 \theta$ & 43.58 & 17.44 & 0.88 & 0.88 \\
\hline $6 \mathrm{H}-1-182$ & 44.32 & 4.28 & $\theta . \theta \theta$ & 8.33 \\
\hline $6 \mathrm{H}-1-142$ & 44.72 & 8.38 & 8.88 & 8.23 \\
\hline $6 \mathrm{H}-2-2 \theta$ & 45.88 & 5.52 & 8.88 & 8.21 \\
\hline $6 \mathrm{H}-2-182$ & 45.82 & 13.18 & $\theta . \theta \theta$ & 8.38 \\
\hline $6 \mathrm{H}-2-128$ & 46.88 & 13.88 & 8.88 & 8.67 \\
\hline $6 \mathrm{H}-2-142$ & 46.28 & 19.58 & 0.88 & 8.64 \\
\hline $6 \mathrm{H}-3-42$ & 46.72 & 8.78 & 8.88 & 8. 25 \\
\hline $6 \mathrm{H}-3-83$ & 47.13 & 6.22 & 0.88 & 8.28 \\
\hline $6 \mathrm{H}-3-128$ & 47.58 & 1.98 & 8.88 & 0.23 \\
\hline $6 \mathrm{H}-4-28$ & 48.88 & 8.72 & 8.80 & 8.23 \\
\hline $6 \mathrm{H}-4-61$ & 48.48 & 6.35 & 8.88 & 8.23 \\
\hline $6 \mathrm{H}-4-182$ & 48.82 & 18.87 & 8.88 & 8. 32 \\
\hline $6 \mathrm{H}-5-42$ & 49.72 & 8.28 & 8.88 & 8. 25 \\
\hline $6 H-5-83$ & 58.13 & 12.28 & 0.80 & 0.31 \\
\hline $6 H-5-128$ & 58.58 & 9.22 & 8.80 & 8.25 \\
\hline $6 \mathrm{H}-6-42$ & 51.22 & 1.88 & 0.88 & 0.15 \\
\hline $6 H-6-68$ & 51.48 & 1.34 & 8.80 & 8.19 \\
\hline $7 \mathrm{H}-1-62$ & 53.42 & 2.78 & 8.88 & 8.14 \\
\hline $7 \mathrm{H}-1-122$ & 54.86 & 1.11 & 5.25 & 8.21 \\
\hline $7 \mathrm{H}-2-41$ & 54.72 & 8.48 & 8.88 & 0.19 \\
\hline $7 \mathrm{H}-2-185$ & 55.35 & 8.71 & 45.39 & 8.26 \\
\hline $7 \mathrm{H}-3-41$ & 56.21 & 1.38 & 18.21 & 8.29 \\
\hline $7 \mathrm{H}-3-82$ & 56.62 & 2.58 & 27.82 & 8.23 \\
\hline $7 \mathrm{H}-3-122$ & 57.82 & 8.84 & 42.57 & 8.27 \\
\hline $7 \mathrm{H}-4-41$ & 57.72 & 1.28 & 38.87 & 8.38 \\
\hline $7 \mathrm{H}-4-83$ & 58.13 & 2.78 & 16.96 & 8.29 \\
\hline $7 H-4-122$ & 58.53 & 1.19 & 9.88 & 8.23 \\
\hline $7 \mathrm{H}-5-41$ & 59.21 & 2.48 & 18.28 & 8.23 \\
\hline $7 \mathrm{H}-5-83$ & 59.62 & 5.57 & 0.88 & 8.17 \\
\hline $7 \mathrm{H}-5-122$ & 68.82 & 2.36 & 8.80 & 8.14 \\
\hline $8 \mathrm{H}-1-35$ & 62.65 & 1.80 & 0.80 & 0.13 \\
\hline $8 \mathrm{H}-2-35$ & 64.15 & 1.47 & 8.80 & 8.12 \\
\hline $8 \mathrm{H}-3-35$ & 65.65 & 0.71 & 8.80 & 8.89 \\
\hline $8 H-4-35$ & 67.15 & 8.72 & 8.88 & 8.11 \\
\hline $8 H-5-35$ & 68.65 & 1.83 & 2.96 & 8.57 \\
\hline $8 H-6-35$ & 78.15 & 8.54 & 8.88 & 8.37 \\
\hline $8 H-C-36$ & 71.66 & 4.24 & 8.88 & 8.30 \\
\hline $9 H-1-48$ & 72.28 & 1.25 & 3.39 & 8.63 \\
\hline $9 H-2-48$ & 73.78 & 8.47 & 5.54 & 8.36 \\
\hline $9 H-3-48$ & 75.28 & 8.89 & 5.63 & 0.19 \\
\hline $9 H-4-48$ & 76.78 & 8.76 & 13.25 & 8.24 \\
\hline $9 \mathrm{H}-5-48$ & 78.28 & 8.80 & 8.03 & 8.26 \\
\hline $9 m-6-48$ & 79.78 & 0.24 & 8.88 & 8.16 \\
\hline $9 H-7-48$ & 81.28 & 8. 22 & 8.39 & 8.32 \\
\hline $18 \mathrm{H}-1-48$ & 81.78 & 8.87 & 13.03 & 8.49 \\
\hline $10+1-2-4 \theta$ & 83.28 & 8.65 & 8.67 & 8. 22 \\
\hline $18 H-3-48$ & 84.78 & 1.89 & 19.22 & 0.28 \\
\hline $18 H-4-48$ & 86.28 & 8.56 & 12.85 & 0.37 \\
\hline $18 H-5-48$ & 87.78 & 0.16 & $\theta .80$ & 0.86 \\
\hline
\end{tabular}

Table 1.3 (continued).

\begin{tabular}{|c|c|c|c|c|}
\hline $\begin{array}{c}\text { Sample } \\
\text { no. }\end{array}$ & $\begin{array}{l}\text { Depth } \\
\text { (mbsf) }\end{array}$ & $\begin{array}{l}>63 \\
\mu \mathrm{m} \\
(\%)\end{array}$ & $\begin{array}{c}\text { Bulk } \\
\mathrm{CaCO}_{3} \\
(\%)\end{array}$ & $\begin{array}{l}\text { TOC } \\
(\%)\end{array}$ \\
\hline $10 H-6-48$ & 89.28 & 8.56 & 8.88 & 0.83 \\
\hline $18 H-7-48$ & 90.78 & 8.68 & 15.86 & 0.29 \\
\hline $11 H-1-4 \theta$ & 91.28 & 8.42 & 4.36 & 8.23 \\
\hline $11 H-2-48$ & 92.78 & 5.16 & 7.61 & 8.24 \\
\hline $11 \mathrm{H}-3-48$ & 94.28 & 8.24 & 3.87 & 8.19 \\
\hline $11 \mathrm{H}-4-4 \theta$ & 95.78 & 8.53 & 8.88 & 0.22 \\
\hline $11 \mathrm{H}-5-4 \theta$ & 97.28 & 0.78 & 16.87 & 0.75 \\
\hline $11 H-6-48$ & $98.7 \theta$ & 1.10 & 28.03 & 8.31 \\
\hline $11 H-7-16$ & 99.96 & 3.73 & 0.88 & 8.16 \\
\hline $12 \mathrm{H}-1-48$ & 180.78 & 0.64 & $\theta . \theta \theta$ & 0.34 \\
\hline $12 \mathrm{H}-2-48$ & 182.28 & 8.77 & 0.80 & 8.63 \\
\hline $12 \mathrm{H}-3-48$ & 183.78 & 5.37 & 0.80 & 0.41 \\
\hline $12 \mathrm{H}-4-48$ & 105.28 & 2.08 & $\theta . \theta 8$ & 1.12 \\
\hline $12 \mathrm{H}-5-48$ & 186.78 & 1.59 & 0.80 & 8.78 \\
\hline $12 \mathrm{H}-6-48$ & 188.28 & 0.58 & 8.88 & 0.65 \\
\hline $13 H-1-48$ & 118.28 & 2.14 & 0.80 & 1.21 \\
\hline $13 H-2-48$ & 111.78 & 1.84 & 0.88 & 1.44 \\
\hline $13 \mathrm{H}-3-4 \theta$ & 113.28 & 0.32 & $\theta . \theta \theta$ & 1.61 \\
\hline $13 H-4-48$ & 114.78 & 1.38 & 0.08 & 8.45 \\
\hline $13 H-5-48$ & 116.28 & 1.59 & $\theta . \theta 8$ & 0.39 \\
\hline $13 H-6-48$ & 117.70 & 8.82 & $8 . \theta 8$ & 0.39 \\
\hline $13 \mathrm{H}-7-4 \theta$ & 119.28 & 1.39 & $8 . \theta 0$ & 0.35 \\
\hline $14 \mathrm{H}-1-4 \theta$ & 119.78 & 8.47 & $\theta . \theta \theta$ & 8.68 \\
\hline $14 \mathrm{H}-2-48$ & 121.28 & 3.39 & 0.80 & 8.31 \\
\hline $14 H-3-48$ & 122.78 & 8.66 & $8 . \theta 8$ & 8.44 \\
\hline $14 \mathrm{H}-4-48$ & 124.28 & 1.78 & 8.80 & 0.74 \\
\hline $14 H-5-48$ & 125.78 & 1.82 & 0.08 & 0.67 \\
\hline $14 H-6-48$ & 127.28 & 1.36 & 8.88 & 1.84 \\
\hline $15 \mathrm{H}-1-39$ & 129.19 & 8.81 & $8 . \theta 8$ & 0.51 \\
\hline $15 \mathrm{H}-2-39$ & 138.69 & 0.54 & 8.80 & 0.46 \\
\hline $15 \mathrm{H}-3-39$ & 132.19 & 8.52 & 8.08 & 0.39 \\
\hline $15 H-4-39$ & 133.69 & 8.27 & $\theta . \theta 8$ & 0.57 \\
\hline $15 \mathrm{H}-5-39$ & 135.19 & 3.12 & 0.88 & 0.68 \\
\hline $15 H-6-39$ & 136.69 & 0.96 & 0.88 & $\theta .30$ \\
\hline $16 \mathrm{H}-1-38$ & 138.68 & 2.58 & 8.88 & 0.26 \\
\hline $16 \mathrm{H}-2-38$ & 148.18 & 5.38 & 8.80 & 8.37 \\
\hline $16 \mathrm{H}-3-38$ & 141.68 & 1.01 & 12.38 & 0.37 \\
\hline $16 \mathrm{H}-4-38$ & 143.18 & 1.31 & 8.08 & 0.35 \\
\hline $16 \mathrm{H}-5-38$ & 144.68 & 1.53 & $8 . \theta 8$ & 8.72 \\
\hline $16 \mathrm{H}-6-38$ & 146.18 & 1.21 & 0.88 & 0.89 \\
\hline $16 \mathrm{H}-7-38$ & 147.68 & 2.81 & $8 . \theta \theta$ & 0.36 \\
\hline $17 x-1-58$ & 148.38 & 1.13 & 8.80 & 0.29 \\
\hline $18 x-1-48$ & 157.78 & 2.21 & 8.08 & 0.32 \\
\hline $18 x-2-4 \theta$ & 159.28 & 8.54 & 8.88 & 8.81 \\
\hline $19 x-1-41$ & 167.21 & 3.57 & 8.80 & 0.24 \\
\hline $19 x-2-41$ & 168.71 & 1.82 & 8.80 & 0.29 \\
\hline $19 x-3-41$ & 178.21 & 1.58 & 0.88 & 0.35 \\
\hline $19 x-4-41$ & 171.71 & 1.55 & 0.80 & 0.31 \\
\hline $19 x-5-41$ & 173.21 & 0.85 & 8.08 & 0.46 \\
\hline $19 x-6-41$ & 174.71 & 8.66 & 8.80 & 0.46 \\
\hline
\end{tabular}


Table 1.3 (continued).

\begin{tabular}{|c|c|c|c|c|}
\hline $\begin{array}{c}\text { Sample } \\
\text { no. }\end{array}$ & $\begin{array}{l}\text { Depth } \\
\text { (mbsf) }\end{array}$ & $\begin{array}{l}>63 \\
\mu \mathrm{m} \\
(\%)\end{array}$ & $\begin{array}{c}\text { Bulk } \\
\mathrm{CaCO}_{3} \\
(\%)\end{array}$ & $\begin{array}{l}\text { TOC } \\
(\%)\end{array}$ \\
\hline $28 x-1-41$ & 176.71 & 0.87 & 8.80 & 0.29 \\
\hline $28 x-2-41$ & 178.21 & 0.30 & 8.80 & 0.45 \\
\hline $28 x-3-41$ & 179.71 & 0.25 & 8.80 & 0.69 \\
\hline $20 x-4-41$ & 181.21 & 1.14 & 0.88 & 0.56 \\
\hline $20 x-5-41$ & 182.71 & 0.36 & 0.80 & 0.62 \\
\hline $28 x-6-41$ & 184.21 & 0.45 & 0.08 & 0.55 \\
\hline $22 x-1-48$ & 195.78 & 0.92 & 0.88 & 8.49 \\
\hline $22 x-2-48$ & 197.28 & 8.60 & 0.88 & 8.62 \\
\hline $22 x-3-48$ & 198.78 & 8.74 & 8.80 & $\theta .78$ \\
\hline $22 x-4-48$ & 280.28 & 0.87 & 0.80 & 8.54 \\
\hline $22 x-5-4 \theta$ & 281.78 & & 0.08 & 8.78 \\
\hline $22 x-6-4 \theta$ & 283.28 & 0.35 & 8.88 & 8.68 \\
\hline $22 x-7-4 \theta$ & 284.70 & 0.83 & 8.88 & 8.73 \\
\hline $23 x-1-39$ & 285.49 & 0.58 & 8.88 & 8.65 \\
\hline $23 x-2-39$ & 286.99 & 0.35 & 0.88 & 0.68 \\
\hline $23 x-3-39$ & 288.49 & 0.42 & 8.88 & 8.68 \\
\hline $23 x-4-39$ & 289.99 & 0.25 & 8.80 & 1.81 \\
\hline $23 x-5-39$ & 211.49 & 8.45 & & 8.54 \\
\hline $23 x-6-39$ & 212.99 & 0.47 & 8.80 & 0.82 \\
\hline $23 x-7-39$ & 214.49 & 1.89 & 8.88 & 0.78 \\
\hline $24 X-1-39$ & 215.29 & 8.47 & 0.88 & 8.44 \\
\hline $24 x-2-39$ & 216.79 & 8.42 & 8.80 & 8.64 \\
\hline $24 x-3-39$ & 218.29 & 0.54 & 0.80 & 8.44 \\
\hline $24 x-4-39$ & 219.79 & 0.61 & 8.88 & 8.46 \\
\hline $24 x-5-39$ & 221.29 & 0.68 & $\theta .80$ & 8.75 \\
\hline $24 x-6-39$ & 222.79 & 0.69 & 8.80 & 8.87 \\
\hline $24 x-7-39$ & 224.29 & 0.32 & 0.80 & 0.87 \\
\hline $25 x-1-50$ & 225.28 & 0.48 & 8.80 & 0.74 \\
\hline $25 x-2-50$ & 226.78 & 0.38 & 0.80 & 8.73 \\
\hline $25 x-3-58$ & 228.28 & 0.56 & 8.80 & 0.66 \\
\hline $25 x-4-58$ & 229.78 & 0.34 & 8.80 & 8.71 \\
\hline $25 x-5-58$ & 231.28 & 1.62 & 0.80 & 0.72 \\
\hline $25 x-6-58$ & 232.78 & 0.93 & 0.80 & 0.58 \\
\hline $25 x-7-58$ & 234.28 & 0.87 & 8.80 & 0.63 \\
\hline $26 x-1-48$ & 234.98 & 0.69 & 8.80 & 8.72 \\
\hline $26 x-2-48$ & 236.48 & 2.12 & 0.80 & 8.92 \\
\hline $26 x-3-48$ & 237.98 & 0.63 & 8.80 & 8.81 \\
\hline $26 x-4-48$ & 239.48 & 2.52 & 8.88 & 0.77 \\
\hline $26 x-5-48$ & 248.98 & 0.72 & 0.80 & 0.65 \\
\hline $26 x-6-48$ & $242.4 \theta$ & 0.61 & 0.80 & 0.79 \\
\hline $27 x-1-48$ & 244.78 & 0.24 & 0.08 & 8.79 \\
\hline $27 x-2-48$ & 246.28 & 0.27 & 0.80 & 0.87 \\
\hline $27 x-3-48$ & 247.78 & 2.65 & 0.80 & 1.87 \\
\hline $27 x-4-48$ & 249.28 & 8.62 & 8.80 & 0.72 \\
\hline $27 x-5-4 \theta$ & 258.78 & 0.27 & 0.88 & 0.82 \\
\hline $27 x-6-4 \theta$ & 252.28 & 0.87 & 8.88 & 0.86 \\
\hline $28 x-1-48$ & 254.58 & 0.66 & 0.80 & 8.84 \\
\hline $28 x-2-48$ & 256.80 & 1.62 & 0.80 & 0.79 \\
\hline $28 x-3-48$ & 257.50 & 8.59 & 8.88 & 1.14 \\
\hline $28 X-4-4 \theta$ & 259.80 & 0.24 & 0.80 & 0.95 \\
\hline $28 X-5-48$ & 268.58 & & 8.88 & 0.75 \\
\hline
\end{tabular}

Table 1.3 (continued).

\begin{tabular}{|c|c|c|c|c|}
\hline $\begin{array}{c}\text { Sample } \\
\text { no. }\end{array}$ & $\begin{array}{l}\text { Depth } \\
\text { (mbsf) }\end{array}$ & $\begin{array}{l}>63 \\
\mu \mathrm{m} \\
(\%)\end{array}$ & $\begin{array}{c}\text { Bulk } \\
\mathrm{CaCO}_{3} \\
(\%)\end{array}$ & $\begin{array}{l}\text { TOC } \\
(\%)\end{array}$ \\
\hline $28 x-6-48$ & 262.88 & & $0 . \theta 8$ & 8. 92 \\
\hline $29 x-1-39$ & 264.29 & & 8.80 & 0.74 \\
\hline $29 x-2-39$ & 265.79 & & 0.80 & 0.82 \\
\hline $29 x-3-39$ & 267.29 & & 0.80 & 0.47 \\
\hline $29 x-4-39$ & 268.79 & & 8.88 & 0.61 \\
\hline $29 x-5-39$ & 278.29 & & 8.88 & 0.68 \\
\hline $29 x-6-39$ & 271.79 & & 8.80 & 0.59 \\
\hline $31 x-2-43$ & 285.43 & & $\theta .08$ & 0.96 \\
\hline $31 x-3-43$ & 286.93 & & 8.68 & 8.67 \\
\hline $31 x-4-43$ & 288.43 & & 0.80 & 8.59 \\
\hline $31 x-5-43$ & 289.93 & & 0.80 & 0.62 \\
\hline $31 x-6-43$ & 291.43 & & 8.80 & 8.88 \\
\hline $32 x-1-43$ & 293.73 & & 0.80 & 1.18 \\
\hline $32 x-2-43$ & 295.23 & & $8 . \theta 8$ & 1.16 \\
\hline $32 x-3-43$ & 296.73 & & 0.80 & 0.76 \\
\hline $33 x-1-62$ & 303.72 & & 0.80 & 1.45 \\
\hline $34 x-1-38$ & 313.28 & & 8.80 & 8. 82 \\
\hline $35 x-1-61$ & 323.31 & & $\theta . \theta \theta$ & 0.56 \\
\hline $36 x-2-4 \theta$ & 332.98 & & $\theta . \theta 8$ & 0.79 \\
\hline $37 x-3-38$ & 342.68 & & 8.80 & 1.27 \\
\hline $38 x-1-46$ & 352.56 & & 8.80 & 1.84 \\
\hline $39 x-1-58$ & 362.38 & & $\theta . \theta \theta$ & 0.35 \\
\hline $41 x-1-93$ & 382.03 & & 8.80 & 0.48 \\
\hline
\end{tabular}


Table 1.3 (continued).

Coarse fraction component analysis (wt $\%$ )

\begin{tabular}{|c|c|c|c|c|c|c|c|c|c|c|c|c|}
\hline \multirow[b]{2}{*}{ Sample no. } & \multirow[b]{2}{*}{$\begin{array}{l}\text { Depth } \\
\text { mbsf }\end{array}$} & \multirow[b]{2}{*}{$\begin{array}{c}>63 \mu \mathrm{m} \\
(\%)\end{array}$} & \multicolumn{10}{|c|}{ Coarse fraction component analysis (wt $\%$ ) } \\
\hline & & & $\begin{array}{l}\text { Benthic } \\
\text { foram. } \\
(\%)\end{array}$ & $\begin{array}{l}\text { Plankt. } \\
\text { foram. } \\
(\%)\end{array}$ & $\begin{array}{c}\text { Biogenic } \\
\text { opal cf } \\
(\%)\end{array}$ & $\begin{array}{c}\text { Quartz } \\
(\%)\end{array}$ & $\begin{array}{c}\text { Feldspar } \\
(\%)\end{array}$ & $\begin{array}{l}\text { Mica } \\
(\%)\end{array}$ & $\begin{array}{c}\text { Rock fr. } \\
(\%)\end{array}$ & $\begin{array}{l}\text { Hev. min. } \\
(\%)\end{array}$ & $\begin{array}{l}\text { Volc. gl. } \\
(\%)\end{array}$ & $\begin{array}{l}\text { Auth. } \\
(\%)\end{array}$ \\
\hline $1 H-1-28$ & 0.28 & 10.81 & 0.25 & 3.99 & 8.83 & 5.59 & 0.25 & 0.03 & 0.66 & 8.80 & 8.68 & 8.88 \\
\hline $1 H-1-4 \theta$ & $8.4 \theta$ & 25.37 & 0.48 & 7.26 & 8.88 & 15.55 & 8.96 & 0.85 & 8.74 & 8.18 & 0.80 & 0.88 \\
\hline $1 H-1-68$ & 8.68 & 4.95 & 0.81 & 8.35 & $\theta .00$ & 3.76 & 0.55 & 8.01 & 8.22 & $\theta .01$ & 8.80 & 8.81 \\
\hline $1 H-1-81$ & 8.80 & 9.81 & 8.05 & 5.17 & 8.88 & 2.96 & 0.54 & 8.85 & 0.15 & 0.87 & 8.88 & 0.08 \\
\hline $1 H-1-1 \theta 1$ & 1.81 & 5.64 & 8.85 & 0.14 & 8.88 & 4.91 & 8.86 & 8.01 & 0.39 & 8.85 & 8.80 & 0.82 \\
\hline $1 H-1-121$ & 1.21 & 21.29 & 8.13 & 7.22 & 8.21 & 11.65 & 0. 38 & 8.38 & 0.94 & 0.13 & 0.80 & 0.21 \\
\hline $1 H-1-141$ & 1.41 & 24.87 & 0.85 & 11.22 & 8.45 & 18.82 & 0.65 & 0.25 & 8.80 & 8.48 & 0.88 & 0.28 \\
\hline $1 \mathrm{H}-2-28$ & 1.78 & 26.81 & 8.88 & 8.85 & 8.88 & 18.84 & 5.39 & 8.19 & 2.49 & 8.64 & 8.88 & 8.88 \\
\hline $1 \mathrm{H}-2-41$ & 1.98 & 19.33 & 8.80 & 0.15 & 0.08 & 11.68 & 4.14 & 0.80 & 2.32 & 0.97 & 0.88 & 0.80 \\
\hline $1 \mathrm{H}-2-64$ & 2.14 & 37.37 & 0.38 & 33.71 & 8.49 & 2.86 & 0.56 & 0.00 & 0.38 & 8.00 & 8.80 & 0.00 \\
\hline $1 \mathrm{H}-2-81$ & 2.31 & 38.63 & 8.83 & 23.89 & 8.48 & 4.59 & 0.12 & 8.80 & 8.55 & 0.12 & 0.80 & 0.12 \\
\hline $1 H-2-101$ & 2.51 & 27.59 & 8.99 & 18.29 & 0.61 & 6.21 & 8.68 & 8.17 & 1.19 & 0.86 & 0.08 & 0.88 \\
\hline $1 \mathrm{H}-3-4 \theta$ & 2.71 & 13.08 & 8.88 & 0.56 & 0.08 & 8.42 & 0.71 & $\theta .8 \theta$ & 3.29 & $\theta . \theta \theta$ & 0.80 & $8 . \theta 8$ \\
\hline $1 \mathrm{H}-3-62$ & 3.48 & 4.32 & 8.81 & 8.38 & 8.68 & 3.16 & 0.33 & 8.82 & 0.48 & 0.81 & 0.80 & 8.82 \\
\hline $1 \mathrm{H}-3-82$ & 3.62 & 21.61 & 8.88 & 8.24 & 8.88 & 18.82 & 0.39 & 0.15 & 2.36 & 0.28 & 0.80 & 0.15 \\
\hline $1 \mathrm{H}-3-101$ & 3.82 & 13.97 & 8.86 & 6.78 & 8.83 & 6.69 & 8.86 & 0.80 & 0.27 & 8.83 & 0.08 & 8.86 \\
\hline $1 \mathrm{H}-3-141$ & 4.41 & 18.33 & 0.84 & 11.77 & 8.04 & 5.43 & 0.33 & 8.87 & 8.57 & 8.89 & 0.88 & 0.08 \\
\hline $1 H-4-21$ & 4.71 & 29.88 & 0.88 & 0.23 & 8.88 & 22.97 & 2.84 & 0.80 & 3.43 & 0.17 & 0.88 & 0.23 \\
\hline $1 H-4-41$ & 4.91 & 16.78 & 8.85 & 1.85 & 8.80 & 12.37 & 1.79 & 0.85 & 1.89 & 0.13 & 8.80 & 0.17 \\
\hline $2 \mathrm{H}-1-21$ & 5.31 & 17.31 & 8.89 & 3.15 & 0.08 & 9.54 & 8.92 & 0.89 & 3.08 & 8.45 & 8.80 & 8.88 \\
\hline $2 \mathrm{H}-1-48$ & 5.78 & 24.82 & 8.18 & 21.42 & 8.80 & 2.41 & 0.17 & 8.12 & 8.62 & 8.80 & 8.88 & 0.88 \\
\hline $2 \mathrm{H}-1-6 \theta$ & 5.98 & 9.54 & 0.84 & 7.52 & 8.88 & 1.54 & 8.18 & 0.10 & 0.21 & 8.82 & 8.80 & 0.82 \\
\hline $2 \mathrm{H}-1-82$ & 6.12 & 18.23 & 0.80 & 8.84 & $\theta .80$ & 18.87 & 2.11 & 0.13 & 4.72 & 0.35 & 8.80 & 8.84 \\
\hline $2 H-1-108$ & 6.30 & 28.98 & 0.13 & 0.80 & 0.80 & 13.48 & 2.89 & 0.88 & 4.97 & 0.18 & 0.08 & 0.15 \\
\hline $2 \mathrm{H}-1-128$ & 6.50 & 5.83 & 8.02 & 4.11 & 8.89 & 1.35 & 8.86 & 8.82 & 0.15 & 0.01 & 0.88 & 0.88 \\
\hline $2 \mathrm{H}-1-142$ & 6.72 & 21.53 & 0.52 & 28.11 & 0.80 & 0.84 & 8.88 & 0.80 & 0.89 & 8.88 & 8.88 & 8.80 \\
\hline $2 \mathrm{H}-2-21$ & 7.81 & 14.82 & 0.29 & 12.62 & 8.88 & 1.81 & 8.88 & $\theta . \theta 0$ & 0.11 & 8.88 & 8.80 & 8.88 \\
\hline $2 \mathrm{H}-2-48$ & 7.28 & 11.13 & 8.47 & 6.99 & 0.80 & 2.83 & 8.14 & 0.18 & 8.49 & 0.01 & 8.80 & 0.80 \\
\hline $2 \mathrm{H}-2-68$ & 7.48 & 13.34 & 0.12 & 6.43 & 8.80 & 5.78 & 0.89 & 0.12 & 0.73 & 8.80 & 8.88 & 0.85 \\
\hline $2 \mathrm{H}-2-82$ & 7.62 & 17.78 & 8.87 & 14.74 & 8.80 & 2.48 & 8.21 & 0.80 & 0.21 & 0.80 & 0.80 & 8.80 \\
\hline $2 \mathrm{H}-2-18 \theta$ & 7.88 & 9.38 & 0.08 & 2.65 & 8.80 & 4.78 & 8.95 & 0.87 & 8.77 & 8.88 & 8.88 & 0.03 \\
\hline $2 \mathrm{H}-2-128$ & 8.80 & 9.17 & 8.80 & 0.04 & 0.80 & 7.21 & 8.29 & 8.82 & 1.58 & 0.87 & 8.82 & 0.82 \\
\hline $2 \mathrm{H}-2-142$ & 8.22 & 3.18 & $\theta . \theta 8$ & 8.81 & 8.80 & 1.99 & 8.69 & 8.82 & 0.37 & 0.82 & 0.80 & 0.81 \\
\hline $2 \mathrm{H}-3-48$ & 8.78 & 2.93 & 0.13 & 2.42 & 8.88 & 8.21 & 8.84 & 8.88 & 8.12 & 8.80 & 8.80 & 0.80 \\
\hline $2 \mathrm{H}-3-68$ & 8.98 & 12.62 & 0.15 & 8.83 & 8.80 & 2.73 & 8.88 & 8.23 & 8.57 & 0.18 & 0.03 & 0.80 \\
\hline $2 \mathrm{H}-3-82$ & 9.12 & 6.83 & 0.85 & 0.92 & 8.80 & 3.71 & 8.79 & $\theta . \theta 0$ & 1.32 & 8.03 & 0.88 & 0.81 \\
\hline $2 \mathrm{H}-3-18 \theta$ & 9.38 & 11.21 & 8.87 & 7.08 & 8.18 & 2.67 & 8.72 & 0.82 & 8.46 & 8.82 & 0.80 & 0.00 \\
\hline $2 \mathrm{H}-3-128$ & 9.58 & 21.16 & 0.23 & 19.15 & 8.02 & 1.31 & 8.15 & 0.86 & 0.17 & 8.80 & 8.88 & 0.00 \\
\hline $2 \mathrm{H}-3-142$ & 9.72 & 16.53 & 8.88 & 13.12 & 8.80 & 2.25 & 0.36 & 0.80 & 8.71 & 8.88 & 8.88 & 8.80 \\
\hline $2 \mathrm{H}-4-21$ & 18.81 & 18.48 & 8.22 & 9.36 & 8.88 & 0.53 & 8.87 & 0.84 & 8.11 & 8.82 & 8.88 & 0.80 \\
\hline $2 \mathrm{H}-4-48$ & 10.28 & 1.48 & 8.28 & 1.88 & $\theta . \theta 8$ & 8.83 & 8.88 & $\theta . \theta 8$ & 0.88 & 8.80 & 0.88 & 0.80 \\
\hline $2 \mathrm{H}-4-6 \theta$ & 18.48 & 8.28 & 0.01 & 0.07 & $\theta . \theta 0$ & 0.86 & 8.81 & 8.80 & 0.01 & 8.88 & 8.03 & 8.08 \\
\hline $2 \mathrm{H}-4-82$ & 18.62 & 24.78 & 0.80 & 0.44 & 8.85 & 28.38 & 1.98 & 0.80 & 1.68 & 0.15 & 8.80 & 0.88 \\
\hline $2 \mathrm{H}-4-106$ & 18.86 & 8.02 & 0.80 & 0.80 & 8.80 & 0.81 & 8.80 & 8.80 & 0.80 & 8.88 & 8.88 & 8.80 \\
\hline $2 \mathrm{H}-4-128$ & 11.88 & 6.98 & 0.03 & 0.21 & 8.03 & 5.11 & 8.85 & 0.85 & 1.19 & 8.85 & 8.18 & 0.89 \\
\hline $2 \mathrm{H}-5-21$ & 11.51 & 14.48 & 0.88 & 8.89 & 8.68 & 11.38 & 0.58 & 8.89 & 2.06 & 8.86 & 8.12 & 0.83 \\
\hline $2 H-5-4 \theta$ & 11.78 & 15.84 & 8.88 & 8.41 & 8.88 & 12.34 & 8.33 & 8.86 & 2.14 & 8.18 & 8.24 & 0.22 \\
\hline $2 \mathrm{H}-5-60$ & 11.98 & 20.95 & 0.08 & 3.27 & 0.88 & 14.81 & 0.31 & 0.19 & 1.68 & 0.08 & 0.42 & 0.13 \\
\hline $2 \mathrm{H}-5-82$ & 12.12 & 13.62 & 8.80 & 11.29 & 0.80 & 1.65 & 8.80 & 8.83 & 8.46 & $\theta .03$ & 0.88 & 8.85 \\
\hline $2 \mathrm{H}-5-18 \theta$ & 12.38 & 18.58 & 8.11 & 9.64 & 8.80 & 8.63 & 8.88 & 8.88 & 0.14 & 8.80 & 8.80 & 0.80 \\
\hline
\end{tabular}


Table 1.3 (continued).

Coarse fraction component analysis $(\mathrm{wt} \%)$

Benthic Plankt. Biogenic

Depth $>63 \mu \mathrm{m}$ foram. foram. opal cf Quartz Feldspar Mica Rock fr. Hev. min. Volc. gl. Auth.

\begin{tabular}{|c|c|c|c|c|c|c|c|c|c|c|c|c|}
\hline Sample no. & mbsf & $(\%)$ & $(\%)$ & $(\%)$ & $(\%)$ & $(\%)$ & $(\%)$ & $(\%)$ & $(\%)$ & $(\%)$ & $(\%)$ & $(\%)$ \\
\hline $2 \mathrm{H}-5-128$ & 12.58 & 11.50 & 0.02 & 0.22 & 0.02 & 9.68 & 0.05 & 8.87 & 1.46 & 8.82 & 0.82 & 0.82 \\
\hline $2 \mathrm{H}-5-142$ & 12.72 & 15.68 & 8.31 & 14.68 & 8.80 & 0.27 & 8.88 & 8.03 & 0.36 & 0.03 & 8.88 & 0.80 \\
\hline $2 \mathrm{H}-6-21$ & 13.81 & 10.87 & 0.26 & 18.34 & 0.80 & 0.17 & 8.88 & 0.80 & 0.10 & 0.80 & 8.88 & 0.88 \\
\hline $2 \mathrm{H}-6-4 \theta$ & 13.28 & $16.4 \theta$ & 0.03 & 1.98 & 0.60 & 11.38 & 0.69 & 0.33 & 1.54 & $\theta . \theta 0$ & 8.26 & 0.18 \\
\hline $2 \mathrm{H}-6-68$ & 13.48 & 2.58 & 8.03 & 1.02 & 0.80 & 1.88 & 8.86 & 0.03 & 0.24 & 8.88 & 8.88 & 0.83 \\
\hline $2 \mathrm{H}-6-82$ & 13.62 & 2.55 & 0.02 & 0.95 & $\theta .08$ & 1.28 & 8.84 & 8. 85 & 8.23 & 0.81 & 8.86 & 8.81 \\
\hline $2 \mathrm{H}-6-111$ & 13.91 & 9.24 & 0.23 & 6.98 & 0.82 & 1.31 & 8.33 & 8.82 & 0.30 & 8.84 & 8.08 & 0.88 \\
\hline $2 H-6-128$ & 14.88 & 6.28 & 0.12 & 1.48 & 8.88 & 3.42 & 0.16 & 0.11 & 8.78 & 8.84 & 8.16 & 0.01 \\
\hline $2 \mathrm{H}-6-142$ & 14.22 & 28.32 & 8.80 & 0.28 & 8.88 & 14.14 & 1.58 & 8.84 & 3.78 & 8.84 & 0.35 & 0.28 \\
\hline $2 \mathrm{H}-7-21$ & 14.51 & 23.48 & $\theta . \theta \theta$ & 0.14 & 0.80 & 19.16 & 1.47 & $\theta . \theta \theta$ & 2.48 & 8.14 & 8.88 & 0.08 \\
\hline $2 \mathrm{H}-7-4 \theta$ & 14.78 & 6.42 & 8.84 & 3.48 & 0.01 & 2.68 & 0.86 & 0.81 & 8.28 & 8.80 & 0.88 & 0.81 \\
\hline $3 H-1-25$ & 15.85 & 18.89 & 0.33 & 14.49 & 0.84 & 2.12 & 0.45 & 0.89 & 0.54 & 0.04 & 8.88 & 8.08 \\
\hline $3 \mathrm{H}-1-42$ & 15.21 & 9.18 & 0.80 & 5.76 & 0.80 & 2.75 & 0.18 & 8.82 & 0.41 & 0.80 & 8.88 & 0.07 \\
\hline $3 H-2-28$ & 16.58 & 8.78 & 8.88 & 7.72 & 0.19 & 0.58 & 8.80 & $\theta . \theta 8$ & 8.21 & 8.88 & 8.80 & 8.02 \\
\hline $3 \mathrm{H}-2-42$ & 16.72 & 11.14 & 0.18 & 9.88 & 0.84 & 1.41 & 0.88 & 8.88 & 8.38 & 8.01 & 8.88 & 8.88 \\
\hline $3 H-2-68$ & 16.98 & 19.61 & 8.16 & 18.26 & 8.84 & 8.73 & 8.88 & 0.12 & 8.28 & 8.88 & 8.88 & 8.80 \\
\hline $3 \mathrm{H}-2-82$ & 17.12 & 15.11 & 8.88 & 18.92 & 8.80 & 3.63 & 8.88 & 0.83 & 8.38 & 8.88 & 8.88 & 0.08 \\
\hline $3 H-2-101$ & 17.31 & 2.63 & 8.80 & 1.18 & 8.80 & 1.21 & 0.02 & 0.03 & 8.17 & 8.80 & 8.88 & 0.82 \\
\hline $3 \mathrm{H}-2-12 \theta$ & 17.58 & 7.11 & $8 . \theta \theta$ & 8.27 & 0.01 & 6.82 & 8.08 & 0.14 & 8.33 & 0.86 & 0.80 & 8.21 \\
\hline $3 H-2-142$ & 17.72 & 28.18 & 8.80 & 0.28 & 8.88 & 17.89 & 0.46 & 0.88 & 1.97 & 0.84 & 0.80 & 0.38 \\
\hline $3 H-3-28$ & 18.80 & 6.84 & 8.80 & 0.33 & 8.03 & 4.45 & 0.38 & 8.80 & 1.37 & 0.84 & 8.80 & 8.18 \\
\hline $3 H-3-25$ & 18.85 & 19.68 & 0.84 & 2.83 & 8.80 & 13.82 & 0.49 & 2.85 & 1.24 & 8.88 & 8.80 & 8.88 \\
\hline $3 \mathrm{H}-3-48$ & 18.28 & 4.48 & 0.01 & 0.51 & 8.01 & 3.25 & 0.15 & 0.02 & 8.42 & 0.02 & 8.08 & 0.83 \\
\hline $3 H-3-58$ & 18.38 & 17.97 & 8.84 & 0.34 & 0.80 & 14.98 & 8.34 & 0.34 & 1.71 & 8.84 & 8.87 & 0.28 \\
\hline $3 H-3-82$ & 18.62 & 0.69 & 0.80 & 0.14 & 8.88 & 0.48 & 8.82 & 0.08 & 0.85 & 8.88 & 0.81 & 0.08 \\
\hline $3 H-3-116$ & 18.96 & 2.80 & 0.81 & 0.17 & 8.80 & 1.46 & 0.16 & 0.80 & 0.17 & 0.01 & $\theta . \theta 0$ & 0.01 \\
\hline $3 \mathrm{H}-3-121$ & 19.83 & 8.92 & 8.88 & 8.88 & 8.88 & 7.56 & 0.46 & 0.80 & 0.78 & 0.85 & 8.88 & 0.85 \\
\hline $3 \mathrm{H}-4-28$ & 19.58 & 16.68 & 8.88 & 8.87 & 8.80 & 11.87 & 1.94 & 0.12 & 2.44 & 0.12 & 8.80 & 8.80 \\
\hline $3 H-4-42$ & 19.72 & 2.68 & 8.81 & 8.21 & 8.82 & 2.83 & 8.84 & 8.85 & 8.28 & 8.01 & $8.8 \theta$ & 8.83 \\
\hline $3 H-4-58$ & 19.88 & 2.38 & 8.88 & 0.18 & 8.80 & 1.53 & 0.16 & 0.81 & 8.38 & 0.01 & 8.88 & 8.82 \\
\hline $3 H-4-82$ & 28.12 & 3.18 & 8.85 & 0.69 & 8.82 & 1.92 & 8.16 & 8.84 & 8.21 & 0.81 & 8.88 & 8.82 \\
\hline $3 H-4-10 \theta$ & 28.38 & 11.76 & 8.82 & 1.49 & 0.80 & 7.94 & 8.94 & 0.89 & 1.12 & 0.14 & 8.80 & 8.82 \\
\hline $3 H-4-122$ & 28.52 & 14.78 & 0.80 & 14.48 & 0.80 & 8.38 & 8.80 & 0.80 & 0.89 & 8.80 & 8.08 & 8.88 \\
\hline $3 H-4-142$ & 28.72 & 6.33 & 0.01 & 2.28 & 8.81 & 3.64 & 8.12 & 0.12 & 8.28 & 0.81 & 0.08 & 0.01 \\
\hline $3 \mathrm{H}-5-28$ & 21.80 & 2.41 & 0.81 & 0.15 & 0.80 & 1.95 & 8.12 & 8.01 & 0.16 & 8.88 & 8.80 & 8.81 \\
\hline $3 H-5-4 \theta$ & 21.28 & 18.41 & 0.24 & 1.95 & 8.80 & 6.93 & 8.16 & 0.41 & 0.68 & 0.82 & 8.80 & 0.82 \\
\hline $3 H-5-58$ & 21.38 & 6.44 & 0.12 & 4.53 & 8.80 & 1.45 & 8.86 & 0.84 & 0.17 & 0.01 & 0.03 & 8.80 \\
\hline $3 H-5-82$ & 21.62 & 6.38 & 0.01 & 0.50 & 8.08 & 4.62 & 0.21 & 0.88 & 0.77 & 0.01 & 0.03 & 8.06 \\
\hline $3 H-5-18 B$ & 21.80 & 4.11 & 0.82 & 0.83 & 0.08 & 2.73 & 0.03 & 0.11 & 0.38 & 0.08 & 8.85 & 0.85 \\
\hline $3 H-5-128$ & 22.80 & 12.18 & 0.18 & 1.19 & 8.80 & 8.79 & $\theta .38$ & 8.87 & 1.58 & 0.87 & 8.80 & 0.16 \\
\hline $3 H-5-148$ & 22.28 & 6.52 & 0.08 & 4.27 & 8.80 & 1.92 & 0.04 & 0.80 & 8.29 & $\theta .80$ & 8.80 & 0.80 \\
\hline $3 H-6-28$ & 22.58 & 5.78 & 0.80 & 2.99 & 0.80 & 2.29 & 0.12 & 0.12 & 0.17 & 0.01 & 0.88 & 8.80 \\
\hline $3 H-6-48$ & 22.78 & 7.78 & 0.32 & 5.91 & 0.88 & 1.14 & 0.28 & 0.82 & 0.12 & 8.88 & 0.80 & 8.80 \\
\hline $3 H-6-58$ & 22.88 & 12.60 & 0.47 & 8.80 & 8.80 & 3.69 & 0.13 & 0.11 & 0.19 & 0.01 & 8.88 & 8.01 \\
\hline $3 H-6-82$ & 23.12 & 6.95 & 8.80 & 0.23 & 0.80 & 4.46 & 0.34 & 0.10 & 1.71 & 0.86 & $8.8 \theta$ & 0.04 \\
\hline $3 H-6-188$ & 23.38 & 2.98 & 0.02 & 0.68 & 8.88 & 1.79 & 8.19 & 0.84 & 0.38 & $\theta .01$ & 8.88 & 0.84 \\
\hline $3 H-6-128$ & 23.58 & 3.98 & 0.04 & 1.72 & 8.80 & 1.74 & 0.21 & 0.12 & 0.10 & 8.02 & 8.88 & 0.83 \\
\hline $3 H-6-148$ & 23.78 & 4.22 & 0.02 & 2.38 & 8.08 & 1.27 & 0.22 & 8.09 & 8.22 & 8.88 & 8.80 & $\theta .03$ \\
\hline $3 H-7-28$ & 24.80 & 9.18 & 8.88 & 6.22 & 0.88 & 2.14 & 0.42 & 0.08 & 0.18 & 0.80 & 8.80 & 8.02 \\
\hline $3 \mathrm{H}-7-48$ & 24.28 & 14.30 & 0.83 & 7.19 & 0.80 & 5.75 & 8.73 & 0.28 & 0.34 & 8.03 & 0.80 & 8.03 \\
\hline
\end{tabular}


Table 1.3 (continued).

\begin{tabular}{|c|c|c|c|c|c|c|c|c|c|c|c|c|}
\hline \multirow[b]{2}{*}{ Sample no. } & \multirow[b]{2}{*}{$\begin{array}{l}\text { Depth } \\
\text { mbsf }\end{array}$} & \multirow[b]{2}{*}{$\begin{array}{c}>63 \mu \mathrm{m} \\
(\%)\end{array}$} & \multicolumn{10}{|c|}{ Coarse fraction component analysis (wt \%) } \\
\hline & & & $\begin{array}{l}\text { Benthic } \\
\text { foram. } \\
(\%)\end{array}$ & $\begin{array}{l}\text { Plankt. } \\
\text { foram. } \\
(\%)\end{array}$ & $\begin{array}{c}\text { Biogenic } \\
\text { opal cf } \\
(\%)\end{array}$ & $\begin{array}{c}\text { Quartz } \\
(\%)\end{array}$ & $\begin{array}{c}\text { Feldspar } \\
(\%)\end{array}$ & $\begin{array}{l}\text { Mica } \\
(\%)\end{array}$ & $\begin{array}{l}\text { Rock fr. } \\
(\%)\end{array}$ & $\begin{array}{l}\text { Hev. min. } \\
(\%)\end{array}$ & $\begin{array}{l}\text { Volc. gl. } \\
(\%)\end{array}$ & $\begin{array}{l}\text { Auth. } \\
(\%)\end{array}$ \\
\hline $3 \mathrm{H}-7-56$ & 24.38 & 13.24 & 8.85 & 5.57 & 8.88 & 6.83 & 8.28 & 8.88 & 8.38 & 8.83 & 8.88 & 8.85 \\
\hline $4 H-1-28$ & 24.58 & 2.18 & 8.80 & 0.34 & $\theta .88$ & 1.46 & 8.11 & 8.08 & 0.85 & 8.81 & 8.80 & 8.03 \\
\hline $4 H-1-48$ & 24.78 & 4.85 & 0.01 & 2.88 & 0.81 & 2.21 & 8.16 & 8.85 & 0.31 & 8.88 & 8.88 & 8.81 \\
\hline $4 H-1-68$ & 24.98 & 8.83 & 0.68 & 8.03 & $\theta .80$ & 0.66 & 8.83 & 8.05 & 0.84 & 0.81 & 8.88 & 0.01 \\
\hline $4 H-1-88$ & 25.18 & 4.88 & 0.82 & 0.87 & 8.80 & 2.85 & 8.89 & 8.82 & 0.12 & 8.88 & 8.88 & 0.03 \\
\hline $4 H-1-94$ & 25.24 & 8.86 & 8.80 & 8.74 & 8.88 & 6.41 & 0.36 & 8.27 & 8.19 & 0.03 & 8.88 & 8.85 \\
\hline $4 H-1-180$ & 25.38 & 7.18 & 0.80 & 8.77 & 8.81 & 5.37 & 8.33 & 8.33 & 0.09 & 8.03 & 8.80 & 0.85 \\
\hline $4 H-1-128$ & 25.58 & 6.58 & 8.88 & 4.34 & 8.88 & 1.67 & 8.21 & 8.18 & 8.16 & 8.88 & 8.88 & 8.81 \\
\hline $4 H-1-148$ & 25.78 & 10.92 & 8.88 & 0.28 & 8.80 & 7.85 & 0.18 & 2.26 & 8.34 & 8.88 & 8.88 & 8.88 \\
\hline $4 H-2-28$ & 26.88 & 5.48 & 0.85 & 0.11 & 8.80 & 4.22 & 0.35 & 0.46 & 0.17 & 0.81 & 8.88 & 0.03 \\
\hline $4 H-2-4 \theta$ & 26.28 & 3.48 & 0.02 & 0.89 & $\theta . \theta 0$ & 2.45 & 8.84 & 0.18 & 0.56 & 8.08 & 8.88 & 0.06 \\
\hline $4 H-2-6 \theta$ & 26.48 & 1.21 & 8.80 & 0.89 & 8.80 & 1.81 & 0.03 & 0.03 & 0.02 & 0.81 & 8.80 & 0.01 \\
\hline $4 H-2-88$ & 26.68 & 2.58 & 8.88 & 8.82 & 8.88 & 2.85 & 8.88 & 0.12 & 0.22 & 0.88 & 8.88 & 8.82 \\
\hline $4 H-2-18 \theta$ & 26.88 & 8.92 & 0.87 & 5.89 & 8.88 & 2.25 & 8.89 & 0.23 & 0.36 & 0.88 & 0.80 & 0.82 \\
\hline $4 H-2-128$ & 27.88 & 6.65 & 0.88 & 2.51 & $\theta .80$ & 3.33 & 8.11 & 8.13 & 0.55 & 8.88 & 8.88 & 8.88 \\
\hline $4 H-2-14 \theta$ & 27.38 & 1.67 & 8.88 & 0.85 & 8.88 & 1.39 & 8.84 & 0.87 & 0.89 & $\theta . \theta \theta$ & 8.80 & $8 . \theta 1$ \\
\hline $4 H-3-28$ & 27.38 & 11.58 & 0.88 & 8.85 & 8.88 & 8.91 & 8.38 & 8.14 & 1.97 & 8.85 & 8.80 & 0.02 \\
\hline $4 \mathrm{H}-3-48$ & $27.6 \theta$ & 2.95 & 0.01 & 0.80 & 0.88 & 2.44 & 8.89 & 0.17 & 8.19 & 0.02 & 0.80 & 8.82 \\
\hline $4 H-3-68$ & 27.88 & 3.80 & 8.08 & 0.76 & 0.08 & 1.52 & 8.54 & 0.01 & 0.13 & 8.84 & 0.80 & 8.88 \\
\hline $4 H-3-8 \theta$ & 28.88 & 15.83 & $\theta . \theta 8$ & 8.08 & 8.88 & 18.48 & 3.15 & 8.80 & 1.35 & 8.71 & 8.88 & 0.22 \\
\hline $4 \mathrm{H}-3-94$ & 28.14 & 7.38 & 8.80 & 0.85 & 8.81 & 4.44 & 1.88 & 8.68 & 0.66 & 8.29 & 8.88 & 8.03 \\
\hline $4 H-3-188$ & 28.28 & 1.72 & 0.88 & 0.12 & 0.01 & 1.36 & 8.85 & 0.86 & 0.12 & 8.88 & 8.88 & 8.80 \\
\hline $4 H-3-128$ & 28.48 & 28.84 & $\theta .80$ & $\theta .80$ & 8.80 & 13.27 & 3.29 & 8.84 & 2.51 & 8.78 & 0.80 & 8.24 \\
\hline $4 H-3-148$ & 28.68 & 14.28 & 8.09 & 11.32 & 8.88 & 1.78 & 8.50 & 8.60 & 0.47 & 8.88 & 8.88 & 8.88 \\
\hline $4 H-4-28$ & 29.80 & 17.18 & $8 . \theta 8$ & 0.07 & 8.88 & 14.95 & 8.34 & 0.38 & 1.89 & 8.19 & $\theta .08$ & 0.07 \\
\hline $4 H-4-4 \theta$ & 29.28 & 18.28 & 8.88 & 8.84 & 8.80 & 16.38 & 0.25 & 8.88 & 1.86 & 0.22 & 8.88 & 0.22 \\
\hline $4 H-4-68$ & 29.48 & 3.38 & 8.88 & 0.18 & 8.08 & 2.68 & 0.03 & 0.85 & 8.58 & 8.81 & 0.88 & 8.03 \\
\hline $4 H-4-8 \theta$ & 29.68 & 18.38 & 8.88 & 7.28 & 8.80 & 2.52 & 0.82 & 8.25 & 0.21 & 8.88 & 8.88 & 8.80 \\
\hline $4 H-4-18 \theta$ & 29.88 & 8.38 & 0.08 & 0.82 & 8.88 & 7.63 & 8.86 & 0.25 & 8.16 & 0.13 & 8.80 & 8.86 \\
\hline $4 H-4-128$ & 38.88 & 6.78 & $\theta . \theta 8$ & 0.83 & 8.81 & 5.55 & 0.14 & 8.22 & 0.73 & 8.81 & 8.88 & 8.80 \\
\hline $4 H-5-18$ & 30.48 & 10.95 & 0.02 & 0.84 & 8.88 & 9.94 & 8.88 & 0.32 & 8.59 & 8.88 & 8.08 & 8.04 \\
\hline $4 \mathrm{H}-5-48$ & 38.78 & 9.04 & 8.82 & 8.48 & 8.80 & 7.86 & 8.23 & 0.11 & 1.17 & 0.84 & 0.80 & 0.84 \\
\hline $4 H-5-94$ & 31.24 & 18.48 & 0.84 & 8.18 & 0.00 & 14.57 & 8.24 & 8.39 & 2.13 & 8.88 & 8.08 & 8.24 \\
\hline $4 \mathrm{H}-5-18 \theta$ & 31.38 & $15.3 \theta$ & 8.08 & 2.38 & 8.88 & 18.86 & 8.15 & 8.03 & 1.79 & 8.86 & 0.08 & 8.86 \\
\hline $4 H-5-128$ & 31.58 & 9.99 & 0.27 & 9.48 & 8.88 & 0.11 & 8.88 & 0.84 & 8.86 & $\theta .88$ & 8.88 & 0.80 \\
\hline $4 H-5-148$ & 31.78 & 4.88 & 0.80 & 0.13 & 0.81 & 3.91 & 8.85 & 0.41 & 8.38 & 8.80 & 0.80 & 8.80 \\
\hline $4 H-6-16$ & 31.96 & 8.38 & $\theta .08$ & 8.12 & $\theta .8 \theta$ & 6.96 & 8.28 & 0.18 & 0.67 & $8 . \theta \theta$ & 8.88 & 0.10 \\
\hline $4 H-6-4 \theta$ & 32.28 & 9.78 & 0.02 & 0.95 & 0.84 & 7.84 & 8.28 & 0.34 & 8.92 & 0.84 & 8.08 & 0.07 \\
\hline $4 H-6-6 \theta$ & 32.48 & 15.28 & 8.88 & 8.36 & 0.86 & 11.52 & 0.47 & 0.14 & 2.33 & 0.88 & 8.80 & $\theta .30$ \\
\hline $4 H-6-8 \theta$ & 32.60 & 6.28 & 8.88 & 0.57 & 8.81 & 4.92 & 0.85 & 0.12 & 8.46 & 8.81 & $\theta . \theta 8$ & 8.85 \\
\hline $4 H-6-10 \theta$ & 32.88 & $5.5 \theta$ & 8.80 & 8.88 & 0.03 & 4.55 & 8.03 & 0.15 & 0.68 & 0.01 & 8.80 & 0.02 \\
\hline $4 H-6-128$ & 33.68 & 12.28 & 8.88 & 0.82 & 0.80 & 10.87 & 8.10 & 8.35 & 1.61 & 8.88 & 8.88 & 8.65 \\
\hline $4 H-6-14 \theta$ & 33.28 & 28.38 & 8.88 & 6.76 & 8.88 & 9.83 & 8.16 & 8.51 & 2.44 & 8.88 & 8.80 & 8.84 \\
\hline $4 \mathrm{H}-7-28$ & 33.58 & 12.48 & 8.80 & 8.82 & 0.02 & 9.97 & 0.12 & 0.83 & 1.28 & 8.18 & 0.88 & 0.18 \\
\hline $4 \mathrm{H}-7-4 \theta$ & 33.78 & $18.4 \theta$ & 8.88 & 8.86 & 8.02 & 8.85 & 8.12 & 0.28 & 1.91 & 8.84 & 0.80 & 0.80 \\
\hline $5 \mathrm{H}-1-17$ & 33.97 & 3.48 & 8.88 & 8.01 & 0.81 & 2.72 & 8.84 & 0.82 & 0.58 & 8.81 & 8.80 & 8.82 \\
\hline $5 H-1-42$ & 34.22 & 2.08 & 8.80 & 0.08 & 8.82 & 1.53 & 8.87 & 8.04 & 0.31 & 8.01 & 8.88 & 0.02 \\
\hline $5 H-1-62$ & 34.42 & 4.38 & 8.88 & 0.82 & $\theta .8 \theta$ & 3.38 & 0.82 & 0.86 & 0.82 & 8.80 & 8.80 & $\theta .01$ \\
\hline $5 H-1-82$ & 34.62 & 9.98 & 8.80 & 8.88 & 0.10 & 7.88 & 0.88 & 8.98 & 8.94 & 8.82 & 8.88 & 8.86 \\
\hline $5 H-1-182$ & 34.82 & 22.18 & 8.80 & 8.88 & 8.88 & 18.65 & 8.66 & 1.84 & 1.66 & 8.84 & 8.88 & 0.8 \\
\hline
\end{tabular}


Table 1.3 (continued).

Coarse fraction component analysis (wt \%)

\begin{tabular}{|c|c|c|c|c|c|c|c|c|c|c|c|c|}
\hline \multirow[b]{2}{*}{ Sample no. } & \multirow[b]{2}{*}{$\begin{array}{l}\text { Depth } \\
\text { mbsf }\end{array}$} & \multirow[b]{2}{*}{$\begin{array}{c}>63 \mu \mathrm{m} \\
(\%)\end{array}$} & \multicolumn{10}{|c|}{ Coarse fraction component analysis (wt \%) } \\
\hline & & & $\begin{array}{l}\text { Benthic } \\
\text { foram. } \\
(\%)\end{array}$ & $\begin{array}{l}\text { Plankt. } \\
\text { foram. } \\
(\%)\end{array}$ & $\begin{array}{c}\text { Biogenic } \\
\text { opal cf } \\
(\%)\end{array}$ & $\begin{array}{c}\text { Quartz } \\
(\%)\end{array}$ & $\begin{array}{c}\text { Feldspar } \\
(\%)\end{array}$ & $\begin{array}{l}\text { Mica } \\
(\%)\end{array}$ & $\begin{array}{c}\text { Rock fr. } \\
(\%)\end{array}$ & $\begin{array}{l}\text { Hev. min. } \\
\quad(\%)\end{array}$ & $\begin{array}{l}\text { Volc. gl. } \\
(\%)\end{array}$ & $\begin{array}{l}\text { Auth. } \\
(\%)\end{array}$ \\
\hline $5 H-1-123$ & 35.83 & 17.88 & 8.80 & 8.80 & 0.80 & 14.97 & 0.28 & 0.64 & 1.89 & 0.84 & $\theta .80$ & 8.87 \\
\hline $5 H-1-142$ & 35.22 & $12.4 \theta$ & 0.82 & 8.88 & 8.82 & 18.24 & 8.29 & 0.11 & 1.36 & 0.21 & 0.80 & 0.14 \\
\hline $5 \mathrm{H}-2-17$ & 35.47 & 11.60 & 8.80 & 0.00 & 8.80 & 9.83 & 0.16 & 0.14 & 1.42 & 8.80 & 8.80 & 0.07 \\
\hline $5 H-2-42$ & 35.72 & 7.58 & 8.80 & 8.80 & 0.82 & 6.52 & 0.11 & 0.33 & 8.58 & 8.80 & 8.80 & 8.82 \\
\hline $5 H-2-62$ & 35.92 & 3.72 & 8.88 & 8.80 & $\theta .80$ & 3.21 & 8.87 & 0.84 & 0.23 & 0.12 & 8.88 & 0.86 \\
\hline $5 H-2-82$ & 36.12 & 11.82 & 0.80 & 0.80 & 0.88 & 8.81 & 0.25 & 0.88 & 1.23 & 1.31 & $8 . \theta 0$ & 0.17 \\
\hline $5 H-2-182$ & 36.32 & 7.80 & 0.80 & 0.88 & 8.80 & 5.94 & 8.13 & 8.25 & 0.59 & 0.03 & 8.80 & 0.06 \\
\hline $5 H-2-123$ & 36.43 & 5.10 & 0.88 & 0.80 & 0.80 & 3.92 & 8.24 & 0.67 & 0.77 & $\theta .07$ & 8.88 & 0.03 \\
\hline $5 H-2-142$ & 36.61 & 7.18 & $8.6 \theta$ & 0.08 & 0.01 & 6.02 & 0.15 & 0.18 & 0.36 & 8.29 & 0.80 & 0.09 \\
\hline $5 \mathrm{H}-3-17$ & 36.97 & 12.18 & 8.08 & 0.02 & 0.02 & 9.68 & 8.73 & 1.13 & 0.36 & 8.85 & 8.08 & 0.12 \\
\hline $5 H-3-42$ & 37.22 & 6.98 & 0.88 & $\theta .80$ & 0.80 & 5.42 & 0.34 & 0.44 & 0.61 & 0.03 & $\theta .80$ & 0.06 \\
\hline $5 H-3-62$ & 37.42 & 5.80 & 8.80 & 0.88 & 0.81 & 4.17 & 0.29 & 0.12 & 8.39 & 0.82 & 8.80 & 0.01 \\
\hline $5 H-3-82$ & 37.62 & 12.78 & $\theta . \theta \theta$ & 8.80 & 0.80 & 10.13 & $\theta .83$ & 0.52 & 1.07 & 0.03 & 0.80 & 0.13 \\
\hline $5 H-3-182$ & 37.82 & 13.63 & 8.80 & 0.80 & 0.80 & 10.75 & 0.83 & 0.25 & 1.46 & 0.00 & 8.80 & 8.35 \\
\hline $5 H-3-142$ & 38.22 & 9.90 & $\theta . \theta 0$ & 8.88 & $\theta . \theta \theta$ & 8.29 & 0.35 & 8.58 & 8.65 & 0.08 & 0.08 & 0.12 \\
\hline $5 \mathrm{H}-4-17$ & 38.47 & 7.41 & 0.48 & 0.01 & 8.80 & 6.34 & 0.15 & 0.11 & 8.16 & 8.16 & 0.80 & 8.87 \\
\hline $5 H-4-42$ & 38.72 & 8.72 & 8.84 & $\theta .80$ & 8.80 & 7.98 & 0.19 & 0.18 & 8.19 & 8.22 & 8.88 & 0.86 \\
\hline $5 H-4-82$ & 39.12 & 8.60 & $\theta . \theta \theta$ & 8.80 & 8.80 & 7.88 & 0.12 & 0.28 & 0.18 & 0.86 & 8.88 & 0.86 \\
\hline $5 H-4-102$ & 39.32 & 6.10 & 0.06 & 8.88 & 0.00 & 5.48 & 8.84 & 0.18 & 0.28 & 0.15 & 0.80 & 0.07 \\
\hline $5 H-4-123$ & 39.43 & 11.48 & 0.02 & 0.08 & 0.80 & 9.77 & 8.11 & 0.22 & 0.68 & 0.43 & 0.00 & 0.24 \\
\hline $5 H-4-143$ & 39.62 & 12.58 & 8.88 & 0.80 & 0.80 & 10.74 & 8.21 & 0.33 & 8.64 & 0.48 & 8.88 & 8.11 \\
\hline $5 H-5-17$ & 39.97 & 10.72 & 0.88 & 0.02 & 0.80 & 9.27 & 0.23 & 0.53 & 0.34 & 0.23 & 0.88 & 0.10 \\
\hline $5 H-5-42$ & 48.22 & 2.42 & 0.80 & 0.80 & 0.00 & 0.88 & 0.80 & $\theta . \theta \theta$ & 0.88 & 8.80 & 2.42 & 8.80 \\
\hline $5 H-5-62$ & 48.42 & 8.88 & 0.08 & 8.00 & 8.80 & 7.57 & 8.87 & 0.84 & 1.12 & 8.84 & 0.80 & 0.85 \\
\hline $5 H-5-82$ & 48.62 & 5.88 & 0.88 & 8.80 & 8.80 & 4.96 & 8.86 & 0.24 & 0.52 & 0.82 & 8.88 & 8.80 \\
\hline $5 H-5-182$ & 48.82 & 6.80 & 8.80 & $0 . \theta \theta$ & 0.88 & 4.93 & 0.89 & 0.25 & 0.72 & 0.81 & $\theta .00$ & 8.88 \\
\hline $5 H-5-123$ & 41.03 & 6.85 & $\theta . \theta \theta$ & 0.80 & 0.80 & 4.76 & 0.16 & 0.28 & 8.81 & 0.00 & 0.11 & 8.01 \\
\hline $5 H-6-17$ & 41.47 & 6.78 & 0.81 & 0.88 & 0.80 & 5.33 & 8.29 & 0.21 & 8.64 & 0.80 & 0.21 & 8.01 \\
\hline $5 H-6-42$ & 41.72 & 7.64 & 0.80 & 8.80 & 8.80 & 6.86 & 8.27 & 0.08 & 1.18 & 8.80 & 0.03 & 8.03 \\
\hline $5 H-6-62$ & 41.92 & 3.89 & 0.08 & 0.80 & 0.87 & 3.03 & 8.89 & 8.18 & 8.45 & 0.82 & 0.11 & 8.82 \\
\hline $5 \mathrm{H}-6-82$ & 42.12 & 4.82 & 0.80 & $\theta . \theta 0$ & 0.83 & 3.17 & 8.84 & 0.08 & 0.68 & 8.80 & 0.02 & 0.82 \\
\hline $5 H-6-102$ & 42.32 & 4.98 & 8.88 & 8.88 & $\theta . \theta \theta$ & 4.24 & 8.87 & 8.86 & 0.43 & 8.84 & 0.01 & 0.86 \\
\hline $5 H-6-122$ & 42.42 & 14.58 & 8.08 & 8.88 & 0.12 & 11.89 & 0.36 & 8.12 & 0.96 & 8.03 & 8.80 & 1.84 \\
\hline $5 H-6-142$ & 42.62 & 18.21 & 8.80 & 8.88 & 0.88 & 8.48 & 8.14 & 0.16 & 1.88 & 8.68 & 8.88 & 8.42 \\
\hline $6 H-1-28$ & 43.58 & 17.44 & 0.80 & 1.13 & 0.26 & 12.84 & 0.19 & 0.52 & 1.46 & 0.12 & 0.88 & 8.91 \\
\hline $6 \mathrm{H}-1-42$ & 43.78 & 18.48 & 8.80 & 0.80 & 8.82 & 7.98 & 8.18 & 0.18 & 8.47 & 0.18 & 8.88 & 1.61 \\
\hline $6 H-1-61$ & 43.91 & 13.38 & 0.08 & 8.88 & 8.80 & 18.99 & 8.28 & 0.31 & 1.26 & 0.88 & 8.80 & 8.47 \\
\hline $6 \mathrm{H}-1-83$ & 44.13 & 13.28 & 0.08 & 8.34 & 0.80 & 11.52 & 8.25 & 0.17 & 8.74 & 0.88 & 8.08 & 8.89 \\
\hline $6 \mathrm{H}-1-102$ & 44.32 & 4.28 & 8.88 & 8.81 & 8.80 & 3.61 & 8.86 & 8.89 & 8.37 & 8.05 & $8.8 \theta$ & 0.82 \\
\hline $6 H-1-128$ & 44.58 & 12.18 & 0.02 & 0.85 & 8.85 & 7.95 & 8.41 & 0.31 & 1.38 & 8.29 & 1.59 & 0.85 \\
\hline $6 \mathrm{H}-1-142$ & 44.72 & 8.30 & 0.88 & 0.80 & 8.18 & 6.54 & 0.12 & 8.55 & 0.52 & 8. 34 & 0.80 & 0.88 \\
\hline $6 \mathrm{H}-2-28$ & 45.88 & 5.52 & 0.88 & 0.88 & 0.03 & 4.33 & 8.11 & 8.38 & 0.63 & 0.02 & 8.80 & 0.82 \\
\hline $6 \mathrm{H}-2-42 ?$ & 45.22 & 3.48 & 0.08 & 0.24 & 0.80 & 2.55 & 0.85 & 0.18 & 0.42 & 0.01 & 0.80 & 0.03 \\
\hline $6 \mathrm{H}-2-61$ & 45.44 & 17.82 & 0.08 & 8.80 & 8.80 & 16.43 & 0.18 & 0.21 & 8.66 & 0.14 & 8.88 & 0.21 \\
\hline $6 \mathrm{H}-2-182$ & 45.82 & 13.10 & 8.80 & 8.80 & 8.83 & 11.88 & 8.39 & 0.41 & 0.93 & 0.89 & 0.88 & 0.17 \\
\hline $6 \mathrm{H}-2-128$ & 46.80 & 13.88 & 8.88 & 8.03 & 8.80 & 11.76 & 0.33 & 0.83 & 0.73 & 8.88 & 8.88 & 0.12 \\
\hline $6 \mathrm{H}-2-142$ & 46.28 & 19.58 & 0.88 & 0.84 & 0.84 & 17.47 & 8.28 & 0.78 & 8.98 & 0.88 & 0.80 & 0.88 \\
\hline $6 \mathrm{H}-3-28$ & 46.50 & 3.65 & 8.80 & 0.88 & $\theta .68$ & 2.81 & 8.84 & 0.49 & 8.38 & 0.01 & 8.80 & 8.81 \\
\hline $6 \mathrm{H}-3-42$ & 46.72 & 8.78 & 8.88 & 8.88 & 8.82 & 7.36 & 8.83 & 8.23 & 8.99 & 0.85 & 8.80 & 8.82 \\
\hline $6 H-3-61$ & 46.98 & 4.78 & 8.80 & 0.80 & $\theta .80$ & 2.73 & 0.87 & 1.68 & 8.29 & $0 . \theta 8$ & 8.80 & 0.81 \\
\hline
\end{tabular}


Table 1.3 (continued).

Coarse fraction component analysis (wt \%)

\begin{tabular}{|c|c|c|c|c|c|c|c|c|c|c|c|c|}
\hline \multirow[b]{2}{*}{ Sample no. } & \multirow[b]{2}{*}{$\begin{array}{l}\text { Depth } \\
\text { mbsf }\end{array}$} & \multirow[b]{2}{*}{$\begin{array}{c}>63 \mu \mathrm{m} \\
(\%)\end{array}$} & \multicolumn{10}{|c|}{ Coarse fraction component analysis (wt \%) } \\
\hline & & & $\begin{array}{c}\text { Benthic } \\
\text { foram. } \\
(\%)\end{array}$ & $\begin{array}{l}\text { Plankt. } \\
\text { foram. } \\
(\%)\end{array}$ & $\begin{array}{c}\text { Biogenic } \\
\text { opal cf } \\
(\%)\end{array}$ & $\begin{array}{c}\text { Quartz } \\
(\%)\end{array}$ & $\begin{array}{c}\text { Feldspar } \\
(\%)\end{array}$ & $\begin{array}{l}\text { Mica } \\
(\%)\end{array}$ & $\begin{array}{c}\text { Rock fr. } \\
(\%)\end{array}$ & $\begin{array}{l}\text { Hev. min. } \\
(\%)\end{array}$ & $\begin{array}{c}\text { Volc. gl. } \\
(\%)\end{array}$ & $\begin{array}{c}\text { Auth. } \\
(\%)\end{array}$ \\
\hline $6 \mathrm{H}-3-83$ & 47.13 & 6.22 & 8.80 & 0.00 & 0.80 & 3.61 & 8.89 & 2.11 & 0.38 & $\theta .80$ & $\theta .80$ & 0.01 \\
\hline $6 \mathrm{H}-3-182$ & 47.32 & 4.48 & 8.80 & 8.88 & 8.88 & 3.62 & 0.18 & 8.12 & 0.55 & 8.88 & 8.88 & 0.81 \\
\hline $6 H-3-128$ & 47.58 & 1.98 & 8.80 & 8.01 & 8.88 & 1.57 & 8.85 & 0.11 & 8.28 & 0.80 & 0.80 & 0.02 \\
\hline $6 H-3-142$ & 47.72 & 4.84 & 8.88 & 8.80 & 8.88 & 3.11 & 8.85 & 0.39 & 8.47 & 0.02 & 0.80 & 0.00 \\
\hline $6 \mathrm{H}-4-28$ & 48.88 & 8.72 & 8.88 & 8.88 & 8.80 & 7.13 & 0.12 & 0.29 & 1.12 & 0.83 & 0.88 & 0.82 \\
\hline $6 \mathrm{H}-4-42$ & 48.22 & 9.78 & 8.80 & 0.88 & 8.88 & 7.95 & 0.82 & 0.19 & 1.35 & 8.82 & 8.88 & 0.17 \\
\hline $6 \mathrm{H}-4-61$ & 48.48 & 6.35 & 0.80 & 0.08 & 0.80 & 5.37 & 0.88 & 0.16 & 8.78 & 8.80 & 8.00 & 8.04 \\
\hline $6 H-4-83$ & 48.63 & 11.98 & 0.80 & 8.80 & 8.88 & 9.50 & 8.32 & 0.18 & 1.76 & 0.12 & 8.80 & 0.12 \\
\hline $6 \mathrm{H}-4-102$ & 48.82 & 18.87 & 0.80 & 0.84 & $\theta . \theta 0$ & 12.59 & 8.25 & 2.28 & 3.64 & 0.80 & 0.00 & 0.88 \\
\hline $6 H-4-128$ & 49.81 & 5.48 & 0.80 & 0.80 & 0.00 & 4.55 & 0.14 & 0.22 & 8.44 & 0.01 & 8.80 & 0.84 \\
\hline $6 \mathrm{H}-5-28$ & 49.58 & 3.42 & 8.88 & $\theta .80$ & 8.81 & 2.82 & 0.06 & 0.28 & 8.38 & 8.83 & 0.88 & 0.80 \\
\hline $6 H-5-42$ & 49.72 & 8.28 & 8.88 & 8.88 & $\theta . \theta \theta$ & 6.89 & 8.18 & 0.23 & 1.56 & 0.82 & 8.88 & 0.13 \\
\hline $6 H-5-61$ & 49.98 & 5.48 & 8.88 & 0.80 & 0.80 & 3.45 & 8.14 & 8.98 & 0.81 & 8.85 & 0.08 & 0.05 \\
\hline $6 \mathrm{H}-5-83$ & 58.13 & 12.28 & 0.88 & 8.80 & 8.80 & 10.58 & 0.28 & 0.57 & 0.82 & 8.80 & 0.00 & 0.85 \\
\hline $6 \mathrm{H}-5-182$ & 58.32 & 6.70 & 8.80 & 8.80 & 8.80 & 5.43 & 0.03 & 0.16 & 1.85 & 8.83 & 0.80 & 0.81 \\
\hline $6 H-5-128$ & 58.58 & 9.22 & 8.88 & 0.88 & 8.88 & 6.98 & 0.29 & 8.76 & 1.80 & 8.88 & 0.88 & 0.19 \\
\hline $6 \mathrm{H}-5-142$ & 50.72 & 7.88 & 8.80 & 8.88 & 0.88 & 5.11 & 8.44 & 8.08 & 1.38 & 8.86 & 8.80 & 0.82 \\
\hline $6 H-6-28$ & 51.80 & 3.44 & 8.88 & 8.80 & 0.18 & 2.15 & 0.24 & 0.89 & 0.78 & 8.88 & 8.88 & 8.88 \\
\hline $6 \mathrm{H}-6-42$ & 51.22 & 1.88 & $\theta .8 \theta$ & $\theta . \theta \theta$ & 0.12 & 1.19 & 0.08 & 8.88 & 0.31 & 8.88 & 0.80 & 0.88 \\
\hline $6 H-6-46$ & 51.26 & 5.28 & 0.00 & 0.81 & 0.08 & 3.73 & 0.13 & 8.25 & 1.03 & 8.84 & 0.88 & 0.01 \\
\hline $6 H-6-68$ & 51.48 & 1.34 & 0.88 & 8.88 & 8.81 & 8.94 & 0.06 & 8.89 & 8.23 & 8.80 & 8.08 & 8.80 \\
\hline $7 \mathrm{H}-1-21$ & 53.01 & 4.78 & 0.02 & 8.80 & 8.31 & 3.89 & 8.17 & 0.16 & 8.79 & 8.82 & 8.80 & 0.14 \\
\hline $7 H-1-41$ & 53.21 & 1.82 & 8.80 & 8.88 & 0.02 & 1.39 & 0.11 & 0.87 & 0.23 & 8.80 & 0.88 & 8.80 \\
\hline $7 H-1-62$ & 53.42 & 2.78 & 8.88 & 8.88 & 8.01 & 1.93 & 8.16 & 8.15 & 0.35 & 0.81 & 0.86 & 0.83 \\
\hline $7 H-1-83$ & 53.62 & 2.83 & 0.80 & $\theta .01$ & 0.07 & 0.93 & 8.11 & 8.07 & 8.87 & 8.88 & 1.55 & 0.83 \\
\hline $7 \mathrm{H}-1-185$ & 53.84 & 0.81 & 0.33 & 0.86 & 0.81 & 0.28 & 8.81 & 0.02 & 0.83 & 8.81 & 0.87 & 0.08 \\
\hline $7 \mathrm{H}-1-122$ & 54.86 & 1.11 & 8.25 & 8.23 & 0.38 & 0.13 & 8.80 & 8.85 & 8.01 & 0.01 & 8.05 & 0.80 \\
\hline $7 \mathrm{H}-1-142$ & 54.22 & 1.38 & 8.68 & 0.19 & 0.23 & 0.88 & 0.88 & 0.84 & 8.03 & 8.82 & 0.82 & 0.80 \\
\hline $7 \mathrm{H}-2-21$ & 54.31 & 0.56 & 8.81 & 8.81 & 0.32 & 8.16 & 8.88 & 8.84 & 0.02 & 8.81 & 0.80 & 8.88 \\
\hline $7 \mathrm{H}-2-41$ & 54.72 & 0.48 & 0.88 & 8.80 & 8.85 & 0.27 & 8.01 & 0.81 & 0.85 & 8.80 & 0.88 & 0.01 \\
\hline $7 \mathrm{H}-2-64$ & 54.98 & 0.64 & 0.38 & 8.86 & 8.82 & 8.14 & 8.88 & 8.88 & 8.82 & 8.80 & 8.80 & 8.81 \\
\hline $7 \mathrm{H}-2-83$ & 55.13 & 0.64 & 0.88 & 0.14 & 0.28 & 8.83 & 8.80 & 8.82 & 8.15 & 0.88 & 8.81 & 8.88 \\
\hline $7 \mathrm{H}-2-185$ & 55.35 & 0.71 & 8.88 & 0.10 & 8.16 & 8.82 & 8.88 & 8.85 & 0.80 & 0.80 & 8.05 & 0.18 \\
\hline $7 \mathrm{H}-2-12 \theta$ & 55.53 & 1.51 & 8.84 & 0.03 & 0.53 & 8.83 & 8.80 & 8.06 & 8.80 & 0.80 & 0.13 & 0.01 \\
\hline $7 \mathrm{H}-2-142$ & 55.72 & 1.48 & 0.28 & 0.84 & 8.21 & 0.87 & 8.80 & 0.86 & $\theta . \theta 8$ & 8.80 & 0.87 & 0.19 \\
\hline $7 \mathrm{H}-3-21$ & 56.81 & 1.84 & 0.87 & 0.81 & 1.15 & 8.81 & 0.01 & 8.83 & $8.8 \theta$ & 0.80 & 0.24 & 0.24 \\
\hline $7 \mathrm{H}-3-41$ & 56.21 & 1.30 & 8.18 & $\theta .03$ & 8.78 & 8.82 & 8.88 & 8.82 & 0.88 & 8.80 & 0.11 & 0.16 \\
\hline $7 \mathrm{H}-3-62$ & 56.42 & 3.22 & 0.68 & 8.73 & 8.47 & 8.03 & 8.88 & 8.86 & 8.81 & 0.13 & B. 42 & 0.14 \\
\hline $7 \mathrm{H}-3-82$ & 56.62 & 2.58 & 0.96 & 0.26 & 0.61 & 8.80 & 8.88 & 0.82 & 8.80 & 8.80 & 8.44 & 0.85 \\
\hline $7 \mathrm{H}-3-122$ & 57.82 & 0.84 & 8.28 & 8.84 & 8.22 & 0.82 & 8.88 & 8.84 & 8.88 & 0.80 & 8.80 & 0.24 \\
\hline $7 \mathrm{H}-4-21$ & 57.51 & 0.86 & 0.13 & 0.13 & 8.45 & 0.01 & 0.80 & $\theta .88$ & 8.04 & 8.80 & 0.81 & 8.89 \\
\hline $7 \mathrm{H}-4-41$ & 57.72 & 1.28 & 0.44 & 0.18 & 0.32 & 8.82 & 8.80 & 8.80 & $8 . \theta 8$ & 8.88 & 0.88 & 8.81 \\
\hline $7 \mathrm{H}-4-62$ & 57.80 & 1.73 & 0.12 & 0.84 & 1.48 & $\theta . \theta 8$ & 8.88 & 0.88 & 0.82 & 8.80 & 0.05 & 8.18 \\
\hline $7 \mathrm{H}-4-83$ & 58.13 & 2.78 & 0.14 & 0.85 & 2.87 & 0.88 & 8.88 & 0.01 & 0.07 & 0.80 & 8.38 & 0.86 \\
\hline $7 \mathrm{H}-4-142$ & 58.72 & 2.18 & 0.48 & 0.78 & 0.73 & 8.02 & 0.80 & 8.88 & 0.01 & 8.88 & 8.16 & 0.88 \\
\hline $7 \mathrm{H}-5-21$ & 59.01 & 3.98 & 1.13 & 8.81 & 8.55 & 0.29 & $8 . \theta 8$ & 8.81 & 0.18 & 8.80 & 1.85 & 8.84 \\
\hline $7 H-5-41$ & 59.21 & 2.48 & 0.27 & 0.21 & 1.34 & 8.85 & 8.80 & $\theta .01$ & 0.88 & $8.8 \theta$ & 8.43 & 0.80 \\
\hline $7 \mathrm{H}-5-62$ & 59.42 & 2.95 & 0.03 & 0.03 & 2.22 & 8.01 & 8.80 & 0.87 & 8.81 & 8.88 & 8.47 & 0.11 \\
\hline $7 H-5-83$ & 59.62 & 5.57 & 8.81 & 8.87 & 8.94 & 8.88 & 8.03 & 0.85 & $\theta . \theta 8$ & 8.03 & 1.41 & 3.83 \\
\hline $7 \mathrm{H}-5-185$ & 59.84 & 1.48 & 0.01 & 0.81 & 0.69 & 0.11 & 0.80 & 0.86 & 0.80 & 8.82 & 0.32 & 0.19 \\
\hline
\end{tabular}


Table 1.3 (continued).

\begin{tabular}{|c|c|c|c|c|c|c|c|c|c|c|c|c|}
\hline \multirow[b]{2}{*}{ Sample no. } & \multirow[b]{2}{*}{$\begin{array}{l}\text { Depth } \\
\text { mbsf }\end{array}$} & \multirow[b]{2}{*}{$\begin{array}{c}>63 \mu \mathrm{m} \\
(\%)\end{array}$} & \multicolumn{10}{|c|}{ Coarse fraction component analysis (wt $\%$ ) } \\
\hline & & & $\begin{array}{l}\text { Benthic } \\
\text { foram. } \\
(\%)\end{array}$ & $\begin{array}{l}\text { Plankt. } \\
\text { foram. } \\
(\%)\end{array}$ & $\begin{array}{c}\text { Biogenic } \\
\text { opal cf } \\
(\%)\end{array}$ & $\begin{array}{c}\text { Quartz } \\
(\%)\end{array}$ & $\begin{array}{c}\text { Feldspar } \\
(\%)\end{array}$ & $\begin{array}{l}\text { Mica } \\
(\%)\end{array}$ & $\begin{array}{c}\text { Rock fr. } \\
(\%)\end{array}$ & $\begin{array}{l}\text { Hev. min. } \\
(\%)\end{array}$ & $\begin{array}{l}\text { Volc. gl. } \\
(\%)\end{array}$ & $\begin{array}{c}\text { Auth. } \\
(\%)\end{array}$ \\
\hline $7 \mathrm{H}-5-122$ & 68.82 & 2.36 & 8.88 & 8.01 & 1.28 & 8.22 & 8.88 & 8.82 & 0.78 & 8.88 & 0.12 & 8.81 \\
\hline $7 \mathrm{H}-6-12$ & 60.42 & 4.88 & 8.08 & 8.80 & 2.38 & 0.15 & 8.80 & 0.02 & 8.44 & 8.88 & 1.89 & 8.01 \\
\hline $8 \mathrm{H}-1-35$ & 62.65 & 1.88 & 0.80 & 0.82 & 8.88 & 8.33 & $\theta . \theta 8$ & 0.88 & 0.23 & 8.81 & 0.19 & 0.80 \\
\hline $8 \mathrm{H}-2-35$ & 64.15 & 1.47 & 8.80 & 0.88 & 8.47 & 0.13 & $8 . \theta 0$ & 0.88 & 0.14 & 8.88 & $\theta .02$ & 8.88 \\
\hline $8 \mathrm{H}-3-35$ & 65.65 & 8.71 & 0.80 & 8.88 & 8.55 & 8.88 & 8.80 & 0.86 & 8.03 & 0.80 & 0.80 & 8.80 \\
\hline $8 H-4-35$ & 67.15 & 8.72 & 8.88 & 8.88 & 0.42 & 8.84 & 8.88 & 8.28 & 8.85 & 8.88 & 8.88 & 8.80 \\
\hline $8 \mathrm{H}-5-35$ & 68.65 & 1.83 & 8.80 & 8.88 & 8.83 & 8.82 & 8.88 & 0.11 & 8.80 & 0.80 & 8.84 & 8.80 \\
\hline $8 \mathrm{H}-6-35$ & 78.15 & 8.54 & 0.80 & 8.88 & 8.26 & 8.82 & $\theta .80$ & 0.87 & 0.02 & 0.80 & 0.17 & 8.80 \\
\hline $8 \mathrm{H}-\mathrm{C}-36$ & 71.66 & 4.24 & 8.80 & 0.08 & 1.88 & 8.39 & 8.80 & 0.78 & 0.13 & 8.88 & 1.16 & 0.80 \\
\hline $9 H-1-4 \theta$ & 72.28 & 1.25 & 0.02 & 0.88 & 0.48 & 0.02 & 8.80 & 0.84 & 0.82 & 8.88 & 0.59 & 8.80 \\
\hline $9 H-2-48$ & 73.78 & 8.47 & 0.01 & 0.82 & 0.35 & 0.81 & 8.88 & 0.01 & 0.82 & $\theta .08$ & 0.86 & 0.80 \\
\hline $9 H-3-48$ & 75.28 & 8.89 & $\theta .8 \theta$ & $\theta .8 \theta$ & 0.85 & 0.80 & 0.80 & 0.01 & 0.80 & $\theta . \theta 0$ & 0.03 & $\theta .80$ \\
\hline $9 H-4-48$ & 76.78 & 8.76 & 8.81 & 8.88 & 8.65 & 8.81 & 8.88 & 8.68 & 8.81 & 8.88 & 8.87 & 8.60 \\
\hline $9 H-5-4 \theta$ & 78.28 & 8.88 & 8.80 & $\theta . \theta 8$ & 8.88 & 8.88 & 8.80 & $\theta . \theta 8$ & 0.80 & 8.80 & 8.80 & 0.80 \\
\hline $9 H-6-48$ & 79.78 & 0.24 & 8.88 & 0.88 & 8.03 & 8.80 & 8.88 & 8.00 & 8.68 & 8.80 & 8.28 & 8.88 \\
\hline $9 H-7-48$ & 81.28 & 8.22 & 0.80 & $\theta .00$ & 0.16 & 0.01 & 8.88 & 8.88 & $\theta .88$ & 8.88 & 8.84 & 8.88 \\
\hline $10 \mathrm{H}-1-4 \theta$ & 81.78 & 8.87 & 8.82 & 0.85 & 0.42 & 0.02 & 8.80 & 0.08 & 0.01 & 8.80 & 0.34 & 8.80 \\
\hline $18 \mathrm{H}-2-48$ & 83.28 & 8.65 & 0.82 & 8.80 & 8.32 & 0.01 & 8.88 & 8.00 & 8.01 & $\theta .08$ & 0.29 & $8.8 \theta$ \\
\hline $18 \mathrm{H}-3-48$ & 84.78 & 1.89 & 8.88 & 8.88 & 8.37 & 8.81 & 8.88 & 8.01 & 8.84 & 8.80 & 1.36 & 8.80 \\
\hline $18 \mathrm{H}-4-48$ & 86.28 & 0.56 & 0.83 & 8.10 & 0.22 & 0.81 & 8.88 & 8.88 & 8.81 & 8.80 & 8.08 & $\theta .88$ \\
\hline $18 \mathrm{H}-5-48$ & 87.78 & 8.16 & 8.88 & $\theta .80$ & 0.13 & 0.88 & 0.88 & 0.01 & 0.60 & $\theta . \theta 8$ & 8.01 & 0.80 \\
\hline $18 \mathrm{H}-6-4 \theta$ & 89.28 & 8.56 & 0.88 & 0.88 & 8.49 & 0.80 & 0.88 & 8.08 & 0.01 & 8.88 & 0.86 & 8.08 \\
\hline $18 \mathrm{H}-7-4 \theta$ & 90.78 & 8.68 & 0.81 & 0.80 & 0.42 & 0.01 & 8.88 & 8.80 & 0.01 & 8.88 & 0.15 & 8.00 \\
\hline $11 H-1-48$ & 91.28 & 8.42 & $\theta . \theta \theta$ & 8.80 & 0.11 & 0.81 & 0.80 & 8.88 & 0.68 & 8.88 & 0.29 & 8.88 \\
\hline $11 \mathrm{H}-2-48$ & 92.78 & 5.16 & 8.81 & 8.88 & 1.44 & 8.33 & 8.88 & 8.81 & 8.88 & 8.88 & 2.85 & 8.88 \\
\hline $11 \mathrm{H}-3-48$ & 94.28 & 0.24 & 0.88 & 0.80 & 8.16 & 8.88 & 8.80 & 8.80 & 8.81 & 0.88 & 8.87 & 8.80 \\
\hline $11 \mathrm{H}-4-48$ & 95.78 & 8.53 & 0.80 & 0.80 & 8.58 & 8.81 & 8.80 & 0.88 & 8.81 & $\theta .80$ & 8.82 & 8.88 \\
\hline $11 \mathrm{H}-5-48$ & 97.28 & 8.78 & 8.80 & 8.88 & 0.55 & 8.88 & 0.88 & 8.88 & 8.80 & 8.80 & 8.17 & 8.88 \\
\hline $11 \mathrm{H}-6-48$ & 98.78 & 1.10 & 0.81 & 0.81 & 0.21 & 8.81 & 8.88 & 0.80 & 8.88 & 8.88 & 8.18 & 8.80 \\
\hline $11 \mathrm{H}-7-16$ & 99.96 & 3.73 & 0.01 & 8.80 & 0.91 & 0.03 & 8.88 & 8.80 & 0.19 & 8.00 & 2.18 & 8.88 \\
\hline $12 \mathrm{H}-1-48$ & 188.78 & 8.64 & 8.88 & 8.88 & 8.41 & 8.84 & 8.88 & 8.88 & 8.84 & 8.88 & 8.14 & 8.88 \\
\hline $12 \mathrm{H}-2-4 \theta$ & $102.2 \theta$ & 0.77 & 0.88 & 0.80 & 8.65 & 0.81 & $\theta .88$ & 0.80 & 0.02 & 8.88 & 8.89 & 0.88 \\
\hline $12 \mathrm{H}-3-48$ & 103.78 & 5.37 & 8.08 & 0.88 & 2.12 & 0.53 & 8.80 & 8.88 & 8.11 & 0.88 & 2.61 & 0.08 \\
\hline $12 \mathrm{H}-4-4 \theta$ & 105.28 & 2.80 & 0.80 & 8.88 & 8.85 & 8.81 & 8.88 & 8.88 & 0.11 & $\theta .88$ & 1.84 & 0.80 \\
\hline $12 \mathrm{H}-5-48$ & 186.78 & 1.59 & 8.88 & 8.80 & 1.35 & 8.82 & 8.88 & 8.88 & 8.80 & 8.80 & 8.21 & 8.80 \\
\hline $12 \mathrm{H}-6-48$ & 108.28 & 8.58 & 8.88 & 0.88 & 0.46 & 0.81 & 8.88 & 8.88 & 8.02 & 0.80 & 0.81 & $\theta .88$ \\
\hline $13 \mathrm{H}-1-48$ & 118.28 & 2.14 & 8.08 & 8.80 & 1.67 & 0.02 & 8.80 & 0.80 & 0.18 & 8.80 & 0.35 & 8.88 \\
\hline $13 \mathrm{H}-2-4 \theta$ & 111.78 & 1.84 & 8.80 & 8.80 & 8.63 & 0.24 & 8.80 & 8.80 & 8.87 & 0.80 & 0.18 & 8.80 \\
\hline $13 \mathrm{H}-3-48$ & 113.28 & 8.32 & 8.88 & 8.80 & 8.26 & 8.01 & 8.80 & 0.08 & 0.01 & 0.80 & 0.04 & 8.88 \\
\hline $13 \mathrm{H}-4-4 \theta$ & 114.78 & 1.38 & 0.00 & 8.88 & 1.32 & 0.01 & 8.88 & 8.80 & 8.88 & 8.08 & 8.85 & 0.08 \\
\hline $13 H-5-4 \theta$ & 116.28 & 1.59 & 0.08 & $\theta .80$ & 1.59 & 8.88 & $\theta .8 \theta$ & 0.80 & 8.80 & 0.08 & 8.80 & 0.08 \\
\hline $13 \mathrm{H}-6-4 \theta$ & 117.78 & 8.82 & 0.80 & 0.83 & 8.65 & 8.88 & 8.88 & 8.88 & 8.82 & 8.80 & 8.11 & 0.80 \\
\hline $13 \mathrm{H}-7-48$ & 119.28 & 1.39 & 0.80 & $\theta .80$ & 0.98 & 0.81 & $\theta .80$ & 8.88 & 0.08 & 8.80 & 0.82 & 0.81 \\
\hline $14 \mathrm{H}-1-48$ & 119.78 & 8.47 & 0.00 & 8.80 & 8.44 & 0.80 & 0.88 & 8.88 & 8.88 & 8.80 & 0.02 & 8.80 \\
\hline $14 \mathrm{H}-2-4 \theta$ & 121.28 & 3.39 & 8.88 & 0.80 & 1.74 & 8.02 & 8.80 & 8.88 & 0.84 & 0.80 & 1.56 & 8.01 \\
\hline $14 \mathrm{H}-3-48$ & 122.78 & 8.66 & 0.08 & 8.88 & 8.64 & 8.80 & 0.88 & 8.88 & 0.88 & 8.80 & 8.82 & 0.80 \\
\hline $14 \mathrm{H}-4-48$ & 124.28 & 1.78 & 0.00 & 8.80 & 1.76 & 0.01 & 0.80 & 0.08 & 8.80 & 0.00 & 8.80 & 8.81 \\
\hline $14 H-5-4 \theta$ & 125.78 & 1.82 & 0.08 & 0.88 & 8.97 & 8.88 & 8.80 & 8.81 & 8.80 & 8.88 & 8.81 & 0.88 \\
\hline $14 H-6-4 \theta$ & 127.28 & 1.36 & 0.80 & 8.80 & 1.34 & 8.88 & 8.88 & 8.88 & 8.88 & 8.80 & 8.82 & 0.88 \\
\hline $15 \mathrm{H}-1-39$ & 129.19 & 8.81 & 8.88 & 8.88 & 8.61 & 8.81 & 8.88 & 8.81 & 8.88 & 8.88 & 0.82 & B. 88 \\
\hline
\end{tabular}


R. HENRICH, T. WOLF, G. BOHRMANN, J. THIEDE

Table 1.3 (continued).

\begin{tabular}{|c|c|c|c|c|c|c|c|c|c|c|c|c|}
\hline \multirow[b]{2}{*}{ Sample no. } & \multirow[b]{2}{*}{$\begin{array}{l}\text { Depth } \\
\text { mbsf }\end{array}$} & \multirow[b]{2}{*}{$\begin{array}{c}>63 \mu \mathrm{m} \\
(\%)\end{array}$} & \multicolumn{10}{|c|}{ Coarse fraction component analysis (wt \%) } \\
\hline & & & $\begin{array}{c}\text { Benthic } \\
\text { foram. } \\
(\%)\end{array}$ & $\begin{array}{c}\text { Plankt. } \\
\text { foram. } \\
(\%)\end{array}$ & $\begin{array}{c}\text { Biogenic } \\
\text { opal cf } \\
(\%)\end{array}$ & $\begin{array}{c}\text { Quartz } \\
(\%)\end{array}$ & $\begin{array}{c}\text { Feldspar } \\
(\%)\end{array}$ & $\begin{array}{l}\text { Mica } \\
(\%)\end{array}$ & $\begin{array}{l}\text { Rock fr. } \\
(\%)\end{array}$ & $\begin{array}{l}\text { Hev. min. } \\
(\%)\end{array}$ & $\begin{array}{l}\text { Volc. gl. } \\
(\%)\end{array}$ & $\begin{array}{c}\text { Auth. } \\
(\%)\end{array}$ \\
\hline 15H-2- 39 & 138.69 & 8.54 & 8.88 & 8.80 & 8.58 & 8.08 & 8.80 & 8.81 & 8.88 & 8.80 & 8.80 & 8.80 \\
\hline $15 \mathrm{H}-3-39$ & 132.19 & 0.52 & 8.68 & 8.80 & 8.48 & 8.88 & 8.80 & 8.88 & 0.88 & 8.80 & 0.81 & 8.88 \\
\hline $15 \mathrm{H}-4-39$ & 133.69 & 0.27 & 0.80 & 8.80 & 8.22 & 8.88 & 8.80 & 8.80 & 8.80 & 8.81 & 8.83 & 0.80 \\
\hline $15 \mathrm{H}-5-39$ & 135.19 & 3.12 & 8.80 & $\theta . \theta 8$ & 1.58 & 8.80 & 8.80 & 8.80 & 0.80 & 8.03 & 1.57 & 0.80 \\
\hline $15 H-6-39$ & 136.69 & 8.96 & 8.81 & 8.88 & 8.48 & 0.81 & 8.08 & 8.88 & 8.81 & 8.88 & 8.32 & 0.88 \\
\hline $16 \mathrm{H}-1-38$ & 138.68 & 2.58 & 8.88 & 0.80 & 2.83 & 8.87 & 8.88 & 0.03 & 8.82 & 0.01 & 0.32 & 0.80 \\
\hline $16 \mathrm{H}-2-38$ & 148.18 & 5.30 & 0.80 & 8.08 & 4.82 & 8.04 & 8.80 & 8.82 & 8.05 & 8.81 & 1.14 & 0.80 \\
\hline $16 \mathrm{H}-3-38$ & 141.68 & 1.81 & 0.01 & 8.88 & 8.96 & 8.08 & 8.80 & 8.08 & 8.88 & $\theta .80$ & 8.83 & 8.88 \\
\hline $16 \mathrm{H}-4-38$ & 143.18 & 1.31 & 0.88 & $\theta .80$ & 1.26 & 8.01 & 8.80 & 8.80 & 8.88 & 8.80 & 0.88 & 8.01 \\
\hline $16 \mathrm{H}-5-38$ & 144.68 & 1.53 & $\theta . \theta 0$ & 8.80 & 1.48 & $\theta .80$ & 8.80 & 8.80 & 8.88 & 8.88 & 8.82 & 0.88 \\
\hline $16 \mathrm{H}-6-38$ & 146.18 & 1.21 & 0.80 & 8.88 & 8.63 & 8.08 & 8.80 & 8.80 & 8.80 & 8.80 & 8.57 & 8.08 \\
\hline $16 \mathrm{H}-7-38$ & 147.68 & 2.81 & 8.80 & 8.88 & 2.65 & $\theta .01$ & 0.80 & $\theta . \theta 8$ & 8.81 & 0.80 & 8.03 & $\theta .08$ \\
\hline $27 x-5-48$ & 258.78 & 8.27 & 0.80 & 8.80 & 0.25 & 0.01 & 8.80 & 0.88 & 8.80 & 8.80 & 0.01 & 0.80 \\
\hline $27 x-6-48$ & 252.28 & 0.87 & 8.80 & 0.80 & 0.87 & 8.80 & 8.80 & 8.80 & 8.80 & 8.88 & 0.80 & 0.88 \\
\hline $28 x-1-48$ & 254.58 & 0.66 & 0.08 & 8.80 & 0.58 & 0.01 & 8.80 & 8.80 & 8.81 & 0.88 & 8.86 & 0.00 \\
\hline $28 x-2-48$ & 256.88 & 1.62 & 0.88 & 8.80 & 1.22 & 0.04 & 0.80 & $\theta .88$ & 8.86 & 8.80 & 0.38 & 0.08 \\
\hline $28 x-3-48$ & 257.58 & 0.59 & 0.80 & $\theta . \theta 8$ & 8.54 & 8.01 & $\theta .80$ & $8.8 \theta$ & 8.82 & 0.80 & 0.02 & 0.00 \\
\hline $28 x-4-48$ & 259.88 & 8.24 & 8.80 & 8.88 & 0.24 & 0.80 & $8 . \theta 8$ & 0.88 & 8.88 & 0.80 & 8.80 & 8.00 \\
\hline
\end{tabular}


Table 1.3 (continued).

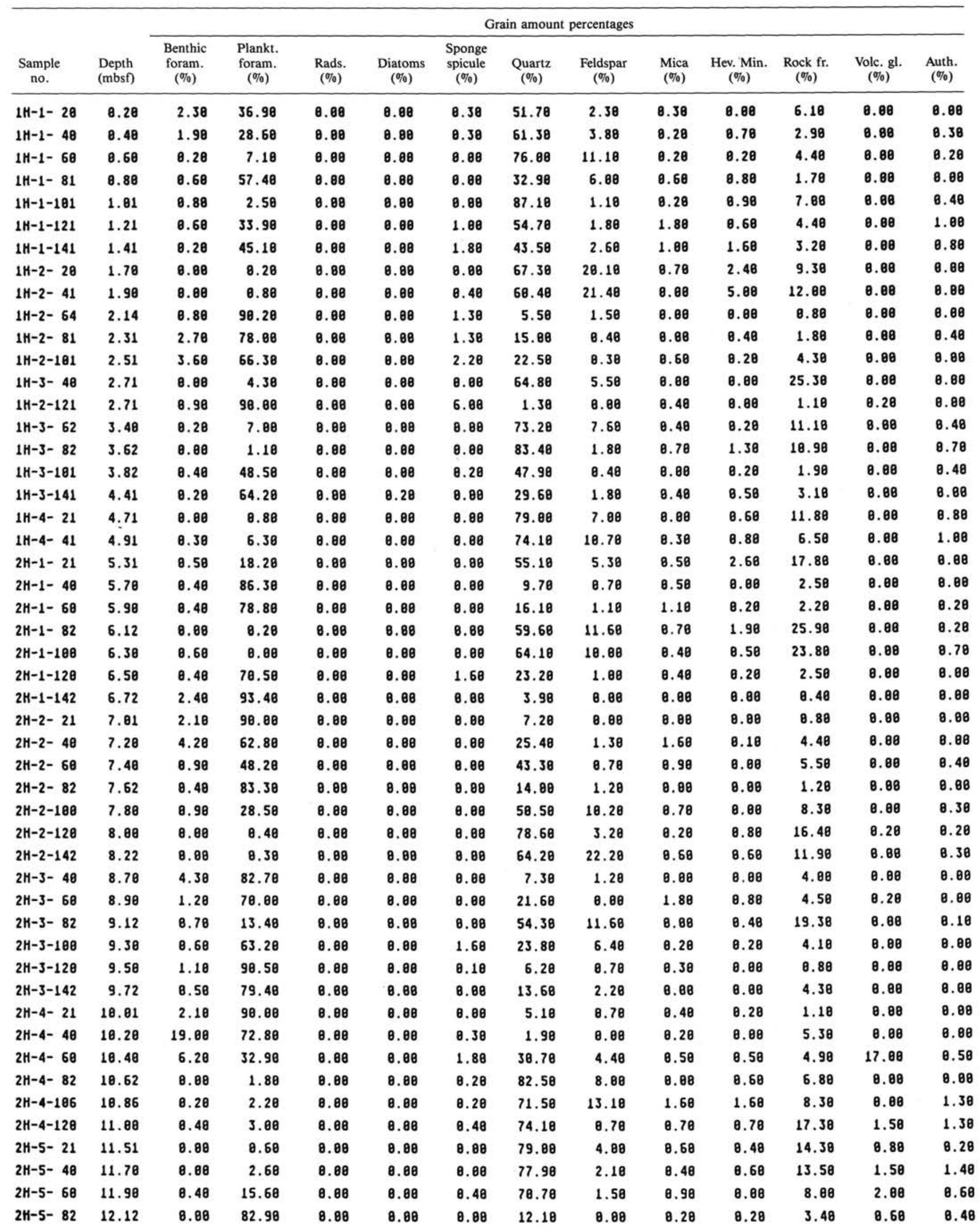


Table 1.3 (continued).

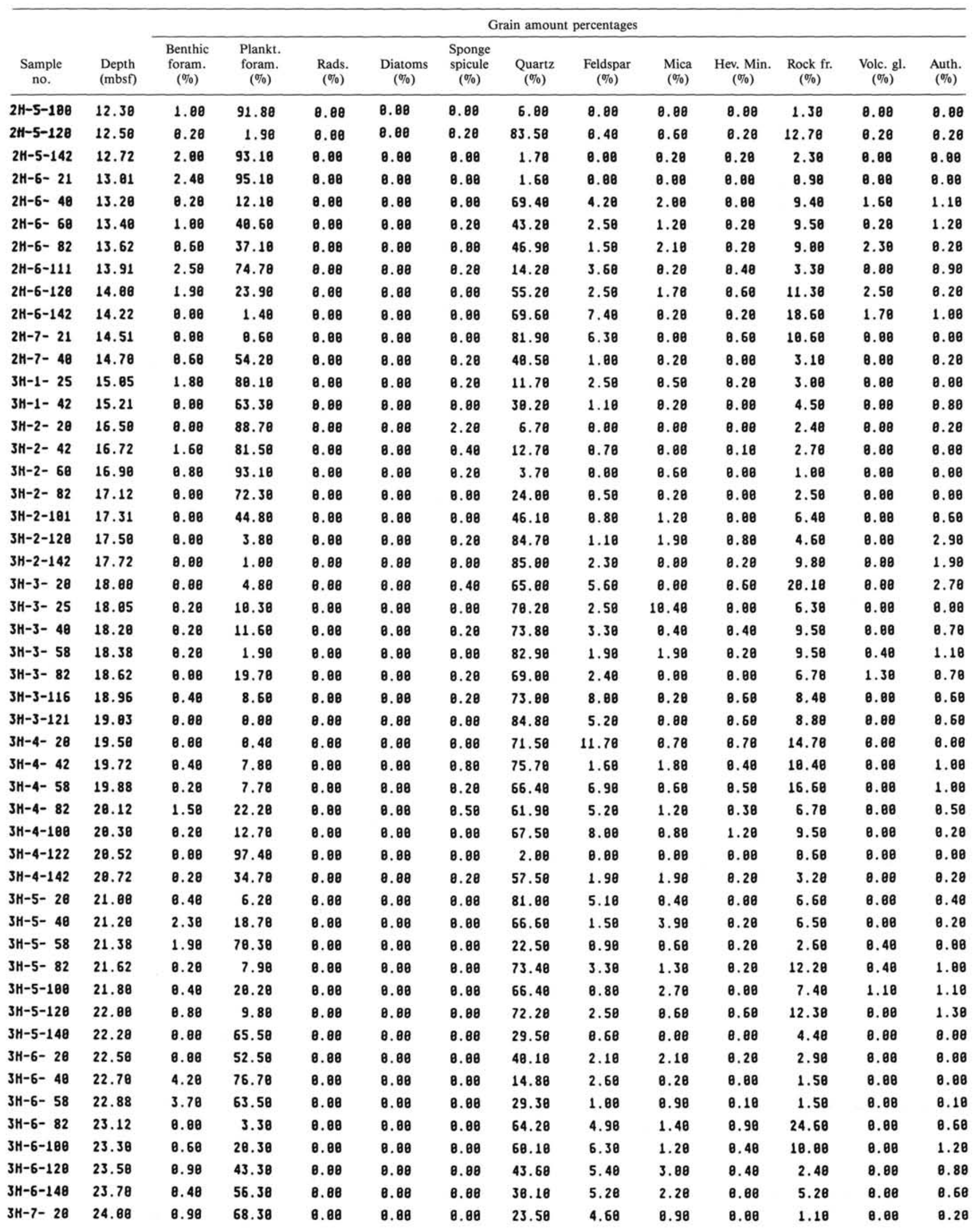


Table 1.3 (continued).

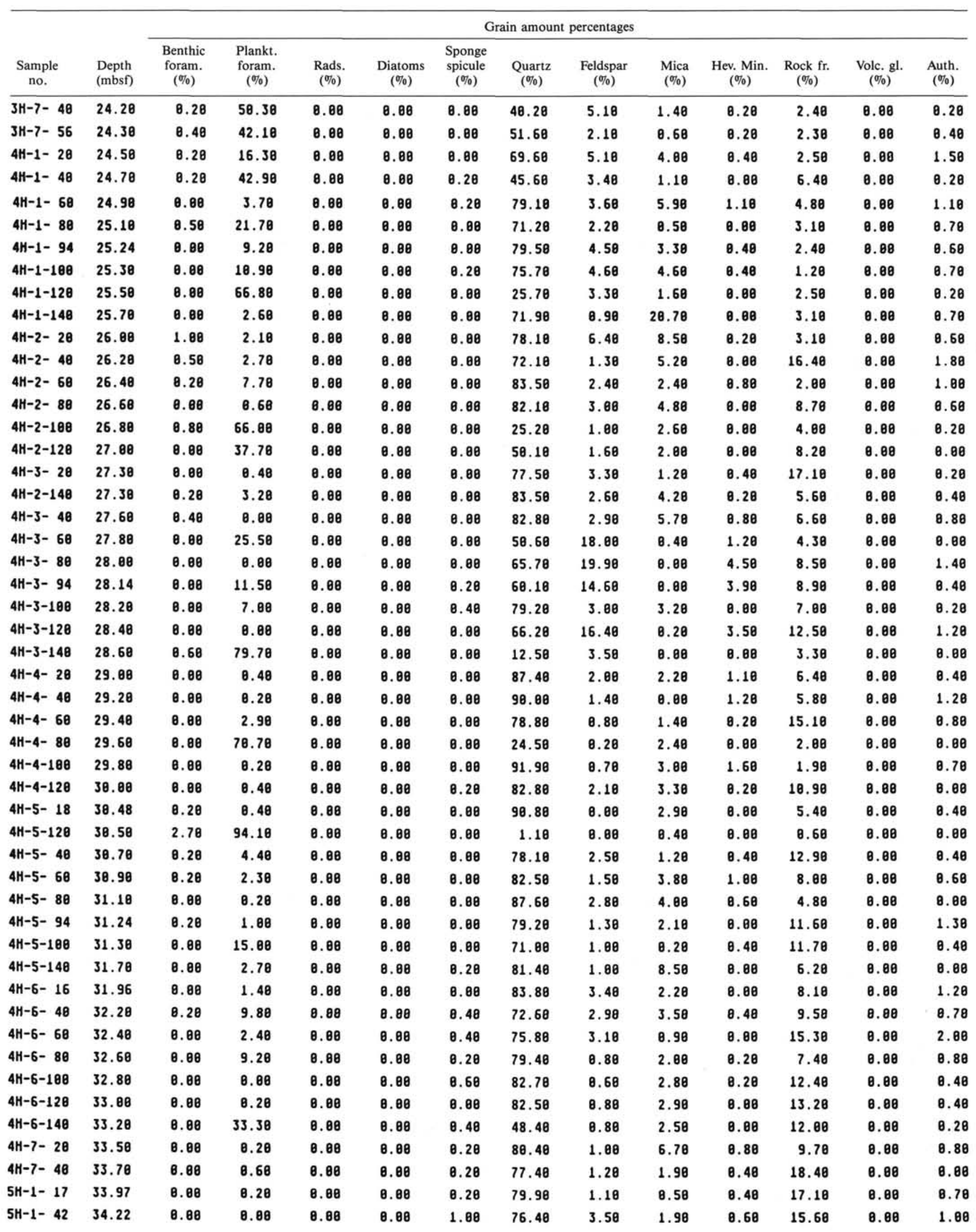


Table 1.3 (continued).




Table 1.3 (continued).

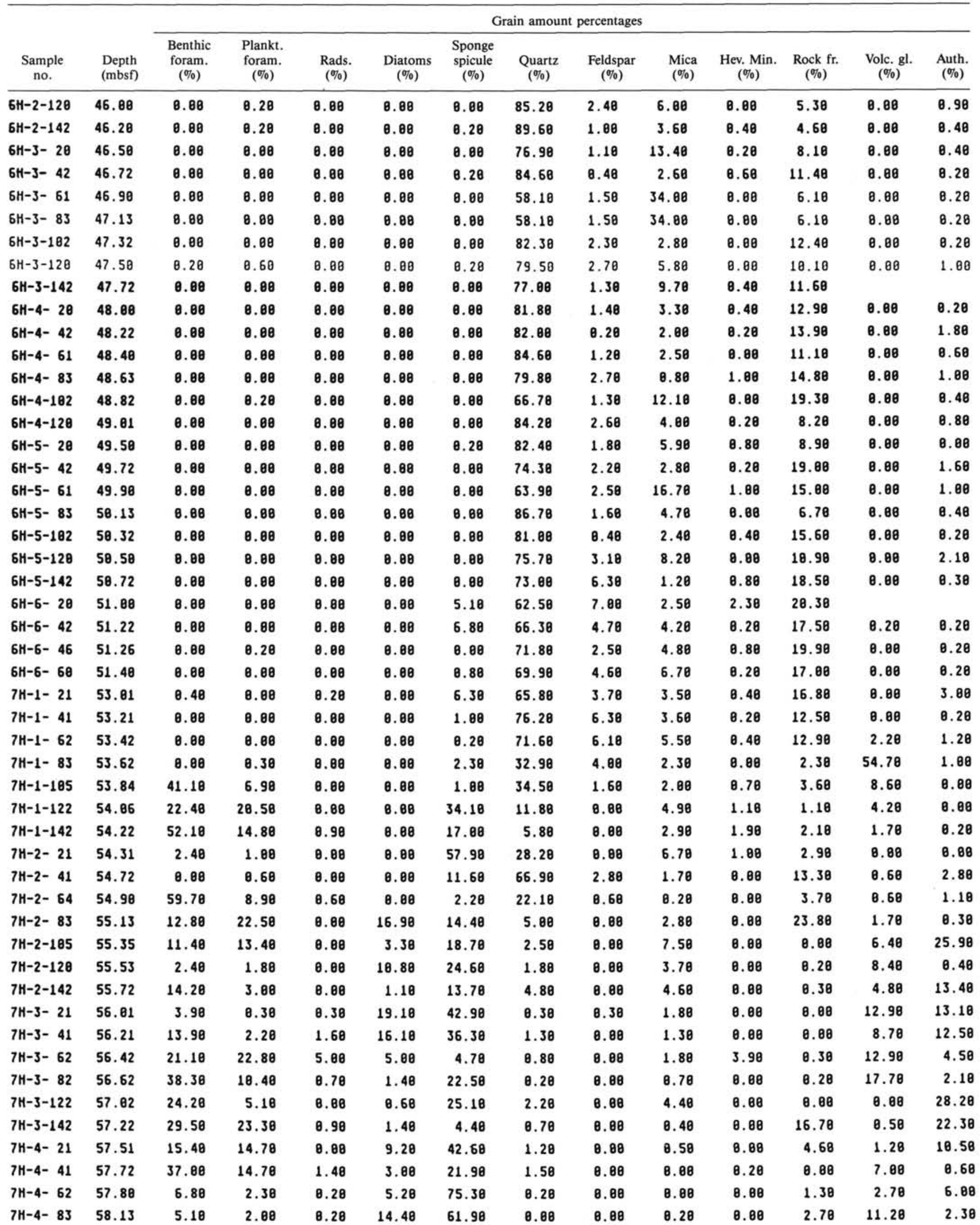


Table 1.3 (continued).

\begin{tabular}{|c|c|c|c|c|c|c|c|c|c|c|c|c|c|}
\hline \multirow[b]{2}{*}{$\begin{array}{c}\text { Sample } \\
\text { no. }\end{array}$} & \multirow[b]{2}{*}{$\begin{array}{l}\text { Depth } \\
\text { (mbsf) }\end{array}$} & \multicolumn{12}{|c|}{ Grain amount percentages } \\
\hline & & $\begin{array}{l}\text { Benthic } \\
\text { foram. } \\
(\%)\end{array}$ & $\begin{array}{l}\text { Plankt. } \\
\text { foram. } \\
(\%)\end{array}$ & $\begin{array}{l}\text { Rads. } \\
(\%)\end{array}$ & $\begin{array}{c}\text { Diatoms } \\
(\%)\end{array}$ & $\begin{array}{l}\text { Sponge } \\
\text { spicule } \\
(\%)\end{array}$ & $\begin{array}{c}\text { Quartz } \\
(\%)\end{array}$ & $\begin{array}{c}\text { Feldspar } \\
(\%)\end{array}$ & $\begin{array}{l}\text { Mica } \\
(\%)\end{array}$ & $\begin{array}{l}\text { Hev. Min. } \\
(\%)\end{array}$ & $\begin{array}{c}\text { Rock fr. } \\
(\%)\end{array}$ & $\begin{array}{l}\text { Volc. gl. } \\
(\%)\end{array}$ & $\begin{array}{l}\text { Auth. } \\
(\%)\end{array}$ \\
\hline $7 \mathrm{H}-4-185$ & 58.34 & 18.98 & 2.78 & 6.80 & 1.98 & 65.38 & 8.08 & 8.88 & 8.88 & 8.68 & 3.88 & 7.78 & 1.98 \\
\hline $7 H-4-122$ & 58.53 & 11.88 & 6.88 & 3.60 & 8.28 & 72.38 & 0.28 & 8.80 & 8.08 & 8.88 & 0.48 & 6.30 & 8.28 \\
\hline $7 \mathrm{H}-4-142$ & 58.72 & 23.80 & 33.38 & 6.28 & 8.88 & 28.78 & 1.80 & 8.88 & 0.80 & 8.08 & 0.58 & 7.48 & 0.28 \\
\hline $7 \mathrm{H}-5-21$ & 59.81 & 28.48 & 28.48 & 2.18 & 0.80 & 11.78 & 7.28 & 0.88 & 8.28 & 8.88 & 2.50 & 26.58 & 1.10 \\
\hline $7 H-5-41$ & 59.21 & 11.48 & 8.80 & 2.48 & 0.80 & 53.60 & 2.80 & 8.80 & 0.48 & 8.80 & 3.58 & 18.80 & 8.80 \\
\hline $7 H-5-62$ & 59.42 & 1.80 & 1.88 & 10.28 & 0.48 & 64.78 & 8.38 & 8.08 & 2.48 & 8.80 & 8.38 & 16.80 & 3.78 \\
\hline $7 H-5-83$ & 59.62 & 0.28 & 1.28 & $\theta .80$ & 14.98 & 1.98 & 0.08 & 8.50 & 8.98 & 8.58 & 8.88 & 25.38 & 54.48 \\
\hline $7 H-5-105$ & 59.84 & 0.88 & 8. 88 & 1.58 & 8.80 & 47.68 & 8.80 & 8.88 & 4.88 & 1.18 & 8.88 & 22.98 & 13.58 \\
\hline $7 H-5-122$ & 60.02 & 8.08 & $0.4 \theta$ & 2.58 & 8.48 & 47.88 & 9.38 & 8.00 & 1.88 & 8.80 & 33.18 & 5.80 & 8.48 \\
\hline $7 H-6-12$ & 68.42 & 0.88 & 0.80 & 1.48 & 8.88 & 57.88 & 3.68 & 0.80 & 8.48 & 8.80 & 18.88 & 26.68 & 8.28 \\
\hline $8 H-1-35$ & 62.65 & 8.88 & 1.28 & 1.80 & 8.08 & 42.58 & 18.38 & 8.28 & 0.28 & 8.48 & 13.80 & 18.68 & 8.88 \\
\hline $8 \mathrm{H}-2-35$ & 64.15 & 0.80 & 0.88 & 2.00 & 8.08 & 38.80 & 8.60 & 8.88 & 5.78 & 8.88 & 9.60 & 1.28 & 0.08 \\
\hline $8 \mathrm{H}-3-35$ & 65.65 & 8.80 & 0.30 & 5.58 & 8.88 & 71.38 & 18.78 & 8.88 & 8.30 & 0.08 & 3.98 & 8.80 & 8.88 \\
\hline $8 H-4-35$ & 67.15 & 0.28 & 8.80 & $5.2 \theta$ & 0.68 & 52.80 & 6.28 & 8.80 & 27.78 & 0.80 & 7.48 & $\theta .80$ & $\theta . \theta 0$ \\
\hline $8 \mathrm{H}-5-35$ & 68.65 & 8.88 & 8.80 & 51.78 & 4.58 & 24.68 & 1.78 & 8.88 & 18.48 & 8.88 & 0.48 & 3.78 & 8.88 \\
\hline $8 H-6-35$ & 78.15 & 0.80 & 0.08 & 18.88 & 0.88 & 28.78 & 3.88 & 8.80 & 13.88 & $8 . \theta 8$ & 3.48 & 31.58 & 8.88 \\
\hline $9 H-7-48$ & 71.28 & 1.88 & $0.2 \theta$ & 55.10 & 8.88 & 18.88 & 3.48 & 8.80 & 0.88 & 0.00 & 1.28 & 28.80 & 8.80 \\
\hline $8 H-C-36$ & 71.66 & 0.88 & 0.08 & 6.10 & $\theta .08$ & 36.48 & 9.18 & 8.88 & 16.58 & 8.80 & 3.88 & 27.38 & 0.80 \\
\hline $9 H-1-48$ & 72.28 & 1.80 & 6.68 & 3.38 & $\theta .88$ & 35.10 & 1.28 & $\theta . \theta 0$ & 2.98 & 0.88 & 1.78 & 47.80 & 8.80 \\
\hline $9 H-2-48$ & 73.78 & 2.78 & 4.08 & 35.10 & 8.80 & 38.78 & 2.10 & 8.88 & 1.78 & 8.80 & 3.88 & 11.88 & 8.80 \\
\hline $9 H-3-48$ & 75.28 & 8.08 & 0.88 & 13.48 & 8.80 & 39.60 & 4.98 & 8.88 & 8.68 & $\theta . \theta 8$ & 3.80 & 38.58 & 8.80 \\
\hline $9 H-4-48$ & 76.78 & 1.88 & 8.88 & 78.98 & 8.68 & 6.88 & 1.78 & 0.88 & 8.68 & 8.88 & 1.78 & 9.48 & 0.88 \\
\hline $9 H-6-48$ & 79.78 & 1.10 & 0.70 & $0.9 \theta$ & 0.08 & 11.60 & 1.60 & 0.80 & 8.58 & 8.88 & 1.88 & 81.78 & $\theta . \theta 0$ \\
\hline $18 \mathrm{H}-1-4 \theta$ & 81.78 & 1.98 & 6.28 & 13.88 & 8.58 & 34.28 & 2.58 & $\theta .8 \theta$ & 0.80 & 8.80 & 1.50 & 38.88 & 0.80 \\
\hline $18 \mathrm{H}-2-48$ & 83.28 & 2.80 & 0.48 & 27.88 & 1.30 & 28.48 & 8.98 & 0.08 & 0.28 & 8.88 & 1.38 & 44.98 & 0.80 \\
\hline $10 \mathrm{H}-3-48$ & 84.78 & 8.80 & 8.80 & 7.58 & 2.60 & 9.30 & 0.58 & 8.80 & 8.60 & 8.08 & 2.28 & 71.98 & 8.80 \\
\hline $18 \mathrm{BH}-4-48$ & 86.28 & 5.98 & 17.78 & 24.28 & 8.88 & 14.28 & 2.68 & 0.80 & 13.68 & $8.8 \theta$ & 1.50 & 14.18 & 8.80 \\
\hline $18 \mathrm{H}-5-48$ & 87.78 & 0.80 & 0.28 & 73.88 & $\theta . \theta \theta$ & 6.90 & 1.88 & 0.80 & 3.98 & 0.80 & 1.78 & 4.58 & $\theta .8 \theta$ \\
\hline $18 \mathrm{H}-6-4 \theta$ & 89.28 & 8.28 & 8.80 & 68.88 & 0.60 & 19.48 & 8.48 & 8.88 & 8.80 & 8.88 & 1.98 & 18.78 & 8.80 \\
\hline $18 \mathrm{H}-7-4 \theta$ & 98.78 & 1.78 & 0.80 & 43.28 & 8.08 & 18.50 & 1.98 & 8.88 & 0.88 & 8.88 & 1.28 & 21.88 & 0.28 \\
\hline $11 \mathrm{H}-1-48$ & 91.28 & 8.80 & 8.80 & 4.98 & 8.88 & 21.98 & 1.60 & 8.88 & 8.68 & 8.08 & 0.48 & 78.28 & 8.80 \\
\hline $11 \mathrm{H}-2-4 \theta$ & 92.78 & 0.28 & 0.80 & 9.10 & 5.98 & 12.98 & 6.38 & 8.88 & 8.28 & 0.80 & 1.58 & 55.38 & 8.80 \\
\hline $11 \mathrm{H}-3-48$ & 94.28 & 0.08 & 0.80 & 37.88 & 8.88 & 28.38 & 1.88 & 8.88 & 8.28 & 0.80 & 3.28 & 29.58 & 0.88 \\
\hline $11 \mathrm{H}-4-48$ & 95.78 & $\theta . \theta 8$ & 0.80 & 85.38 & $\theta .88$ & 8.58 & 1.10 & 8.88 & 0.28 & 8.80 & 1.18 & 3.28 & 0.88 \\
\hline $11 \mathrm{H}-5-4 \theta$ & 97.28 & $\theta .08$ & 0.80 & $32.6 \theta$ & 2.88 & 35.38 & 0.48 & 8.08 & 0.00 & 8.80 & 8.48 & 21.48 & 8.80 \\
\hline $11 \mathrm{H}-6-48$ & 98.70 & 0.60 & 8.60 & 2.68 & 2.28 & 14.28 & 0.60 & 8.88 & 8.80 & 0.00 & 0.28 & 9.18 & 0.88 \\
\hline $11 \mathrm{H}-7-16$ & 99.96 & 0.28 & 8.80 & 4.68 & 2.88 & 17.78 & 0.78 & 8.88 & 0.88 & 8.80 & 5.18 & 56.38 & 8.88 \\
\hline $12 \mathrm{H}-1-4 \theta$ & 180.78 & 8.08 & 0.80 & 24.68 & 0.38 & 39.88 & 6.10 & 8.88 & 0.80 & $\theta .80$ & 6.80 & 22.58 & 0.80 \\
\hline $12 \mathrm{H}-2-4 \theta$ & 102.28 & 8.88 & 8.88 & 43.98 & 1.38 & 39.58 & 0.80 & 0.08 & $\theta .80$ & 8.88 & 2.78 & 11.78 & 0.80 \\
\hline $12 \mathrm{H}-3-48$ & 103.78 & 8.80 & 8.88 & 18.48 & 6.28 & 22.98 & 9.80 & 8.88 & 8.80 & 8.88 & 2.88 & 48.68 & 8.80 \\
\hline $12 \mathrm{H}-4-48$ & 105.28 & 0.68 & 0.80 & 9.38 & 9.98 & 23.28 & 0.48 & 0.88 & 8.08 & 8.88 & 5.38 & 52.88 & 0.80 \\
\hline $12 \mathrm{H}-5-48$ & 106.78 & $0.2 \theta$ & 0.80 & 27.88 & 34.48 & 22.88 & 1.58 & 8. 88 & 8.88 & 8.88 & 8.80 & 13.38 & 8.88 \\
\hline $12 \mathrm{H}-6-48$ & 108.28 & 0.88 & 0.80 & 71.18 & 18.78 & 9.38 & 2.80 & 8.88 & 8.80 & 8.88 & 3.28 & 2.88 & 8.28 \\
\hline $13 \mathrm{H}-1-48$ & 118.28 & 8.88 & 0.80 & 29.98 & 25.98 & 22.28 & 1.18 & 0.80 & 8.80 & 8.80 & 4.68 & 16.38 & 8.08 \\
\hline $13 \mathrm{H}-2-48$ & 111.78 & 0.08 & 0.88 & 38.50 & 13.58 & 16.28 & 23.30 & $8 . \theta 0$ & 0.88 & 0.28 & 6.68 & 9.78 & 8.80 \\
\hline $13 \mathrm{H}-3-48$ & 113.28 & 8.80 & 0.88 & 78.58 & 2.28 & 1.98 & 2.78 & 8.80 & 8.80 & 0.28 & 3.18 & 11.48 & 8.88 \\
\hline $13 \mathrm{H}-4-48$ & 114.78 & $\theta . \theta \theta$ & 0.88 & 32.58 & 31.28 & 32.38 & 8.48 & 0.80 & 0.80 & 8.88 & 8.28 & 3.30 & 8.88 \\
\hline $13 H-5-48$ & 116.28 & $\theta .08$ & 0.80 & 27.88 & 35.58 & 36.78 & $\theta . \theta 8$ & 8.80 & 8.88 & 0.08 & 8.80 & 0.88 & 8.88 \\
\hline $13 \mathrm{H}-6-48$ & 117.78 & 0.08 & 3.10 & 53.58 & 4.38 & 21.78 & 0.30 & 0.80 & 8.88 & 8.00 & 3.80 & 13.98 & 8.08 \\
\hline $13 \mathrm{H}-7-48$ & 119.28 & 0.28 & 0.88 & 35.98 & 24.98 & 9.58 & 8.50 & 8.88 & 8.80 & 8.88 & 8.88 & 1.18 & 8.58 \\
\hline
\end{tabular}


Table 1.3 (continued).

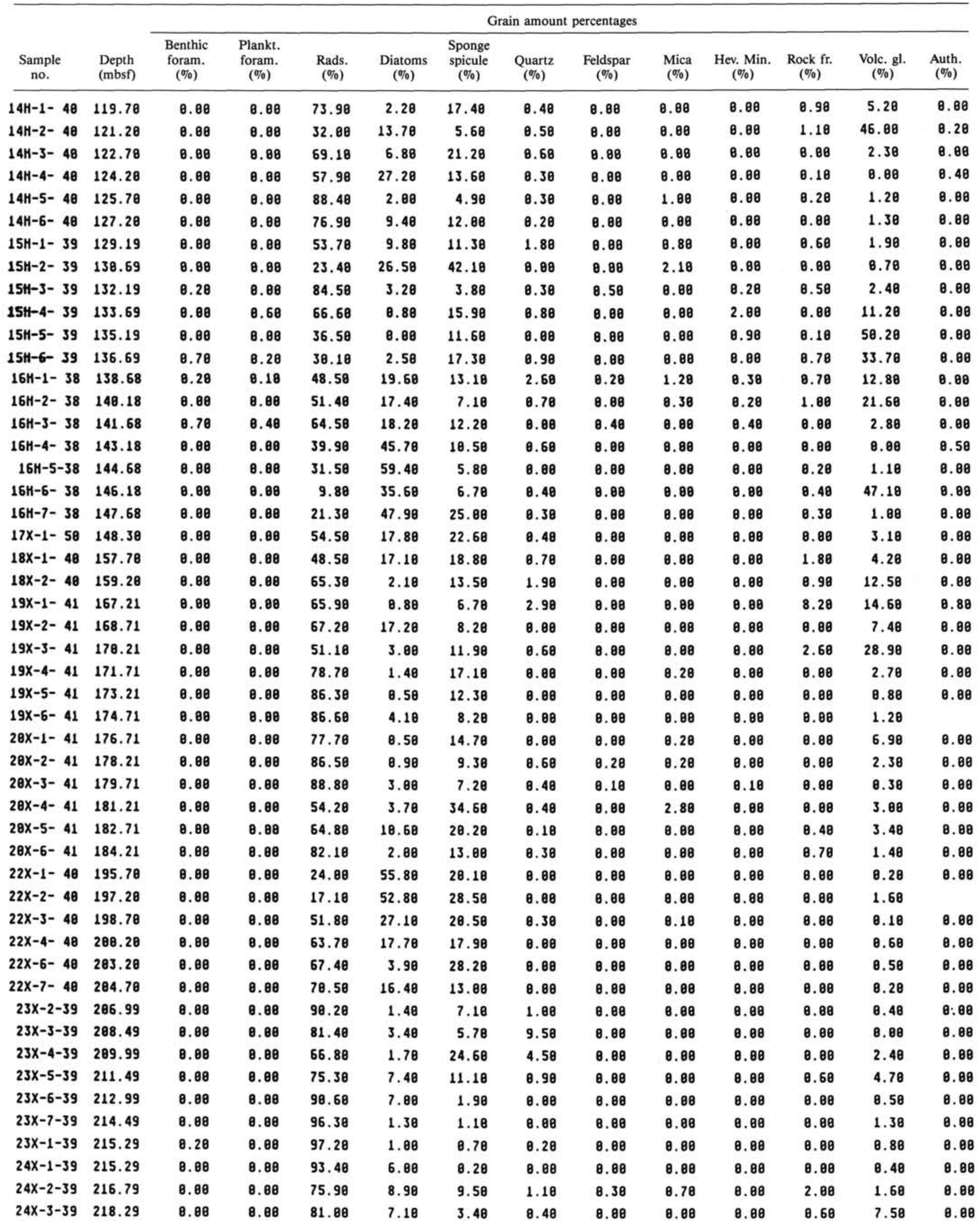


Table 1.3 (continued).

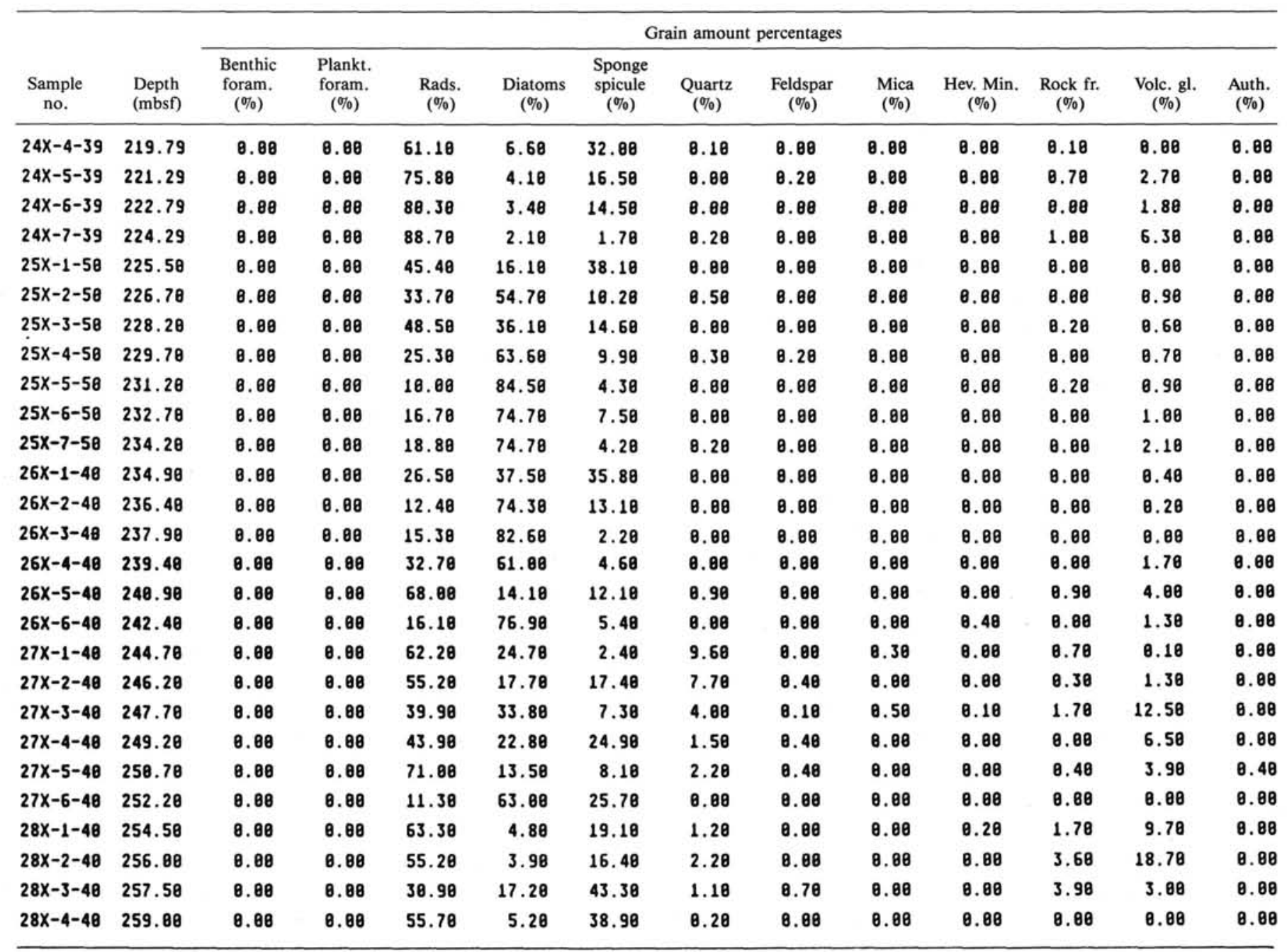


Table 1.3 (continued).

\begin{tabular}{|c|c|c|c|c|c|c|}
\hline \multirow[b]{2}{*}{$\begin{array}{c}\text { Sample } \\
\text { no. }\end{array}$} & \multirow[b]{2}{*}{$\begin{array}{l}\text { Depth } \\
\text { (mbsf) }\end{array}$} & \multicolumn{5}{|c|}{ Subfraction of coarse fraction $(\mu \mathrm{m})$} \\
\hline & & $\begin{array}{l}>63 \\
(\%)\end{array}$ & $\begin{array}{c}63-125 \\
(\%)\end{array}$ & $\begin{array}{c}125-500 \\
(\%)\end{array}$ & $\begin{array}{c}500-1000 \\
(\%)\end{array}$ & $\begin{array}{c}>1000 \\
(\%)\end{array}$ \\
\hline $1 H-1-28$ & 0.28 & 18.81 & 49.78 & 47.57 & 2.24 & 0.48 \\
\hline $1 H-1-48$ & 0.48 & 25.37 & 58.21 & 45.44 & 2.67 & 1.68 \\
\hline $1 H-1-68$ & 0.68 & 4.95 & 74.72 & 24.15 & 1.11 & 8.82 \\
\hline $1 H-1-81$ & 8.88 & 9.81 & 65.72 & 32.95 & 1.21 & 0.12 \\
\hline $1 H-1-181$ & 1.01 & 5.64 & 62.28 & 33.56 & 3.81 & 0.43 \\
\hline $1 H-1-121$ & 1.21 & 21.29 & 56.81 & 42.98 & 0.88 & 0.21 \\
\hline $1 H-1-141$ & 1.41 & 24.87 & 33.67 & 64.78 & 0.71 & 0.84 \\
\hline $1 \mathrm{H}-2-28$ & 1.70 & 26.81 & 79.38 & 28.28 & 0.28 & 0.22 \\
\hline $1 \mathrm{H}-2-41$ & 1.98 & 19.33 & 71.48 & 27.34 & 1.89 & 0.89 \\
\hline $1 \mathrm{H}-2-64$ & 2.14 & 37.37 & 54.38 & 45.84 & 0.17 & 0.49 \\
\hline $1 \mathrm{H}-2-81$ & 2.31 & 38.63 & 17.23 & 81.48 & 0.78 & B. 51 \\
\hline $1 \mathrm{H}-2-101$ & 2.51 & 27.59 & 19.72 & 78.32 & 1.86 & 0.89 \\
\hline $1 H-3-62$ & 3.62 & 21.61 & 94.11 & 5.18 & 0.48 & 0.30 \\
\hline $1 H-3-101$ & 4.81 & 13.29 & 51.88 & 44.37 & 2.71 & 1.12 \\
\hline $1 \mathrm{H}-3-121$ & 4.21 & 15.44 & 59.66 & 34.79 & 4.48 & 1.15 \\
\hline $1 \mathrm{H}-3-141$ & 4.41 & 18.33 & 66.92 & 25.17 & 8.75 & 7.15 \\
\hline $1 H-4-21$ & 4.71 & 29.08 & 58.98 & 46.86 & 1.61 & 0.55 \\
\hline $1 \mathrm{H}-4-41$ & 4.91 & 16.78 & 58.64 & 43.86 & 4.98 & 8.68 \\
\hline $2 \mathrm{H}-1-21$ & 5.31 & 17.31 & 53.88 & 45.13 & 1.59 & 0.28 \\
\hline $2 \mathrm{H}-1-48$ & 5.78 & 24.82 & 25.17 & 78.73 & 1.38 & 2.73 \\
\hline $2 H-1-68$ & 5.98 & 9.54 & 34.26 & 64.60 & 1.00 & 0.14 \\
\hline $2 H-1-82$ & 6.12 & 18.23 & 66.30 & 38.16 & 1.96 & 1.58 \\
\hline $2 \mathrm{H}-1-18 \theta$ & 6.30 & 28.98 & 58.84 & 36.56 & 3.38 & 1.21 \\
\hline $2 H-1-128$ & 6.58 & 5.83 & 36.52 & 62.13 & 1.27 & 0.08 \\
\hline $2 \mathrm{H}-1-142$ & 6.72 & 21.53 & 14.77 & 84.68 & 8.58 & 0.85 \\
\hline $2 \mathrm{H}-2-4 \theta$ & 7.28 & 11.13 & 41.27 & 57.76 & 0.95 & 0.02 \\
\hline $2 \mathrm{H}-2-68$ & 7.48 & 13.34 & 66.74 & 38.97 & 1.88 & 0.41 \\
\hline $2 \mathrm{H}-2-82$ & 7.62 & 17.78 & 26.77 & 71.68 & 1.44 & 0.19 \\
\hline $2 \mathrm{H}-2-18 \theta$ & 7.80 & 9.38 & 59.57 & 38.31 & 1.97 & 0.15 \\
\hline $2 \mathrm{H}-2-128$ & 8.80 & 9.17 & 63.84 & 33.02 & 2.54 & 8.68 \\
\hline $2 \mathrm{H}-3-21$ & 8.51 & 1.18 & 99.96 & 8.88 & 8.80 & 8.84 \\
\hline $2 \mathrm{H}-3-48$ & 8.78 & 2.93 & 44.64 & 55.16 & 0.28 & 8.88 \\
\hline $2 \mathrm{H}-3-6 \theta$ & 8.98 & 12.62 & 31.98 & 65.68 & 2.26 & 0.15 \\
\hline $2 \mathrm{H}-3-82$ & 9.12 & 6.83 & 3.93 & 38.76 & 56.26 & 1.85 \\
\hline $2 \mathrm{H}-3-18 \theta$ & 9.38 & 11.21 & 45.59 & 53.32 & 1.04 & 8.85 \\
\hline $2 \mathrm{H}-3-128$ & 9.58 & 21.16 & 28.36 & 69.36 & 0.68 & $1.6 \theta$ \\
\hline $2 \mathrm{H}-3-142$ & 9.72 & 16.53 & 45.21 & 58.98 & 2.80 & 1.81 \\
\hline $2 \mathrm{H}-4-21$ & 10.81 & 10.48 & 32.44 & 67.06 & 0.49 & 0.01 \\
\hline $2 H-4-4 \theta$ & 18.28 & 1.48 & 58.47 & 41.53 & 0.88 & 8.88 \\
\hline $2 \mathrm{H}-4-6 \theta$ & 10.48 & 8.28 & 52.74 & 47.26 & 8.88 & $\theta . \theta 8$ \\
\hline $2 \mathrm{H}-4-82$ & 10.62 & 24.78 & 45.89 & 52.75 & 1.86 & 0.38 \\
\hline $2 H-4-128$ & 11.88 & 6.98 & 59.79 & 33.61 & 5.86 & 8.74 \\
\hline $2 \mathrm{H}-5-21$ & 11.51 & 14.48 & 58.98 & 48.64 & 8.34 & 0.12 \\
\hline $2 \mathrm{H}-5-48$ & 11.78 & 15.84 & 62.75 & 34.97 & 1.98 & 0.38 \\
\hline $2 \mathrm{H}-5-68$ & 11.98 & 28.95 & 98.53 & 0.88 & 1.28 & 0.19 \\
\hline $2 H-5-82$ & 12.12 & 13.62 & 38.37 & 57.12 & 1.83 & 2.68 \\
\hline $2 H-5-18 \theta$ & 12.38 & 10.58 & 17.87 & 80.66 & 1.38 & 0.17 \\
\hline $2 H-5-12 \theta$ & 12.58 & 11.58 & 53.88 & 44.22 & 2.32 & 0.46 \\
\hline $2 H-5-142$ & 12.72 & 15.68 & 15.28 & 83.84 & 0.94 & 8.82 \\
\hline $2 H-6-21$ & 13.01 & 18.87 & 38.78 & 68.94 & 0.28 & 8.88 \\
\hline $2 \mathrm{H}-6-4 \mathrm{~B}$ & 13.28 & 16.48 & 49.19 & 58.25 & 0.57 & 0.88 \\
\hline $2 \mathrm{H}-6-68$ & 13.48 & 2.58 & 60.13 & 37.65 & 2.22 & 0.08 \\
\hline
\end{tabular}


Table 1.3 (continued).

\begin{tabular}{|c|c|c|c|c|c|c|}
\hline \multirow[b]{2}{*}{$\begin{array}{c}\text { Sample } \\
\text { no. }\end{array}$} & \multirow[b]{2}{*}{$\begin{array}{l}\text { Depth } \\
\text { (mbsf) }\end{array}$} & \multicolumn{5}{|c|}{ Subfraction of coarse fraction $(\mu \mathrm{m})$} \\
\hline & & $\begin{array}{l}>63 \\
(\%)\end{array}$ & $\begin{array}{c}63-125 \\
(\%)\end{array}$ & $\begin{array}{c}125-500 \\
(\%)\end{array}$ & $\begin{array}{c}500-1000 \\
(\%)\end{array}$ & $\begin{array}{c}>1000 \\
(\%)\end{array}$ \\
\hline $2 \mathrm{H}-6-82$ & 13.62 & 2.55 & 47.75 & 49.46 & 2.88 & 8.88 \\
\hline $2 \mathrm{H}-6-111$ & 13.91 & 9.24 & 46.86 & 58.97 & 1.85 & 8. 32 \\
\hline $2 H-6-12 \theta$ & 14.08 & 6.20 & 76.98 & 22.07 & 8.95 & 0.88 \\
\hline $2 \mathrm{H}-6-142$ & 14.22 & 28.32 & 71.12 & 26.52 & 1.52 & 0.84 \\
\hline $2 \mathrm{H}-7-21$ & 14.51 & 23.48 & 52.95 & 46.78 & 0.31 & 0.04 \\
\hline $2 \mathrm{H}-7-48$ & 14.78 & 6.42 & 68.71 & 35.78 & 2.63 & 8.88 \\
\hline $3 H-1-25$ & 15.85 & 18.89 & 29.38 & 69.55 & 0.74 & 8.33 \\
\hline $3 H-1-42$ & 15.21 & 9.10 & 48.24 & 49.56 & 1.96 & 0.24 \\
\hline $3 \mathrm{H}-2-28$ & 16.50 & 8.78 & 25.79 & 73.87 & 1.13 & 8.82 \\
\hline $3 H-2-42$ & 16.72 & 11.14 & 37.02 & 58.74 & 3.48 & 8.75 \\
\hline $3 H-2-6 \theta$ & 16.98 & 19.61 & 27.25 & 78.95 & 1.78 & 0.18 \\
\hline $3 H-2-82$ & 17.12 & 15.11 & 42.87 & 56.81 & 1.83 & 8.89 \\
\hline $3 H-2-101$ & 17.31 & 2.63 & 65.58 & 33.88 & 1.58 & 8.01 \\
\hline $3 H-2-12 \theta$ & 17.58 & 7.11 & 58.43 & 42.84 & 6.46 & 0.27 \\
\hline $3 H-2-142$ & 17.72 & 28.18 & 51.28 & 44.81 & 3.11 & 1.68 \\
\hline $3 \mathrm{H}-3-28$ & 18.80 & 6.84 & 63.28 & 34.16 & 2.44 & 0.28 \\
\hline $3 H-3-25$ & 18.85 & 19.68 & 88.99 & 18.72 & 0.26 & 0.84 \\
\hline $3 H-3-48$ & $18.2 \theta$ & 4.48 & 53.71 & 44.74 & 1.41 & 8.14 \\
\hline $3 \mathrm{H}-3-58$ & 18.38 & 17.97 & 44.68 & 53.37 & 1.73 & 8.38 \\
\hline $3 \mathrm{H}-3-82$ & 18.62 & 8.69 & 58.98 & 39.69 & 1.33 & 0.01 \\
\hline $3 H-3-18 \theta$ & 18.80 & 1.45 & 72.32 & 24.65 & 2.79 & 0.24 \\
\hline $3 H-3-116$ & 18.96 & 2.80 & 73.31 & 26.69 & 8.80 & 0.88 \\
\hline $3 H-3-121$ & 19.83 & 8.92 & 54.29 & 42.92 & 2.52 & 0.27 \\
\hline $3 H-4-28$ & 19.58 & 16.68 & 43.49 & 58.54 & 5.16 & 8.81 \\
\hline $3 \mathrm{H}-4-42$ & 19.72 & 2.68 & 65.46 & 31.86 & 3.29 & 8.19 \\
\hline $3 H-4-58$ & 19.88 & 2.38 & 88.32 & 17.66 & 1.65 & 0.37 \\
\hline $3 H-4-82$ & 28.12 & 3.10 & 88.54 & 18.04 & 1.42 & 8.80 \\
\hline $3 H-4-188$ & 28.38 & 11.76 & 57.37 & 39.21 & 3.11 & 8.32 \\
\hline $3 \mathrm{H}-4-122$ & 28.52 & 14.78 & 15.60 & 83.99 & 8.38 & 0.83 \\
\hline $3 H-4-142$ & 28.72 & 6.33 & 77.84 & 22.84 & 8.88 & 0.84 \\
\hline $3 H-5-28$ & 21.88 & 2.41 & 64.28 & 32.86 & 2.94 & 8.88 \\
\hline $3 H-5-4 \theta$ & 21.28 & 10.41 & 67.88 & 31.85 & 0.94 & 8.14 \\
\hline $3 \mathrm{H}-5-58$ & 21.38 & 6.44 & 15.67 & 81.61 & 2.64 & 0.88 \\
\hline $3 H-5-82$ & 21.62 & 6.38 & 78.39 & 19.48 & 2.86 & 0.87 \\
\hline $3 H-5-18 \theta$ & 21.80 & 4.11 & 65.48 & 32.83 & 1.62 & 0.08 \\
\hline $3 H-5-128$ & 22.80 & 12.18 & 64.46 & 33.25 & 1.86 & 0.43 \\
\hline $3 H-5-148$ & 22.28 & 6.52 & 49.39 & 49.23 & 1.30 & 8.09 \\
\hline $3 H-6-28$ & 22.58 & 5.78 & 44.63 & 53.34 & 1.98 & 8.85 \\
\hline $3 H-6-4 \theta$ & 22.78 & 7.78 & 44.53 & 58.68 & 4.85 & 8.82 \\
\hline $3 H-6-58$ & 22.88 & $12.6 \theta$ & 67.88 & 31.88 & 1.76 & 8.16 \\
\hline $3 H-6-82$ & 23.12 & 6.95 & 66.72 & 30.72 & 2.41 & 0.15 \\
\hline $3 \mathrm{H}-6-188$ & 23.38 & 2.98 & 65.51 & 31.93 & 2.51 & 0.85 \\
\hline $3 H-6-128$ & 23.58 & 3.98 & 72.88 & 26.43 & 1.31 & 0.18 \\
\hline $3 H-6-148$ & 23.78 & 4.22 & 59.32 & 39.42 & 1.23 & 0.03 \\
\hline $3 H-7-2 \theta$ & 24.80 & 9.10 & 43.38 & 54.77 & 1.82 & 0.11 \\
\hline $3 H-7-48$ & 24.28 & 14.38 & 46.45 & 51.35 & 1.93 & 8.26 \\
\hline $3 H-7-56$ & 24.38 & 13.24 & 45.56 & 52.81 & 1.57 & 8.85 \\
\hline $4 H-1-28$ & 24.58 & 2.18 & 71.16 & 24.52 & 4.25 & 8.07 \\
\hline $4 H-1-48$ & 24.78 & 4.85 & 54.89 & 41.79 & 2.77 & 0.56 \\
\hline $4 H-1-6 \theta$ & 24.98 & 0.83 & 71.47 & 25.43 & 3.18 & 8.80 \\
\hline $4 H-1-88$ & 25.18 & 4.68 & 78.62 & 25.46 & 3.38 & 0.53 \\
\hline $4 t t-1-94$ & 25.24 & 8.86 & 88.27 & 11.22 & 8.48 & 8.83 \\
\hline
\end{tabular}


Table 1.3 (continued).

\begin{tabular}{|c|c|c|c|c|c|c|}
\hline \multirow[b]{2}{*}{$\begin{array}{c}\text { Sample } \\
\text { no. }\end{array}$} & \multirow[b]{2}{*}{$\begin{array}{l}\text { Depth } \\
\text { (mbsf) }\end{array}$} & \multicolumn{5}{|c|}{ Subfraction of coarse fraction $(\mu \mathrm{m})$} \\
\hline & & $\begin{array}{l}>63 \\
(\%)\end{array}$ & $\begin{array}{c}63-125 \\
(\%)\end{array}$ & $\begin{array}{c}125-500 \\
(\%)\end{array}$ & $\begin{array}{c}500-1000 \\
(\%)\end{array}$ & $\begin{array}{c}>1000 \\
(\%)\end{array}$ \\
\hline $4 H-1-18 \theta$ & 25.38 & 7.18 & 82.87 & 17.12 & 8.88 & 0.01 \\
\hline $4 H-1-128$ & 25.50 & 6.58 & 78.53 & 21.47 & 8.88 & 0.80 \\
\hline $4 H-1-148$ & 25.78 & 18.92 & 88.84 & 11.57 & 0.39 & 0.80 \\
\hline $4 \mathrm{H}-2-28$ & 26.80 & 5.48 & 84.62 & 15.34 & 8.88 & 0.04 \\
\hline $4 H-2-48$ & 26.28 & 3.48 & 66.85 & 26.83 & 6.18 & 0.21 \\
\hline $4 H-2-6 \theta$ & 26.48 & 1.21 & 31.47 & 32.28 & 36.24 & $\theta .01$ \\
\hline $4 H-2-88$ & $26.6 \theta$ & 2.58 & 62.88 & 37.15 & 8.80 & 8.85 \\
\hline $4 \mathrm{H}-2-10 \theta$ & 26.88 & 8.92 & 52.89 & 47.25 & 8.63 & 0.03 \\
\hline $4 H-2-12 \theta$ & 27.88 & 6.65 & 62.39 & 37.57 & 8.88 & 8.84 \\
\hline $4 H-2-148$ & 27.38 & 1.67 & 74.67 & 25.28 & 8.80 & 8.85 \\
\hline $4 \mathrm{H}-3-28$ & 27.58 & 11.58 & 69.85 & 28.56 & 1.85 & 0.55 \\
\hline $4 H-3-4 \theta$ & 27.68 & 2.95 & 74.41 & 23.46 & 2.09 & 8.84 \\
\hline $4 \mathrm{H}-3-68$ & 27.88 & 3.88 & 66.85 & 32.97 & 8.98 & 8.88 \\
\hline $4 H-3-88$ & 28.88 & 15.83 & 57.98 & 39.51 & 2.28 & 8.23 \\
\hline $4 H-3-94$ & 28.14 & 7.38 & 59.36 & 36.97 & 3.48 & 8.19 \\
\hline $4 H-3-10 \theta$ & 28.28 & 1.72 & 67.31 & 32.66 & 8.08 & 0.83 \\
\hline $4 H-3-12 \theta$ & 28.48 & 28.04 & 64.27 & 33.32 & 2.87 & 0.34 \\
\hline $4 H-3-148$ & 28.68 & 14.28 & 29.17 & 69.53 & 1.29 & 0.01 \\
\hline $4 H-4-28$ & 29.80 & 17.10 & 79.78 & 19.73 & 8.45 & 0.12 \\
\hline $4 H-4-48$ & 29.28 & 18.28 & 71.85 & 26.71 & 1.19 & 8.24 \\
\hline $4 H-4-68$ & 29.48 & 3.38 & 73.74 & 26.18 & 8.88 & 8.88 \\
\hline $4 H-4-88$ & 29.68 & 18.38 & 44.66 & 55.88 & 8.88 & 8.26 \\
\hline $4 H-4-18 \theta$ & 29.88 & 8.38 & 54.25 & 43.16 & 2.19 & 8.48 \\
\hline $4 H-4-128$ & 30.80 & 6.78 & 74.47 & 25.34 & 8.17 & 8.81 \\
\hline $4 H-5-18$ & 38.48 & 18.95 & 68.54 & 37.72 & 1.62 & 8.13 \\
\hline $4 H-5-48$ & 38.78 & 9.84 & 63.80 & 34.63 & 10 & 8.27 \\
\hline $4 H-5-6 \theta$ & 38.98 & 17.34 & 59.52 & 38.78 & $\theta . \theta 8$ & 1.78 \\
\hline $4 H-5-8 \theta$ & 31.18 & 15.17 & 72.38 & 26.49 & 8.83 & 0.39 \\
\hline $4 H-5-94$ & 31.24 & 18.40 & 98.28 & 8.08 & 8.80 & 1.72 \\
\hline $4 H-5-108$ & 31.38 & $15.3 \theta$ & 92.28 & 7.41 & 8.88 & 8.39 \\
\hline $4 H-5-148$ & 31.78 & 4.88 & 41.99 & 57.98 & $8.8 \theta$ & 8.83 \\
\hline $4 H-6-16$ & 31.96 & 8.38 & 66.48 & 33.51 & 8.88 & 0.01 \\
\hline $4 H-6-48$ & 32.28 & 9.78 & 69.22 & 38.58 & $8.8 \theta$ & 8.28 \\
\hline $4 H-6-6 \theta$ & 32.48 & 15.28 & 82.14 & 17.51 & 8.88 & 0.35 \\
\hline $4 H-6-8 \theta$ & 32.68 & 6.28 & 68.48 & 31.51 & 8.80 & 8.89 \\
\hline $4 H-6-10 \theta$ & 32.88 & 5.58 & 79.81 & 19.28 & 1.57 & 8.14 \\
\hline $4 H-6-128$ & 33.80 & 12.28 & 46.27 & 47.56 & 5.32 & 8.85 \\
\hline $4 H-6-148$ & 33.28 & 28.38 & 62.59 & 36.64 & 8.88 & 0.77 \\
\hline $4 H-7-28$ & 33.58 & 12.48 & 64.68 & 92 & .26 & 8.14 \\
\hline $4 H-7-48$ & 33.78 & 18.48 & 68.87 & 29.41 & 2.33 & 0.19 \\
\hline $5 \mathrm{H}-1-17$ & 33.97 & 3.48 & 56.57 & 43.38 & 8.88 & 0.13 \\
\hline $5 H-1-42$ & 34.22 & 2.08 & 65.66 & 33.87 & 1.18 & 0.16 \\
\hline $5 H-1-62$ & 34.42 & 4.38 & 56.64 & 48.18 & 3.86 & 8.12 \\
\hline $5 H-1-82$ & 34.62 & 9.98 & 61.49 & 36.85 & 1.66 & 0.81 \\
\hline $5 H-1-182$ & 34.82 & 22.10 & 49.84 & 49.12 & 8.91 & 8.13 \\
\hline $5 H-1-123$ & 35.83 & 17.80 & 68.48 & 28.61 & 2.43 & 0.56 \\
\hline $5 H-1-142$ & 35.22 & 12.48 & 78.28 & 27.72 & 1.71 & 8.29 \\
\hline $5 H-2-17$ & 35.47 & 11.68 & 68.53 & 38.98 & 8.48 & 8.89 \\
\hline $5 H-2-42$ & 35.72 & 7.58 & 59.88 & 37.83 & 1.98 & 8.38 \\
\hline $5 \mathrm{H}-2-62$ & 35.92 & 3.72 & 57.77 & 39.92 & 2.26 & 8.85 \\
\hline $5 H-2-82$ & 36.12 & 11.82 & 54.89 & 39.28 & 4.98 & 8.93 \\
\hline $5 H-2-102$ & 36.32 & 7.80 & 51.94 & 45.94 & 2.87 & 8.84 \\
\hline
\end{tabular}


Table 1.3 (continued).

\begin{tabular}{|c|c|c|c|c|c|c|}
\hline \multirow[b]{2}{*}{$\begin{array}{c}\text { Sample } \\
\text { no. }\end{array}$} & \multirow[b]{2}{*}{$\begin{array}{l}\text { Depth } \\
\text { (mbsf) }\end{array}$} & \multicolumn{5}{|c|}{ Subfraction of coarse fraction $(\mu \mathrm{m})$} \\
\hline & & $\begin{array}{l}>63 \\
(\%)\end{array}$ & $\begin{array}{c}63-125 \\
(\%)\end{array}$ & $\begin{array}{c}125-500 \\
(\%)\end{array}$ & $\begin{array}{c}500-1000 \\
(\%)\end{array}$ & $\begin{array}{c}>1000 \\
(\%)\end{array}$ \\
\hline $5 H-2-123$ & 36.43 & 5.10 & 72.73 & 27.11 & 0.13 & 8.03 \\
\hline $5 H-2-142$ & 36.61 & 7.10 & 68.77 & 38.38 & 0.98 & 8.84 \\
\hline $5 \mathrm{H}-3-17$ & 36.97 & 12.10 & 67.66 & 31.62 & 8.67 & 8.85 \\
\hline $5 H-3-42$ & 37.22 & 6.98 & 58.84 & 39.54 & 1.55 & 8. 86 \\
\hline $5 \mathrm{H}-3-62$ & 37.42 & 5.80 & 78.54 & 27.87 & 1.59 & 8.08 \\
\hline $5 H-3-82$ & 37.62 & 12.78 & 59.82 & 39.82 & 8.88 & 8. 36 \\
\hline $5 H-3-102$ & 37.82 & 13.63 & 67.98 & 29.63 & 1.97 & 8.51 \\
\hline $5 H-3-142$ & 38.22 & 9.98 & 68.52 & 36.98 & 2.33 & 0.17 \\
\hline $5 H-4-17$ & 38.47 & 7.41 & 78.27 & 28.97 & 0.75 & $\theta .01$ \\
\hline $5 H-4-42$ & 38.72 & 8.72 & 55.88 & 48.78 & 2.77 & 0.65 \\
\hline $5 H-4-62$ & 38.92 & 8.57 & 65.54 & 33.83 & 1.18 & 8.25 \\
\hline $5 H-4-82$ & 39.12 & 8.60 & 78.11 & 21.02 & 0.63 & 0.25 \\
\hline $5 H-4-182$ & 39.32 & 6.18 & 44.33 & 53.38 & 2.37 & 8.80 \\
\hline $5 H-4-123$ & 39.43 & 11.48 & 67.43 & 30.42 & 1.99 & 8.16 \\
\hline $5 H-4-143$ & 39.62 & 12.58 & 69.35 & 28.97 & 1.39 & 8.29 \\
\hline $5 H-5-17$ & 39.97 & 18.72 & 58.52 & 47.57 & 1.89 & 8.02 \\
\hline $5 H-5-62$ & 48.42 & 8.88 & 66.66 & 31.19 & 1.48 & 8.68 \\
\hline $5 \mathrm{H}-5-82$ & 48.62 & 5.88 & 53.92 & 43.86 & 2.80 & 0.21 \\
\hline $5 H-5-102$ & 48.82 & 6.88 & 64.87 & 34.81 & 1.13 & 0.88 \\
\hline $5 H-5-123$ & 41.83 & 6.05 & 65.74 & 32.66 & 1.58 & 0.10 \\
\hline $5 H-6-17$ & 41.47 & 6.78 & 66.16 & 32.42 & 1.37 & 8.85 \\
\hline $5 H-6-42$ & 41.72 & 7.64 & 76.52 & 23.42 & 8.88 & 0.86 \\
\hline $5 H-6-62$ & 41.92 & 3.89 & 77.96 & 21.71 & 0.33 & 0.80 \\
\hline $5 H-6-82$ & 42.12 & 4.82 & 2.78 & 93.76 & 3.27 & 0.19 \\
\hline $5 H-6-102$ & 42.32 & 4.90 & 55.83 & 40.94 & 3.16 & 0.86 \\
\hline $5 H-6-122$ & 42.42 & 14.58 & 65.62 & 38.32 & 1.67 & 2.48 \\
\hline $5 H-6-142$ & 42.62 & 18.21 & 68.69 & 34.93 & 3.14 & 1.24 \\
\hline $6 H-1-2 \theta$ & 43.58 & 17.44 & 55.49 & 33.97 & 7.50 & 3.03 \\
\hline $6 \mathrm{H}-1-42$ & 43.78 & 18.48 & 55.51 & 37.98 & 5.61 & 0.98 \\
\hline $6 \mathrm{H}-1-61$ & 43.91 & 13.38 & 63.18 & 34.13 & 2.48 & 0.29 \\
\hline $6 \mathrm{H}-1-83$ & 44.13 & 13.28 & 99.49 & 8.80 & 8.88 & 0.51 \\
\hline $6 \mathrm{H}-1-182$ & 44.32 & 4.28 & 56.18 & 41.88 & 1.78 & 0.16 \\
\hline $6 \mathrm{H}-1-128$ & 44.58 & 12.18 & 78.87 & 19.96 & 1.81 & 0.16 \\
\hline $6 H-1-142$ & 44.72 & 8.30 & 69.28 & 29.86 & 8.94 & 8.80 \\
\hline $6 \mathrm{H}-2-28$ & 45.80 & 5.52 & 79.14 & 19.92 & 0.91 & 8.03 \\
\hline $6 \mathrm{H}-2-61$ & 45.44 & 17.82 & 86.35 & 8.24 & 8.80 & 5.41 \\
\hline $6 \mathrm{H}-2-182$ & 45.82 & 13.10 & 69.98 & 28.61 & 1.27 & 0.14 \\
\hline $6 \mathrm{H}-2-12 \theta$ & 46.88 & 13.88 & 34.27 & 64.64 & 1.05 & 0.84 \\
\hline $6 \mathrm{H}-2-142$ & 46.28 & 19.58 & 74.58 & 23.59 & 1.63 & 8.28 \\
\hline $6 \mathrm{H}-3-28$ & 46.58 & 3.65 & 67.77 & 31.17 & 1.87 & 8.68 \\
\hline $6 \mathrm{H}-3-42$ & 46.72 & 8.70 & 95.96 & 0.80 & 3.81 & 0.23 \\
\hline $6 \mathrm{H}-3-61$ & 46.98 & 4.78 & 72.81 & 25.87 & 1.26 & 0.86 \\
\hline $6 \mathrm{H}-3-83$ & 47.13 & 6.22 & 76.27 & 21.48 & 2.25 & 8.01 \\
\hline $6 \mathrm{H}-3-182$ & 47.32 & 4.48 & 64.33 & 34.84 & 1.54 & 8.18 \\
\hline $6 \mathrm{H}-3-12 \theta$ & 47.58 & 1.98 & 69.41 & 30.59 & 0.80 & 8.88 \\
\hline $6 \mathrm{H}-3-142$ & 47.72 & 4.04 & 54.85 & 43.93 & 1.94 & 8.89 \\
\hline $6 \mathrm{H}-4-28$ & 48.08 & 8.72 & 72.85 & 25.18 & 1.98 & 0.87 \\
\hline $61-4-42$ & 48.22 & 9.78 & 98.88 & 9.69 & 8.29 & 8.82 \\
\hline $6 \mathrm{H}-4-61$ & 48.48 & 6.35 & 57.11 & 42.84 & 0.68 & 0.17 \\
\hline $6 \mathrm{H}-4-83$ & 48.63 & 11.98 & 55.85 & 43.25 & 1.44 & 0.27 \\
\hline $6 t-4-102$ & 48.82 & 18.87 & 53.96 & 44.47 & 1.19 & 0.38 \\
\hline $6 H-4-128$ & 49.81 & 5.48 & 53.26 & 44.23 & 2.38 & 0.13 \\
\hline
\end{tabular}


Table 1.3 (continued).

\begin{tabular}{|c|c|c|c|c|c|c|}
\hline \multirow[b]{2}{*}{$\begin{array}{l}\text { Sample } \\
\text { no. }\end{array}$} & \multirow[b]{2}{*}{$\begin{array}{l}\text { Depth } \\
\text { (mbsf) }\end{array}$} & \multicolumn{5}{|c|}{ Subfraction of coarse fraction $(\mu \mathrm{m})$} \\
\hline & & $\begin{array}{l}>63 \\
(\%)\end{array}$ & $\begin{array}{c}63-125 \\
(\%)\end{array}$ & $\begin{array}{c}125-500 \\
(\%)\end{array}$ & $\begin{array}{c}500-1000 \\
(\%)\end{array}$ & $\begin{array}{c}>1000 \\
(\%)\end{array}$ \\
\hline $6 H-5-28$ & 49.50 & 3.42 & 84.12 & 15.31 & 8.57 & 8.80 \\
\hline $6 H-5-42$ & 49.72 & 8.28 & 57.85 & 48.03 & 1.89 & 8.23 \\
\hline $6 H-5-61$ & 49.98 & 5.48 & 68.28 & 36.79 & 2.35 & 8.58 \\
\hline $6 H-5-83$ & 58.13 & 12.28 & 53.78 & 44.56 & 1.69 & 8.05 \\
\hline $6 \mathrm{H}-5-182$ & 58.32 & 6.78 & 64.95 & 33.89 & 1.88 & 8.88 \\
\hline $6 H-5-128$ & 58.58 & 9.22 & 61.39 & 36.97 & 1.29 & 8.35 \\
\hline $6 H-5-142$ & 58.72 & 7.88 & 63.46 & 34.93 & 1.58 & 8.12 \\
\hline $6 H-6-28$ & 51.88 & 3.44 & 75.47 & 24.19 & 8.25 & 8.89 \\
\hline $6 H-6-42$ & 51.22 & 1.88 & 77.67 & 22.33 & 8.88 & 0.88 \\
\hline $6 H-6-46$ & 51.26 & 5.28 & 99.86 & 0.80 & 8.68 & 0.14 \\
\hline $6 H-6-68$ & 51.48 & 1.34 & 85.86 & 14.94 & 8.88 & 8.88 \\
\hline $6 H-6-83$ & 51.63 & 2.78 & 188.08 & 8.80 & $\theta . \theta \theta$ & 8.80 \\
\hline $7 \mathrm{H}-1-21$ & 53.01 & 4.78 & 68.06 & 27.69 & 3.79 & 0.46 \\
\hline $7 \mathrm{H}-1-41$ & 53.21 & 1.82 & 79.58 & 19.12 & 1.87 & 8.23 \\
\hline $7 \mathrm{H}-1-62$ & 53.42 & 2.78 & 88.49 & 18.89 & 8.62 & 8.88 \\
\hline $7 H-1-83$ & 53.62 & 2.83 & 98.27 & 8.89 & 8.84 & 8.80 \\
\hline $7 \mathrm{H}-1-185$ & 53.84 & 8.81 & 74.44 & 22.28 & 3.37 & 0.88 \\
\hline $7 \mathrm{H}-1-122$ & 54.86 & 1.11 & 97.48 & 2.68 & 8.00 & 8.80 \\
\hline $7 \mathrm{H}-1-142$ & 54.22 & 1.38 & 97.47 & 2.34 & 0.19 & 8.80 \\
\hline $7 \mathrm{H}-2-21$ & 54.31 & 8.56 & 95.62 & 4.38 & 8.88 & 8.88 \\
\hline $7 H-2-41$ & 54.72 & $0.4 \theta$ & 88.18 & 19.90 & $\theta .80$ & 8.80 \\
\hline $7 \mathrm{H}-2-64$ & 54.98 & 8.64 & 98.01 & 7.66 & 2.33 & 8.88 \\
\hline $7 \mathrm{H}-2-83$ & 55.13 & 0.64 & 188.88 & 8.88 & 8.68 & 8.88 \\
\hline $7 \mathrm{H}-2-185$ & 55.35 & 8.71 & 180.80 & 8.80 & 8.80 & 8.80 \\
\hline $7 \mathrm{H}-2-123$ & 55.53 & 1.51 & 87.13 & 12.78 & 0.17 & 8.80 \\
\hline $7 \mathrm{H}-2-142$ & 55.72 & 1.48 & 180.80 & 8.88 & $\theta .08$ & 8.08 \\
\hline $7 \mathrm{H}-3-21$ & 56.81 & 1.84 & 87.08 & 10.22 & 2.78 & 8.80 \\
\hline $7 H-3-41$ & 56.21 & 1.38 & 72.37 & 28.97 & 6.61 & 0.85 \\
\hline $7 \mathrm{H}-3-62$ & 56.42 & 3.22 & 95.85 & 4.51 & 8.43 & 0.81 \\
\hline $7 \mathrm{H}-3-82$ & 56.62 & 2.50 & 74.42 & 23.47 & 2.11 & 8.80 \\
\hline $7 \mathrm{H}-3-122$ & 57.82 & 8.84 & 188.88 & 8.88 & 8.88 & 8.88 \\
\hline $7 \mathrm{H}-3-142$ & 57.22 & 0.87 & 180.08 & 8.80 & 0.80 & 8.88 \\
\hline $7 \mathrm{H}-4-21$ & 57.51 & 8. 86 & 83.78 & 14.83 & 1.38 & 8.88 \\
\hline $7 \mathrm{H}-4-41$ & 57.72 & 1.28 & 99.84 & 8.80 & 0.08 & 0.16 \\
\hline $7 \mathrm{H}-4-62$ & 57.88 & 1.73 & 88.18 & 11.35 & 8.55 & 8.80 \\
\hline $7 \mathrm{H}-4-83$ & 58.13 & 2.78 & 76.46 & 22.49 & 1.85 & 8.08 \\
\hline $7 \mathrm{H}-4-185$ & 58.34 & 1.97 & 87.16 & 12.29 & 8.52 & 8.03 \\
\hline $7 \mathrm{H}-4-122$ & 58.53 & 1.19 & 180.88 & $\theta .88$ & 8.08 & 8.88 \\
\hline $7 H-4-142$ & 58.72 & 2.18 & 78.46 & 12.79 & 8.74 & 8.88 \\
\hline $7 H-5-21$ & 59.81 & 3.98 & 83.37 & 15.65 & 1.43 & 0.15 \\
\hline $7 \mathrm{H}-5-41$ & 59.21 & 2.48 & 94.12 & 5.26 & 8.62 & 8.88 \\
\hline $7 \mathrm{H}-5-62$ & 59.42 & 2.95 & 92.46 & 7.11 & 8.41 & 0.82 \\
\hline $7 \mathrm{H}-5-83$ & 59.62 & 5.57 & 76.45 & 22.21 & 8.83 & 8.51 \\
\hline $7 \mathrm{H}-5-185$ & 59.84 & 1.48 & 81.35 & 17.94 & 8.71 & 8.88 \\
\hline $7 H-5-122$ & 60.02 & 2.36 & 96.88 & 3.92 & 8.80 & 8.80 \\
\hline $7 \mathrm{H}-6-12$ & 68.42 & 4.88 & 92.98 & 6.61 & 0.49 & 8.80 \\
\hline $8 H-1-35$ & 62.65 & 1.8 & 95.14 & 4.86 & 0.88 & 8.80 \\
\hline $8 \mathrm{H}-2-35$ & 64.15 & 1.47 & 89.87 & 9.35 & 0.78 & 8.80 \\
\hline $8 \mathrm{H}-3-35$ & 65.65 & 8.71 & 86.62 & 13.38 & $\theta .8 \theta$ & 8.88 \\
\hline $8 \mathrm{H}-4-35$ & 67.15 & 0.72 & 92.68 & 6.34 & 6. 98 & 8.88 \\
\hline $8 \mathrm{H}-5-35$ & 68.65 & 1.83 & 86.03 & 13.97 & 8.80 & 8.80 \\
\hline $8 \mathrm{H}-6-35$ & 78.15 & 8.54 & 88.03 & 11.11 & 8.85 & 8.88 \\
\hline
\end{tabular}


Table 1.3 (continued).

\begin{tabular}{|c|c|c|c|c|c|c|}
\hline \multirow[b]{2}{*}{$\begin{array}{c}\text { Sample } \\
\text { no. }\end{array}$} & \multirow[b]{2}{*}{$\begin{array}{l}\text { Depth } \\
\text { (mbsf) }\end{array}$} & \multicolumn{5}{|c|}{ Subfraction of coarse fraction $(\mu \mathrm{m})$} \\
\hline & & $\begin{array}{l}>63 \\
(\%)\end{array}$ & $\begin{array}{c}63-125 \\
(\%)\end{array}$ & $\begin{array}{c}125-500 \\
(\%)\end{array}$ & $\begin{array}{c}500-1000 \\
(\%)\end{array}$ & $\begin{array}{c}>1000 \\
(\%)\end{array}$ \\
\hline $9 H-1-48$ & 72.28 & 1.25 & 98.68 & 9.48 & 8.88 & 8.80 \\
\hline $9 \mathrm{H}-2-48$ & 73.78 & 0.47 & 78.76 & 19.47 & 1.77 & 8.80 \\
\hline $9 H-3-48$ & 75.28 & 0.89 & 67.50 & 38.80 & 2.58 & 8.80 \\
\hline $9 H-4-4 \theta$ & $76.7 \theta$ & 0.76 & 69.17 & 27.88 & 3.75 & 8.80 \\
\hline $9 H-6-4 \theta$ & 79.78 & 0.24 & 53.85 & 41.54 & 4.62 & $\theta .00$ \\
\hline $9 H-7-4 \theta$ & 71.28 & 8.22 & 78.77 & 29.23 & 8.88 & 8.88 \\
\hline $18 \mathrm{BH}-1-48$ & 81.78 & 0.87 & 58.28 & 31.84 & 17.88 & 8.80 \\
\hline $10 \mathrm{H}-2-48$ & 83.28 & 0.65 & 66.89 & 23.65 & 9.46 & 0.80 \\
\hline $18 \mathrm{H}-3-48$ & 84.78 & 1.89 & 78.21 & 28.92 & 8.87 & 8.80 \\
\hline $18 \mathrm{H}-4-48$ & 86.28 & 0.56 & 82.14 & 17.86 & 8.88 & 8.88 \\
\hline $10 \mathrm{H}-5-4 \theta$ & 87.78 & 8.16 & 53.33 & 46.67 & 8.80 & 8.88 \\
\hline $18 \mathrm{H}-6-4 \theta$ & 89.28 & 0.56 & 69.54 & 38.46 & 8.88 & 8.88 \\
\hline $18 \mathrm{H}-7-48$ & 98.78 & 0.68 & 88.38 & 11.78 & $\theta .08$ & 8.88 \\
\hline $11 H-1-48$ & 91.28 & 8.42 & 87.93 & 12.87 & 8.88 & 8.88 \\
\hline $11 \mathrm{H}-2-48$ & 92.78 & 5.16 & 83.93 & 15.58 & 8.57 & 8.88 \\
\hline $11 \mathrm{H}-3-48$ & 94.28 & 0.24 & 71.79 & 17.95 & 18.26 & 8.80 \\
\hline $11 \mathrm{H}-4-4 \theta$ & 95.78 & 8.53 & 71.23 & 28.77 & 8.08 & 8.08 \\
\hline $11 \mathrm{H}-5-4 \theta$ & 97.28 & 8.78 & 25.67 & 74.33 & 8.88 & $\theta .80$ \\
\hline $11 \mathrm{H}-6-4 \theta$ & 98.78 & 1.1 & 22.22 & 77.78 & 8.88 & 8.80 \\
\hline $11 \mathrm{H}-7-16$ & 99.96 & 3.73 & 46.18 & 52.66 & 8.80 & 1.16 \\
\hline $12 \mathrm{H}-1-48$ & 188.78 & 0.64 & 15.66 & 67.47 & 4.82 & 12.85 \\
\hline $12 \mathrm{H}-2-48$ & 182.28 & 0.77 & 21.57 & 78.43 & 8.88 & 8.88 \\
\hline $12 \mathrm{H}-3-48$ & 183.78 & 5.37 & 73.94 & 25.93 & 0.14 & 0.80 \\
\hline $12 \mathrm{H}-4-48$ & 185.28 & 2 & 62.36 & 37.64 & 8.80 & 8.80 \\
\hline $12 \mathrm{H}-5-48$ & 186.78 & 1.59 & 43.21 & 47.22 & 4.45 & 5.12 \\
\hline $12 \mathrm{H}-6-4 \theta$ & 188.28 & 8.5 & 54.39 & 45.61 & 0.80 & 0.80 \\
\hline $13 H-1-48$ & 118.28 & 2.14 & 44.88 & 53.98 & 1.38 & 8.88 \\
\hline $13 \mathrm{H}-2-48$ & 111.78 & 1.84 & 48.45 & 59.55 & 8.88 & 8.88 \\
\hline $13 H-3-4 \theta$ & 113.28 & 0.32 & 58.68 & 58.80 & 8.80 & 8.80 \\
\hline $13 H-4-48$ & 114.78 & 1.38 & 43.69 & 56.31 & 8.88 & 8.88 \\
\hline $13 H-5-4 \theta$ & 116.28 & 1.59 & 57.68 & 36.88 & 6.48 & 0.88 \\
\hline $13 H-6-4 \theta$ & 117.78 & 0.82 & 88.42 & 19.58 & 8.88 & 8.80 \\
\hline $13 \mathrm{H}-7-48$ & 119.28 & 1.39 & 65.75 & 38.88 & 4.25 & 8.88 \\
\hline $14 H-1-4 \theta$ & 119.78 & 0.47 & 53.48 & 48.78 & 0.88 & 5.83 \\
\hline $14 \mathrm{H}-2-4 \theta$ & 121.28 & 3.39 & 78.43 & 29.57 & 8.88 & 0.88 \\
\hline $14 \mathrm{H}-3-48$ & 122.78 & 8.66 & 62.86 & 37.14 & 8.80 & 8.88 \\
\hline $14 H-4-4 \theta$ & 124.28 & 1.78 & 56.46 & 41.95 & 1.58 & 0.80 \\
\hline $14 H-5-48$ & 125.78 & 2.82 & 52.85 & 45.68 & 1.55 & 8.88 \\
\hline $14 H-6-48$ & 127.28 & 1.36 & 61.25 & 33.85 & 5.78 & 8.88 \\
\hline $15 \mathrm{H}-1-39$ & 129.19 & 0.81 & 84.96 & 15.84 & 8.80 & 0.88 \\
\hline $15 \mathrm{H}-2-39$ & 138.69 & 0.54 & 28.83 & 56.76 & 14.41 & 8.08 \\
\hline $15 \mathrm{H}-3-39$ & 132.19 & 0.52 & 59.15 & 33.88 & 7.84 & 0.00 \\
\hline $15 \mathrm{H}-4-39$ & 133.69 & 8.27 & 5.38 & 13.91 & 6.62 & 74.17 \\
\hline $15 H-5-39$ & 135.19 & 3.12 & 62.94 & 33.56 & 3.58 & $8 . \theta 8$ \\
\hline $15 H-6-39$ & 136.69 & 0.96 & 63.53 & 31.31 & 5.17 & 8.88 \\
\hline $16 \mathrm{H}-1-38$ & 138.68 & 2.5 & 77.88 & 28.16 & 2.85 & 8.88 \\
\hline $16 H-2-38$ & 148.18 & 5.3 & 79.89 & 28.48 & 0.44 & 8.80 \\
\hline $16 \mathrm{Ht}-3-38$ & 141.68 & 1.81 & 74.18 & 25.82 & 8.80 & 8.80 \\
\hline $16 \mathrm{H}-4-38$ & 143.18 & 1.31 & 69.39 & 27.78 & 2.98 & 8.80 \\
\hline $16 H-5-38$ & 144.68 & 1.53 & 78.89 & 21.91 & 8.80 & 8.80 \\
\hline $16 \mathrm{H}-6-38$ & 146.18 & 1.21 & 94.86 & 5.37 & 8.88 & 8.57 \\
\hline $16 \mathrm{H}-7-38$ & 147.68 & 2.81 & 94.88 & 5.12 & 0.88 & 8.88 \\
\hline
\end{tabular}


Table 1.3 (continued).

\begin{tabular}{|c|c|c|c|c|c|c|}
\hline \multirow[b]{2}{*}{$\begin{array}{c}\text { Sample } \\
\text { no. }\end{array}$} & \multirow[b]{2}{*}{$\begin{array}{l}\text { Depth } \\
\text { (mbsf) }\end{array}$} & \multicolumn{5}{|c|}{ Subfraction of coarse fraction $(\mu \mathrm{m})$} \\
\hline & & $\begin{array}{l}>63 \\
(\%)\end{array}$ & $\begin{array}{c}63-125 \\
(\%)\end{array}$ & $\begin{array}{c}125-500 \\
(\%)\end{array}$ & $\begin{array}{c}500-1000 \\
(\%)\end{array}$ & $\begin{array}{c}>1000 \\
(\%)\end{array}$ \\
\hline $17 x-1-58$ & 148.30 & 1.13 & 78.16 & 17.85 & 5.25 & 7.54 \\
\hline $18 x-1-48$ & 157.78 & 2.21 & 28.11 & 5.33 & 3.56 & 71.88 \\
\hline $18 X-2-48$ & 159.28 & 8.54 & 83.58 & 16.58 & $\theta .88$ & $\theta . \theta 0$ \\
\hline $19 x-1-41$ & 167.21 & 3.57 & 46.36 & 12.28 & 21.88 & 28.29 \\
\hline $19 x-2-41$ & 168.71 & 1.82 & 72.83 & 26.91 & 1.06 & 0.80 \\
\hline $19 x-3-41$ & 178.21 & 1.5 & 92.93 & 6.58 & 8.57 & 8.80 \\
\hline $19 x-4-41$ & 171.71 & 1.55 & 86.85 & 13.28 & 0.67 & 8.80 \\
\hline $19 x-5-41$ & 173.21 & 8.85 & 75.80 & 25.88 & 0.80 & 0.80 \\
\hline $19 x-6-41$ & 174.71 & 0.66 & 73.55 & 26.45 & 0.00 & 8.80 \\
\hline $28 x-1-41$ & 176.71 & 0.87 & 64.72 & 35.28 & 0.80 & 8.88 \\
\hline $28 x-2-41$ & 178.21 & 0.3 & 83.75 & 16.25 & 8.88 & 8.88 \\
\hline $28 x-3-41$ & 179.71 & 0.25 & 61.98 & 38.82 & 0.80 & 0.80 \\
\hline $20 x-4-41$ & 181.21 & 1.14 & 30.78 & 51.78 & 17.44 & 0.00 \\
\hline $28 x-5-41$ & 182.71 & 8.36 & 46.52 & 53.48 & 8.80 & 0.80 \\
\hline $20 X-6-41$ & 184.21 & 8.45 & 69.47 & 38.53 & 8.80 & 0.80 \\
\hline $22 x-1-4 \theta$ & 195.78 & 0.92 & 75.24 & 24.76 & 0.88 & 0.80 \\
\hline $22 x-2-4 \theta$ & 197.28 & 0.6 & 73.48 & 26.52 & 8.88 & 0.80 \\
\hline $22 x-3-4 \theta$ & 198.78 & 8.74 & 68.83 & 31.97 & 8.08 & 0.80 \\
\hline $22 x-4-4 \theta$ & 280.28 & 8.87 & 73.58 & 26.42 & 8.80 & 8.88 \\
\hline $22 x-5-48$ & 281.78 & 8.35 & 41.83 & 58.17 & 8.80 & 8.88 \\
\hline $22 x-6-48$ & 203.28 & 0.35 & 41.83 & 58.17 & 8.88 & 8.88 \\
\hline $22 x-7-48$ & 284.78 & 8.83 & 77.83 & 22.97 & 0.80 & 0.08 \\
\hline $23 x-1-39$ & 285.49 & 0.5 & 64.62 & 35.38 & 8.88 & 0.88 \\
\hline $23 x-2-39$ & 286.99 & 8.35 & 57.84 & 42.16 & 8.88 & 0.08 \\
\hline $23 x-3-39$ & 288.49 & 0.42 & 44.81 & 55.19 & 8.80 & 8.80 \\
\hline $23 x-4-39$ & 289.99 & 0.25 & 65.71 & 34.29 & 8.88 & 8.88 \\
\hline $23 x-5-39$ & 211.49 & 8.45 & 73.77 & 26.23 & 8.88 & 8.88 \\
\hline $23 x-6-39$ & 212.99 & 0.47 & 74.48 & 25.68 & 8.88 & 8.80 \\
\hline $23 x-7-39$ & 214.49 & 1.89 & 88.78 & 11.22 & 8.88 & 8.80 \\
\hline $24 X-1-39$ & 215.29 & 8.47 & 65.19 & 34.81 & 8.88 & 0.80 \\
\hline $24 X-2-39$ & 216.79 & 0.42 & 83.82 & 16.18 & 0.80 & 0.88 \\
\hline $24 x-3-39$ & 218.29 & 8.54 & 85.88 & 15.88 & $\theta .88$ & 8.80 \\
\hline $24 x-4-39$ & 219.79 & 8.61 & 74.58 & 25.58 & 8.88 & 0.88 \\
\hline $24 x-5-39$ & 221.29 & 0.6 & 69.43 & 38.57 & 8.88 & 0.80 \\
\hline $24 x-6-39$ & 222.79 & 0.69 & 72.11 & 27.89 & 8.80 & 0.80 \\
\hline $24 x-7-39$ & 224.29 & 8.32 & 72.86 & 27.14 & 8.80 & 0.80 \\
\hline $25 x-1-58$ & 225.28 & 0.48 & 77.12 & 22.88 & 8.88 & 8.80 \\
\hline $25 x-2-58$ & 226.70 & 0.38 & 39.39 & 60.61 & 8.08 & 8.80 \\
\hline $25 x-3-58$ & 228.28 & 8.56 & 83.67 & 16.33 & 8.88 & 8.88 \\
\hline $25 x-4-50$ & 229.78 & 8.34 & 48.23 & 59.77 & 0.80 & 0.88 \\
\hline $25 x-5-58$ & 231.28 & 1.62 & 12.81 & 87.19 & 0.88 & 0.88 \\
\hline $25 x-6-50$ & 232.78 & 0.93 & 65.31 & 34.69 & 0.80 & 0.80 \\
\hline $25 x-7-58$ & 234.28 & 0.87 & 60.38 & 39.78 & 0.00 & 0.88 \\
\hline $26 x-1-4 \theta$ & 234.98 & 8.69 & 58.24 & 41.76 & $\theta .8 \theta$ & 0.80 \\
\hline $26 x-2-4 \theta$ & 236.48 & 2.12 & 58.47 & 49.38 & 8.16 & 8.88 \\
\hline $26 x-3-48$ & 237.98 & 0.63 & 71.43 & 28.57 & 0.88 & 8.88 \\
\hline $26 x-4-48$ & 239.48 & 2.52 & 86.71 & 12.97 & 8.32 & 8.80 \\
\hline $26 x-5-4 \theta$ & 248.98 & 0.72 & 97.26 & 2.74 & 0.00 & 0.88 \\
\hline $26 x-6-48$ & 242.48 & 0.61 & 33.33 & 66.67 & 8.80 & 8.88 \\
\hline $27 x-1-48$ & 244.78 & 0.24 & 58.80 & 58.88 & 8.88 & 0.80 \\
\hline $27 x-2-48$ & 246.28 & 0.27 & 58.80 & 58.80 & $\theta .8 \theta$ & 0.88 \\
\hline $27 x-3-48$ & 247.78 & 2.65 & 93.79 & 5.97 & 0.24 & 0.88 \\
\hline
\end{tabular}


R. HENRICH, T. WOLF, G. BOHRMANN, J. THIEDE

Table 1.3 (continued).

\begin{tabular}{|c|c|c|c|c|c|c|}
\hline \multirow[b]{2}{*}{$\begin{array}{l}\text { Sample } \\
\text { no. }\end{array}$} & \multirow[b]{2}{*}{$\begin{array}{l}\text { Depth } \\
\text { (mbsf) }\end{array}$} & \multicolumn{5}{|c|}{ Subfraction of coarse fraction $(\mu \mathrm{m})$} \\
\hline & & $\begin{array}{l}>63 \\
(\%)\end{array}$ & $\begin{array}{c}63-125 \\
(\%)\end{array}$ & $\begin{array}{c}125-500 \\
(\%)\end{array}$ & $\begin{array}{c}500-1000 \\
(\%)\end{array}$ & $\begin{array}{c}>1000 \\
(\%)\end{array}$ \\
\hline $27 x-4-48$ & 249.28 & 0.62 & 57.92 & 42.68 & $\theta . \theta 0$ & 0.88 \\
\hline $27 x-5-48$ & 258.78 & 8.27 & 54.76 & 45.24 & 8.80 & 8.80 \\
\hline $27 x-6-48$ & 252.28 & 0.87 & 47.98 & 52.82 & 8.80 & 8. 80 \\
\hline $28 x-1-48$ & 254.58 & 0.66 & 62.21 & 37.79 & 8.80 & 8. 88 \\
\hline $28 x-2-4 \theta$ & 256.80 & 1.62 & 65.97 & 34.03 & 8.80 & $8.8 \theta$ \\
\hline $28 x-3-4 \theta$ & 257.58 & 0.59 & 88.87 & 19.13 & $\theta .08$ & 0.80 \\
\hline $28 x-4-48$ & 259.80 & 0.24 & 67.29 & 32.71 & 0.08 & 8.88 \\
\hline $28 x-5-48$ & 268.50 & 3.22 & 95.32 & 4.48 & 8.19 & 0.80 \\
\hline $28 X-6-48$ & 262.80 & 1.55 & 78.67 & 21.33 & 8.08 & 8.88 \\
\hline $29 x-1-39$ & 264.29 & 1.18 & 57.63 & 33.98 & 8.47 & 0.88 \\
\hline $29 x-2-39$ & 265.79 & 8.56 & 38.57 & 61.43 & 0.88 & 8.88 \\
\hline $29 x-3-39$ & 267.29 & 0.85 & 69.16 & 38.84 & $8.8 \theta$ & 8.80 \\
\hline $29 x-4-39$ & 268.79 & 0.55 & 108.08 & 8.80 & $\theta .08$ & 0.88 \\
\hline
\end{tabular}


Table 1.4. Sedimentological data from Hole 644A. Analysis of coarsefraction components.

\begin{tabular}{|c|c|c|c|c|}
\hline $\begin{array}{c}\text { Sample } \\
\text { no. }\end{array}$ & $\begin{array}{l}\text { Depth } \\
\text { (mbsf) }\end{array}$ & $\begin{array}{l}>63 \\
\mu \mathrm{m} \\
(\%)\end{array}$ & $\begin{array}{c}\text { Bulk } \\
\mathrm{CaCO}_{3} \\
(\%)\end{array}$ & $\begin{array}{c}\text { TOC } \\
(\%)\end{array}$ \\
\hline $1 H-1-22$ & 8.22 & 9.52 & 7.22 & 0.65 \\
\hline $1 H-1-42$ & 8.42 & 2.74 & 6.38 & \\
\hline $1 H-1-59$ & 8.59 & 8.85 & 7.88 & 0.44 \\
\hline $1 H-1-82$ & 8.82 & 4.64 & 9.93 & \\
\hline $1 H-1-182$ & 1.82 & 7.16 & 18.90 & 8.33 \\
\hline $1 H-1-122$ & 1.22 & 10.94 & 18.21 & \\
\hline $1 H-1-142$ & 1.42 & 13.96 & 9.88 & 0.31 \\
\hline $1 \mathrm{H}-2-22$ & 1.72 & 4.35 & 10.23 & 8.48 \\
\hline $1 \mathrm{H}-2-42$ & 1.92 & 5.75 & 11.24 & \\
\hline $1 \mathrm{H}-2-59$ & 2.89 & 28.05 & 8.33 & 8.33 \\
\hline $1 H-2-82$ & 2.38 & 7.43 & 9.21 & \\
\hline $1 \mathrm{H}-2-182$ & 2.52 & 13.25 & 18.04 & 0.35 \\
\hline $1 \mathrm{H}-2-122$ & 2.72 & 5.47 & 10.03 & \\
\hline $1 \mathrm{H}-2-142$ & 2.98 & 6.08 & 8.77 & 8.38 \\
\hline $1 \mathrm{H}-3-22$ & 3.22 & 5.36 & 8.14 & 0.43 \\
\hline $1 \mathrm{H}-3-42$ & 3.42 & 4.95 & 9.49 & \\
\hline $1 \mathrm{H}-3-59$ & 3.59 & 8.69 & 18.49 & 8.33 \\
\hline $1 \mathrm{H}-3-82$ & 3.82 & 7.31 & 9.78 & \\
\hline $1 \mathrm{H}-3-182$ & 4.82 & 4.78 & 8.82 & 0.39 \\
\hline $1 \mathrm{H}-3-122$ & 4.22 & 2.19 & 8.25 & \\
\hline $1 \mathrm{H}-3-142$ & 4.42 & 6.88 & 7.78 & 8.41 \\
\hline $1 H-4-22$ & 4.72 & 3.82 & 6.73 & 0.38 \\
\hline $1 \mathrm{H}-4-42$ & 4.92 & 4.52 & 8.85 & 8.35 \\
\hline $1 H-4-59$ & 5.89 & 3.79 & 7.39 & 8.26 \\
\hline $1 \mathrm{H}-4-82$ & 5.30 & 3.98 & 6.92 & 0.36 \\
\hline $1 \mathrm{H}-4-182$ & 5.52 & 7.11 & 7.87 & 8.48 \\
\hline $1 \mathrm{H}-4-122$ & 5.72 & 4.81 & 6.66 & 0.37 \\
\hline $1 \mathrm{H}-5-22$ & 6.22 & 4.84 & 7.58 & 8.35 \\
\hline $1 \mathrm{H}-5-42$ & 6.42 & 4.98 & 6.59 & \\
\hline $1 \mathrm{H}-5-59$ & 6.59 & 6.99 & 6.25 & 8.43 \\
\hline $1 \mathrm{H}-5-82$ & 6.82 & 1.87 & 6.78 & \\
\hline $1 \mathrm{H}-5-102$ & 7.82 & 3.27 & 5.52 & 8.31 \\
\hline $1 \mathrm{H}-5-122$ & 7.22 & 3.28 & 7.23 & \\
\hline $1 \mathrm{H}-5-142$ & 7.42 & 4.54 & 10.57 & 0.48 \\
\hline $1 \mathrm{H}-6-22$ & 7.72 & 5.22 & 11.02 & 0.53 \\
\hline $1 H-6-42$ & 7.92 & 2.58 & 18.99 & \\
\hline $1 H-6-59$ & 8.84 & 6.42 & 9.65 & 8.54 \\
\hline $1 \mathrm{H}-6-82$ & 8.38 & 1.76 & 8.84 & \\
\hline $1 H-6-182$ & 8.52 & 4.85 & 7.26 & 8.48 \\
\hline $1 \mathrm{H}-6-122$ & 8.72 & 2.71 & 18.68 & \\
\hline $1 \mathrm{H}-6-142$ & 8.98 & 5.85 & 8.93 & 0.62 \\
\hline $2 \mathrm{H}-1-22$ & 9.42 & 3.81 & 11.84 & 8.47 \\
\hline $2 \mathrm{H}-1-42$ & 9.62 & 2.83 & 6.28 & \\
\hline $2 \mathrm{H}-1-62$ & 9.82 & 15.28 & 0.62 & 1.89 \\
\hline $2 \mathrm{H}-1-82$ & 18.82 & 6.64 & 7.23 & \\
\hline $2 \mathrm{H}-1-104$ & 18.24 & 9.85 & 11.54 & 8.31 \\
\hline $2 \mathrm{H}-1-122$ & 18.42 & 8.93 & 5.87 & \\
\hline $2 \mathrm{H}-1-142$ & 18.62 & 12.26 & 23.86 & 0.27 \\
\hline $2 \mathrm{H}-2-22$ & 10.92 & 14.23 & 29.76 & 8.45 \\
\hline $2 \mathrm{H}-2-42$ & 11.12 & 8.23 & 15.13 & \\
\hline $2 \mathrm{H}-2-62$ & 11.32 & 7.42 & 8.67 & 0.48 \\
\hline
\end{tabular}

Table 1.4 (continued).

\begin{tabular}{|c|c|c|c|c|}
\hline $\begin{array}{c}\text { Sample } \\
\text { no. }\end{array}$ & $\begin{array}{l}\text { Depth } \\
\text { (mbsf) }\end{array}$ & $\begin{array}{l}>63 \\
\mu \mathrm{m} \\
(\%)\end{array}$ & $\begin{array}{c}\text { Bulk } \\
\mathrm{CaCO}_{3} \\
(\%)\end{array}$ & $\begin{array}{c}\text { TOC } \\
(\%)\end{array}$ \\
\hline $2 \mathrm{H}-2-82$ & 11.52 & 18.75 & 7.31 & \\
\hline $2 \mathrm{H}-2-184$ & 11.74 & 7.59 & 22.23 & 8.45 \\
\hline $2 \mathrm{H}-2-122$ & 11.98 & 16.88 & 14.36 & \\
\hline $2 \mathrm{H}-2-142$ & 12.12 & 3.22 & 11.13 & 0.41 \\
\hline $2 \mathrm{H}-3-22$ & 12.42 & 6.87 & 13.96 & 8.45 \\
\hline $2 \mathrm{H}-3-42$ & 12.62 & 4.39 & 19.59 & \\
\hline $2 \mathrm{H}-3-62$ & 12.82 & 2.48 & 6.73 & 0.56 \\
\hline $2 \mathrm{H}-3-82$ & 13.82 & 24.19 & 4.47 & \\
\hline $2 \mathrm{H}-3-184$ & 13.22 & 23.68 & 6.63 & 0.34 \\
\hline $2 \mathrm{H}-3-122$ & 13.42 & 17.31 & 8.36 & \\
\hline $2 \mathrm{H}-4-22$ & 13.92 & 14.82 & 15.15 & 0.27 \\
\hline $2 \mathrm{H}-4-42$ & 14.12 & 6.39 & 17.03 & \\
\hline $2 \mathrm{H}-4-62$ & 14.32 & 4.61 & 18.21 & 8.36 \\
\hline $2 \mathrm{H}-4-82$ & 14.52 & 8.53 & 18.01 & \\
\hline $2 \mathrm{H}-4-184$ & 14.74 & 24.75 & 8.73 & 0.98 \\
\hline $2 \mathrm{H}-4-122$ & 14.98 & 12.87 & 11.91 & \\
\hline $2 \mathrm{H}-4-142$ & 15.12 & 18.56 & 16.84 & 8.41 \\
\hline $2 \mathrm{H}-5-22$ & 15.42 & 9.88 & 8.61 & 8.47 \\
\hline $2 \mathrm{H}-5-42$ & 15.62 & 9.88 & 10.77 & \\
\hline $2 \mathrm{H}-5-62$ & 15.82 & 9.98 & 18.59 & 0.33 \\
\hline $3 H-1-22$ & 16.42 & 6.22 & 11.92 & \\
\hline $3 \mathrm{H}-1-42$ & 16.62 & 4.78 & 8.76 & 0.43 \\
\hline $3 \mathrm{H}-1-62$ & 16.82 & 3.93 & 11.85 & \\
\hline $3 H-1-82$ & 17.02 & 14.38 & 4.77 & 8.51 \\
\hline $3 H-1-182$ & 17.22 & 6.85 & 9.26 & \\
\hline $3 H-1-122$ & $17.4 \theta$ & 9.58 & 6.80 & 0.29 \\
\hline $3 H-1-142$ & $17.6 \theta$ & 14.27 & 8.28 & \\
\hline $3 \mathrm{H}-2-22$ & 17.92 & 11.98 & 7.81 & 8.42 \\
\hline $3 \mathrm{H}-2-42$ & 18.12 & 12.51 & & \\
\hline $3 \mathrm{H}-2-62$ & 18.32 & 11.21 & 7.83 & 8.29 \\
\hline $3 H-2-82$ & 18.52 & 7.78 & & \\
\hline $3 H-2-182$ & 18.72 & 4.86 & 7.52 & 8.29 \\
\hline $3 \mathrm{H}-2-122$ & 18.98 & 8.63 & & \\
\hline $3 H-2-142$ & 19.12 & 2.21 & 5.63 & 0.26 \\
\hline $3 \mathrm{H}-3-22$ & 19.42 & 6.52 & 5.79 & 0.27 \\
\hline $3 \mathrm{H}-3-42$ & 19.62 & 1.96 & & \\
\hline $3 \mathrm{H}-3-62$ & 19.82 & 3.76 & 8.29 & 8.31 \\
\hline $3 \mathrm{H}-3-82$ & 28.82 & 3.23 & & \\
\hline $3 \mathrm{H}-3-102$ & 28.28 & 6.19 & 6.87 & $\theta .30$ \\
\hline $3 \mathrm{H}-4-22$ & 28.42 & 8.02 & 1.33 & 0.65 \\
\hline $3 \mathrm{H}-4-42$ & 21.12 & 3.57 & & \\
\hline $3 H-4-62$ & 21.32 & 4.91 & 5.89 & 0.37 \\
\hline $3 \mathrm{H}-4-82$ & 21.52 & 3.28 & & \\
\hline $3 \mathrm{H}-4-182$ & 21.72 & 5.87 & 3.45 & 0.45 \\
\hline $3 H-4-122$ & 21.98 & 3.71 & & \\
\hline $3 \mathrm{H}-5-22$ & 22.43 & 5.14 & 7.69 & 0.33 \\
\hline $3 H-5-42$ & 22.62 & 2.64 & & \\
\hline $3 H-5-62$ & 22.82 & 18.33 & 2.16 & 0.31 \\
\hline $3 H-5-82$ & 23.82 & 18.21 & & \\
\hline $3 H-5-102$ & 23.22 & 2.81 & 2.96 & 8.36 \\
\hline $3 H-5-122$ & 23.48 & 0.62 & 3.22 & \\
\hline
\end{tabular}


Table 1.4 (continued).

\begin{tabular}{|c|c|c|c|c|}
\hline $\begin{array}{c}\text { Sample } \\
\text { no. }\end{array}$ & $\begin{array}{l}\text { Depth } \\
\text { (mbsf) }\end{array}$ & $\begin{array}{l}>63 \\
\mu \mathrm{m} \\
(\%)\end{array}$ & $\begin{array}{c}\text { Bulk } \\
\mathrm{CaCO}_{3} \\
(\%)\end{array}$ & $\begin{array}{l}\text { TOC } \\
(\%)\end{array}$ \\
\hline $3 H-5-142$ & 23.68 & 2.75 & 9.23 & 8.32 \\
\hline $3 H-6-22$ & 23.99 & 14.07 & 4.59 & 0.34 \\
\hline $3 H-7-22$ & 25.42 & 6.82 & 9.94 & 8.33 \\
\hline $4 H-1-22$ & 25.92 & 9.98 & 7.14 & 8.41 \\
\hline $4 H-1-42$ & 26.12 & 5.68 & & \\
\hline $4 H-1-62$ & 26.32 & 4.83 & 18.61 & 8.58 \\
\hline $4 H-1-82$ & 26.52 & 5.45 & & \\
\hline $4 H-1-102$ & 26.72 & 8.99 & 6.35 & 8.56 \\
\hline $4 H-1-122$ & 26.98 & 1.86 & & \\
\hline $4 H-1-142$ & 27.12 & 3.42 & 6.68 & 0.51 \\
\hline $4 \mathrm{H}-2-22$ & 27.42 & 7.60 & 5.63 & 0.24 \\
\hline $4 H-2-42$ & 27.62 & 5.48 & & \\
\hline $4 H-2-62$ & 27.82 & 7.87 & 3.36 & 8.38 \\
\hline $4 H-2-82$ & 28.82 & 5.15 & & \\
\hline $4 \mathrm{H}-2-182$ & 28.22 & 6.63 & 4.88 & 8.37 \\
\hline $4 \mathrm{H}-2-122$ & 28.48 & 18.33 & & \\
\hline $4 \mathrm{H}-2-142$ & 28.68 & 4.45 & 5.69 & 8.41 \\
\hline $4 H-3-22$ & 28.92 & 2.85 & & \\
\hline $4 H-3-42$ & 29.12 & 3.99 & 0.80 & 8.36 \\
\hline $4 H-3-62$ & 29.32 & 12.42 & & \\
\hline $4 H-3-82$ & 29.52 & 7.23 & 3.36 & 8.45 \\
\hline $4 \mathrm{H}-3-102$ & 29.72 & 15.48 & & \\
\hline $4 H-3-122$ & 29.98 & 16.18 & 0.95 & 8.93 \\
\hline $4 \mathrm{H}-3-142$ & 38.12 & 2.25 & & \\
\hline $4 \mathrm{H}-4-22$ & 38.42 & 3.32 & 12.86 & 8.56 \\
\hline $4 H-4-42$ & 38.62 & 5.25 & & \\
\hline $4 H-4-62$ & 38.82 & 2.38 & 9.64 & 8.54 \\
\hline $4 H-4-82$ & 31.82 & 1.96 & 9.88 & 0.57 \\
\hline $4 H-4-182$ & 31.22 & 25.51 & 8.17 & 8.51 \\
\hline $4 H-4-122$ & 31.48 & 5.27 & 8.64 & 0.57 \\
\hline $4 H-4-142$ & 31.68 & 5.97 & 9.97 & 0.43 \\
\hline $4 H-5-22$ & 31.92 & 4.03 & 9.23 & 8.44 \\
\hline $4 \mathrm{H}-5-42$ & 32.12 & 2.64 & & \\
\hline $4 H-5-62$ & 32.32 & 8.66 & 3.57 & 8.64 \\
\hline $4 H-5-82$ & 32.52 & 13.86 & & \\
\hline $4 H-5-102$ & 32.72 & 12.68 & 8.38 & 8.69 \\
\hline $4 H-5-122$ & 32.92 & 6.59 & & \\
\hline $4 H-5-142$ & 33.12 & 5.93 & 4.53 & 8.27 \\
\hline $4 H-6-22$ & 33.42 & 2.72 & 3.87 & 8.31 \\
\hline $4 H-6-42$ & 33.62 & 4.29 & & \\
\hline $4 H-6-62$ & 33.82 & 3.58 & 4.33 & 8.57 \\
\hline $4 H-6-82$ & 34.82 & 1.62 & & \\
\hline $5 H-1-22$ & 35.42 & 4.25 & 8.73 & 8.45 \\
\hline $5 H-1-42$ & 35.62 & 3.94 & & \\
\hline $5 H-1-62$ & 35.82 & 13.77 & 6.63 & 0.76 \\
\hline $5 H-1-82$ & 36.82 & 4.58 & & \\
\hline $5 H-1-102$ & 36.22 & 14.13 & 8.27 & 0.70 \\
\hline $5 H-1-122$ & 36.48 & 15.93 & & \\
\hline $5 H-1-141$ & 36.31 & 28.53 & 1.29 & 8.82 \\
\hline $5 \mathrm{H}-2-22$ & 36.92 & 15.98 & 1.01 & 8.41 \\
\hline $5 \mathrm{H}-2-42$ & 37.12 & 15.66 & & \\
\hline
\end{tabular}

Table 1.4 (continued).

\begin{tabular}{|c|c|c|c|c|}
\hline $\begin{array}{c}\text { Sample } \\
\text { no. }\end{array}$ & $\begin{array}{l}\text { Depth } \\
\text { (mbsf) }\end{array}$ & $\begin{array}{l}>63 \\
\mu \mathrm{m} \\
(\%)\end{array}$ & $\begin{array}{c}\text { Bulk } \\
\mathrm{CaCO}_{3} \\
(\%)\end{array}$ & $\begin{array}{l}\text { TOC } \\
(\%)\end{array}$ \\
\hline $5 H-2-62$ & 37.32 & 1.45 & 11.88 & 0.75 \\
\hline $5 \mathrm{H}-2-82$ & 37.52 & 6.82 & 15.41 & \\
\hline $5 H-2-182$ & 37.72 & 14.28 & 18.34 & 8.33 \\
\hline $5 H-2-122$ & 37.98 & 5.64 & & \\
\hline $5 \mathrm{H}-2-137$ & 38.87 & 8.79 & 18.82 & 8.66 \\
\hline $5 \mathrm{H}-3-22$ & 38.42 & 14.16 & 7.82 & 0.51 \\
\hline $5 \mathrm{H}-3-42$ & 38.62 & 7.96 & & \\
\hline $5 \mathrm{H}-3-62$ & 38.82 & 3.97 & 18.38 & 0.65 \\
\hline $5 \mathrm{H}-3-82$ & 39.82 & 11.81 & & \\
\hline $5 H-3-182$ & 39.22 & 3.81 & 14.09 & 0.57 \\
\hline $5 \mathrm{H}-3-122$ & 39.48 & 4.21 & & \\
\hline $5 \mathrm{H}-3-137$ & 39.60 & 3.26 & 9.78 & 8.59 \\
\hline $5 \mathrm{H}-4-22$ & 39.92 & 2.26 & 12.75 & 0.92 \\
\hline $5 H-4-42$ & 48.12 & 2.58 & 18.86 & \\
\hline $5 H-4-62$ & 48.32 & 6.28 & 18.82 & 0.66 \\
\hline $5 H-4-82$ & 48.52 & 1.56 & & \\
\hline $5 H-4-182$ & 48.72 & 3.42 & 11.85 & 8.88 \\
\hline $5 H-4-122$ & 48.98 & 5.94 & & \\
\hline $5 H-4-137$ & 41.87 & 9.71 & 9.04 & 0.57 \\
\hline $5 H-5-22$ & 41.42 & 9.77 & 12.41 & 0.67 \\
\hline $5 H-5-42$ & 41.62 & 7.08 & & \\
\hline $5 H-5-62$ & 41.82 & 6.85 & 13.48 & 0.86 \\
\hline $5 \mathrm{H}-5-82$ & 42.82 & 23.15 & & \\
\hline $5 H-5-182$ & 42.22 & 9.93 & 12.12 & 8.94 \\
\hline $5 H-5-122$ & 42.48 & 5.19 & & \\
\hline $5 H-5-141$ & 42.64 & 26.89 & 9.32 & 0.48 \\
\hline $6 \mathrm{H}-1-22$ & 44.92 & 6.18 & 11.92 & 0.39 \\
\hline $6 H-1-59$ & 45.29 & 6.18 & & \\
\hline $6 H-1-78$ & 45.48 & 31.58 & 0.78 & 0.75 \\
\hline $6 H-1-101$ & 45.71 & 36.15 & & \\
\hline $6 H-1-122$ & 45.98 & 32.86 & 8.39 & 0.78 \\
\hline $6 H-1-142$ & 46.12 & 6.19 & & \\
\hline $6 \mathrm{H}-2-22$ & 46.42 & 2.82 & 9.25 & 0.58 \\
\hline $6 \mathrm{H}-2-59$ & 46.79 & 12.36 & & \\
\hline $6 \mathrm{H}-2-78$ & 46.92 & 11.12 & 8.97 & 0.31 \\
\hline $6 \mathrm{H}-2-181$ & 47.21 & 2.71 & 14.47 & \\
\hline $6 H-2-122$ & 47.48 & 1.87 & 9.25 & 8.58 \\
\hline $6 \mathrm{H}-2-142$ & 47.62 & 9.78 & & \\
\hline $6 H-3-22$ & 47.92 & 2.21 & 5.57 & 0.38 \\
\hline $6 \mathrm{H}-3-59$ & 48.29 & 6.62 & & \\
\hline $6 \mathrm{H}-3-78$ & 48.48 & 5.89 & 8.46 & 0.45 \\
\hline $6 \mathrm{H}-3-181$ & 48.71 & 35.88 & 4.78 & \\
\hline $6 \mathrm{H}-3-122$ & 48.98 & 2.89 & & 0.29 \\
\hline $6 H-3-142$ & 49.12 & 3.46 & & \\
\hline $6 \mathrm{H}-4-22$ & 49.42 & 7.85 & 23.81 & 0.25 \\
\hline $6 \mathrm{H}-4-42$ & 49.62 & 5.81 & & \\
\hline $6 H-4-59$ & 49.79 & 2.54 & 12.11 & 8.34 \\
\hline $6 \mathrm{H}-4-78$ & 49.98 & 6.84 & & \\
\hline $6 \mathrm{H}-4-101$ & 58.21 & 4.46 & 10.55 & 8.51 \\
\hline $6 \mathrm{H}-4-117$ & 58.37 & 4.67 & & \\
\hline $6 H-5-22$ & 50.12 & 5.28 & 5.18 & 8.27 \\
\hline
\end{tabular}


Table 1.4 (continued).

\begin{tabular}{|c|c|c|c|c|}
\hline $\begin{array}{c}\text { Sample } \\
\text { no. }\end{array}$ & $\begin{array}{l}\text { Depth } \\
\text { (mbsf) }\end{array}$ & $\begin{array}{l}>63 \\
\mu \mathrm{m} \\
(\%)\end{array}$ & $\begin{array}{c}\text { Bulk } \\
\mathrm{CaCO}_{3} \\
(\%)\end{array}$ & $\begin{array}{l}\text { TOC } \\
(\%)\end{array}$ \\
\hline $6 H-5-59$ & 58.29 & 5.10 & & \\
\hline $6 H-5-78$ & 58.48 & 7.88 & 4.82 & 8.63 \\
\hline $5 H-5-101$ & 58.71 & 12.48 & & \\
\hline $6 \mathrm{H}-5-122$ & 58.98 & 27.91 & 8.28 & 8.74 \\
\hline $6 H-5-142$ & 51.12 & 4.31 & & \\
\hline $6 H-6-22$ & 52.42 & 15.90 & 0.83 & 0.71 \\
\hline $6 H-6-42$ & 52.62 & 16.15 & & \\
\hline $6 \mathrm{H}-6-59$ & 52.79 & 6.03 & 11.44 & 8.58 \\
\hline $6 \mathrm{H}-6-78$ & 52.98 & 9.60 & & \\
\hline $6 H-6-181$ & 53.21 & 3.83 & 17.17 & 8.25 \\
\hline $7 \mathrm{H}-1-22$ & 54.42 & 15.11 & 1.19 & 0.68 \\
\hline $7 \mathrm{H}-1-42$ & 54.62 & 12.43 & & \\
\hline $7 H-1-64$ & 54.85 & 11.38 & 0.39 & 8.31 \\
\hline $7 H-1-83$ & 55.83 & 7.94 & & \\
\hline $7 \mathrm{H}-2-22$ & 55.42 & 12.76 & 7.58 & 1.12 \\
\hline $7 \mathrm{H}-2-42$ & 56.13 & 18.24 & & \\
\hline $7 \mathrm{H}-2-63$ & 56.33 & 2.78 & 11.19 & 0.93 \\
\hline $7 \mathrm{H}-2-83$ & 56.53 & 28.54 & 18.98 & \\
\hline $7 \mathrm{H}-3-22$ & 57.42 & 3.72 & 13.38 & 1.84 \\
\hline $7 \mathrm{H}-3-42$ & 57.62 & 1.91 & & \\
\hline $7 \mathrm{H}-3-64$ & 57.85 & 25.73 & 8.89 & 0.51 \\
\hline $7 \mathrm{H}-3-83$ & 58.03 & 2.84 & & \\
\hline $7 \mathrm{H}-4-22$ & 58.92 & 28.16 & 1.18 & 0.80 \\
\hline $7 \mathrm{H}-4-42$ & 59.13 & 1.82 & & \\
\hline $7 \mathrm{H}-4-65$ & 59.35 & 2.22 & 11.97 & 8.98 \\
\hline $7 H-4-86$ & 59.56 & 8.62 & & \\
\hline $8 \mathrm{H}-1-21$ & 63.91 & 9.56 & 7.89 & 8.63 \\
\hline $8 H-1-48$ & 64.11 & 13.72 & & \\
\hline $8 H-1-58$ & 64.29 & 3.61 & 11.88 & 0.46 \\
\hline $8 H-1-182$ & 64.72 & 7.58 & & \\
\hline $8 \mathrm{H}-2-21$ & 65.41 & 6.73 & 12.66 & 8.54 \\
\hline $8 H-2-48$ & 65.60 & 9.62 & & \\
\hline $8 \mathrm{H}-2-59$ & 65.78 & 4.13 & 11.46 & 0.58 \\
\hline $8 \mathrm{H}-2-82$ & 66.88 & 12.86 & & \\
\hline $8 \mathrm{H}-2-182$ & 66.22 & 3.08 & 11.19 & 0.58 \\
\hline $8 H-2-122$ & 66.42 & 3.05 & & \\
\hline $8 \mathrm{H}-2-142$ & 66.62 & 2.98 & 9.98 & 8.52 \\
\hline $8 \mathrm{H}-3-28$ & 66.98 & 3.88 & & \\
\hline $8 H-3-48$ & 67.11 & 13.98 & 8.89 & 0.74 \\
\hline $8 \mathrm{H}-3-68$ & 67.37 & 5.88 & & \\
\hline $8 \mathrm{H}-3-82$ & 67.58 & 5.94 & 9.76 & 0.46 \\
\hline $8 \mathrm{H}-3-182$ & 67.72 & 8.08 & & \\
\hline $8 \mathrm{H}-3-122$ & 67.98 & 7.68 & 9.39 & 0.36 \\
\hline $8 \mathrm{H}-3-142$ & 68.18 & 1.16 & & \\
\hline $8 H-4-22$ & 68.43 & 9.84 & 7.11 & 8.54 \\
\hline $8 \mathrm{H}-4-42$ & 68.62 & 13.66 & & \\
\hline $8 \mathrm{H}-5-22$ & 69.92 & 4.98 & 1.45 & 8.64 \\
\hline $8 H-5-42$ & 78.13 & 11.74 & & \\
\hline $8 H-5-68$ & 78.38 & 23.73 & 0.15 & 0.73 \\
\hline $8 H-5-82$ & 78.52 & 2.31 & & \\
\hline $8 \mathrm{H}-5-122$ & 78.92 & 6.47 & 2.73 & 0.27 \\
\hline
\end{tabular}

Table 1.4 (continued).

\begin{tabular}{|c|c|c|c|c|}
\hline $\begin{array}{c}\text { Sample } \\
\text { no. }\end{array}$ & $\begin{array}{l}\text { Depth } \\
\text { (mbsf) }\end{array}$ & $\begin{array}{l}>63 \\
\mu \mathrm{m} \\
(\%)\end{array}$ & $\begin{array}{c}\text { Bulk } \\
\mathrm{CaCO}_{3} \\
(\%)\end{array}$ & $\begin{array}{l}\text { TOC } \\
(\%)\end{array}$ \\
\hline $8 \mathrm{H}-5-142$ & 71.12 & 6.72 & & \\
\hline $8 \mathrm{H}-6-22$ & 71.42 & 2.44 & 7.67 & 0.69 \\
\hline $8 \mathrm{H}-6-42$ & 71.62 & 3.15 & 1.86 & \\
\hline $8 \mathrm{H}-6-68$ & 71.88 & 0.57 & 5.16 & 0.57 \\
\hline $9 \mathrm{H}-1-22$ & 73.43 & 2.86 & 11.63 & 8.54 \\
\hline $9 H-1-48$ & 73.68 & 2.23 & & \\
\hline $9 H-1-59$ & 73.79 & 3.95 & 16.95 & 8.72 \\
\hline $9 H-1-82$ & 74.82 & 1.53 & & \\
\hline $9 H-1-182$ & 74.22 & 0.67 & 7.86 & 8.65 \\
\hline $9 \mathrm{H}-1-123$ & 74.43 & 8.37 & & \\
\hline $9 H-1-142$ & 74.62 & 17.89 & 2.67 & 8.29 \\
\hline $9 \mathrm{H}-2-23$ & 74.93 & 6.32 & & \\
\hline $9 H-2-4 \theta$ & 75.18 & 3.92 & 4.26 & 0.30 \\
\hline $9 H-2-59$ & 75.29 & 1.94 & & \\
\hline $9 \mathrm{H}-2-82$ & 75.52 & 2.52 & 5.99 & 8. 31 \\
\hline $9 \mathrm{H}-2-182$ & 75.72 & 5.52 & & \\
\hline $9 \mathrm{H}-2-123$ & 75.93 & 5.72 & 4.98 & 0.51 \\
\hline $9 H-2-142$ & 76.12 & 13.96 & 1.83 & 0.39 \\
\hline $9 \mathrm{H}-3-23$ & 76.43 & 1.43 & 7.81 & 0.81 \\
\hline $9 H-3-48$ & 76.68 & 0.80 & & \\
\hline $9 \mathrm{H}-3-59$ & 76.79 & 1.49 & 3.39 & 0.42 \\
\hline $9 H-3-82$ & 77.02 & 2.31 & 7.81 & \\
\hline $9 \mathrm{H}-3-182$ & 77.22 & 1.87 & 6.08 & 0.78 \\
\hline $9 \mathrm{H}-3-123$ & 77.43 & 1.05 & & \\
\hline $9 \mathrm{H}-3-142$ & 77.62 & 22.37 & 1.04 & 0.78 \\
\hline $9 \mathrm{H}-4-23$ & 78.43 & 4.21 & & \\
\hline $9 \mathrm{H}-4-48$ & 78.68 & 27.21 & 1.73 & 0.63 \\
\hline $9 \mathrm{H}-4-59$ & 78.79 & 6.92 & & \\
\hline $9 \mathrm{H}-4-82$ & 79.82 & 4.61 & 3.68 & 8.38 \\
\hline $9 \mathrm{H}-4-182$ & 79.22 & 5.38 & 2.51 & 0.34 \\
\hline $9 \mathrm{H}-4-123$ & 79.43 & 1.48 & & \\
\hline $9 \mathrm{H}-5-23$ & 79.93 & 1.03 & 4.94 & 0.68 \\
\hline $9 \mathrm{H}-5-4 \theta$ & 88.18 & 14.57 & 8.77 & 0.56 \\
\hline $9 \mathrm{H}-5-59$ & 88.29 & 5.85 & & \\
\hline $9 H-5-82$ & 88.52 & 5.68 & 4.41 & 0.34 \\
\hline $9 H-5-182$ & 88.72 & 9.26 & & \\
\hline $9 H-5-123$ & 88.93 & 2.88 & 1.89 & 0.77 \\
\hline $10 \mathrm{H}-2-22$ & 84.42 & 1.78 & 16.84 & 0.58 \\
\hline $18 \mathrm{H}-2-41$ & 84.61 & 0.81 & 9.46 & 0.74 \\
\hline $18 \mathrm{H}-2-57$ & 84.78 & 2.28 & & \\
\hline $10 \mathrm{H}-2-121$ & 85.42 & 1.75 & 17.34 & 0.68 \\
\hline $18 \mathrm{H}-2-142$ & 85.63 & 0.72 & & \\
\hline $10 \mathrm{H}-3-22$ & 85.92 & 8.26 & 10.74 & 0.62 \\
\hline $18 \mathrm{H}-3-41$ & 86.11 & 4.92 & & \\
\hline $18 \mathrm{H}-3-57$ & 86.28 & 1.83 & 14.85 & 0.78 \\
\hline $10 \mathrm{H}-3-78$ & 86.48 & 1.93 & & \\
\hline $18 \mathrm{H}-3-101$ & 86.71 & 8.93 & 12.98 & 0.54 \\
\hline $18 \mathrm{H}-3-121$ & 86.92 & 2.52 & & \\
\hline $10 \mathrm{H}-3-142$ & 87.12 & 8.11 & 8.54 & 0.28 \\
\hline $10 \mathrm{H}-4-21$ & 87.42 & 1.84 & & \\
\hline $10 \mathrm{H}-4-41$ & 87.61 & 0.93 & 17.99 & 8.63 \\
\hline
\end{tabular}


Table 1.4 (continued).

\begin{tabular}{|c|c|c|c|c|}
\hline $\begin{array}{c}\text { Sample } \\
\text { no. }\end{array}$ & $\begin{array}{l}\text { Depth } \\
\text { (mbsf) }\end{array}$ & $\begin{array}{l}>63 \\
\mu \mathrm{m} \\
(\%)\end{array}$ & $\begin{array}{c}\text { Bulk } \\
\mathrm{CaCO}_{3} \\
(\%)\end{array}$ & $\begin{array}{l}\text { TOC } \\
(\%)\end{array}$ \\
\hline $10 \mathrm{H}-4-57$ & 87.78 & 28.98 & & \\
\hline $18 \mathrm{H}-4-78$ & 87.98 & 28.55 & 5.33 & 8.25 \\
\hline $10 \mathrm{H}-4-181$ & 88.22 & 27.55 & & \\
\hline $18 \mathrm{H}-4-121$ & 88.41 & 11.32 & 28.63 & 0.36 \\
\hline $10 \mathrm{H}-5-21$ & 88.91 & 8.81 & 15.34 & 8.71 \\
\hline $18 \mathrm{H}-5-41$ & 89.11 & 3.22 & & \\
\hline $18 \mathrm{H}-5-57$ & 89.28 & 1.39 & 14.82 & 8.64 \\
\hline $10 \mathrm{H}-5-78$ & 89.48 & 9.51 & & \\
\hline $18 \mathrm{H}-5-101$ & 89.71 & 5.11 & 12.93 & 8.51 \\
\hline $18 \mathrm{H}-5-121$ & 89.92 & 3.17 & 15.15 & \\
\hline $18 \mathrm{H}-6-21$ & 98.42 & 14.21 & 6.24 & 8.28 \\
\hline $10 H-6-41$ & 98.62 & 5.79 & 6.55 & 8.50 \\
\hline $11 H-1-22$ & 92.42 & 3.98 & 10.68 & 8.32 \\
\hline $11 \mathrm{H}-1-42$ & 92.62 & 3.83 & & \\
\hline $11 H-1-6 \theta$ & 92.88 & 2.79 & 11.69 & 8.29 \\
\hline $11 \mathrm{H}-1-81$ & 93.81 & 13.63 & & \\
\hline $11 H-1-10 \theta$ & 93.28 & 8.94 & 4.49 & 8.44 \\
\hline $11 H-1-122$ & 93.48 & 2.37 & & \\
\hline $11 H-1-142$ & 93.62 & 5.62 & 3.43 & 0.45 \\
\hline $11 \mathrm{H}-2-22$ & 93.92 & 5.96 & & \\
\hline $11 \mathrm{H}-2-39$ & 94.89 & 3.42 & 9.81 & 8.59 \\
\hline $11 \mathrm{H}-2-63$ & 94.33 & 2.16 & & \\
\hline $11 \mathrm{H}-2-81$ & 94.51 & 1.22 & 4.74 & 0.59 \\
\hline $11 \mathrm{H}-2-108$ & 94.78 & 1.99 & & \\
\hline $11 \mathrm{H}-2-122$ & 94.92 & 8.99 & 11.92 & 8.66 \\
\hline $11 \mathrm{H}-2-142$ & 95.14 & 1.51 & & \\
\hline $11 \mathrm{H}-3-22$ & 95.42 & 1.78 & 11.38 & 8.53 \\
\hline $11 \mathrm{H}-3-42$ & 95.62 & 5.82 & & \\
\hline $11 \mathrm{H}-3-6 \theta$ & 95.88 & 2.91 & 22.29 & 8.35 \\
\hline $11 \mathrm{H}-3-81$ & 96.81 & 13.87 & & \\
\hline $11 \mathrm{H}-3-180$ & 96.28 & 11.55 & 2.49 & 1.86 \\
\hline $11 \mathrm{H}-3-122$ & 96.48 & 3.94 & & \\
\hline $11 \mathrm{H}-3-142$ & 96.62 & 5.38 & 28.86 & 0.36 \\
\hline $11 \mathrm{H}-4-22$ & 96.92 & 3.88 & & \\
\hline $11 \mathrm{H}-4-42$ & 97.14 & 3.15 & 17.18 & 8.39 \\
\hline $11 H-4-68$ & 97.38 & 2.57 & & \\
\hline $11 \mathrm{H}-4-81$ & 97.51 & 1.29 & 18.66 & 0.43 \\
\hline $11 \mathrm{H}-4-180$ & 97.78 & 7.52 & 4.98 & \\
\hline $11 \mathrm{H}-4-122$ & 97.92 & 28.32 & 8.84 & 8.61 \\
\hline $11 \mathrm{H}-4-142$ & 97.14 & 4.27 & & \\
\hline $11 \mathrm{H}-5-22$ & 98.42 & 8.66 & 5.41 & 8.58 \\
\hline $11 \mathrm{H}-5-42$ & 98.62 & 17.98 & & \\
\hline $11 \mathrm{H}-5-68$ & 98.88 & 8.69 & 1.83 & 8.58 \\
\hline $11 \mathrm{H}-5-88$ & 99.88 & 8.38 & & \\
\hline $11 \mathrm{H}-5-100$ & 99.38 & 8.51 & 0.81 & 8.45 \\
\hline $11 \mathrm{H}-5-122$ & 99.52 & 2.28 & & \\
\hline $11 \mathrm{H}-6-22$ & 99.92 & 1.72 & 7.58 & 0.54 \\
\hline $11 \mathrm{H}-6-42$ & 180.14 & 8.91 & & \\
\hline $11 \mathrm{H}-6-68$ & 180.38 & 1.78 & 13.58 & 8.72 \\
\hline $11 \mathrm{H}-6-88$ & 180.58 & 1.58 & & \\
\hline $11 H-6-18 \theta$ & 100.70 & 1.57 & 13.28 & 0.75 \\
\hline
\end{tabular}

Table 1.4 (continued).

\begin{tabular}{|c|c|c|c|c|}
\hline $\begin{array}{c}\text { Sample } \\
\text { no. }\end{array}$ & $\begin{array}{l}\text { Depth } \\
\text { (mbsf) }\end{array}$ & $\begin{array}{l}>63 \\
\mu \mathrm{m} \\
(\%)\end{array}$ & $\begin{array}{c}\text { Bulk } \\
\mathrm{CaCO}_{3} \\
(\%)\end{array}$ & $\begin{array}{l}\text { TOC } \\
(\%)\end{array}$ \\
\hline $11 H-6-122$ & 180.92 & 1.28 & & \\
\hline $11 \mathrm{H}-6-142$ & 181.14 & 5.73 & 15.68 & 0.73 \\
\hline $11 \mathrm{H}-7-22$ & 101.42 & 1.16 & 13.18 & 0.62 \\
\hline $12 \mathrm{H}-1-22$ & 181.91 & 15.99 & 1.80 & 8.33 \\
\hline $12 \mathrm{H}-1-42$ & 102.10 & 1.46 & & \\
\hline $12 \mathrm{H}-1-62$ & 182.38 & 4.56 & 6.76 & 8.67 \\
\hline $12 \mathrm{H}-1-82$ & 102.49 & 1.78 & & \\
\hline $12 \mathrm{H}-1-102$ & 102.68 & 1.87 & 3.33 & 8.58 \\
\hline $12 \mathrm{H}-1-122$ & 102.87 & 2.45 & & \\
\hline $12 \mathrm{H}-1-142$ & 183.86 & 4.87 & 1.54 & 8.49 \\
\hline $12 \mathrm{H}-2-22$ & 103.35 & 8.08 & & \\
\hline $12 \mathrm{H}-2-42$ & 103.54 & 0.58 & 0.75 & 8.60 \\
\hline $12 \mathrm{H}-2-62$ & 183.74 & 2.65 & & \\
\hline $12 \mathrm{H}-2-82$ & 103.93 & 5.65 & 8.52 & 8.56 \\
\hline $12 \mathrm{H}-2-182$ & 184.12 & 1.92 & & \\
\hline $12 \mathrm{H}-2-122$ & 184.31 & 1.38 & 13.16 & 0.71 \\
\hline $12 \mathrm{H}-2-142$ & 184.58 & 2.85 & & \\
\hline $12 \mathrm{H}-3-22$ & 184.79 & 6.92 & 8.44 & 8.41 \\
\hline $12 \mathrm{H}-3-42$ & 184.98 & 1.24 & & \\
\hline $12 \mathrm{H}-3-62$ & 185.18 & 1.04 & 8.41 & 8.47 \\
\hline $12 \mathrm{H}-3-82$ & 105.37 & 1.84 & & \\
\hline $12 \mathrm{H}-3-182$ & 105.56 & 0.79 & 8.16 & 8.59 \\
\hline $12 \mathrm{H}-3-122$ & 185.75 & 0.39 & & \\
\hline $12 \mathrm{H}-3-142$ & 105.94 & 4.66 & 0.23 & 0. 28 \\
\hline $12 \mathrm{H}-4-22$ & 106.23 & 3.76 & & \\
\hline $12 \mathrm{H}-4-42$ & 186.42 & 8.94 & 6.17 & 8.51 \\
\hline $12 \mathrm{H}-4-62$ & 106.62 & 1.17 & & \\
\hline $12 \mathrm{H}-4-82$ & 106.81 & 3.81 & 8.63 & 8.55 \\
\hline $12 \mathrm{H}-4-102$ & 187.08 & 5.94 & 8.88 & \\
\hline $12 \mathrm{H}-5-22$ & 187.67 & 16.86 & 1.96 & 1.88 \\
\hline $12 \mathrm{H}-5-42$ & 187.86 & 6.27 & & \\
\hline $12 \mathrm{H}-5-62$ & 108.06 & 3.14 & 3.91 & 8.75 \\
\hline $12 \mathrm{H}-5-82$ & 188.25 & 2.66 & & \\
\hline $12 \mathrm{H}-5-182$ & 188.44 & 2.55 & 8.87 & 8.34 \\
\hline $12 \mathrm{H}-5-122$ & 188.63 & 4.37 & & \\
\hline $12 \mathrm{H}-5-142$ & 108.82 & 7.43 & 8.41 & 0.33 \\
\hline $12 \mathrm{H}-6-22$ & 189.11 & 4.64 & & \\
\hline $12 \mathrm{H}-6-42$ & 189.38 & 8.61 & 8. 26 & 8.51 \\
\hline $12 \mathrm{H}-6-62$ & 109.58 & 1.41 & & \\
\hline $12 \mathrm{H}-6-82$ & 189.69 & 1.58 & 6.55 & 8.68 \\
\hline $12 \mathrm{H}-6-182$ & 109.88 & 3.78 & & \\
\hline $12 \mathrm{H}-6-122$ & 110.87 & 5.18 & 29.23 & 0.71 \\
\hline $12 \mathrm{H}-6-142$ & 110.26 & 1.77 & & \\
\hline $12 \mathrm{H}-7-22$ & 110.55 & 0.88 & 3.45 & 8.88 \\
\hline $12 \mathrm{H}-7-42$ & 110.74 & 1.54 & 9.48 & 0.79 \\
\hline $13 \mathrm{H}-1-62$ & 111.81 & 1.88 & 7.81 & 8.78 \\
\hline $13 \mathrm{H}-1-82$ & 112.80 & 1.23 & & \\
\hline $13 \mathrm{H}-1-182$ & 112.28 & 8.79 & 6.22 & 0.74 \\
\hline $13 \mathrm{H}-1-122$ & $112.4 \theta$ & 0.72 & & \\
\hline $13 \mathrm{H}-1-145$ & 112.62 & 2.87 & 3.76 & 8.68 \\
\hline $13 \mathrm{H}-2-21$ & 112.88 & 18.42 & & \\
\hline
\end{tabular}


Table 1.4 (continued).

\begin{tabular}{|c|c|c|c|c|}
\hline $\begin{array}{c}\text { Sample } \\
\text { no. }\end{array}$ & $\begin{array}{l}\text { Depth } \\
\text { (mbsf) }\end{array}$ & $\begin{array}{l}>63 \\
\mu \mathrm{m} \\
(\%)\end{array}$ & $\begin{array}{c}\text { Bulk } \\
\mathrm{CaCO}_{3} \\
(\%)\end{array}$ & $\begin{array}{l}\text { TOC } \\
(\%)\end{array}$ \\
\hline $13 \mathrm{H}-2-42$ & 113.88 & 5.24 & 0.34 & 0.26 \\
\hline $13 \mathrm{H}-2-62$ & 113.28 & 4.58 & & \\
\hline $13 \mathrm{H}-2-82$ & 113.47 & 1.03 & 0.48 & 0.58 \\
\hline $13 H-2-182$ & 113.67 & 5.88 & 0.29 & \\
\hline $13 H-2-122$ & 113.87 & 1.56 & 4.44 & 8.67 \\
\hline $13 \mathrm{H}-3-21$ & 114.35 & 3.38 & 2.34 & 8.33 \\
\hline $13 H-3-145$ & 114.58 & 9.74 & 5.65 & 8.64 \\
\hline $13 H-4-21$ & 115.82 & 18.35 & & \\
\hline $13 \mathrm{H}-4-42$ & 116.82 & 0.75 & 4.49 & 0.61 \\
\hline $13 H-4-62$ & 116.22 & 3.87 & & \\
\hline $13 \mathrm{H}-4-82$ & 116.41 & 3.96 & 10.21 & 8.39 \\
\hline $13 H-4-182$ & 116.61 & 2.89 & & \\
\hline $13 H-4-122$ & 116.81 & 15.82 & 4.91 & 0.79 \\
\hline $13 H-5-21$ & 117.29 & 4.28 & & \\
\hline $13 \mathrm{H}-5-42$ & 117.49 & 7.28 & 3.47 & 0.66 \\
\hline $13 H-5-62$ & 117.69 & 6.42 & & \\
\hline $13 H-5-86$ & 117.92 & 6.61 & 8.30 & 0.45 \\
\hline $13 H-6-21$ & 118.76 & 1.22 & & \\
\hline $13 H-6-42$ & 118.96 & 1.84 & 9.29 & 8.76 \\
\hline $13 H-6-62$ & 119.16 & 8.79 & & \\
\hline $13 H-6-82$ & 119.35 & 1.16 & 5.84 & 8.65 \\
\hline $13 H-7-21$ & 119.56 & 1.77 & & \\
\hline $13 \mathrm{H}-7-42$ & 119.77 & 13.74 & 1.13 & 0.35 \\
\hline $13 H-7-62$ & 119.96 & 0.67 & & \\
\hline $13 \mathrm{H}-7-82$ & 128.16 & 1.31 & 6.18 & 0.78 \\
\hline $13 H-7-102$ & 128.35 & 8.48 & & \\
\hline $13 H-7-122$ & 120.55 & 0.73 & 5.45 & 0.76 \\
\hline $13 H-C-21$ & 120.76 & 8.78 & 1.36 & B. 87 \\
\hline $14 \mathrm{H}-2-82$ & 123.02 & 6.28 & 0.63 & 0.39 \\
\hline $14 \mathrm{H}-2-183$ & 123.23 & 4.25 & & \\
\hline $14 \mathrm{H}-2-123$ & 123.43 & 2.82 & 5.79 & B. 30 \\
\hline $14 \mathrm{H}-2-142$ & 123.62 & 24.58 & & \\
\hline $14 \mathrm{H}-3-21$ & 123.91 & 24.52 & 1.84 & 0.91 \\
\hline $14 \mathrm{H}-3-41$ & 124.11 & 6.98 & & \\
\hline $14 \mathrm{H}-3-62$ & 124.32 & 2.72 & 0.83 & 0.58 \\
\hline $14 \mathrm{H}-3-82$ & 123.52 & 3.15 & & \\
\hline $14 H-3-103$ & 124.72 & 3.87 & 0.55 & 0.37 \\
\hline $14 \mathrm{H}-3-123$ & 124.93 & 3.38 & 8.88 & \\
\hline $14 \mathrm{H}-4-21$ & 125.41 & 8.41 & 8.42 & 8.67 \\
\hline $14 \mathrm{H}-4-41$ & 125.61 & 2.21 & & \\
\hline $14 \mathrm{H}-4-62$ & 125.82 & 2.22 & 8.80 & 0.69 \\
\hline $14 H-4-82$ & 126.82 & 8.26 & & \\
\hline $14 \mathrm{H}-4-183$ & 126.22 & 1.97 & 8.44 & 0.78 \\
\hline $14 \mathrm{H}-4-123$ & 126.38 & 1.85 & & \\
\hline $14 \mathrm{H}-5-21$ & 126.91 & 2.82 & 3.93 & 8.81 \\
\hline $14 H-5-41$ & 127.11 & 18.84 & & \\
\hline $14 \mathrm{H}-5-62$ & 127.32 & 21.53 & 8.08 & 0.78 \\
\hline $14 H-5-82$ & 127.52 & 8.73 & & \\
\hline $14 \mathrm{H}-5-102$ & 127.72 & 1.37 & 5.31 & 8.71 \\
\hline $14 H-5-123$ & 127.93 & 1.88 & & \\
\hline $14 \mathrm{H}-5-142$ & 128.12 & 5.85 & 5.12 & 8.81 \\
\hline
\end{tabular}

Table 1.4 (continued).

\begin{tabular}{|c|c|c|c|c|}
\hline $\begin{array}{c}\text { Sample } \\
\text { no. }\end{array}$ & $\begin{array}{l}\text { Depth } \\
\text { (mbsf) }\end{array}$ & $\begin{array}{l}>63 \\
\mu \mathrm{m} \\
(\%)\end{array}$ & $\begin{array}{c}\text { Bulk } \\
\mathrm{CaCO}_{3} \\
(\%)\end{array}$ & $\begin{array}{l}\text { TOC } \\
(\%)\end{array}$ \\
\hline $14 \mathrm{H}-\mathrm{C}-15$ & 128.35 & 4.81 & 6.27 & 8.67 \\
\hline $15 \mathrm{H}-1-22$ & 130.42 & 3.35 & 154.80 & 0.56 \\
\hline $15 \mathrm{H}-1-42$ & 130.62 & 0.93 & & \\
\hline $15 H-1-62$ & 138.82 & 6.57 & 2.23 & 8.98 \\
\hline $15 H-1-82$ & 131.82 & 6.39 & 8.53 & \\
\hline $15 t-1-182$ & 131.22 & 6.96 & 3.87 & 0.83 \\
\hline $15 t-1-122$ & 131.42 & 1.96 & & \\
\hline $15 H-1-142$ & 131.62 & 1.65 & 8.88 & 0.62 \\
\hline $15 H-2-22$ & 131.92 & 1.28 & & \\
\hline $15 \mathrm{H}-2-42$ & 132.12 & 4.86 & 0.11 & 0.43 \\
\hline $15 \mathrm{H}-2-62$ & 132.32 & 4.03 & & \\
\hline $15 \mathrm{H}-2-82$ & 132.52 & 5.65 & 8.88 & 8.53 \\
\hline $15 H-2-182$ & 132.72 & 3.59 & & \\
\hline $15 \mathrm{H}-2-122$ & 132.92 & 9.75 & 1.80 & 0.81 \\
\hline $15 H-2-142$ & 133.12 & 1.17 & 0.80 & 0.88 \\
\hline $15 H-3-42$ & 133.42 & 1.34 & 7.38 & 8.93 \\
\hline $15 H-3-62$ & 133.62 & 1.32 & & \\
\hline $15 H-3-82$ & 133.82 & 1.35 & 4.61 & 0.77 \\
\hline $15 \mathrm{H}-3-182$ & 134.82 & 0.99 & & \\
\hline $15 \mathrm{H}-3-122$ & 134.22 & 1.56 & 1.78 & 1.09 \\
\hline $15 H-3-142$ & 134.42 & 1.74 & & \\
\hline $15 \mathrm{H}-4-22$ & 134.92 & 1.37 & 2.88 & 0.86 \\
\hline $15 H-4-42$ & 135.12 & 1.88 & & \\
\hline $15 \mathrm{H}-4-62$ & 135.32 & 1.72 & 8.54 & 8.65 \\
\hline $15 \mathrm{H}-4-82$ & 135.52 & 0.71 & & \\
\hline $15 \mathrm{H}-4-182$ & 135.72 & 1.87 & 8.27 & 0.63 \\
\hline $15 H-4-122$ & 135.92 & 8.52 & 8.67 & 8.57 \\
\hline $15 \mathrm{H}-5-22$ & 136.42 & 1.00 & 5.85 & 8.86 \\
\hline $15 H-5-62$ & 136.62 & 6.82 & & \\
\hline $15 \mathrm{H}-5-82$ & 136.82 & 8.99 & 7.84 & 0.77 \\
\hline $15 \mathrm{H}-5-182$ & 137.82 & 0.73 & & \\
\hline $15 H-5-122$ & 137.22 & 8.75 & 7.22 & 8.78 \\
\hline $15 H-5-142$ & 137.42 & 1.62 & & \\
\hline $15 H-6-22$ & 137.92 & 1.53 & 7.42 & 8.73 \\
\hline $15 H-6-42$ & 138.12 & 0.61 & & \\
\hline $15 \mathrm{H}-6-62$ & 138.32 & 7.44 & 8.80 & 0.86 \\
\hline $15 H-6-82$ & 138.52 & 1.71 & & \\
\hline $15 \mathrm{H}-7-22$ & 139.42 & 2.11 & 0.18 & 8.64 \\
\hline $15 \mathrm{H}-7-42$ & 139.62 & 0.88 & & \\
\hline $15 \mathrm{H}-7-62$ & 139.82 & 7.22 & 8.23 & 0.58 \\
\hline $16 \mathrm{H}-1-23$ & 139.93 & 8.73 & 8.78 & 8.75 \\
\hline $16 \mathrm{H}-1-102$ & 140.72 & 1.18 & 24.28 & 8.67 \\
\hline $16 \mathrm{H}-1-128$ & 148.98 & 1.11 & 34.68 & 8.65 \\
\hline $16 \mathrm{H}-1-142$ & 141.12 & 1.41 & 23.21 & 0.52 \\
\hline $16 \mathrm{H}-2-23$ & 141.43 & 9.88 & 19.51 & 0.55 \\
\hline $16 \mathrm{H}-2-182$ & 142.82 & 0.78 & 2.68 & 0.31 \\
\hline $16 \mathrm{H}-2-128$ & 142.28 & 2.15 & 2.17 & 8.38 \\
\hline $16 \mathrm{H}-2-142$ & 142.42 & 6.39 & 6.31 & 0.39 \\
\hline $16 \mathrm{H}-3-23$ & 142.93 & 3.37 & 8.69 & 8.61 \\
\hline $16 \mathrm{H}-3-182$ & 143.72 & 1.11 & 6.85 & 0.74 \\
\hline $16 \mathrm{H}-3-128$ & 143.98 & 1.36 & & \\
\hline
\end{tabular}


Table 1.4 (continued).

\begin{tabular}{|c|c|c|c|c|}
\hline $\begin{array}{c}\text { Sample } \\
\text { no. }\end{array}$ & $\begin{array}{l}\text { Depth } \\
\text { (mbsf) }\end{array}$ & $\begin{array}{l}>63 \\
\mu \mathrm{m} \\
(\%)\end{array}$ & $\begin{array}{c}\text { Bulk } \\
\mathrm{CaCO}_{3} \\
(\%)\end{array}$ & $\begin{array}{l}\text { TOC } \\
(\%)\end{array}$ \\
\hline $16 \mathrm{H}-3-142$ & 144.12 & 8.84 & 5.89 & 0.85 \\
\hline $16 \mathrm{H}-4-23$ & 144.43 & 1.15 & 1.39 & 0.83 \\
\hline $16 \mathrm{H}-4-102$ & 145.02 & 1.45 & 5.47 & 0.78 \\
\hline $16 \mathrm{H}-4-128$ & 145.28 & 1.48 & 4.42 & 0.75 \\
\hline $16 \mathrm{H}-5-23$ & 145.93 & 8. 82 & 8.94 & 8.64 \\
\hline $16 \mathrm{H}-5-102$ & 146.72 & 8.75 & 18.86 & 0.86 \\
\hline $16 \mathrm{H}-5-12 \theta$ & 146.92 & 1.29 & & \\
\hline $16 \mathrm{H}-5-142$ & 147.12 & 8.85 & 14.37 & 8.87 \\
\hline $16 \mathrm{H}-6-23$ & 147.43 & 4.68 & 1.21 & 0.32 \\
\hline $17 \mathrm{H}-1-23$ & 149.42 & 1.39 & 8.80 & 0.84 \\
\hline $17 \mathrm{H}-1-42$ & 149.62 & 1.87 & & \\
\hline $17 \mathrm{H}-1-122$ & 158.42 & 1.24 & 8.17 & 0.63 \\
\hline $17 H-1-142$ & 150.62 & 3.85 & & \\
\hline $17 \mathrm{H}-2-22$ & 150.92 & 2.85 & 27.88 & 8.73 \\
\hline $17 \mathrm{H}-2-42$ & 151.12 & 8.97 & & \\
\hline $17 \mathrm{H}-2-62$ & 151.22 & 4.74 & 8.49 & 0.78 \\
\hline $17 \mathrm{H}-3-22$ & 152.42 & 0.82 & 14.86 & 0.75 \\
\hline $17 \mathrm{H}-3-42$ & 152.62 & 1.38 & & \\
\hline $17 \mathrm{H}-3-62$ & 152.82 & 2.16 & 5.88 & B. 77 \\
\hline $17 \mathrm{H}-4-22$ & 153.92 & 8.89 & 8.80 & 0.79 \\
\hline $17 \mathrm{H}-4-42$ & 154.12 & 2.59 & 1.53 & \\
\hline $17 \mathrm{H}-4-102$ & 154.32 & 1.89 & & 8.86 \\
\hline $17 \mathrm{H}-4-122$ & 154.52 & 2.39 & & \\
\hline $17 \mathrm{H}-4-142$ & 154.72 & 1.12 & 4.58 & 8.69 \\
\hline $17 \mathrm{H}-5-22$ & 155.42 & 1.65 & 32.11 & 0.78 \\
\hline $17 \mathrm{H}-5-122$ & 156.42 & 1.44 & 7.98 & 8.78 \\
\hline $17 \mathrm{H}-6-22$ & 157.42 & 1.58 & 8.56 & 8.69 \\
\hline $17 \mathrm{H}-6-62$ & 157.62 & 8.24 & B. 80 & 8.69 \\
\hline $18 \mathrm{H}-1-42$ & 159.12 & 1.46 & 8.80 & 8. 58 \\
\hline $18 H-1-58$ & 159.38 & 2.61 & & \\
\hline $18 \mathrm{H}-1-102$ & 159.52 & 1.35 & 3.22 & 0.86 \\
\hline $18 \mathrm{H}-1-122$ & 159.72 & 8.99 & & \\
\hline $18 H-1-142$ & 159.92 & 0.98 & 5.13 & 0.75 \\
\hline $18 \mathrm{H}-2-42$ & 160.62 & 1.31 & 8.45 & 8.64 \\
\hline $18 \mathrm{H}-2-58$ & 168.88 & 15.19 & & \\
\hline $18 \mathrm{H}-2-182$ & 161.28 & 1.48 & 8.39 & 8.57 \\
\hline $18 \mathrm{H}-2-122$ & 161.42 & 3.75 & & \\
\hline $18 \mathrm{H}-2-142$ & 161.62 & 1.93 & 1.64 & 8.66 \\
\hline $18 \mathrm{H}-3-42$ & 162.12 & 8.42 & 1.82 & 0.60 \\
\hline $18 \mathrm{H}-3-58$ & 162.38 & 14.85 & 0.88 & \\
\hline $18 \mathrm{H}-3-182$ & 162.52 & 18.57 & 9.83 & 8.56 \\
\hline $18 \mathrm{H}-3-122$ & 162.72 & 0.45 & & \\
\hline $18 \mathrm{H}-3-142$ & 162.92 & 3.73 & 0.80 & 0.34 \\
\hline $18 \mathrm{H}-4-42$ & 163.62 & 2.34 & 8.80 & 8.76 \\
\hline $18 \mathrm{H}-4-58$ & 163.88 & 3.68 & & \\
\hline $18 \mathrm{H}-4-182$ & 164.28 & 0.46 & 0.88 & 8.62 \\
\hline $18 \mathrm{H}-5-42$ & 165.12 & 8.58 & 4.85 & 8.88 \\
\hline $18 H-5-58$ & 165.38 & 1.61 & & \\
\hline $18 \mathrm{H}-5-182$ & 165.52 & 0.86 & 2.78 & 8.37 \\
\hline $18 H-5-122$ & 165.72 & 1.89 & & \\
\hline $18 \mathrm{H}-5-142$ & 165.92 & 0.64 & 9.88 & 8.63 \\
\hline
\end{tabular}

Table 1.4 (continued).

\begin{tabular}{|c|c|c|c|c|}
\hline $\begin{array}{c}\text { Sample } \\
\text { no. }\end{array}$ & $\begin{array}{l}\text { Depth } \\
\text { (mbsf) }\end{array}$ & $\begin{array}{l}>63 \\
\mu \mathrm{m} \\
(\%)\end{array}$ & $\begin{array}{c}\text { Bulk } \\
\mathrm{CaCO}_{3} \\
(\%)\end{array}$ & $\begin{array}{l}\text { TOC } \\
(\%)\end{array}$ \\
\hline $18 \mathrm{H}-6-42$ & 166.62 & 8.48 & 2.37 & 8.41 \\
\hline $18 \mathrm{H}-6-58$ & 166.88 & 1.72 & 3.88 & 0.58 \\
\hline $18 \mathrm{H}-7-42$ & 168.12 & 13.95 & 8.47 & 1.48 \\
\hline $18 \mathrm{H}-7-58$ & 168.38 & 8.36 & 1.21 & 0.76 \\
\hline $19 \mathrm{H}-2-22$ & 169.92 & 1.81 & 5.38 & 8.63 \\
\hline $19 \mathrm{H}-2-82$ & 178.52 & 18.41 & & \\
\hline $19 \mathrm{H}-2-10 \theta$ & 178.78 & 1.51 & 1.08 & 0.51 \\
\hline $19 \mathrm{H}-3-22$ & 171.42 & 1.35 & 1.35 & 8.88 \\
\hline $19 \mathrm{H}-3-82$ & 172.82 & 2.29 & 8.58 & \\
\hline $19 \mathrm{H}-3-98$ & 172.22 & $1.5 \theta$ & 18.18 & 0.76 \\
\hline $19 \mathrm{H}-3-128$ & 172.48 & 8.89 & & \\
\hline $19 H-3-142$ & 172.62 & 8.86 & 3.64 & 8.74 \\
\hline $19 H-4-22$ & 172.92 & 1.37 & 1.89 & 0.87 \\
\hline $19 H-4-82$ & 173.52 & 8.78 & & \\
\hline $19 H-4-100$ & 173.78 & 2.61 & 2.92 & 0.69 \\
\hline $19 \mathrm{H}-4-142$ & 174.12 & 8.37 & 8.78 & 8.77 \\
\hline $28 \mathrm{H}-1-42$ & 178.12 & 2.88 & 0.80 & 0.49 \\
\hline $28 \mathrm{BH}-1-82$ & 178.22 & 9.48 & & \\
\hline $28 \mathrm{H}-1-102$ & 178.42 & 0.82 & 13.34 & 0.81 \\
\hline $28 \mathrm{BH}-1-122$ & 178.62 & 8.40 & & \\
\hline $2 \mathrm{BH}-1-142$ & 178.82 & 0.52 & 5.22 & 0.71 \\
\hline $28 \mathrm{H}-2-42$ & 179.68 & 7.78 & 8.84 & 8.52 \\
\hline $28 \mathrm{H}-2-82$ & 179.88 & 1.91 & & \\
\hline $28 \mathrm{H}-2-182$ & 188.88 & 1.82 & 15.48 & 8.66 \\
\hline $28 \mathrm{H}-2-122$ & 188.28 & 1.73 & & \\
\hline $28 \mathrm{H}-2-142$ & 188.48 & 1.99 & 12.78 & 0.62 \\
\hline $28 \mathrm{H}-3-42$ & 181.18 & 0.31 & 8.88 & 8.65 \\
\hline $28 \mathrm{H}-3-82$ & 181.38 & 8.65 & 4.38 & 0.72 \\
\hline $28 \mathrm{H}-3-182$ & 181.58 & 1.52 & 5.42 & \\
\hline $28 \mathrm{H}-3-122$ & 181.78 & 8.45 & 8.75 & 8.65 \\
\hline $28 \mathrm{H}-4-42$ & 182.68 & 1.76 & 4.22 & 8.77 \\
\hline $2 \mathrm{OH}-4-82$ & 182.88 & 1.29 & 8.80 & 0.73 \\
\hline $28 \mathrm{H}-4-182$ & 183.88 & 8.66 & & \\
\hline $28 \mathrm{H}-4-122$ & 183.28 & 8.38 & 8.27 & 0.75 \\
\hline $28 \mathrm{H}-5-42$ & 184.18 & 1.41 & 8.33 & 0.91 \\
\hline $28 \mathrm{H}-5-82$ & 184.38 & 8.68 & & \\
\hline $28 \mathrm{H}-5-182$ & 184.48 & 0.65 & 8.88 & 8.50 \\
\hline $21 \mathrm{H}-1-22$ & 185.01 & 2.88 & 8.29 & 8.77 \\
\hline $21 \mathrm{H}-1-42$ & 185.28 & 1.32 & & \\
\hline $21 \mathrm{H}-1-62$ & 185.39 & 1.38 & 8.80 & 8.83 \\
\hline $21 \mathrm{H}-1-82$ & 185.58 & 0.55 & & \\
\hline $21 \mathrm{H}-1-182$ & 185.77 & 1.21 & 8.80 & 8.92 \\
\hline $21 \mathrm{H}-1-122$ & 185.96 & 1.58 & & \\
\hline $21 \mathrm{H}-1-142$ & 186.15 & 2.14 & 8.22 & 8.95 \\
\hline $22 \mathrm{H}-1-22$ & 187.01 & 3.44 & 8.52 & 8.85 \\
\hline $22 \mathrm{H}-1-42$ & 187.28 & 0.55 & & \\
\hline $22 \mathrm{H}-1-182$ & 187.77 & 8.88 & 2.96 & 8.69 \\
\hline $22 \mathrm{H}-1-122$ & 187.96 & 8.65 & 8.88 & \\
\hline $22 \mathrm{H}-2-42$ & 188.62 & 0.86 & 1.41 & 8.65 \\
\hline $22 \mathrm{H}-2-62$ & 188.81 & 8.95 & & \\
\hline $22 \mathrm{H}-2-82$ & 189.88 & 1.41 & & \\
\hline
\end{tabular}


Table 1.4 (continued).

\begin{tabular}{|c|c|c|c|c|}
\hline $\begin{array}{c}\text { Sample } \\
\text { no. }\end{array}$ & $\begin{array}{l}\text { Depth } \\
\text { (mbsf) }\end{array}$ & $\begin{array}{l}>63 \\
\mu \mathrm{m} \\
(\%)\end{array}$ & $\begin{array}{c}\text { Bulk } \\
\mathrm{CaCO}_{3} \\
(\%)\end{array}$ & $\begin{array}{l}\text { TOC } \\
(\%)\end{array}$ \\
\hline $22 \mathrm{H}-2-182$ & 189.19 & 6.83 & 0.21 & 0.77 \\
\hline $22 \mathrm{H}-3-22$ & 189.86 & 1.10 & 8.88 & 8.46 \\
\hline $22 \mathrm{H}-3-42$ & 190.85 & 2.74 & & \\
\hline $22 \mathrm{H}-3-62$ & 198.24 & 3.25 & 8.88 & 8.53 \\
\hline $22 \mathrm{H}-3-182$ & 198.62 & 2.41 & & \\
\hline $22 \mathrm{H}-3-122$ & 198.81 & 4.65 & 8.80 & 8.48 \\
\hline $22 \mathrm{H}-4-22$ & 191.28 & 13.32 & 8.88 & 0.34 \\
\hline $22 \mathrm{H}-4-42$ & 191.47 & 1.97 & & \\
\hline $22 \mathrm{H}-4-62$ & 191.66 & 1.15 & 12.98 & 8.81 \\
\hline $22 \mathrm{H}-4-182$ & 192.84 & 8.71 & 8.80 & \\
\hline $22 \mathrm{H}-4-121$ & 192.22 & 2.82 & 8.88 & 0.68 \\
\hline $22 \mathrm{H}-5-42$ & 192.98 & 2.59 & 8.88 & 0.75 \\
\hline $22 \mathrm{H}-5-62$ & 193.89 & 8.48 & & \\
\hline $22 \mathrm{H}-5-82$ & 193.28 & 0.49 & & \\
\hline $22 \mathrm{H}-5-98$ & 193.43 & 4.78 & 8.80 & 8.37 \\
\hline $23 \mathrm{H}-1-39$ & 194.45 & 1.66 & 7.83 & 0.73 \\
\hline $23 \mathrm{H}-2-42$ & 195.81 & 0.36 & 0.99 & 0.76 \\
\hline $23 \mathrm{H}-2-102$ & 196.34 & 1.88 & 0.80 & $0.7 \theta$ \\
\hline $23 \mathrm{H}-2-122$ & 196.52 & 2.82 & & \\
\hline $23 \mathrm{H}-2-142$ & 196.78 & 27.31 & 1.19 & 0.98 \\
\hline $23 \mathrm{H}-3-42$ & 197.14 & 1.25 & 8.50 & 8.91 \\
\hline $23 \mathrm{H}-3-182$ & 197.68 & 4.26 & 0.18 & 8.59 \\
\hline $23 \mathrm{H}-3-122$ & 197.86 & 4.48 & & \\
\hline $23 \mathrm{H}-3-142$ & 198.03 & 1.37 & 0.80 & 8.59 \\
\hline $23 \mathrm{H}-4-42$ & 198.48 & 1.86 & 8.26 & 0.31 \\
\hline $23 \mathrm{H}-4-182$ & 199.81 & 0.64 & 8.80 & 8.89 \\
\hline $23 \mathrm{H}-4-122$ & 199.19 & 0.34 & 0.80 & \\
\hline $23 \mathrm{H}-4-142$ & 199.37 & 8.31 & 8.80 & 0.71 \\
\hline $23 \mathrm{H}-5-42$ & 199.81 & 3.58 & 8.80 & 8.85 \\
\hline $23 \mathrm{H}-5-182$ & 288.35 & 0.83 & 0.22 & 0.72 \\
\hline $23 \mathrm{H}-5-122$ & 288.53 & 1.86 & & \\
\hline $23 \mathrm{H}-5-142$ & 288.78 & 4.58 & 8.88 & 8.44 \\
\hline $24 \mathrm{H}-1-28$ & 281.38 & 0.68 & 0.88 & 8.65 \\
\hline $24 \mathrm{H}-1-65$ & 201.74 & 19.13 & 8.88 & 0.42 \\
\hline $24 \mathrm{H}-1-82$ & 281.98 & 1.73 & & \\
\hline $24 \mathrm{H}-1-182$ & 282.18 & 8.38 & 8.08 & 8.83 \\
\hline $24 \mathrm{H}-1-122$ & 282.38 & 8.76 & 8.19 & \\
\hline $24 \mathrm{H}-1-134$ & 282.41 & 8.69 & 8.88 & 0.75 \\
\hline $24 \mathrm{H}-2-65$ & 283.21 & 8.68 & 1.87 & 0.72 \\
\hline $24 \mathrm{H}-2-182$ & 283.57 & 17.53 & & \\
\hline $24 \mathrm{H}-2-128$ & 283.75 & 17.95 & 2.16 & 8.83 \\
\hline $24 \mathrm{H}-3-65$ & 284.68 & $1.6 \theta$ & 8.88 & 0.74 \\
\hline $24 \mathrm{H}-3-82$ & 284.84 & 0.45 & & \\
\hline $24 \mathrm{H}-3-182$ & 285.84 & 8.62 & 1.56 & 8.92 \\
\hline $24 \mathrm{H}-3-122$ & 285.27 & 0.71 & & \\
\hline $24 \mathrm{H}-3-142$ & 285.43 & 0.26 & 8.15 & 0.86 \\
\hline $24 \mathrm{H}-4-28$ & 285.71 & 0.55 & 0.35 & 0.68 \\
\hline $25 \mathrm{H}-1-22$ & 286.28 & 3.73 & 8.19 & 8.65 \\
\hline $25 \mathrm{H}-1-42$ & 286.48 & 1.86 & & \\
\hline $25 \mathrm{H}-1-62$ & 286.68 & 1.39 & & \\
\hline $25 \mathrm{H}-1-82$ & 286.88 & 8.46 & 0.84 & 8.83 \\
\hline
\end{tabular}

Table 1.4 (continued).

\begin{tabular}{|c|c|c|c|c|}
\hline $\begin{array}{c}\text { Sample } \\
\text { no. }\end{array}$ & $\begin{array}{l}\text { Depth } \\
\text { (mbsf) }\end{array}$ & $\begin{array}{l}>63 \\
\mu \mathrm{m} \\
(\%)\end{array}$ & $\begin{array}{c}\text { Bulk } \\
\mathrm{CaCO}_{3} \\
(\%)\end{array}$ & $\begin{array}{l}\text { TOC } \\
(\%)\end{array}$ \\
\hline $25 \mathrm{H}-1-182$ & 287.88 & 8.47 & 8.80 & \\
\hline $25 \mathrm{H}-1-122$ & 287.28 & 8.28 & 8.88 & 0.75 \\
\hline $25 \mathrm{H}-2-22$ & 287.78 & 0.46 & 2.93 & 0.68 \\
\hline $25 \mathrm{H}-2-42$ & 287.98 & 1.27 & & \\
\hline $25 \mathrm{H}-2-62$ & 288.12 & 8.76 & & \\
\hline $25 \mathrm{H}-2-82$ & 288.32 & 4.84 & 8.80 & 0.34 \\
\hline $25 \mathrm{H}-2-182$ & 288.52 & 8.85 & & \\
\hline $25 \mathrm{H}-2-142$ & 289.18 & 8.44 & 8.08 & 0.71 \\
\hline $25 \mathrm{H}-3-22$ & 289.28 & 1.38 & 0.08 & 8.58 \\
\hline $25 \mathrm{H}-3-42$ & 289.48 & 8.71 & & \\
\hline $25 \mathrm{H}-3-62$ & 289.68 & 1.62 & 8.88 & 8.68 \\
\hline $26 \mathrm{H}-1-22$ & 210.71 & 1.41 & 0.82 & 0.83 \\
\hline $26 \mathrm{H}-1-42$ & 210.91 & 1.34 & & \\
\hline $26 \mathrm{H}-1-62$ & 211.18 & 8.72 & $8 . \theta 8$ & 0.66 \\
\hline $26 \mathrm{H}-1-82$ & 211.38 & 0.91 & & \\
\hline $26 \mathrm{H}-2-22$ & 212.17 & 4.51 & 6.84 & 8.76 \\
\hline $26 \mathrm{H}-2-42$ & 212.36 & 14.84 & & \\
\hline $26 \mathrm{H}-2-62$ & 212.56 & 8.88 & 8.41 & 8.88 \\
\hline $26 \mathrm{H}-2-82$ & 212.75 & 3.88 & & \\
\hline $26 \mathrm{H}-2-182$ & 212.94 & 1.28 & 8.80 & 0.64 \\
\hline $26 \mathrm{H}-2-122$ & 213.14 & 8.84 & 8.26 & \\
\hline $26 \mathrm{H}-2-142$ & 213.33 & 8.79 & $\theta . \theta 0$ & 0.64 \\
\hline $26 \mathrm{H}-3-22$ & 213.62 & 2.37 & & 0.98 \\
\hline $26 \mathrm{H}-3-42$ & 213.82 & 8.77 & 0.88 & 8. 81 \\
\hline $27 \mathrm{H}-1-182$ & 215.38 & 1.33 & 0.28 & 8.88 \\
\hline $27 \mathrm{H}-1-122$ & 215.58 & 4. 91 & $8.8 \theta$ & 0.63 \\
\hline $28 \mathrm{H}-1-182$ & 217.05 & 4.93 & 5.49 & 8.86 \\
\hline $28 \mathrm{H}-1-122$ & 217.23 & 8.39 & 0.88 & \\
\hline $28 \mathrm{H}-1-142$ & 217.42 & 8.69 & 8.21 & 8.61 \\
\hline $28 \mathrm{H}-2-182$ & 218.44 & 12.87 & 8.80 & 0.71 \\
\hline $28 \mathrm{H}-2-122$ & 218.63 & 8.46 & & \\
\hline $28 \mathrm{H}-2-142$ & 218.82 & 1.34 & 8.88 & 8. 65 \\
\hline $28 \mathrm{H}-3-182$ & 219.84 & 8.38 & B. 51 & 8.43 \\
\hline $29 \mathrm{H}-1-182$ & 222.18 & 8.57 & 8.88 & 8.88 \\
\hline $29 \mathrm{H}-1-122$ & 222.38 & 8.78 & 8.62 & \\
\hline $29 \mathrm{H}-1-142$ & 222.58 & 8.47 & 8.80 & 8.76 \\
\hline $29 \mathrm{H}-2-102$ & 223.68 & 8. 51 & 8.88 & 8.68 \\
\hline $29 \mathrm{H}-2-122$ & 223.88 & 8.81 & & \\
\hline $29 \mathrm{H}-2-142$ & 223.98 & 8.63 & 8.80 & 0.62 \\
\hline $29 \mathrm{H}-3-182$ & 225.18 & 1.83 & $\theta .88$ & 0.78 \\
\hline $29 \mathrm{H}-3-122$ & 225.38 & 2.22 & 8.88 & 8.57 \\
\hline $38 \mathrm{H}-1-182$ & 227.12 & 3.52 & 8.80 & 0.68 \\
\hline $38 \mathrm{H}-1-122$ & 227.32 & 4.27 & & \\
\hline $38 \mathrm{H}-1-142$ & 227.51 & 1.14 & 8.80 & 0.73 \\
\hline $30 \mathrm{H}-2-182$ & 228.68 & 1.16 & 1.12 & 8.71 \\
\hline $38 \mathrm{H}-2-122$ & 228.82 & 1.85 & & \\
\hline зен-2-142 & 229.82 & 1.78 & 4.86 & 8.77 \\
\hline зен-3-182 & 230.12 & 8.84 & 0.80 & 8.68 \\
\hline зөн-3-122 & 238.32 & 1.98 & & \\
\hline зен-3-142 & 238.52 & 3.27 & $\theta . \theta 8$ & 0.55 \\
\hline $3 \theta \mathrm{BH}-4-102$ & 231.68 & 1.25 & 0.41 & 8. 56 \\
\hline
\end{tabular}


R. HENRICH, T. WOLF, G. BOHRMANN, J. THIEDE

Table 1.4 (continued).

\begin{tabular}{|c|c|c|c|c|}
\hline $\begin{array}{c}\text { Sample } \\
\text { no. }\end{array}$ & $\begin{array}{l}\text { Depth } \\
\text { (mbsf) }\end{array}$ & $\begin{array}{l}>63 \\
\mu \mathrm{m} \\
(\%)\end{array}$ & $\begin{array}{c}\text { Bulk } \\
\mathrm{CaCO}_{3} \\
(\%)\end{array}$ & $\begin{array}{l}\text { TOC } \\
(\%)\end{array}$ \\
\hline $30 \mathrm{H}-4-122$ & 231.82 & 0.76 & 8. 95 & \\
\hline $38 \mathrm{H}-4-142$ & 232.82 & 2.59 & 8.88 & 8.49 \\
\hline $31 \mathrm{H}-1-182$ & 233.18 & 18.19 & 8.08 & 8. 39 \\
\hline $31 H-1-142$ & 233.32 & 2.13 & 8.80 & 0.68 \\
\hline $31 \mathrm{H}-2-182$ & 234.68 & 1.51 & 8.88 & 0. 81 \\
\hline $31 \mathrm{H}-2-142$ & 234.92 & 1.28 & 8.88 & 0.82 \\
\hline $31 \mathrm{H}-3-182$ & 236.12 & 0.84 & 0.88 & 0.66 \\
\hline $31 \mathrm{H}-3-122$ & 236.32 & 1.24 & 8.68 & 8.84 \\
\hline $32 \mathrm{H}-1-102$ & 239.12 & 2.11 & 5.76 & 0.92 \\
\hline $32 \mathrm{H}-2-182$ & 248.62 & 1.18 & 3.88 & 0.87 \\
\hline $32 \mathrm{H}-2-122$ & 248.82 & 1.36 & 18.56 & \\
\hline $32 t 1-2-142$ & 241.82 & 1.36 & 6.28 & 0.81 \\
\hline $32 \mathrm{H}-3-102$ & 242.12 & 1.88 & 7.92 & 8.81 \\
\hline $32 \mathrm{H}-3-122$ & 242.32 & 2.88 & 6.57 & \\
\hline $32 \mathrm{H}-3-142$ & 242.52 & 2.36 & 5.78 & 8.76 \\
\hline $32 \mathrm{H}-4-182$ & 243.68 & 1.35 & 11.75 & 0.72 \\
\hline $32 \mathrm{H}-4-122$ & 243.82 & 1.13 & 14.48 & 0.69 \\
\hline $33 H-1-182$ & 244.72 & 3.98 & 16.27 & 8.63 \\
\hline $33 H-1-122$ & 244.92 & 1.28 & 17.41 & \\
\hline $33 H-1-142$ & 245.12 & 1.66 & 26.59 & 0.68 \\
\hline $34 H-1-102$ & 246.86 & 2.62 & 0.80 & 0.63 \\
\hline $34 \mathrm{H}-1-122$ & 247.82 & 1.82 & 2.87 & \\
\hline $34 H-1-142$ & 247.19 & 1.24 & 0.80 & 0.67 \\
\hline $34 \mathrm{H}-2-102$ & 248.12 & 8.80 & 1.68 & 0.62 \\
\hline $34 \mathrm{H}-2-122$ & 248.28 & 0.77 & & \\
\hline $34 \mathrm{H}-2-142$ & 248.45 & 1.37 & 8.80 & 0.65 \\
\hline $34 \mathrm{H}-4-102$ & 258.64 & 1.25 & 8.80 & 0.75 \\
\hline $34 H-4-122$ & 258.88 & 0.80 & 2.39 & 0.77 \\
\hline $34 H-5-102$ & 251.98 & 1.81 & 15.48 & 0.86 \\
\hline $34 H-5-122$ & 252.86 & 1.81 & & \\
\hline $34 H-5-142$ & 252.23 & 1.84 & 3.64 & 8.94 \\
\hline $34 H-6-182$ & 253.16 & & 22.73 & 1.80 \\
\hline $34 H-6-142$ & 253.49 & & 16.82 & 0.95 \\
\hline
\end{tabular}


Table 1.4 (continued).

\begin{tabular}{|c|c|c|c|c|c|c|c|c|c|c|c|c|}
\hline \multirow[b]{2}{*}{ Sample no. } & \multirow[b]{2}{*}{$\begin{array}{l}\text { Depth } \\
\text { mbsf }\end{array}$} & \multirow[b]{2}{*}{$\begin{array}{c}>63 \mu \mathrm{m} \\
(\%)\end{array}$} & \multicolumn{10}{|c|}{ Coarse fraction component analysis (wt \%) } \\
\hline & & & $\begin{array}{l}\text { Benthic } \\
\text { foram. } \\
(\%)\end{array}$ & $\begin{array}{l}\text { Plankt. } \\
\text { foram. } \\
(\%)\end{array}$ & $\begin{array}{c}\text { Biogenic } \\
\text { opal cf } \\
(\%)\end{array}$ & $\begin{array}{c}\text { Quartz } \\
(\%)\end{array}$ & $\begin{array}{c}\text { Feldspar } \\
(\%)\end{array}$ & $\begin{array}{c}\text { Mica } \\
(\%)\end{array}$ & $\begin{array}{l}\text { Hev. min. } \\
(\%)\end{array}$ & $\begin{array}{c}\text { Rock fr. } \\
(\%)\end{array}$ & $\begin{array}{l}\text { Volc. gl. } \\
(\%)\end{array}$ & $\begin{array}{c}\text { Auth. } \\
(\%)\end{array}$ \\
\hline $1 H-1-22$ & 0.22 & 9.52 & 0.03 & 8.32 & 8.80 & 8.84 & 8.58 & 0.80 & 0.10 & 8.47 & 8.80 & 8.85 \\
\hline $1 H-1-42$ & 0.42 & 2.74 & 0.28 & 8.42 & 0.83 & 1.64 & 0.88 & 8.02 & 0.83 & 0.31 & 0.81 & 0.82 \\
\hline $1 H-1-59$ & 0.59 & 8.85 & 8.10 & 0.64 & 8.80 & 7.85 & 8.41 & 8.84 & 8.81 & 0.54 & 8.80 & 0.04 \\
\hline $1 H-1-82$ & 0.82 & 4.64 & 0.86 & 0.75 & 8.82 & 3.31 & 8.21 & 0.85 & 0.82 & 0.16 & 8.82 & 0.86 \\
\hline $1 H-1-182$ & 1.82 & 7.16 & 0.21 & 8.87 & 0.01 & 5.38 & 0.27 & 0.08 & 8.87 & 0.23 & 0.88 & 0.01 \\
\hline $1 H-1-122$ & 1.22 & 18.94 & 0.82 & 0.31 & 0.80 & 9.87 & 0.75 & 0.08 & 8.10 & 0.51 & 0.86 & 0.10 \\
\hline $1 H-1-142$ & 1.42 & 13.96 & 8.37 & 8.54 & 8.80 & 11.81 & 8.95 & 0.12 & 0.12 & 0.73 & 8.88 & 8.87 \\
\hline $1 \mathrm{H}-2-22$ & 1.72 & 4.35 & 8.15 & 8.84 & 0.81 & 2.88 & 8.18 & 8.81 & 8.84 & 0.22 & 8.80 & 0.03 \\
\hline $1 H-2-59$ & 2.89 & 28.85 & 0.12 & 8.65 & 8.80 & 16.35 & 0.91 & 8.35 & 0.21 & 1.23 & 8.88 & 0.18 \\
\hline $1 \mathrm{H}-2-82$ & 2.38 & 7.43 & 0.19 & 8.69 & 0.81 & 5.71 & 8.41 & 8.02 & 8.82 & 8.31 & 8.08 & 8.85 \\
\hline $1 H-2-182$ & 2.52 & 13.25 & 0.38 & 1.53 & 8.80 & 18.63 & 0.28 & 0.80 & 8.87 & 8.28 & 8.80 & 8.87 \\
\hline $1 \mathrm{H}-2-122$ & 2.72 & 5.47 & 0.26 & 1.24 & 0.80 & 3.22 & 0.16 & 0.05 & 8.82 & 8.29 & 8.81 & $0.2 \theta$ \\
\hline $1 \mathrm{H}-2-142$ & 2.98 & 6.08 & 0.23 & 1.47 & 0.00 & 3.71 & 0.12 & 8.84 & 8.84 & 8.37 & 0.80 & 0.10 \\
\hline $1 H-3-22$ & 3.22 & 5.36 & 8.26 & 8.77 & 8.81 & 3.79 & 0.18 & 8.81 & 8.81 & 8.24 & 8.80 & 0.16 \\
\hline $1 H-3-42$ & 3.42 & 4.95 & 8.33 & 8.58 & 0.03 & 3.37 & 0.22 & 0.87 & 8.85 & 0.22 & 0.80 & 0.13 \\
\hline $1 \mathrm{H}-3-59$ & 3.59 & 8.69 & 0.23 & 1.33 & 8.03 & 5.68 & 0.55 & 0.88 & 0.12 & 8.61 & $0.8 \theta$ & 0.08 \\
\hline $1 \mathrm{H}-3-82$ & 3.82 & 7.31 & 0.88 & 1.76 & 0.80 & 4.77 & 0.23 & 0.88 & 8.87 & 0.36 & $\theta .80$ & 0.84 \\
\hline $1 \mathrm{H}-3-102$ & 4.82 & 4.78 & 8.83 & 8.13 & 8.88 & 3.81 & 0.36 & 0.03 & 8.63 & 8.27 & 0.88 & 0.03 \\
\hline $1 H-3-122$ & 4.22 & 2.19 & 0.84 & 0.23 & 8.88 & 1.57 & 0.12 & 8.83 & 8.82 & 8.11 & 0.88 & 0.86 \\
\hline $1 \mathrm{H}-3-142$ & 4.42 & 6.88 & 0.30 & 8.95 & 0.80 & 4.36 & 0.85 & 8.82 & 0.34 & 8.80 & 8.80 & 8.86 \\
\hline $1 \mathrm{H}-4-22$ & 4.72 & 3.82 & 8.86 & 8.93 & 0.08 & 1.77 & 0.88 & 8.02 & 8.82 & 0.12 & 0.82 & 0.88 \\
\hline $1 \mathrm{H}-4-42$ & 4.92 & 4.52 & 0.88 & 8.62 & 0.80 & 3.29 & 0.38 & 8.80 & 8.84 & 0.15 & 8.81 & 8.84 \\
\hline $1 \mathrm{H}-4-59$ & 5.89 & 3.79 & 0.87 & 8.88 & 0.80 & 2.32 & 0.28 & 0.84 & 0.01 & 0.16 & 8.80 & 0.01 \\
\hline $1 \mathrm{H}-4-82$ & 5.38 & 3.98 & 0.14 & 1.80 & 8.88 & 2.56 & 0.89 & 0.83 & 8.80 & 8.14 & 8.80 & 0.02 \\
\hline $1 H-4-182$ & 5.52 & 7.11 & 0.18 & 1.56 & 0.80 & 4.79 & 8.88 & 0.88 & 0.83 & 8.42 & 0.83 & 0.83 \\
\hline $1 \mathrm{H}-4-122$ & 5.72 & 4.81 & 0.18 & 1.18 & 8.80 & 3.19 & 8.07 & 0.12 & 0.83 & 8.89 & 8.80 & 0.03 \\
\hline $1 H-5-22$ & 6.22 & 4.84 & 0.03 & 1.32 & 8.08 & 3.24 & 0.03 & 8.04 & 0.83 & 0.14 & 8.80 & 0.82 \\
\hline $1 \mathrm{H}-5-42$ & 6.42 & 4.98 & 0.85 & 1.47 & 8.80 & 3.21 & 8.88 & 8.82 & 8.84 & 8.11 & 8.88 & 8.01 \\
\hline $1 \mathrm{H}-5-59$ & 6.59 & 6.99 & 0.08 & 1.56 & 0.01 & 4.59 & 8.24 & 0.13 & 0.83 & 0.32 & 8.80 & 0.83 \\
\hline $1 \mathrm{H}-5-82$ & 6.82 & 1.87 & 0.83 & 8.62 & 8.88 & 0.36 & 0.82 & 8.82 & 8.88 & 8.82 & 8.80 & 8.88 \\
\hline $1 H-5-182$ & 7.82 & 3.27 & 8.83 & 8.41 & 8.88 & 2.52 & 0.15 & 8.08 & 8.81 & 0.86 & 8.80 & $\theta .8 \theta$ \\
\hline $1 H-5-122$ & 7.22 & 3.28 & 0.13 & 8.44 & 8.88 & 2.27 & 8.84 & 8.81 & 0.88 & 8.23 & 8.80 & 8.81 \\
\hline $1 \mathrm{H}-5-142$ & 7.42 & 4.54 & 0.12 & 1.91 & 8.08 & 2.38 & 0.88 & 0.82 & 8.81 & 8.10 & 8.88 & 0.08 \\
\hline $1 \mathrm{H}-6-22$ & 7.72 & 5.22 & 0.11 & 1.39 & 8.88 & 3.41 & 8.88 & 0.16 & 0.80 & 0.87 & 0.80 & 0.81 \\
\hline $1 H-6-42$ & 7.92 & 2.58 & 0.87 & 8.72 & 8.08 & 1.57 & 8.85 & 0.83 & 0.81 & 0.85 & 8.88 & 0.80 \\
\hline $1 H-6-59$ & 8.84 & 6.42 & 8.15 & 1.19 & 8.80 & 4.66 & 8.18 & 0.89 & 0.05 & 8.86 & 8.80 & 0.82 \\
\hline $1 H-6-82$ & 8.30 & 1.76 & 0.15 & 8.64 & 0.80 & 8.87 & 0.04 & 8.82 & 8.80 & 0.84 & 8.88 & 0.80 \\
\hline $1 \mathrm{H}-6-102$ & 8.52 & 4.06 & 8.28 & 1.85 & 0.01 & 2.62 & 8.07 & 0.85 & 8.82 & 0.03 & 8.80 & 0.81 \\
\hline $1 \mathrm{H}-6-122$ & 8.72 & 2.71 & 8.82 & 8.54 & 8.80 & 1.72 & 0.17 & 8.81 & 0.82 & 0.22 & 0.88 & 0.80 \\
\hline $1 H-6-142$ & 8.98 & 5.85 & 0.11 & 2.39 & 8.84 & 2.98 & 0.18 & 0.84 & 8.81 & 8.16 & 0.80 & 8.81 \\
\hline $2 \mathrm{H}-1-22$ & 9.42 & 3.81 & 0.12 & 1.66 & 8.80 & 1.58 & 0.06 & 0.04 & 8.84 & 0.36 & 8.88 & 0.02 \\
\hline $2 \mathrm{H}-1-42$ & 9.62 & 2.83 & 0.87 & 1.80 & 0.88 & 1.52 & 0.05 & 0.83 & 8.82 & 0.14 & 8.88 & 0.80 \\
\hline $2 \mathrm{H}-1-62$ & 9.82 & 15.28 & 0.80 & 0.88 & 8.80 & 12.57 & 8.69 & 0.19 & 8.25 & 1.27 & 8.88 & 0.22 \\
\hline $2 \mathrm{H}-1-82$ & 18.82 & 6.64 & 0.89 & 2.12 & 8.81 & 4.87 & 0.11 & 8.85 & 8.86 & 8.89 & $\theta .08$ & 0.05 \\
\hline $2 \mathrm{H}-1-184$ & 18.24 & 9.85 & 0.26 & 3.75 & 8.15 & 4.74 & 0.16 & 0.89 & 8.11 & 8.46 & 0.88 & 8.84 \\
\hline $2 \mathrm{H}-1-122$ & 18.42 & 8.93 & 0.12 & 5.55 & 8.80 & 2.85 & 0.14 & 8.88 & 8.82 & 0.23 & 8.08 & 8.88 \\
\hline $2 \mathrm{H}-1-142$ & 18.62 & 12.26 & 8.31 & 18.58 & 8.88 & 1.25 & 0.02 & 8.85 & 8.80 & 0.85 & 0.88 & 8.88 \\
\hline $2 \mathrm{H}-2-22$ & 10.92 & 14.23 & 0.36 & 13.46 & 8.83 & 8.33 & 0.80 & 8.03 & 8.88 & 0.03 & 0.80 & 8.88 \\
\hline $2 \mathrm{H}-2-62$ & 11.32 & 7.42 & 0.17 & 3.28 & 8.80 & 3.48 & 0.25 & 0.02 & 0.12 & 0.26 & $0 . \theta 8$ & 8.80 \\
\hline $2 \mathrm{H}-2-82$ & 11.52 & 18.75 & 8.12 & 3.81 & 0.08 & 6.45 & 8.84 & 0.82 & 0.84 & 0.23 & 8.88 & 8.84 \\
\hline
\end{tabular}


Table 1.4 (continued).

Coarse fraction component analysis (wt $\%)$

\begin{tabular}{|c|c|c|c|c|c|c|c|c|c|c|c|c|}
\hline \multirow[b]{2}{*}{ Sample no. } & \multirow[b]{2}{*}{$\begin{array}{c}\text { Depth } \\
\text { mbsf }\end{array}$} & \multirow[b]{2}{*}{$\begin{array}{c}>63 \mu \mathrm{m} \\
(\%)\end{array}$} & \multicolumn{10}{|c|}{ Coarse fraction component analysis (wt \%) } \\
\hline & & & $\begin{array}{c}\text { Benthic } \\
\text { foram. } \\
(\%)\end{array}$ & $\begin{array}{l}\text { Plankt. } \\
\text { foram. } \\
(\%)\end{array}$ & $\begin{array}{c}\text { Biogenic } \\
\text { opal cf } \\
(\%)\end{array}$ & $\begin{array}{c}\text { Quartz } \\
(\%)\end{array}$ & $\begin{array}{c}\text { Feldspar } \\
(\%)\end{array}$ & $\begin{array}{l}\text { Mica } \\
(\%)\end{array}$ & $\begin{array}{l}\text { Hev. min. } \\
(\%)\end{array}$ & $\begin{array}{c}\text { Rock fr. } \\
(\%)\end{array}$ & $\begin{array}{c}\text { Volc. gl. } \\
(\%)\end{array}$ & $\begin{array}{c}\text { Auth. } \\
(\%)\end{array}$ \\
\hline $2 \mathrm{H}-2-104$ & 11.74 & 7.59 & 0.25 & 7.12 & 0.01 & 0.18 & 0.80 & 8.80 & 0.03 & 8.88 & $8 . \theta 8$ & 0.80 \\
\hline $2 \mathrm{H}-2-122$ & 11.98 & 16.88 & 0.25 & 18.88 & 8.83 & 5.23 & 8.16 & 8.89 & 8.86 & 8.03 & 8.88 & 0.86 \\
\hline $2 \mathrm{H}-2-142$ & 12.12 & 3.22 & 0.86 & 2.74 & $\theta .08$ & 0.32 & 0.01 & 0.89 & 0.00 & $8 . \theta 8$ & 0.88 & 8.80 \\
\hline $2 \mathrm{H}-3-22$ & 12.42 & 6.07 & 0.24 & 5.32 & 0.82 & 0.48 & $8 . \theta \theta$ & 0.88 & 0.80 & 0.89 & 8.08 & 0.80 \\
\hline $2 \mathrm{H}-3-42$ & 12.62 & 4.39 & 0.24 & 3.85 & 8.04 & 0.17 & 0.80 & 8.84 & 8.01 & 0.03 & $0.0 \theta$ & 0.01 \\
\hline $2 \mathrm{H}-3-62$ & 12.82 & 2.48 & 0.89 & 1.15 & 0.03 & 1.02 & 0.80 & 0.04 & 0.80 & 8.87 & 8.08 & 8.88 \\
\hline $2 \mathrm{H}-3-82$ & 13.82 & 24.19 & 8.04 & 2.69 & 0.04 & 17.42 & 1.38 & 1.78 & 0.26 & 0.48 & 0.08 & 0.17 \\
\hline $2 \mathrm{H}-3-184$ & 13.22 & 23.68 & 8.84 & 0.73 & 8.80 & 18.88 & 1.51 & 1.38 & 0.35 & 0.86 & 0.88 & 0.89 \\
\hline $2 \mathrm{H}-3-122$ & 13.42 & 17.31 & 0.89 & 0.50 & 0.88 & 13.38 & 1.46 & 1.18 & 0.12 & 8.58 & 8.88 & 0.09 \\
\hline $2 \mathrm{H}-4-22$ & 13.92 & 14.82 & 8.80 & 8.39 & 0.88 & 11.78 & 8.79 & 1.38 & 0.14 & 0.28 & 8.60 & 0.14 \\
\hline $2 \mathrm{H}-4-42$ & 14.12 & 6.39 & 0.10 & 0.97 & 0.88 & 4.29 & 8.28 & 8.37 & 8.86 & 8.18 & 8.80 & 8.14 \\
\hline $2 \mathrm{H}-4-62$ & 14.32 & 4.61 & 0.07 & 0.84 & 8.88 & 3.29 & 8.84 & 0.28 & 0.84 & 8.88 & 8.80 & 8.86 \\
\hline $2 \mathrm{H}-4-82$ & 14.52 & 8.53 & 0.26 & 0.73 & 8.08 & 6.92 & 8.18 & 0.28 & 0.85 & 0.16 & 8.80 & 0.03 \\
\hline $2 \mathrm{H}-4-104$ & 14.74 & 24.75 & 8.80 & 0.80 & 8.80 & 21.93 & 8.35 & 0.18 & 8.48 & 1.88 & 0.00 & 0.10 \\
\hline $2 \mathrm{H}-4-122$ & 14.98 & 12.87 & 8.67 & 2.83 & 8.88 & 6.29 & 8.13 & 2.84 & 8.18 & 0.48 & 0.80 & 0.07 \\
\hline $2 \mathrm{H}-4-142$ & 15.12 & 10.56 & 0.16 & 1.17 & 0.80 & 8.33 & 8.11 & 8.68 & 0.04 & 8.13 & 8.88 & 0.82 \\
\hline $2 \mathrm{H}-5-22$ & 15.42 & 9.88 & 8.03 & 1.21 & 0.80 & 5.68 & 0.03 & 1.61 & 0.10 & 8.36 & 8.80 & 0.12 \\
\hline $2 \mathrm{H}-5-42$ & 15.62 & 9.88 & 8.24 & 0.98 & 8.80 & 7.48 & 0.10 & 8.27 & 8.87 & 0.75 & 8.80 & 8.07 \\
\hline $2 \mathrm{H}-5-62$ & 15.82 & 9.98 & 0.14 & 2.89 & 8.02 & 6.38 & 8.23 & 8.66 & 0.88 & 0.33 & 8.80 & 0.86 \\
\hline $3 H-1-22$ & 16.42 & 6.22 & 0.29 & 1.58 & 0.81 & 3.51 & 0.88 & 0.26 & 8.06 & 0.37 & $0 . \theta 8$ & 0.85 \\
\hline $3 \mathrm{H}-1-42$ & 16.62 & 4.78 & 0.07 & 1.24 & 8.80 & 2.96 & 8.88 & 0.17 & 0.02 & 0.22 & 8.80 & 0.82 \\
\hline $3 H-1-62$ & 16.82 & 3.93 & 0.17 & 1.78 & 8.88 & 1.58 & 8.84 & 8.19 & 0.85 & 8.28 & 8.80 & 8.81 \\
\hline $3 H-1-82$ & 17.82 & 14.38 & 0.83 & 1.87 & 0.08 & 11.18 & 0.21 & 0.32 & 0.85 & 1.17 & 8.80 & 0.35 \\
\hline $3 H-1-182$ & 17.22 & 6.85 & 8.36 & 1.98 & 0.08 & 3.48 & 8.87 & 8.89 & 8.84 & 0.18 & 8.88 & 0.81 \\
\hline $3 H-1-122$ & 17.48 & 9.58 & 8.18 & 4.48 & 8.80 & 4.88 & 0.10 & 0.55 & 0.06 & 0.16 & 8.88 & 0.85 \\
\hline $3 H-1-142$ & 17.68 & 14.27 & 8.16 & 0.83 & 8.80 & 12.52 & 0.16 & 8.08 & 0.08 & 0.39 & 8.80 & 0.85 \\
\hline $3 \mathrm{H}-2-22$ & 17.92 & 11.98 & 0.14 & 0.87 & 0.82 & 9.46 & 0.23 & 0.48 & 8.87 & 8.53 & 0.80 & 0.11 \\
\hline $3 \mathrm{H}-2-42$ & 18.12 & 12.51 & 0.14 & 1.15 & 0.82 & 9.82 & 8.21 & 8.58 & 0.23 & 0.34 & $0 . \theta 8$ & 8.89 \\
\hline $3 \mathrm{H}-2-62$ & 18.32 & 11.21 & 0.02 & 2.88 & 8.86 & 7.76 & 8.28 & 8.68 & 0.02 & 0.21 & 8.80 & 0.89 \\
\hline $3 \mathrm{H}-2-82$ & 18.52 & 7.78 & 0.01 & 1.50 & 0.03 & 5.39 & 8.10 & 0.15 & 8.84 & 8.46 & 8.80 & 0.88 \\
\hline $3 H-2-182$ & 18.72 & 4.86 & 0.19 & 1.56 & 0.88 & 2.18 & 8.03 & 8.83 & 8.88 & 0.84 & 8.80 & 8.82 \\
\hline $3 H-2-122$ & 18.90 & 0.63 & 8.82 & 0.51 & 0.08 & 0.86 & 8.80 & 0.08 & 8.88 & 0.82 & 8.08 & 0.80 \\
\hline $3 \mathrm{H}-2-142$ & 19.12 & 2.21 & 8.86 & 8.29 & 0.80 & 1.54 & 8.07 & 8.87 & 8.03 & 0.13 & 8.80 & 8.02 \\
\hline $3 \mathrm{H}-3-22$ & 19.42 & 6.52 & 0.84 & 1.47 & 0.80 & 4.61 & 0.15 & 0.85 & 8.81 & 0.19 & 8.88 & 0.08 \\
\hline $3 \mathrm{H}-3-42$ & 19.62 & 1.96 & 8.82 & 1.28 & 8.88 & 8.52 & 8.88 & 8.85 & 8.08 & 8.18 & 0.88 & 0.06 \\
\hline $3 \mathrm{H}-3-62$ & 19.82 & 3.76 & 0.05 & 2.27 & 8.81 & 1.11 & 0.84 & 0.07 & 8.01 & 0.13 & 0.80 & 0.87 \\
\hline $3 H-3-82$ & 28.82 & 3.23 & 0.18 & 2.69 & 8.02 & 8.28 & 8.80 & 0.04 & 0.80 & 8.68 & 0.80 & 8.01 \\
\hline $3 \mathrm{H}-3-182$ & 28.28 & 6.19 & 8.89 & 3.49 & 8.88 & 1.89 & 8.84 & 8.42 & 8.87 & 8.18 & 8.80 & 0.01 \\
\hline $3 \mathrm{H}-4-22$ & 28.42 & 8.82 & 0.01 & 8.53 & 0.80 & 4.94 & 8.19 & 8.15 & 8.87 & 2.83 & 0.88 & 0.89 \\
\hline $3 H-4-42$ & 21.12 & 3.57 & 0.04 & 1.41 & 8.80 & 1.81 & 0.85 & 0.02 & $\theta .01$ & 0.17 & 8.88 & 0.80 \\
\hline $3 \mathrm{H}-4-62$ & 21.32 & 4.91 & 8.09 & 1.17 & 8.80 & 3.87 & 8.87 & 0.11 & 8.10 & 8.25 & 8.80 & 0.06 \\
\hline $3 H-4-82$ & 21.52 & 3.28 & 0.84 & 0.68 & 0.80 & 2.15 & 8.86 & 0.86 & 0.03 & 0.16 & 8.88 & 0.02 \\
\hline $3 \mathrm{H}-4-182$ & 21.72 & 5.87 & 8.18 & 1.48 & 8.80 & 3.29 & 0.88 & 8.03 & 0.86 & 8.80 & 0.80 & 8.81 \\
\hline $3 \mathrm{H}-4-122$ & 21.98 & 3.71 & 8.87 & 2.25 & 0.81 & 1.23 & 8.81 & 0.85 & 8.81 & 8.89 & 8.80 & 0.80 \\
\hline $3 \mathrm{H}-5-22$ & 22.43 & 5.14 & 8.28 & 4.17 & 8.83 & 8.57 & 8.81 & 8.88 & 8.88 & 8.88 & 8.88 & 8.88 \\
\hline $3 \mathrm{H}-5-42$ & 22.62 & 2.64 & 8.81 & 0.96 & 8.80 & 1.89 & 8.01 & 8.81 & 8.01 & 8.58 & 0.88 & 0.88 \\
\hline $3 H-5-62$ & 22.82 & 10.33 & 8.82 & 0.86 & 8.86 & 8.52 & 0.26 & 1.88 & 8.84 & 8.24 & 8.80 & 0.84 \\
\hline $3 \mathrm{H}-5-82$ & 23.82 & 10.21 & 0.29 & 2.02 & 0.80 & 6.86 & 0.13 & 0.48 & 8.82 & 0.39 & 0.08 & 0.82 \\
\hline $3 \mathrm{H}-5-182$ & 23.22 & 2.81 & 0.80 & 0.27 & 0.80 & 2.18 & 0.06 & 0.10 & 0.02 & 0.15 & 0.88 & 0.02 \\
\hline $3 H-5-122$ & 23.48 & 8.62 & 8.88 & 8.83 & 8.88 & 8.51 & 8.81 & 8.82 & 8.81 & 0.03 & 0.80 & 8.80 \\
\hline
\end{tabular}


Table 1.4 (continued).

\begin{tabular}{|c|c|c|c|c|c|c|c|c|c|c|c|c|}
\hline \multirow[b]{2}{*}{ Sample no. } & \multirow[b]{2}{*}{$\begin{array}{c}\text { Depth } \\
\text { mbsf }\end{array}$} & \multirow[b]{2}{*}{$\begin{array}{c}>63 \mu \mathrm{m} \\
(\%)\end{array}$} & \multicolumn{10}{|c|}{ Coarse fraction component analysis (wt \%) } \\
\hline & & & $\begin{array}{l}\text { Benthic } \\
\text { foram. } \\
(\%)\end{array}$ & $\begin{array}{l}\text { Plankt. } \\
\text { foram. } \\
(\%)\end{array}$ & $\begin{array}{c}\text { Biogenic } \\
\text { opal cf } \\
(\%)\end{array}$ & $\begin{array}{c}\text { Quartz } \\
(\%)\end{array}$ & $\begin{array}{c}\text { Feldspar } \\
(\%)\end{array}$ & $\begin{array}{l}\text { Mica } \\
(\%)\end{array}$ & $\begin{array}{l}\text { Hev. min. } \\
(\%)\end{array}$ & $\begin{array}{c}\text { Rock fr. } \\
(\%)\end{array}$ & $\begin{array}{l}\text { Volc. gl. } \\
\qquad(\%)\end{array}$ & $\begin{array}{l}\text { Auth. } \\
(\%)\end{array}$ \\
\hline $3 H-5-142$ & 23.68 & 2.75 & 8.85 & 8.78 & 8.84 & 1.53 & 8.80 & 8.14 & 8.80 & 8.83 & 8.88 & 8.16 \\
\hline $3 H-6-22$ & 23.99 & 14.87 & 8.85 & 8.05 & 8.88 & 11.97 & 8.23 & 0.43 & 0.05 & 1.24 & 8.80 & 8.85 \\
\hline $3 H-7-22$ & 25.42 & 6.02 & 8.42 & 2.19 & $\theta .01$ & 2.91 & 8.89 & 0.85 & 8.03 & 8.27 & 8.08 & 8.83 \\
\hline $4 t 1-1-22$ & 25.92 & 9.98 & 8.28 & 8.57 & 0.08 & 7.97 & 8.27 & 0.15 & 8.87 & 8.66 & 8.88 & 0.68 \\
\hline $4 H-1-42$ & 26.12 & 5.68 & 8.24 & 1.80 & 8.68 & 4.88 & 0.80 & 8.24 & 8.08 & 8.82 & 8.88 & 8.82 \\
\hline $4 H-1-62$ & 26.32 & 4.83 & 8.29 & 1.48 & 8.88 & 2.43 & 8.87 & 8.82 & 8.88 & 8.48 & 8.88 & 0.21 \\
\hline $4 H-1-82$ & 26.52 & 5.45 & 8.59 & 2.48 & 8.88 & 2.87 & 8.05 & 0.16 & 8.82 & 8.88 & 0.80 & 0.01 \\
\hline $4 H-1-182$ & 26.72 & 8.99 & 8.18 & 8.58 & 8.88 & 8.24 & 0.88 & 8.03 & 8.08 & 0.81 & 0.80 & 0.82 \\
\hline $4 H-1-122$ & 26.98 & 1.86 & 8.86 & 8.25 & 8.80 & 0.59 & 8.02 & 0.82 & 0.81 & 0.87 & 8.80 & 0.85 \\
\hline $4 H-1-142$ & 27.12 & 3.42 & 0.19 & 8.87 & $8.8 \theta$ & 1.91 & 0.85 & 8.88 & 8.88 & 8.32 & 8.84 & 0.82 \\
\hline $4 H-2-22$ & 27.42 & 7.68 & 0.85 & 2.48 & 8.06 & 4.33 & 0.22 & 0.14 & 8.82 & 8.36 & 8.08 & 8.80 \\
\hline $4 \mathrm{H}-2-42$ & 27.62 & 5.48 & 8.81 & 1.87 & 8.80 & 3.64 & 0.28 & 0.89 & 0.85 & 8.38 & 8.88 & 0.04 \\
\hline $4 \mathrm{H}-2-62$ & 27.82 & 7.87 & 0.80 & 8.27 & 8.88 & 6.28 & 8.27 & 0.17 & 8.03 & 8.87 & 8.80 & 8.02 \\
\hline $4 H-2-82$ & 28.82 & 5.15 & 0.22 & 3.98 & 8.88 & 0.76 & 8.82 & 8.84 & 8.88 & 8.12 & 8.88 & 0.00 \\
\hline $4 \mathrm{H}-2-182$ & 28.22 & 6.63 & 0.11 & 2.38 & $\theta .08$ & 3.64 & 0.11 & 0.22 & 0.81 & 0.24 & 0.88 & 8.80 \\
\hline $4 \mathrm{H}-2-122$ & 28.48 & 10.33 & 8.21 & 5.89 & 0.08 & 3.46 & 0.13 & 8.13 & 8.84 & 8.42 & 8.88 & 0.02 \\
\hline $4 H-2-142$ & 28.60 & 4.45 & 0.16 & 8.27 & 8.80 & 3.42 & 0.18 & 0.03 & 8.82 & $8.4 \theta$ & 0.88 & 8.85 \\
\hline $4 \mathrm{H}-3-22$ & 28.92 & 2.85 & 8.88 & 8.80 & 8.88 & 1.78 & 8.86 & 0.02 & 8.88 & 8.15 & 0.08 & 0.84 \\
\hline $4 \mathrm{H}-3-42$ & 29.12 & 3.99 & 8.80 & 8.88 & 8.88 & 3.22 & 8.12 & 0.86 & 8.82 & 8.21 & 8.88 & 8.36 \\
\hline $4 H-3-62$ & 29.32 & 12.42 & 8.88 & $8.8 \theta$ & 8.82 & 10.82 & 8.17 & 1.47 & 8.85 & 0.32 & 8.80 & 8.37 \\
\hline $4 H-3-82$ & 29.52 & 7.23 & 8.26 & 8.51 & 8.88 & 5.56 & 0.86 & 8.58 & 8.11 & 8.87 & 8.88 & 0.88 \\
\hline $4 \mathrm{H}-3-182$ & 29.72 & 15.48 & 0.21 & 8.78 & 0.03 & 13.27 & 8.12 & 0.67 & 8.89 & 8.18 & 8.08 & 0.12 \\
\hline $4 \mathrm{H}-3-122$ & 29.98 & 16.18 & 8.08 & 8.80 & 8.88 & 13.48 & 0.89 & 1.80 & 8.33 & 8.49 & 8.80 & 8.79 \\
\hline $4 \mathrm{H}-3-142$ & 30.12 & 2.25 & 8.16 & 8.14 & 8.88 & 1.82 & 8.88 & 8.02 & 8.82 & 8.88 & 8.88 & 0.81 \\
\hline $4 \mathrm{H}-4-22$ & 38.42 & 3.32 & 8.29 & 8.44 & 8.88 & 1.76 & 8.82 & 8.82 & 8.83 & 8.37 & 8.88 & 8.38 \\
\hline $4 H-4-42$ & 38.62 & 5.25 & 0.12 & 8.37 & 0.80 & 4.37 & $\theta .8 \theta$ & 0.05 & 8.82 & 8.17 & 8.88 & 8.15 \\
\hline $4 H-4-62$ & 38.82 & 2.38 & 0.28 & 8.21 & 8.80 & 1.46 & 8.86 & 8.82 & 0.01 & 8.14 & 8.88 & 0.18 \\
\hline $4 H-4-82$ & 31.82 & 1.96 & 8.72 & 0.31 & 8.88 & 8.51 & 8.81 & 0.01 & 8.88 & 0.11 & 8.88 & 8.28 \\
\hline $4 \mathrm{H}-4-102$ & 31.22 & 25.51 & 1.14 & 3.48 & 0.88 & 16.68 & 8.38 & 8.13 & 0.25 & 2.85 & 8.88 & 1.23 \\
\hline $4 H-4-122$ & 31.48 & 5.27 & 8.01 & 0.83 & 8.68 & 4.82 & 8.28 & 0.81 & 8.84 & 8.75 & 8.81 & 8.12 \\
\hline $4 H-4-142$ & 31.68 & 5.97 & 8.11 & 8.82 & 8.88 & 3.88 & 0.12 & 0.13 & 8.83 & 8.45 & 8.08 & 8.42 \\
\hline $4 H-5-22$ & 31.92 & 4.03 & 8.38 & 8.62 & 8.88 & 2.58 & 8.81 & 0.86 & $\theta .03$ & 8.41 & 8.88 & 8.18 \\
\hline $4 \mathrm{H}-5-42$ & 32.12 & 2.64 & 8.24 & 1.11 & 8.88 & 1.23 & 8.88 & 8.88 & 8.88 & 8.84 & 8.88 & 8.81 \\
\hline $4 H-5-62$ & 32.32 & 8.66 & 1.25 & 2.28 & 8.88 & 2.88 & 8.83 & 8.86 & 8.83 & 1.63 & 8.88 & 8.49 \\
\hline $4 H-5-82$ & 32.52 & 13.86 & 8.17 & 0.62 & 8.85 & 11.75 & 8.08 & 0.18 & 8.87 & 8.05 & 8.80 & 0.15 \\
\hline $4 H-5-182$ & 32.72 & 12.60 & 0.02 & 0.82 & 8.88 & 11.28 & 8.22 & 0.80 & 8.22 & 8.73 & 8.88 & 8.18 \\
\hline $4 H-5-122$ & 32.92 & 6.59 & 0.03 & 8.24 & 0.08 & 6.87 & 8.80 & 8.84 & 8.84 & 8.14 & 8.88 & 0.04 \\
\hline $4 H-5-142$ & 33.12 & 5.93 & 0.01 & 8.81 & 8.88 & 5.34 & 8.87 & 0.19 & 8.89 & 8.21 & 8.88 & 0.81 \\
\hline $5 H-1-22$ & 35.42 & 4.25 & 8.17 & 1.28 & 8.88 & 2.34 & 8.82 & 0.19 & 0.82 & 8.31 & 8.88 & 8.81 \\
\hline $5 H-1-42$ & 35.62 & 3.94 & 8.08 & 1.97 & 8.88 & 1.71 & 8.88 & 0.05 & 8.81 & 8.12 & 0.88 & 8.88 \\
\hline $5 H-1-62$ & 35.82 & 13.77 & 0.85 & 8.21 & 8.80 & 12.46 & 8.18 & 8.34 & 8.26 & 8.21 & 8.80 & 8.85 \\
\hline $5 H-1-82$ & 36.82 & 4.58 & 0.01 & 8.86 & 8.80 & 4.17 & 8.08 & 0.12 & 0.81 & 8.18 & 0.80 & 0.03 \\
\hline $5 H-1-182$ & 36.22 & 14.13 & 0.80 & 8.80 & 0.88 & 11.36 & 0.17 & 0.19 & 8.17 & 8.64 & 8.80 & 1.58 \\
\hline $5 H-1-122$ & 36.48 & 15.93 & $\theta .80$ & 0.03 & 0.08 & 14.92 & 8.08 & 8.88 & 8.88 & 8.12 & 8.03 & 8.74 \\
\hline $5 H-1-141$ & 36.68 & 28.53 & 0.80 & 8.88 & 0.80 & 17.65 & 0.12 & 1.38 & 8.16 & 8.63 & 0.80 & 8.59 \\
\hline $5 H-2-22$ & 36.92 & 15.98 & 0.08 & 8.80 & 8.88 & 13.88 & 0.16 & 8.42 & 8.18 & 0.78 & 0.83 & 1.49 \\
\hline $5 H-2-42$ & 37.12 & 15.66 & 0.80 & 8.88 & 8.88 & 14.29 & 8.88 & 0.88 & 8.80 & 8.86 & 0.83 & 1.11 \\
\hline $5 H-2-62$ & 37.32 & 1.45 & 0.88 & 0.22 & 0.88 & 1.87 & 8.80 & 8.85 & 8.88 & 8.82 & 8.80 & 8.82 \\
\hline $5 H-2-82$ & 37.52 & 6.82 & 0.08 & 8.88 & 8.88 & 5.83 & 8.81 & 8.88 & 8.88 & 8.03 & 8.80 & 0.75 \\
\hline $5 H-2-102$ & 37.72 & 14.28 & 8.88 & 0.22 & 8.08 & 12.41 & 0.16 & 0.30 & 8.14 & 0.78 & 8.88 & 0.16 \\
\hline
\end{tabular}


Table 1.4 (continued).

\begin{tabular}{|c|c|c|c|c|c|c|c|c|c|c|c|c|}
\hline \multirow[b]{2}{*}{ Sample no. } & \multirow[b]{2}{*}{$\begin{array}{c}\text { Depth } \\
\text { mbsf }\end{array}$} & \multirow[b]{2}{*}{$\begin{array}{c}>63 \mu \mathrm{m} \\
(\%)\end{array}$} & \multicolumn{10}{|c|}{ Coarse fraction component analysis (wt \%) } \\
\hline & & & $\begin{array}{l}\text { Benthic } \\
\text { foram. } \\
(\%)\end{array}$ & $\begin{array}{l}\text { Plankt. } \\
\text { foram. } \\
(\%)\end{array}$ & $\begin{array}{c}\text { Biogenic } \\
\text { opal cf } \\
(\%)\end{array}$ & $\begin{array}{c}\text { Quartz } \\
(\%)\end{array}$ & $\begin{array}{c}\text { Feldspar } \\
(\%)\end{array}$ & $\begin{array}{c}\text { Mica } \\
(\%)\end{array}$ & $\begin{array}{l}\text { Hev. min. } \\
(\%)\end{array}$ & $\begin{array}{c}\text { Rock fr. } \\
(\%)\end{array}$ & $\begin{array}{l}\text { Volc. gl. } \\
(\%)\end{array}$ & $\begin{array}{c}\text { Auth. } \\
(\%)\end{array}$ \\
\hline $5 H-2-137$ & 38.87 & 8.79 & 8.85 & 8.29 & 8.82 & 6.97 & 8.18 & 8.68 & 8.85 & 8.66 & 8.88 & 0.54 \\
\hline $5 \mathrm{H}-3-22$ & 38.42 & 14.16 & 8.82 & 8.71 & 8.80 & 18.17 & 8.28 & 0.33 & 8.87 & 1.89 & 8.80 & 1.52 \\
\hline $5 H-3-42$ & 38.62 & 7.96 & 0.80 & 8.59 & 0.88 & 6.66 & 8.88 & 0.86 & 8.80 & 0.12 & 0.81 & 8.58 \\
\hline $5 H-3-62$ & 38.82 & 3.97 & 8.84 & 8.45 & 8.80 & 2.14 & 8.84 & 8.82 & 0.84 & 0.39 & 8.08 & 8. 84 \\
\hline $5 H-3-82$ & 39.82 & 11.81 & 8.82 & 0.87 & 0.88 & 10.88 & 8.88 & 8.17 & 0.08 & 0.82 & 8.82 & 1.43 \\
\hline $5 H-3-182$ & 39.22 & 3.81 & 8.87 & 8.26 & 8.81 & 2.89 & 0.81 & 8.87 & 8.82 & 8.26 & 8.88 & 8.21 \\
\hline $5 H-3-122$ & 39.48 & 4.21 & 0.84 & 0.15 & 0.00 & 3.47 & 0.80 & 0.02 & 0.80 & 0.17 & 8.60 & 0.36 \\
\hline $5 \mathrm{H}-3-137$ & 39.68 & 3.26 & 0.83 & 0.32 & $0.8 \theta$ & 2.88 & 8.05 & 0.84 & 0.03 & 0.27 & 8.80 & 8.53 \\
\hline $5 H-4-22$ & 39.92 & 2.26 & 8.04 & 0.15 & 8.80 & 1.66 & 8.01 & 8.84 & 0.81 & 0.12 & 8.80 & 0.22 \\
\hline $5 H-4-42$ & 48.12 & 2.58 & 8.03 & 0.18 & 8.88 & 1.31 & 8.88 & 0.80 & 0.80 & 0.98 & 0.81 & 0.87 \\
\hline $5 H-4-62$ & 48.32 & 6.28 & 8.87 & 8.28 & 8.88 & 3.93 & 8.23 & 0.17 & 8.82 & 8.87 & 8.88 & 8.78 \\
\hline $5 H-4-82$ & 48.52 & 1.56 & 0.10 & 8.18 & 0.01 & 8.98 & 0.88 & 0.81 & 8.88 & 8.27 & 8.80 & 0.10 \\
\hline $5 H-4-182$ & 48.72 & 3.42 & 8.61 & 0.03 & 0.88 & 8.55 & 0.81 & 0.81 & 8.81 & 0.15 & 8.00 & 2.63 \\
\hline $5 H-4-122$ & 48.98 & 5.94 & 0.89 & 0.32 & 8.08 & 5.84 & 0.08 & 0.81 & 8.88 & 8.22 & 8.80 & 8.26 \\
\hline $5 H-4-137$ & 41.87 & 9.71 & 0.13 & 0.32 & 0.88 & 7.19 & 8.06 & 0.17 & 8.02 & 8.64 & 0.08 & 1.11 \\
\hline $5 H-5-22$ & 41.42 & 9.77 & 8.88 & 0.28 & 8.80 & 7.88 & 8.89 & 0.38 & 8.84 & 8.72 & 8.87 & 0.39 \\
\hline $5 H-5-42$ & 41.62 & 7.68 & 0.08 & 0.29 & 0.80 & 5.89 & 8.88 & 0.18 & 8.08 & 0.85 & 0.00 & 0.59 \\
\hline $5 H-5-62$ & 41.82 & 6.85 & 0.80 & 0.28 & 0.80 & 4.78 & 8.05 & 0.16 & 0.88 & 8.58 & 0.88 & 0.19 \\
\hline $5 H-5-82$ & 42.82 & 23.15 & 0.85 & 8.88 & 0.88 & 22.47 & 0.88 & 0.80 & 8.89 & 0.85 & 8.00 & 8.58 \\
\hline $5 H-5-182$ & 42.22 & 9.93 & 0.08 & 8.88 & 0.00 & 8.91 & 8.12 & 0.16 & 8.86 & 8.68 & 0.80 & 0.08 \\
\hline $5 H-5-122$ & 42.48 & 5.19 & 0.01 & 0.01 & 0.80 & 4.80 & 8.80 & 0.03 & 8.88 & 0.04 & 8.82 & 8.29 \\
\hline $5 H-5-141$ & 42.64 & 26.09 & 0.80 & 8.85 & 0.88 & 23.76 & 0.41 & 0.62 & 8.18 & 1.84 & 8.80 & 0.10 \\
\hline $6 \mathrm{H}-1-22$ & 44.92 & 6.18 & 0.14 & 1.51 & 0.88 & 3.63 & 8.85 & 0.36 & 8.88 & 0.31 & 0.08 & 0.16 \\
\hline $6 H-1-59$ & 45.29 & 6.18 & 0.05 & 0.48 & 0.08 & 5.47 & 8.80 & 0.02 & 8.80 & 0.85 & 0.88 & 0.84 \\
\hline $6 H-1-78$ & 45.48 & 31.58 & 8.80 & 8.08 & 0.80 & 28.83 & 8.12 & 0.29 & 8.53 & 1.11 & 8.88 & 0.78 \\
\hline $6 \mathrm{H}-1-181$ & 45.71 & 36.15 & 8.08 & 8.00 & 0.88 & 34.38 & 8.88 & 0.80 & $0 . \theta 0$ & 0.78 & 8.87 & 8.92 \\
\hline $6 H-1-122$ & 45.98 & 32.86 & 8.88 & 8.80 & 0.08 & 29.48 & 1.48 & 0.25 & 8.13 & 1.46 & 8.80 & 0.13 \\
\hline $6 \mathrm{H}-1-142$ & 46.12 & 6.19 & 8.83 & 0.28 & 0.80 & 5.08 & 8.88 & 0.82 & 8.80 & 0.86 & 8.88 & 0.72 \\
\hline $6 \mathrm{H}-2-22$ & 46.42 & 2.82 & 0.26 & 1.16 & 0.80 & 1.22 & 0.01 & 0.11 & 8.88 & 0.85 & 8.88 & 0.81 \\
\hline $6 \mathrm{H}-2-59$ & 46.79 & 12.36 & 8.80 & 8.82 & 0.88 & 11.78 & 8.88 & 0.12 & 8.80 & 8.85 & 8.88 & 8.46 \\
\hline $6 \mathrm{H}-2-78$ & 46.92 & 11.12 & 8.84 & 8.64 & 0.80 & 9.77 & 8.89 & 8.17 & 8.86 & 0.38 & 0.08 & 8.84 \\
\hline $6 \mathrm{H}-2-182$ & 47.21 & 2.71 & 0.28 & 0.93 & 0.00 & 1.33 & 8.80 & 0.01 & 0.80 & 0.80 & 0.80 & 0.16 \\
\hline $6 \mathrm{H}-2-122$ & 47.48 & 1.87 & 0.18 & 1.07 & 8.88 & 0.49 & 8.81 & 8.82 & 8.80 & 8.11 & 0.80 & 8.86 \\
\hline $6 \mathrm{H}-2-142$ & 47.62 & 9.78 & 0.38 & 4.39 & 0.80 & 4.82 & 8.88 & 0.88 & 8.84 & 8.88 & 0.88 & 8.23 \\
\hline $6 \mathrm{H}-3-22$ & 47.92 & 2.21 & 8.82 & 8.79 & 0.80 & 1.28 & 8.81 & 0.86 & 8.81 & 8.12 & 8.88 & 0.88 \\
\hline $6 \mathrm{H}-3-59$ & 48.29 & 6.62 & 8.05 & 8.65 & 0.80 & 5.69 & 0.81 & 8.85 & 8.88 & 8.80 & 8.80 & 0.17 \\
\hline $6 \mathrm{H}-3-78$ & 48.48 & 5.89 & 0.17 & 2.81 & 0.88 & 2.73 & 8.86 & 8.84 & 8.81 & 8.84 & 8.80 & 8.82 \\
\hline $6 \mathrm{H}-3-181$ & 48.71 & 35.88 & 8.88 & 8.88 & 8.80 & 32.88 & 8.71 & 0.71 & 0.35 & 0.43 & 0.80 & 0.88 \\
\hline $6 \mathrm{H}-3-122$ & 48.98 & 2.89 & 0.02 & 1.87 & 0.82 & 1.61 & 8.82 & 8.89 & 8.81 & 8.83 & 8.88 & 8.81 \\
\hline $6 H-3-142$ & 49.12 & 3.46 & 0.05 & 8.52 & 0.08 & 2.88 & 0.81 & 0.01 & 8.80 & 8.80 & 8.88 & 0.08 \\
\hline $6 \mathrm{H}-4-22$ & 49.42 & 7.85 & 8. 28 & 4.68 & 8.88 & 2.71 & 8.03 & 0.03 & 8.83 & 8.83 & 0.88 & 0.88 \\
\hline $6 \mathrm{H}-4-42$ & 49.62 & 5.81 & 0.13 & 4.37 & 8.80 & 0.42 & 8.80 & 0.84 & 0.80 & 8.88 & 8.88 & 8.85 \\
\hline $6 H-4-59$ & 49.79 & 2.54 & 8.85 & 1.54 & 8.82 & 0.83 & 8.01 & 8.08 & 0.81 & 0.82 & 8.88 & 8.03 \\
\hline $6 \mathrm{H}-4-78$ & 49.98 & 6.84 & 8.38 & 6.32 & 8.82 & 8.86 & 8.80 & 8.80 & 8.80 & 8.80 & 8.88 & 8.81 \\
\hline $6 H-4-181$ & 50.21 & 4.46 & 8.36 & 3.64 & 8.80 & 0.37 & 8.88 & 0.87 & 8.80 & 8.80 & 8.80 & 0.81 \\
\hline $6 \mathrm{H}-4-117$ & 58.37 & 4.67 & 8.14 & 4.39 & 8.80 & 0.12 & 8.80 & 0.08 & 8.88 & 8.80 & 8.80 & 0.01 \\
\hline $6 H-5-22$ & 58.92 & 5.28 & 0.16 & 8.78 & 0.80 & 4.14 & 8.01 & 0.87 & 0.01 & 8.89 & 8.88 & 0.03 \\
\hline $6 \mathrm{H}-5-59$ & 51.29 & 5.18 & 8.16 & 1.31 & 0.81 & 3.48 & 0.80 & 0.80 & 0.80 & 0.08 & 8.88 & 8.15 \\
\hline $6 \mathrm{H}-5-78$ & 51.48 & 7.88 & 0.29 & 8.47 & 8.88 & 5.89 & 0.82 & 0.15 & 8.82 & 8.18 & 8.80 & 0.12 \\
\hline $6 H-5-181$ & 51.71 & 12.48 & 1.81 & 2.35 & 8.88 & 8.79 & 8.80 & 8.88 & 8.88 & 8.80 & 8.08 & 0.33 \\
\hline
\end{tabular}




\begin{tabular}{|c|c|c|c|c|c|c|c|c|c|c|c|c|}
\hline \multirow[b]{2}{*}{ Sample no. } & \multirow[b]{2}{*}{$\begin{array}{c}\text { Depth } \\
\text { mbsf }\end{array}$} & \multirow[b]{2}{*}{$\begin{array}{c}>63 \mu \mathrm{m} \\
(\%)\end{array}$} & \multicolumn{10}{|c|}{ Coarse fraction component analysis (wt\%) } \\
\hline & & & $\begin{array}{l}\text { Benthic } \\
\text { foram. } \\
(\%)\end{array}$ & $\begin{array}{c}\text { Plankt. } \\
\text { foram. } \\
(\%)\end{array}$ & $\begin{array}{c}\text { Biogenic } \\
\text { opal cf } \\
(\%)\end{array}$ & $\begin{array}{c}\text { Quartz } \\
(\%)\end{array}$ & $\begin{array}{c}\text { Feldspar } \\
(\%)\end{array}$ & $\begin{array}{c}\text { Mica } \\
(\%)\end{array}$ & $\begin{array}{l}\text { Hev. min. } \\
\quad(\%)\end{array}$ & $\begin{array}{c}\text { Rock fr. } \\
(\%)\end{array}$ & $\begin{array}{l}\text { Volc. gl. } \\
(\%)\end{array}$ & $\begin{array}{c}\text { Auth. } \\
(\%)\end{array}$ \\
\hline $6 H-5-122$ & 51.98 & 27.91 & 8.85 & 0.27 & 8.88 & 25.25 & 0.16 & 0.27 & 0.32 & 8. 32 & 8.80 & 1.28 \\
\hline $6 H-5-142$ & 52.12 & 4.31 & 0.51 & 1.22 & 8.81 & 2.42 & 8.08 & 8.08 & $\theta . \theta 8$ & 8.80 & $\theta .08$ & 0.16 \\
\hline $6 \mathrm{H}-6-22$ & 52.42 & 15.98 & 0.80 & 8.08 & $\theta . \theta 0$ & 14.66 & 8.16 & 8.30 & 0.16 & 0.16 & 8.80 & 8.38 \\
\hline $6 \mathrm{H}-6-42$ & 52.62 & 16.15 & 8.89 & 8.86 & 8.88 & 15.28 & 8.88 & 8.88 & 8.86 & 8.83 & 8.88 & 8.62 \\
\hline $6 \mathrm{H}-6-59$ & 52.79 & 6.03 & 0.78 & 4.88 & 0.80 & 1.17 & 0.80 & 8.86 & 8.08 & 8.05 & 0.08 & 0.82 \\
\hline $6 \mathrm{H}-6-78$ & 52.98 & 9.68 & 0.36 & 7.27 & 0.80 & 1.93 & 0.80 & 8.88 & 8.80 & 8.80 & $\theta . \theta 8$ & 0.04 \\
\hline $6 H-6-181$ & 53.21 & 3.83 & 0.23 & 2.79 & 8.88 & 8.64 & $8 . \theta 8$ & 0.03 & 0.81 & 8.81 & 8.88 & 8.86 \\
\hline $7 H-1-22$ & 54.42 & 15.11 & 8.11 & 1.99 & 8.88 & 11.26 & 8.85 & 1.28 & 8.88 & 8.27 & 8. 88 & 8.19 \\
\hline $7 \mathrm{H}-1-42$ & 54.62 & 12.43 & 0.85 & 8.87 & 0.80 & 12.81 & 8.80 & 8.80 & 0.80 & 8.88 & 8.80 & 8.29 \\
\hline $7 H-1-64$ & 54.85 & 11.30 & 8.88 & 8.88 & 0.88 & 9.44 & 0.06 & 1.54 & 8.02 & 0.13 & 0.08 & 8. 82 \\
\hline $7 \mathrm{H}-1-83$ & 55.83 & 7.94 & 0.80 & 8.15 & 8.80 & 7.60 & 0.88 & 8.80 & 8.80 & 0.80 & 0.80 & 0.18 \\
\hline 7H-2- 22 & 55.42 & 12.76 & 0.85 & 0.12 & 0.02 & 10.16 & 8.85 & 0.71 & 0.14 & 0.12 & 8.80 & 1.38 \\
\hline $7 \mathrm{H}-2-42$ & 56.13 & 18.24 & $0 . \theta 8$ & 0.08 & 8.80 & 17.71 & 0.08 & 0.88 & 8.80 & 0.11 & 8.88 & 0.36 \\
\hline $7 \mathrm{H}-2-63$ & 56.33 & 2.78 & 0.27 & 0.63 & 8.80 & 1.58 & 8.08 & 8.17 & 8.82 & 0.84 & 8.08 & 0.08 \\
\hline $7 \mathrm{H}-2-83$ & 56.53 & 28.54 & 0.08 & 8.80 & 8.88 & 19.88 & 0.88 & 0.80 & 0.00 & 8.84 & 8.80 & 8.51 \\
\hline $7 \mathrm{H}-3-22$ & 57.42 & 3.72 & 0.12 & 0.84 & 8.88 & 2.48 & 8.80 & 0.15 & 8.80 & 0.85 & 8.80 & 0.16 \\
\hline $7 \mathrm{H}-3-42$ & 57.62 & 1.91 & 0.23 & 8.67 & 8.88 & 0.74 & 8.88 & 0.01 & 0.08 & 8.08 & 0.08 & 0.26 \\
\hline $7 \mathrm{H}-3-64$ & 57.85 & 25.73 & 0.80 & 0.00 & 0.85 & 23.83 & 0.15 & 0.78 & 8.28 & 0.10 & 0.80 & 0.63 \\
\hline $7 \mathrm{H}-3-83$ & 58.03 & 2.48 & 0.19 & 0.42 & 8.80 & 1.54 & 8.88 & 0.84 & 8.80 & 0.08 & 8.80 & 8.28 \\
\hline $7 \mathrm{H}-4-22$ & 58.92 & 28.16 & $0 . \theta 0$ & 8.80 & 0.80 & 26.65 & 8.86 & 0.95 & 0.11 & 0.34 & 0.88 & 0.86 \\
\hline $7 \mathrm{H}-4-42$ & 59.13 & 1.82 & 0.31 & 8.77 & 8.88 & 0.63 & $\theta . \theta \theta$ & 0.80 & 0.00 & 0.00 & 8.80 & 0.18 \\
\hline $7 \mathrm{H}-4-65$ & 59.35 & 2.22 & 8.89 & 8.42 & 8.80 & 1.56 & 8.80 & 0.03 & 8.82 & 0.82 & 8.08 & 8.06 \\
\hline $7 H-4-86$ & 59.56 & 8.62 & 1.00 & 1.48 & 8.82 & 5.83 & 8.88 & 8.88 & 8.88 & 8.80 & 8.88 & 0. 37 \\
\hline $8 H-1-21$ & 63.91 & 9.56 & 0.34 & 2.86 & 8.80 & 6.59 & $8 . \theta 8$ & 8.10 & 8.03 & 0.15 & 8.80 & 0.22 \\
\hline $8 \mathrm{H}-1-48$ & 64.11 & 13.72 & 0.16 & 0.47 & 0.80 & 12.78 & 8.80 & 0.03 & 8.88 & 0.08 & $8 . \theta 0$ & 0.29 \\
\hline $8 H-1-58$ & 64.29 & 3.61 & 0.48 & 2.27 & 8.68 & 8.78 & 8.68 & 8.83 & 8.81 & 0.01 & 0.80 & 8.88 \\
\hline $8 H-1-182$ & 64.72 & 7.58 & 0.87 & 0.28 & 0.88 & 7.18 & 8.08 & 8.80 & 8.88 & 0.88 & 0.08 & 0.13 \\
\hline $8 \mathrm{H}-2-21$ & 65.41 & 6.73 & 8.48 & 0.85 & 8.88 & 4.88 & 0.01 & 0.58 & 8.82 & 0.86 & 8.68 & 0.01 \\
\hline $8 \mathrm{H}-2-48$ & 65.68 & 9.62 & 0.34 & 3.89 & 8.88 & 5.99 & 8.88 & 8.02 & 8.88 & 0.83 & 0.88 & 0.12 \\
\hline $8 \mathrm{H}-2-59$ & 65.78 & 4.13 & 8.56 & 3.13 & 8.88 & 0.19 & 8.80 & 0.26 & 8.88 & 8.88 & 0.80 & 8.08 \\
\hline $8 \mathrm{H}-2-82$ & 66.88 & 12.86 & 0.18 & 8.67 & 8.88 & 18.98 & 8.88 & 0.88 & 8.88 & 0.80 & 8.80 & 0.31 \\
\hline $8 \mathrm{H}-2-182$ & 66.22 & 3.88 & 0.17 & 1.27 & 8.88 & 1.68 & 8.88 & 8. 88 & 8.82 & 0.81 & 8.88 & 0.01 \\
\hline $8 \mathrm{H}-2-122$ & 66.42 & 3.85 & 0.31 & 1.37 & 0.80 & 1.23 & 8.80 & 0.01 & 8.80 & 0.80 & 8.08 & 0.89 \\
\hline $8 \mathrm{H}-2-142$ & 66.62 & 2.98 & 0.13 & 1.23 & 0.80 & 1.68 & 8.81 & 0.01 & 0.81 & 8.88 & 8.88 & 0.81 \\
\hline $8 \mathrm{H}-3-28$ & 66.98 & 3.80 & 0.23 & 1.22 & $8 . \theta 8$ & 1.47 & 8.80 & 0.01 & 0.80 & 8.01 & 8.80 & 0.85 \\
\hline $8 \mathrm{H}-3-48$ & 67.11 & 13.98 & 0.85 & 8.62 & 8.88 & 12.82 & 8.05 & 8.75 & 8.03 & 8.11 & 8.88 & 0.32 \\
\hline $8 H-3-68$ & 67.37 & $5.8 \theta$ & 8.68 & 2.99 & 8.80 & 1.99 & 8.08 & 8.88 & 8.88 & 0.08 & 0.00 & 0.13 \\
\hline $8 \mathrm{H}-3-82$ & 67.58 & 5.94 & 8.45 & 2.12 & 8.80 & 3.19 & 0.01 & 0.11 & 8.88 & 0.03 & 0.81 & 0.81 \\
\hline $8 \mathrm{H}-3-182$ & 67.72 & 8.88 & B. 24 & 1.75 & 8.80 & 4.93 & 8.86 & 0.16 & 8.86 & 8.72 & 8.88 & 0.13 \\
\hline $8 H-3-122$ & 67.98 & 7.68 & 8.42 & 1.58 & 8.80 & 4.68 & 0.88 & 0.39 & 8.84 & 8.58 & 0.88 & 8.87 \\
\hline $8 \mathrm{H}-3-142$ & 68.10 & 1.16 & 8.88 & 8.95 & 8.80 & 0.11 & 8.88 & 0.08 & 8.88 & 0.80 & 8.88 & 0.01 \\
\hline $8 H-4-22$ & 68.43 & 9.84 & 0.23 & 8.75 & 8.80 & 8.14 & 0.06 & 0.84 & 0.82 & 0.58 & 8.08 & 0.11 \\
\hline $8 \mathrm{H}-4-42$ & 68.62 & 13.66 & 0.13 & 0.65 & 8.80 & 12.52 & 8.88 & 8.85 & 8.83 & $8 . \theta 0$ & 8.08 & 0.26 \\
\hline $8 H-5-22$ & 69.92 & 4.98 & 0.18 & 0.16 & 8.88 & 4.21 & 0.83 & 8.82 & 8.03 & 8.26 & 0.80 & 0.09 \\
\hline $8 \mathrm{H}-5-42$ & 78.13 & 11.47 & 0.11 & 8.82 & 8.80 & 18.23 & 8.80 & 0.84 & 8.88 & 8.88 & 8.08 & 0.24 \\
\hline $8 H-5-68$ & 78.38 & 23.73 & 8.80 & 8.84 & 8.80 & 28.99 & 8.13 & 0.89 & 0.18 & 1.84 & 8.80 & 0.45 \\
\hline $8 H-5-82$ & 78.52 & 2.31 & 0.03 & 0.81 & 8.08 & 2.83 & 8.80 & 8.01 & 8.88 & 0.08 & 8.80 & 0.22 \\
\hline $8 \mathrm{H}-5-102$ & 78.72 & 4.98 & 0.43 & 1.68 & 8.08 & 2.62 & 0.88 & 0.02 & 8.81 & $0 . \theta \theta$ & 8.80 & 0.15 \\
\hline $8 \mathrm{H}-5-122$ & 78.92 & 6.47 & 0.06 & 0.89 & 8.81 & 5.59 & 0.12 & 0.86 & 0.03 & 0.45 & 8.88 & 8.04 \\
\hline $8 H-5-142$ & 71.12 & 6.72 & 0.13 & 0.74 & $8 . \theta 8$ & 5.67 & 8.80 & 8.83 & 0.08 & 8.80 & $\theta . \theta 8$ & 8.16 \\
\hline
\end{tabular}


Table 1.4 (continued).

\begin{tabular}{|c|c|c|c|c|c|c|c|c|c|c|c|c|}
\hline \multirow[b]{2}{*}{ Sample no. } & \multirow[b]{2}{*}{$\begin{array}{l}\text { Depth } \\
\text { mbsf }\end{array}$} & \multirow[b]{2}{*}{$\begin{array}{c}>63 \mu \mathrm{m} \\
(\%)\end{array}$} & \multicolumn{10}{|c|}{ Coarse fraction component analysis (wt \%) } \\
\hline & & & $\begin{array}{l}\text { Benthic } \\
\text { foram. } \\
(\%)\end{array}$ & $\begin{array}{c}\text { Plankt. } \\
\text { foram. } \\
(\%)\end{array}$ & $\begin{array}{c}\text { Biogenic } \\
\text { opal cf } \\
(\%)\end{array}$ & $\begin{array}{c}\text { Quartz } \\
(\%)\end{array}$ & $\begin{array}{c}\text { Feldspar } \\
(\%)\end{array}$ & $\begin{array}{c}\text { Mica } \\
(\%)\end{array}$ & $\begin{array}{l}\text { Hev. min. } \\
(\%)\end{array}$ & $\begin{array}{c}\text { Rock fr. } \\
(\%)\end{array}$ & $\begin{array}{l}\text { Volc. gl. } \\
(\%)\end{array}$ & $\begin{array}{l}\text { Auth. } \\
(\%)\end{array}$ \\
\hline $8 H-6-22$ & 71.42 & 2.44 & 8.29 & 0.71 & 8.88 & 1.17 & 8. 81 & 8.82 & 8.84 & 8.17 & 8.88 & 8.03 \\
\hline $8 H-6-42$ & 71.62 & 3.15 & 8.15 & 0.48 & 8.08 & 2.41 & 0.81 & 8.81 & 8.80 & 8.88 & 8.88 & 8.89 \\
\hline $8 H-6-68$ & 71.80 & 8.57 & 0.88 & 8.33 & 8.81 & 8.86 & $\theta . \theta 8$ & 8.88 & 8.88 & 8.82 & 8.88 & 8.86 \\
\hline $9 H-1-22$ & 73.43 & 2.86 & 8.86 & 1.68 & 0.88 & 0.25 & 8.82 & 8.88 & 8.80 & 0.88 & 8.80 & 8.84 \\
\hline $9 H-1-48$ & 73.68 & 2.23 & 0.02 & 1.36 & 8.80 & 0.77 & 8.88 & 0.88 & 8.80 & 8.80 & 0.08 & 0.07 \\
\hline $9 H-1-59$ & 73.79 & 3.95 & 8.15 & 3.65 & 8.03 & 0.03 & 8.80 & 8.88 & 0.00 & 8.88 & 0.80 & 0.85 \\
\hline $9 H-1-82$ & 74.82 & 1.53 & 0.10 & 1.34 & $\theta .08$ & 0.03 & 8.88 & 0.80 & 0.80 & 8.88 & 8.80 & 0.85 \\
\hline $9 H-1-182$ & 74.22 & 0.67 & 8.86 & 0.50 & 8.88 & 0.02 & 8.80 & 0.80 & 0.08 & 8.68 & $\theta . \theta 8$ & 0.89 \\
\hline $9 H-1-123$ & 74.43 & 8.37 & 8.85 & 8.07 & $8.0 \theta$ & 0.07 & 8.08 & $8.8 \theta$ & 8.88 & 0.80 & 8.80 & 0.18 \\
\hline $9 H-1-142$ & 74.62 & 17.89 & 8.16 & 0.48 & 0.88 & 13.14 & 8.16 & 2.84 & 0.21 & 0.88 & 8.88 & 0.16 \\
\hline $9 H-2-23$ & 74.93 & 6.32 & 0.82 & 0.31 & 8.88 & 5.57 & 8.80 & 0.81 & 0.84 & 0.80 & 0.08 & 0.35 \\
\hline $9 H-2-48$ & 75.18 & 3.92 & 0.11 & 8.63 & 0.88 & 3.18 & 8.82 & 8.80 & 8.88 & 0.04 & 0.88 & 0.01 \\
\hline $9 \mathrm{H}-2-59$ & 75.29 & 1.94 & 0.82 & 0.18 & 8.88 & 1.66 & 8.80 & 0.81 & 0.01 & 8.88 & 8.80 & 0.86 \\
\hline $9 H-2-82$ & 75.52 & 2.52 & 8.82 & 0.11 & $8 . \theta 0$ & 2.21 & 0.82 & 0.12 & 0.01 & 0.81 & 8.80 & 0.02 \\
\hline $9 \mathrm{H}-2-102$ & 75.72 & 5.52 & 0.12 & 0.68 & $\theta . \theta \theta$ & 4.62 & 0.80 & 0.01 & 0.81 & 0.02 & 0.08 & 0.12 \\
\hline $9 \mathrm{H}-2-123$ & 75.93 & 5.72 & 0.15 & 2.81 & 0.80 & 3.84 & 0.81 & 0.25 & 8.01 & 8.89 & 0.80 & 0.15 \\
\hline $9 H-2-142$ & 76.12 & 13.96 & 8.84 & 0.96 & 8.80 & 11.31 & 0.82 & 1.43 & 0.08 & 0.89 & $8 . \theta 8$ & 8.89 \\
\hline $9 \mathrm{H}-3-23$ & 76.43 & 1.43 & 0.12 & 1.10 & 8.82 & 0.82 & 8.88 & 8.86 & 8.80 & 8.81 & 8.08 & 0.08 \\
\hline $9 \mathrm{H}-3-48$ & 76.68 & 0.88 & 0.85 & 8.45 & 8.88 & 8.82 & 8.80 & 8.88 & 8.80 & 8.80 & 0.88 & 0.28 \\
\hline $9 H-3-59$ & 76.79 & 1.49 & 8.06 & 0.83 & 8.81 & 8.32 & 8.88 & 8.02 & 8.80 & 8.82 & 8.80 & 0.22 \\
\hline $9 H-3-82$ & 77.82 & 2.31 & 0.88 & 1.13 & 8.88 & 8.81 & 8.80 & $8 . \theta 8$ & 8.80 & 0.80 & 8.68 & 8.29 \\
\hline $9 \mathrm{H}-3-182$ & 77.22 & 1.87 & 0.87 & 8.47 & 8.81 & 0.15 & 8.88 & 0.81 & 8.80 & 0.81 & 0.80 & 0.35 \\
\hline $9 \mathrm{H}-3-123$ & 77.43 & 1.85 & 0.15 & 8.64 & 8.88 & 8.16 & 8.88 & 8.88 & 8.88 & 8.88 & 8.88 & 0.18 \\
\hline $9 \mathrm{H}-3-142$ & 77.62 & 22.37 & 8.08 & 0.08 & 0.08 & 28.81 & 0.21 & 0.50 & $\theta . \theta 0$ & 1.16 & $\theta . \theta \theta$ & 8.41 \\
\hline $9 \mathrm{H}-4-23$ & 78.43 & 4.21 & 8.82 & 1.33 & 8.80 & 2.66 & 8.80 & 8.81 & 8.81 & 8.88 & 8.01 & 0.18 \\
\hline $9 H-4-4 \theta$ & 78.60 & 27.21 & 8.10 & 0.15 & 0.80 & 23.88 & 8.38 & 0.28 & 8.28 & 1.75 & 8.88 & 0.78 \\
\hline $9 H-4-59$ & 78.79 & 6.92 & 8.85 & 1.88 & 8.80 & 5.31 & 8.80 & 0.15 & 8.08 & 8.80 & 0.05 & 0.27 \\
\hline $9 H-4-82$ & 79.82 & 4.61 & 8.88 & 0.51 & 8.01 & 3.63 & 8.82 & 8.18 & 8.80 & 8.16 & 8.88 & 0.88 \\
\hline $9 H-4-182$ & 79.22 & 5.38 & 0.03 & 8.12 & 8.80 & 4.45 & 8.85 & 0.87 & 0.85 & 8.33 & 0.08 & 0.28 \\
\hline $9 \mathrm{H}-5-23$ & 79.93 & 1.03 & 0.11 & 0.62 & $8.8 \theta$ & 8.88 & $0 . \theta 0$ & 0.03 & 8.88 & 8.81 & 0.68 & 0.17 \\
\hline $9 \mathrm{H}-5-48$ & 80.10 & 14.57 & 0.64 & 0.64 & $\theta .8 \theta$ & 18.21 & 8.88 & 0.17 & 8.82 & 0.12 & 0.80 & 2.32 \\
\hline $9 \mathrm{H}-5-59$ & 80.29 & 5.85 & 0.80 & 8.84 & 8.80 & 4.55 & 8.82 & 0.26 & 8.01 & 8.01 & 0.80 & 0.16 \\
\hline $9 \mathrm{H}-5-82$ & 88.52 & 5.68 & 0.81 & 8.86 & 8.80 & 4.85 & 8.87 & 8.89 & 8.87 & 8.39 & 8.80 & 0.07 \\
\hline $9 H-5-182$ & 88.72 & 9.26 & 8.80 & 8.85 & 8.88 & 8.21 & 0.03 & 8.36 & 8.82 & 8.88 & 8.88 & 0.56 \\
\hline $9 H-5-123$ & 88.93 & 2.88 & 0.80 & 8.01 & 8.88 & 2.24 & 0.01 & 0.84 & 8.84 & 0.43 & 0.80 & 0.02 \\
\hline $18 \mathrm{H}-2-22$ & 84.42 & 1.78 & 0.86 & 0.95 & 8.80 & 0.59 & 8.80 & 0.01 & 8.01 & 0.11 & 8.88 & 0.84 \\
\hline $10 \mathrm{H}-2-41$ & 84.61 & 8.81 & 0.08 & 0.38 & 0.88 & 0.34 & $\theta .80$ & $\theta . \theta 8$ & 8.88 & 0.01 & 8.88 & 0.88 \\
\hline $10 \mathrm{H}-2-57$ & 84.78 & 2.28 & 0.11 & 1.75 & 8.81 & 8.25 & 8.88 & 8.82 & 8.88 & 0.80 & 8.68 & 8.84 \\
\hline $10 \mathrm{H}-2-101$ & 85.22 & 1.98 & 0.81 & 0.29 & $8 . \theta 0$ & 1.52 & 8.88 & 8.81 & 8.80 & 0.08 & 0.80 & 0.86 \\
\hline $18 \mathrm{H}-2-121$ & 85.42 & 1.75 & 0.02 & 1.38 & 8.88 & 8.39 & 8.88 & 8.81 & 8.88 & 0.80 & 8.08 & 8.82 \\
\hline $18 \mathrm{H}-2-142$ & 85.63 & 8.72 & 8.81 & 8.68 & 8.88 & 8.81 & 8.88 & 8.88 & 8.88 & $\theta . \theta 8$ & 0.80 & 0.82 \\
\hline $18 \mathrm{H}-3-22$ & 85.92 & 8.26 & 8.86 & 0.12 & 8.08 & 8.01 & 8.80 & 8.80 & 8.80 & 8.80 & 8.08 & 8.86 \\
\hline $18 \mathrm{H}-3-41$ & 86.11 & 4.92 & 8.23 & 0.41 & 8.88 & 8.25 & 8.88 & 8.81 & 0.88 & 8.08 & 8.80 & 3.98 \\
\hline $18 \mathrm{H}-3-57$ & 86.28 & 1.83 & 0.11 & 0.89 & 8.80 & 8.80 & 8.80 & 8.80 & 8.80 & 8.80 & 0.80 & 0.82 \\
\hline $18 \mathrm{H}-3-78$ & 86.48 & 1.93 & 0.14 & 0.73 & 8.88 & 8.36 & 8.88 & 8.88 & 8.80 & 0.80 & 8.88 & 0.66 \\
\hline $18 \mathrm{H}-3-181$ & 86.71 & 8.93 & 8.83 & 0.84 & 8.80 & 0.01 & $\theta . \theta \theta$ & 8.80 & 8.08 & 8.80 & 0.88 & 8.84 \\
\hline $18 \mathrm{H}-3-121$ & 86.92 & 2.52 & 0.87 & 1.78 & 8.86 & 0.03 & 8.88 & 8.88 & 8.88 & 8.80 & 8.88 & 8.53 \\
\hline $18 \mathrm{H}-3-142$ & 87.12 & 8.11 & 8.88 & 8.83 & 8.84 & 6.47 & 8.84 & 1.12 & 8.83 & 8.24 & 0.88 & 8.13 \\
\hline $18 \mathrm{H}-4-21$ & 87.42 & 1.84 & 8.81 & 8.99 & 8.80 & 8.80 & 8.88 & 8.80 & 8.80 & 8.88 & 8.80 & 8.83 \\
\hline $18 \mathrm{H}-4-41$ & 87.61 & 0.93 & 8.28 & 0.35 & 8.88 & 0.89 & 8.80 & 8.84 & $\theta . \theta 8$ & 8.88 & 8.88 & 0.15 \\
\hline
\end{tabular}


Table 1.4 (continued).

\begin{tabular}{|c|c|c|c|c|c|c|c|c|c|c|c|c|}
\hline \multirow[b]{2}{*}{ Sample no. } & \multirow[b]{2}{*}{$\begin{array}{l}\text { Depth } \\
\text { mbsf }\end{array}$} & \multirow[b]{2}{*}{$\begin{array}{c}>63 \mu \mathrm{m} \\
(\%)\end{array}$} & \multicolumn{10}{|c|}{ Coarse fraction component analysis (wt \%) } \\
\hline & & & $\begin{array}{l}\text { Benthic } \\
\text { foram. } \\
(\%)\end{array}$ & $\begin{array}{l}\text { Plankt. } \\
\text { foram. } \\
(\%)\end{array}$ & $\begin{array}{c}\text { Biogenic } \\
\text { opal cf } \\
(\%)\end{array}$ & $\begin{array}{c}\text { Quartz } \\
(\%)\end{array}$ & $\begin{array}{c}\text { Feldspar } \\
(\%)\end{array}$ & $\begin{array}{l}\text { Mica } \\
(\%)\end{array}$ & $\begin{array}{l}\text { Hev. min. } \\
(\%)\end{array}$ & $\begin{array}{l}\text { Rock fr. } \\
(\%)\end{array}$ & $\begin{array}{l}\text { Volc. gl. } \\
(\%)\end{array}$ & $\begin{array}{c}\text { Auth. } \\
(\%)\end{array}$ \\
\hline $10 \mathrm{H}-4-57$ & 87.78 & 28.98 & 8.84 & 8.88 & 8.84 & 19.45 & 8.88 & 0.88 & 0.80 & 8.88 & 8.84 & 1.25 \\
\hline $18 \mathrm{H}-4-78$ & 87.98 & 28.55 & 8.18 & 0.69 & 8.14 & 19.68 & $\theta . \theta 8$ & 7.39 & 8.14 & 0.14 & 0.88 & 8.18 \\
\hline $10 \mathrm{H}-4-101$ & 88.22 & 27.55 & 8.32 & 0.32 & 8.85 & 25.72 & 8.80 & 0.59 & 8.88 & 0.88 & 8.80 & 8.54 \\
\hline $18 \mathrm{H}-4-121$ & 88.41 & 11.32 & 8.11 & 0.22 & 8.88 & 9.81 & 8.82 & 8.13 & 8.84 & 0.24 & 8.80 & 8.73 \\
\hline $10 \mathrm{H}-5-21$ & 88.91 & 8.81 & 8.89 & 8.52 & 8.88 & 0.89 & 8.88 & 8.87 & 8.88 & 8.82 & 8.88 & 8.82 \\
\hline $18 \mathrm{H}-5-41$ & 89.11 & 3.22 & 8.82 & 8.14 & 8.80 & 2.82 & 8.88 & 8.82 & 8.08 & 8.80 & 8.88 & 8.28 \\
\hline $18 \mathrm{H}-5-57$ & 89.28 & 1.39 & 0.13 & 1.84 & 8.88 & 0.17 & 0.80 & 0.81 & 8.80 & 8.81 & 0.80 & 8.81 \\
\hline $18 \mathrm{H}-5-78$ & 89.48 & 9.51 & 0.29 & 1.39 & 0.82 & 5.45 & 8.88 & 8.82 & 8.80 & 8.88 & 8.88 & 2.38 \\
\hline $10 \mathrm{H}-5-101$ & 89.71 & 5.11 & 0.13 & 1.17 & 8.81 & 3.43 & 8.82 & 0.15 & 8.03 & 8.86 & 8.88 & 8.11 \\
\hline $10 \mathrm{H}-5-121$ & 89.92 & 3.17 & 0.41 & 1.12 & 8.08 & 1.24 & 8.88 & 0.81 & 0.88 & 8.81 & 0.08 & 0.33 \\
\hline $10 \mathrm{H}-6-21$ & 98.42 & 14.21 & 0.85 & 0.75 & 0.03 & 10.27 & 0.85 & 2.81 & 8.88 & 0.13 & $\theta . \theta \theta$ & 0.85 \\
\hline $18 \mathrm{H}-6-41$ & 90.62 & 5.79 & 0.24 & 1.88 & 8.80 & 2.96 & 8.81 & 8.64 & 8.83 & 0.02 & 8.88 & 8.83 \\
\hline $11 \mathrm{H}-1-22$ & 92.42 & 3.98 & 8.84 & 0.07 & 8.88 & 3.52 & 8.82 & 8.08 & 0.81 & 0.86 & $\theta . \theta \theta$ & 8.87 \\
\hline $11 \mathrm{H}-1-42$ & 92.62 & 3.03 & 8.82 & 0.03 & 8.84 & 2.88 & 8.88 & 0.03 & 8.80 & 0.01 & 8.88 & 8.69 \\
\hline $11 H-1-6 \theta$ & 92.88 & 2.79 & 8.02 & 0.01 & 8.88 & 2.45 & 8.82 & 0.18 & 8.04 & 8.04 & $\theta .00$ & 8.04 \\
\hline $11 H-1-81$ & 93.01 & 13.63 & 0.05 & 8.80 & 8.83 & 12.52 & 8.88 & 0.88 & 8.80 & 8.08 & 8.88 & 8.80 \\
\hline $11 \mathrm{H}-1-180$ & 93.28 & 0.94 & 0.82 & 0.49 & 8.88 & 8.37 & 8.88 & 0.81 & 8.80 & 8.81 & 8.88 & 8.83 \\
\hline $11 \mathrm{H}-1-122$ & 93.48 & 2.37 & 0.86 & 0.25 & 8.81 & 1.98 & 8.88 & 0.01 & 8.08 & 8.80 & 8.80 & 0.13 \\
\hline $11 \mathrm{H}-1-142$ & 93.62 & 5.62 & 0.21 & 0.66 & 8.88 & 4.32 & 0.01 & 0.89 & 0.85 & 0.06 & 8.88 & 0.28 \\
\hline $11 \mathrm{H}-2-22$ & 93.92 & 5.96 & 0.28 & 3.03 & 8.87 & 2.21 & 8.80 & 8.01 & 8.88 & 8.88 & $8.8 \theta$ & 8.42 \\
\hline $11 \mathrm{H}-2-39$ & 94.89 & 3.42 & 0.05 & 3.23 & 8.88 & 8.18 & 8.88 & 0.83 & 8.88 & 8.80 & 8.80 & 0.01 \\
\hline $11 \mathrm{H}-2-63$ & 94.33 & 2.16 & 0.85 & 1.67 & 8.81 & 8.37 & 8.80 & 0.08 & 8.80 & 0.80 & 8.80 & 0.05 \\
\hline $11 \mathrm{H}-2-81$ & 94.51 & 1.22 & 0.88 & 8.55 & 8.88 & 0.55 & 8.80 & 8.80 & 8.88 & 8.88 & 8.08 & 8.82 \\
\hline $11 \mathrm{H}-2-18 \theta$ & 94.78 & 1.99 & 0.85 & 8.87 & 0.81 & 1.88 & 8.88 & 8.80 & 8.88 & 0.80 & 8.88 & 0.86 \\
\hline $11 \mathrm{H}-2-122$ & 94.92 & 8.99 & 0.16 & 8.72 & 8.88 & 8.87 & 8.88 & 8.88 & 8.80 & 8.80 & 8.88 & 0.03 \\
\hline $11 \mathrm{H}-2-142$ & 95.14 & 1.51 & 0.36 & 0.57 & 8.88 & 8.25 & 8.88 & $8.8 \theta$ & 8.88 & 8.80 & 8.88 & 0.29 \\
\hline $11 \mathrm{H}-3-22$ & 95.42 & 1.78 & 0.33 & 8.35 & 8.88 & 8.96 & 8.88 & 8.82 & 8.80 & 8.82 & 8.80 & 0.18 \\
\hline $11 \mathrm{H}-3-42$ & 95.62 & 5.82 & 0.21 & 8.18 & 8.80 & 5.10 & 8.80 & 8.07 & 8.88 & 8.88 & 8.88 & 8.26 \\
\hline $11 \mathrm{H}-3-68$ & 95.88 & 2.91 & 8.12 & 8.07 & 8.08 & 2.47 & 8.88 & 0.19 & 0.03 & 8.03 & 8.80 & 8.01 \\
\hline $11 \mathrm{H}-3-81$ & 96.01 & 13.87 & 8.03 & 8.05 & 8.88 & 13.18 & 8.88 & 0.11 & 8.08 & 8.03 & 8.88 & 8.56 \\
\hline $11 \mathrm{H}-3-10 \theta$ & 96.28 & 11.55 & 8.84 & 8.08 & 8.88 & 18.55 & 8.82 & 0.27 & 0.86 & 8.42 & 0.08 & 0.19 \\
\hline $11 \mathrm{H}-3-122$ & 96.48 & 3.94 & 8.85 & 0.80 & 8.80 & 3.73 & 8.88 & 0.02 & 8.80 & 8.88 & 8.88 & 8.14 \\
\hline $11 \mathrm{H}-3-142$ & 96.62 & 5.38 & 8.18 & 0.13 & 8.80 & 4.46 & 8.80 & 8.22 & 0.86 & 8.14 & 0.88 & 8.12 \\
\hline $11 \mathrm{H}-4-22$ & 96.92 & 3.88 & 8.13 & 0.82 & 8.80 & 1.88 & 8.88 & 8.81 & 0.81 & 8.88 & 0.88 & 8.10 \\
\hline $11 \mathrm{H}-4-42$ & 97.14 & 3.15 & 0.16 & 0.81 & 8.81 & 1.79 & 8.02 & 0.27 & 0.81 & 0.85 & 8.88 & 8.81 \\
\hline $11 \mathrm{H}-4-68$ & 97.38 & 2.57 & 0.12 & 0.66 & 8.88 & 1.57 & 8.88 & 8.88 & 8.88 & 8.88 & 0.08 & 0.28 \\
\hline $11 \mathrm{H}-4-81$ & 97.51 & 1.29 & 8.11 & 0.88 & 8.80 & 0.28 & 8.88 & 0.07 & 8.88 & 8.03 & 0.88 & 0.08 \\
\hline $11 \mathrm{H}-4-18 \theta$ & 97.78 & 7.52 & 8.88 & 0.01 & 8.88 & 7.03 & 8.80 & 0.08 & 8.88 & 0.01 & 0.84 & 0.34 \\
\hline $11 \mathrm{H}-4-122$ & 97.92 & 28.32 & 8.84 & 0.80 & 8.88 & 18.78 & 0.88 & 0.87 & 8.88 & 0.46 & 0.80 & 8.88 \\
\hline $11 \mathrm{H}-4-142$ & 98.14 & 4.27 & 8.82 & 8.18 & 8.81 & 4.81 & 0.88 & 8.84 & 8.08 & 8.88 & 8.81 & 8.88 \\
\hline $11 \mathrm{H}-5-22$ & 98.42 & 8.66 & 0.85 & 0.38 & 8.80 & 0.19 & 8.80 & 0.01 & 8.01 & 8.82 & 8.88 & 8.88 \\
\hline $11 \mathrm{H}-5-42$ & 98.62 & 17.98 & 0.84 & 0.04 & 8.88 & 17.38 & 8.80 & 0.11 & 0.84 & 8.80 & 8.88 & 0.39 \\
\hline $11 \mathrm{H}-5-68$ & 98.88 & 8.69 & 8.85 & 0.42 & 0.80 & 0.19 & 8.80 & 0.01 & 8.88 & 8.82 & 0.88 & 8.08 \\
\hline $11 \mathrm{H}-5-88$ & 99.08 & 8.38 & 8.88 & 0.17 & 8.88 & 7.88 & 8.88 & 0.03 & 8.88 & 8.08 & 0.82 & 8.28 \\
\hline $11 \mathrm{H}-5-18 \theta$ & 99.38 & 0.51 & 8.88 & 8.80 & 8.88 & 8.45 & 8.80 & 8.81 & 8.80 & 8.03 & 8.88 & 8.81 \\
\hline $11 \mathrm{H}-5-122$ & 99.52 & 2.28 & 8.82 & 0.18 & 8.88 & 1.94 & 8.88 & 8.88 & 8.88 & 8.88 & 8.88 & 8.11 \\
\hline $11 \mathrm{H}-6-22$ & 99.92 & 1.72 & 8.28 & 1.27 & 8.88 & 8.19 & 8.88 & 8.82 & 8.88 & 8.88 & 8.80 & 0.03 \\
\hline $11 \mathrm{H}-6-42$ & 108.14 & 0.91 & 8.81 & 8.05 & 8.80 & 0.76 & 8.80 & 8.88 & 8.80 & 8.80 & 0.81 & 8.86 \\
\hline $11 H-6-68$ & 180.38 & 1.78 & 8.15 & 1.54 & 8.88 & 8.82 & 0.80 & 8.80 & 8.08 & 0.80 & 0.80 & 8.85 \\
\hline $11 \mathrm{H}-6-88$ & 188.58 & 1.58 & 0.15 & 1.26 & 8.81 & 0.01 & 0.80 & 8.80 & 8.88 & 8.88 & 8.88 & 0.86 \\
\hline
\end{tabular}


Table 1.4 (continued).

\begin{tabular}{|c|c|c|c|c|c|c|c|c|c|c|c|c|}
\hline \multirow[b]{2}{*}{ Sample no. } & \multirow[b]{2}{*}{$\begin{array}{c}\text { Depth } \\
\text { mbsf }\end{array}$} & \multirow[b]{2}{*}{$\begin{array}{c}>63 \mu \mathrm{m} \\
(\%)\end{array}$} & \multicolumn{10}{|c|}{ Coarse fraction component analysis (wt $\%)$} \\
\hline & & & $\begin{array}{c}\text { Benthic } \\
\text { foram. } \\
(\%)\end{array}$ & $\begin{array}{l}\text { Plankt. } \\
\text { foram. } \\
(\%)\end{array}$ & $\begin{array}{c}\text { Biogenic } \\
\text { opal cf } \\
(\%)\end{array}$ & $\begin{array}{c}\text { Quartz } \\
(\%)\end{array}$ & $\begin{array}{c}\text { Feldspar } \\
(\%)\end{array}$ & $\begin{array}{c}\text { Mica } \\
(\%)\end{array}$ & $\begin{array}{c}\text { Hev. min. } \\
(\%)\end{array}$ & $\begin{array}{c}\text { Rock fr. } \\
(\%)\end{array}$ & $\begin{array}{c}\text { Volc. gl. } \\
(\%)\end{array}$ & $\begin{array}{c}\text { Auth. } \\
(\%)\end{array}$ \\
\hline $11 \mathrm{H}-6-122$ & 108.92 & 1.28 & 0.12 & 0.89 & 8.81 & 8.81 & 8.80 & 8.80 & 0.80 & 8.80 & 0.80 & 0.17 \\
\hline $11 H-6-142$ & 101.14 & 5.73 & 8.78 & 4.48 & 8.03 & 0.82 & 8.88 & 8.08 & 8.88 & 8.80 & 8.80 & 0.39 \\
\hline $11 \mathrm{H}-7-22$ & 101.42 & 1.16 & 0.14 & 8.87 & 0.82 & 8.81 & 8.88 & 8.88 & 8.88 & 8.88 & 8.88 & 8.18 \\
\hline $12 \mathrm{H}-1-22$ & 101.91 & 15.99 & 8.89 & 8.86 & 8.89 & 15.89 & 8.80 & 8.46 & 0.80 & 8.12 & 8.88 & 0.86 \\
\hline $12 \mathrm{H}-1-42$ & 182.10 & 1.46 & 8.01 & 0.01 & 0.80 & 1.28 & $\theta . \theta 0$ & 8.81 & 0.80 & $\theta . \theta 1$ & 8.88 & 0.14 \\
\hline $12 \mathrm{H}-1-62$ & 182.38 & 4.56 & 0.38 & 2.31 & 8.81 & 1.68 & 0.88 & 8.02 & 0.88 & 8.81 & 0.88 & 0.10 \\
\hline $12 \mathrm{H}-1-82$ & 102.49 & 1.78 & 8.15 & 1.36 & 0.81 & 0.28 & 8.80 & 8.08 & 8.80 & 0.88 & 8.80 & 0.86 \\
\hline $12 \mathrm{H}-1-102$ & 182.68 & 1.87 & 0.18 & 0.76 & 0.80 & 8.89 & 8.88 & $\theta . \theta \theta$ & 8.88 & 0.80 & 8.88 & 0.12 \\
\hline $12 \mathrm{H}-1-122$ & 182.87 & 2.45 & 0.82 & 0.21 & 0.80 & 1.85 & $\theta .80$ & 0.88 & 0.80 & 0.16 & 8.01 & 0.28 \\
\hline $12 \mathrm{H}-1-143$ & 183.86 & 4.87 & 8.80 & 0.09 & 8.08 & 3.76 & 0.82 & 0.81 & 8.80 & $8 . \theta 8$ & 0.01 & 0.17 \\
\hline $12 \mathrm{H}-2-22$ & 103.35 & 8.08 & $0.8 \theta$ & 0.80 & 8.80 & 7.61 & 8.80 & 0.28 & 0.80 & 0.88 & 0.81 & 0.25 \\
\hline $12 \mathrm{H}-2-42$ & 103.54 & 0.58 & 0.08 & 0.08 & 0.80 & 0.32 & 8.80 & 0.88 & 8.80 & 8.80 & 0.80 & 0.26 \\
\hline $12 \mathrm{H}-2-62$ & 103.74 & 2.65 & 0.80 & 0.80 & 0.81 & 2.22 & 8.08 & $\theta . \theta \theta$ & 8.88 & 0.17 & 0.88 & 8.24 \\
\hline $12 \mathrm{H}-2-82$ & 103.93 & 5.65 & 8.80 & 0.82 & 0.88 & 5.19 & 8.88 & 0.08 & 8.80 & 8.80 & 0.82 & 8.41 \\
\hline $12 \mathrm{H}-2-182$ & 104.12 & 1.92 & 0.88 & 1.59 & 0.80 & 8.86 & 8.88 & 0.88 & 8.88 & 0.01 & 0.84 & 0.13 \\
\hline $12 \mathrm{H}-2-122$ & 184.31 & 1.38 & 8.81 & 1.18 & 0.81 & 8.82 & 8.80 & 0.88 & 8.80 & 0.88 & 0.80 & 0.23 \\
\hline $12 \mathrm{H}-2-142$ & 184.58 & 2.85 & 0.07 & 8.18 & 0.02 & 8.99 & 0.80 & 0.88 & 0.80 & 0.02 & 8.81 & 0.75 \\
\hline $12 \mathrm{H}-3-22$ & 184.79 & 6.92 & 0.88 & 8.85 & 0.80 & 6.45 & 0.81 & $\theta . \theta 0$ & 0.80 & 8.81 & 8.81 & 0.39 \\
\hline $12 \mathrm{H}-3-42$ & 184.98 & 1.24 & 8.84 & 8.54 & 8.88 & 8.53 & 0.88 & 8.82 & 8.81 & 8.85 & 0.80 & 8.84 \\
\hline $12 \mathrm{H}-3-62$ & 185.18 & 1.84 & 8.80 & 8.08 & 8.88 & 8.64 & 8.88 & 8.88 & 8.08 & 8.80 & 0.80 & 0.32 \\
\hline $12 \mathrm{H}-3-82$ & 185.37 & 1.84 & 8.88 & 8.80 & 0.80 & 8.78 & 8.80 & 8.01 & 0.80 & 8.03 & 0.01 & 0.29 \\
\hline $12 \mathrm{H}-3-102$ & 105.56 & 0.79 & 0.80 & 0.80 & 0.00 & 8.33 & 8.80 & 0.88 & 0.80 & 8.80 & 0.00 & 0.45 \\
\hline $12 \mathrm{H}-3-122$ & 185.75 & 0.39 & 0.87 & 8.05 & 8.01 & 0.81 & 8.88 & 8.88 & 8.00 & 8.80 & 0.80 & 8.23 \\
\hline $12 \mathrm{H}-3-142$ & 105.94 & 4.66 & 8.80 & 0.80 & 8.80 & 4.25 & 8.80 & 0.03 & 8.88 & 0.03 & 0.08 & 0.36 \\
\hline $12 \mathrm{H}-4-22$ & 186.23 & 3.76 & 0.48 & 0.78 & 0.28 & 8.88 & 8.88 & 0.03 & 8.80 & 8.00 & 8.80 & 2.26 \\
\hline $12 \mathrm{H}-4-42$ & 186.42 & 0.94 & 8.12 & 0.29 & 8.82 & 8.88 & 0.80 & 8.88 & 0.68 & 8.68 & 8.88 & 0.43 \\
\hline $12 \mathrm{H}-4-62$ & 186.62 & 1.17 & 8.12 & 0.59 & 0.86 & 8.81 & 8.88 & 8.82 & 8.80 & 8.88 & 8.81 & 0.34 \\
\hline $12 \mathrm{H}-4-82$ & 186.81 & 3.01 & 1.53 & 8.64 & 8.31 & 8.21 & 8.80 & 0.82 & 0.88 & 0.08 & 0.81 & 0.22 \\
\hline $12 \mathrm{H}-4-102$ & 107.80 & 5.94 & 8.86 & 0.88 & 0.06 & 5.34 & 8.80 & 8.82 & 8.82 & 8.18 & 0.80 & 0.27 \\
\hline $12 \mathrm{H}-5-22$ & 187.67 & 16.86 & 0.88 & 0.80 & 0.86 & 15.75 & 0.80 & 0.86 & 8.86 & 0.38 & 8.83 & 0.51 \\
\hline $12 \mathrm{H}-5-42$ & 187.86 & 6.27 & 0.12 & 8.23 & 0.87 & 5.89 & 0.80 & 8.82 & 0.85 & 0.88 & 0.88 & 0.61 \\
\hline $12 \mathrm{H}-5-62$ & 188.86 & 3.14 & 0.86 & 0.24 & 0.10 & 2.17 & 0.80 & 0.80 & 8.88 & 0.81 & 0.80 & 8.56 \\
\hline $12 \mathrm{H}-5-82$ & 188.25 & 2.66 & 0.10 & 0.16 & 8.18 & 0.18 & 8.68 & 0.88 & $0 . \theta 8$ & 0.88 & 1.75 & 8.45 \\
\hline $12 \mathrm{H}-5-102$ & 188.44 & 2.55 & 0.01 & 8.08 & 0.83 & 2.10 & 8.80 & 0.82 & 0.80 & 0.01 & 0.83 & 0.37 \\
\hline $12 \mathrm{H}-5-122$ & 188.63 & 4.37 & 0.01 & 0.01 & 0.89 & 3.63 & 8.80 & 0.03 & 8.08 & 0.81 & 0.80 & 0.61 \\
\hline $12 \mathrm{H}-5-142$ & 188.82 & 7.43 & 8.08 & 0.81 & 8.08 & 7.17 & 8.88 & 8.88 & 8.84 & 0.87 & 0.80 & 0.14 \\
\hline $12 \mathrm{H}-6-22$ & 189.11 & 4.64 & 0.08 & 0.07 & 8.88 & 4.18 & 0.80 & 0.84 & 8.81 & 0.81 & 8.80 & 0.25 \\
\hline $12 \mathrm{H}-6-42$ & 109.30 & 0.61 & 8.80 & 0.08 & 8.80 & 0.56 & 8.80 & $\theta . \theta \theta$ & 8.80 & 0.08 & 8.80 & 0.84 \\
\hline $12 \mathrm{H}-6-62$ & 189.50 & 1.41 & 0.81 & 0.23 & 0.68 & 0.88 & 8.80 & 8.88 & 8.08 & 8.08 & 8.08 & 0.29 \\
\hline $12 \mathrm{H}-6-82$ & 189.69 & 1.58 & 0.17 & 1.17 & 0.81 & 8.84 & 8.80 & 0.80 & 0.88 & 8.88 & 8.80 & 8.18 \\
\hline $12 \mathrm{H}-6-186$ & 189.88 & 3.78 & 8.16 & 3.39 & 0.85 & 8.80 & 8.80 & 8.80 & 0.88 & 8.80 & 0.01 & 8.13 \\
\hline $12 \mathrm{H}-6-122$ & 110.07 & 5.18 & 8.12 & 4.64 & 8.16 & 0.82 & 8.80 & 0.80 & 8.80 & 8.88 & 8.81 & 0.15 \\
\hline $12 \mathrm{H}-6-142$ & 118.26 & 1.77 & 8.86 & 1.52 & 0.81 & 0.81 & 8.88 & 8.88 & 8.88 & 8.88 & 8.88 & B. 12 \\
\hline $12 \mathrm{H}-7-22$ & 118.55 & 0.88 & 0.82 & 0.54 & 8.88 & 8.80 & 0.88 & 0.80 & 8.80 & 8.88 & 8.88 & 0.38 \\
\hline $12 \mathrm{H}-7-42$ & 110.74 & 1.54 & 8.84 & 1.16 & 8.80 & 8.88 & 8.88 & 0.80 & 0.80 & 0.88 & 0.88 & 0.31 \\
\hline $13 H-1-62$ & 111.81 & 1.88 & 0.29 & 1.11 & 8.80 & 8.18 & 8.88 & $\theta .8 \theta$ & 0.88 & 8.80 & 0.80 & 0.29 \\
\hline $13 \mathrm{H}-1-82$ & 112.80 & 1.23 & 0.23 & 8.74 & 0.08 & 8.89 & 0.08 & 8.80 & 8.88 & 8.88 & 8.08 & 0.17 \\
\hline $13 \mathrm{H}-1-182$ & 112.28 & 0.79 & 8.18 & 8.36 & 0.81 & 8.85 & 8.88 & 8.88 & 8.88 & 0.83 & 0.80 & 0.15 \\
\hline $13 H-1-122$ & 112.48 & 0.72 & 8.89 & 8.27 & 0.88 & 0.17 & 8.88 & 8.08 & 8.80 & 8.83 & 8.80 & 8.15 \\
\hline $13 H-1-145$ & 112.62 & 2.87 & 8.73 & 1.88 & 8.81 & 8.62 & 8.81 & 8.08 & 8.88 & 0.87 & 8.08 & 0.31 \\
\hline
\end{tabular}


Table 1.4 (continued).

\begin{tabular}{|c|c|c|c|c|c|c|c|c|c|c|c|c|}
\hline \multirow[b]{2}{*}{ Sample no. } & \multirow[b]{2}{*}{$\begin{array}{l}\text { Depth } \\
\text { mbsf }\end{array}$} & \multirow[b]{2}{*}{$\begin{array}{c}>63 \mu \mathrm{m} \\
(\%)\end{array}$} & \multicolumn{10}{|c|}{ Coarse fraction component analysis $(w t \%)$} \\
\hline & & & $\begin{array}{c}\text { Benthic } \\
\text { foram. } \\
(\%)\end{array}$ & $\begin{array}{c}\text { Plankt. } \\
\text { foram. } \\
(\%)\end{array}$ & $\begin{array}{c}\text { Biogenic } \\
\text { opal cf } \\
(\%)\end{array}$ & $\begin{array}{c}\text { Quartz } \\
(\%)\end{array}$ & $\begin{array}{c}\text { Feldspar } \\
(\%)\end{array}$ & $\begin{array}{l}\text { Mica } \\
(\%)\end{array}$ & $\begin{array}{l}\text { Hev. min. } \\
(\%)\end{array}$ & $\begin{array}{c}\text { Rock fr. } \\
(\%)\end{array}$ & $\begin{array}{c}\text { Volc. gl. } \\
(\%)\end{array}$ & $\begin{array}{l}\text { Auth. } \\
(\%)\end{array}$ \\
\hline $13 \mathrm{H}-2-21$ & 112.88 & 10.42 & 8.88 & 8.88 & 0.82 & 18.89 & 8.80 & 8.84 & 0.02 & 8.12 & 8.80 & 0.84 \\
\hline $13 \mathrm{H}-2-42$ & 113.88 & 5.24 & 8.88 & 8.88 & 8.88 & 5.87 & 0.88 & 8.81 & 8.84 & 8.89 & 8.80 & 0.02 \\
\hline $13 \mathrm{H}-2-62$ & 113.28 & 4.58 & 8.28 & 8.16 & 8.84 & 4.82 & 0.88 & 8.01 & 8.81 & 8.81 & 8.80 & 8.84 \\
\hline $13 \mathrm{H}-2-82$ & 113.47 & 1.83 & 8.82 & 8.81 & 8.80 & 0.51 & 0.80 & 8.88 & 8.88 & 8.80 & 8.81 & 0. 48 \\
\hline $13 \mathrm{H}-2-182$ & 113.67 & 5.88 & 8.85 & 8.88 & 0.01 & 5.53 & 0.88 & 0.88 & 8.08 & 8.88 & 0.80 & 0.29 \\
\hline $13 \mathrm{H}-2-122$ & 113.87 & 1.56 & 0.38 & 0.89 & 8.80 & 0.87 & 8.80 & 8.81 & 0.80 & 8.01 & 0.80 & 0.28 \\
\hline $13 \mathrm{H}-3-21$ & 114.35 & 3.38 & 8.81 & 0.81 & 8.08 & 3.16 & 8.80 & 8.88 & 8.03 & 8.82 & 8.88 & 8.87 \\
\hline $13 \mathrm{H}-3-145$ & 114.58 & 9.74 & 8.88 & 0.02 & 8.06 & 8.68 & 8.80 & 0.11 & 8.88 & 8.69 & 8.80 & 0.19 \\
\hline $13 \mathrm{H}-4-21$ & 115.82 & 18.35 & 0.14 & 8.80 & 8.80 & 9.83 & 8.80 & 8.80 & 0.16 & 0.16 & 0.80 & 0.88 \\
\hline $13 \mathrm{H}-4-42$ & 116.82 & 0.75 & 8.17 & 8.08 & 8.80 & 8.44 & 0.88 & 8.08 & 8.88 & 0.81 & 0.08 & 8.84 \\
\hline $13 \mathrm{H}-4-62$ & 116.22 & 3.87 & 8.64 & 1.13 & 8.00 & 2.01 & 8.80 & 8.81 & 0.03 & $8 . \theta 8$ & 8.88 & 0.84 \\
\hline $13 H-4-82$ & 116.41 & 3.96 & 8.89 & 0.02 & 8.08 & 3.73 & 8.88 & 8.82 & 8.85 & 0.03 & 8.80 & 0.82 \\
\hline $13 \mathrm{H}-4-102$ & 116.61 & 2.89 & 0.22 & 8.14 & 8.80 & 2.49 & 0.88 & 0.01 & 0.03 & 0.01 & 8.80 & 0.88 \\
\hline $13 \mathrm{H}-4-122$ & 116.81 & 15.82 & 8.03 & 8.80 & 8.03 & 14.53 & 0.88 & 8.89 & 0.21 & 8.89 & 8.80 & 0.85 \\
\hline $13 H-5-21$ & 117.29 & 4.28 & 0.68 & 0.13 & 8.80 & 1.93 & 8.81 & 8.80 & 0.02 & 0.25 & 0.88 & 1.18 \\
\hline $13 \mathrm{H}-5-42$ & 117.49 & 7.28 & 8.28 & 0.08 & 8.88 & 5.35 & 0.15 & 8.88 & 0.13 & 8.97 & 8.81 & 8.31 \\
\hline $13 \mathrm{H}-5-62$ & 117.69 & 6.42 & 0.23 & 0.81 & $\theta .8 \theta$ & 4.99 & 0.37 & 0.25 & 0.18 & 0.36 & 8.80 & 0.12 \\
\hline $13 \mathrm{H}-5-86$ & 117.92 & 6.61 & 8.80 & 0.81 & $8 . \theta 8$ & 6.35 & 8.80 & $\theta .08$ & 0.84 & 0.18 & 8.88 & 0.10 \\
\hline $13 H-6-21$ & 118.76 & 1.22 & 0.37 & 0.10 & $\theta . \theta \theta$ & 0.39 & 8.80 & $\theta . \theta 8$ & 0.80 & 0.08 & 0.00 & 0.36 \\
\hline $13 \mathrm{H}-6-42$ & 118.96 & 1.84 & 0.24 & 0.85 & 8.80 & 0.12 & 0.80 & 0.88 & 8.80 & 8.88 & 8.80 & 0.63 \\
\hline $13 \mathrm{H}-6-62$ & 119.16 & 0.79 & 8.33 & 0.13 & 8.80 & 8.87 & 0.08 & 8.88 & 8.88 & 0.80 & $\theta .08$ & 0.26 \\
\hline $13 H-6-82$ & 119.35 & 1.16 & 8.27 & 0.16 & 0.80 & 0.83 & 8.08 & $8 . \theta 8$ & 8.88 & 8.80 & 8.88 & 0.68 \\
\hline $13 \mathrm{H}-7-21$ & 119.56 & 1.77 & 8.86 & 8. 26 & 8.03 & 0.31 & 8.88 & 8.88 & 8.01 & 8.01 & 0.08 & 1.88 \\
\hline $13 \mathrm{H}-7-42$ & 119.77 & 13.74 & 8.80 & 8.82 & 8.88 & 12.56 & 0.80 & 8.16 & 8.89 & 8.23 & 8.09 & 0.58 \\
\hline $13 \mathrm{H}-7-62$ & 119.96 & 8.67 & 0.89 & 8.31 & $\theta . \theta 8$ & 0.01 & 0.80 & 8.80 & 8.81 & 0.88 & 8.80 & 0.24 \\
\hline $13 \mathrm{H}-7-84$ & 128.16 & 1.31 & 8.86 & 8.67 & 0.08 & 0.87 & 8.08 & 8.80 & 8.80 & 8.80 & 8.08 & 0.51 \\
\hline $13 \mathrm{H}-7-182$ & 128.35 & 8.48 & 0.15 & 0.21 & 0.80 & 0.84 & 0.80 & 8.88 & 8.80 & $\theta . \theta \theta$ & 8.80 & 0.07 \\
\hline $13 \mathrm{H}-7-122$ & 128.55 & 8.73 & 8.24 & 0.32 & 8.80 & 0.81 & 8.00 & 0.88 & 0.08 & 0.00 & 0.80 & 0.15 \\
\hline $14 \mathrm{H}-1-21$ & 128.91 & 1.62 & 8.17 & 8.86 & 8.88 & 1.19 & 0.80 & 8.88 & 0.00 & 0.01 & 8.08 & 0.19 \\
\hline $14 H-1-41$ & 121.11 & 1.31 & 8.01 & 8.80 & 8.88 & 1.22 & 8.08 & 8.88 & 0.88 & 0.02 & 8.88 & 0.06 \\
\hline $14 \mathrm{H}-1-62$ & 121.32 & 3.46 & 8.88 & 0.88 & 8.80 & 3.28 & $\theta .8 \theta$ & $\theta . \theta \theta$ & 8.82 & 0.81 & $\theta . \theta \theta$ & 0.16 \\
\hline $14 \mathrm{H}-2-82$ & 123.82 & 6.28 & 0.02 & 8.80 & 8.80 & 4.79 & 0.23 & 0.18 & 8.87 & 0.55 & 0.01 & 0.35 \\
\hline $14 \mathrm{H}-2-183$ & 123.23 & 4.25 & 0.81 & 0.02 & 8.88 & 3.52 & 0.19 & 0.16 & 8.83 & 0.26 & 8.80 & 0.86 \\
\hline $14 \mathrm{H}-2-123$ & 123.43 & 2.82 & 0.02 & 8.88 & 8.88 & 2.35 & 0.12 & 8.86 & 0.88 & 8.21 & 8.00 & 0.85 \\
\hline $14 \mathrm{H}-2-142$ & 123.62 & 1.57 & 0.18 & 8.80 & 0.80 & 1.42 & 8.80 & 8.08 & 0.02 & 8.02 & 0.00 & 0.01 \\
\hline $14 \mathrm{H}-3-21$ & 123.91 & 24.52 & 8.08 & 8.80 & 8.88 & 23.38 & 0.14 & 8.18 & 8.24 & 8.43 & 0.80 & 0.24 \\
\hline $14 \mathrm{H}-3-41$ & 124.11 & 6.98 & 0.88 & 8.88 & 0.83 & 6.63 & 8.88 & 0.84 & 8.88 & 8.84 & 0.88 & 8.14 \\
\hline $14 \mathrm{H}-3-62$ & 124.32 & 2.72 & 8.16 & 0.01 & 8.88 & 1.89 & 0.08 & 8.08 & 0.82 & 0.08 & 0.80 & B. 64 \\
\hline $14 \mathrm{H}-3-82$ & 124.52 & 3.15 & 0.02 & 0.88 & 0.01 & 2.82 & 8.80 & 0.04 & 0.03 & 0.02 & 0.08 & 8.22 \\
\hline $14 \mathrm{H}-3-183$ & 124.72 & 3.87 & 8.81 & 0.88 & 0.01 & 2.58 & 0.83 & 0.84 & 8.83 & 0.28 & $\theta .08$ & 8.10 \\
\hline $14 \mathrm{H}-3-123$ & 124.93 & 3.30 & 0.77 & 8.08 & 0.82 & 0.61 & $\theta .01$ & 8.86 & 8.08 & 0.83 & 8.80 & 1.80 \\
\hline $14 \mathrm{H}-4-21$ & 125.41 & 0.41 & 8.80 & 0.88 & 8.80 & 8.35 & 8.81 & 0.01 & 0.80 & 0.03 & 8.80 & 0.01 \\
\hline $14 \mathrm{H}-4-62$ & 125.82 & 2.22 & 0.81 & 8.80 & $\theta .08$ & 1.88 & 8.02 & 8.01 & 8.02 & 0.13 & 8.80 & 0.23 \\
\hline $14 \mathrm{H}-4-82$ & 126.82 & 8.26 & 8.14 & 0.01 & 8.80 & 0.82 & 8.80 & 8.80 & 8.08 & 8.81 & 8.88 & 8.88 \\
\hline $14 \mathrm{H}-5-21$ & 126.91 & 2.82 & 0.49 & 8.87 & 0.80 & 1.88 & 8.80 & 8.88 & 0.81 & 8.86 & 8.80 & 1.88 \\
\hline $14 H-5-41$ & 127.11 & 10.84 & 8.52 & 8.82 & 0.02 & 7.69 & 8.11 & 0.10 & 0.21 & 8.68 & 8.80 & 0.76 \\
\hline $14 \mathrm{H}-5-62$ & 127.32 & 21.53 & 8.08 & 8.08 & 8.80 & 17.59 & 0.58 & 8.39 & 8.23 & 2.52 & 0.03 & 8.19 \\
\hline $14 \mathrm{H}-5-82$ & 127.52 & 8.73 & 8.24 & 0.80 & 0.80 & 6.68 & 0.15 & 0.80 & 0.04 & 1.21 & 8.80 & 8.41 \\
\hline $14 \mathrm{H}-5-103$ & 127.72 & 1.37 & 0.58 & 0.81 & 0.80 & 8.33 & 8.81 & 8.01 & 8.88 & 8.03 & 8.80 & 8.35 \\
\hline $14 \mathrm{H}-5-123$ & 127.93 & 1.88 & 8.48 & 0.81 & 8.88 & 1.35 & 8.80 & 8.88 & 8.08 & 8.81 & 8.80 & 8.82 \\
\hline
\end{tabular}


Table 1.4 (continued).

\begin{tabular}{|c|c|c|c|c|c|c|c|c|c|c|c|c|}
\hline \multirow[b]{2}{*}{ Sample no. } & \multirow[b]{2}{*}{$\begin{array}{c}\text { Depth } \\
\text { mbsf }\end{array}$} & \multirow[b]{2}{*}{$\begin{array}{c}>63 \mu \mathrm{m} \\
(\%)\end{array}$} & \multicolumn{10}{|c|}{ Coarse fraction component analysis (wt \%) } \\
\hline & & & $\begin{array}{c}\text { Benthic } \\
\text { foram. } \\
(\%)\end{array}$ & $\begin{array}{c}\text { Plankt. } \\
\text { foram. } \\
(\%)\end{array}$ & $\begin{array}{c}\text { Biogenic } \\
\text { opal cf } \\
(\%)\end{array}$ & $\begin{array}{c}\text { Quartz } \\
(\%)\end{array}$ & $\begin{array}{c}\text { Feldspar } \\
(\%)\end{array}$ & $\begin{array}{c}\text { Mica } \\
(\%)\end{array}$ & $\begin{array}{l}\text { Hev. min. } \\
\quad(\%)\end{array}$ & $\begin{array}{c}\text { Rock fr. } \\
(\%)\end{array}$ & $\begin{array}{l}\text { Volc. gl. } \\
(\%)\end{array}$ & $\begin{array}{c}\text { Auth. } \\
(\%)\end{array}$ \\
\hline $14 \mathrm{H}-5-142$ & 128.12 & 5.85 & 0.31 & 0.80 & 8.88 & 4.57 & 8.88 & 8.03 & 8.83 & 0.84 & 8.80 & 0.88 \\
\hline $14 \mathrm{H}-\mathrm{C}-15$ & 128.35 & 4.81 & 0.16 & 8.80 & 8.88 & 3.68 & 8.85 & 8.81 & 8.03 & 8.47 & 8.80 & 0.41 \\
\hline $15 \mathrm{H}-1-22$ & 138.42 & 3.35 & 0.11 & 8.82 & 0.88 & 2.92 & 8.88 & 8.01 & 8.88 & 0.86 & 8.14 & 8.89 \\
\hline $15 \mathrm{H}-1-42$ & 138.62 & 8.93 & 0.15 & 8.18 & 8.88 & 0.21 & 8.80 & 8.88 & 0.88 & 8.88 & 8.88 & 0.45 \\
\hline $15 H-1-62$ & 130.82 & 6.57 & 0.11 & 8.80 & 8.88 & 5.13 & 8.85 & 0.88 & 8.89 & 8.58 & 8.00 & 0.61 \\
\hline $15 \mathrm{H}-1-82$ & 131.02 & 6.39 & 0.07 & 8.81 & 8.01 & 5.44 & 8.82 & 0.82 & 0.08 & 8.56 & 0.80 & 0.16 \\
\hline $15 \mathrm{H}-1-182$ & 131.22 & 6.96 & 8.45 & 8.85 & 8.26 & 3.67 & 8.85 & 0.86 & 8.84 & 0.19 & 0.01 & 2.12 \\
\hline $15 \mathrm{H}-1-122$ & 131.42 & 1.96 & 0.21 & 0.03 & 8.83 & 8.68 & 0.82 & 0.80 & 8.88 & 8.03 & 0.80 & 0.95 \\
\hline $15 H-1-142$ & 131.62 & 1.65 & 8.00 & 0.82 & 8.81 & 1.17 & 8.83 & 8.88 & 8.81 & 8.88 & 8.88 & 0.33 \\
\hline $15 \mathrm{H}-2-22$ & 131.92 & 1.28 & 0.81 & 0.81 & 8.80 & 8.78 & 8.01 & 0.88 & 8.80 & 0.85 & 8.80 & 8.33 \\
\hline $15 \mathrm{H}-2-42$ & 132.12 & 4.86 & 8.88 & 0.01 & 8.88 & 3.52 & 8.18 & 0.01 & 0.81 & 0.23 & 0.88 & 8.19 \\
\hline $15 \mathrm{H}-2-62$ & 132.32 & 4.83 & 8.86 & 8.80 & 0.01 & 2.83 & 0.82 & 0.82 & $\theta . \theta 0$ & 0.89 & 0.14 & 1.66 \\
\hline $15 \mathrm{H}-2-82$ & 132.52 & 5.65 & 8.80 & 0.08 & 8.88 & 2.65 & 8.89 & 8.82 & 0.82 & 8.86 & 0.11 & 2.71 \\
\hline $15 \mathrm{H}-2-102$ & 132.72 & 3.59 & 8.80 & 8.80 & 0.80 & 2.87 & 8.86 & 0.82 & 0.83 & 0.18 & 0.02 & 0.42 \\
\hline $15 H-2-122$ & 132.92 & 9.75 & 0.08 & 0.02 & 8.80 & 7.31 & 0.08 & 0.85 & 8.84 & 8.88 & 0.88 & 2.33 \\
\hline $15 t-2-142$ & 133.12 & 1.17 & 0.80 & 0.88 & 8.81 & 8.59 & 0.88 & 0.88 & 0.08 & 8.88 & 8.80 & 0.56 \\
\hline $15 H-3-42$ & 133.42 & 1.34 & 0.18 & 0.69 & 0.88 & 0.83 & 0.80 & 0.88 & 0.88 & 8.88 & 8.88 & 0.42 \\
\hline $15 \mathrm{H}-3-62$ & 133.62 & 1.32 & 0.42 & 8.54 & 8.01 & 8.83 & $\theta .0 \theta$ & 0.88 & 8.80 & 8.81 & 0.88 & 0.38 \\
\hline $15 \mathrm{H}-3-82$ & 133.82 & 1.35 & 0.38 & 0.28 & 0.88 & 8.84 & 0.88 & 8.88 & 8.88 & 8.88 & 0.26 & 0.41 \\
\hline $15 \mathrm{H}-3-102$ & 134.82 & 0.99 & 0.18 & 0.32 & 8.01 & 8.04 & 0.80 & 0.80 & 0.80 & $8 . \theta 0$ & 0.01 & 0.49 \\
\hline $15 \mathrm{H}-3-122$ & 134.22 & 1.56 & 0.87 & 8.89 & 8.81 & 1.12 & 0.80 & 8.80 & 8.80 & 0.03 & 8.80 & 0.24 \\
\hline $15 \mathrm{H}-3-142$ & 134.42 & 1.74 & 8.01 & 0.86 & 8.82 & 0.52 & 0.81 & 8.80 & 0.82 & 8.83 & 8.88 & 1.86 \\
\hline $15 \mathrm{H}-4-22$ & 134.92 & 1.37 & 0.07 & 0.01 & 8.14 & 0.43 & 8.88 & 0.81 & 0.81 & 8.80 & $\theta . \theta 0$ & 0.78 \\
\hline $15 \mathrm{H}-4-42$ & 135.12 & 1.88 & 8.80 & 0.80 & 8.80 & 8.25 & 8.68 & 8.80 & 8.80 & 0.02 & 0.88 & 1.48 \\
\hline $15 \mathrm{H}-4-62$ & 135.32 & 1.72 & 0.84 & 0.81 & 8.80 & 1.22 & 0.82 & 8.88 & 0.81 & 8.11 & 0.81 & 0.28 \\
\hline $15 \mathrm{H}-4-82$ & 135.52 & 8.71 & 0.82 & 8.88 & 0.81 & 8.34 & 8.01 & 8.88 & 8.80 & 0.05 & 0.01 & 0.26 \\
\hline $15 \mathrm{H}-4-102$ & 135.72 & 1.07 & 8.81 & 8.81 & $\theta . \theta 8$ & 8.76 & 8.81 & 8.88 & 8.01 & 0.08 & 8.80 & 0.28 \\
\hline $15 H-4-122$ & 135.92 & 0.52 & 0.01 & 0.08 & 8.81 & 8.85 & 0.80 & 8.08 & 0.00 & 0.80 & 0.01 & 0.37 \\
\hline $15 \mathrm{H}-5-22$ & 136.42 & 1.08 & 0.11 & 8.87 & 0.81 & 0.86 & 0.88 & 0.88 & 0.80 & 8.80 & 8.80 & 8.75 \\
\hline $15 \mathrm{H}-5-62$ & 136.62 & 6.82 & 8.58 & 1.69 & 0.86 & 8.18 & 8.88 & 8.88 & 0.88 & 8.80 & 8.80 & 4.27 \\
\hline $15 \mathrm{H}-5-82$ & 136.82 & 8.99 & 0.11 & 0.19 & 0.01 & 0.82 & 8.80 & 8.80 & 0.88 & 8.80 & 8.80 & 8.65 \\
\hline $15 \mathrm{H}-5-182$ & 137.82 & 8.73 & 0.89 & 8.84 & 0.81 & 0.02 & 8.80 & 8.80 & 0.80 & 0.80 & 8.80 & 8.57 \\
\hline $15 H-5-122$ & 137.22 & 0.75 & 8.10 & 8.82 & 8.82 & 8.82 & 8.80 & $\theta .80$ & 0.88 & 0.80 & 8.08 & 8.68 \\
\hline $15 \mathrm{H}-5-142$ & 137.42 & 1.62 & 8.86 & 8.02 & 8.03 & 0.15 & 0.01 & 8.80 & 0.88 & 0.80 & 0.80 & 1.35 \\
\hline $15 \mathrm{H}-6-22$ & 137.92 & 1.53 & 8.16 & 0.11 & 8.81 & 0.39 & 0.82 & 8.80 & 0.02 & 8.84 & 0.25 & 0.53 \\
\hline $15 H-6-42$ & 138.12 & 0.61 & 0.84 & 0.03 & 8.81 & 0.12 & 8.01 & 0.80 & 8.08 & $\theta .01$ & 0.80 & 0.39 \\
\hline $15 \mathrm{H}-6-62$ & 138.32 & 7.44 & 8.11 & 0.03 & 8.68 & 5.91 & 0.21 & 8.88 & 8.87 & 8.74 & 0.80 & 0.34 \\
\hline $15 \mathrm{H}-6-82$ & 138.52 & 1.71 & 8.03 & 8.81 & 0.80 & 1.26 & 8.03 & 8.88 & 8.83 & 0.11 & 0.81 & 0.23 \\
\hline $15 \mathrm{H}-7-22$ & 139.42 & 2.11 & 8.84 & 0.81 & 8.80 & 1.14 & 0.82 & 0.82 & 0.84 & 0.11 & 0.82 & 0.78 \\
\hline $15 \mathrm{H}-7-42$ & 139.62 & 0.88 & 8.88 & 0.08 & 0.80 & 0.65 & 8.81 & 8.80 & 8.81 & 8.87 & 0.08 & 8.15 \\
\hline $15 \mathrm{H}-7-62$ & 139.82 & 7.22 & 8.88 & 0.80 & 8.01 & 6.53 & 0.18 & 8.01 & 8.18 & 0.33 & 0.80 & 0.15 \\
\hline $16 \mathrm{H}-1-23$ & 139.93 & 8.73 & 0.11 & 0.88 & 8.88 & 7.76 & 8.06 & 0.03 & 8.88 & 0.34 & 8.80 & 0.34 \\
\hline $16 \mathrm{H}-1-102$ & 148.72 & 1.18 & 8.13 & 0.53 & 8.80 & 0.82 & 8.88 & 0.81 & 0.88 & 0.02 & 0.80 & 0.14 \\
\hline $16 \mathrm{H}-1-128$ & 148.98 & 1.11 & 0.19 & 8.71 & 8.88 & 0.80 & 8.88 & 8.88 & 8.88 & 8.88 & 0.88 & 0.11 \\
\hline $16 \mathrm{H}-1-142$ & 141.12 & 1.41 & 0.19 & 0.80 & 8.82 & 8.80 & 0.88 & 0.00 & 8.08 & 0.88 & 8.88 & 8.27 \\
\hline $16 \mathrm{H}-2-23$ & 141.43 & 9.88 & 0.88 & 0.87 & 8.88 & 6.98 & 8.11 & 0.58 & 0.82 & 0.11 & 0.88 & 2.17 \\
\hline $16 \mathrm{H}-2-182$ & 142.82 & 0.78 & 8.88 & 8.48 & 8.88 & 0.81 & 8.08 & 0.88 & 8.00 & 8.80 & 0.88 & 0.28 \\
\hline $16 \mathrm{H}-2-128$ & 142.28 & 2.15 & 0.04 & 8.86 & 8.80 & 1.38 & 8.84 & 0.02 & 0.80 & 0.17 & 0.08 & 0.44 \\
\hline $16 \mathrm{H}-2-142$ & 142.42 & 6.39 & 8.13 & 8.84 & 8.80 & 5.34 & 0.84 & 8.25 & 8.87 & 0.87 & 0.80 & 0.46 \\
\hline $16 \mathrm{H}-3-23$ & 142.93 & 3.37 & 8.84 & 8.14 & 8.81 & 1.79 & 8.82 & 8.81 & 8.84 & 8.34 & 0.87 & 0.92 \\
\hline
\end{tabular}


Table 1.4 (continued).

\begin{tabular}{|c|c|c|c|c|c|c|c|c|c|c|c|c|}
\hline \multirow[b]{2}{*}{ Sample no. } & \multirow[b]{2}{*}{$\begin{array}{c}\text { Depth } \\
\text { mbsf }\end{array}$} & \multirow[b]{2}{*}{$\begin{array}{c}>63 \mu \mathrm{m} \\
(\%)\end{array}$} & \multicolumn{10}{|c|}{ Coarse fraction component analysis (wt \%) } \\
\hline & & & $\begin{array}{l}\text { Benthic } \\
\text { foram. } \\
(\%)\end{array}$ & $\begin{array}{l}\text { Plankt. } \\
\text { foram. } \\
(\%)\end{array}$ & $\begin{array}{c}\text { Biogenic } \\
\text { opal cf } \\
(\%)\end{array}$ & $\begin{array}{c}\text { Quartz } \\
(\%)\end{array}$ & $\begin{array}{c}\text { Feldspar } \\
(\%)\end{array}$ & $\begin{array}{l}\text { Mica } \\
(\%)\end{array}$ & $\begin{array}{l}\text { Hev. min. } \\
(\%)\end{array}$ & $\begin{array}{l}\text { Rock fr. } \\
(\%)\end{array}$ & $\begin{array}{l}\text { Volc. gl. } \\
(\%)\end{array}$ & $\begin{array}{l}\text { Auth. } \\
(\%)\end{array}$ \\
\hline $16 \mathrm{H}-3-182$ & 143.72 & 1.11 & 8.16 & 0.15 & 8.82 & 8.32 & 8.88 & 8.81 & 8.81 & 8.86 & 8.02 & 8.34 \\
\hline $16 \mathrm{H}-3-128$ & 143.98 & 1.36 & 0.14 & 8.11 & 0.58 & 0.81 & 8.80 & 8.81 & 8.88 & 8.82 & 0.81 & 8.46 \\
\hline $16 \mathrm{H}-3-142$ & 144.12 & 0.84 & 0.10 & 0.15 & 0.19 & 0.82 & 8.80 & 8.80 & 0.80 & 8.02 & 8.08 & 0.35 \\
\hline $16 \mathrm{H}-4-23$ & 144.43 & 1.15 & 0.26 & 0.84 & 8.17 & 8.83 & 8.88 & $8 . \theta 8$ & 8.80 & 0.81 & 8.80 & 0.63 \\
\hline $16 \mathrm{H}-4-182$ & 145.82 & 1.45 & 0.05 & 0.71 & 8.84 & 0.81 & 8.80 & 8.08 & 8.80 & 0.81 & $\theta .80$ & 0.61 \\
\hline $16 \mathrm{H}-4-128$ & 145.28 & 1.48 & 8.89 & 0.59 & 8.03 & 0.31 & 8.81 & 8.80 & 0.80 & 8.83 & 8.80 & 8.39 \\
\hline $16 \mathrm{H}-5-23$ & 145.93 & 0.82 & 8.81 & 0.21 & 8.82 & 0.36 & 8.01 & 8.80 & 8.08 & 8.84 & 8.88 & 8.16 \\
\hline $16 \mathrm{H}-5-182$ & 146.72 & 8.75 & 8.28 & 0.14 & 0.03 & 0.84 & 8.88 & 8.88 & 0.88 & 0.81 & 8.88 & 8. 32 \\
\hline $16 \mathrm{H}-5-128$ & 146.92 & 1.29 & 8.46 & 0.38 & 8.88 & 0.85 & 8.80 & 8.88 & 8.88 & 0.86 & 8.80 & 8.43 \\
\hline $16 \mathrm{H}-5-142$ & 147.12 & 8.85 & 8.12 & 8.48 & 8.82 & 8.81 & 8.88 & 8.80 & 0.88 & 0.01 & 0.08 & 8.28 \\
\hline $16 \mathrm{H}-6-23$ & 147.43 & 4.68 & 8.88 & 8.84 & $\theta .88$ & 3.72 & 0.89 & 8.85 & 8.82 & 8.32 & $\theta .8 \theta$ & 8.36 \\
\hline $17 \mathrm{H}-1-23$ & 149.42 & 1.39 & 8.88 & 0.01 & 8.84 & 0.64 & 8.83 & 8.81 & 0.81 & 8.87 & 8.81 & 8.57 \\
\hline $17 \mathrm{H}-1-42$ & 149.62 & 18.78 & 0.88 & 8.88 & 8.95 & 3.88 & $8.8 \theta$ & 8.89 & 0.85 & 8.79 & 0.18 & 4.75 \\
\hline $17 \mathrm{H}-1-122$ & 158.42 & 1.24 & 8.88 & 8.80 & 8.82 & 8.47 & 8.81 & 8.68 & 0.01 & 8.85 & $\theta . \theta 8$ & 8.68 \\
\hline $17 H-1-142$ & 150.62 & 3.85 & 0.80 & 0.80 & 0.82 & 2.74 & 0.80 & 8.23 & 0.82 & 8.18 & 0.19 & 8.47 \\
\hline $17 \mathrm{H}-2-22$ & 158.92 & 2.05 & 8.47 & 0.77 & 8.80 & 0.36 & $\theta .01$ & 8.80 & 8.81 & 8.18 & 0.85 & 8.28 \\
\hline $17 \mathrm{H}-2-42$ & 151.12 & 8.97 & 8.18 & 0.68 & 8.13 & 8.81 & 8.88 & 8.81 & 8.80 & 8.88 & 0.82 & 8.89 \\
\hline $17 \mathrm{H}-2-62$ & 151.22 & 4.74 & 0.83 & 8.45 & 8.45 & 0.88 & 8.88 & 8.88 & 8.88 & 8.80 & 3.78 & 8.18 \\
\hline $17 \mathrm{H}-3-22$ & 152.42 & 8.82 & 0.03 & 0.85 & 8.17 & 0.03 & 0.80 & $\theta .08$ & 0.08 & 8.80 & 8.87 & 8.47 \\
\hline $17 H-3-42$ & 152.62 & 1.38 & 0.33 & 8.85 & 8.84 & 0.23 & 8.80 & 8.88 & 0.80 & 8.82 & 0.04 & 0.58 \\
\hline $17 \mathrm{H}-3-62$ & 152.82 & 2.16 & 8.06 & 0.83 & 0.84 & 1.89 & 0.02 & 8.80 & 0.02 & 0.15 & 8.16 & 0.58 \\
\hline $17 \mathrm{H}-4-22$ & 153.92 & 8.89 & 8.82 & 0.80 & 0.28 & 5.02 & 0.11 & 8.88 & 8.03 & 0.83 & 8.83 & 2.63 \\
\hline $17 \mathrm{H}-4-42$ & 154.12 & 2.59 & 8.02 & 0.03 & 8.08 & 1.67 & 8.84 & 8.01 & 8.82 & 8.33 & 8.86 & 8.41 \\
\hline $17 \mathrm{H}-4-182$ & 154.32 & 1.09 & 8.88 & 8.28 & 0.83 & 8.88 & $\theta .08$ & 8.88 & 0.80 & 0.80 & 8.84 & 8.59 \\
\hline $17 H-4-122$ & 154.52 & 2.39 & 0.37 & 0.18 & 8.23 & 1.11 & 8.88 & 8.03 & 0.82 & 8.08 & 0.87 & 8.38 \\
\hline $17 \mathrm{H}-4-142$ & 154.72 & 1.12 & 8.84 & 0.32 & 8.81 & 0.15 & 8.80 & 8.80 & 8.81 & 0.03 & 8.80 & 8.57 \\
\hline $17 \mathrm{H}-5-22$ & 155.42 & 1.65 & 0.05 & 8.25 & 8.80 & 8.49 & 0.01 & 8.08 & 8.01 & 8.84 & 0.03 & 0.77 \\
\hline $17 H-5-122$ & 156.42 & 1.44 & 8.21 & 8.17 & 8.45 & 0.83 & 8.88 & 8.81 & 8.88 & 8.88 & 8.61 & 8.54 \\
\hline $18 \mathrm{H}-1-42$ & 159.12 & 1.46 & 8.01 & 0.88 & 8.85 & 8.97 & 8.82 & $\theta .01$ & 8.88 & 0.17 & 8.80 & 8.22 \\
\hline $18 \mathrm{H}-1-58$ & 159.30 & 2.61 & 8.08 & $\theta .8 \theta$ & 0.88 & 2.82 & 0.80 & 8.08 & 0.80 & 0.19 & 8.08 & 0.39 \\
\hline $18 \mathrm{H}-1-182$ & 159.52 & 1.35 & 0.06 & 8.83 & 8.17 & 0.18 & $\theta .80$ & 8.81 & 0.80 & 0.81 & 8.80 & 8.96 \\
\hline $18 \mathrm{H}-1-122$ & 159.72 & 8.99 & 0.31 & 0.21 & 0.88 & 8.03 & 8.88 & 8.81 & 0.08 & 0.07 & 8.80 & 8.28 \\
\hline $18 H-1-142$ & 159.92 & 8.98 & 8.32 & 8.86 & 8.84 & 8.88 & 8.08 & 8.80 & 8.80 & 8.81 & 0.08 & 8.38 \\
\hline $18 \mathrm{H}-2-42$ & 160.62 & 1.31 & 8.80 & 8.80 & 8.02 & 0.74 & 0.02 & 8.88 & $\theta .80$ & 0.11 & 0.88 & 8.41 \\
\hline $18 \mathrm{H}-2-58$ & 168.88 & 15.19 & 8.88 & 8.88 & 8.05 & 9.68 & 8.80 & 8.32 & 8.80 & 5.14 & 8.88 & 8.80 \\
\hline $18 \mathrm{H}-2-102$ & 161.28 & 1.48 & 8.01 & 0.80 & 8.80 & 1.83 & 8.84 & 8.83 & 0.03 & 8.18 & 8.88 & 0.08 \\
\hline $18 \mathrm{H}-2-122$ & 161.42 & 3.75 & 8.89 & 0.80 & 0.88 & 2.63 & $8 . \theta 0$ & 8.21 & 0.02 & 8.68 & 8.88 & 8.21 \\
\hline $18 \mathrm{H}-2-142$ & 161.62 & 1.93 & 8.82 & $\theta . \theta \theta$ & 0.80 & 1.32 & 8.88 & 0.02 & 8.85 & 0.19 & 8.88 & 8.25 \\
\hline $18 \mathrm{H}-3-42$ & 162.12 & 0.42 & 0.83 & 0.84 & 8.88 & 8.12 & 8.88 & 0.88 & 0.80 & 0.01 & 8.80 & 0.21 \\
\hline $18 \mathrm{H}-3-58$ & 162.38 & 14.05 & 8.88 & $\theta .8 \theta$ & 8.88 & 11.49 & 8.58 & 8.10 & 8.88 & 1.57 & 8.88 & 0.31 \\
\hline $18 \mathrm{H}-3-182$ & 162.52 & 18.57 & 1.84 & 8.87 & 8.88 & 7.32 & 8.29 & 0.11 & 0.89 & 1.17 & 8.88 & 0.48 \\
\hline $18 \mathrm{H}-3-122$ & 162.72 & 0.45 & 0.15 & 8.08 & 8.88 & 0.88 & 8.80 & 0.81 & 0.88 & 0.83 & 8.08 & 8.89 \\
\hline $18 \mathrm{H}-3-142$ & 162.92 & 3.73 & 8.81 & 8.88 & 8.88 & 2.94 & 8.86 & 8.03 & 0.85 & 8.39 & 0.80 & 0.25 \\
\hline $18 \mathrm{H}-4-42$ & 163.62 & 2.34 & 0.41 & 0.03 & 8.08 & 0.68 & 8.82 & 8.80 & 8.88 & 0.11 & 8.84 & 1.84 \\
\hline $18 \mathrm{H}-4-58$ & 163.88 & 3.68 & 8.33 & 0.08 & 8.88 & 0.79 & 8.80 & 8.80 & 8.81 & 0.21 & 0.88 & 2.17 \\
\hline $18 \mathrm{H}-4-182$ & 164.28 & 8.46 & 0.80 & 8.00 & 8.88 & 0.83 & 8.80 & 8.88 & 8.80 & 0.01 & 8.08 & 0.41 \\
\hline $18 \mathrm{H}-5-42$ & 165.12 & 8.58 & 0.03 & 0.22 & 8.01 & 0.02 & 8.88 & 8.80 & 8.00 & 8.08 & 8.88 & 0.22 \\
\hline $18 \mathrm{H}-5-58$ & 165.38 & 1.61 & 0.88 & 0.52 & 8.87 & 8.84 & 8.88 & 8.85 & 8.88 & 0.83 & 8.88 & 8.82 \\
\hline $18 \mathrm{H}-5-182$ & 165.52 & 8.86 & 8.82 & 8.53 & 8.02 & 0.82 & 8.88 & 8.80 & 8.80 & 0.02 & 0.83 & 8.23 \\
\hline $18 \mathrm{H}-5-122$ & 165.72 & 1.89 & 8.21 & 0.28 & 8.88 & 8.03 & 8.88 & 8.88 & 8.88 & 8.01 & 0.01 & 0.42 \\
\hline
\end{tabular}


Table 1.4 (continued).

Coarse fraction component analysis (wt \%)

\begin{tabular}{|c|c|c|c|c|c|c|c|c|c|c|c|c|}
\hline \multirow[b]{2}{*}{ Sample no. } & \multirow[b]{2}{*}{$\begin{array}{c}\text { Depth } \\
\text { mbsf }\end{array}$} & \multirow[b]{2}{*}{$\begin{array}{c}>63 \mu \mathrm{m} \\
(\%)\end{array}$} & \multicolumn{10}{|c|}{ Coarse fraction component analysis (wt \%) } \\
\hline & & & $\begin{array}{c}\text { Benthic } \\
\text { foram. } \\
(\%)\end{array}$ & $\begin{array}{l}\text { Plankt. } \\
\text { foram. } \\
(\%)\end{array}$ & $\begin{array}{l}\text { Biogenic } \\
\text { opal cf } \\
(\%)\end{array}$ & $\begin{array}{c}\text { Quartz } \\
(\%)\end{array}$ & $\begin{array}{c}\text { Feldspar } \\
(\%)\end{array}$ & $\begin{array}{l}\text { Mica } \\
(\%)\end{array}$ & $\begin{array}{l}\text { Hev. min. } \\
(\%)\end{array}$ & $\begin{array}{c}\text { Rock fr. } \\
(\%)\end{array}$ & $\begin{array}{l}\text { Volc. gl. } \\
(\%)\end{array}$ & $\begin{array}{c}\text { Auth. } \\
(\%)\end{array}$ \\
\hline $18 \mathrm{H}-5-142$ & 165.92 & 8.64 & 8.88 & 8.32 & 0.01 & 8.81 & 8.80 & 8.80 & 8.88 & 8.81 & 8.80 & 8.28 \\
\hline $18 \mathrm{H}-6-42$ & 166.62 & 0.48 & 0.02 & 8.01 & 0.01 & 8.02 & 8.80 & 0.80 & 0.80 & 8.88 & 0.88 & 0.33 \\
\hline $18 \mathrm{H}-6-58$ & 166.88 & 1.72 & 8.02 & 8.81 & 8.82 & 0.89 & 8.80 & 8.88 & 0.88 & 0.03 & 8.80 & 1.55 \\
\hline $18 \mathrm{H}-7-42$ & 168.12 & 13.95 & 8.81 & 8.22 & 8.88 & 9.88 & 8.88 & 8.86 & 8.03 & 2.27 & 0.80 & 1.48 \\
\hline $18 \mathrm{H}-7-58$ & 168.38 & 8.36 & 8.88 & 8.80 & 0.80 & 0.33 & 0.80 & 0.88 & 0.88 & 8.83 & 8.88 & 8.88 \\
\hline $19 \mathrm{H}-2-22$ & 169.92 & 1.81 & 8.52 & 0.37 & 0.03 & 0.15 & 0.08 & 8.88 & 0.08 & 0.01 & 8.08 & 8.73 \\
\hline $19 \mathrm{H}-2-82$ & 178.52 & 18.41 & 8.80 & 0.80 & 0.46 & 9.65 & 0.80 & 8.18 & 0.88 & 8.84 & 0.88 & 8.16 \\
\hline $19 H-2-180$ & 178.78 & 1.51 & 0.81 & 0.80 & 0.80 & 1.15 & 8.88 & 8.81 & 8.01 & 0.03 & $8 . \theta \theta$ & 0.31 \\
\hline $19 \mathrm{H}-3-22$ & 171.42 & 1.35 & 0.87 & 8.80 & 0.80 & 0.26 & 0.08 & 8.81 & 0.01 & $\theta .08$ & 8.80 & 1.80 \\
\hline $19 \mathrm{H}-3-82$ & 172.02 & 2.29 & 8.28 & 8.81 & 0.88 & 0.81 & 0.08 & 8.88 & 0.01 & 0.01 & 0.00 & 1.16 \\
\hline $19 \mathrm{H}-3-98$ & 172.22 & 1.50 & 8.26 & 8.18 & 8.25 & 8.87 & 8.08 & 8.01 & 0.88 & 8.01 & 8.88 & 8.71 \\
\hline $19 H-3-128$ & 172.48 & 8.89 & 8.82 & 0.17 & 0.01 & 0.83 & 8.08 & 8.88 & 8.80 & 0.01 & 0.25 & 0.39 \\
\hline $19 H-3-142$ & 172.62 & 0.86 & 0.12 & 8.15 & 8.14 & 0.84 & 8.88 & $\theta . \theta 0$ & 0.08 & 0.00 & 0.80 & 8.48 \\
\hline $19 \mathrm{H}-4-22$ & 172.92 & 1.37 & 8.89 & 0.83 & 8.84 & 0.72 & 8.88 & 8.88 & 8.88 & 8.88 & 0.80 & 0.49 \\
\hline $19 \mathrm{H}-4-82$ & 173.52 & 8.78 & 0.85 & 8.16 & 8.84 & 8.84 & 8.80 & 8.80 & 0.80 & 8.08 & 0.88 & 8.48 \\
\hline $19 \mathrm{H}-4-188$ & 173.78 & 2.61 & 0.13 & 0.85 & 8.18 & 0.22 & 0.88 & 0.81 & 0.80 & 0.88 & 0.97 & 1.14 \\
\hline $28 \mathrm{H}-1-42$ & 178.12 & 2.88 & 8.80 & 8.80 & 8.03 & 2.42 & 0.81 & 8.87 & 8.82 & 8.86 & 0.87 & 8.13 \\
\hline $28 \mathrm{H}-1-82$ & 178.28 & 9.48 & 8.25 & 8.88 & 0.19 & 8.84 & 8.80 & 8.89 & 8.80 & 8.84 & 0.80 & B. 88 \\
\hline $28 \mathrm{H}-1-102$ & 178.42 & 8.82 & 0.17 & 0.25 & 0.01 & 0.89 & 0.88 & 8.88 & 0.80 & 8.80 & 8.88 & 8.26 \\
\hline $28 \mathrm{H}-1-122$ & 178.62 & 8.48 & 8.87 & 8.15 & 0.01 & 0.82 & 8.80 & 8.88 & 0.80 & 8.80 & 0.88 & 0.14 \\
\hline $28 \mathrm{H}-1-142$ & 178.82 & 0.52 & 0.18 & 0.22 & $\theta .0 \theta$ & 0.82 & 8.80 & 8.80 & 8.68 & 8.80 & 8.88 & 0.18 \\
\hline $2 \mathrm{OH}^{-2}-42$ & 179.68 & 7.78 & 8.88 & 8.80 & 0.83 & 6.95 & 0.81 & 8.16 & 0.80 & 8.21 & 0.07 & 8.34 \\
\hline $28 \mathrm{H}-2-82$ & 179.88 & 1.91 & 0.61 & 8.22 & 8.04 & 0.99 & 8.00 & 0.80 & 0.88 & 0.80 & 8.08 & 0.06 \\
\hline $2 \theta \mathrm{H}-2-102$ & 188.08 & 1.82 & 8.67 & 8.29 & 0.80 & 8.29 & 0.80 & 8.81 & 8.08 & 0.01 & 0.80 & 8.54 \\
\hline $28 \mathrm{H}-2-122$ & 188.28 & 1.73 & 8.68 & 8.78 & 8.81 & 8.36 & 0.08 & 8.88 & 0.80 & 8.88 & 8.80 & 8.87 \\
\hline $28 \mathrm{H}-2-142$ & 188.48 & 1.99 & 8.95 & 8.46 & 0.19 & 0.01 & $\theta .08$ & 0.81 & $\theta .01$ & 8.80 & 8.80 & 8.37 \\
\hline $28 \mathrm{H}-3-42$ & 181.10 & 8.31 & $8 . \theta 8$ & 0.81 & 0.03 & 0.11 & 8.88 & 8.80 & 8.88 & 0.80 & 8.81 & 0.15 \\
\hline $28 \mathrm{H}-3-82$ & 181.38 & 8.65 & 0.28 & 0.18 & 0.80 & 0.89 & 8.88 & 8.80 & 8.80 & 8.01 & 8.80 & 0.24 \\
\hline $28 \mathrm{H}-3-182$ & 181.58 & 1.52 & 8.66 & 0.21 & 8.88 & 0.18 & 8.88 & 8.88 & 8.80 & 0.01 & 8.80 & 8.53 \\
\hline $28 \mathrm{H}-3-122$ & 181.78 & 8.45 & 0.27 & 8.02 & 8.08 & 8.82 & $\theta . \theta 8$ & 8.88 & 8.80 & 0.01 & 8.80 & 0.13 \\
\hline $28 \mathrm{H}-4-42$ & 182.68 & 1.76 & 0.13 & 0.08 & 0.80 & 0.68 & 8.81 & 8.88 & 0.02 & 0.83 & 0.13 & 0.67 \\
\hline $28 \mathrm{H}-4-82$ & 182.88 & 1.29 & 8.84 & 8.88 & 8.26 & 8.18 & 8.80 & 8.88 & 8.81 & 0.18 & 0.01 & 0.76 \\
\hline $28 \mathrm{H}-4-122$ & 183.28 & 0.38 & 0.01 & 0.80 & 8.86 & 8.85 & 0.88 & 8.81 & 8.88 & 8.88 & 8.80 & 8.26 \\
\hline $28 \mathrm{H}-5-42$ & 184.18 & 1.41 & 0.15 & 0.81 & 8.81 & 0.97 & 8.80 & 8.81 & 8.01 & 0.02 & 0.02 & 8.22 \\
\hline $28 \mathrm{H}-5-82$ & 184.38 & 0.68 & 0.83 & 0.81 & 8.84 & 0.19 & 8.88 & 8.88 & 8.88 & 8.08 & 0.88 & 0.41 \\
\hline $28 \mathrm{H}-5-102$ & $184.4 \theta$ & 8.65 & 0.80 & 8.88 & 8.88 & 8.32 & 8.88 & 8.80 & 8.80 & 8.80 & 8.80 & 0.32 \\
\hline $21 \mathrm{H}-1-22$ & 185.81 & 2.08 & 0.09 & 8.89 & 8.48 & 8.82 & 8.80 & 8.88 & 8.88 & 8.80 & 8.08 & 0.68 \\
\hline $21 \mathrm{H}-1-42$ & 185.28 & 1.32 & 8.80 & 8.82 & 0.68 & 0.83 & 8.80 & 8.88 & 8.88 & 0.88 & 8.81 & 0.59 \\
\hline $21 \mathrm{H}-1-62$ & 185.39 & 1.30 & 0.80 & 0.88 & 0.24 & 0.02 & 0.80 & 8.88 & 8.80 & 0.81 & 8.80 & 1.02 \\
\hline $21 \mathrm{H}-1-82$ & 185.58 & 0.55 & 0.80 & 0.80 & 0.30 & 0.02 & 0.08 & 8.81 & 8.80 & 0.01 & 0.18 & 8.82 \\
\hline $21 \mathrm{H}-1-182$ & 185.77 & 1.21 & 0.80 & 8.88 & 8.38 & 0.82 & 0.80 & 8.88 & 8.80 & 0.01 & 0.01 & 8.87 \\
\hline $21 \mathrm{H}-1-122$ & 185.96 & 1.58 & 8.08 & 8.88 & 0.93 & 8.03 & 0.88 & 8.82 & 0.02 & 0.06 & 0.48 & 0.84 \\
\hline $21 \mathrm{H}-1-142$ & 186.15 & 2.14 & 0.82 & 8.82 & 1.18 & 0.05 & 0.88 & 8.88 & 8.80 & 0.81 & 0.88 & 0.94 \\
\hline $22 \mathrm{H}-1-22$ & 187.01 & 3.44 & 8.11 & 8.81 & 0.12 & 2.45 & 8.01 & 8.88 & 8.88 & 0.00 & 0.00 & 0.73 \\
\hline $22 \mathrm{H}-1-182$ & 187.77 & 8.88 & 0.24 & 0.12 & 8.84 & 0.28 & 0.88 & 8.88 & 8.88 & 8.80 & 0.80 & 0.28 \\
\hline $22 \mathrm{H}-1-122$ & 187.96 & 0.65 & 0.02 & 8.80 & 8.86 & 0.11 & 0.80 & 8.88 & 8.08 & 8.80 & $\theta . \theta 8$ & 8.46 \\
\hline $22 \mathrm{H}-2-42$ & 188.62 & 0.86 & 0.82 & 0.80 & 0.80 & 8.03 & 0.88 & 8.88 & 0.88 & 0.88 & 0.80 & 0.81 \\
\hline $22 \mathrm{H}-2-62$ & 188.81 & 8.95 & 0.36 & 0.80 & 0.80 & 8.10 & 8.88 & 8.88 & 0.01 & 0.88 & 8.80 & 0.47 \\
\hline $22 \mathrm{H}-2-82$ & 189.08 & 1.41 & 0.81 & 8.88 & 0.01 & 0.37 & 8.88 & 8.86 & 0.88 & 8.88 & 8.80 & 8. 96 \\
\hline $22 \mathrm{H}-2-102$ & 189.19 & 6.03 & 8.18 & 8.80 & 0.82 & 5.59 & 8.80 & 8.81 & 8.80 & 8.81 & 0.80 & 0.29 \\
\hline
\end{tabular}


Table 1.4 (continued).

\begin{tabular}{|c|c|c|c|c|c|c|c|c|c|c|c|c|}
\hline \multirow[b]{2}{*}{ Sample no. } & \multirow[b]{2}{*}{$\begin{array}{c}\text { Depth } \\
\text { mbsf }\end{array}$} & \multirow[b]{2}{*}{$\begin{array}{c}>63 \mu \mathrm{m} \\
(\%)\end{array}$} & \multicolumn{10}{|c|}{ Coarse fraction component analysis (wt $\%)$} \\
\hline & & & $\begin{array}{c}\text { Benthic } \\
\text { foram. } \\
(\%)\end{array}$ & $\begin{array}{c}\text { Plankt. } \\
\text { foram. } \\
(\%)\end{array}$ & $\begin{array}{c}\text { Biogenic } \\
\text { opal cf } \\
(\%)\end{array}$ & $\begin{array}{c}\text { Quartz } \\
(\%)\end{array}$ & $\begin{array}{c}\text { Feldspar } \\
(\%)\end{array}$ & $\begin{array}{l}\text { Mica } \\
(\%)\end{array}$ & $\begin{array}{l}\text { Hev. min. } \\
(\%)\end{array}$ & $\begin{array}{c}\text { Rock fr. } \\
(\%)\end{array}$ & $\begin{array}{c}\text { Volc. gl. } \\
(\%)\end{array}$ & $\begin{array}{l}\text { Auth. } \\
(\%)\end{array}$ \\
\hline $22 \mathrm{H}-3-22$ & 189.86 & 1.10 & 0.08 & 8.80 & 0.80 & 1.84 & 0.81 & 0.01 & 8.88 & 8.01 & 8.88 & 0.83 \\
\hline $22 \mathrm{H}-3-42$ & 198.85 & 2.74 & 8.80 & 8.88 & 8.80 & 2.36 & 0.88 & 0.84 & 0.84 & 8.03 & 8.80 & 0.27 \\
\hline $22 \mathrm{H}-3-62$ & 198.24 & 3.25 & 0.81 & 0.08 & $8 . \theta 8$ & 3.86 & 0.81 & 8.81 & 0.03 & 8.85 & 8.80 & 0.88 \\
\hline $22 \mathrm{H}-3-182$ & 198.62 & 2.41 & 0.88 & 8.88 & 8.88 & 1.53 & 0.80 & 0.11 & 8.84 & 8.83 & 0.88 & 0.69 \\
\hline $22 \mathrm{H}-3-122$ & 198.81 & 4.65 & 0.80 & 8.80 & 8.80 & 4.44 & 8.83 & 0.89 & 8.82 & 0.85 & 0.88 & 0.03 \\
\hline $22 \mathrm{H}-4-22$ & 191.28 & 13.32 & 8.88 & 8.80 & 0.87 & 12.58 & 8.80 & 0.15 & 8.82 & 8.49 & 8.85 & 0.85 \\
\hline $22 \mathrm{H}-4-42$ & 191.47 & 1.97 & 0.81 & 0.80 & 0.80 & 1.60 & $0 . \theta \theta$ & 8.82 & 8.84 & 8.81 & 8.80 & 0.27 \\
\hline $22 \mathrm{H}-4-62$ & 191.66 & 1.15 & 0.78 & 8.23 & 8.81 & 0.16 & 8.88 & 0.01 & 0.80 & 0.01 & 8.80 & 8.82 \\
\hline $22 \mathrm{H}-4-102$ & 192.04 & 0.71 & 0.84 & 8.06 & 0.02 & 0.21 & 8.80 & 8.80 & 8.80 & 8.80 & 0.80 & 0.37 \\
\hline $22 \mathrm{H}-4-121$ & 192.22 & 2.82 & 0.80 & 8.88 & 0.81 & 1.21 & 8.88 & 8.01 & 0.81 & 0.81 & $8.8 \theta$ & 1.57 \\
\hline $22 \mathrm{H}-5-42$ & 192.98 & 2.59 & 0.02 & 8.01 & 0.80 & 0.34 & 8.08 & 8.88 & 8.80 & 0.81 & 0.80 & 2.22 \\
\hline $22 \mathrm{H}-5-98$ & 193.43 & 4.78 & 8.80 & $\theta . \theta 8$ & 8.83 & 4.18 & 0.82 & 0.02 & 0.01 & 8.85 & 0.08 & 0.48 \\
\hline $23 \mathrm{H}-1-39$ & 194.45 & 1.66 & 0.26 & 0.15 & 8.31 & 0.19 & 8.80 & 0.01 & $8.8 \theta$ & 0.01 & 0.80 & 0.72 \\
\hline $23 \mathrm{H}-2-42$ & 195.81 & 8.36 & 8.82 & 0.86 & 8.81 & 8.88 & 8.88 & 8.88 & 8.88 & 8.88 & 8.88 & 8.17 \\
\hline $23 \mathrm{H}-2-182$ & 196.34 & 1.88 & 8.26 & 0.14 & 8.01 & 0.85 & 8.80 & 8.80 & 8.68 & 0.84 & 0.80 & 0.49 \\
\hline $23 \mathrm{H}-2-122$ & 196.52 & 2.82 & 8.80 & 0.88 & 0.80 & 1.74 & $\theta . \theta \theta$ & 8.86 & 8.83 & 0.03 & 8.02 & 0.14 \\
\hline $23 \mathrm{H}-2-142$ & 196.78 & 27.31 & 0.10 & 0.88 & $0.8 \theta$ & 26.82 & 0.80 & $\theta . \theta \theta$ & 0.41 & 0.15 & 0.80 & 0.62 \\
\hline $23 \mathrm{H}-3-42$ & 197.14 & 1.25 & 0.33 & 8.84 & 0.03 & 0.58 & 8.08 & 0.80 & 8.80 & 8.80 & 0.88 & 0.27 \\
\hline $23 H-3-182$ & 197.68 & 4.26 & 8.88 & 8.08 & 8.82 & 3.93 & $\theta . \theta 8$ & 8.80 & 8.85 & 8.83 & 8.88 & 8.23 \\
\hline $23 \mathrm{H}-3-122$ & 197.86 & 4.48 & 8.82 & 8.80 & 0.81 & 4.12 & 8.80 & 0.84 & 8.87 & 8.13 & 8.80 & 0.81 \\
\hline $23 \mathrm{H}-3-142$ & 198.03 & 1.37 & 8.88 & 8.88 & 8.81 & 8.68 & 8.88 & $\theta .8 \theta$ & 8.01 & 0.81 & 8.80 & 8.75 \\
\hline $23 \mathrm{H}-4-42$ & 198.48 & 1.86 & 0.88 & 0.08 & 0.88 & 1.88 & 0.80 & 0.88 & 0.81 & $8 . \theta 8$ & 8.80 & 0.05 \\
\hline $23 \mathrm{H}-4-182$ & 199.81 & 8.64 & 0.01 & 0.01 & 0.88 & 0.38 & 8.88 & 8.88 & 0.80 & 8.81 & 8.81 & 8.23 \\
\hline $23 \mathrm{H}-4-122$ & 199.19 & 0.34 & 8.88 & $\theta .88$ & 8.80 & 0.11 & 8.80 & 0.88 & 8.80 & 8.14 & 8.88 & 0.09 \\
\hline $23 \mathrm{H}-4-142$ & 199.37 & 0.31 & 8.81 & 8.88 & 8.68 & 0.87 & 8.68 & 8.68 & 8.68 & 8.68 & 0.80 & 8.22 \\
\hline $23 \mathrm{H}-5-42$ & 199.81 & 3.58 & 0.88 & 8.08 & 0.81 & 2.86 & 8.88 & $\theta . \theta 0$ & 0.80 & 8.86 & 8.84 & 0.61 \\
\hline $23 \mathrm{H}-5-182$ & 280.35 & 0.83 & 0.02 & 8.82 & 0.08 & 0.15 & 0.60 & 8.80 & 8.88 & 8.08 & 0.01 & 8.62 \\
\hline $23 \mathrm{H}-5-122$ & 288.53 & 1.86 & 8.84 & 0.53 & $\theta .01$ & 0.02 & 0.80 & 0.80 & 0.80 & 0.08 & 0.80 & 0.46 \\
\hline $23 H-5-142$ & 280.78 & 4.58 & 0.03 & 8.85 & 8.08 & 3.85 & 0.02 & 0.86 & 0.80 & 0.03 & 0.80 & 8.47 \\
\hline $24 \mathrm{H}-1-28$ & 281.38 & 0.68 & 0.80 & 0.81 & 0.81 & 0.48 & $8 . \theta 8$ & $0 . \theta \theta$ & 8.80 & 0.08 & 0.08 & 0.17 \\
\hline $24 \mathrm{H}-1-65$ & 281.74 & 19.13 & 8.88 & 8.88 & 0.88 & 16.19 & 8.08 & 0.84 & 8.21 & 0.18 & 8.08 & 2.51 \\
\hline $24 H-1-82$ & 281.98 & 1.73 & 0.68 & 8.80 & 8.82 & 8.52 & 0.88 & 8.87 & 0.03 & 0.01 & 8.80 & 1.89 \\
\hline $24 \mathrm{H}-1-182$ & 282.18 & 0.38 & 0.88 & 8.88 & 0.80 & 0.12 & 0.80 & 8.88 & 8.88 & 8.80 & $8 . \theta 8$ & 0.25 \\
\hline $24 H-1-122$ & 282.30 & 8.76 & 0.08 & 8.80 & 0.82 & 0.18 & 0.08 & 0.03 & 8.80 & 8.80 & 8.80 & 0.53 \\
\hline $24 \mathrm{H}-1-134$ & 282.41 & 0.69 & 0.03 & 0.02 & 0.82 & 0.83 & 8.80 & 0.08 & 0.80 & 8.00 & 8.80 & 0.58 \\
\hline $24 \mathrm{H}-2-65$ & 283.21 & 0.68 & 0.84 & 0.03 & 8.80 & 8.15 & 0.08 & 0.88 & 8.80 & 8.80 & 0.80 & 0.37 \\
\hline $24 \mathrm{H}-2-182$ & 283.57 & 17.53 & 0.88 & 8.88 & 8.03 & 15.68 & 8.88 & 0.47 & 8.47 & 0.03 & 8.80 & 8.85 \\
\hline $24 H-2-128$ & 283.75 & 17.95 & 8.80 & 8.80 & 8.83 & 17.86 & 0.03 & 0.16 & 8.87 & 8.16 & 0.83 & 8.39 \\
\hline $24 \mathrm{H}-3-65$ & 284.68 & 1.68 & 8.08 & 8.80 & 0.88 & 0.43 & 8.88 & 0.00 & 8.88 & 8.80 & 0.80 & 1.17 \\
\hline $24 H-3-82$ & 284.84 & 0.45 & 0.88 & 8.88 & 8.81 & 0.87 & 8.88 & 0.88 & 8.68 & 8.88 & 8.80 & 8.37 \\
\hline $24 \mathrm{H}-3-102$ & 285.84 & 0.62 & 0.17 & 0.05 & 8.80 & 8.82 & 0.08 & 0.88 & 8.80 & 0.80 & 0.08 & 0.37 \\
\hline $24 \mathrm{H}-3-122$ & 285.27 & 8.71 & 8.81 & 0.88 & 0.80 & 8.88 & 0.88 & 0.08 & 8.68 & 8.88 & 0.80 & 0.62 \\
\hline $24 \mathrm{H}-3-142$ & 285.43 & 0.26 & 0.80 & 8.80 & 0.80 & 8.82 & 0.88 & 0.88 & 0.80 & 8.88 & 0.80 & 0.23 \\
\hline $24 H-4-28$ & 285.71 & 8.55 & 8.01 & 8.01 & 0.80 & 0.16 & 8.80 & 0.80 & 8.80 & 0.00 & 8.08 & B. 36 \\
\hline $25 \mathrm{H}-1-22$ & 286.28 & 3.73 & 0.13 & 0.11 & 8.80 & 2.52 & 8.88 & 0.80 & 8.80 & 0.80 & 0.02 & 0.95 \\
\hline $25 \mathrm{H}-1-42$ & 286.48 & 1.86 & 0.00 & 0.88 & 0.81 & 0.39 & 8.88 & 8.83 & 8.82 & 0.08 & 0.85 & 8. 56 \\
\hline $25 \mathrm{H}-1-62$ & 286.68 & 1.39 & 0.88 & 0.80 & 0.81 & 8.76 & 0.80 & 8.86 & 8.81 & 0.81 & 0.01 & 0.53 \\
\hline $25 \mathrm{H}-1-82$ & 286.88 & 8.46 & 0.80 & 0.08 & 0.82 & 8.82 & 0.88 & 0.80 & 8.80 & 0.80 & 8.00 & 0.42 \\
\hline $25 \mathrm{H}-1-182$ & 287.80 & 8.47 & 8.82 & 0.02 & 0.01 & 0.02 & 0.08 & 0.08 & 0.00 & 0.08 & 8.08 & 8.48 \\
\hline $25 \mathrm{H}-1-122$ & 287.28 & 8.28 & 8.80 & 8.80 & 0.80 & 0.03 & 0.00 & 0.80 & 8.80 & 0.80 & 8.80 & 0.17 \\
\hline
\end{tabular}


Table 1.4 (continued).

\begin{tabular}{|c|c|c|c|c|c|c|c|c|c|c|c|c|}
\hline \multirow[b]{2}{*}{ Sample no. } & \multirow[b]{2}{*}{$\begin{array}{c}\text { Depth } \\
\text { mbsf }\end{array}$} & \multirow[b]{2}{*}{$\begin{array}{c}>63 \mu \mathrm{m} \\
(\%)\end{array}$} & \multicolumn{10}{|c|}{ Coarse fraction component analysis (wt \%) } \\
\hline & & & $\begin{array}{l}\text { Benthic } \\
\text { foram. } \\
(\%)\end{array}$ & $\begin{array}{l}\text { Plankt. } \\
\text { foram. } \\
(\%)\end{array}$ & $\begin{array}{l}\text { Biogenic } \\
\text { opal cf } \\
(\%)\end{array}$ & $\begin{array}{c}\text { Quartz } \\
(\%)\end{array}$ & $\begin{array}{c}\text { Feldspar } \\
(\%)\end{array}$ & $\begin{array}{c}\text { Mica } \\
(\%)\end{array}$ & $\begin{array}{l}\text { Hev. min. } \\
(\%)\end{array}$ & $\begin{array}{c}\text { Rock fr. } \\
(\%)\end{array}$ & $\begin{array}{c}\text { Volc. gl. } \\
(\%)\end{array}$ & $\begin{array}{l}\text { Auth. } \\
(\%)\end{array}$ \\
\hline $25 \mathrm{H}-2-22$ & 287.78 & 8.46 & 8.03 & 0.12 & 8.88 & 8.81 & 8.80 & 8.00 & 8.88 & 8.88 & 0.88 & 0.38 \\
\hline $25 \mathrm{H}-2-42$ & 287.98 & 1.27 & 8.82 & 0.02 & 8.84 & 8.43 & 8.88 & 0.02 & 0.80 & 8.84 & 8.88 & 0.78 \\
\hline $25 \mathrm{H}-2-62$ & 288.12 & 8.76 & 8.89 & 0.89 & 8.83 & 0.11 & 8.80 & 0.08 & 8.80 & 8.84 & 8.08 & 0.48 \\
\hline $25 \mathrm{H}-2-82$ & 288.32 & 4.84 & 8.88 & 8.88 & 0.81 & 3.38 & 8.88 & 0.21 & 0.02 & 8.81 & 0.01 & 0.48 \\
\hline $25 \mathrm{H}-2-182$ & 208.52 & 8.85 & 8.88 & 0.80 & 8.88 & 0.19 & 8.88 & 8.80 & 0.88 & 8.86 & 8.82 & 0.58 \\
\hline $25 \mathrm{H}-2-142$ & 209.18 & 0.44 & 8.08 & 8.88 & 8.80 & 8.85 & 8.80 & 8.80 & 8.88 & 8.88 & 8.88 & 0.38 \\
\hline $25 \mathrm{H}-3-22$ & 289.28 & 1.38 & 8.08 & 8.80 & 8.88 & 0.74 & 8.88 & 0.80 & 8.88 & 8.88 & 0.08 & 0.55 \\
\hline $25 \mathrm{H}-3-42$ & 289.48 & 0.71 & 8.88 & 0.88 & 8.80 & 8.83 & 8.80 & 0.80 & 0.80 & 8.85 & 8.80 & 8.47 \\
\hline $25 \mathrm{H}-3-62$ & 289.68 & 1.62 & 0.03 & $\theta .00$ & 0.83 & 1.23 & 8.88 & 0.81 & 8.80 & 8.80 & 0.80 & 8.33 \\
\hline $26 \mathrm{H}-1-22$ & 218.71 & 1.41 & 0.82 & 0.01 & 8.81 & 8.56 & 8.88 & 0.01 & 8.01 & 0.00 & 0.80 & 0.81 \\
\hline $26 \mathrm{H}-1-42$ & 210.91 & 1.34 & 8.01 & 8.81 & 8.88 & 8.58 & 8.88 & 8.88 & 8.88 & 8.13 & 8.88 & 8.61 \\
\hline $26 \mathrm{H}-1-62$ & 211.10 & 0.72 & 8.80 & 0.08 & 8.81 & 0.13 & 0.88 & 8.80 & 8.08 & 8.80 & 8.80 & 0.57 \\
\hline $26 \mathrm{H}-1-82$ & 211.38 & 8.91 & 0.02 & 0.08 & 0.85 & 8.83 & 8.88 & 8.81 & 8.80 & 8.84 & 8.81 & 8.76 \\
\hline $26 \mathrm{H}-2-22$ & 212.17 & 4.51 & 0.71 & 0.28 & 8.80 & 1.65 & 8.80 & 0.81 & 0.80 & 0.80 & 8.80 & 1.85 \\
\hline $26 \mathrm{H}-2-42$ & 212.36 & 14.84 & 0.80 & 0.86 & 8.88 & 11.67 & 8.80 & 0.86 & 8.80 & 1.67 & 8.31 & 0.28 \\
\hline $26 \mathrm{H}-2-62$ & 212.56 & 0.88 & 0.14 & 0.88 & 0.88 & 0.41 & 0.80 & 0.80 & 8.88 & 8.80 & 8.80 & 8.24 \\
\hline $26 \mathrm{H}-2-82$ & 212.75 & 3.88 & 0.80 & 8.01 & 8.88 & 2.93 & 8.80 & 8.88 & 8.81 & 8.78 & 8.14 & 0.81 \\
\hline $26 \mathrm{H}-2-182$ & 212.94 & 1.28 & 8.88 & 8.88 & 0.80 & 0.82 & 8.88 & 8.80 & 8.88 & 8.88 & 8.80 & 8.38 \\
\hline $26 \mathrm{H}-2-122$ & 213.14 & 0.84 & 8.84 & 8.81 & 8.88 & 0.56 & 8.88 & 0.88 & 8.88 & 8.88 & 8.88 & 8.22 \\
\hline $26 \mathrm{H}-2-142$ & 213.33 & 8.79 & 8.88 & 8.82 & 8.88 & 0.31 & 8.80 & 8.80 & 8.80 & 8.88 & 8.80 & 8.46 \\
\hline $26 \mathrm{H}-3-22$ & 213.62 & 2.37 & 8.88 & 0.80 & 8.03 & 1.38 & 0.80 & 8.88 & 0.82 & 8.86 & 8.80 & 0.87 \\
\hline $26 \mathrm{H}-3-42$ & 213.82 & 8.77 & 8.80 & 0.88 & 0.81 & 0.18 & 8.80 & $\theta . \theta 0$ & 0.88 & 8.80 & 8.80 & 8.58 \\
\hline $27 \mathrm{H}-1-102$ & 215.38 & 1.33 & 0.12 & 0.86 & 8.80 & 8.75 & 8.88 & 8.80 & 8.88 & 8.88 & 8.88 & 0.41 \\
\hline $27 \mathrm{H}-1-122$ & 215.50 & 4.91 & 0.81 & 8.88 & 0.88 & 4.39 & 8.88 & 0.03 & 0.80 & 0.81 & 8.82 & 0.46 \\
\hline $28 \mathrm{H}-1-182$ & 217.85 & 4.93 & 8.80 & 0.80 & 8.88 & 4.48 & 8.80 & 0.81 & 8.88 & 0.01 & 8.80 & 8.43 \\
\hline $28 \mathrm{H}-1-122$ & 217.23 & 8.39 & 8.04 & 8.05 & 8.88 & 8.86 & 8.80 & 8.88 & $8 . \theta 8$ & 0.80 & 8.88 & 0.24 \\
\hline $28 \mathrm{H}-1-142$ & 217.42 & 8.69 & 8.89 & 0.82 & 8.80 & 8.44 & 8.80 & 8.08 & 8.80 & 0.81 & 8.08 & 0.12 \\
\hline $28 \mathrm{H}-2-182$ & 218.44 & 12.87 & 0.02 & 0.80 & 8.08 & 11.38 & 8.00 & 0.88 & 8.88 & 8.82 & 0.02 & 8.78 \\
\hline $28 \mathrm{H}-2-122$ & 218.63 & 0.46 & 0.17 & 8.81 & 8.80 & 8.16 & 8.80 & 0.88 & 0.88 & 0.03 & 8.08 & 0.08 \\
\hline $28 \mathrm{H}-2-142$ & 218.82 & 1.34 & 0.08 & $\theta .80$ & 8.80 & 8.68 & 8.80 & 0.08 & 8.80 & 8.80 & 0.80 & 0.72 \\
\hline $28 \mathrm{H}-3-182$ & 219.84 & 8.38 & 0.85 & 8.80 & 8.08 & 6.69 & 8.80 & 0.15 & $\theta .88$ & $\theta .01$ & 0.80 & 1.47 \\
\hline $29 \mathrm{H}-1-182$ & 222.10 & 8.57 & 8.82 & 8.81 & 8.00 & 8.87 & 8.88 & 0.80 & 8.80 & 8.88 & 8.80 & 8.46 \\
\hline $29 H-1-122$ & 222.38 & 0.78 & 8.08 & 0.00 & 0.81 & 0.18 & 8.80 & 0.80 & 0.80 & 8.80 & 0.08 & 8.58 \\
\hline $29 H-1-142$ & 222.50 & 8.47 & 0.80 & 0.08 & 8.81 & 0.07 & 0.88 & 0.88 & 0.08 & 8.88 & 8.80 & 0.38 \\
\hline $29 \mathrm{H}-2-182$ & 223.68 & 8.51 & 8.88 & 0.88 & 8.81 & 0.03 & 8.88 & 0.01 & 0.00 & 8.80 & 0.08 & 0.47 \\
\hline $29 \mathrm{H}-2-122$ & 223.75 & 0.78 & 0.82 & 0.80 & 0.03 & 0.01 & 0.82 & 8.01 & 0.88 & 8.82 & 0.01 & 0.64 \\
\hline $29 H-2-142$ & 223.90 & 0.63 & 8.80 & 8.80 & 0.01 & 0.15 & 0.80 & 0.88 & 0.80 & 8.80 & 0.80 & 0.47 \\
\hline $29 H-3-182$ & 225.10 & 1.83 & 0.81 & 8.80 & 0.80 & 1.46 & 8.81 & 0.88 & 0.01 & 8.83 & 8.80 & 0.30 \\
\hline $29 \mathrm{H}-3-122$ & 225.38 & 2.22 & 8.80 & 8.88 & 8.80 & 1.52 & 0.80 & 8.80 & 8.80 & 8.88 & 8.88 & 8.78 \\
\hline зөн-1-102 & 227.12 & 3.52 & 0.80 & 0.00 & 8.88 & 2.80 & 0.01 & 0.88 & 0.08 & 8.87 & 8.80 & 8.64 \\
\hline $38 \mathrm{~B}-1-122$ & 227.32 & 4.27 & 0.88 & 0.80 & 0.85 & 2.39 & 8.80 & 0.82 & 8.80 & 8.46 & 0.89 & 1.27 \\
\hline $38 \mathrm{H}-1-142$ & 227.51 & 1.14 & 0.80 & 0.80 & 0.80 & 8.15 & 0.80 & 8.80 & 0.88 & 8.88 & 0.08 & 8.98 \\
\hline $38 \mathrm{H}-2-182$ & 228.68 & 1.16 & 0.13 & 0.16 & 0.03 & 0.01 & $8.8 \theta$ & 0.80 & 8.08 & 8.80 & 8.80 & 0.84 \\
\hline $38 \mathrm{H}-2-122$ & 228.82 & 1.85 & 0.18 & 8.05 & 0.03 & 8.02 & 8.80 & 0.01 & 0.07 & 0.88 & 0.02 & 8.75 \\
\hline $38 \mathrm{H}-2-142$ & 229.82 & 1.78 & 0.02 & 8.83 & 0.17 & 0.81 & 8.80 & $\theta .80$ & 8.80 & 8.88 & 8.88 & 1.54 \\
\hline $30 \mathrm{H}-3-182$ & 238.12 & 0.84 & 8.81 & 8.80 & 0.04 & 0.02 & 8.80 & 8.80 & 0.08 & 8.88 & 8.80 & 8.77 \\
\hline $38 \mathrm{H}-3-122$ & 238.32 & 1.98 & 0.80 & 8.88 & 8.21 & 8.29 & 8.88 & 8.85 & 0.80 & 8.15 & 0.30 & 8.89 \\
\hline $38 \mathrm{H}-3-142$ & 238.52 & 3.27 & 8.80 & 8.81 & 8.87 & 2.81 & 8.88 & 8.83 & 8.81 & 8.84 & 0.02 & 8.29 \\
\hline $38 \mathrm{BH}-4-182$ & 231.60 & 1.25 & 0.88 & 8.88 & 0.82 & 0.89 & 0.08 & $8.8 \theta$ & 8.80 & 8.08 & 8.88 & 1.14 \\
\hline $38 \mathrm{H}-4-122$ & 231.82 & 0.76 & 0.80 & 8.80 & 0.80 & 0.35 & 8.08 & $\theta .08$ & 8.88 & 8.88 & 0.08 & 0.41 \\
\hline
\end{tabular}


Table 1.4 (continued).

\begin{tabular}{|c|c|c|c|c|c|c|c|c|c|c|c|c|}
\hline \multirow[b]{2}{*}{ Sample no. } & \multirow[b]{2}{*}{$\begin{array}{c}\text { Depth } \\
\text { mbsf }\end{array}$} & \multirow[b]{2}{*}{$\begin{array}{c}>63 \mu \mathrm{m} \\
(\%)\end{array}$} & \multicolumn{10}{|c|}{ Coarse fraction component analysis (wt \%) } \\
\hline & & & $\begin{array}{l}\text { Benthic } \\
\text { foram. } \\
(\%)\end{array}$ & $\begin{array}{l}\text { Plankt. } \\
\text { foram. } \\
(\%)\end{array}$ & $\begin{array}{l}\text { Biogenic } \\
\text { opal cf } \\
(\%)\end{array}$ & $\begin{array}{c}\text { Quartz } \\
(\%)\end{array}$ & $\begin{array}{c}\text { Feldspar } \\
(\%)\end{array}$ & $\begin{array}{l}\text { Mica } \\
(\%)\end{array}$ & $\begin{array}{l}\text { Hev. min. } \\
(\%)\end{array}$ & $\begin{array}{c}\text { Rock fr. } \\
(\%)\end{array}$ & $\begin{array}{l}\text { Volc. gl. } \\
\text { (\%) }\end{array}$ & $\begin{array}{l}\text { Auth. } \\
(\%)\end{array}$ \\
\hline $38 \mathrm{H}-4-142$ & 232.82 & 2.59 & 8.88 & 8.80 & 8.88 & 2.28 & 8.88 & 8.88 & 8.88 & 8.03 & 8.80 & 8.35 \\
\hline $31 \mathrm{H}-1-182$ & 233.18 & 18.19 & 8.80 & 8.80 & 0.88 & 9.58 & 8. 80 & $\theta .80$ & 8.82 & 8.88 & 8.88 & 8.43 \\
\hline $31 \mathrm{H}-1-142$ & 233.32 & 2.13 & 8.88 & 0.80 & 8.88 & 1.45 & $\theta .80$ & 8.80 & 8.80 & 0.81 & 8.80 & 8.67 \\
\hline $31 \mathrm{H}-2-182$ & 234.68 & 1.51 & 8.80 & 8.88 & 8.36 & 8.19 & 8.00 & 0.01 & 8.80 & 0.82 & 8.88 & 0.93 \\
\hline $31 \mathrm{H}-2-142$ & 234.92 & 1.28 & 0.88 & 8.88 & 8.26 & 8.83 & 8.88 & 8.88 & 8.88 & 0.81 & 8.88 & 0.98 \\
\hline $31 \mathrm{H}-3-182$ & 236.12 & 8.84 & 0.88 & 8.88 & 0.11 & 0.12 & 0.88 & 0.88 & 0.80 & 8.88 & 8.80 & 8.68 \\
\hline $31 \mathrm{H}-3-122$ & 236.32 & 1.24 & 8.88 & 0.80 & 8.17 & 8.03 & 8.80 & 8.88 & 8.80 & 8.80 & 0.08 & 1.84 \\
\hline $32 \mathrm{H}-2-102$ & 248.62 & 1.18 & 0.87 & 8.87 & 0.18 & 8.82 & 0.80 & 0.00 & 8.88 & 8.88 & 8.80 & 0.82 \\
\hline $32 \mathrm{H}-2-122$ & 248.82 & 1.36 & 0.18 & 8.29 & 0.82 & 8.81 & 8.88 & 8.88 & 8.80 & 8.08 & 8.80 & 0.88 \\
\hline $32 \mathrm{H}-2-142$ & 241.82 & 1.36 & 0.16 & 0.15 & 0.13 & 8.83 & 8.88 & 0.88 & 8.88 & 8.88 & 8.88 & 8.88 \\
\hline $32 \mathrm{H}-3-182$ & 242.12 & 1.88 & 8.12 & 8.17 & 8.33 & 8.83 & 8.80 & 0.80 & 8.88 & 8.88 & 8.88 & 1.14 \\
\hline $32 \mathrm{H}-3-122$ & 242.32 & 2.88 & 0.10 & 8.11 & 8.44 & 8.82 & 8.88 & 8.08 & 8.80 & 8.88 & 0.81 & 1.39 \\
\hline $32 \mathrm{H}-3-142$ & 242.52 & 2.36 & 8.84 & 0.85 & 8.55 & 8.88 & 8.08 & 8.08 & 8.88 & 8.88 & 8.88 & 1.78 \\
\hline $32 \mathrm{H}-4-182$ & 243.68 & 1.35 & 0.12 & 8.12 & 0.12 & 0.81 & 8.80 & 0.88 & 8.88 & 0.88 & 8.88 & 0.95 \\
\hline $32 \mathrm{H}-4-122$ & 243.82 & 1.13 & 8.12 & 0.10 & 0.31 & 8.08 & 8.80 & 8.80 & 0.80 & 8.80 & 8.88 & 0.59 \\
\hline $33 H-1-182$ & 244.72 & 3.98 & 8.48 & 8.12 & 0.52 & 8.88 & $\theta . \theta 8$ & 8.03 & 8.88 & $\theta .00$ & 8.80 & 2.65 \\
\hline $33 H-1-122$ & 244.92 & 1.28 & 0.18 & 8.87 & 8.17 & 8.01 & 8.88 & 8.88 & 8.88 & 8.88 & $\theta .08$ & 8.86 \\
\hline $33 H-1-142$ & 245.12 & 1.66 & 8.16 & 8.87 & 0.17 & 0.85 & 8.88 & 8.68 & 0.88 & 8.80 & 8.88 & 1.22 \\
\hline $34 \mathrm{H}-1-182$ & 246.86 & 2.62 & 0.88 & 8.81 & 1.72 & 8.84 & 8.88 & 8.83 & 8.88 & 8.80 & 8.80 & 0.82 \\
\hline $34 H-1-122$ & 247.82 & 1.82 & 8.80 & 0.01 & 1.39 & 8.03 & $\theta .08$ & 0.83 & 8.88 & $\theta .80$ & 0.88 & 8.36 \\
\hline $34 \mathrm{H}-1-142$ & 247.19 & 1.24 & 0.82 & 0.02 & 8.55 & 8.83 & 8.88 & 8.82 & 8.88 & 8.88 & 8.80 & 8.61 \\
\hline $34 \mathrm{H}-2-102$ & 248.12 & 8.88 & 0.82 & 8.80 & 8.34 & 8.01 & 8.88 & 0.88 & 8.88 & 8.88 & 8.88 & 8.43 \\
\hline $34 H-2-122$ & 248.28 & 0.97 & 0.05 & 0.83 & 0.49 & 0.01 & $\theta . \theta 8$ & 0.83 & 8.88 & 8.01 & 8.03 & 8.32 \\
\hline $34 \mathrm{H}-2-142$ & 248.45 & 1.37 & 8.80 & 8.80 & 8.22 & 8.88 & 8.88 & 0.01 & 8.88 & 8.88 & 8.88 & 1.14 \\
\hline $34 \mathrm{H}-4-182$ & 258.64 & 1.25 & 0.88 & 8.88 & 8.82 & 8.83 & 8.88 & 8.88 & 0.01 & 8.88 & 8.88 & 8. 38 \\
\hline $34 \mathrm{H}-4-122$ & 258.88 & 8.88 & 8.85 & 0.13 & 8.81 & 8.83 & 0.80 & 8.88 & 8.08 & 8.80 & 8.88 & 8.58 \\
\hline $34 H-5-182$ & 251.98 & 1.81 & 0.16 & 0.23 & 0.28 & 8.02 & 8.80 & 0.80 & 0.08 & 0.80 & 8.88 & 1.28 \\
\hline $34 H-5-122$ & 252.06 & 1.23 & 0.12 & 0.85 & 8.66 & 8.83 & 8.80 & 8.81 & 8.80 & $\theta . \theta \theta$ & 0.01 & 0.34 \\
\hline $34 H-5-142$ & 252.23 & 1.84 & 8.82 & 8.84 & 0.16 & 8.88 & 8.88 & $\theta .08$ & 8.88 & 0.88 & 8.80 & 8.81 \\
\hline $34 \mathrm{H}-6-182$ & 253.16 & 1.82 & 0.87 & 0.29 & 0.19 & 8.81 & 8.80 & $\theta .08$ & 8.88 & 8.88 & 0.80 & 8.47 \\
\hline $34 H-6-142$ & 253.49 & 1.81 & 8.04 & 0.16 & 0.13 & 8.88 & 8.88 & 0.00 & 8.80 & 8.88 & 0.88 & 0.67 \\
\hline
\end{tabular}


Table 1.4 (continued).

\begin{tabular}{|c|c|c|c|c|c|c|c|c|c|c|c|c|c|}
\hline \multirow[b]{2}{*}{$\begin{array}{c}\text { Sample } \\
\text { no. }\end{array}$} & \multirow[b]{2}{*}{$\begin{array}{l}\text { Depth } \\
\text { (mbsf) }\end{array}$} & \multicolumn{12}{|c|}{ Grain amount percentages } \\
\hline & & $\begin{array}{l}\text { Benthic } \\
\text { foram. } \\
(\%)\end{array}$ & $\begin{array}{l}\text { Plankt. } \\
\text { foram. } \\
(\%)\end{array}$ & $\begin{array}{l}\text { Rads. } \\
(\%)\end{array}$ & $\begin{array}{c}\text { Diatoms } \\
(\%)\end{array}$ & $\begin{array}{l}\text { Sponge } \\
\text { spicule } \\
(\%)\end{array}$ & $\begin{array}{l}\text { Quartz } \\
(\%)\end{array}$ & $\begin{array}{l}\text { Feldspar } \\
(\%)\end{array}$ & $\begin{array}{l}\text { Mica } \\
(\%)\end{array}$ & $\begin{array}{l}\text { Hev. Min. } \\
(\%)\end{array}$ & $\begin{array}{l}\text { Rock fr. } \\
(\%)\end{array}$ & $\begin{array}{l}\text { Volc. gl. } \\
(\%)\end{array}$ & $\begin{array}{l}\text { Auth. } \\
(\%)\end{array}$ \\
\hline $1 \mathrm{H}-1-22$ & 0.22 & 8.35 & 3.36 & 0.88 & 8.08 & 8.88 & 84.45 & 5.38 & 0.08 & 1.86 & 4.95 & 0.80 & 0.53 \\
\hline $1 H-1-42$ & 0.42 & 7.28 & 15.37 & $\theta .88$ & 8.80 & 0.97 & 59.73 & 2.92 & 0.58 & 1.17 & 11.28 & 0.19 & 0.58 \\
\hline $1 H-1-59$ & 0.59 & 1.13 & 7.26 & 8.88 & 8.88 & $\theta . \theta 8$ & 79.68 & 4.68 & 0.48 & 0.16 & 6.13 & 8.80 & 8.48 \\
\hline $1 H-1-82$ & 0.82 & 1.31 & 16.87 & $\theta . \theta \theta$ & $\theta .80$ & 0.33 & 71.31 & 4.59 & 8.98 & 8.33 & 3.44 & 8.33 & 1.31 \\
\hline $1 H-1-182$ & 1.82 & 2.92 & 12.18 & $\theta .80$ & 8.88 & 0.16 & 75.16 & 3.73 & 1.14 & 8. 97 & 3.25 & $\theta . \theta \theta$ & 8.16 \\
\hline $1 H-1-122$ & 1.22 & 0.18 & 2.87 & 8.80 & 0.88 & $\theta .80$ & 82.94 & 6.82 & 8.08 & 8.98 & 4.67 & 0.54 & 8.98 \\
\hline $1 H-1-142$ & 1.42 & 2.62 & 3.85 & 0.80 & 0.80 & 8.80 & 78.85 & 6.82 & 0.87 & 8.87 & 5.24 & 8.80 & 0.52 \\
\hline $1 \mathrm{H}-2-22$ & 1.72 & 3.51 & 19.37 & $0.8 \theta$ & 0.88 & 8.17 & 66.11 & 4.17 & 0.17 & 8.83 & 5.81 & 8.80 & 0.67 \\
\hline $1 \mathrm{H}-2-59$ & 2.89 & 0.59 & 3.23 & 0.88 & 0.80 & 8.80 & 81.52 & 4.55 & 1.76 & 1.83 & 6.16 & 8.88 & 8.88 \\
\hline $1 \mathrm{H}-2-82$ & 2.30 & 2.52 & 9.24 & 0.88 & 0.88 & 0.17 & 76.81 & 5.55 & 0.34 & 0.34 & 4.28 & 8.88 & 8.67 \\
\hline $1 \mathrm{H}-2-182$ & 2.52 & 2.85 & 11.57 & $\theta .80$ & 0.80 & 8.80 & 88.25 & 2.14 & 8.80 & 0.53 & 2.14 & 8.88 & 8.53 \\
\hline $1 \mathrm{H}-2-122$ & 2.72 & 4.78 & 22.73 & 8.88 & 8.88 & 0.80 & 58.81 & 2.97 & 8.99 & 8.33 & 5.27 & 8.16 & 3.62 \\
\hline $1 \mathrm{H}-2-142$ & 2.98 & 3.71 & 24.11 & 0.88 & $\theta . \theta \theta$ & $\theta . \theta 8$ & 61.05 & 2.82 & 0.67 & 8.67 & 6.87 & 8.88 & 1.69 \\
\hline $1 \mathrm{H}-3-22$ & 3.22 & 4.79 & 14.36 & 8.88 & 8.88 & 8.17 & 78.79 & 1.82 & 0.17 & 8.17 & 4.46 & 8.88 & 2.97 \\
\hline $1 \mathrm{H}-3-42$ & 3.42 & 6.62 & 10.82 & 0.88 & 8.88 & 8.57 & 68.85 & 4.54 & 1.51 & 8. 95 & 4.54 & 8.88 & 2.65 \\
\hline $1 \mathrm{H}-3-59$ & 3.59 & 2.61 & 15.33 & $\theta . \theta \theta$ & 8.80 & 0.35 & 65.33 & 6.27 & 0.87 & 1.39 & 6.97 & 8.88 & 8.87 \\
\hline $1 \mathrm{H}-3-82$ & 3.82 & 1.84 & 24.83 & 8.88 & 0.88 & 8.80 & 65.22 & 3.13 & 0.88 & 8.98 & 4.93 & 8.88 & 8.68 \\
\hline $1 \mathrm{H}-3-1 \theta 2$ & 4.82 & 8.74 & 2.88 & 8.88 & 8.88 & 8.80 & 81.15 & 7.66 & 0.59 & 8.59 & 5.74 & 8.80 & 8.74 \\
\hline $1 \mathrm{H}-3-122$ & 4.22 & 2.84 & 18.53 & 0.88 & 8.88 & 0.16 & 71.86 & 5.66 & 1.26 & 8.94 & 4.87 & 8.88 & 2.67 \\
\hline $1 H-3-142$ & 4.42 & 4.87 & 15.59 & 8.80 & 8.88 & 8.88 & 71.73 & 8.78 & 8.39 & 5.65 & 8.88 & 8.88 & 8.97 \\
\hline $1 \mathrm{H}-4-22$ & 4.72 & 2.03 & 38.81 & $\theta .8 \theta$ & 0.88 & 8.88 & 58.49 & 2.58 & 8.74 & 8.55 & 4.86 & 0.74 & 8.88 \\
\hline $1 \mathrm{H}-4-42$ & 4.92 & 1.79 & 13.69 & $8.8 \theta$ & 0.88 & 8.88 & 72.82 & 6.55 & $\theta .88$ & 0.79 & 3.37 & 8.28 & 8.79 \\
\hline $1 \mathrm{H}-4-59$ & 5.89 & 1.96 & 23.18 & 8.88 & 8.88 & 8.88 & 61.38 & 7.47 & 8.98 & 8.39 & 4.32 & 8.88 & 8.39 \\
\hline $1 \mathrm{H}-4-82$ & 5.30 & 3.41 & 25.19 & 8.88 & 0.80 & $\theta .8 \theta$ & 64.28 & 2.27 & 8.76 & 8.80 & 3.68 & 8.88 & 8.57 \\
\hline $1 \mathrm{H}-4-182$ & 5.52 & 1.37 & 21.88 & $\theta . \theta \theta$ & 0.88 & $\theta . \theta \theta$ & 67.38 & 1.17 & 1.17 & 8.39 & 5.86 & 8.39 & 8.39 \\
\hline $1 \mathrm{H}-4-122$ & 5.72 & 2.13 & 24.61 & 0.80 & 8.80 & $\theta . \theta 8$ & 66.28 & 1.36 & 2.52 & 0.58 & 1.94 & $\theta .88$ & 8.58 \\
\hline $1 H-5-22$ & 6.22 & 0.57 & 27.27 & 8.88 & 8.88 & 0.88 & 67.85 & 8.57 & 0.76 & 0.57 & 2.84 & 8.88 & 8. 38 \\
\hline $1 \mathrm{H}-5-42$ & 6.42 & 0.97 & 29.46 & 8.88 & 0.88 & 8.88 & 64.53 & 1.55 & 8.39 & 8.78 & 2.13 & 8.88 & 8.19 \\
\hline $1 H-5-59$ & 6.59 & 1.14 & 22.35 & 8.88 & 0.88 & 0.19 & 65.72 & 3.41 & 1.89 & 8. 38 & 4.55 & 8.80 & 8. 38 \\
\hline $1 H-5-82$ & 6.82 & 2.52 & 57.77 & 8.80 & 8.88 & 8.88 & 34.83 & 1.68 & 1.47 & 8.88 & 2.31 & 8.88 & 8. 21 \\
\hline $1 \mathrm{H}-5-182$ & 7.02 & 8.98 & 12.52 & 8.88 & 0.80 & $\theta . \theta \theta$ & 77.18 & 4.58 & 2.54 & 0.28 & 1.76 & 8.88 & 8.88 \\
\hline $1 \mathrm{H}-5-122$ & 7.22 & 4.85 & 13.71 & 8.88 & 8.88 & 8.88 & 78.85 & 1.16 & 8.39 & 2.51 & 7.14 & 8.88 & 8.19 \\
\hline $1 \mathrm{H}-5-142$ & 7.42 & 2.69 & 42.12 & 8.80 & 8.80 & 8.88 & 58.77 & 1.73 & 0.38 & 8.19 & 2.12 & 8.88 & 8.88 \\
\hline $1 \mathrm{H}-6-22$ & 7.72 & 2.87 & 26.55 & 8.88 & 8.88 & 8.88 & 65.35 & 1.51 & 3.81 & 8.88 & 1.32 & 8.88 & 8.19 \\
\hline $1 \mathrm{H}-6-42$ & 7.92 & 2.63 & 28.98 & 8.80 & 8.88 & 8.16 & 62.73 & 1.97 & 1.15 & 8.33 & 1.81 & 8.88 & 8.16 \\
\hline $1 H-6-59$ & 8.84 & 2.29 & 18.55 & 8.80 & 8.88 & 8.88 & 72.66 & 2.87 & 1.34 & 8.76 & 8.96 & 8.88 & 8. 38 \\
\hline $1 H-6-82$ & 8.30 & 8.69 & 36.23 & 8.88 & 0.88 & $\theta . \theta 8$ & 49.54 & 2.83 & 8.92 & 8.18 & 2.22 & 8.88 & 8.88 \\
\hline $1 \mathrm{H}-6-102$ & 8.52 & 4.91 & 25.98 & 0.88 & 8.88 & 8.19 & 64.46 & 1.78 & 1.32 & B. 57 & 8.76 & 8.88 & 8.19 \\
\hline $1 \mathrm{H}-6-122$ & 8.72 & 0.86 & 19.83 & 8.88 & 0.88 & 8.88 & 63.62 & 6.38 & 8.34 & 8.86 & 8.18 & 8.88 & 8.88 \\
\hline $1 H-6-142$ & 8.98 & 1.93 & 48.98 & 8.88 & 8.80 & 8.64 & 49.68 & 3.86 & 0.64 & 8.16 & 2.74 & 8.88 & 8.16 \\
\hline $2 \mathrm{H}-1-22$ & 9.42 & 3.86 & 43.55 & 8.88 & 0.88 & 8.88 & 39.35 & 1.61 & 8.97 & 8.97 & 9.52 & 8.88 & 0.65 \\
\hline $2 H-1-42$ & 9.62 & 2.54 & 35.51 & 8.88 & 8.88 & 8.88 & 53.88 & 1.63 & 1.89 & 8. 54 & 4.89 & 8.80 & 8.88 \\
\hline $2 \mathrm{H}-\mathrm{I}-62$ & 9.82 & 0.80 & 0.54 & 8.88 & 0.88 & $\theta .8 \theta$ & 82.25 & 4.53 & 1.27 & 1.63 & 8.33 & $\theta .88$ & 1.45 \\
\hline $2 H-1-82$ & 10.02 & 1.28 & 31.87 & 0.88 & 8.88 & 0.18 & 61.36 & 1.65 & 0.73 & 8.92 & 1.28 & 8.88 & 8.73 \\
\hline $2 \mathrm{H}-1-184$ & 18.24 & 2.68 & 38.18 & 8.88 & $\theta .8 \theta$ & 1.49 & 48.14 & 1.67 & 8.93 & 1.12 & 4.65 & 8.80 & 8.37 \\
\hline $2 \mathrm{H}-1-122$ & 18.42 & 1.36 & 62.18 & 8.88 & 8.88 & 8.88 & 31.97 & 1.56 & 8.88 & 8.19 & 2.53 & 8.88 & 8.88 \\
\hline $2 \mathrm{H}-1-142$ & 18.62 & 2.55 & 86.27 & 8.88 & 8.88 & $8.8 \theta$ & 18.28 & 8.28 & 0.39 & 8.88 & 0.39 & 0.88 & 8.88 \\
\hline $2 \mathrm{H}-2-22$ & 10.92 & 2.52 & 94.68 & 0.88 & 0.80 & 8.18 & 2.34 & 8.88 & 0.18 & 8.88 & 0.18 & $\theta .8 \theta$ & $8 . \theta 8$ \\
\hline $2 \mathrm{H}-2-62$ & 11.32 & 2.32 & 43.12 & 8.08 & 8.88 & 8.88 & 45.77 & 3.32 & 0.33 & 1.66 & 3.48 & 8.88 & 8.88 \\
\hline $2 \mathrm{H}-2-82$ & 11.52 & 1.89 & 35.45 & 0.08 & 8.88 & $\theta . \theta 0$ & 68.88 & 8.36 & 0.18 & 0. 36 & 2.18 & 8.88 & 8. 36 \\
\hline
\end{tabular}


Table 1.4 (continued).




Table 1.4 (continued).






\begin{tabular}{|c|c|c|c|c|c|c|c|c|c|c|c|c|c|}
\hline \multirow[b]{2}{*}{$\begin{array}{c}\text { Sample } \\
\text { no. }\end{array}$} & \multirow[b]{2}{*}{$\begin{array}{l}\text { Depth } \\
\text { (mbsf) }\end{array}$} & \multicolumn{12}{|c|}{ Grain amount percentages } \\
\hline & & $\begin{array}{l}\text { Benthic } \\
\text { foram. } \\
(\%)\end{array}$ & $\begin{array}{l}\text { Plankt. } \\
\text { foram. } \\
(\%)\end{array}$ & $\begin{array}{l}\text { Rads. } \\
(\%)\end{array}$ & $\begin{array}{c}\text { Diatoms } \\
(\%)\end{array}$ & $\begin{array}{l}\text { Sponge } \\
\text { spicule } \\
(\%)\end{array}$ & $\begin{array}{c}\text { Quartz } \\
(\%)\end{array}$ & $\begin{array}{c}\text { Feldspar } \\
(\%)\end{array}$ & $\begin{array}{c}\text { Mica } \\
(\%)\end{array}$ & $\begin{array}{l}\text { Hev. Min. } \\
(\%)\end{array}$ & $\begin{array}{c}\text { Rock fr. } \\
(\%)\end{array}$ & $\begin{array}{l}\text { Volc. gl. } \\
(\%)\end{array}$ & $\begin{array}{l}\text { Auth. } \\
(\%)\end{array}$ \\
\hline $5 H-2-137$ & 38.87 & 8.57 & 3.26 & 0.88 & 8.80 & 8.19 & 79.31 & 1.15 & 0.96 & 8.57 & 7.47 & 8.80 & 6.13 \\
\hline $5 H-3-22$ & 38.42 & 0.16 & 5.84 & 8.88 & 8.88 & 8.88 & 71.81 & 1.42 & 2.36 & 8.47 & 7.72 & 8.88 & 10.71 \\
\hline $5 H-3-42$ & 38.62 & 8.80 & 7.43 & $\theta . \theta \dot{\theta}$ & 0.80 & 8.80 & 83.78 & 8.88 & 0.72 & 8.80 & 1.45 & 0.18 & 6.34 \\
\hline $5 H-3-62$ & 38.82 & 0.98 & 11.26 & 0.80 & 8.80 & 8.88 & 53.98 & 1.85 & 8.68 & 1.85 & 9.76 & 8.80 & 21.17 \\
\hline $5 H-3-82$ & 39.82 & $0.2 \theta$ & 8.60 & $\theta .00$ & 0.88 & 8.88 & 85.31 & 8.88 & 1.41 & 8.80 & 8.28 & 8.28 & 12.07 \\
\hline $5 H-3-182$ & 39.22 & 2.43 & 8.52 & 8.88 & $\theta . \theta 0$ & 8.28 & 69.57 & 8.41 & 2.43 & 8.61 & 8.72 & 8.88 & 7.18 \\
\hline $5 H-3-122$ & 39.40 & 8.92 & 3.51 & 8.88 & 8.80 & 0.80 & 82.47 & 0.80 & 8.55 & 8.88 & 4.86 & 8.88 & 8.49 \\
\hline $5 H-3-137$ & 39.68 & 1.86 & 9.73 & 8.80 & 0.88 & 0.00 & 61.24 & 1.59 & 1.24 & 0.88 & 8.14 & 0.08 & 16.11 \\
\hline $5 \mathrm{H}-4-22$ & 39.92 & 1.56 & 6.84 & 0.08 & $8 . \theta 8$ & 0.80 & 73.63 & 0.59 & 1.95 & 0.39 & 5.27 & 0.28 & 9.57 \\
\hline $5 H-4-42$ & 48.12 & 1.13 & 7.32 & $\theta .8 \theta$ & 8.80 & 0.80 & 52.35 & 0.88 & 0.80 & 8.80 & 36.82 & 0.38 & 2.81 \\
\hline $5 H-4-62$ & 48.32 & 1.11 & 3.14 & 0.08 & 8.80 & 0.88 & 62.55 & 3.69 & 2.77 & 8.37 & 13.84 & 8.80 & 12.36 \\
\hline $5 H-4-82$ & 48.52 & 6.68 & 6.41 & 0.39 & 8.80 & 8.88 & 62.52 & 8.88 & 8.39 & 8.80 & 17.89 & 8.88 & 6.60 \\
\hline $5 H-4-102$ & 48.72 & 0.39 & 8.97 & 8.08 & 8.80 & 8.88 & 16.15 & 0.19 & 0.39 & 8.19 & 4.47 & 8.80 & 77.84 \\
\hline $5 H-4-122$ & 48.98 & 1.47 & 5.34 & 8.80 & 8.88 & 8.80 & 84.98 & 8.88 & 0.18 & 0.88 & 3.68 & 8.88 & 4.42 \\
\hline $5 H-4-137$ & 41.87 & 1.35 & 3.29 & 0.88 & 8.88 & 8.80 & 74.88 & 8.58 & 1.74 & 8.19 & 6.58 & 8.77 & 11.41 \\
\hline $5 H-5-22$ & 41.42 & 0.80 & 2.84 & 0.80 & 8.80 & 0.80 & 80.68 & 0.95 & 3.83 & 0.38 & 7.39 & 8.76 & 3.98 \\
\hline $5 H-5-42$ & 41.62 & 1.12 & 4.18 & $\theta . \theta 0$ & 8.80 & 0.88 & 84.14 & 8.08 & 1.49 & $8 . \theta 8$ & 0.75 & 0.80 & $8.4 \theta$ \\
\hline $5 H-5-62$ & 41.82 & $\theta .80$ & 4.78 & 0.80 & 8.88 & 0.88 & 78.95 & 8.75 & 2.63 & 1.32 & 8.27 & 8.80 & 3.28 \\
\hline $5 H-5-82$ & 42.82 & $0.2 \theta$ & 8.88 & 0.00 & 0.88 & 0.08 & 97.84 & 8.08 & 0.88 & 8.39 & 8.28 & 8.88 & 2.17 \\
\hline $5 H-5-182$ & 42.22 & 8.88 & 8.80 & 8.80 & 8.80 & 8.88 & 89.76 & 1.28 & 1.61 & 8.68 & 6.02 & 8.88 & 8.88 \\
\hline $5 \mathrm{H}-5-122$ & 42.48 & 0.18 & 8.18 & 8.88 & 8.88 & 8.80 & 92.42 & 8.80 & 0.54 & 8.08 & 8.72 & 8.36 & 5.68 \\
\hline $5 H-5-141$ & 42.64 & 8.80 & 0.28 & 8.08 & 8.80 & 8.88 & 91.87 & 1.59 & 2.38 & 8.48 & 3.97 & 8.08 & $0.4 \theta$ \\
\hline $6 \mathrm{H}-1-22$ & 44.92 & 2.19 & 24.50 & 0.00 & 8.80 & 8.80 & 58.76 & 0.88 & 5.78 & 8.88 & 4.98 & 8.88 & 2.59 \\
\hline $6 H-1-59$ & 45.29 & 8.77 & 7.88 & 8.88 & 8.88 & $\theta .08$ & 89.62 & 8.88 & 0.38 & 8.88 & 8.77 & 8.88 & 8.58 \\
\hline $6 H-1-78$ & 45.48 & 8.88 & 8.88 & 8.80 & 8.88 & 0.80 & 91.28 & 0.37 & 0.93 & 1.67 & 3.53 & 0.88 & 2.23 \\
\hline $6 H-1-101$ & 45.71 & 8.88 & 8.88 & 0.88 & 8.08 & 8.88 & 95.11 & 8.08 & 8.88 & 8.88 & 2.15 & 8.28 & 2.54 \\
\hline $6 \mathrm{H}-1-122$ & 45.98 & 8.80 & 0.08 & 0.88 & 8.88 & 8.00 & 89.73 & 4.26 & 0.78 & 8.39 & 4.46 & 8.80 & 8.39 \\
\hline $6 \mathrm{H}-1-142$ & 46.12 & 0.56 & 4.45 & 8.88 & 8.80 & 8.80 & 82.88 & 8.88 & 0.37 & 8.80 & 8.93 & 8.80 & 11.69 \\
\hline $6 \mathrm{H}-2-22$ & 46.42 & 9.15 & 41.16 & 0.80 & 8.80 & 0.08 & 43.24 & 8.42 & 3.95 & 8.80 & 1.87 & 8.08 & 0.21 \\
\hline $6 \mathrm{H}-2-59$ & 46.79 & 8.80 & 0.28 & 8.88 & 8.88 & 0.88 & 94.69 & $\quad 8.88$ & 0.98 & 8.88 & 0.39 & 8.80 & 3.74 \\
\hline $6 \mathrm{H}-2-78$ & 46.92 & 0.39 & 5.79 & 0.08 & 8.88 & 8.80 & 87.84 & 8.77 & 1.54 & 0.58 & 2.78 & 8.00 & 0.39 \\
\hline $6 \mathrm{H}-2-102$ & 47.21 & 18.46 & 34.22 & 0.88 & 0.88 & 8.88 & 49.85 & 8.80 & 0.38 & 8.88 & 8.80 & 8.80 & 5.89 \\
\hline $6 \mathrm{H}-2-122$ & 47.48 & 5.44 & 57.28 & 8.80 & 8.88 & 0.88 & 26.41 & 8.39 & 0.97 & 8.19 & 6.82 & 8.80 & 3.38 \\
\hline $6 \mathrm{H}-2-142$ & 47.62 & 3.18 & 44.89 & 8.80 & 8.80 & 8.88 & 49.27 & 8.88 & 8.88 & 0.36 & 8.88 & 8.08 & 2.37 \\
\hline $6 \mathrm{H}-3-22$ & 47.92 & 0.98 & 35.55 & 8.80 & 8.80 & 0.88 & 54.10 & 8.59 & 2.73 & 8.39 & 5.27 & 8.88 & 8.28 \\
\hline $6 \mathrm{H}-3-59$ & 48.29 & 0.73 & 9.87 & 8.88 & 0.88 & 8.08 & 85.92 & 8.18 & 8.73 & 0.08 & 8.80 & 8.80 & 2.56 \\
\hline $6 \mathrm{H}-3-78$ & 48.48 & 2.84 & 47.78 & 0.88 & 0.88 & 8.88 & 46.36 & 1.87 & 0.71 & 0.18 & 8.71 & 8.88 & 0.36 \\
\hline $6 \mathrm{H}-3-181$ & 48.71 & 0.80 & 0.80 & 0.08 & 8.80 & 8.88 & 93.74 & 2.82 & 2.82 & 1.01 & 1.21 & 8.88 & 8.88 \\
\hline $6 \mathrm{H}-3-122$ & 48.98 & 0.73 & 37.16 & 0.88 & 8.80 & 8.73 & 55.56 & 8.73 & 3.18 & 0.36 & 0.91 & 8.80 & 8.36 \\
\hline $6 \mathrm{H}-3-142$ & 49.12 & 1.34 & 14.97 & 8.80 & 8.88 & 8.88 & 81.80 & 0.19 & 0.19 & 8.88 & 8.88 & 8.80 & 2.38 \\
\hline $6 \mathrm{H}-4-22$ & 49.42 & 3.68 & 59.66 & 0.88 & 8.88 & 8.88 & 34.47 & 8.38 & 0.38 & 0.38 & 0.38 & 8.88 & 8.08 \\
\hline $6 \mathrm{H}-4-42$ & 49.62 & 2.58 & 87.31 & 0.88 & 8.80 & 0.80 & 8.46 & 8.80 & 8.77 & 8.80 & 0.80 & 8.80 & 8.96 \\
\hline $6 \mathrm{H}-4-59$ & 49.79 & 1.95 & 68.75 & 0.88 & 8.80 & 0.81 & 32.74 & 0.33 & 0.80 & 8.33 & 0.65 & 0.08 & 1.14 \\
\hline $6 \mathrm{H}-4-78$ & 49.98 & 5.62 & 92.39 & $\theta .8 \theta$ & 8.80 & 0.36 & 0.91 & 8.80 & 8.88 & 0.80 & $8.8 \theta$ & 0.08 & 8.18 \\
\hline $6 \mathrm{H}-4-181$ & 58.21 & 8.02 & 81.56 & 8.88 & 8.88 & 8.88 & 8.22 & 8.88 & 1.68 & 8.88 & 8.80 & $\theta .80$ & $\theta .2 \theta$ \\
\hline $6 \mathrm{H}-4-117$ & 58.37 & 3.09 & 94.88 & 8.80 & 8.88 & 8.80 & 2.51 & 0.08 & 8.80 & 8.88 & 8.80 & 8.88 & 0.19 \\
\hline $6 \mathrm{H}-5-22$ & 58.92 & 2.99 & 14.76 & 8.80 & $\theta .08$ & 0.80 & 78.44 & 8.17 & 1.33 & 0.17 & 1.66 & 0.80 & 8.58 \\
\hline $6 H-5-59$ & 51.29 & 3.89 & 25.64 & 8.80 & 8.88 & 8.18 & 68.18 & 0.88 & 8.88 & 0.88 & 8.80 & 8.80 & 2.91 \\
\hline $6 \mathrm{H}-5-78$ & 51.48 & 4.86 & 6.78 & 0.88 & 8.88 & $\theta .80$ & 83.25 & 8.35 & 2.12 & 0.35 & 1.41 & 8.88 & 1.76 \\
\hline $6 \mathrm{H}-5-181$ & 51.71 & 8.18 & 18.83 & 0.88 & 8.88 & 0.88 & 78.43 & 8.88 & 8.88 & 8.88 & 8.88 & 8.88 & 2.64 \\
\hline
\end{tabular}


Table 1.4 (continued).




Table 1.4 (continued).

\begin{tabular}{|c|c|c|c|c|c|c|c|c|c|c|c|c|c|}
\hline \multirow[b]{2}{*}{$\begin{array}{c}\text { Sample } \\
\text { no. }\end{array}$} & \multirow[b]{2}{*}{$\begin{array}{l}\text { Depth } \\
\text { (mbsf) }\end{array}$} & \multicolumn{12}{|c|}{ Grain amount percentages } \\
\hline & & $\begin{array}{l}\text { Benthic } \\
\text { foram. } \\
(\%)\end{array}$ & $\begin{array}{c}\text { Plankt. } \\
\text { foram. } \\
(\%)\end{array}$ & $\begin{array}{c}\text { Rads. } \\
(\%)\end{array}$ & $\begin{array}{c}\text { Diatoms } \\
(\%)\end{array}$ & $\begin{array}{l}\text { Sponge } \\
\text { spicule } \\
(\%)\end{array}$ & $\begin{array}{c}\text { Quartz } \\
(\%)\end{array}$ & $\begin{array}{c}\text { Feldspar } \\
(\%)\end{array}$ & $\begin{array}{c}\text { Mica } \\
(\%)\end{array}$ & $\begin{array}{l}\text { Hev. Min. } \\
(\%)\end{array}$ & $\begin{array}{c}\text { Rock fr. } \\
(\%)\end{array}$ & $\begin{array}{l}\text { Volc. gl. } \\
(\%)\end{array}$ & $\begin{array}{l}\text { Auth. } \\
(\%)\end{array}$ \\
\hline $8 \mathrm{H}-6-22$ & 71.42 & 11.85 & 29.08 & 8.08 & 8.88 & 8.88 & 48.88 & 0.31 & 8.77 & 1.54 & 6.92 & 8.88 & 1.88 \\
\hline $8 H-6-42$ & 71.62 & 4.75 & 15.14 & 8.80 & 8.88 & 0.88 & 76.41 & 8.35 & 8.18 & 8.88 & 0.80 & 8.88 & 2.99 \\
\hline $8 H-6-68$ & 71.88 & 14.62 & 58.35 & 0.80 & 8.80 & 8.89 & 18.64 & 0.15 & 8.30 & 8.08 & 3.25 & 8.88 & 18.34 \\
\hline $9 \mathrm{H}-1-22$ & 73.43 & 3.03 & 77.78 & 8.80 & 0.80 & 8.80 & 12.12 & 8.87 & 8.08 & 0.80 & 3.75 & 8.88 & 2.16 \\
\hline $9 H-1-48$ & 73.68 & 0.97 & 61.81 & 8.80 & 0.08 & $\theta .08$ & 34.78 & $\theta .80$ & $\theta .8 \theta$ & $\theta .08$ & 8.80 & 8.88 & 2.92 \\
\hline $9 H-1-59$ & 73.79 & 3.87 & 92.44 & 0.18 & 8.88 & 0.78 & 8.78 & 8.88 & 0.80 & 0.08 & 8.80 & 8.88 & 1.23 \\
\hline $9 H-1-82$ & 74.82 & 6.30 & 87.57 & 0.80 & 8.80 & 8.80 & 1.93 & 0.88 & 8.08 & 8.88 & 8.80 & 8.88 & 2.98 \\
\hline $9 H-1-102$ & 74.22 & 8.52 & 75.19 & $0 . \theta 0$ & 8.80 & 8.80 & 2.27 & 0.80 & 0.80 & 8.88 & 0.57 & 8.80 & 12.88 \\
\hline $9 H-1-123$ & 74.43 & 13.67 & 17.97 & 0.80 & 8.80 & 0.80 & 18.36 & 8.88 & $8.0 \theta$ & 0.88 & 0.88 & 8.68 & 49.62 \\
\hline $9 H-1-142$ & 74.62 & 0.98 & 2.25 & 8.88 & 8.80 & 8.80 & 73.46 & 0.98 & 15.89 & 1.28 & 4.50 & 8.80 & 8.98 \\
\hline $9 \mathrm{H}-2-23$ & 74.93 & 8.38 & 4.95 & 0.80 & 8.88 & 0.80 & 88.19 & 0.80 & 0.19 & 0.57 & 0.80 & $\theta . \theta 8$ & 5.52 \\
\hline $9 H-2-48$ & 75.18 & 2.81 & 16.14 & 0.80 & $\theta . \theta 8$ & 0.80 & 79.17 & 0.56 & 0.80 & 0.08 & 8.94 & 8.80 & 8.19 \\
\hline $9 \mathrm{H}-2-59$ & 75.29 & 0.95 & 9.51 & 0.80 & $\theta . \theta \theta$ & 0.88 & 85.36 & 8.88 & 8.76 & 0.38 & 0.00 & $\theta .80$ & 3.84 \\
\hline $9 H-2-82$ & 75.52 & 0.74 & 4.28 & $\theta . \theta \theta$ & 8.88 & 8.08 & 87.71 & 8.74 & 4.84 & 8.56 & 0.37 & $8 . \theta \theta$ & 0.74 \\
\hline $9 H-2-182$ & 75.72 & 2.89 & 18.82 & 0.88 & 8.88 & 8.08 & 83.68 & 8.08 & 0.19 & 0.19 & 8.38 & 0.80 & 2.89 \\
\hline $9 H-2-123$ & 75.93 & 2.55 & 35.89 & 0.88 & 0.88 & 0.88 & 53.11 & 0.16 & 4.31 & 0.16 & 1.59 & 0.80 & 2.55 \\
\hline $9 H-2-142$ & 76.12 & 0.32 & 6.87 & 8.08 & 8.80 & 0.80 & 80.99 & 8.16 & 18.22 & 0.08 & 0.64 & 0.88 & 0.64 \\
\hline $9 H-3-23$ & 76.43 & 8.43 & 77.25 & $\theta . \theta 8$ & $\theta .80$ & 1.57 & 1.18 & 8.68 & 4.12 & 8.80 & 0.78 & 8.80 & 5.29 \\
\hline $9 \mathrm{H}-3-48$ & 76.60 & 6.76 & 55.67 & 8.80 & 0.80 & 0.80 & 1.99 & 0.80 & 0.08 & 8.80 & 8.80 & 8.80 & 35.19 \\
\hline $9 H-3-59$ & 76.79 & 4.18 & 55.61 & 8.80 & $0 . \theta 0$ & 0.36 & 21.75 & 8.80 & 1.25 & 8.80 & 1.87 & 0.80 & 14.97 \\
\hline $9 H-3-82$ & 77.82 & 3.33 & 48.92 & 8.80 & 0.80 & 0.80 & 35.83 & 0.80 & 0.08 & 0.08 & 8.80 & 0.80 & 12.72 \\
\hline $9 \mathrm{H}-3-182$ & 77.22 & 6.21 & 44.33 & 0.18 & 8.88 & 0.35 & 14.81 & 8.18 & 0.71 & 0.18 & 0.71 & 8.80 & 33.16 \\
\hline $9 \mathrm{H}-3-123$ & 77.43 & 13.98 & 61.14 & 0.80 & 8.80 & 8.80 & 15.85 & 0.80 & 8.19 & 0.80 & 8.80 & 8.80 & 9.71 \\
\hline $9 \mathrm{H}-3-142$ & 77.62 & 8.88 & $8.8 \theta$ & 0.80 & 0.80 & 0.37 & 89.44 & 8.93 & 2.22 & 0.88 & 5.19 & 8.80 & 1.85 \\
\hline $9 \mathrm{H}-4-23$ & 78.43 & 8.38 & 31.57 & 0.88 & 0.88 & 8.80 & 63.14 & 0.80 & 8.19 & 0.19 & 0.88 & 0.19 & 4.35 \\
\hline $9 H-4-48$ & 78.68 & 0.37 & 0.55 & 8.80 & 0.88 & 0.88 & 87.48 & 1.18 & 8.74 & 0.74 & 6.45 & 0.88 & 2.58 \\
\hline $9 H-4-59$ & 78.79 & 8.78 & 15.63 & 8.88 & 8.88 & 0.80 & 76.76 & 8.80 & 2.15 & 0.88 & 8.80 & 0.78 & 3.91 \\
\hline $9 H-4-82$ & 79.82 & 1.83 & 11.16 & 8.80 & 8.80 & 0.28 & 78.78 & 8.41 & 2.23 & 8.00 & 3.45 & 8.88 & 1.83 \\
\hline $9 \mathrm{H}-4-182$ & 79.22 & 0.56 & 2.23 & $\theta .08$ & 0.88 & 0.80 & 84.81 & 0.93 & 1.38 & 0.93 & 6.32 & $\theta . \theta 8$ & 3.72 \\
\hline $9 \mathrm{H}-5-23$ & 79.93 & 10.73 & 68.15 & 0.00 & 0.88 & 0.08 & 7.85 & 0.80 & 3.07 & 0.19 & 8.57 & 8.80 & 16.67 \\
\hline $9 H-5-48$ & 80.18 & 4.48 & 4.48 & 8.80 & 8.88 & 8.00 & 78.05 & 8.80 & 1.18 & 0.17 & 0.85 & 8.80 & 15.91 \\
\hline $9 \mathrm{H}-5-59$ & 88.29 & 8.08 & 0.78 & 8.88 & 8.88 & 8.80 & 98.12 & 0.39 & 5.23 & 8.19 & 0.19 & 8.80 & 3.18 \\
\hline $9 H-5-82$ & 80.52 & 0.17 & 1.84 & 0.08 & 8.88 & 0.80 & 72.37 & 1.21 & 15.89 & 1.21 & 6.91 & 0.88 & 1.21 \\
\hline $9 H-5-182$ & 88.72 & 8.80 & 0.55 & 8.80 & 8.88 & 8.88 & 88.64 & 8.37 & 3.85 & 0.18 & 8.88 & 8.08 & 6.84 \\
\hline $9 H-5-123$ & 88.93 & 0.17 & 0.33 & 0.80 & 0.08 & 0.88 & 88.87 & 8.58 & 1.33 & 1.58 & 15.28 & 0.88 & 0.83 \\
\hline $18 \mathrm{H}-2-22$ & 84.42 & 3.15 & 53.58 & 8.80 & 8.80 & 0.80 & 32.87 & 0.17 & 0.35 & 0.35 & 6.12 & 8.08 & 2.27 \\
\hline $18 \mathrm{H}-2-41$ & 84.61 & 10.31 & 46.58 & 8.08 & 0.88 & 8.88 & 41.44 & 0.80 & 0.19 & 0.19 & 0.78 & 8.88 & 8.39 \\
\hline $18 \mathrm{H}-2-57$ & 84.78 & 5.18 & 79.65 & 0.38 & 8.88 & 0.80 & 11.52 & 8.80 & 8.96 & 8.88 & 8.88 & 0.88 & 1.92 \\
\hline $10 \mathrm{H}-2-101$ & 85.22 & 8.57 & 15.28 & 0.19 & 8.88 & 0.00 & 79.81 & 0.80 & 0.57 & 0.80 & 0.19 & 0.08 & 3.40 \\
\hline $18 \mathrm{H}-2-121$ & 85.42 & 1.32 & 74.18 & 8.88 & 8.88 & 8.88 & 22.84 & 8.80 & 0.66 & 0.80 & 0.16 & 0.88 & 1.15 \\
\hline $18 \mathrm{H}-2-142$ & 85.63 & 8.77 & 94.23 & 0.38 & 8.88 & 0.19 & 0.96 & 0.80 & 0.19 & 8.88 & 8.88 & 8.88 & 2.88 \\
\hline $10 \mathrm{H}-3-22$ & 85.92 & 24.25 & 46.43 & 0.80 & 8.80 & 0.38 & 2.63 & 0.08 & 8.56 & 8.08 & 0.38 & 0.80 & 24.62 \\
\hline $10 \mathrm{H}-3-41$ & 86.11 & 4.75 & 8.37 & 8.80 & 0.80 & 8.80 & 5.13 & 0.80 & 0.19 & 8.88 & 8.88 & 8.88 & 88.99 \\
\hline $10 \mathrm{H}-3-57$ & 86.28 & 10.52 & 86.81 & 8.88 & 8.88 & 8.88 & 8.88 & 8.88 & 0.18 & 8.88 & 8.88 & 8.80 & 1.68 \\
\hline $18 \mathrm{H}-3-78$ & 86.48 & 7.29 & 38.84 & 0.23 & 8.88 & 0.80 & 18.91 & 0.08 & 8.23 & 8.88 & 8.88 & 0.88 & 34.48 \\
\hline $18 \mathrm{H}-3-181$ & 86.71 & 2.93 & 98.63 & 8.08 & 8.80 & 0.28 & 0.59 & 8.80 & 0.39 & 8.80 & 0.88 & 8.80 & 4.69 \\
\hline $18 \mathrm{H}-3-121$ & 86.92 & 2.97 & 78.69 & 0.19 & 0.88 & 2.23 & 1.11 & 8.88 & $8 . \theta \theta$ & 8.80 & 0.80 & 8.80 & 28.96 \\
\hline $10 \mathrm{H}-3-142$ & 87.12 & 0.80 & 0.36 & 8.80 & 8.88 & 0.55 & 79.78 & 8.55 & 13.84 & 8.36 & 2.91 & 8.80 & 1.64 \\
\hline $18 \mathrm{H}-4-21$ & 87.42 & 0.99 & 95.28 & 8.80 & 8.88 & 8.88 & 0.33 & 8.88 & 8.80 & 8.80 & 8.88 & 8.88 & 2.81 \\
\hline $18 H-4-41$ & 87.61 & 38.21 & 37.67 & 8.08 & 8.88 & 8.19 & 18.13 & 8.80 & 4.21 & 8.88 & 8. 38 & 8.88 & 15.68 \\
\hline
\end{tabular}


Table 1.4 (continued).

\begin{tabular}{|c|c|c|c|c|c|c|c|c|c|c|c|c|c|}
\hline \multirow[b]{2}{*}{$\begin{array}{c}\text { Sample } \\
\text { no. }\end{array}$} & \multirow[b]{2}{*}{$\begin{array}{l}\text { Depth } \\
\text { (mbsf) }\end{array}$} & \multicolumn{12}{|c|}{ Grain amount percentages } \\
\hline & & $\begin{array}{l}\text { Benthic } \\
\text { foram. } \\
(\%)\end{array}$ & $\begin{array}{l}\text { Plankt. } \\
\text { foram. } \\
(\%)\end{array}$ & $\begin{array}{l}\text { Rads. } \\
(\%)\end{array}$ & $\begin{array}{c}\text { Diatoms } \\
(\%)\end{array}$ & $\begin{array}{c}\text { Sponge } \\
\text { spicule } \\
(\%)\end{array}$ & $\begin{array}{c}\text { Quartz } \\
(\%)\end{array}$ & $\begin{array}{c}\text { Feldspar } \\
(\%)\end{array}$ & $\begin{array}{l}\text { Mica } \\
(\%)\end{array}$ & $\begin{array}{l}\text { Hev. Min. } \\
(\%)\end{array}$ & $\begin{array}{l}\text { Rock fr. } \\
(\%)\end{array}$ & $\begin{array}{l}\text { Volc. gl. } \\
(\%)\end{array}$ & $\begin{array}{l}\text { Auth. } \\
(\%)\end{array}$ \\
\hline $18 \mathrm{H}-4-57$ & 87.78 & 0.19 & 8.39 & 8.88 & 8.08 & 0.19 & 93.06 & 8.60 & 8.80 & 8.88 & 8.88 & 8.19 & 5.97 \\
\hline $18 \mathrm{H}-4-78$ & 87.98 & 0.65 & 2.43 & 8.80 & 8.80 & 8.49 & 68.93 & 0.88 & 25.89 & 8.49 & 8.49 & 8.88 & 8.65 \\
\hline $18 \mathrm{H}-4-181$ & 88.22 & 1.17 & 1.17 & 8.88 & 8.88 & 8.28 & 93.36 & 8.88 & 2.15 & 8.88 & 8.88 & 8.80 & 1.95 \\
\hline $18 \mathrm{H}-4-121$ & 88.41 & 8.98 & 1.96 & 8.80 & 8.80 & 8.08 & 86.69 & 0.28 & 1.17 & 8.39 & 2.15 & 0.88 & 6.46 \\
\hline $10 \mathrm{H}-5-21$ & 88.91 & 18.87 & 64.89 & 8.88 & 8.88 & 0.18 & 18.68 & 8.88 & 8.66 & 8.55 & 2.58 & 0.88 & 2.21 \\
\hline $10 \mathrm{H}-5-41$ & 89.11 & 0.58 & 4.22 & 8.80 & 8.80 & $\theta . \theta \theta$ & 87.72 & 0.80 & 8.77 & 8.80 & 8.80 & $\theta .80$ & 6.14 \\
\hline $10 \mathrm{H}-5-57$ & 89.28 & 9.63 & 75.88 & 0.16 & 8.08 & 8.08 & 12.11 & 8.88 & 0.78 & 8.16 & 8.47 & 0.80 & 0.78 \\
\hline $18 \mathrm{H}-5-78$ & 89.48 & 3.85 & 14.67 & 8.80 & 8.88 & 8.19 & 57.33 & 8.68 & 0.19 & 8.88 & 8.88 & 8.88 & 24.19 \\
\hline $10 \mathrm{H}-5-181$ & 89.71 & 2.55 & 22.99 & 0.88 & 8.80 & 0.18 & 67.15 & 8.36 & 2.92 & 8.55 & 1.89 & 0.80 & 2.19 \\
\hline $18 \mathrm{H}-5-121$ & 89.92 & 12.96 & 35.38 & 8.88 & 0.88 & 8.80 & 39.28 & 8.80 & 8.17 & 8.80 & 0.17 & 8.88 & 18.38 \\
\hline $18 \mathrm{H}-6-21$ & 98.42 & 8.36 & 5.25 & 8.88 & 8.80 & 8.18 & 72.28 & 0.36 & 19.75 & 0.54 & 8.91 & 0.80 & 8.36 \\
\hline $18 \mathrm{H}-6-41$ & 98.62 & 4.14 & 31.16 & 8.88 & 8.88 & 0.88 & 51.88 & 8.28 & 11.05 & 8.59 & 0.39 & 8.80 & 0.59 \\
\hline $11 H-1-22$ & 92.42 & 0.95 & 1.89 & $\theta . \theta 0$ & 8.80 & 0.80 & 98.36 & 0.57 & 2.88 & 0.38 & 1.51 & 8.88 & 1.89 \\
\hline $11 H-1-42$ & 92.62 & 8.77 & 1.15 & 8.80 & 8.80 & 1.35 & 92.58 & 8.00 & 1.15 & 8.80 & 0.19 & 8.88 & 2.88 \\
\hline $11 \mathrm{H}-1-68$ & 92.88 & 0.77 & 0.38 & 8.88 & 8.88 & 0.08 & 87.74 & 0.57 & 6.51 & 1.34 & 1.34 & 8.08 & 1.34 \\
\hline $11 \mathrm{H}-1-81$ & 93.81 & 0.38 & 8.08 & 8.68 & 8.88 & 0.19 & 91.83 & 0.57 & 8.57 & 8.80 & 8.57 & 8.80 & 5.89 \\
\hline $11 H-1-180$ & 93.28 & 1.93 & 52.53 & $8 . \theta 0$ & 0.80 & 0.30 & 39.58 & 0.15 & 1.84 & 8.30 & 1.84 & 0.80 & 2.98 \\
\hline $11 \mathrm{H}-1-122$ & 93.48 & 2.67 & 10.58 & 8.80 & 8.80 & 8.38 & 88.34 & 0.19 & 8.38 & 8.08 & 8.80 & 0.00 & 5.53 \\
\hline $11 \mathrm{H}-1-142$ & 93.62 & 3.77 & 11.88 & 8.88 & 8.88 & 8.88 & 76.89 & 8.16 & 1.64 & 8.82 & 1.15 & 8.88 & 3.61 \\
\hline $11 \mathrm{H}-2-22$ & 93.92 & 3.37 & 58.89 & 8.80 & 8.80 & 1.19 & 37.83 & $8 . \theta 8$ & 8.28 & 8.88 & 8.80 & 8.08 & 7.13 \\
\hline $11 \mathrm{H}-2-39$ & 94.89 & 1.37 & 94.51 & 0.80 & 0.08 & 8.88 & 2.94 & 8.08 & 0.78 & 8.88 & 8.08 & $\theta .80$ & 0.28 \\
\hline $11 \mathrm{H}-2-63$ & 94.33 & 2.32 & 77.18 & 8.80 & 8.88 & 0.58 & 17.21 & 0.80 & 8.19 & 8.00 & 0.88 & 0.80 & 2.13 \\
\hline $11 \mathrm{H}-2-81$ & 94.51 & 6.91 & 45.38 & 8.88 & 8.88 & 0.88 & 45.38 & 8.88 & 0.38 & 8.19 & 0.19 & 8.80 & 1.54 \\
\hline $11 \mathrm{H}-2-100$ & 94.78 & 2.61 & 43.47 & 0.88 & 8.88 & 8.75 & 58.37 & 8.68 & 8.88 & 8.80 & 8.80 & 0.80 & 2.88 \\
\hline $11 \mathrm{H}-2-122$ & 94.92 & 16.31 & 72.82 & 8.80 & 8.80 & 0.16 & 6.59 & 0.88 & 8.33 & 8.88 & 0.49 & 8.80 & 3.13 \\
\hline $11 \mathrm{H}-2-142$ & 95.14 & 24.13 & 37.85 & 8.88 & 8.88 & 8.17 & 16.69 & 8.80 & 8.17 & 8.88 & 8.80 & 0.17 & 19.81 \\
\hline $11 \mathrm{H}-3-22$ & 95.42 & 18.59 & 19.57 & 8.80 & 8.88 & 8.88 & 53.78 & 8.88 & 1.15 & 0.16 & 0.99 & $\theta .8 \theta$ & 5.76 \\
\hline $11 \mathrm{H}-3-42$ & 95.62 & 3.64 & 3.87 & 8.88 & 8.88 & 0.08 & 87.55 & 8.88 & 1.15 & 0.88 & 0.80 & 8.08 & 4.41 \\
\hline $11 \mathrm{H}-3-68$ & 95.80 & 3.96 & 2.52 & 8.88 & 0.08 & 8.88 & 84.89 & 8.88 & 6.47 & 8.98 & 8.98 & 8.80 & 8. 18 \\
\hline $11 \mathrm{H}-3-81$ & 96.01 & 0.19 & 0.38 & 8.88 & 8.80 & 8.88 & 94.43 & 8.88 & 8.77 & 8.88 & 0.19 & 8.80 & 4.83 \\
\hline $11 \mathrm{H}-3-180$ & 96.28 & 8.36 & 8.08 & 8.88 & 8.88 & 8.80 & 91.37 & 0.18 & 2.34 & 8.54 & 3.68 & 8.88 & 1.62 \\
\hline $11 \mathrm{H}-3-122$ & 96.48 & 1.35 & 0.08 & 8.80 & 8.88 & 0.80 & 94.61 & 0.88 & 8.51 & 8.88 & 0.08 & 0.88 & 3.54 \\
\hline $11 \mathrm{H}-3-142$ & 96.62 & 3.48 & 2.38 & 8.88 & 8.88 & 8.88 & 84.87 & 0.88 & 4.21 & 1.18 & 2.56 & 8.80 & 2.28 \\
\hline $11 \mathrm{H}-4-22$ & 96.92 & 4.17 & 27.46 & 8.88 & 8.80 & 0.88 & 62.69 & 8.80 & 8.19 & 0.19 & 8.80 & 0.88 & 3.22 \\
\hline $11 \mathrm{H}-4-42$ & 97.14 & 5.21 & 25.85 & 8.08 & 8.80 & 0.36 & 56.73 & 0.54 & 8.44 & 0.36 & 1.44 & 8.80 & 8.36 \\
\hline $11 \mathrm{H}-4-68$ & 97.38 & 4.84 & 25.58 & 8.88 & 8.88 & 8.88 & 61.24 & 0.88 & 8.19 & 8.08 & 0.08 & 8.19 & 7.95 \\
\hline $11 H-4-81$ & 97.51 & 8.63 & 62.85 & 8.80 & 0.88 & 8.88 & 22.89 & $\theta .08$ & 5.22 & 8.80 & 2.81 & 8.88 & 8.88 \\
\hline $11 \mathrm{H}-4-188$ & 97.78 & 0.80 & 8.19 & 8.88 & 8.80 & 0.88 & 93.43 & 8.80 & 1.13 & 8.88 & 8.19 & 8.56 & 4.58 \\
\hline $11 \mathrm{H}-4-122$ & 97.92 & 0.28 & 0.80 & 0.88 & 8.88 & 0.88 & 92.43 & 0.80 & 4.29 & 8.41 & 2.25 & 0.80 & 8.41 \\
\hline $11 \mathrm{H}-4-142$ & 98.14 & 8.58 & 2.31 & 8.88 & 8.88 & 0.19 & 93.85 & 8.88 & 0.96 & 8.80 & 0.88 & 0.19 & 1.92 \\
\hline $11 \mathrm{H}-5-22$ & 98.42 & 7.88 & 58.18 & 0.88 & 8.88 & 8.88 & 28.28 & $\theta . \theta 0$ & 2.02 & 8.81 & 2.83 & 8.80 & 8.80 \\
\hline $11 \mathrm{H}-5-42$ & 98.62 & 8.28 & 8.28 & 8.80 & 8.80 & 0.88 & 96.67 & 8.80 & 8. 59 & 8.28 & 8.88 & 8.80 & 2.16 \\
\hline $11 \mathrm{H}-5-68$ & 98.88 & 6.97 & 61.22 & 8.88 & 8.88 & 8.13 & 27.76 & 0.88 & 8.89 & 8.25 & 2.28 & 0.13 & 8.38 \\
\hline $11 H-5-88$ & 99.88 & 8.93 & 2.84 & 8.88 & 8.88 & 8.88 & 94.86 & 8.88 & 8.37 & 8.88 & 8.88 & 0.19 & 2.41 \\
\hline $11 \mathrm{H}-5-188$ & 99.30 & 0.19 & 8.56 & 8.80 & 8.80 & 0.88 & 88.87 & 0.93 & 1.48 & 8.74 & 5.57 & 0.19 & 1.48 \\
\hline $11 \mathrm{H}-5-122$ & 99.52 & 1.88 & 8.88 & 8.88 & 8.88 & 0.80 & 85.18 & 0.18 & 8.88 & 8.80 & 8.80 & 0.80 & 4.67 \\
\hline $11 \mathrm{H}-6-22$ & 99.92 & 11.86 & 73.98 & 8.88 & 0. 80 & 8.86 & 10.88 & 0.80 & 1.86 & 0.80 & 0.08 & 8.88 & 1.77 \\
\hline $11 \mathrm{H}-6-42$ & 180.14 & 1.31 & 5.79 & 8.88 & 0.80 & 8.88 & 83.55 & 8.37 & 8.88 & 8.80 & 8.80 & 1.31 & 7.18 \\
\hline $11 \mathrm{H}-6-68$ & 180.38 & 8.78 & 86.58 & 8.88 & 8.88 & $8 . \theta 8$ & 0.95 & 8.08 & 8.80 & 8.88 & $8 . \theta 0$ & 0.80 & 2.84 \\
\hline $11 \mathrm{H}-6-88$ & 180.58 & 18.19 & 83.96 & 0.19 & 8.88 & 0.38 & 0.57 & 8.80 & 8.80 & 8.80 & 8.88 & 8.88 & 3.96 \\
\hline
\end{tabular}


Table 1.4 (continued).

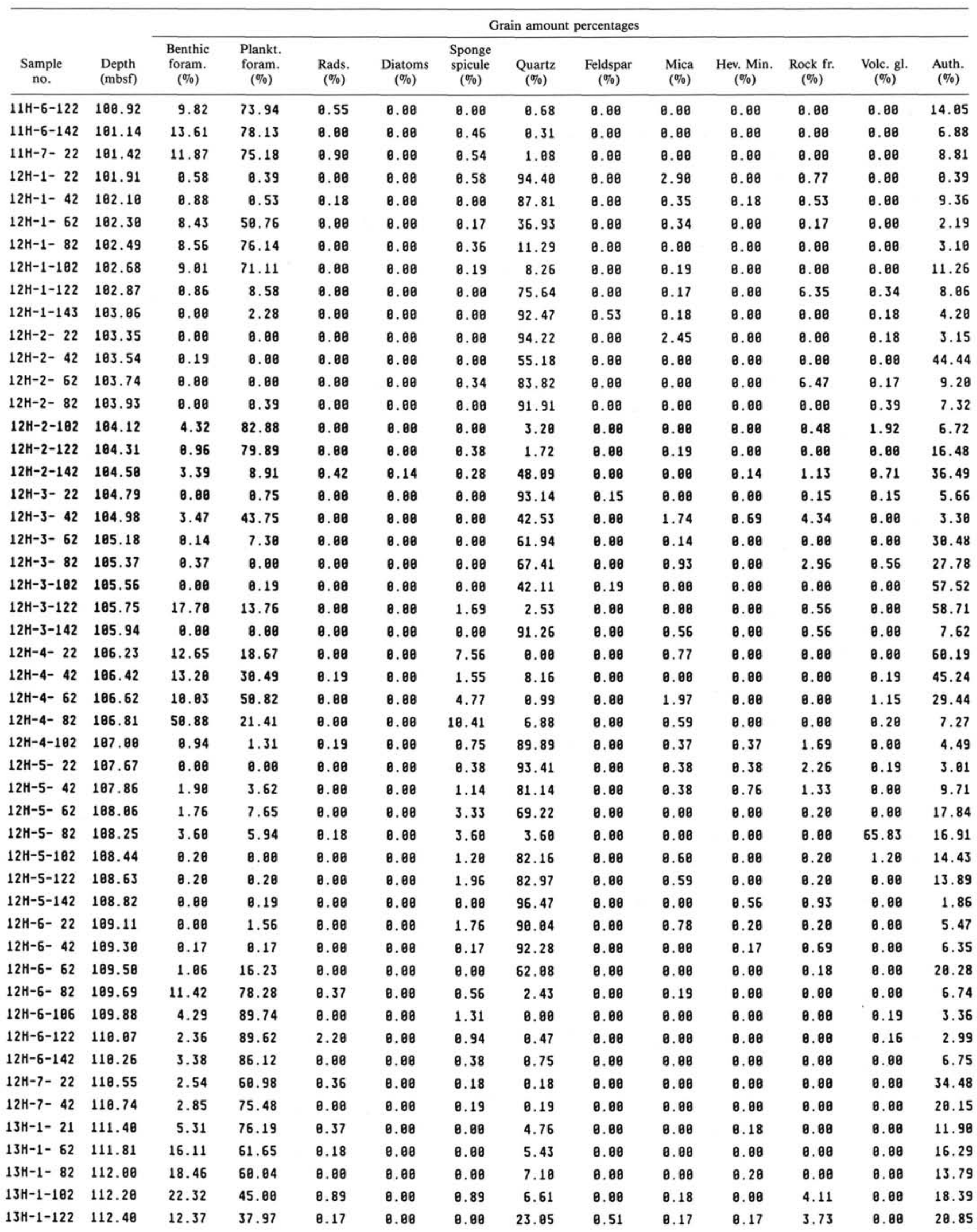


Table 1.4 (continued).

\begin{tabular}{|c|c|c|c|c|c|c|c|c|c|c|c|c|c|}
\hline \multirow[b]{2}{*}{$\begin{array}{c}\text { Sample } \\
\text { no. }\end{array}$} & \multirow[b]{2}{*}{$\begin{array}{l}\text { Depth } \\
\text { (mbsf) }\end{array}$} & \multicolumn{12}{|c|}{ Grain amount percentages } \\
\hline & & $\begin{array}{l}\text { Benthic } \\
\text { foram. } \\
(\%)\end{array}$ & $\begin{array}{l}\text { Plankt. } \\
\text { foram. } \\
(\%)\end{array}$ & $\begin{array}{l}\text { Rads. } \\
(\%)\end{array}$ & $\begin{array}{c}\text { Diatoms } \\
(\%)\end{array}$ & $\begin{array}{c}\text { Sponge } \\
\text { spicule } \\
(\%)\end{array}$ & $\begin{array}{l}\text { Quartz } \\
(\%)\end{array}$ & $\begin{array}{l}\text { Feldspar } \\
(\%)\end{array}$ & $\begin{array}{l}\text { Mica } \\
(\%)\end{array}$ & $\begin{array}{l}\text { Hev. Min. } \\
\quad(\%)\end{array}$ & $\begin{array}{l}\text { Rock fr. } \\
(\%)\end{array}$ & $\begin{array}{l}\text { Volc. gl. } \\
(\%)\end{array}$ & $\begin{array}{l}\text { Auth. } \\
(\%)\end{array}$ \\
\hline $13 \mathrm{H}-1-145$ & 112.62 & 25.53 & 37.88 & 0.32 & 8.08 & 0.16 & 21.65 & 0.48 & 0.80 & 8.80 & 2.58 & 8.88 & 18.66 \\
\hline $13 \mathrm{H}-2-21$ & 112.88 & 0.79 & 0.80 & 8.88 & 8.88 & 0.28 & 96.83 & 0.80 & 8.48 & 8.28 & 1.19 & 8.00 & 8.48 \\
\hline $13 \mathrm{H}-2-42$ & 113.88 & 8.88 & 0.80 & 0.88 & 8.00 & 8.88 & 96.79 & 0.08 & 0.28 & 8.88 & 1.81 & 8.88 & 8.48 \\
\hline $13 \mathrm{H}-2-62$ & 113.28 & 6.02 & 3.41 & 0.88 & 8.88 & 8.98 & 87.88 & 8.08 & 8.33 & 8.16 & 8.16 & 8.88 & 8.98 \\
\hline $13 \mathrm{H}-2-82$ & 113.47 & 1.91 & 1.34 & 8.19 & 8. 88 & 8.19 & 49.43 & 8.88 & 8.80 & 8.88 & 8.80 & 8.57 & 46.37 \\
\hline $13 \mathrm{H}-2-182$ & 113.67 & 8.79 & 8.88 & 8.28 & 8.88 & 8.88 & 94.86 & 8.88 & 8.80 & 8.88 & 8.88 & 8.08 & 4.95 \\
\hline $13 \mathrm{H}-2-122$ & 113.87 & 19.58 & 5.88 & 8.68 & 8.80 & 0.88 & 55.88 & 8.60 & 0.67 & 8.17 & 0.34 & 8.80 & 17.65 \\
\hline $13 \mathrm{H}-3-21$ & 114.35 & 0.28 & 0.28 & 0.80 & 8.80 & 0.80 & 95.84 & 8.00 & 8.80 & 0.99 & 8.59 & $\theta . \theta 0$ & 2.18 \\
\hline $13 H-3-145$ & 114.58 & 0.80 & 0.28 & 0.59 & 8.00 & 8.88 & 88.26 & 0.88 & 1.17 & 8.78 & 7.85 & 8.80 & 1.96 \\
\hline $13 \mathrm{H}-4-21$ & 115.82 & 1.31 & $8 . \theta 8$ & 0.88 & $\theta .80$ & 8.88 & 94.93 & 8.88 & 8.80 & 1.58 & 1.58 & 8.80 & 0.75 \\
\hline $13 \mathrm{H}-4-42$ & 116.02 & 23.23 & 18.24 & 0.88 & 8.88 & 8.08 & 58.27 & 0.00 & 8.80 & 0.39 & 1.77 & 8.08 & 5.71 \\
\hline $13 \mathrm{H}-4-62$ & 116.22 & 16.63 & 29.25 & 0.80 & 8.88 & 0.88 & 51.82 & 8.08 & 0.19 & 8.76 & 8.88 & 8.80 & 8.96 \\
\hline $13 \mathrm{H}-4-82$ & 116.41 & 2.34 & 0.39 & 8.88 & 8.88 & 8.88 & 94.14 & 0.80 & 0.59 & 1.17 & 8.78 & 8.00 & 8.59 \\
\hline $13 H-4-182$ & 116.61 & 7.62 & 4.83 & $\theta . \theta 8$ & 8.88 & 8.88 & 86.86 & 8.08 & 0.19 & 8.93 & 0.37 & 8.88 & 0.80 \\
\hline $13 \mathrm{H}-4-122$ & 116.81 & 0.19 & 8.80 & 0.19 & 8.08 & 0.88 & 91.85 & 8.88 & 0.56 & 1.38 & 8.56 & $\theta .8 \theta$ & 5.37 \\
\hline $13 \mathrm{H}-5-21$ & 117.29 & 16.28 & 3.82 & $\theta . \theta \theta$ & 8.08 & 8.88 & 45.97 & 8.17 & 0.88 & 8.50 & 6.84 & 8.88 & 28.82 \\
\hline $13 \mathrm{H}-5-42$ & 117.49 & 3.88 & 8.80 & 0.88 & 0.88 & 8.88 & 74.31 & 2.03 & $8.8 \theta$ & 1.85 & 13.49 & 0.18 & 4.25 \\
\hline $13 H-5-62$ & 117.69 & 3.51 & 0.15 & 8.88 & 8.88 & 8.88 & 77.71 & 5.88 & 3.82 & 1.53 & 5.65 & 8.88 & 1.83 \\
\hline $13 \mathrm{H}-5-86$ & 117.92 & 8.88 & 0.19 & 8.88 & 0.80 & 8.88 & 96.11 & 8.88 & 8.80 & 8.58 & 1.56 & 8.08 & 1.56 \\
\hline $13 \mathrm{H}-6-21$ & 118.76 & 30.80 & 7.88 & 8.80 & 8.80 & 8.88 & 32.31 & 8.80 & 0.08 & 8.00 & 8.08 & 8.08 & 29.42 \\
\hline $13 \mathrm{H}-6-42$ & 118.96 & 22.84 & 5.84 & 0.88 & $\theta . \theta 8$ & 0.88 & 11.33 & 0.88 & 0.88 & 8.68 & 8.80 & 8.88 & 68.79 \\
\hline $13 \mathrm{H}-6-62$ & 119.16 & 41.46 & 16.78 & 8.88 & 8.88 & 8.88 & 8.44 & 8.08 & 0.19 & 8.88 & 8.88 & 0.80 & 32.65 \\
\hline $13 \mathrm{H}-6-82$ & 119.35 & 23.57 & 14.14 & 0.88 & 8.88 & 8.41 & 2.46 & 8.88 & 0.08 & 8.88 & $\theta .80$ & 8.88 & 59.82 \\
\hline $13 \mathrm{H}-7-21$ & 119.56 & 3.65 & 14.74 & 0.15 & 8.15 & 1.46 & 17.66 & 0.00 & 0.15 & 8.44 & 8.44 & 0.15 & 61.82 \\
\hline $13 \mathrm{H}-7-42$ & 119.77 & $\theta . \theta 8$ & 0.17 & 0.88 & 8.80 & 8.88 & 91.39 & 8.88 & 1.18 & 0.68 & 1.69 & 8.68 & 4.22 \\
\hline $13 \mathrm{H}-7-62$ & 119.96 & 12.85 & 46.67 & 0.68 & 8.80 & 8.88 & 1.63 & 0.80 & 8.49 & 0.98 & 0.16 & 0.16 & 35.77 \\
\hline $13 \mathrm{H}-7-84$ & 128.16 & 4.50 & 51.33 & 8.08 & 8.88 & 8.80 & 5.17 & 8.80 & 0.08 & 0.80 & 0.17 & 8.08 & 38.83 \\
\hline $13 \mathrm{H}-7-182$ & 128.35 & 31.88 & 44.58 & 0.88 & 8.80 & 8.42 & 8.54 & 8.80 & 8.80 & 8.63 & 8.21 & 8.80 & 13.75 \\
\hline $13 \mathrm{H}-7-122$ & 128.55 & 32.61 & 43.81 & 0.80 & 8.80 & 8.28 & 8. 98 & 0.08 & 0.08 & 8.80 & 0.80 & 8.80 & 28.63 \\
\hline $14 \mathrm{H}-1-21$ & 128.91 & 18.53 & 3.83 & 0.00 & 8.80 & 8.88 & 73.39 & 0.80 & 8.08 & 8.28 & 8.48 & $\theta .00$ & 11.69 \\
\hline $14 \mathrm{H}-1-41$ & 121.11 & 0.74 & 0.18 & 8.68 & 8.88 & 8.88 & 92.99 & 8.88 & 8.80 & 8.88 & 1.29 & 8.88 & 4.88 \\
\hline $14 \mathrm{H}-1-62$ & 121.32 & 0.80 & 0.80 & 8.08 & 8.88 & 0.88 & 94.68 & 0.08 & 0.80 & 0.57 & 0.19 & 8.88 & 4.56 \\
\hline $14 \mathrm{H}-1-82$ & 121.52 & $\theta .88$ & 0.14 & 0.08 & 0.08 & 0.80 & 74.84 & 2.88 & 1.24 & 8.69 & 8.10 & 8.69 & 12.23 \\
\hline $14 \mathrm{H}-1-182$ & 121.73 & 1.92 & 8.59 & 8.88 & 8.88 & 8.88 & 65.24 & 1.33 & 0.15 & 1.84 & 9.82 & 8.38 & 28.41 \\
\hline $14 \mathrm{H}-1-123$ & 121.93 & 0.13 & $\theta . \theta \theta$ & $8 . \theta \theta$ & 0.88 & $\theta .88$ & 71.31 & 3.89 & 1.74 & 8.27 & 6.97 & 8.88 & 15.68 \\
\hline $14 \mathrm{H}-2-82$ & 123.02 & 0.36 & 0.00 & 0.08 & 8.08 & 0.88 & 77.31 & 3.63 & 2.98 & 1.89 & 8.89 & 0.18 & 5.63 \\
\hline $14 \mathrm{H}-2-103$ & 123.23 & 0.15 & 0.45 & 8.08 & 8.80 & 0.88 & 82.93 & 4.38 & 3.78 & 0.60 & 6.84 & 0.80 & 1.51 \\
\hline $14 \mathrm{H}-2-123$ & 123.43 & 0.58 & 8.80 & 8.80 & 8.88 & $\theta .08$ & 83.31 & 4.32 & 2.81 & 0.14 & 7.34 & 0.80 & 1.87 \\
\hline $14 \mathrm{H}-2-142$ & 123.62 & 6.41 & 8.88 & 8.88 & 8.88 & 8.88 & 98.29 & 0.80 & 8.80 & 0.97 & 1.55 & 8.88 & 8.78 \\
\hline $14 \mathrm{H}-3-21$ & 123.91 & 8.88 & 8.08 & 8.08 & 8.80 & 8.88 & 95.33 & 0.58 & 0.39 & 0.97 & 1.75 & 8.80 & 8.97 \\
\hline $14 \mathrm{H}-3-41$ & 124.11 & 1.28 & 8.88 & 8.80 & 8.88 & 8.48 & 95.81 & 8.88 & 0.68 & 8.08 & 0.68 & 8.00 & 2.08 \\
\hline $14 \mathrm{H}-3-62$ & 124.32 & 5.73 & 8.48 & 0.08 & 8.08 & 8.88 & 69.37 & 8.88 & 0.08 & 8.59 & 8.88 & 8.88 & 23.52 \\
\hline $14 \mathrm{H}-3-82$ & 124.52 & 0.68 & 8.88 & $\theta . \theta \theta$ & 8.88 & 8.48 & 89.38 & 8.88 & 1.28 & 8.88 & 8.68 & 8.88 & 7.81 \\
\hline $14 \mathrm{H}-3-103$ & 124.72 & 0.19 & 0.00 & 8.80 & 8.08 & 0.19 & 84.03 & 8.95 & 1.33 & 8.95 & 9.13 & 0.88 & 3.23 \\
\hline $14 \mathrm{H}-3-123$ & 124.93 & 23.21 & 0.12 & 0.80 & 8.88 & 8.49 & 18.35 & 8.24 & 1.94 & 8.88 & 0.97 & 8.88 & 54.68 \\
\hline $14 \mathrm{H}-4-21$ & 125.41 & 0.15 & 8.88 & 8.88 & 8.08 & 8.31 & 85.47 & 1.53 & 1.38 & 8.76 & 7.34 & 8.88 & 3.86 \\
\hline $14 \mathrm{H}-4-62$ & 125.82 & 8.44 & 8.15 & 8.80 & 8.80 & 8.88 & 81.87 & 8.89 & 0.44 & 0.74 & 6.87 & 8.08 & 18.21 \\
\hline $14 H-4-82$ & 126.82 & 52.84 & 3.18 & 8.80 & 8.88 & 0.88 & 7.69 & 8.08 & 8.33 & 8.58 & 3.51 & 8.88 & 29.93 \\
\hline $14 \mathrm{H}-5-21$ & 126.91 & 17.21 & 2.58 & 8.17 & 8.88 & 8.88 & 38.38 & 0.17 & 0.17 & 8.34 & 2.87 & 8.88 & 38.21 \\
\hline $14 \mathrm{H}-5-41$ & 127.11 & 5.22 & 8.16 & 0.88 & 8.16 & 8.88 & 76.58 & 1.11 & 8.95 & 2.86 & 6.01 & 8.88 & 7.59 \\
\hline
\end{tabular}


Table 1.4 (continued).

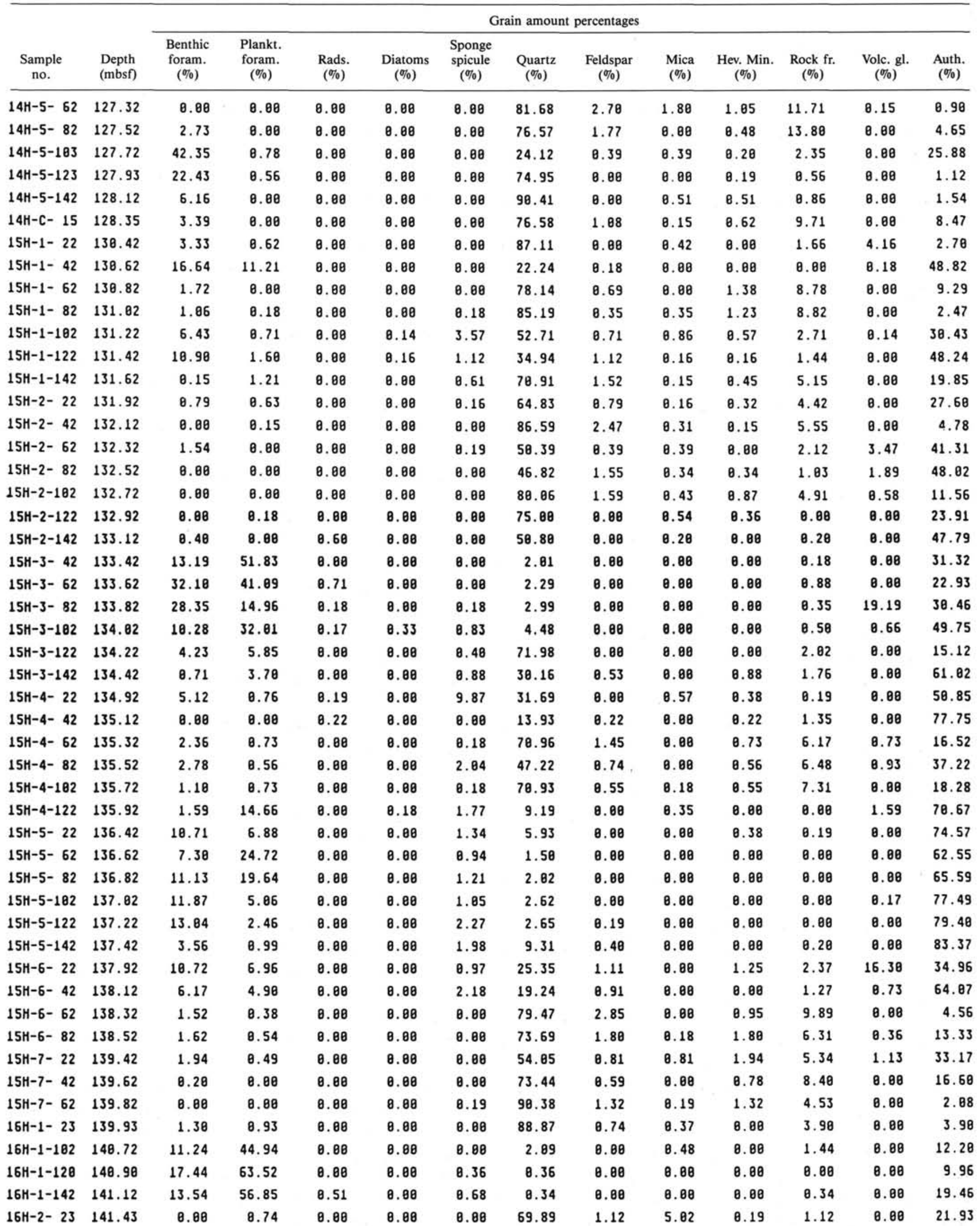


Table 1.4 (continued).

\begin{tabular}{|c|c|c|c|c|c|c|c|c|c|c|c|c|c|}
\hline \multirow[b]{2}{*}{$\begin{array}{c}\text { Sample } \\
\text { no. }\end{array}$} & \multirow[b]{2}{*}{$\begin{array}{l}\text { Depth } \\
\text { (mbsf) }\end{array}$} & \multicolumn{12}{|c|}{ Grain amount percentages } \\
\hline & & $\begin{array}{l}\text { Benthic } \\
\text { foram. } \\
(\%)\end{array}$ & $\begin{array}{l}\text { Plankt. } \\
\text { foram. } \\
(\%)\end{array}$ & $\begin{array}{l}\text { Rads. } \\
(\%)\end{array}$ & $\begin{array}{c}\text { Diatoms } \\
(\%)\end{array}$ & $\begin{array}{l}\text { Sponge } \\
\text { spicule } \\
(\%)\end{array}$ & $\begin{array}{c}\text { Quartz } \\
(\%)\end{array}$ & $\begin{array}{c}\text { Feldspar } \\
(\%)\end{array}$ & $\begin{array}{c}\text { Mica } \\
(\%)\end{array}$ & $\begin{array}{l}\text { Hev. Min. } \\
\quad(\%)\end{array}$ & $\begin{array}{c}\text { Rock fr. } \\
(\%)\end{array}$ & $\begin{array}{l}\text { Volc. gl. } \\
(\%)\end{array}$ & $\begin{array}{l}\text { Auth. } \\
(\%)\end{array}$ \\
\hline $16 \mathrm{H}-2-102$ & 142.82 & 18.71 & 60.97 & 8.88 & 8.88 & 8.80 & 1.55 & 8.88 & 0.80 & 0.80 & 8.17 & 8.88 & 26.88 \\
\hline $16 \mathrm{H}-2-128$ & 142.28 & 1.72 & 2.66 & 0.80 & 8.88 & 0.80 & 64.32 & 1.88 & 0.94 & 8.80 & 7.82 & 8.88 & 28.58 \\
\hline $16 \mathrm{H}-2-142$ & 142.42 & 2.82 & 0.55 & 8.88 & 8.80 & 8.80 & 83.64 & 8.55 & 3.86 & 1.10 & 1.18 & 8.80 & 7.17 \\
\hline $16 \mathrm{H}-3-23$ & 142.93 & 1.13 & 4.21 & 8.80 & 8.88 & 0.16 & 53.87 & 8.65 & 0.16 & 1.29 & 18.83 & 2.10 & 27.18 \\
\hline $16 \mathrm{H}-3-182$ & 143.72 & 14.58 & 13.89 & 8.88 & 8.11 & 1.84 & 29.28 & 0.23 & 8.46 & 8.46 & 5.86 & 1.72 & 38.28 \\
\hline $16 \mathrm{H}-3-128$ & 143.98 & 10.61 & 8.44 & 8.41 & 8.14 & 42.18 & 0.82 & 8.08 & 1.89 & 0.14 & 1.22 & 8.41 & 34.81 \\
\hline $16 \mathrm{H}-3-142$ & 144.12 & 12.31 & 17.68 & 0.80 & 8.80 & 23.65 & 1.91 & 8.80 & 0.35 & 8.88 & 2.43 & 0.88 & 41.77 \\
\hline $16 \mathrm{H}-4-23$ & 144.43 & 22.49 & 3.72 & 8.88 & 0.80 & 14.98 & 2.29 & 8.80 & 0.29 & 8.88 & 0.57 & 0.14 & 54.58 \\
\hline $16 \mathrm{H}-4-182$ & 145.82 & 3.76 & 49.86 & 8.88 & 8.88 & 2.56 & 1.03 & 8.88 & 0.17 & 8.88 & 0.51 & 0.80 & 42.22 \\
\hline $16 H-4-128$ & 145.28 & 6.86 & 39.93 & 8.53 & 0.88 & 1.43 & 28.68 & 0.71 & 0.18 & 8.80 & 1.96 & 8.08 & 26.38 \\
\hline $16 \mathrm{H}-5-23$ & 145.93 & 1.22 & 26.15 & 8.80 & 0.88 & 1.99 & 44.58 & 1.87 & 0.80 & 0.31 & 5.28 & 8.15 & 19.11 \\
\hline $16 \mathrm{H}-5-182$ & 146.72 & 26.66 & 18.05 & 0.88 & 8.80 & 3.48 & 5.79 & 0.58 & 8.88 & 8.80 & 1.82 & 8.50 & 42.88 \\
\hline $16 \mathrm{H}-5-128$ & 146.92 & 35.60 & 23.19 & 0.08 & 0.80 & $\theta .80$ & 3.55 & 8.88 & 8.88 & 0.88 & 4.58 & $\theta . \theta 8$ & 33.89 \\
\hline $16 \mathrm{H}-5-142$ & 147.12 & 14.48 & 46.55 & 8.80 & 0.80 & 2.93 & 1.21 & 8.88 & 8.17 & 0.80 & 1.38 & 8.80 & 33.18 \\
\hline $16 \mathrm{H}-6-23$ & 147.43 & 1.73 & 8.86 & 0.88 & 8.88 & 8.08 & 79.45 & 1.98 & 1.84 & 0.52 & 6.74 & 8.88 & 7.77 \\
\hline $17 \mathrm{H}-1-23$ & 149.42 & 8.16 & 0.47 & 8.88 & 8.88 & 2.85 & 46.04 & 2.37 & 0.47 & 0.47 & 5.38 & 8.47 & 41.38 \\
\hline $17 \mathrm{H}-1-42$ & 149.62 & 8.80 & $\theta .08$ & 8.88 & 8.88 & 8.91 & 36.38 & 8.08 & 0.83 & 0.58 & 7.43 & 1.65 & 44.39 \\
\hline $17 \mathrm{H}-1-122$ & 158.42 & 0.37 & 8.80 & 8.80 & 8.08 & 1.49 & 37.87 & 8.75 & 0.37 & 8.56 & 3.92 & 8.88 & 54.66 \\
\hline $17 \mathrm{H}-1-142$ & 158.62 & 8.08 & 0.08 & 8.80 & 0.80 & 8.54 & 71.10 & 0.08 & 5.92 & 0.54 & 4.67 & 5.03 & 12.21 \\
\hline $17 \mathrm{H}-2-22$ & 158.92 & 22.83 & 37.32 & 8.88 & 8.88 & 8.16 & 17.48 & 0.31 & 0.16 & 0.47 & 4.88 & 2.52 & 13.86 \\
\hline $17 \mathrm{H}-2-42$ & 151.12 & 18.38 & 62.11 & $8 . \theta 8$ & 0.80 & 13.49 & 1.04 & 8.80 & 1.84 & 0.35 & 0.00 & 2.88 & 9.34 \\
\hline $17 \mathrm{H}-2-62$ & 151.22 & 8.72 & 9.47 & 0.14 & 0.14 & 9.18 & 8.80 & 8.80 & 8.88 & $\theta .80$ & 8.88 & 78.85 & 2.15 \\
\hline $17 \mathrm{H}-3-22$ & 152.42 & 3.74 & 5.69 & 0.88 & 0.80 & 28.46 & 3.28 & 0.80 & 8.08 & 0.88 & 0.18 & 9.87 & 57.12 \\
\hline $17 \mathrm{H}-3-42$ & 152.62 & 25.61 & 3.94 & 8.88 & 8.88 & 2.73 & 18.03 & $\theta .80$ & 8.80 & 0.38 & 1.82 & 2.73 & 44.55 \\
\hline $17 \mathrm{H}-3-62$ & 152.82 & 2.97 & 1.32 & 8.88 & 8.88 & 1.82 & 58.58 & 1.16 & 0.17 & 0.83 & 6.77 & 7.26 & 26.98 \\
\hline $17 \mathrm{H}-4-22$ & 153.92 & 8.18 & 8.88 & 0.80 & 8.88 & 2.29 & 56.51 & 1.23 & 8.80 & 8.35 & 9.33 & 0.35 & 29.58 \\
\hline $17 \mathrm{H}-4-42$ & 154.12 & 0.80 & 1.01 & 0.88 & 0.08 & 8.00 & 64.59 & 1.61 & 0.28 & 8.88 & 12.68 & 2.41 & 15.98 \\
\hline $17 \mathrm{H}-4-182$ & 154.32 & 6.93 & 25.65 & 0.68 & 8.88 & 2.95 & 7.11 & 8.88 & 8.88 & 0.08 & 0.00 & 3.29 & 54.87 \\
\hline $17 \mathrm{H}-4-122$ & 154.52 & 15.37 & 7.43 & 0.08 & 8.88 & 9.46 & 46.62 & 0.80 & 1.35 & 8.68 & 3.38 & 3.84 & 12.67 \\
\hline $17 \mathrm{H}-4-142$ & 154.72 & 3.63 & $28.4 \theta$ & 0.80 & 8.80 & 0.54 & 13.19 & 0.27 & 0.88 & 8.67 & 2.29 & 8. 27 & 58.74 \\
\hline $17 \mathrm{H}-5-22$ & 155.42 & 3.23 & 15.32 & $\theta . \theta \theta$ & 8.80 & 0.80 & 29.84 & 0.48 & 8.80 & 8.32 & 2.58 & 1.77 & 46.45 \\
\hline $17 \mathrm{H}-5-122$ & 156.42 & 14.82 & 11.75 & 0.34 & 0.80 & 31.18 & 2.39 & 8.80 & 8.85 & 8.34 & 0.00 & 8.51 & 37.65 \\
\hline $18 \mathrm{H}-1-42$ & 159.12 & 8.92 & 0.00 & 8.88 & 8.88 & 3.48 & 66.67 & 1.47 & 0.55 & 8.88 & 11.72 & 0.18 & 15.82 \\
\hline $18 \mathrm{H}-1-58$ & 159.38 & 0.88 & 8.88 & 8.80 & 0.88 & 8.00 & 77.28 & 0.80 & 0.19 & 8.19 & 7.26 & 8.80 & 15.08 \\
\hline $18 \mathrm{H}-1-182$ & 159.52 & 4.53 & 2.19 & 8.88 & 8.88 & 12.55 & 7.45 & 8.15 & 0.88 & 0.15 & 0.58 & 0.29 & 71.24 \\
\hline $18 \mathrm{H}-1-122$ & 159.72 & 38.89 & 21.87 & 8.80 & 8.88 & 7.86 & 3.21 & 8.80 & 8.89 & 0.18 & 6.96 & 0.08 & 28.57 \\
\hline $18 \mathrm{H}-1-142$ & 159.92 & 35.21 & 6.76 & 0.80 & 8.88 & 4.93 & 9.44 & 8.80 & 0.80 & 0.14 & 1.27 & 8.88 & 42.25 \\
\hline $18 \mathrm{H}-2-42$ & 168.62 & 8.35 & 8.18 & 8.88 & 8.88 & 1.48 & 56.39 & 1.23 & 8.35 & 0.35 & 8.76 & 8.88 & 31.80 \\
\hline $18 \mathrm{H}-2-58$ & 168.88 & $\theta .08$ & 0.80 & $\theta . \theta 8$ & 0.88 & 0.32 & 63.72 & 8.80 & 2.89 & 0.80 & 33.87 & 8.88 & 8.88 \\
\hline $18 \mathrm{H}-2-182$ & 161.28 & 0.87 & 8.00 & 8.80 & 0.88 & 8.35 & 73.31 & 2.68 & 2.88 & 2.88 & 13.17 & 0.80 & 5.55 \\
\hline $18 \mathrm{H}-2-122$ & 161.42 & 2.29 & 0.80 & 0.80 & 0.88 & 8.08 & 78.04 & 0.88 & 5.53 & 0.57 & 16.03 & 8.80 & 5.53 \\
\hline $18 \mathrm{H}-2-142$ & 161.62 & 1.18 & 0.80 & 8.80 & 8.88 & 8.88 & 68.58 & 4.25 & 8.94 & 2.36 & 10.88 & 0.80 & 12.76 \\
\hline $18 \mathrm{H}-3-42$ & 162.12 & 6.97 & 10.28 & 0.88 & 8.80 & 8.08 & 28.91 & 0.34 & 0.17 & 8.34 & 3.86 & 8.08 & 58.80 \\
\hline $18 \mathrm{H}-3-58$ & 162.38 & 8.88 & 8.08 & 8.88 & 8.88 & 8.80 & 81.75 & 3.54 & 8.74 & 0.56 & 11.17 & 8.88 & 2.23 \\
\hline $18 \mathrm{H}-3-182$ & 162.52 & 9.85 & 8.68 & 0.88 & 8.88 & 8.08 & 69.27 & 2.72 & 1.82 & 8.85 & 11.84 & $\theta . \theta 0$ & 4.58 \\
\hline $18 \mathrm{H}-3-122$ & 162.72 & 33.33 & 17.77 & 0.80 & 0.80 & 8.08 & 17.58 & 8.88 & 2.56 & 8.88 & 7.69 & 8.88 & 21.86 \\
\hline $18 \mathrm{H}-3-142$ & 162.92 & 0.36 & 0.08 & 0.80 & 0.88 & 8.00 & 78.74 & 1.62 & 0.98 & 1.26 & 10.45 & 8.88 & 6.67 \\
\hline $18 \mathrm{H}-4-42$ & 163.62 & 17.32 & 1.37 & 8.88 & 8.88 & 8.17 & 28.99 & 0.69 & 0.17 & 0.17 & 4.88 & 1.72 & 44.68 \\
\hline $18 \mathrm{H}-4-58$ & 163.88 & 8.93 & 2.28 & 0.80 & 8.80 & 8.00 & 21.54 & 0.80 & 8.80 & 8.35 & 5.78 & 2.10 & 59.82 \\
\hline $18 \mathrm{H}-4-182$ & 164.28 & 0.39 & 0.39 & 8.88 & 8.88 & 8.59 & 6.65 & 0.88 & 0.28 & 8.28 & 2.94 & 8.80 & 88.65 \\
\hline
\end{tabular}


Table 1.4 (continued).

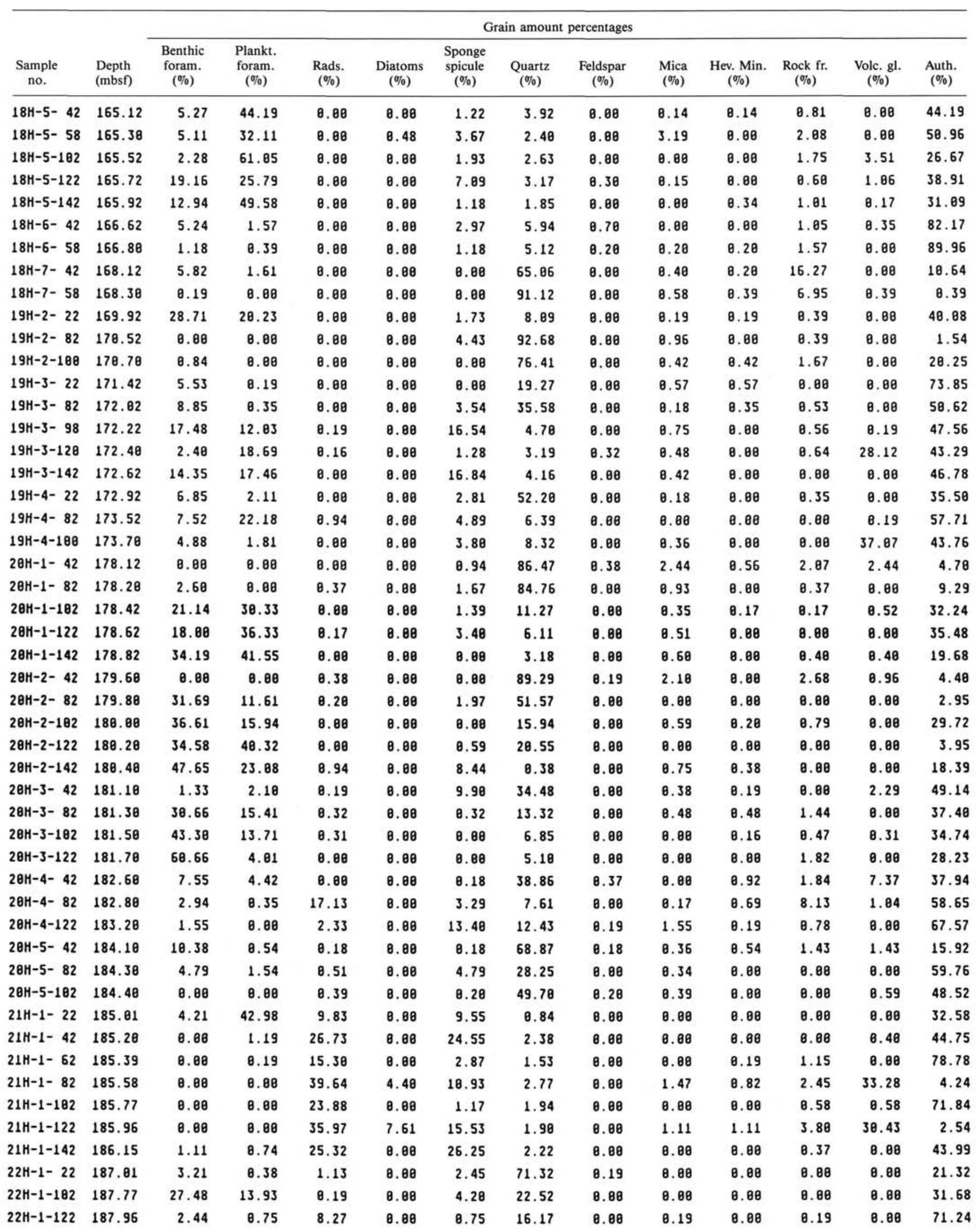


Table 1.4 (continued).

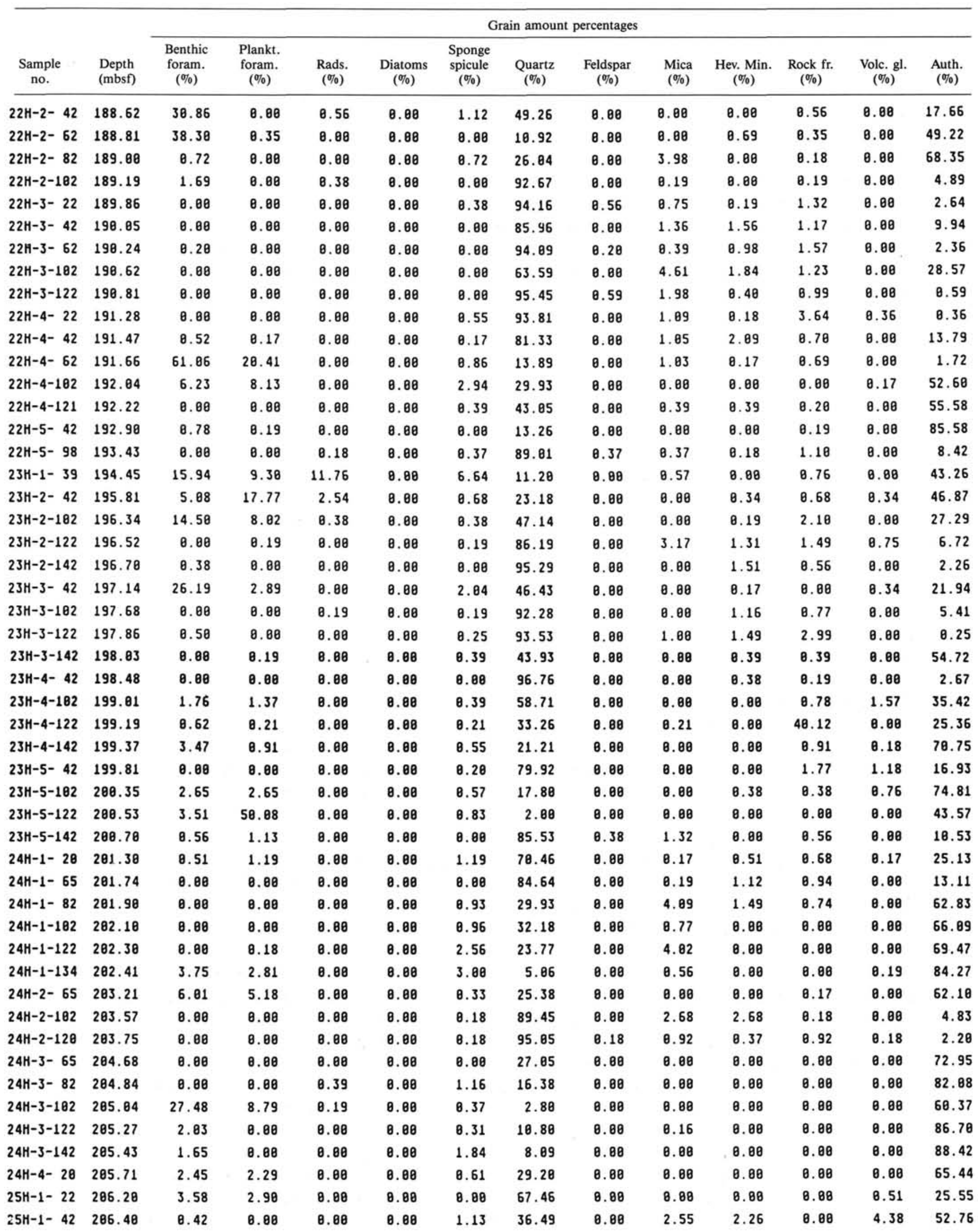


Table 1.4 (continued).

\begin{tabular}{|c|c|c|c|c|c|c|c|c|c|c|c|c|c|}
\hline \multirow[b]{2}{*}{$\begin{array}{c}\text { Sample } \\
\text { no. }\end{array}$} & \multirow[b]{2}{*}{$\begin{array}{l}\text { Depth } \\
\text { (mbsf) }\end{array}$} & \multicolumn{12}{|c|}{ Grain amount percentages } \\
\hline & & $\begin{array}{l}\text { Benthic } \\
\text { foram. } \\
(\%)\end{array}$ & $\begin{array}{l}\text { Plankt. } \\
\text { foram. } \\
(\%)\end{array}$ & $\begin{array}{c}\text { Rads. } \\
(\%)\end{array}$ & $\begin{array}{c}\text { Diatoms } \\
(\%)\end{array}$ & $\begin{array}{c}\text { Sponge } \\
\text { spicule } \\
(\%)\end{array}$ & $\begin{array}{l}\text { Quartz } \\
(\%)\end{array}$ & $\begin{array}{c}\text { Feldspar } \\
(\%)\end{array}$ & $\begin{array}{c}\text { Mica } \\
(\%)\end{array}$ & $\begin{array}{l}\text { Hev. Min. } \\
(\%)\end{array}$ & $\begin{array}{c}\text { Rock fr. } \\
(\%)\end{array}$ & $\begin{array}{l}\text { Volc. gl. } \\
(\%)\end{array}$ & $\begin{array}{c}\text { Auth. } \\
(\%)\end{array}$ \\
\hline $25 \mathrm{H}-1-62$ & 286.68 & 0.80 & 0.08 & 0.80 & 0.88 & 0.68 & 54.57 & 8.80 & 4.35 & 1.85 & 8.45 & 1.85 & 37.93 \\
\hline $25 \mathrm{H}-1-82$ & 286.80 & 0.19 & 8.19 & 3.77 & 0.80 & 1.13 & 3.58 & 8.08 & 8.08 & 8.80 & 0.80 & 0.19 & 98.96 \\
\hline $25 \mathrm{H}-1-102$ & 287.88 & 3.66 & 4.76 & 1.28 & 0.80 & 8.55 & 3.38 & 8.80 & 8.18 & 8.80 & 0.80 & 0.37 & 85.98 \\
\hline $25 \mathrm{H}-1-122$ & 287.28 & 0.19 & 1.15 & 8.80 & 8.88 & 0.38 & 14.81 & 8.80 & 8.88 & 0.08 & $\theta . \theta 0$ & 0.38 & 83.88 \\
\hline $25 \mathrm{H}-2-22$ & 287.78 & 6.72 & 25.17 & 8.80 & 8.08 & 0.80 & 2.87 & 8.88 & 8.80 & 8.80 & 0.80 & 8.88 & 65.80 \\
\hline $25 \mathrm{H}-2-42$ & 287.98 & 1.21 & 1.73 & 0.80 & 8.80 & 3.11 & 33.74 & 8.80 & 1.98 & 0.80 & 3.29 & 0.08 & 55.82 \\
\hline $25 \mathrm{H}-2-62$ & 288.12 & 11.33 & 11.51 & 0.88 & 0.88 & 3.96 & 15.11 & 8.80 & 0.88 & 8.88 & 5.48 & 0.80 & 52.78 \\
\hline $25 \mathrm{H}-2-82$ & 208.32 & 8.80 & 0.80 & 0.08 & 0.08 & 0.15 & 81.76 & 0.80 & 5.26 & 8.46 & 8.31 & 8.15 & 11.98 \\
\hline $25 \mathrm{H}-2-182$ & 288.52 & 0.80 & 0.80 & $0 . \theta 0$ & 8.80 & 0.35 & 22.88 & 0.80 & 0.18 & 0.08 & 7.24 & 2.12 & 68.02 \\
\hline $25 \mathrm{H}-2-142$ & 289.10 & $8 . \theta 8$ & 0.88 & 0.88 & 0.00 & 0.41 & 12.89 & 8.80 & 8.28 & 8.88 & 8.80 & 8.80 & 87.30 \\
\hline $25 \mathrm{H}-3-22$ & 289.28 & 8.88 & 8.88 & 0.88 & 0.88 & 0.88 & 56.87 & 0.88 & 8.80 & 8.17 & 8.34 & 0.80 & 42.61 \\
\hline $25 \mathrm{H}-3-42$ & 289.48 & 11.73 & 11.33 & 8.80 & 8.80 & 0.88 & 4.37 & 8.80 & $\theta .08$ & 0.88 & 6.76 & 0.80 & 65.81 \\
\hline $25 \mathrm{H}-3-62$ & 289.68 & 1.78 & 0.28 & 0.80 & 8.80 & 1.58 & 75.69 & 0.80 & 8.59 & 0.80 & 8.80 & 0.80 & 28.16 \\
\hline $26 \mathrm{H}-1-22$ & 218.71 & 1.13 & 0.38 & 8.80 & 0.80 & 0.38 & 39.66 & 8.80 & 0.38 & 0. 38 & 8.80 & 0.80 & 57.71 \\
\hline $26 \mathrm{H}-1-42$ & 218.91 & 8.56 & 8.56 & 8.88 & 8.88 & 0.80 & 43.23 & 8.88 & 8.88 & 8.88 & 9.46 & 8.37 & 45.83 \\
\hline $26 \mathrm{H}-1-62$ & 211.18 & 0.19 & 8.80 & 8.88 & 8.88 & 2.88 & 17.92 & 8.88 & 8.19 & 0.80 & 0.80 & 0.80 & 79.62 \\
\hline $26 \mathrm{H}-1-82$ & 211.38 & 2.84 & 8.19 & 8.56 & 0.08 & 5.19 & 2.97 & 8.80 & 0.56 & $\theta . \theta 8$ & 3.98 & 0.74 & 83.67 \\
\hline $26 \mathrm{H}-1-182$ & 211.52 & 5.88 & 34.59 & 8.88 & $\theta .8 \theta$ & 0.80 & 15.23 & 0.80 & 0.38 & 8.88 & 8.88 & 0.80 & 44.74 \\
\hline $26 \mathrm{H}-1-142$ & 211.92 & 7.51 & 5.72 & 8.88 & 8.88 & 8.88 & 66.81 & 0.80 & 8.18 & 8.80 & 0.88 & 8.88 & 28.57 \\
\hline $26 \mathrm{H}-2-22$ & 212.17 & 15.81 & 6.22 & 0.08 & 0.80 & 8.88 & 36.59 & 0.88 & 8.18 & 8.88 & 8.08 & 0.88 & 41.83 \\
\hline $26 \mathrm{H}-2-42$ & 212.36 & 0.08 & 8.48 & 8.80 & 8.80 & $\theta . \theta 8$ & 83.18 & 8.88 & 0.48 & 8.80 & 11.87 & 2.21 & 2.81 \\
\hline $26 \mathrm{H}-2-62$ & 212.56 & 16.41 & 8.81 & 0.80 & 0.80 & $\theta . \theta 8$ & 46.98 & 0.80 & $\theta .00$ & 0.17 & 8.80 & 0.00 & 27.63 \\
\hline $26 \mathrm{H}-2-82$ & 212.75 & 0.80 & 0.18 & 8.88 & 0.80 & 8.80 & 75.57 & 0.80 & 1.93 & 0.35 & 17.93 & 3.69 & 0.35 \\
\hline $26 \mathrm{H}-2-102$ & 212.94 & 0.80 & 0.80 & 8.80 & 8.08 & 8.80 & 67.92 & 0.00 & 0.80 & 0.88 & 8.28 & 8.08 & 31.88 \\
\hline $26 \mathrm{H}-2-122$ & 213.14 & 5.88 & 1.41 & 8.88 & 8.88 & 8.14 & 67.14 & $\theta . \theta \theta$ & 8.80 & 8.80 & 0.28 & $\theta . \theta \theta$ & 25.95 \\
\hline $26 \mathrm{H}-2-142$ & 213.33 & 8.37 & 2.59 & 8.80 & 8.88 & 8.80 & 38.89 & $8.8 \theta$ & $\theta .80$ & $8.8 \theta$ & 0.37 & 8.80 & 57.78 \\
\hline $26 \mathrm{H}-3-22$ & 213.62 & $8 . \theta \theta$ & 0.08 & $\theta . \theta 8$ & 8.80 & 1.32 & 58.89 & 0.88 & 0.17 & 0.99 & 2.64 & 8.88 & 36.88 \\
\hline $26 \mathrm{H}-3-42$ & 213.82 & 8.80 & 0.80 & 0.80 & 8.88 & 1.12 & 23.32 & 0.88 & 0.19 & 8.88 & 0.88 & $8 . \theta 8$ & 75.37 \\
\hline $27 \mathrm{H}-1-102$ & 215.38 & 8.80 & 4.15 & 0.17 & $8 . \theta \theta$ & 0.88 & 56.31 & 8.08 & 0.80 & 8.88 & 0.80 & 0.08 & 30.56 \\
\hline $27 \mathrm{H}-1-122$ & 215.58 & 0.19 & 8.80 & 8.88 & 8.80 & 0.80 & 89.37 & 0.08 & 0.56 & 8.80 & 0.19 & 0.37 & 9.33 \\
\hline $28 \mathrm{H}-1-102$ & 217.85 & $\theta .00$ & 8.80 & 0.80 & 8.80 & 0.80 & 98.91 & 8.88 & 0.28 & 8.08 & 0.14 & 8.08 & 8.67 \\
\hline $28 \mathrm{H}-1-122$ & 217.23 & 9.52 & 13.23 & 0.32 & 0.80 & 0.32 & 15.81 & 8.88 & 8.80 & 8.88 & 8.80 & 8.80 & 68.81 \\
\hline $28 \mathrm{H}-1-142$ & 217.42 & 12.88 & 3.35 & 8.80 & 0.80 & 8.80 & 63.78 & 0.08 & 0.39 & 8.88 & 1.77 & 0.80 & 17.91 \\
\hline $28 \mathrm{H}-2-182$ & 218.44 & 0.19 & 0.80 & 0.88 & 0.08 & $\theta . \theta \theta$ & 93.58 & 0.80 & 0.08 & 0.80 & 0.19 & 0.19 & 5.84 \\
\hline $28 \mathrm{H}-2-122$ & 218.63 & 38.04 & 3.85 & 8.88 & 8.80 & 8.88 & 33.87 & 0.80 & 8.80 & 8.16 & 6.98 & 0.80 & 17.98 \\
\hline $28 \mathrm{H}-2-142$ & 218.82 & 8.35 & 8.88 & 0.80 & 8.88 & 0.35 & 44.86 & 8.88 & 0.18 & 0.80 & 8.88 & 0.18 & 54.88 \\
\hline $28 \mathrm{H}-3-182$ & 219.84 & 0.64 & 8.88 & 0.80 & 0.88 & 0.88 & 79.84 & 8.88 & 1.76 & 0.08 & 8.16 & 8.88 & 17.60 \\
\hline $29 \mathrm{H}-1-182$ & 222.10 & 4.12 & 2.86 & 8.80 & 0.08 & 8.17 & 12.37 & 8.88 & 0.34 & 0.60 & 8.80 & 0.80 & 88.93 \\
\hline $29 \mathrm{H}-1-122$ & 222.38 & 0.39 & 8.80 & 0.80 & 8.80 & 0.97 & 23.55 & 8.88 & 0.19 & 8.08 & 0.08 & 8.88 & 74.98 \\
\hline $29 \mathrm{H}-1-142$ & 222.58 & 8.59 & 0.80 & 8.28 & 8.80 & 2.56 & 14.76 & 8.88 & 0.80 & 8.88 & 0.39 & 0.88 & 81.50 \\
\hline $29 \mathrm{H}-2-102$ & 223.68 & 8.80 & 8.80 & 8.08 & 0.80 & 2.18 & 5.53 & 8.88 & 1.15 & 0.60 & 0.00 & 0.80 & 91.22 \\
\hline $29 \mathrm{H}-2-122$ & 223.75 & 2.88 & 0.45 & 8.22 & 0.80 & 4.23 & 1.78 & 2.80 & 1.78 & 0.45 & 3.12 & 1.78 & 82.18 \\
\hline $29 \mathrm{H}-2-142$ & 223.98 & 8.80 & 0.08 & 8.88 & 0.08 & 1.02 & 23.98 & 8.80 & 0.80 & 0.17 & 0.80 & 8.88 & 74.92 \\
\hline $29 \mathrm{H}-3-102$ & 225.18 & 8.38 & 8.88 & 8.88 & 0.88 & 8.88 & 88.88 & 8.57 & 0.19 & 8.75 & 1.89 & 8.80 & 16.23 \\
\hline $29 \mathrm{H}-3-122$ & 225.30 & 8.88 & 8.88 & 0.08 & 8.88 & 8.88 & 68.52 & 8.80 & 8.80 & 8.08 & $\theta .08$ & 8.08 & 31.48 \\
\hline $38 \mathrm{H}-1-182$ & 227.12 & 0.80 & 8.80 & 8.08 & 8.80 & 8.88 & 79.52 & $0.4 \theta$ & 0.80 & 0.00 & 1.99 & 8.80 & 18.89 \\
\hline $38 \mathrm{H}-1-122$ & 227.32 & 0.88 & 0.80 & 0.80 & 8.80 & 1.13 & 55.91 & 8.80 & 0.38 & 8.68 & 10.69 & 2.86 & 29.83 \\
\hline зөн-1-142 & 227.51 & $\theta .00$ & 8.80 & 8.80 & $\theta . \theta 0$ & 8.80 & 13.28 & 0.80 & 0.39 & 0.80 & 8.80 & 0.08 & 86.33 \\
\hline $38 \mathrm{H}-2-182$ & $228.6 \theta$ & 18.81 & 13.51 & 1.44 & 8.80 & 8.72 & 1.88 & 8.88 & 8.80 & 8.88 & 0.80 & 0.08 & 72.43 \\
\hline зөн-2-122 & 228.82 & 9.51 & 4.66 & $\theta . \theta 8$ & 1.12 & 1.49 & 2.85 & 8.88 & 8.93 & 7.89 & 0.80 & 1.87 & 71.27 \\
\hline
\end{tabular}


R. HENRICH, T. WOLF, G. BOHRMANN, J. THIEDE

Table 1.4 (continued).

\begin{tabular}{|c|c|c|c|c|c|c|c|c|c|c|c|c|c|}
\hline \multirow[b]{2}{*}{$\begin{array}{c}\text { Sample } \\
\text { no. }\end{array}$} & \multirow[b]{2}{*}{$\begin{array}{l}\text { Depth } \\
\text { (mbsf) }\end{array}$} & \multicolumn{12}{|c|}{ Grain amount percentages } \\
\hline & & $\begin{array}{l}\text { Benthic } \\
\text { foram. } \\
(\%)\end{array}$ & $\begin{array}{l}\text { Plankt. } \\
\text { foram. } \\
(\%)\end{array}$ & $\begin{array}{c}\text { Rads. } \\
(\%)\end{array}$ & $\begin{array}{c}\text { Diatoms } \\
(\%)\end{array}$ & $\begin{array}{c}\text { Sponge } \\
\text { spicule } \\
(\%)\end{array}$ & $\begin{array}{c}\text { Quartz } \\
(\%)\end{array}$ & $\begin{array}{c}\text { Feldspar } \\
(\%)\end{array}$ & $\begin{array}{l}\text { Mica } \\
(\%)\end{array}$ & $\begin{array}{l}\text { Hev. Min. } \\
(\%)\end{array}$ & $\begin{array}{c}\text { Rock fr. } \\
(\%)\end{array}$ & $\begin{array}{l}\text { Volc. gl. } \\
(\%)\end{array}$ & $\begin{array}{l}\text { Auth. } \\
(\%)\end{array}$ \\
\hline $38 \mathrm{H}-2-142$ & 229.02 & 1.28 & 1.44 & 6.38 & 8.80 & 3.35 & 0.80 & 8.80 & 8.80 & 8.80 & 8.80 & $\theta . \theta 0$ & 86.76 \\
\hline $38 \mathrm{H}-3-182$ & 230.12 & 0.77 & 8.88 & 0.77 & 8.88 & 4.43 & 1.93 & 8.88 & 8.80 & 0.80 & 8.80 & 8.88 & 92.18 \\
\hline $38 \mathrm{H}-3-122$ & 238.32 & 0.08 & 8.80 & 0.93 & 8.08 & 18.37 & 15.19 & 8.88 & 2.59 & 8.80 & 8.15 & 15.74 & 47.84 \\
\hline $30 \mathrm{H}-3-142$ & 238.52 & 0.80 & 0.19 & 0.19 & 8.80 & 2.86 & 85.98 & 8.80 & 0.93 & 0.19 & 1.12 & 0.56 & 8.79 \\
\hline $38 \mathrm{H}-4-182$ & 231.60 & 0.37 & 0.80 & 8.80 & 8.80 & 1.31 & 7.10 & 8.80 & 8.80 & 0.19 & 0.08 & 0.08 & 91.03 \\
\hline $38 \mathrm{H}-4-122$ & 231.82 & 0.28 & 8.80 & 0.08 & 8.80 & 0.28 & 45.88 & 8.88 & 8.88 & 0.88 & 0.80 & 0.88 & 53.88 \\
\hline $30 \mathrm{H}-4-142$ & 232.02 & 8.19 & 0.08 & 8.08 & 8.88 & 0.19 & 84.87 & $\theta .88$ & $\theta .80$ & 0.88 & 1.34 & 8.80 & 13.41 \\
\hline $31 \mathrm{H}-1-182$ & 233.18 & 0.80 & 8.88 & 0.80 & 8.88 & 8.77 & 94.88 & 8.80 & $\theta .80$ & 0.19 & 0.77 & 8.80 & 4.26 \\
\hline $31 \mathrm{H}-1-142$ & 233.32 & 0.18 & 8.88 & 8.88 & 8.88 & 8.80 & 67.87 & 8.88 & 0.18 & 8.88 & 8.36 & 0.18 & 31.23 \\
\hline $31 \mathrm{H}-2-182$ & 234.68 & 0.08 & 8.19 & 2.82 & 0.08 & 21.89 & 12.81 & 8.88 & 0.38 & 0.88 & 1.13 & 0.88 & 61.58 \\
\hline $31 \mathrm{H}-2-142$ & 234.92 & 8.88 & 0.80 & 3.32 & 0.88 & 18.15 & 2.62 & 8.88 & 8.08 & 8.88 & 1.85 & 8.88 & 74.87 \\
\hline $31 \mathrm{H}-3-102$ & 236.12 & 0.08 & 0.08 & 7.45 & 0.88 & 6.18 & 14.73 & 8.88 & 0.18 & 8.88 & 8.80 & 8.80 & 71.45 \\
\hline $31 \mathrm{H}-3-122$ & 236.32 & 0.80 & 8.88 & 3.41 & 0.80 & 18.22 & 2.28 & 8.88 & 8.88 & 0.88 & 0.88 & 0.80 & 84.17 \\
\hline $32 \mathrm{H}-2-182$ & 248.62 & 5.82 & 6.16 & 3.16 & 0.88 & 12.15 & 1.58 & 8.88 & 8.80 & 0.80 & 0.00 & 0.88 & 69.22 \\
\hline $32 \mathrm{H}-2-122$ & 240.82 & 7.71 & 21.51 & 8.18 & 8.88 & 1.88 & 0.54 & 8.88 & 8.88 & 0.08 & 0.80 & 8.88 & 64.78 \\
\hline $32 \mathrm{H}-2-142$ & 241.02 & 12.85 & 18.89 & 0.66 & 0.33 & 8.91 & 2.15 & 8.80 & 8.80 & 8.88 & 0.80 & 8.88 & 64.69 \\
\hline $32 \mathrm{H}-3-182$ & 242.12 & 6.68 & 9.35 & 1.88 & 1.80 & 16.19 & 1.58 & 8.88 & 8.88 & 8.80 & 8.80 & 8.08 & 63.61 \\
\hline $32 \mathrm{H}-3-122$ & 242.32 & 4.84 & 5.51 & 1.17 & 1.17 & 18.78 & 1.17 & 8.88 & 8.88 & 8.88 & 8.80 & 8.58 & 66.94 \\
\hline $32 \mathrm{H}-3-142$ & 242.52 & 1.72 & 2.30 & 0.19 & 8.96 & 22.83 & 0.19 & 8.88 & 8.19 & 0.19 & 8.88 & 0.19 & 72.03 \\
\hline $32 \mathrm{H}-4-102$ & 243.68 & 8.74 & 8.74 & 1.38 & $0 . \theta 0$ & 7.43 & 8.93 & $\theta .88$ & 0.19 & 0.08 & 8.00 & 8.88 & 78.63 \\
\hline $32 \mathrm{H}-4-122$ & 243.82 & 10.82 & 9.14 & 1.87 & 8.75 & 24.63 & 8.37 & 8.88 & 8.19 & 8.88 & 8.88 & 8.19 & 52.85 \\
\hline $33 \mathrm{H}-1-182$ & 244.72 & 12.43 & 3.15 & 1.48 & 0.35 & 11.56 & 2.18 & 0.80 & 0.78 & 0.88 & $\theta .8 \theta$ & 8.88 & 67.95 \\
\hline $33 \mathrm{H}-1-122$ & 244.92 & 8.12 & 5.78 & 2.17 & 0.18 & 11.81 & 8.98 & 8.80 & 0.36 & 8.88 & $\theta .8 \theta$ & 8.88 & 67.51 \\
\hline $33 H-1-142$ & 245.12 & 9.38 & 4.23 & 2.76 & 0.80 & 7.54 & 2.76 & 8.80 & 0.80 & 8.80 & 8.08 & 8.88 & 73.35 \\
\hline $34 H-1-182$ & 246.86 & 0.88 & 0.35 & $1.4 \theta$ & 0.00 & 64.21 & 1.48 & 0.80 & 1.23 & 8.80 & 0.08 & 8.80 & 31.48 \\
\hline $34 H-1-122$ & 247.82 & 8.80 & 8.69 & 1.56 & 8.35 & 74.74 & 1.56 & 0.88 & 1.38 & 0.80 & 0.08 & 0.88 & 19.72 \\
\hline $34 H-1-142$ & 247.19 & 1.21 & 1.21 & 8.17 & 0.88 & 44.19 & 2.25 & 0.88 & 1.21 & 8.80 & 8.88 & 0.35 & 49.39 \\
\hline $34 H-2-182$ & 248.12 & 2.28 & 8.17 & 1.18 & 8.80 & 41.46 & 0.68 & 8.88 & 8.51 & 8.88 & 8.08 & 0.08 & 53.81 \\
\hline $34 \mathrm{H}-2-122$ & 248.28 & 5.13 & 2.93 & 5.68 & 4.03 & 48.84 & 8.92 & $\theta . \theta 8$ & 3.48 & 8.80 & B. 55 & 3.48 & 32.97 \\
\hline $34 \mathrm{H}-2-142$ & 248.45 & 0.80 & 8.80 & 0.37 & 0.88 & 15.61 & 8.19 & 8.88 & 0.74 & 8.80 & 0.08 & 8.88 & 83.89 \\
\hline $34 \mathrm{H}-4-182$ & 258.64 & 0.88 & 8.80 & 8.19 & 8.88 & 1.17 & 66.47 & 8.88 & 0.39 & 0.78 & 0.80 & 8.39 & 38.68 \\
\hline $34 \mathrm{H}-4-122$ & 258.88 & 5.66 & 16.84 & 0.19 & 8.88 & 0.94 & 3.77 & $\theta .8 \theta$ & 0. 38 & 8.88 & 0.00 & 0.80 & 73.02 \\
\hline
\end{tabular}


Table 1.4 (continued).

\begin{tabular}{|c|c|c|c|c|c|c|}
\hline \multirow[b]{2}{*}{$\begin{array}{c}\text { Sample } \\
\text { no. }\end{array}$} & \multirow[b]{2}{*}{$\begin{array}{l}\text { Depth } \\
\text { (mbsf) }\end{array}$} & \multicolumn{5}{|c|}{ Subfraction of coarse fraction $(\mu \mathrm{m})$} \\
\hline & & $\begin{array}{l}>63 \\
(\%)\end{array}$ & $\begin{array}{c}63-125 \\
(\%)\end{array}$ & $\begin{array}{c}125-500 \\
(\%)\end{array}$ & $\begin{array}{c}500-1000 \\
(\%)\end{array}$ & $\begin{array}{c}>1000 \\
(\%)\end{array}$ \\
\hline $1 H-1-22$ & 0.22 & 9.52 & 2.24 & 7.19 & 0.07 & 0.82 \\
\hline $1 H-1-42$ & 8.42 & 2.74 & 1.51 & 1.15 & 0.07 & 0.01 \\
\hline $1 H-1-59$ & 8.59 & 8.85 & 5.71 & 2.68 & 8.19 & 0.27 \\
\hline $1 H-1-82$ & 0.82 & 4.64 & 2.56 & 1.83 & 0.23 & 8. 82 \\
\hline $1 H-1-182$ & 1.82 & 7.16 & 3.99 & 3.03 & 0.13 & 0.81 \\
\hline $1 H-1-122$ & 1.22 & 18.94 & 6.31 & 3.76 & 0.32 & 8.55 \\
\hline $1 H-1-142$ & 1.42 & 13.96 & 7.63 & 5.36 & 0.76 & 0.21 \\
\hline $1 H-2-22$ & 1.72 & 4.35 & 1.97 & 2.23 & 8.11 & 8.84 \\
\hline $1 \mathrm{H}-2-59$ & 2.89 & 28.85 & 11.89 & 5.52 & 2.02 & 0.62 \\
\hline $1 \mathrm{H}-2-82$ & 2.30 & 7.43 & 4.81 & 1.96 & 0.16 & 0.58 \\
\hline $1 \mathrm{H}-2-182$ & 2.52 & 13.25 & 5.43 & 5.97 & 8.64 & 1.21 \\
\hline $1 \mathrm{H}-2-122$ & 2.72 & 5.47 & 2.87 & 2.28 & 0.47 & 0.65 \\
\hline $1 \mathrm{H}-2-142$ & 2.98 & 6.88 & 2.74 & 2.98 & 0.23 & 0.13 \\
\hline $1 H-3-22$ & 3.22 & 5.36 & 2.71 & 2.81 & 0.14 & 8.50 \\
\hline $1 \mathrm{H}-3-42$ & 3.42 & 4. 95 & 2.16 & 2.10 & 0.19 & 0.58 \\
\hline $1 \mathrm{H}-3-59$ & 3.59 & 8.69 & 3.77 & 4.87 & 0.43 & 0.42 \\
\hline $1 \mathrm{H}-3-82$ & 3.82 & 7.31 & 3.62 & 3.14 & 0.18 & 8.37 \\
\hline $1 \mathrm{H}-3-182$ & 4.02 & 4.78 & 3.26 & 1.27 & 8.16 & 0.81 \\
\hline $1 \mathrm{H}-3-122$ & 4.22 & 2.19 & 1.54 & 0.28 & 0.37 & 8.88 \\
\hline $1 \mathrm{H}-3-142$ & 4.42 & 6.88 & 3.72 & 1.13 & 0.88 & 1.15 \\
\hline $1 H-4-22$ & 4.72 & 3.82 & 2.13 & 0.84 & 0.85 & 0.80 \\
\hline $1 \mathrm{H}-4-42$ & 4.92 & 4.52 & 1.87 & 1.48 & 8.89 & 1.16 \\
\hline $1 H-4-59$ & 5.09 & 3.79 & 2.22 & 1.34 & 8.17 & 0.86 \\
\hline $1 H-4-82$ & 5.38 & 3.98 & 2.23 & 1.49 & 0.23 & 0.03 \\
\hline $1 \mathrm{H}-4-102$ & 5.52 & 7.11 & 4.52 & 2.27 & 0.13 & 0.19 \\
\hline $1 \mathrm{H}-4-122$ & 5.72 & 4.81 & 2.53 & 1.95 & 0.89 & 0.24 \\
\hline $1 \mathrm{H}-5-22$ & 6.22 & 4.84 & 4.58 & 8.25 & 0.81 & 0.80 \\
\hline $1 \mathrm{H}-5-42$ & 6.42 & 4.98 & 3.23 & 1.78 & 0.85 & 0.88 \\
\hline $1 \mathrm{H}-5-59$ & 6.59 & 6.99 & 4.10 & 2.75 & 0.05 & 0.89 \\
\hline $1 \mathrm{H}-5-82$ & 6.82 & 1.87 & 0.58 & 0.53 & 0.84 & 0.00 \\
\hline $1 \mathrm{H}-5-102$ & 7.02 & 3.27 & 1.91 & 1.24 & 0.87 & 0.05 \\
\hline $1 H-5-122$ & 7.22 & 3.28 & 1.83 & 1.35 & 0.82 & 8.88 \\
\hline $1 H-5-142$ & 7.42 & 4.54 & 1.77 & 2.51 & 8.86 & 0.28 \\
\hline $1 H-6-22$ & 7.72 & 5.22 & 2.35 & 2.25 & 8.09 & 8.53 \\
\hline $1 H-6-42$ & 7.92 & 2.50 & 1.16 & 1.26 & 8.01 & 8.87 \\
\hline $1 \mathrm{H}-6-59$ & 8.04 & 6.42 & 3.13 & 3.18 & 8.88 & 8.11 \\
\hline $1 H-6-82$ & 8.38 & 1.76 & 8.99 & 0.75 & 8.82 & 0.80 \\
\hline $1 H-6-182$ & 8.52 & 4.86 & 2.15 & 1.59 & 8.18 & 0.22 \\
\hline $1 H-6-122$ & 8.72 & 2.71 & 1.56 & 1.15 & 8.88 & 8.88 \\
\hline $1 H-6-142$ & 8.98 & 5.85 & 3.20 & 2.48 & 8.88 & 0.17 \\
\hline $2 \mathrm{H}-1-22$ & 9.42 & 3.81 & 2.08 & 1.42 & 8.88 & 0.23 \\
\hline $2 \mathrm{H}-1-42$ & 9.62 & 2.83 & 1.71 & 1.07 & 0.02 & 0.03 \\
\hline $2 \mathrm{H}-1-62$ & 9.82 & 15.28 & 11.85 & 3.21 & 0.12 & 0.18 \\
\hline $2 \mathrm{H}-1-82$ & 18.82 & 6.64 & 4.31 & 2.18 & 8.15 & 0.88 \\
\hline $2 \mathrm{H}-1-184$ & 18.24 & 9.85 & 3.79 & 3.95 & 8.42 & 1.69 \\
\hline $2 \mathrm{H}-1-122$ & 10.42 & 8.93 & 4.51 & 4.28 & 0.11 & 8.03 \\
\hline $2 \mathrm{H}-1-142$ & 10.62 & 12.26 & 4.86 & 5.61 & 2.57 & 0.82 \\
\hline $2 \mathrm{H}-2-22$ & 10.92 & 14.23 & 1.61 & 12.55 & 8.80 & 0.87 \\
\hline $2 \mathrm{H}-2-62$ & 11.32 & 7.42 & 3.84 & 3.49 & 8.88 & 8.81 \\
\hline $2 \mathrm{H}-2-82$ & 11.52 & 18.75 & 7.29 & 3.34 & 0.86 & 8.86 \\
\hline $2 \mathrm{H}-2-184$ & 11.74 & 7.59 & 2.68 & 4.84 & 8.82 & 0.85 \\
\hline $2 \mathrm{H}-2-122$ & 11.98 & 16.88 & 12.48 & 4.23 & 8.13 & 0.84 \\
\hline
\end{tabular}


Table 1.4 (continued).

\begin{tabular}{|c|c|c|c|c|c|c|}
\hline \multirow[b]{2}{*}{$\begin{array}{l}\text { Sample } \\
\text { no. }\end{array}$} & \multirow[b]{2}{*}{$\begin{array}{l}\text { Depth } \\
\text { (mbsf) }\end{array}$} & \multicolumn{5}{|c|}{ Subfraction of coarse fraction $(\mu \mathrm{m})$} \\
\hline & & $\begin{array}{l}>63 \\
(\%)\end{array}$ & $\begin{array}{c}63-125 \\
(\%)\end{array}$ & $\begin{array}{c}125-500 \\
(\%)\end{array}$ & $\begin{array}{c}500-1000 \\
(\%)\end{array}$ & $\begin{array}{c}>1000 \\
(\%)\end{array}$ \\
\hline $2 \mathrm{H}-2-142$ & 12.12 & 3.22 & 1.44 & 1.71 & 0.85 & $\theta .02$ \\
\hline $2 \mathrm{H}-3-22$ & 12.42 & 6.87 & 2.26 & 3.78 & 8.03 & 8.80 \\
\hline $2 \mathrm{H}-3-42$ & 12.62 & 4.39 & 2.32 & 2.07 & 8.88 & $\theta . \theta \theta$ \\
\hline $2 \mathrm{H}-3-62$ & 12.82 & 2.48 & 1.67 & 8.71 & 0.82 & 8.88 \\
\hline $2 \mathrm{H}-3-82$ & 13.02 & 24.19 & 18.13 & 5.84 & 0.07 & 0.15 \\
\hline $2 \mathrm{H}-3-184$ & 13.22 & 23.68 & 18.78 & 4.47 & 0.89 & 0.34 \\
\hline $2 \mathrm{H}-3-122$ & 13.42 & 17.31 & 14.14 & 3.13 & 8.84 & 8.80 \\
\hline $2 \mathrm{H}-4-22$ & 13.92 & 14.82 & 11.43 & 2.97 & 8.16 & 8.26 \\
\hline $2 \mathrm{H}-4-42$ & 14.12 & 6.39 & 3.82 & 2.80 & 0.26 & 0.31 \\
\hline $2 \mathrm{H}-4-62$ & 14.32 & 4.61 & 2.21 & 1.68 & 0.19 & 0.53 \\
\hline $2 \mathrm{H}-4-82$ & 14.52 & 8.53 & 4.41 & 3.57 & 8.24 & 0.31 \\
\hline $2 \mathrm{H}-4-184$ & 14.74 & 24.75 & 13.51 & 18.64 & 0.35 & 0.25 \\
\hline $2 \mathrm{H}-4-122$ & 14.98 & 12.87 & 9.03 & 2.66 & 0.16 & 8.22 \\
\hline $2 \mathrm{H}-4-142$ & 15.12 & 18.56 & 5.64 & 3.95 & 0.38 & 0.59 \\
\hline $2 \mathrm{H}-5-22$ & 15.42 & 9.88 & 6.80 & 2.62 & 0.21 & 0.25 \\
\hline $2 \mathrm{H}-5-42$ & 15.62 & 9.88 & 4.85 & 3.57 & 0.49 & 8.97 \\
\hline $2 \mathrm{H}-5-62$ & 15.82 & 9.98 & 3.12 & 3.62 & 0.42 & 2.82 \\
\hline $3 H-1-22$ & 16.42 & 6.22 & 3.28 & 2.42 & 0.25 & 0.27 \\
\hline $3 \mathrm{H}-1-42$ & 16.62 & 4.78 & 2.49 & 1.94 & 0.21 & 8.14 \\
\hline $3 H-1-62$ & 16.82 & 3.93 & 2.16 & 1.23 & 0.18 & 0.44 \\
\hline $3 \mathrm{H}-1-82$ & 17.82 & 14.38 & 7.92 & 4.25 & 0.38 & 1.83 \\
\hline $3 \mathrm{H}-1-182$ & 17.22 & 6.85 & 3.91 & 1.91 & 0.12 & 0.11 \\
\hline $3 H-1-122$ & 17.48 & 9.58 & 5.86 & 3.34 & 8.27 & 0.11 \\
\hline $3 \mathrm{H}-1-142$ & 17.68 & 14.27 & 8.18 & 5.49 & 0.28 & 0.32 \\
\hline $3 H-2-22$ & 17.92 & 11.98 & 5.46 & 5.83 & 8.79 & 0.62 \\
\hline $3 H-2-42$ & 18.12 & 12.51 & 5.89 & 5.47 & 0.82 & 1.13 \\
\hline $3 \mathrm{H}-2-62$ & 18.32 & 11.21 & 5.31 & 4.47 & 8.54 & 0.89 \\
\hline $3 H-2-82$ & 18.52 & 7.78 & 3.28 & 2.26 & 8.43 & 1.81 \\
\hline $3 \mathrm{H}-2-102$ & 18.72 & 4.86 & 2.93 & 0.99 & 0.87 & 8.87 \\
\hline $3 \mathrm{H}-2-122$ & 18.98 & 0.63 & 0.36 & 0.14 & 0.01 & 8.12 \\
\hline $3 \mathrm{H}-2-142$ & 19.12 & 2.21 & 1.67 & 8.47 & 8.84 & 8.03 \\
\hline $3 \mathrm{H}-3-22$ & 19.42 & 6.52 & 2.93 & 2.84 & 8.29 & 8.46 \\
\hline $3 \mathrm{H}-3-42$ & 19.62 & 1.96 & 1.01 & 8.79 & 8.84 & 8.12 \\
\hline $3 \mathrm{H}-3-62$ & 19.82 & 3.76 & 1.34 & 2.28 & 8.11 & 8.11 \\
\hline $3 \mathrm{H}-3-82$ & 28.82 & 3.23 & 1.84 & 2.14 & 8.83 & 8.82 \\
\hline $3 \mathrm{H}-3-102$ & 28.28 & 6.19 & 2.83 & 3.25 & 8.08 & 8.03 \\
\hline $3 \mathrm{H}-4-22$ & 28.42 & 8.82 & 4.63 & 2.64 & 8.32 & 8.43 \\
\hline $3 \mathrm{H}-4-42$ & 21.12 & 3.57 & 1.46 & 1.41 & 8.28 & 8.58 \\
\hline $3 H-4-62$ & 21.32 & 4.91 & 2.52 & 1.99 & 0.13 & 0.27 \\
\hline $3 \mathrm{H}-4-82$ & 21.52 & 3.28 & 1.63 & 1.25 & 8.85 & 0.27 \\
\hline $3 H-4-182$ & 21.72 & 5.87 & 2.53 & 2.14 & 8.11 & 8.29 \\
\hline $3 \mathrm{H}-4-122$ & 21.98 & 3.71 & 1.72 & 1.56 & 0.11 & 0.32 \\
\hline $3 \mathrm{H}-5-22$ & 22.43 & 5.14 & 2.11 & 2.74 & 0.84 & 0.25 \\
\hline $3 H-5-42$ & 22.62 & 2.64 & 1.64 & 0.88 & 0.03 & 8.89 \\
\hline $3 H-5-62$ & 22.82 & 10.33 & 6.88 & 3.54 & 0.78 & 0.01 \\
\hline $3 \mathrm{H}-5-82$ & 23.82 & 18.21 & 5.73 & 4.35 & 0.12 & 0.81 \\
\hline $3 \mathrm{H}-5-182$ & 23.22 & 2.81 & 1.64 & 1.15 & 0.02 & 0.88 \\
\hline $3 \mathrm{H}-5-122$ & 23.48 & 0.62 & 8.36 & 0.26 & 0.80 & 0.80 \\
\hline $3 H-5-142$ & 23.68 & 2.75 & 1.80 & 0.94 & 8.80 & 0.01 \\
\hline $3 H-6-22$ & 23.99 & 14.87 & 8.46 & 4.85 & 8.69 & 8.87 \\
\hline $3 \mathrm{H}-7-22$ & 25.42 & 6.02 & 2.62 & 1.83 & 8.85 & 1.52 \\
\hline $4 \mathrm{H}-1-22$ & 25.92 & 9.98 & 6.66 & 3.84 & 0.12 & 0.16 \\
\hline
\end{tabular}


Table 1.4 (continued).

\begin{tabular}{|c|c|c|c|c|c|c|}
\hline \multirow[b]{2}{*}{$\begin{array}{l}\text { Sample } \\
\text { no. }\end{array}$} & \multirow[b]{2}{*}{$\begin{array}{l}\text { Depth } \\
\text { (mbsf) }\end{array}$} & \multicolumn{5}{|c|}{ Subfraction of coarse fraction $(\mu \mathrm{m})$} \\
\hline & & $\begin{array}{l}>63 \\
(\%)\end{array}$ & $\begin{array}{c}63-125 \\
(\%)\end{array}$ & $\begin{array}{c}125-500 \\
(\%)\end{array}$ & $\begin{array}{c}500-1000 \\
(\%)\end{array}$ & $\begin{array}{c}>1000 \\
(\%)\end{array}$ \\
\hline $4 H-1-42$ & 26.12 & 5.68 & 3.11 & 2.19 & 0.14 & 0.16 \\
\hline $4 H-1-62$ & 26.32 & 4.83 & 2.88 & 1.85 & 8.11 & 8.87 \\
\hline $4 H-1-82$ & 26.52 & 5.45 & 2.84 & 2.29 & 8.15 & 8.17 \\
\hline $4 H-1-102$ & 26.72 & 0.99 & 0.46 & 0.46 & 8.03 & 0.84 \\
\hline $4 H-1-122$ & 26.98 & 1.86 & 0.63 & 0.41 & 8.82 & 8.80 \\
\hline $4 H-1-142$ & 27.12 & 3.42 & 1.68 & 1.59 & 8.11 & 0.84 \\
\hline $4 \mathrm{H}-2-22$ & 27.42 & 7.68 & 4.16 & 2.99 & 0.13 & 0.32 \\
\hline $4 H-2-42$ & 27.62 & 5.48 & 3.13 & 1.92 & 0.19 & 0.16 \\
\hline $4 \mathrm{H}-2-62$ & 27.82 & 7.87 & 5.57 & 2.19 & 0.85 & 8.06 \\
\hline $4 \mathrm{H}-2-82$ & 28.82 & 5.15 & 2.78 & 2.32 & 0.84 & 0.09 \\
\hline $4 \mathrm{H}-2-182$ & 28.22 & 6.63 & 2.91 & 3.57 & 0.15 & 8.80 \\
\hline $4 \mathrm{H}-2-122$ & 28.48 & 10.33 & 4.45 & 3.86 & 8.79 & 1.23 \\
\hline $4 \mathrm{H}-2-142$ & 28.68 & 4.45 & 2.16 & 1.51 & 8.87 & 0.71 \\
\hline $4 \mathrm{H}-3-22$ & 28.92 & 2.85 & 1.15 & 0.73 & 0.03 & 8.14 \\
\hline $4 \mathrm{H}-3-42$ & 29.12 & 3.99 & 2.42 & 1.28 & 8.87 & 8.38 \\
\hline $4 \mathrm{H}-3-62$ & 29.32 & 12.42 & 8.37 & 3.81 & 8.14 & 0.10 \\
\hline $4 \mathrm{H}-3-82$ & 29.52 & 7.23 & 6.88 & 8.26 & 0.82 & 0.87 \\
\hline $4 \mathrm{H}-3-182$ & 29.72 & 15.48 & 6.73 & 6.66 & 8.66 & 1.35 \\
\hline $4 \mathrm{H}-3-122$ & 29.98 & 16.18 & 11.14 & 4.27 & 8. 31 & 0.38 \\
\hline $4 \mathrm{H}-3-142$ & 30.12 & 2.25 & 1.80 & 0.98 & 0.14 & 0.13 \\
\hline $4 H-4-22$ & 38.42 & 3.32 & 1.58 & 1.34 & 0.15 & 0.33 \\
\hline $4 H-4-42$ & 38.62 & 5.25 & 2.58 & 1.33 & 8.23 & 1.19 \\
\hline $4 H-4-62$ & 38.82 & 2.38 & 1.19 & 0.74 & 8.88 & 0.37 \\
\hline $4 H-4-82$ & 31.82 & 1.96 & 0.76 & 0.36 & 8.39 & 0.45 \\
\hline $4 \mathrm{H}-4-102$ & 31.22 & 25.51 & 7.82 & 18.30 & 1.28 & 6.19 \\
\hline $4 H-4-122$ & 31.48 & 5.27 & 2.25 & 2.12 & 8.41 & 8.49 \\
\hline $4 \mathrm{H}-4-142$ & 31.68 & 5.97 & 2.47 & 2.35 & 0.23 & 0.92 \\
\hline $4 H-5-22$ & 31.92 & 4.83 & 2.33 & 1.50 & 0.12 & 0.88 \\
\hline $4 \mathrm{H}-5-42$ & 32.12 & 2.64 & 8.48 & 0.43 & 0.82 & 1.79 \\
\hline $4 H-5-62$ & 32.32 & 8.66 & 5.71 & 2.47 & 0.41 & 0.87 \\
\hline $4 \mathrm{H}-5-82$ & 32.52 & 13.86 & 7.59 & 4.47 & 8.35 & 0.65 \\
\hline $4 \mathrm{H}-5-182$ & 32.72 & 12.68 & 7.31 & 4.37 & 8.29 & 8.63 \\
\hline $4 H-5-122$ & 32.92 & 6.59 & 3.93 & 2.48 & 8.21 & 8.85 \\
\hline $4 H-5-142$ & 33.12 & 5.93 & 3.25 & 2.11 & 8.28 & 0.37 \\
\hline $5 \mathrm{H}-1-22$ & 35.42 & 4.25 & 1.89 & 2.81 & 8.19 & 8.16 \\
\hline $5 H-1-42$ & 35.62 & 3.94 & 1.86 & 1.82 & 8.86 & 8.28 \\
\hline $5 H-1-62$ & 35.82 & 13.77 & 5.98 & 7.31 & 8.16 & 0.48 \\
\hline $5 H-1-82$ & 36.02 & 4.58 & 2.13 & 1.98 & 8.36 & 0.11 \\
\hline $5 H-1-182$ & 36.22 & 14.13 & 8.27 & 3.63 & 1.85 & 0.38 \\
\hline $5 H-1-122$ & 36.48 & 15.93 & 8.81 & 6.46 & 8.41 & 8.25 \\
\hline $5 H-1-141$ & 36.31 & 28.53 & 8.85 & 8.89 & 0.84 & 2.75 \\
\hline $5 \mathrm{H}-2-22$ & 36.92 & 15.98 & 9.89 & 2.73 & 2.67 & 8.69 \\
\hline $5 \mathrm{H}-2-42$ & 37.12 & 15.66 & 7.51 & 6.63 & 0.93 & 8.59 \\
\hline $5 H-2-62$ & 37.32 & 1.45 & 8.77 & 8.68 & 0.04 & 0.84 \\
\hline $5 H-2-82$ & 37.52 & 6.82 & 0.87 & 4.99 & 0.13 & 8.03 \\
\hline $5 \mathrm{H}-2-102$ & 37.72 & 14.28 & 6.81 & 6.34 & 8.91 & 1.82 \\
\hline $5 \mathrm{H}-2-122$ & 37.98 & 5.64 & 1.97 & 2.73 & 0.45 & 0.49 \\
\hline $5 H-2-137$ & 38.87 & 8.79 & 3.22 & 4.39 & 0.38 & 0.88 \\
\hline $5 \mathrm{H}-3-22$ & 38.42 & 14.16 & 6.81 & 5.68 & 0.46 & 1.21 \\
\hline $5 t-3-42$ & 38.62 & 7.96 & 2.48 & 3.97 & 8.73 & 8.86 \\
\hline $5 H-3-62$ & 38.82 & 3.97 & 1.18 & 1.59 & 8.23 & 8.97 \\
\hline $5 \mathrm{H}-3-82$ & 39.82 & 11.81 & 5.45 & 4.74 & 8.59 & 1.83 \\
\hline
\end{tabular}


Table 1.4 (continued).

\begin{tabular}{|c|c|c|c|c|c|c|}
\hline \multirow[b]{2}{*}{$\begin{array}{c}\text { Sample } \\
\text { no. }\end{array}$} & \multirow[b]{2}{*}{$\begin{array}{l}\text { Depth } \\
\text { (mbsf) }\end{array}$} & \multicolumn{5}{|c|}{ Subfraction of coarse fraction $(\mu \mathrm{m})$} \\
\hline & & $\begin{array}{l}>63 \\
(\%)\end{array}$ & $\begin{array}{c}63-125 \\
(\%)\end{array}$ & $\begin{array}{c}125-500 \\
(\%)\end{array}$ & $\begin{array}{c}500-1000 \\
(\%)\end{array}$ & $\begin{array}{c}>1000 \\
(\%)\end{array}$ \\
\hline $5 H-3-182$ & 39.22 & 3.01 & 1.89 & 1.49 & 8.19 & 0.24 \\
\hline $5 H-3-122$ & 39.48 & 4.21 & 1.95 & 1.74 & 8.25 & 0.27 \\
\hline $5 H-3-137$ & 39.60 & 3.26 & 8.98 & 1.44 & 8.17 & 0.67 \\
\hline $5 H-4-22$ & 39.92 & 2.26 & 8.67 & 0.87 & 0.21 & 8.51 \\
\hline $5 H-4-42$ & 48.12 & 2.50 & 0.62 & 0.84 & 0.34 & 0.78 \\
\hline $5 H-4-62$ & 48.32 & 6.28 & 2.58 & 2.29 & 8.32 & 1.89 \\
\hline $5 H-4-82$ & $48: 52$ & 1.56 & 8.71 & 8.67 & 8.12 & 8.86 \\
\hline $5 H-4-182$ & 48.72 & 3.42 & 8.31 & 8.94 & 8.32 & 1.85 \\
\hline $5 H-4-122$ & 48.98 & 5.94 & 1.67 & 2.52 & 0.25 & 1.58 \\
\hline $5 H-4-137$ & 41.87 & 9.71 & 3.17 & 4.82 & 8.21 & 1.51 \\
\hline $5 \mathrm{H}-5-22$ & 41.42 & 9.77 & 1.83 & 2.61 & 8.58 & 4.83 \\
\hline $5 H-5-42$ & 41.62 & 7.08 & 2.73 & 3.52 & 0.45 & 0.38 \\
\hline $5 H-5-62$ & 41.82 & 6.85 & 2.36 & 2.56 & 8.44 & 0.69 \\
\hline $5 \mathrm{H}-5-82$ & 42.82 & 23.15 & 7.72 & 12.34 & 1.27 & 1.82 \\
\hline $5 H-5-182$ & 42.22 & 9.93 & 4.24 & 5.84 & 8.31 & 8.34 \\
\hline $5 H-5-122$ & 42.48 & 5.19 & 2.28 & 2.43 & 8.38 & 8.26 \\
\hline $5 H-5-141$ & 42.64 & 26.89 & 9.85 & 12.77 & 1.25 & 2.22 \\
\hline $6 \mathrm{H}-1-22$ & 44.92 & 6.18 & 4.29 & 1.34 & 8.19 & 0.36 \\
\hline $6 \mathrm{H}-1-59$ & 45.29 & 6.18 & 4.97 & 1.82 & 8.83 & 8.88 \\
\hline $6 H-1-78$ & 45.48 & 31.58 & 12.74 & 18.38 & 0.28 & 0.26 \\
\hline $6 H-1-181$ & 45.71 & 36.15 & 15.44 & 19.63 & 8.38 & 0.78 \\
\hline $6 \mathrm{H}-1-122$ & 45.98 & 32.86 & 14.46 & 17.31 & 8.26 & 8.83 \\
\hline $6 \mathrm{H}-1-142$ & 46.12 & 6.19 & 4.71 & 1.42 & 8.85 & 0.81 \\
\hline $6 \mathrm{H}-2-22$ & 46.42 & 2.82 & 1.86 & 0.86 & 0.38 & 0.52 \\
\hline $6 \mathrm{H}-2-59$ & 46.79 & 12.36 & 7.91 & 3.68 & 0.28 & 8.57 \\
\hline $6 \mathrm{H}-2-78$ & 46.92 & 11.12 & 2.38 & 3.57 & 0.38 & 4.87 \\
\hline $6 \mathrm{H}-2-181$ & 47.21 & 2.71 & 1.39 & 8.91 & 8.14 & 8.27 \\
\hline $6 \mathrm{H}-2-122$ & 47.48 & 1.87 & 8.75 & 8.67 & 0.87 & 0.38 \\
\hline $6 \mathrm{H}-2-142$ & 47.62 & 9.78 & 5.83 & 3.97 & 8.31 & 8.47 \\
\hline $6 \mathrm{H}-3-22$ & 47.92 & 2.21 & 1.21 & 8.98 & 0.85 & 0.85 \\
\hline $6 \mathrm{H}-3-59$ & 48.29 & 6.62 & 3.84 & 1.74 & 0.14 & 1.78 \\
\hline $6 \mathrm{H}-3-78$ & 48.48 & 5.89 & 2.96 & 2.01 & 0.18 & 0.82 \\
\hline $6 H-3-181$ & 48.71 & 35.88 & 14.38 & 28.37 & 8.18 & 8.15 \\
\hline $6 \mathrm{H}-3-122$ & 48.98 & 2.89 & 1.55 & 1.89 & 8.87 & 8.18 \\
\hline $6 \mathrm{H}-3-142$ & 49.12 & 3.46 & 2.88 & 1.28 & 8.18 & 8.08 \\
\hline $6 \mathrm{H}-4-22$ & 49.42 & 7.85 & 3.58 & 4.83 & 8.23 & 8.81 \\
\hline $6 \mathrm{H}-4-42$ & 49.62 & 5.01 & 2.22 & 2.77 & 8.82 & 8.88 \\
\hline $6 \mathrm{H}-4-59$ & 49.79 & 2.54 & 1.39 & 1.18 & 0.85 & 8.80 \\
\hline $6 H-4-78$ & 49.98 & 6.84 & 2.16 & 4.63 & 8.82 & 0.83 \\
\hline $6 \mathrm{H}-4-101$ & 58.21 & 4.46 & 1.98 & 2.42 & 8.85 & 0.89 \\
\hline $6 H-4-117$ & 58.37 & 4.67 & 1.46 & 3.88 & 8.02 & 8.11 \\
\hline $6 \mathrm{H}-5-22$ & 50.12 & 5.28 & 3.88 & 2.18 & 8.81 & 8.81 \\
\hline $6 \mathrm{H}-5-59$ & 58.29 & 5.18 & 2.76 & 2.86 & 8.11 & 0.17 \\
\hline $6 \mathrm{H}-5-78$ & 58.48 & 7.08 & 4.13 & 2.88 & 0.88 & 8.87 \\
\hline $6 \mathrm{H}-5-181$ & 50.71 & 12.48 & 5.61 & 6.56 & 0.14 & 0.17 \\
\hline $6 \mathrm{H}-5-122$ & 50.98 & 27.91 & 11.38 & 15.24 & 8.36 & 1.81 \\
\hline $6 \mathrm{H}-5-142$ & 51.12 & 4.31 & 2.47 & 1.61 & 0.18 & 0.13 \\
\hline $6 \mathrm{H}-6-22$ & 52.42 & 15.98 & 8.95 & 5.43 & 0.44 & 1.08 \\
\hline $6 \mathrm{H}-6-42$ & 52.62 & 16.15 & 8.96 & 5.57 & 8.37 & 1.25 \\
\hline $6 \mathrm{H}-6-59$ & 52.79 & 6.03 & 2.67 & 2.98 & 8.12 & 8.26 \\
\hline $6 \mathrm{H}-6-78$ & 52.98 & 9.68 & 3.93 & 5.21 & 0.22 & 8.24 \\
\hline $6 H-6-181$ & 53.21 & 3.83 & 1.51 & 2.22 & 8.18 & $\theta . \theta 8$ \\
\hline
\end{tabular}


Table 1.4 (continued).

\begin{tabular}{|c|c|c|c|c|c|c|}
\hline \multirow[b]{2}{*}{$\begin{array}{c}\text { Sample } \\
\text { no. }\end{array}$} & \multirow[b]{2}{*}{$\begin{array}{l}\text { Depth } \\
\text { (mbsf) }\end{array}$} & \multicolumn{5}{|c|}{ Subfraction of coarse fraction $(\mu \mathrm{m})$} \\
\hline & & $\begin{array}{l}>63 \\
(\%)\end{array}$ & $\begin{array}{c}63-125 \\
(\%)\end{array}$ & $\begin{array}{c}125-500 \\
(\%)\end{array}$ & $\begin{array}{c}500-1000 \\
(\%)\end{array}$ & $\begin{array}{c}>1000 \\
(\%)\end{array}$ \\
\hline $7 \mathrm{H}-1-22$ & 54.42 & 15.11 & 8.78 & 5.98 & 8.25 & 8.18 \\
\hline $7 H-1-42$ & 54.62 & 12.43 & 6.37 & 5.28 & 0.19 & 0.59 \\
\hline $7 H-1-64$ & 54.85 & 11.38 & 5.39 & 5.58 & 8.26 & 8.15 \\
\hline $7 \mathrm{H}-1-83$ & 55.63 & 7.94 & 5.12 & 2.57 & 0.14 & 0.11 \\
\hline $7 \mathrm{H}-2-22$ & 55.42 & 12.76 & 5.68 & 4.33 & 8.62 & 2.13 \\
\hline $7 \mathrm{H}-2-42$ & 56.13 & 18.24 & 7.87 & 8.72 & 8.98 & 8.67 \\
\hline $7 \mathrm{H}-2-63$ & 56.33 & 2.78 & 1.31 & 1.85 & 8.17 & 8.25 \\
\hline $7 \mathrm{H}-2-83$ & 56.53 & 28.54 & 9.24 & 9.82 & 1.08 & 1.28 \\
\hline $7 \mathrm{H}-3-22$ & 57.42 & 3.72 & 1.63 & 1.27 & 8.21 & 8.61 \\
\hline $7 \mathrm{H}-3-42$ & 57.62 & 1.91 & 0.63 & 8.44 & 8.28 & 8.56 \\
\hline $7 \mathrm{H}-3-64$ & 57.85 & 25.73 & 8.72 & 10.69 & 1.32 & 5.80 \\
\hline $7 \mathrm{H}-3-83$ & 58.03 & 2.84 & 1.21 & 1.88 & 0.30 & 8.33 \\
\hline $7 \mathrm{H}-4-22$ & 58.92 & 28.16 & 13.84 & 13.51 & 0.48 & 8.33 \\
\hline $7 \mathrm{H}-4-42$ & 59.13 & 1.82 & 8.69 & 8.64 & 8.13 & 8.36 \\
\hline $7 \mathrm{H}-4-65$ & 59.35 & 2.22 & 1.16 & 8.71 & 8.11 & 8.24 \\
\hline $7 \mathrm{H}-4-86$ & 59.56 & 8.62 & 5.65 & 2.88 & 0.32 & 8.57 \\
\hline $8 \mathrm{H}-1-21$ & 63.91 & 9.56 & 6.91 & 2.21 & 8.48 & 8.84 \\
\hline $8 H-1-48$ & 64.11 & 13.72 & 6.66 & 5.71 & 0.74 & 8.61 \\
\hline $8 \mathrm{H}-1-58$ & 64.29 & 3.61 & 1.84 & 1.18 & 0.15 & 8.52 \\
\hline $8 \mathrm{H}-1-182$ & 64.72 & 7.58 & 6.42 & 8.95 & 8.89 & 8.12 \\
\hline $8 \mathrm{H}-2-21$ & 65.41 & 6.73 & 5.04 & 1.33 & 0.13 & 0.23 \\
\hline $8 \mathrm{H}-2-48$ & 65.68 & 9.62 & 4.26 & 3.27 & 8.85 & 1.24 \\
\hline $8 \mathrm{H}-2-59$ & 65.78 & 4.13 & 2.63 & 8.97 & 0.23 & 0.38 \\
\hline $8 \mathrm{H}-2-82$ & 66.80 & 12.86 & 6.85 & 4.76 & 0.65 & 8.68 \\
\hline $8 \mathrm{H}-2-182$ & 66.22 & 3.88 & 1.22 & 1.41 & 0.18 & 8.27 \\
\hline $8 \mathrm{H}-2-122$ & 66.42 & 3.85 & 1.24 & 1.27 & 8.38 & 8.24 \\
\hline $8 \mathrm{H}-2-142$ & 66.62 & 2.98 & 1.21 & 1.32 & 8.21 & 8.24 \\
\hline $8 \mathrm{H}-3-28$ & 66.98 & 3.80 & 1.61 & 0.72 & 0.24 & 8.43 \\
\hline $8 \mathrm{H}-3-48$ & 67.11 & 13.98 & 7.44 & 4.78 & 8.55 & 1.21 \\
\hline $8 \mathrm{H}-3-68$ & 67.37 & 5.88 & 3.54 & 1.94 & 8.19 & 8.13 \\
\hline $8 \mathrm{H}-3-82$ & 67.58 & 5.94 & 3.94 & 1.77 & 0.11 & 8.12 \\
\hline $8 \mathrm{H}-3-182$ & 67.72 & 8.08 & 3.68 & 3.87 & 8.56 & 8.85 \\
\hline $8 \mathrm{H}-3-122$ & 67.98 & 7.68 & 5.52 & 1.88 & 0.13 & 0.15 \\
\hline $8 \mathrm{H}-3-142$ & 68.18 & 1.16 & 0.32 & 0.43 & 8.13 & 0.28 \\
\hline $8 \mathrm{H}-4-22$ & 68.43 & 9.84 & 5.61 & 3.90 & 8.17 & 0.16 \\
\hline $8 \mathrm{H}-4-42$ & 68.62 & 13.66 & 7.57 & 4.99 & 0.32 & 8. 78 \\
\hline $8 H-5-22$ & 69.92 & 4.98 & 2.39 & 2.87 & 0.17 & 8.27 \\
\hline $8 \mathrm{H}-5-42$ & 78.13 & 11.74 & 7.38 & 3.67 & 8.49 & 0.28 \\
\hline $8 H-5-6 \theta$ & 78.38 & 23.73 & 6.83 & 7.94 & 0.46 & 8.50 \\
\hline $8 H-5-82$ & 78.52 & 2.31 & 1.48 & 0.62 & 0.24 & 8.85 \\
\hline $8 \mathrm{H}-5-122$ & 78.92 & 6.47 & 4.29 & 1.97 & 0.15 & 0.86 \\
\hline $8 H-5-142$ & 71.12 & 6.72 & 4.36 & 2.18 & 0.21 & 8.85 \\
\hline $8 \mathrm{H}-6-22$ & 71.42 & 2.44 & 1.39 & 0.82 & 0.88 & 0.15 \\
\hline $8 H-6-42$ & 71.62 & 3.15 & 2.26 & 8.80 & 8.85 & 8.84 \\
\hline $8 H-6-68$ & 71.88 & 8.57 & 8.37 & 8.16 & 8.84 & 0.88 \\
\hline $9 H-1-22$ & 73.43 & 2.86 & 0.94 & 0.99 & 0.82 & 0.11 \\
\hline $9 H-1-48$ & 73.68 & 2.23 & 1.08 & 1.87 & 8.84 & 8.84 \\
\hline $9 H-1-59$ & 73.79 & 3.95 & 1.34 & 2.58 & 0.01 & 8.82 \\
\hline $9 H-1-82$ & 74.82 & 1.53 & 0.71 & 8.81 & 8.88 & 8.81 \\
\hline $9 H-1-182$ & 74.22 & 8.67 & 8.35 & 0.29 & 8.82 & 8.01 \\
\hline $9 H-1-123$ & 74.43 & 8.37 & 8.22 & 8.13 & 8.82 & 8.68 \\
\hline $9 \mathrm{H}-1-142$ & 74.62 & 17.89 & 15.16 & 2.62 & 8.89 & 8.82 \\
\hline
\end{tabular}


R. HENRICH, T. WOLF, G. BOHRMANN, J. THIEDE

Table 1.4 (continued).

\begin{tabular}{|c|c|c|c|c|c|c|}
\hline \multirow[b]{2}{*}{$\begin{array}{l}\text { Sample } \\
\text { no. }\end{array}$} & \multirow[b]{2}{*}{$\begin{array}{l}\text { Depth } \\
\text { (mbsf) }\end{array}$} & \multicolumn{5}{|c|}{ Subfraction of coarse fraction $(\mu \mathrm{m})$} \\
\hline & & $\begin{array}{l}>63 \\
(\%)\end{array}$ & $\begin{array}{c}63-125 \\
(\%)\end{array}$ & $\begin{array}{c}125-500 \\
(\%)\end{array}$ & $\begin{array}{c}500-1000 \\
(\%)\end{array}$ & $\begin{array}{c}>1000 \\
(\%)\end{array}$ \\
\hline $9 \mathrm{H}-2-23$ & 74.93 & 6.32 & 4.72 & 1.38 & 8.89 & 0.13 \\
\hline $9 H-2-48$ & 75.10 & 3.92 & 2.91 & 8.89 & 0.12 & ө. \\
\hline $9 H-2-59$ & 75.29 & 1.94 & 1.48 & 8.35 & 8.18 & 8.01 \\
\hline $9 H-2-82$ & 75.52 & 2.52 & 1.68 & 8.75 & 0.87 & 0.02 \\
\hline $9 H-2-182$ & 75.72 & 5.52 & 3.38 & 1.75 & 0.13 & 0.26 \\
\hline $9 \mathrm{H}-2-123$ & 75.93 & 5.72 & 3.52 & 2.11 & 0.88 & $\theta .01$ \\
\hline $9 H-2-142$ & 76.12 & 13.96 & 8.94 & 4.46 & 8.11 & 8.45 \\
\hline $9 \mathrm{H}-3-23$ & 76.43 & 1.43 & 8.58 & 8.74 & 8.87 & 8.84 \\
\hline $9 \mathrm{H}-3-4 \theta$ & 76.68 & 0.80 & 8.35 & 8.38 & 8.87 & 0.08 \\
\hline $9 H-3-59$ & 76.79 & 1.49 & 8.77 & 8.78 & 8.02 & 0.08 \\
\hline $9 \mathrm{H}-3-82$ & 77.82 & 2.31 & 8.93 & 8.64 & 8.85 & 8.69 \\
\hline $9 H-3-182$ & 77.22 & 1.87 & 8.48 & 8.49 & 8.89 & 8.81 \\
\hline $9 \mathrm{H}-3-123$ & 77.43 & 1.05 & 8.52 & 8.38 & 8.08 & 0.15 \\
\hline $9 \mathrm{H}-3-142$ & 77.62 & 22.37 & 18.68 & 11.45 & 8.21 & 8.11 \\
\hline $9 H-4-23$ & 78.43 & 4.21 & 2.71 & 1.39 & 0.86 & 8.85 \\
\hline $9 H-4-48$ & 78.68 & 27.21 & 22.22 & 3.98 & 0.35 & 8.66 \\
\hline $9 H-4-59$ & 78.79 & 6.92 & 3.98 & 2.17 & 0.16 & 8.69 \\
\hline $9 H-4-82$ & 79.82 & 4.61 & 2.62 & 1.87 & 8.11 & 8.81 \\
\hline $9 \mathrm{H}-4-182$ & 79.22 & 5.30 & 3.14 & 2.82 & 0.87 & 8.87 \\
\hline $9 \mathrm{H}-5-23$ & 79.93 & 1.63 & 8.67 & 8.32 & 8.03 & 8.01 \\
\hline $9 H-5-4 \theta$ & 80.10 & 14.57 & 11.22 & 3.25 & 0.18 & 0.80 \\
\hline $9 H-5-59$ & 88.29 & 5.85 & 4.18 & 8.81 & 8.84 & 8.82 \\
\hline $9 H-5-82$ & 88.52 & 5.60 & 4.44 & 0.98 & 8.85 & 0.13 \\
\hline $9 \mathrm{H}-5-182$ & 88.72 & 9.26 & 5.94 & 3.87 & 8.28 & 0.05 \\
\hline $9 H-5-123$ & 88.93 & 2.88 & 1.15 & 1.84 & 8.11 & 8.58 \\
\hline $10 \mathrm{H}-2-22$ & 84.42 & 1.78 & 8.98 & 8.65 & 8.83 & 8.28 \\
\hline $18 \mathrm{H}-2-41$ & 84.61 & 0.81 & 8.48 & 0.30 & 8.03 & 0.88 \\
\hline $18 \mathrm{H}-2-57$ & 84.78 & 2.28 & 1.11 & 1.82 & 8.07 & 8.80 \\
\hline $10 \mathrm{H}-2-121$ & 85.42 & 1.75 & 0.78 & 1.05 & 8.80 & 0.08 \\
\hline $10 \mathrm{H}-2-142$ & 85.63 & 8.72 & 8.48 & 8.38 & 0.82 & 8.88 \\
\hline $18 \mathrm{H}-3-22$ & 85.92 & 0.26 & 8.16 & 8.18 & 8.80 & 8.80 \\
\hline $18 \mathrm{H}-3-41$ & 86.11 & 4.92 & 8.38 & 1.52 & 8.33 & 2.77 \\
\hline $18 \mathrm{H}-3-57$ & 86.28 & 1.03 & 8.44 & 8.57 & 0.80 & 8.82 \\
\hline $18 \mathrm{H}-3-78$ & 86.48 & 1.93 & 8.62 & 8.83 & 8.18 & 8.38 \\
\hline $18 \mathrm{H}-3-181$ & 86.71 & 8.93 & 8.56 & 0.34 & 8.82 & 8.81 \\
\hline $10 \mathrm{H}-3-121$ & 86.92 & 2.52 & 0.85 & 1.26 & 8.15 & 0.26 \\
\hline $10 \mathrm{H}-3-142$ & 87.12 & 8.11 & 4.11 & 3.68 & 0.18 & 0. 22 \\
\hline $10 \mathrm{H}-4-21$ & 87.42 & 1.84 & 8.55 & 8.47 & 0.81 & 8.81 \\
\hline $18 \mathrm{H}-4-41$ & 87.61 & 8.93 & 8.54 & 8.27 & 8.84 & 8.88 \\
\hline $10 \mathrm{H}-4-57$ & 87.78 & 28.98 & 16.69 & 3.52 & 8.29 & 8.48 \\
\hline $10 \mathrm{H}-4-78$ & 87.98 & 28.55 & 22.45 & 5.62 & 0.28 & 0.28 \\
\hline $10 \mathrm{H}-4-101$ & 88.22 & 27.55 & 21.23 & 5.85 & 0.28 & 8.27 \\
\hline $10 \mathrm{H}-4-121$ & 88.41 & 11.32 & 5.69 & 4.28 & 8.66 & 8.69 \\
\hline $10 \mathrm{H}-5-21$ & 88.91 & 0.81 & 0.28 & 0.37 & 8.88 & 8.88 \\
\hline $18 \mathrm{H}-5-41$ & 89.11 & 3.22 & 1.88 & 1.12 & 0.12 & 8.18 \\
\hline $18 \mathrm{H}-5-57$ & 89.28 & 1.39 & 8.22 & 8.41 & 8.18 & 8.66 \\
\hline $18 \mathrm{H}-5-78$ & 89.48 & 9.51 & 2.15 & 1.88 & 8.67 & 5.61 \\
\hline $18 \mathrm{H}-5-181$ & 89.71 & 5.11 & 2.88 & 1.23 & 8.22 & 1.58 \\
\hline $10 \mathrm{H}-5-121$ & 89.92 & 3.17 & 1.45 & 0.58 & 8.11 & 1.83 \\
\hline $18 \mathrm{H}-6-21$ & 98.42 & 14.21 & 12.36 & 1.80 & 8.84 & 0.01 \\
\hline $10 \mathrm{H}-6-41$ & 98.62 & 5.79 & 4.34 & 1.86 & 0.12 & 8. 27 \\
\hline $11 \mathrm{H}-1-22$ & 92.42 & 3.98 & 2.87 & 8.86 & 8.86 & 8.11 \\
\hline
\end{tabular}


Table 1.4 (continued).

\begin{tabular}{|c|c|c|c|c|c|c|}
\hline \multirow[b]{2}{*}{$\begin{array}{c}\text { Sample } \\
\text { no. }\end{array}$} & \multirow[b]{2}{*}{$\begin{array}{l}\text { Depth } \\
\text { (mbsf) }\end{array}$} & \multicolumn{5}{|c|}{ Subfraction of coarse fraction $(\mu \mathrm{m})$} \\
\hline & & $\begin{array}{l}>63 \\
(\%)\end{array}$ & $\begin{array}{c}63-125 \\
(\%)\end{array}$ & $\begin{array}{c}125-500 \\
(\%)\end{array}$ & $\begin{array}{c}500-1000 \\
(\%)\end{array}$ & $\begin{array}{c}>1000 \\
(\%)\end{array}$ \\
\hline $11 \mathrm{H}-1-42$ & 92.62 & 3.03 & 1.83 & 8.79 & 8.17 & 0.24 \\
\hline $11 H-1-6 \theta$ & 92.80 & 2.79 & 1.67 & 0.92 & 8.85 & 8.15 \\
\hline $11 H-1-81$ & 93.01 & 13.63 & 7.98 & 4.64 & 8.38 & 8.71 \\
\hline $11 \mathrm{H}-1-108$ & 93.28 & 0.94 & 0.54 & 0.37 & 8. 82 & 0.81 \\
\hline $11 \mathrm{H}-1-122$ & 93.40 & 2.37 & 1.67 & 0.61 & $8 . \theta 8$ & 0.89 \\
\hline $11 H-1-142$ & 93.62 & 5.62 & 3.34 & 1.58 & 0.14 & 0.64 \\
\hline $11 \mathrm{H}-2-22$ & 93.92 & 5.96 & 1.64 & 1.37 & 0.33 & 2.62 \\
\hline $11 \mathrm{H}-2-39$ & 94.89 & 3.42 & 1.99 & 1.24 & 8.07 & 0.12 \\
\hline $11 \mathrm{H}-2-63$ & 94.33 & 2.16 & 0.95 & 1.15 & 0.04 & 8.82 \\
\hline $11 \mathrm{H}-2-81$ & 94.51 & 1.22 & 0.54 & 8.50 & 0.03 & 0.15 \\
\hline $11 H-2-188$ & 94.78 & 1.99 & 0.93 & 0.83 & 0.84 & 8.19 \\
\hline $11 \mathrm{H}-2-122$ & 94.92 & 0.99 & 8.35 & 0.48 & 8.81 & 0.23 \\
\hline $11 \mathrm{H}-2-142$ & 95.14 & 1.51 & 0.55 & 0.37 & 0.03 & 0.56 \\
\hline $11 \mathrm{H}-3-22$ & 95.42 & 1.78 & 0.99 & 0.69 & 0.03 & 0.87 \\
\hline $11 \mathrm{H}-3-42$ & 95.62 & 5.82 & 4.17 & 1.88 & 0.12 & 8.45 \\
\hline $11 \mathrm{H}-3-6 \theta$ & 95.88 & 2.91 & 2.03 & 8.77 & 0.86 & 0.85 \\
\hline $11 \mathrm{H}-3-81$ & 96.81 & 13.87 & 8.92 & 4.52 & 0.33 & 0.18 \\
\hline $11 \mathrm{H}-3-180$ & 96.28 & 11.55 & 7.66 & 3.57 & 0.26 & 0.86 \\
\hline $11 \mathrm{H}-3-122$ & 96.48 & 3.94 & 2.88 & 0.94 & 8.89 & 0.83 \\
\hline $11 \mathrm{H}-3-142$ & 96.62 & 5.38 & 3.66 & 1.43 & 0.15 & 0.86 \\
\hline $11 \mathrm{H}-4-22$ & 96.92 & 3.08 & 1.34 & 1.84 & 0.89 & 0.53 \\
\hline $11 \mathrm{H}-4-42$ & 97.14 & 3.15 & 1.83 & 1.03 & 0.11 & 0.18 \\
\hline $11 \mathrm{H}-4-68$ & 97.38 & 2.57 & 1.42 & 8.65 & 8.18 & 0.32 \\
\hline $11 \mathrm{H}-4-81$ & 97.51 & 1.29 & 0.55 & 8.53 & 8.87 & 0.14 \\
\hline $11 \mathrm{H}-4-122$ & 97.92 & 28.32 & 18.58 & 7.68 & 8.72 & 1.42 \\
\hline $11 H-4-142$ & 97.14 & 4.27 & 2.27 & 1.56 & 0.18 & 0.26 \\
\hline $11 \mathrm{H}-5-22$ & 98.42 & 8.66 & 8.24 & 0.22 & 0.07 & 0.13 \\
\hline $11 \mathrm{H}-5-42$ & 98.62 & 17.98 & 11.95 & $5.4 \theta$ & 0.23 & 0.32 \\
\hline $11 \mathrm{H}-5-68$ & 98.88 & 8.69 & 8.23 & 8.12 & 8.83 & 0.31 \\
\hline $11 \mathrm{H}-5-88$ & 99.08 & 8.38 & 4.88 & 3.83 & 8.22 & 0.33 \\
\hline $11 \mathrm{H}-5-108$ & 99.38 & 8.51 & 0.31 & 0.18 & 8.82 & 0.08 \\
\hline $11 \mathrm{H}-5-122$ & 99.52 & 2.28 & 0.87 & 0.61 & 0.11 & 0.69 \\
\hline $11 \mathrm{H}-6-22$ & 99.92 & 1.72 & 0.72 & 8.54 & 8.82 & 8. 44 \\
\hline $11 \mathrm{H}-6-42$ & 108.14 & 0.91 & 8.57 & 0.25 & 0.02 & 0.07 \\
\hline $11 \mathrm{H}-6-68$ & 180.38 & 1.78 & 8.64 & 8.51 & 0.81 & 8.62 \\
\hline $11 H-6-88$ & 180.58 & 1.58 & 0.62 & 8.83 & 8.02 & 0.83 \\
\hline $11 \mathrm{H}-6-122$ & 188.92 & 1.28 & 8.57 & 8.57 & 0.83 & 0.83 \\
\hline $11 \mathrm{H}-6-142$ & 181.14 & 5.73 & 5.03 & 0.56 & 0.01 & 0.13 \\
\hline $11 \mathrm{H}-7-22$ & 181.42 & 1.16 & 0.49 & 0.61 & 0.03 & 0.83 \\
\hline $12 \mathrm{H}-1-22$ & 181.91 & 15.99 & 8.49 & 6.16 & 8.75 & 0.59 \\
\hline $12 \mathrm{H}-1-42$ & 102.18 & 1.46 & 0.85 & 0.56 & 0.84 & 8.01 \\
\hline $12 \mathrm{H}-1-62$ & 102.30 & 4.56 & 2.11 & 1.48 & 0.12 & 0.85 \\
\hline $12 \mathrm{H}-1-82$ & 182.49 & 1.78 & 1.22 & 0.46 & 8.81 & 0.89 \\
\hline $12 \mathrm{H}-1-102$ & 182.68 & 1.87 & 0.51 & 8.43 & 8.84 & 8.89 \\
\hline $12 \mathrm{H}-1-122$ & 182.87 & 2.45 & 1.38 & 1.14 & 8.81 & 0.80 \\
\hline $12 \mathrm{H}-1-142$ & 183.86 & 4.07 & 1.85 & 2.84 & 8.87 & 0.11 \\
\hline $12 \mathrm{H}-2-22$ & 183.35 & 8.08 & 4.29 & 3.63 & 0.12 & 0.84 \\
\hline $12 \mathrm{H}-2-42$ & 183.54 & 0. 58 & 0.32 & 0.23 & 0.03 & 0.00 \\
\hline $12 \mathrm{H}-2-62$ & 103.74 & 2.65 & 1.64 & 8.97 & 0.84 & 8.80 \\
\hline $12 \mathrm{H}-2-82$ & 183.93 & 5.65 & 3.98 & 1.72 & 8.83 & 8.88 \\
\hline $12 \mathrm{H}-2-102$ & 104.12 & 1.92 & 1.08 & 0.79 & 8.88 & 0.05 \\
\hline $12 \mathrm{H}-2-122$ & 104.31 & 1.38 & 0.82 & 0.48 & 0.01 & 0.07 \\
\hline
\end{tabular}


Table 1.4 (continued).

\begin{tabular}{|c|c|c|c|c|c|c|}
\hline \multirow[b]{2}{*}{$\begin{array}{c}\text { Sample } \\
\text { no. }\end{array}$} & \multirow[b]{2}{*}{$\begin{array}{l}\text { Depth } \\
\text { (mbsf) }\end{array}$} & \multicolumn{5}{|c|}{ Subfraction of coarse fraction $(\mu \mathrm{m})$} \\
\hline & & $\begin{array}{l}>63 \\
(\%)\end{array}$ & $\begin{array}{c}63-125 \\
(\%)\end{array}$ & $\begin{array}{c}125-500 \\
(\%)\end{array}$ & $\begin{array}{c}500-1000 \\
(\%)\end{array}$ & $\begin{array}{c}>1000 \\
(\%)\end{array}$ \\
\hline $12 \mathrm{H}-2-142$ & 104.58 & 2.85 & 1.86 & 0.82 & 0.03 & 0.14 \\
\hline $12 \mathrm{H}-3-22$ & 184.79 & 6.92 & 3.62 & 2.65 & 8.85 & 8.68 \\
\hline $12 \mathrm{H}-3-42$ & 184.98 & 1.24 & 8.88 & 0.38 & 8.82 & 8.84 \\
\hline $12 \mathrm{H}-3-62$ & 105.18 & 1.84 & 8.53 & 0.48 & 0.88 & 0.83 \\
\hline $12 \mathrm{H}-3-82$ & 185.37 & 1.04 & 0.51 & 0.58 & 8.03 & 8.80 \\
\hline $12 \mathrm{H}-3-182$ & 105.56 & 8.79 & 8.37 & 0.41 & 0.01 & 8.80 \\
\hline $12 \mathrm{H}-3-122$ & 105.75 & 0.39 & 0.15 & 0.15 & 0.03 & 8.86 \\
\hline $12 \mathrm{H}-3-142$ & 185.94 & 4.66 & 2.78 & 1.78 & 0.18 & 8.88 \\
\hline $12 \mathrm{H}-4-22$ & 186.23 & 3.76 & 0.78 & 0.65 & 0.11 & 2.22 \\
\hline $12 \mathrm{H}-4-42$ & 186.42 & 0.94 & 0.44 & 0.26 & 0.06 & 0.18 \\
\hline $12 \mathrm{H}-4-62$ & 186.62 & 1.17 & 0.59 & 8.58 & 0.84 & 8.84 \\
\hline $12 \mathrm{H}-4-82$ & 186.81 & 3.01 & 8.62 & 2.06 & 0.29 & 0.84 \\
\hline $12 \mathrm{H}-4-102$ & 107.80 & 5.94 & 3.36 & 2.29 & 0.15 & 0.14 \\
\hline $12 \mathrm{H}-5-22$ & 107.67 & 16.86 & 18.28 & 5.11 & 8.43 & 1.12 \\
\hline $12 \mathrm{H}-5-42$ & 107.86 & 6.27 & 3.13 & 2.71 & 0.86 & 0.37 \\
\hline $12 \mathrm{H}-5-62$ & 188.86 & 3.14 & 1.18 & 1.78 & 8.24 & 0.02 \\
\hline $12 \mathrm{H}-5-82$ & 108.25 & 2.66 & 1.77 & 0.84 & 0.03 & 0.82 \\
\hline $12 \mathrm{H}-5-102$ & 188.44 & 2.55 & 1.71 & 0.79 & 8.05 & 8.80 \\
\hline $12 \mathrm{H}-5-122$ & 188.63 & 4.37 & 2.25 & 1.34 & 0.89 & 0.69 \\
\hline $12 \mathrm{H}-5-142$ & 188.82 & 7.43 & 4.93 & 2.13 & 8.85 & 8.32 \\
\hline $12 \mathrm{H}-6-22$ & 189.11 & 4.64 & 2.53 & 2.82 & 8.87 & 8.82 \\
\hline $12 \mathrm{H}-6-42$ & 189.38 & 0.61 & 8.68 & 0.81 & 8.88 & 8.88 \\
\hline $12 \mathrm{H}-6-62$ & 189.58 & 1.41 & 8.94 & 0.39 & 0.03 & 0.85 \\
\hline $12 \mathrm{H}-6-82$ & 189.69 & 1.58 & 0.77 & 8.66 & 0.85 & 8.82 \\
\hline $12 \mathrm{H}-6-182$ & 109.88 & 3.78 & 1.17 & 1.96 & 0.11 & 8.54 \\
\hline $12 \mathrm{H}-6-122$ & 110.87 & 5.18 & 1.65 & 3.84 & 0.11 & 0.38 \\
\hline $12 \mathrm{H}-6-142$ & 118.26 & 1.77 & 0.84 & 8.65 & 8.88 & 0.28 \\
\hline $12 \mathrm{H}-7-22$ & 110.55 & 0.88 & 0.32 & 8.28 & 0.07 & 0.21 \\
\hline $12 \mathrm{H}-7-42$ & 110.74 & 1.54 & 8.77 & 0.66 & 0.10 & 8.81 \\
\hline $13 \mathrm{H}-1-62$ & 111.81 & 1.88 & 0.86 & 0.27 & 0.07 & 0.68 \\
\hline $13 H-1-82$ & 112.88 & 1.23 & 8.78 & 0.35 & 8.01 & 0.89 \\
\hline $13 H-1-182$ & 112.28 & 0.79 & 0.52 & 0.26 & 0.01 & 0.80 \\
\hline $13 H-1-122$ & 112.48 & 0.72 & 8.48 & 8.23 & 8.88 & 8.81 \\
\hline $13 H-1-145$ & 112.62 & 2.87 & 0.39 & 0.28 & 8.08 & 2.28 \\
\hline $13 \mathrm{H}-2-21$ & 112.88 & 18.42 & 5.56 & 4.68 & 8.17 & 8.89 \\
\hline $13 \mathrm{H}-2-42$ & 113.88 & 5.24 & 2.91 & 2.87 & 0.14 & 0.12 \\
\hline $13 \mathrm{H}-2-62$ & 113.28 & 4.58 & 2.52 & 1.82 & 0.11 & 0.13 \\
\hline $13 \mathrm{H}-2-82$ & 113.47 & 1.03 & 8.44 & 0.53 & 0.05 & 0.01 \\
\hline $13 \mathrm{H}-2-182$ & 113.67 & 5.88 & 3.99 & 1.74 & 0.03 & 8.12 \\
\hline $13 H-2-122$ & 113.87 & 1.56 & 8.78 & 8.88 & 8.81 & 0.85 \\
\hline $13 \mathrm{H}-3-21$ & 114.35 & 3.30 & 1.48 & 1.71 & 8.10 & 8.89 \\
\hline $13 H-3-145$ & 114.58 & 9.74 & 4.46 & 4.39 & 0.59 & 0.30 \\
\hline $13 H-4-21$ & 115.82 & 18.35 & 5.81 & 4.86 & 0.17 & 0.31 \\
\hline $13 \mathrm{H}-4-42$ & 116.82 & 8.75 & 8.39 & 8.17 & 8.86 & 8.13 \\
\hline $13 H-4-62$ & 116.22 & 3.87 & 2.26 & 1.44 & 0.08 & 0.89 \\
\hline $13 \mathrm{H}-4-82$ & 116.41 & 3.96 & 2.52 & 1.26 & 0.13 & 0.85 \\
\hline $13 \mathrm{H}-4-102$ & 116.61 & 2.89 & 1.86 & 8.94 & 0.87 & 0.82 \\
\hline $13 H-4-122$ & 116.81 & 15.82 & 7.69 & 2.55 & 0.38 & 5.28 \\
\hline $13 H-5-21$ & 117.29 & 4.28 & 1.14 & 1.28 & 0.61 & 1.17 \\
\hline $13 H-5-42$ & 117.49 & 7.28 & 2.65 & 1.78 & 0.19 & 2.66 \\
\hline $13 H-5-62$ & 117.69 & 6.42 & 5.18 & 1.18 & 0.03 & 0.03 \\
\hline $13 t-5-86$ & 117.92 & 6.61 & 4.80 & 2.13 & 0.18 & B. 38 \\
\hline
\end{tabular}


Table 1.4 (continued).

\begin{tabular}{|c|c|c|c|c|c|c|}
\hline \multirow[b]{2}{*}{$\begin{array}{c}\text { Sample } \\
\text { no. }\end{array}$} & \multirow[b]{2}{*}{$\begin{array}{l}\text { Depth } \\
\text { (mbsf) }\end{array}$} & \multicolumn{5}{|c|}{ Subfraction of coarse fraction $(\mu \mathrm{m})$} \\
\hline & & $\begin{array}{l}>63 \\
(\%)\end{array}$ & $\begin{array}{c}63-125 \\
(\%)\end{array}$ & $\begin{array}{c}125-500 \\
(\%)\end{array}$ & $\begin{array}{c}500-1000 \\
(\%)\end{array}$ & $\begin{array}{c}>1000 \\
(\%)\end{array}$ \\
\hline $13 H-6-21$ & 118.76 & 1.22 & 8.29 & 8.21 & 0.83 & 8.69 \\
\hline $13 H-6-42$ & 118.96 & 1.84 & 0.58 & 0.35 & 0.82 & 8.89 \\
\hline $13 H-6-62$ & 119.16 & 8.79 & 8.32 & 8.42 & 8.85 & 8.88 \\
\hline $13 \mathrm{H}-6-82$ & 119.35 & 1.16 & 8.65 & 8.46 & 8.84 & $\theta .01$ \\
\hline $13 \mathrm{H}-7-21$ & 119.56 & 1.77 & 8.52 & 8.51 & 8.12 & 8.62 \\
\hline $13 \mathrm{H}-7-42$ & 119.77 & 13.74 & 5.39 & 7.83 & 0.24 & 1.88 \\
\hline $13 \mathrm{H}-7-62$ & 119.96 & 8.67 & 8.31 & 8.29 & 8.06 & 8.81 \\
\hline $13 \mathrm{H}-7-82$ & 128.16 & 1.31 & 8.78 & 8.39 & 8.89 & 8.13 \\
\hline $13 \mathrm{H}-7-182$ & 128.35 & 8.48 & 8.31 & 8.11 & 8.85 & 0.81 \\
\hline $13 \mathrm{H}-7-122$ & 128.55 & 0.73 & 0.24 & 8.36 & 8.13 & 8. 80 \\
\hline $14 \mathrm{H}-2-82$ & 123.02 & 6.28 & 3.58 & 2.44 & 8.17 & 8.01 \\
\hline $14 \mathrm{H}-2-103$ & 123.23 & 4.25 & 2.46 & 1.72 & 8.85 & 8.82 \\
\hline $14 \mathrm{H}-2-123$ & 123.43 & 2.82 & 1.43 & 1.26 & 8.12 & 8.81 \\
\hline $14 \mathrm{H}-2-142$ & 123.62 & 24.58 & 9.78 & 12.55 & 1.53 & 8.64 \\
\hline $14 \mathrm{H}-3-21$ & 123.91 & 24.52 & 9.88 & 13.76 & 8.32 & 8.64 \\
\hline $14 \mathrm{H}-3-41$ & 124.11 & 6.98 & 3.63 & 2.91 & 0.22 & 8.22 \\
\hline $14 \mathrm{H}-3-62$ & 124.32 & 2.72 & 1.18 & 8.96 & 8.28 & 8. 38 \\
\hline $14 \mathrm{H}-3-82$ & 123.52 & 3.15 & 1.18 & 8.78 & 0.29 & 8. 98 \\
\hline $14 \mathrm{H}-3-183$ & 124.72 & 3.07 & 1.16 & 0.91 & 0.05 & 0.95 \\
\hline $14 \mathrm{H}-3-123$ & 124.93 & 3.38 & 1.75 & 1.52 & 0.08 & 0.83 \\
\hline $14 \mathrm{H}-4-21$ & 125.41 & 8.41 & 0.17 & 8.23 & 8.81 & 8.88 \\
\hline $14 \mathrm{H}-4-62$ & 125.82 & 2.22 & 1.42 & 8.75 & 0.85 & 8.88 \\
\hline $14 \mathrm{H}-4-82$ & 126.02 & 8.26 & 8.13 & 8.12 & 8.80 & 8.81 \\
\hline $14 \mathrm{H}-5-21$ & 126.91 & 2.82 & 8.55 & 8.49 & 0.25 & 1.53 \\
\hline $14 \mathrm{H}-5-41$ & 127.11 & 18.84 & 5.87 & 3.31 & 8.47 & 1.19 \\
\hline $14 \mathrm{H}-5-62$ & 127.32 & 21.53 & 11.38 & 9.77 & 0.15 & 8.23 \\
\hline $14 \mathrm{H}-5-82$ & 127.52 & 8.73 & 5.30 & 2.76 & 8.21 & 8.46 \\
\hline $14 \mathrm{H}-5-182$ & 127.72 & 1.37 & 8.28 & 8.43 & 8.15 & 8.51 \\
\hline $14 \mathrm{H}-5-123$ & 127.93 & 1.88 & 8.79 & 8.62 & 0.03 & 8.36 \\
\hline $14 \mathrm{H}-5-142$ & 128.12 & 5.85 & 2.84 & 2.82 & 0.87 & 8.12 \\
\hline $14 \mathrm{H}-\mathrm{C}-15$ & 128.35 & 4.81 & 2.29 & 2.18 & 8.15 & 8.19 \\
\hline $15 \mathrm{H}-1-22$ & 130.42 & 3.35 & 1.19 & 1.48 & 8.45 & 8.31 \\
\hline $15 \mathrm{H}-1-42$ & 138.62 & 8.93 & 0.41 & 8.33 & 8.14 & 8.85 \\
\hline $15 \mathrm{H}-1-62$ & 130.82 & 6.57 & 2.84 & 3.82 & 8.33 & 8. 38 \\
\hline $15 \mathrm{H}-1-82$ & 131.82 & 6.39 & 2.93 & 3.33 & 0.89 & 8.84 \\
\hline $15 \mathrm{H}-1-182$ & 131.22 & 6.96 & 3.89 & 3.38 & 8.41 & 8.88 \\
\hline $15 \mathrm{H}-1-122$ & 131.42 & 1.96 & 8.88 & 0.82 & 0.18 & 8.88 \\
\hline $15 \mathrm{H}-1-142$ & 131.62 & 1.65 & 8.35 & 8. 88 & 8.89 & 8. 41 \\
\hline $15 \mathrm{H}-2-22$ & 131.92 & 1.28 & 0.71 & 8.32 & 8.12 & 8.85 \\
\hline $15 \mathrm{H}-2-42$ & 132.12 & 4.86 & 2.23 & 1.69 & 0.85 & 8.89 \\
\hline $15 \mathrm{H}-2-62$ & 132.32 & 4.03 & 2.21 & 1.47 & 0.26 & 8.89 \\
\hline $15 \mathrm{H}-2-82$ & 132.52 & 5.65 & 8.64 & 3.84 & 8.87 & 8.38 \\
\hline $15 \mathrm{H}-2-102$ & 132.72 & 3.59 & 1.87 & 1.46 & 8.13 & 0.13 \\
\hline $15 \mathrm{H}-2-122$ & 132.92 & 9.75 & 5.31 & 2.48 & 0.30 & 1.66 \\
\hline $15 \mathrm{H}-2-142$ & 133.12 & 1.17 & 0.53 & 8.28 & 8.86 & 8.38 \\
\hline $15 \mathrm{H}-3-42$ & 133.42 & 1.34 & 8. 57 & 8.45 & 0.18 & 8.14 \\
\hline $15 \mathrm{H}-3-62$ & 133.62 & 1.32 & 8.75 & 8.34 & 0.18 & 0.85 \\
\hline $15 \mathrm{H}-3-82$ & 133.82 & 1.35 & 8.47 & 8.18 & 0.16 & 8.54 \\
\hline $15 \mathrm{H}-3-182$ & 134.82 & 8.99 & 8.34 & 8.28 & 8.05 & 8.48 \\
\hline $15 \mathrm{H}-3-122$ & 134.22 & 1.56 & 8.79 & 8.75 & 8.82 & 8.88 \\
\hline $15 \mathrm{H}-3-142$ & 134.42 & 1.74 & 8.77 & 8.78 & 8.14 & 8.85 \\
\hline $15 \mathrm{H}-4-22$ & 134.92 & 1.37 & 8.72 & 0.52 & 0.12 & 8.01 \\
\hline
\end{tabular}


Table 1.4 (continued).

\begin{tabular}{|c|c|c|c|c|c|c|}
\hline \multirow[b]{2}{*}{$\begin{array}{l}\text { Sample } \\
\text { no. }\end{array}$} & \multirow[b]{2}{*}{$\begin{array}{l}\text { Depth } \\
\text { (mbsf) }\end{array}$} & \multicolumn{5}{|c|}{ Subfraction of coarse fraction $(\mu \mathrm{m})$} \\
\hline & & $\begin{array}{l}>63 \\
(\%)\end{array}$ & $\begin{array}{c}63-125 \\
(\%)\end{array}$ & $\begin{array}{c}125-500 \\
(\%)\end{array}$ & $\begin{array}{c}500-1000 \\
(\%)\end{array}$ & $\begin{array}{c}>1000 \\
(\%)\end{array}$ \\
\hline $15 \mathrm{H}-4-42$ & 135.12 & 1.88 & 8.55 & 8.58 & 8.29 & 8.38 \\
\hline $15 H-4-62$ & 135.32 & 1.72 & 0.94 & 8.73 & 0.85 & 8.80 \\
\hline $15 H-4-82$ & 135.52 & 0.71 & 0.48 & 8.31 & 8.88 & 8.80 \\
\hline $15 \mathrm{H}-4-182$ & 135.72 & 1.87 & 0.65 & 8.37 & 8.80 & 0.85 \\
\hline $15 H-4-122$ & 135.92 & 8.52 & 0.31 & 0.17 & 8.84 & $\theta .88$ \\
\hline $15 \mathrm{H}-5-22$ & 136.42 & 1.08 & 0.62 & 0.18 & 8.11 & 8.09 \\
\hline $15 \mathrm{H}-5-62$ & 136.62 & 6.82 & 8.36 & 0.69 & 8.44 & 5.33 \\
\hline $15 \mathrm{H}-5-82$ & 136.82 & 0.99 & 0.36 & 8.45 & 0.13 & 0.85 \\
\hline $15 \mathrm{H}-5-182$ & 137.82 & 8.73 & 8.34 & 0.34 & 8.04 & 8.81 \\
\hline $15 \mathrm{H}-5-122$ & 137.22 & 0.75 & 0.36 & 0.34 & 8.85 & 8.88 \\
\hline $15 \mathrm{H}-5-142$ & 137.42 & 1.62 & 8.42 & 0.35 & 0.15 & 8.78 \\
\hline $15 H-6-22$ & 137.92 & 1.53 & 0.81 & 8.55 & 0.88 & 8.69 \\
\hline $15 \mathrm{H}-6-42$ & 138.12 & 8.61 & 0.29 & 0.25 & 8.85 & 0.82 \\
\hline $15 H-6-62$ & 138.32 & 7.44 & 3.58 & 3.16 & 8.24 & 8.54 \\
\hline $15 H-6-82$ & 138.52 & 1.71 & 8.72 & 8.87 & 0.88 & 8.84 \\
\hline $15 \mathrm{H}-7-22$ & 139.42 & 2.11 & 8.19 & 8.18 & 8.15 & 1.59 \\
\hline $15 \mathrm{H}-7-42$ & 139.62 & 8.88 & 8.29 & 8.21 & 8.88 & 8. 38 \\
\hline $15 \mathrm{H}-7-62$ & 139.82 & 7.22 & 3.99 & 3.87 & 0.12 & 8.84 \\
\hline $16 \mathrm{H}-1-23$ & 139.93 & 8.73 & 4.32 & 3.38 & 8.36 & 8.75 \\
\hline $16 \mathrm{H}-1-182$ & 148.72 & 1.18 & 0.51 & 0.48 & 8.10 & 8.89 \\
\hline $16 \mathrm{H}-1-128$ & 148.98 & 1.11 & 8.52 & 8.47 & 8.86 & 8.86 \\
\hline $16 \mathrm{H}-1-142$ & 141.12 & 1.41 & 8.46 & 8.32 & 8.86 & 8.57 \\
\hline $16 \mathrm{H}-2-23$ & 141.43 & 9.88 & 4.72 & 4.11 & 8.43 & 8.62 \\
\hline $16 \mathrm{H}-2-102$ & 142.02 & 8.78 & 8.48 & 8.32 & 8.85 & 0.01 \\
\hline $16 \mathrm{H}-2-128$ & 142.28 & 2.15 & 1.24 & 8.72 & 8.12 & 8.87 \\
\hline $16 \mathrm{H}-2-142$ & 142.42 & 6.39 & 4.86 & 2.13 & 0.12 & 0.88 \\
\hline $16 \mathrm{H}-3-23$ & 142.93 & 3.37 & 1.94 & 1.23 & 8.15 & 0.85 \\
\hline $16 \mathrm{H}-3-182$ & 143.72 & 1.11 & 0.63 & 0.25 & 8.89 & 0.14 \\
\hline $16 \mathrm{H}-3-128$ & 143.98 & 1.36 & 0.81 & 0.48 & 8.88 & 8.87 \\
\hline $16 \mathrm{H}-3-142$ & 144.12 & 0.84 & 8.29 & 8.33 & 0.19 & 8.83 \\
\hline $16 \mathrm{H}-4-23$ & 144.43 & 1.15 & 8.74 & 8.29 & 8.12 & 8.88 \\
\hline $16 \mathrm{H}-4-182$ & 145.82 & 1.45 & B. 41 & 8.42 & 0.14 & 0.48 \\
\hline $16 \mathrm{H}-4-128$ & 145.28 & 1.48 & 0.49 & 8.48 & 8.84 & 8.47 \\
\hline $16 \mathrm{H}-5-23$ & 145.93 & 0.82 & 0.37 & 8.37 & 8.03 & 8.85 \\
\hline $16 \mathrm{H}-5-182$ & 146.72 & 0.75 & 0.47 & 0.25 & 8.83 & 8.88 \\
\hline $16 \mathrm{H}-5-128$ & 146.92 & 1.29 & 0.58 & 0.49 & 8.88 & 8.14 \\
\hline $16 \mathrm{H}-5-142$ & 147.12 & 8.85 & 0.38 & 0.37 & 0.89 & 8.81 \\
\hline $16 \mathrm{H}-6-23$ & 147.43 & 4.68 & 2.34 & 1.87 & 8.22 & 8.25 \\
\hline $17 \mathrm{H}-1-23$ & 149.42 & 1.39 & 8.84 & 8.45 & 8.85 & 8.85 \\
\hline $17 \mathrm{H}-1-42$ & 149.62 & 1.87 & 0.57 & 8.45 & 8.85 & 8.88 \\
\hline $17 \mathrm{H}-1-122$ & 158.42 & 1.24 & B. 48 & 0.69 & 8.87 & 8.80 \\
\hline $17 H-1-142$ & 150.62 & 3.85 & 1.31 & 1.68 & 8.81 & 0.13 \\
\hline $17 \mathrm{H}-2-22$ & 158.92 & 2.85 & 1.28 & 8.61 & 8.86 & 0.18 \\
\hline $17 \mathrm{H}-2-42$ & 151.12 & 8.97 & 0.89 & 8.86 & 8.81 & 0.01 \\
\hline $17 \mathrm{H}-2-62$ & 151.22 & 4.74 & 3.45 & 1.25 & 0.82 & 8.82 \\
\hline $17 \mathrm{H}-3-22$ & 152.42 & 8.82 & 0.36 & 8.33 & 8.89 & 8.84 \\
\hline $17 \mathrm{H}-3-42$ & 152.62 & 1.38 & 8.54 & 8.37 & 8.86 & 0.33 \\
\hline $17 \mathrm{H}-3-62$ & 152.82 & 2.16 & 1.41 & 8.58 & 0.89 & 0.88 \\
\hline $17 \mathrm{H}-4-22$ & 153.92 & 8.89 & 3.32 & 1.72 & 2.45 & 1.48 \\
\hline $17 \mathrm{H}-4-42$ & 154.12 & 2.59 & 1.74 & 8.83 & 8.01 & 8.81 \\
\hline $17 \mathrm{H}-4-102$ & 154.32 & 1.09 & 8.73 & 0.28 & 8.88 & 8.88 \\
\hline $17 \mathrm{H}-4-122$ & 154.52 & 2.39 & 1.21 & 1.83 & 8.88 & 8.07 \\
\hline
\end{tabular}


Table 1.4 (continued).

\begin{tabular}{|c|c|c|c|c|c|c|}
\hline \multirow[b]{2}{*}{$\begin{array}{c}\text { Sample } \\
\text { no. }\end{array}$} & \multirow[b]{2}{*}{$\begin{array}{l}\text { Depth } \\
\text { (mbsf) }\end{array}$} & \multicolumn{5}{|c|}{ Subfraction of coarse fraction $(\mu \mathrm{m})$} \\
\hline & & $\begin{array}{l}>63 \\
(\%)\end{array}$ & $\begin{array}{c}63-125 \\
(\%)\end{array}$ & $\begin{array}{c}125-500 \\
(\%)\end{array}$ & $\begin{array}{c}500-1000 \\
(\%)\end{array}$ & $\begin{array}{c}>1000 \\
(\%)\end{array}$ \\
\hline $17 \mathrm{H}-4-142$ & 154.72 & 1.12 & 8.68 & 0.34 & 0.13 & 8.85 \\
\hline $17 \mathrm{H}-5-22$ & 155.42 & 1.65 & 0.77 & 0.46 & 8.89 & 0.33 \\
\hline $17 H-5-122$ & 156.42 & 1.44 & 0.75 & 8.47 & 8.17 & 0.85 \\
\hline $18 \mathrm{H}-1-42$ & 159.12 & 1.46 & 1.84 & 0.36 & 0.81 & 8.85 \\
\hline $18 \mathrm{H}-1-58$ & 159.30 & 2.61 & 1.54 & 8.66 & 8.86 & 8.35 \\
\hline $18 \mathrm{H}-1-182$ & 159.52 & 1.35 & 8.77 & 0.37 & 0.86 & 0.15 \\
\hline $18 \mathrm{H}-1-122$ & 159.72 & 8.99 & 0.78 & 8.24 & 8.84 & 0.81 \\
\hline $18 \mathrm{H}-1-142$ & 159.92 & 8.98 & 8.47 & 8.19 & 8.86 & 0.18 \\
\hline $18 \mathrm{H}-2-42$ & 168.62 & 1.31 & 8.73 & 0.58 & 8.83 & 0.85 \\
\hline $18 \mathrm{H}-2-58$ & 168.88 & 15.19 & 9.68 & 4.72 & 0.33 & 0.46 \\
\hline $18 \mathrm{H}-2-182$ & 161.28 & 1.48 & 8.82 & 8.51 & 8.85 & 8.82 \\
\hline $18 \mathrm{H}-2-122$ & 161.42 & 3.75 & 2.16 & 1.45 & 8.14 & 8.80 \\
\hline $18 \mathrm{H}-2-142$ & 161.62 & 1.93 & 8.97 & 0.85 & 8.18 & 8.01 \\
\hline $18 \mathrm{H}-3-42$ & 162.12 & 0.42 & 0.29 & 0.11 & 0.01 & 0.81 \\
\hline $18 \mathrm{H}-3-58$ & 162.38 & 14.85 & 6.89 & 6.63 & 0.32 & 0.21 \\
\hline $18 \mathrm{H}-3-182$ & 162.52 & 18.57 & 6.23 & 2.62 & 0.21 & 1.51 \\
\hline $18 \mathrm{H}-3-122$ & 162.72 & 0.45 & 8.33 & 0.11 & 0.01 & 0.88 \\
\hline $18 \mathrm{H}-3-142$ & 162.92 & 3.73 & 1.28 & 1.59 & 0.18 & 8.68 \\
\hline $18 \mathrm{H}-4-42$ & 163.62 & 2.34 & 8.64 & 8.71 & 8.89 & 8.98 \\
\hline $18 \mathrm{H}-4-58$ & 163.88 & 3.68 & 8.77 & 0.82 & 0.10 & 1.99 \\
\hline $18 \mathrm{H}-4-182$ & 164.28 & 8.46 & 8.24 & 0.28 & 8.81 & 0.81 \\
\hline $18 \mathrm{H}-5-42$ & 165.12 & 0.58 & 8.23 & 0.17 & 0.07 & 8.03 \\
\hline $18 \mathrm{H}-5-58$ & 165.38 & 1.61 & 8.50 & 8.78 & 0.28 & 0.85 \\
\hline $18 \mathrm{H}-5-102$ & 165.52 & 8.86 & 8.43 & 0.28 & 8.89 & 0.86 \\
\hline $18 \mathrm{H}-5-122$ & 165.72 & 1.89 & 8.37 & 8.38 & 8.17 & 0.17 \\
\hline $18 \mathrm{H}-5-142$ & 165.92 & 8.64 & 0.18 & 0.32 & 0.09 & 0.05 \\
\hline $18 \mathrm{H}-6-42$ & 166.62 & 8.48 & 8.21 & 8.14 & 8.81 & 8.84 \\
\hline $18 H-6-58$ & 166.88 & 1.72 & 8.69 & 0.68 & 0.22 & 0.21 \\
\hline $18 \mathrm{H}-7-42$ & 168.12 & 13.95 & 7.89 & 3.68 & 0.13 & 3.85 \\
\hline $18 \mathrm{H}-7-58$ & 168.38 & 8.36 & 8.21 & 0.14 & 0.81 & 0.80 \\
\hline $19 \mathrm{H}-2-22$ & 169.92 & 1.81 & 8.39 & 8.26 & 0.83 & 1.13 \\
\hline $19 H-2-82$ & 178.52 & 18.41 & 4.12 & 5.44 & 0.32 & 0.53 \\
\hline $19 H-2-180$ & 178.78 & 1.51 & 8.61 & 8.76 & 8.18 & 0.84 \\
\hline $19 \mathrm{H}-3-22$ & 171.42 & 1.35 & 8.48 & 0.57 & 0.08 & 8.22 \\
\hline $19 H-3-82$ & 172.02 & 2.29 & 1.07 & 0.67 & 0.10 & 0.45 \\
\hline $19 H-3-98$ & 172.22 & 1.58 & 0.65 & 8.41 & 8.21 & 0.23 \\
\hline $19 H-3-128$ & 172.48 & 0.89 & 0.58 & 0.28 & 0.12 & 0.07 \\
\hline $19 H-3-142$ & 172.62 & 8.86 & 8.46 & 8.27 & 0.86 & 0.87 \\
\hline $19 H-4-22$ & 172.92 & 1.37 & 0.78 & 0.32 & 0.13 & 0.14 \\
\hline $19 \mathrm{H}-4-82$ & 173.52 & 0.70 & 8.55 & 8.15 & 8.88 & 8.80 \\
\hline $19 H-4-188$ & 173.78 & 2.61 & 2.28 & 0.26 & 0.85 & 0.82 \\
\hline $28 \mathrm{H}-1-42$ & 178.12 & 2.88 & 1.44 & 1.28 & 0.11 & 0.85 \\
\hline $28 \mathrm{H}-1-82$ & 178.22 & 9.48 & 4.41 & 4.19 & 0.27 & 8.61 \\
\hline $2 \theta \mathrm{H}-1-182$ & 178.42 & 0.82 & 0.46 & 8.38 & 8.86 & 0.80 \\
\hline $28 \mathrm{H}-1-122$ & 178.62 & 8.48 & 0.19 & 8.28 & 8.81 & 0.80 \\
\hline $28 \mathrm{H}-1-142$ & 178.82 & 0.52 & 0.34 & 8.17 & 8.01 & 0.88 \\
\hline $28 \mathrm{BH}-2-42$ & 179.68 & 7.78 & 2.96 & 3.76 & 0.38 & 0.76 \\
\hline $28 \mathrm{H}-2-82$ & 179.80 & 1.91 & 1.16 & 0.64 & 8.08 & 0.83 \\
\hline $28 \mathrm{H}-2-182$ & 188.80 & 1.82 & 8.86 & 8.59 & 8.17 & 0.28 \\
\hline $28 \mathrm{H}-2-122$ & 188.28 & 1.73 & 1.34 & 8.34 & 8.83 & 0.82 \\
\hline $28 \mathrm{H}-2-142$ & 188.48 & 1.99 & 1.26 & 0.51 & 0.02 & 8.28 \\
\hline $28 \mathrm{H}-3-42$ & 181.18 & 8.31 & 0.28 & 0.18 & 8.01 & 8.80 \\
\hline
\end{tabular}


Table 1.4 (continued).

\begin{tabular}{|c|c|c|c|c|c|c|}
\hline \multirow[b]{2}{*}{$\begin{array}{l}\text { Sample } \\
\text { no. }\end{array}$} & \multirow[b]{2}{*}{$\begin{array}{l}\text { Depth } \\
\text { (mbsf) }\end{array}$} & \multicolumn{5}{|c|}{ Subfraction of coarse fraction $(\mu \mathrm{m})$} \\
\hline & & $\begin{array}{l}>63 \\
(\%)\end{array}$ & $\begin{array}{c}63-125 \\
(\%)\end{array}$ & $\begin{array}{c}125-500 \\
(\%)\end{array}$ & $\begin{array}{c}500-1000 \\
(\%)\end{array}$ & $\begin{array}{l}>1000 \\
(\%)\end{array}$ \\
\hline $28 \mathrm{BH}-3-82$ & 181.30 & 8.65 & 0.32 & 8.14 & 0.03 & 8.16 \\
\hline $28 \mathrm{H}-3-182$ & 181.50 & 1.52 & 8.36 & 0.29 & 0.22 & 8.65 \\
\hline $28 \mathrm{H}-3-122$ & 181.78 & 0.45 & 0.16 & 0.17 & 0.84 & 8.08 \\
\hline $28 \mathrm{H}-4-42$ & 182.68 & 1.76 & 8.61 & 0.34 & 8.84 & 8.77 \\
\hline $28 \mathrm{H}-4-82$ & 182.88 & 1.29 & 0.53 & 0.22 & 8.16 & 8.38 \\
\hline $28 \mathrm{H}-4-122$ & 183.28 & 0.38 & 0.29 & 0.08 & 0.81 & $\theta . \theta \theta$ \\
\hline $28 \mathrm{H}-5-42$ & 184.18 & 1.41 & 1.80 & 0.39 & $\theta .02$ & 8.80 \\
\hline $28 \mathrm{H}-5-82$ & 184.38 & 8.68 & 0.32 & 0.13 & 8.81 & 0.22 \\
\hline $28 \mathrm{H}-5-102$ & 184.48 & 8.65 & 0.29 & 0.28 & 8.05 & 0.03 \\
\hline $21 \mathrm{H}-1-22$ & 185.81 & 2.88 & 0.79 & 1.86 & 0.08 & 0.15 \\
\hline $21 \mathrm{H}-1-42$ & $185.2 \theta$ & 1.32 & 8.68 & 0.48 & 0.14 & 0.02 \\
\hline $21 \mathrm{H}-1-62$ & 185.39 & 1.38 & 8.54 & 0.63 & 8.13 & 0.08 \\
\hline $21 \mathrm{H}-1-82$ & 185.58 & 0.55 & 8.48 & 0.14 & 8.81 & 0.80 \\
\hline $21 \mathrm{H}-1-102$ & 185.77 & 1.21 & 1.89 & 0.10 & 0.82 & 8.80 \\
\hline $21 \mathrm{H}-1-122$ & 185.96 & 1.58 & 8.68 & 8.76 & 0.13 & 0.81 \\
\hline $21 H-1-142$ & 186.15 & 2.14 & 8.67 & 0.69 & 8.86 & 0.72 \\
\hline $22 \mathrm{H}-1-22$ & 187.81 & 3.44 & 1.45 & 1.69 & 8.18 & 8.12 \\
\hline $22 \mathrm{H}-1-42$ & 187.28 & 8.55 & 0.24 & 8.29 & 0.82 & 0.88 \\
\hline $22 \mathrm{H}-1-182$ & 187.77 & 8.88 & 0.52 & 0.33 & 0.82 & 0.01 \\
\hline $22 \mathrm{H}-1-122$ & 187.96 & 0.65 & 8.41 & 8.28 & 0.84 & 8.88 \\
\hline $22 \mathrm{H}-2-42$ & 188.62 & 8.86 & 0.84 & 0.02 & 0.88 & 8.80 \\
\hline $22 \mathrm{H}-2-62$ & 188.81 & 8.95 & 0.28 & 0.52 & 0.82 & 8.13 \\
\hline $22 \mathrm{H}-2-82$ & 189.88 & 1.41 & 0.38 & 0.79 & 0.19 & 0.85 \\
\hline $22 \mathrm{H}-2-182$ & 189.19 & 6.03 & 2.56 & 2.57 & 0.12 & 8.78 \\
\hline $22 \mathrm{H}-3-22$ & 189.86 & 1.10 & 0.29 & 8.32 & 8.05 & 8.44 \\
\hline $22 \mathrm{H}-3-42$ & 198.85 & 2.74 & 1.16 & 1.17 & 0.16 & 0.25 \\
\hline $22 \mathrm{H}-3-62$ & 198.24 & 3.25 & 1.41 & 1.44 & 0.14 & 8.26 \\
\hline $22 \mathrm{H}-3-182$ & 198.62 & 2.41 & 1.08 & 1.17 & 8.18 & 8.86 \\
\hline $22 \mathrm{H}-3-122$ & 198.81 & 4.65 & 2.16 & 2.12 & 8.18 & 0.27 \\
\hline $22 \mathrm{H}-4-22$ & 191.28 & 13.32 & 4.47 & 5.48 & 0.35 & 3.82 \\
\hline $22 \mathrm{H}-4-42$ & 191.47 & 1.97 & 8.81 & 1.03 & 0.87 & 0.06 \\
\hline $22 \mathrm{H}-4-62$ & 191.66 & 1.15 & 8.36 & 0.78 & 8.89 & 8.80 \\
\hline $22 \mathrm{H}-4-182$ & 192.84 & 8.71 & 8.89 & 8.43 & 0.15 & 8.84 \\
\hline $22 \mathrm{H}-4-121$ & 192.22 & 2.82 & 8.46 & 8.59 & 0.88 & 8.89 \\
\hline $22 \mathrm{H}-5-42$ & 192.98 & 2.59 & 0.18 & 8.46 & 8.36 & 1.59 \\
\hline $22 \mathrm{H}-5-62$ & 193.89 & 8.48 & 0.16 & 0.29 & 8.83 & 8.80 \\
\hline $22 \mathrm{H}-5-82$ & 193.28 & 8.49 & 8.21 & 8.27 & 8.81 & 8.88 \\
\hline $22 \mathrm{H}-5-98$ & 193.43 & 4.78 & 2.28 & 2.24 & 0.13 & 8.85 \\
\hline $23 \mathrm{H}-1-39$ & 194.45 & 1.66 & 8.63 & 0.83 & 0.14 & 8.86 \\
\hline $23 \mathrm{H}-2-42$ & 195.81 & 8.36 & 8.28 & 0.13 & 0.03 & 8.08 \\
\hline $23 \mathrm{H}-2-182$ & 196.34 & 1.88 & 0.88 & 8.88 & 0.87 & 0.85 \\
\hline $23 \mathrm{H}-2-122$ & 196.52 & 2.82 & 1.23 & 0.67 & 0.11 & 0.01 \\
\hline $23 \mathrm{H}-2-142$ & 196.78 & 27.31 & 9.41 & 16.99 & 0.48 & 8.43 \\
\hline $23 \mathrm{H}-3-42$ & 197.14 & 1.25 & 0.11 & 8.27 & 0.28 & 8.59 \\
\hline $23 \mathrm{H}-3-182$ & 197.68 & 4.26 & 1.66 & 2.24 & 8.22 & 0.14 \\
\hline $23 \mathrm{H}-3-122$ & 197.86 & 4.48 & 1.98 & 1.86 & 0.22 & 8. 42 \\
\hline $23 \mathrm{H}-3-142$ & 198.03 & 1.37 & 0.48 & 8.64 & 0.21 & 8.84 \\
\hline $23 \mathrm{H}-4-42$ & 198.48 & 1.86 & 8. 91 & 0.89 & 0.86 & 0.80 \\
\hline $23 \mathrm{H}-4-102$ & 199.81 & 8.64 & 8.46 & 0.16 & 0.82 & 0.80 \\
\hline $23 \mathrm{H}-4-122$ & 199.19 & 8.34 & 8.22 & 0.08 & 0.03 & 0.01 \\
\hline $23 \mathrm{H}-4-142$ & 199.37 & 0.31 & 0.14 & 0.11 & 8.84 & 0.02 \\
\hline $23 \mathrm{H}-5-42$ & 199.81 & 3.58 & 2.12 & 1.35 & 8.86 & 0.85 \\
\hline
\end{tabular}


Table 1.4 (continued).

\begin{tabular}{|c|c|c|c|c|c|c|}
\hline \multirow[b]{2}{*}{$\begin{array}{c}\text { Sample } \\
\text { no. }\end{array}$} & \multirow[b]{2}{*}{$\begin{array}{c}\text { Depth } \\
\text { (mbsf) }\end{array}$} & \multicolumn{5}{|c|}{ Subfraction of coarse fraction $(\mu \mathrm{m})$} \\
\hline & & $\begin{array}{l}>63 \\
(\%)\end{array}$ & $\begin{array}{c}63-125 \\
(\%)\end{array}$ & $\begin{array}{c}125-500 \\
(\%)\end{array}$ & $\begin{array}{c}500-1000 \\
(\%)\end{array}$ & $\begin{array}{l}>1000 \\
(\%)\end{array}$ \\
\hline $23 \mathrm{H}-5-102$ & 280.35 & 8.83 & 0.34 & 0.33 & 8.18 & 8.86 \\
\hline $23 \mathrm{H}-5-122$ & 280.53 & 1.06 & 0.22 & 0.28 & 0.28 & 8.44 \\
\hline $23 \mathrm{H}-5-142$ & 280.78 & 8.68 & 8.38 & 0.22 & $\theta . \theta 3$ & 0.85 \\
\hline $24 \mathrm{H}-1-28$ & 281.38 & 4.58 & 1.65 & 2.02 & 0.28 & 8.55 \\
\hline $24 \mathrm{H}-1-65$ & 201.74 & 19.13 & 2.97 & 0.26 & 0.02 & 15.88 \\
\hline $24 H-1-82$ & 201.98 & 1.73 & 0.96 & 0.53 & 0.22 & 0.82 \\
\hline $24 \mathrm{H}-1-102$ & 282.18 & 0.38 & 8.16 & 0.18 & 8.85 & 0.07 \\
\hline $24 \mathrm{H}-1-122$ & 282.38 & 8.76 & 0.56 & 0.12 & 8.82 & 0.06 \\
\hline $24 \mathrm{H}-1-134$ & 202.41 & 0.69 & 8.49 & 0.17 & 0.83 & 8.08 \\
\hline $24 \mathrm{H}-2-65$ & 203.21 & 0.68 & 0.27 & 0.31 & 0.82 & 0.80 \\
\hline $24 \mathrm{H}-2-102$ & 283.57 & 17.53 & 18.18 & 6.38 & 0.39 & 8.66 \\
\hline $24 H-2-128$ & 283.75 & 17.95 & 18.44 & 5.98 & 8.54 & 1.87 \\
\hline $24 \mathrm{H}-3-65$ & 284.68 & 1.60 & 0.42 & 8.24 & 0.81 & 0.93 \\
\hline $24 \mathrm{H}-3-82$ & 284.84 & 8.45 & 8.18 & 0.25 & 0.82 & 8.08 \\
\hline $24 \mathrm{H}-3-182$ & 205.84 & 8.62 & 0.22 & 0.33 & 8.87 & 8.88 \\
\hline $24 \mathrm{H}-3-122$ & 285.27 & 0.71 & 8.23 & 0.34 & 0.14 & 8.88 \\
\hline $24 \mathrm{H}-3-142$ & 285.43 & 0.26 & 0.17 & 0.89 & 0.08 & 8.80 \\
\hline $24 \mathrm{H}-4-28$ & 205.71 & 0.55 & 0.26 & 0.22 & 0.85 & 8.82 \\
\hline $25 \mathrm{H}-1-22$ & 286.28 & 3.73 & 0. 58 & 0.49 & 8.85 & 2.61 \\
\hline $25 \mathrm{H}-1-42$ & 286.48 & 1.86 & 8.46 & 0.47 & 8.88 & 0.05 \\
\hline $25 \mathrm{H}-1-62$ & 286.60 & 1.39 & 8.71 & 0.61 & 0.87 & 0.08 \\
\hline $25 \mathrm{H}-1-82$ & 286.80 & 0.46 & 0.32 & 0.14 & 8.80 & 8.80 \\
\hline $25 \mathrm{H}-1-182$ & 287.88 & 0.47 & 0.32 & 0.14 & 8.01 & 0.88 \\
\hline $25 H-1-122$ & 287.28 & 0.28 & 8.08 & 0.89 & 8.83 & 8.88 \\
\hline $25 \mathrm{H}-2-22$ & 287.78 & 0.46 & 0.32 & 0.12 & 8.02 & 8.80 \\
\hline $25 \mathrm{H}-2-42$ & 287.98 & 1.27 & 0.72 & 0.46 & 8.88 & 8.81 \\
\hline $25 \mathrm{H}-2-62$ & 288.12 & 8.76 & 8.36 & 0.34 & 8.86 & 8.88 \\
\hline $25 \mathrm{H}-2-82$ & 288.32 & 4.84 & 1.66 & 1.74 & 8.12 & 0.52 \\
\hline $25 \mathrm{H}-2-182$ & 288.52 & 8.85 & 8.28 & 0.46 & 0.07 & 8.84 \\
\hline $25 \mathrm{H}-2-142$ & 289.10 & 8.44 & 8.27 & 0.10 & 0.01 & 8.86 \\
\hline $25 \mathrm{H}-3-22$ & 289.28 & 1.38 & 8.69 & 0.38 & 0.18 & 8.13 \\
\hline $25 \mathrm{H}-3-42$ & 289.48 & 8.71 & 0.18 & 0.28 & 8.87 & 0.26 \\
\hline $25 \mathrm{H}-3-62$ & 289.68 & 1.62 & 0.68 & 8.76 & 8.85 & 0.13 \\
\hline $26 \mathrm{H}-1-22$ & 218.71 & 1.41 & 0.63 & 8.55 & 8.28 & 0.83 \\
\hline $26 \mathrm{H}-1-42$ & 218.91 & 1.34 & 0.66 & 8.58 & 8.88 & 8.02 \\
\hline $26 \mathrm{H}-1-62$ & 211.18 & 8.72 & 0.28 & 0.36 & 8.87 & 8.81 \\
\hline $26 \mathrm{H}-1-82$ & 211.38 & 8.91 & 0.18 & 8.38 & 8.17 & 8.18 \\
\hline $26 \mathrm{H}-2-22$ & 212.17 & 4.51 & 8.78 & 1.89 & 8.42 & 2.38 \\
\hline $26 \mathrm{H}-2-42$ & 212.36 & 14.84 & 7.61 & 6.12 & 0.11 & 0.28 \\
\hline $26 \mathrm{H}-2-62$ & 212.56 & 0.88 & 8.36 & 0.34 & 0.85 & 8.13 \\
\hline $26 \mathrm{H}-2-82$ & 212.75 & 3.88 & 1.87 & 1.35 & 8.15 & 8.51 \\
\hline $26 \mathrm{H}-2-102$ & 212.94 & 1.28 & 8.53 & 0.68 & 0.07 & 8.08 \\
\hline $26 \mathrm{H}-2-122$ & 213.14 & 0.84 & 0.28 & 0.42 & 8.89 & 8.05 \\
\hline $26 \mathrm{H}-2-142$ & 213.33 & 0.79 & 0.22 & 8.38 & 8.84 & 0.23 \\
\hline $26 \mathrm{H}-3-22$ & 213.62 & 2.37 & 0.92 & 0.66 & 8.38 & 0. 41 \\
\hline $26 \mathrm{H}-3-42$ & 213.82 & 8.77 & 8.28 & 0.35 & 8.89 & 8.05 \\
\hline $27 \mathrm{H}-1-182$ & 215.38 & 1.33 & 0.64 & 0.55 & 0.11 & 8.83 \\
\hline $27 \mathrm{H}-1-122$ & 215.50 & 4.91 & 1.89 & 2.28 & 8.31 & 8.43 \\
\hline $28 \mathrm{H}-1-182$ & 217.85 & 4.93 & 1.43 & 2.43 & 8.16 & 8.91 \\
\hline $28 \mathrm{H}-1-122$ & 217.23 & 0.39 & 0.17 & 8.28 & 0.82 & 8.80 \\
\hline $28 \mathrm{H}-1-142$ & 217.42 & 0.69 & 0.89 & 8.14 & 8.86 & 8.48 \\
\hline $28 \mathrm{H}-2-182$ & 218.44 & 12.87 & 6.57 & 4.82 & 0.34 & 0.34 \\
\hline
\end{tabular}


R. HENRICH, T. WOLF, G. BOHRMANN, J. THIEDE

Table 1.4 (continued).

\begin{tabular}{|c|c|c|c|c|c|c|}
\hline \multirow[b]{2}{*}{$\begin{array}{l}\text { Sample } \\
\text { no. }\end{array}$} & \multirow[b]{2}{*}{$\begin{array}{l}\text { Depth } \\
\text { (mbsf) }\end{array}$} & \multicolumn{5}{|c|}{ Subfraction of coarse fraction $(\mu \mathrm{m})$} \\
\hline & & $\begin{array}{l}>63 \\
(\%)\end{array}$ & $\begin{array}{c}63-125 \\
(\%)\end{array}$ & $\begin{array}{c}125-500 \\
(\%)\end{array}$ & $\begin{array}{c}500-1000 \\
(\%)\end{array}$ & $\begin{array}{l}>1000 \\
(\%)\end{array}$ \\
\hline $28 \mathrm{H}-2-122$ & 218.63 & 8.46 & 8.15 & 0.13 & 8.85 & 0.13 \\
\hline $28 \mathrm{H}-2-142$ & 218.82 & 1.34 & 0.18 & 8.28 & 8.88 & 0.88 \\
\hline $28 \mathrm{H}-3-182$ & 219.84 & 8.38 & 3.49 & 3.91 & 8.44 & 8.54 \\
\hline $29 \mathrm{H}-1-182$ & 222.18 & 8.57 & 0.21 & 8.24 & 8.87 & 0.85 \\
\hline $29 \mathrm{H}-1-122$ & 222.38 & 0.78 & 0.27 & 0.38 & 0.18 & 0.03 \\
\hline $29 H-1-142$ & 222.58 & 0.47 & 8.28 & 8. 22 & 8.04 & 0.81 \\
\hline $29 \mathrm{H}-2-182$ & 223.68 & 8.51 & 0.13 & 8.29 & 0.05 & 8.84 \\
\hline $29 \mathrm{H}-2-122$ & 223.88 & 0.81 & 8.25 & 8.49 & 0.86 & 8.81 \\
\hline $29 \mathrm{H}-2-142$ & 223.98 & 0.63 & 8.27 & 8.32 & 8.84 & 0.80 \\
\hline $29 \mathrm{H}-3-182$ & 225.10 & 1.83 & 0.78 & 0.41 & 0.13 & 0.51 \\
\hline $29 \mathrm{H}-3-122$ & 225.30 & 2.22 & 8.72 & 8.64 & 8.24 & 0.62 \\
\hline $38 \mathrm{H}-1-182$ & 227.12 & 3.52 & 1.28 & 1.34 & 8.18 & 8.72 \\
\hline $30 \mathrm{H}-1-122$ & 227.32 & 4.27 & 0.95 & 8.95 & 0.25 & 2.12 \\
\hline зөн-1-142 & 227.51 & 1.14 & 0.34 & 8.58 & 0.18 & 0.12 \\
\hline $38 \mathrm{H}-2-182$ & 228.68 & 1.16 & 8.83 & 0.27 & 8.86 & 8.80 \\
\hline зөн-2-122 & 228.82 & 1.85 & 8.43 & 0.31 & 0.18 & 0.13 \\
\hline зөн-2-142 & 229.82 & 1.78 & 8.48 & 0.67 & 0.12 & 0.59 \\
\hline $30 \mathrm{H}-3-182$ & 230.12 & 8.84 & 0.24 & 0.46 & 0.11 & 0.03 \\
\hline $30 \mathrm{H}-3-122$ & 230.32 & 1.98 & 0.27 & 8.73 & 8.22 & 0.68 \\
\hline $38 \mathrm{H}-3-142$ & 238.52 & 3.27 & 1.74 & 1.48 & 0.11 & 8.82 \\
\hline зен-4-182 & 231.68 & 1.25 & 0.15 & 0.28 & 0.15 & 0.67 \\
\hline $38 \mathrm{H}-4-122$ & 231.82 & 8.76 & 0.33 & 8.33 & 0.86 & 8.84 \\
\hline $38 \mathrm{H}-4-142$ & 232.82 & 2.59 & 1.11 & 1.88 & 0.48 & 0.08 \\
\hline $31 \mathrm{H}-1-182$ & 233.18 & 18.19 & 4.43 & 4.57 & 8.32 & 0.87 \\
\hline $31 H-1-142$ & 233.32 & 2.13 & 0.85 & 8.88 & 0.15 & 8.33 \\
\hline $31 \mathrm{H}-2-182$ & 234.68 & 1.51 & 0.85 & 8.56 & 0.18 & 0.80 \\
\hline $31 H-2-142$ & 234.92 & 1.28 & 8.79 & 8. 33 & 0.88 & 8.80 \\
\hline $31 \mathrm{H}-3-102$ & 236.12 & 0.84 & 0.47 & 0.34 & 8.03 & 8.88 \\
\hline $31 \mathrm{H}-3-122$ & 236.32 & 1.24 & 0.75 & 0.39 & 0.18 & 0.80 \\
\hline $32 \mathrm{H}-2-102$ & 248.62 & 1.18 & 8.75 & 8.48 & 0.03 & 8.88 \\
\hline $32 \mathrm{H}-2-122$ & 248.82 & 1.36 & 0.64 & 0.54 & 0.85 & 8.13 \\
\hline $32 \mathrm{H}-2-142$ & 241.82 & 1.36 & 8.66 & 8.49 & 8.85 & 0.16 \\
\hline $32 \mathrm{H}-3-102$ & 242.12 & 1.88 & 8.78 & 8.55 & 8.48 & 0.15 \\
\hline $32 \mathrm{H}-3-122$ & 242.32 & 2.88 & 8.69 & 0.95 & 8.31 & 0.13 \\
\hline $32 \mathrm{H}-3-142$ & 242.52 & 2.36 & 8.88 & 1.88 & 8.32 & 0.16 \\
\hline $32 \mathrm{H}-4-182$ & 243.68 & 1.35 & 0.69 & 8.47 & 0.19 & 8.88 \\
\hline $32 \mathrm{H}-4-122$ & 243.82 & 1.13 & 0.57 & 8.48 & 8.88 & 0.80 \\
\hline $33 H-1-182$ & 244.72 & 3.98 & 1.83 & 1.45 & 0.28 & 1.14 \\
\hline $33 \mathrm{H}-1-122$ & 244.92 & 1.28 & 0.60 & 0.52 & 8.16 & 0.88 \\
\hline $33 H-1-142$ & 245.12 & 1.66 & 8.47 & 8.49 & 0.51 & 0.19 \\
\hline $34 H-1-102$ & 246.86 & 2.62 & 0.69 & 0.86 & 8.30 & 8.77 \\
\hline $34 \mathrm{H}-1-122$ & 247.82 & 1.82 & 0.94 & 0.53 & 8.21 & 8.14 \\
\hline $34 \mathrm{H}-1-142$ & 247.19 & 1.24 & 8.66 & 0.49 & 8.09 & 8.80 \\
\hline $34 \mathrm{H}-2-102$ & 248.12 & 0.88 & 8.46 & 0.29 & 8.85 & 8.88 \\
\hline $34 \mathrm{H}-2-122$ & 248.28 & 0.77 & 0.37 & 0.21 & 8.87 & 8.12 \\
\hline $34 \mathrm{H}-2-142$ & 248.45 & 1.37 & 1.85 & 0.22 & 0.84 & 8.06 \\
\hline $34 \mathrm{H}-4-182$ & 258.64 & 1.25 & 8.66 & 8.57 & 0.82 & 8.88 \\
\hline $34 \mathrm{H}-4-122$ & 258.88 & 8.80 & 0.24 & 8.33 & 0.14 & 8.89 \\
\hline $34 \mathrm{H}-5-102$ & 251.98 & 1.81 & 1.80 & 8.49 & 0.23 & 8.89 \\
\hline $34 \mathrm{H}-5-122$ & 252.86 & 1.81 & 0.78 & 0.22 & 0.09 & 8.88 \\
\hline $34 \mathrm{H}-5-142$ & 252.23 & 1.04 & 0.65 & 0.33 & 8.86 & 8.88 \\
\hline
\end{tabular}

